\title{
Neuroepigenomics in Alzheimer's disease
}

\author{
Citation for published version (APA):
}

Riemens, R. J. M. (2021). Neuroepigenomics in Alzheimer's disease: the single cell ADds. [Doctoral Thesis, Maastricht University, University of Würzburg]. Ipskamp. https://doi.org/10.26481/dis.20211105rr

Document status and date:

Published: 01/01/2021

DOI:

10.26481/dis.20211105rr

Document Version:

Publisher's PDF, also known as Version of record

\section{Please check the document version of this publication:}

- A submitted manuscript is the version of the article upon submission and before peer-review. There can be important differences between the submitted version and the official published version of record.

People interested in the research are advised to contact the author for the final version of the publication, or visit the DOI to the publisher's website.

- The final author version and the galley proof are versions of the publication after peer review.

- The final published version features the final layout of the paper including the volume, issue and page numbers.

Link to publication

\footnotetext{
General rights rights.

- You may freely distribute the URL identifying the publication in the public portal. please follow below link for the End User Agreement:

www.umlib.nl/taverne-license

Take down policy

If you believe that this document breaches copyright please contact us at:

repository@maastrichtuniversity.nl

providing details and we will investigate your claim.
}

Copyright and moral rights for the publications made accessible in the public portal are retained by the authors and/or other copyright owners and it is a condition of accessing publications that users recognise and abide by the legal requirements associated with these

- Users may download and print one copy of any publication from the public portal for the purpose of private study or research.

- You may not further distribute the material or use it for any profit-making activity or commercial gain

If the publication is distributed under the terms of Article $25 \mathrm{fa}$ of the Dutch Copyright Act, indicated by the "Taverne" license above, 
Neuroepigenomics in Alzheimer's disease: The single cell ADds 
COPYRIGHT: @ Renzo J.M. Riemens, Maastricht 2021

Neuroepigenomics in Alzheimer's disease: The single cell ADds

All rights reserved. No part of this thesis may be reproduced, distributed, or transmitted in any form or by any means, without the prior written permission of the copyright holder.

Lay-Out: Renzo J.M. Riemens and Ipskamp Maastricht

Cover Design: Renzo J.M. Riemens

Published By: Maastricht University

Isbn: 978-94-6423-524-1

Printed By: Ipskamp Maastricht 


\title{
Neuroepigenomics in Alzheimer's disease: The single cell ADds
}

Neuroepigenomik bei der Alzheimer-Krankheit: Die Einzelzell ADds

\section{DISSERTATION}

\author{
To obtain the degree of \\ Doctor at Maastricht University, \\ on the authority of the Rector Magnificus, \\ Prof. dr. Rianne M. Letschert, \\ in accordance with the decision of the Board of Deans
}

and

to obtain the degree of Doctor of Philosophy in Natural Sciences

Dr. rer. Nat. at Julius-Maximilians-Universität Würzburg,

on the authority of the President Magnificus,

Prof. dr. Alfred Forchel,

in accordance with the decision of the Graduate School of Life Sciences

Common Graduation Commission

To be defended in public on Friday 5 November 2021, at 10.00 hours

by

Renzo J.M. Riemens

This thesis was written under a jointly supervised PhD degree programme of Maastricht University, Maastricht, the Netherlands and Julius-MaximiliansUniversität Würzburg, Würzburg, Germany. 
Maastricht University

Promotors

Prof. Dr. Daniël L.A. van den Hove

Prof. Dr. Bart P.F. Rutten

\section{Co-Promotor}

Dr. Gunter Kenis

Julius-Maximilians-Universität Würzburg

Promotor

Prof. Dr. Thomas Haaf (Primary supervisor)

Co-promotors

Prof. Dr. Charlotte Förster (Second supervisor)

Prof. Dr. Klaus-Peter Lesch (Third supervisor)

Prof. Dr. Daniël L.A. van den Hove (Fourth supervisor)

Graduate School of Life Sciences (GSLS) chairperson

Prof. Dr. Christian Wegener

\section{Assessment Committee}

Prof. Dr. Jos H.H.J. Prickaerts (Maastricht University) (Chair)

Dr. Angelika Schmitt-Böhrer (Julius Maximilian University, Germany)

Prof. Dr. Charlotte Förster (Julius Maximilian University, Germany)

Prof. Dr. Geraldine Zimmer-Bensch (Aachen University, Germany)

Prof. Dr. Paul J. Lucassen (University of Amsterdam, the Netherlands)

Dr. Tim Vanmierlo (Maastricht University) 
Table of contents

Chapter 1 General introduction

Chapter 2 Epigenome-wide association studies in Alzheimer's

Disease; Achievements and challenges

Chapter 3 Targeted detection of unmodified cytosine, 5-methylcytosine and 5-hydroxymethylcytosine levels at single CpG sites by oxidative bisulfite pyrosequencing

Chapter 4 Targeted methylation profiling of single laser-capture microdissected post-mortem brain cells by adapted limiting dilution bisulfite pyrosequencing (LDBSP)

Chapter 5 Brain-region- and cell type-specific epigenetic profiling strongly implicates a role for dysregulation of TNXB and other loci in the brainstem in Alzheimer's disease

Chapter 6 Human-induced pluripotent stem cells as a model for sporadic Alzheimer's disease

Chapter 7 Directing neuronal cell fate in vitro: Achievements and challenges

Chapter 8 Establishment and characterization of a human neuronal in vitro model system for Alzheimer's disease using induced pluripotent stem cells: An exploratory approach

Chapter 9 General discussion

Chapter 10 Summary

Chapter 11 Samenvatting

Chapter 12 Zusammenfassung

Chapter 13 Impact paragraph

Chapter 14 Curriculum vitae 355

$\begin{array}{lll}\text { Chapter } 15 & \text { List of publications } & 361\end{array}$

Chapter 16 Statement of individual author contributions 365

$\begin{array}{lll}\text { Chapter } 17 \text { Affidavit } & 385\end{array}$

$\begin{array}{lll}\text { Chapter } 18 & \text { Acknowledgements } & 389\end{array}$ 

Chapter 1

General introduction 



\section{Alzheimer's disease}

Even though the first records of Alzheimer's disease (AD) date back to millennia ago, our knowledge of its underlying pathophysiology is little more than a century old [1]. The German psychiatrist and neuropathologist Alois Alzheimer published his now famous case study of Auguste Deter in 1906 [2]. Since then, we have witnessed an outburst of basic and translational research into the underlying molecular mechanisms, risk factors and potential treatment strategies for $A D$. Research into AD began with profiling of the disease-associated neuropsychological deficits. Further efforts lead to e.g. the identification of cognitive mechanisms affected by the neuropathological hallmarks observed in AD brains, the discovery of genetic, as well as environmental risk factors contributing to the disease, and the development of transgenic animal models harboring AD hallmarks.

Despite the considerable advances in more than a century, gaps of knowledge on the etiopathophysiology of $A D$ continue to exist. In fact, $A D$ nowadays ranks among the most challenging health care problems worldwide, as it remains incurable and impossible to detect in a timely manner that could improve treatment outcome. It has been estimated that $A D$ and related dementias currently affect around 50 million people worldwide, a figure that is projected to double every 20 years to 100 million cases in 2040 [3]. This exponential increase, both in developed and developing countries, possesses a huge social burden on both patients, their direct family members, caregivers and society as a whole. The progressive nature of $A D$, leading to severe functional deterioration and a need for constant care, is one of the major determinants of this burden. Consequently, the magnitude of this disease has a devastating societal and financial impact on caregivers and the healthcare system resources. Unless an effective treatment will be developed, the medical, as well as non-medical costs, are estimated to surpass one trillion euro by 2050 [3]. As such, the need for a better understanding, more advanced AD models, better diagnostic tools and more effective therapeutics today is more urgent than ever, given the anticipated, alarming increase in incidence in our everaging population.

As it is currently understood, AD is as a chronic, progressive neurodegenerative disease associated with memory loss and cognitive decline [4]. Other behavioral and psychological symptoms of $A D$, which might vary with time and per individual, include agitation, apathy, disturbances in motor behavior, deficiencies in language, elation, irritability, disinhibition, delusions, hallucinations, changes in sleep or appetite, and anxiety and depression [5]. The main pathological hallmarks observed in $A D$ brains are depositions of extracellular amyloid beta $(A B)$ plaques and intracellular neurofibrillary tangles consisting of hyperphosphorylated tau protein [6]. Substantial evidence supports that these depositions are central to the 
neurobiology of the disease, as they are causally related to the neurodegenerative processes leading to cortical and subcortical atrophy typically observed in $A D$ brains [7]. Other molecular hallmarks of $A D$ include oxidative stress, dysfunctional calcium homeostasis and neuroinflammation, ultimately contributing to the loss of neurons and synapses [8-10]. This loss in neuronal integrity is known to contribute to a disequilibrium in neurotransmitter systems, affecting most, if eventually not all, systems found in the human brain, including glutamate, acetylcholine, dopamine, noradrenaline and serotonin [11]. It has been proposed that a region-specific disbalance in these neurotransmitter systems underlies the behavioral changes observed in $A D$ patients.

It is worth mentioning that aberrant $A \beta$ and tau proteins already start accumulating in the brain long before the manifestation of the first clinical symptoms [12]. The pathological process spans decades and, during this time, the distribution pattern of neurofibrillary tangles appears to develop according to a hierarchical temporospatial pattern [13]. Although $A \beta$ burden has been associated with the neurobiological basis of the disease, only tau pathology has been shown to be directly correlated with progression of cognitive deterioration and clinical symptoms in AD. Braak and Braak therefore introduced a staging system based on aberrant tau, i.e. Braak staging, which describes the initial appearance of tauopathy in the transentorhinal regions (stages I and II) that spreads to limbic regions (stages III and IV) and finally to neocortical areas (stages $\mathrm{V}$ and $\mathrm{VI}$ ) [13]. However, more recent studies suggested that tauopathy may begin earlier than previously thought and possibly in the lower brainstem rather than in the transentorhinal regions $[14,15]$. Furthermore, the occurrence of various non-cognitive behavioral and neuropsychological symptoms in AD suggests brainstem involvement, specifically that of the dorsal raphe nuclei (DRN) and the locus coeruleus (LC). Even though one cannot ignore the later involvement of entorhinal, limbic and cortical neurodegeneration in relation to the cognitive symptoms, critical questions on the temporal and spatial causality did arise in this respect during the last years. Research on the pathogenesis of $A D$ is therefore currently expanding to areas such as the DRN and the LC.

An individual person's risk of developing AD pathology is determined by genetic, environmental and demographic risk factors, as well as the complex interactions between them. From a genetic point of view, $A D$ can be classified into two forms of dementia: familial $A D(f A D)$ and sporadic $A D(S A D)$. Although both types appear identical in light of clinical features and neuropathological hallmarks, they are distinguishable based on to their etiology and time of onset. Generally, fAD is referred to as the early onset form of $A D$, occurring between 30 and 60 years of age, although later development in life has also been observed in patients. 
The most critical difference when compared to $S A D$, is that fAD is inherited in a mendelian fashion, resulting from autosomal-dominant mutations in genes involved in $A \beta$ processing that lead to increased neurotoxic $A \beta$ aggregation, including amyloid precursor protein (APP), presenilin 1 (PSEN1) and presenilin 2 (PSEN2) [16]. Notably, it is estimated that fAD patients only represent less than $5 \%$ of all $A D$ cases. $S A D$, representing the most common variant of $A D$, is estimated to affect more than $95 \%$ of all cases, while having a time of onset starting at around the age of 65 years, with a doubling likelihood of developing the disease every 5 years thereafter [17]. SAD has a less obvious and more diffuse, polygenic, genetic underpinning when compared to $f A D$ and is known to be multifactorial with a life-long etiopathogenesis. However, since theories on contributing factors and the preliminary appearance of pathological hallmarks are constantly changing, a thorough understanding of its etiology remains largely elusive, and a definite, unifying hypothesis on the exact cause-effect relationships of these hallmarks, despite decades of research, remains to be formulated.

Over the last years, large genome wide-association studies (GWAS) have identified genetic risk, as well as protective, loci for the development of $S A D$, including ABCA7, BIN1, TREM2, CD33, CLU, CRI, EPHA1, MS4A, PICALM, and SORL1, among others [18-24]. These genes have been associated with $A \beta$ and tau processing, lipid metabolism, immune activation and synaptic function, which all have been linked to AD [25]. Nonetheless, the risk effects exerted by these individual genes are small, that is they confer only a $\sim 0.10$ - to 0.15 -fold increase in carriers versus non-carriers [16]. As such, the impact of genetic variation around these loci remains significantly lower than that of $A P O E$, which for many years was the only major gene known to increase SAD risk, but on itself not sufficient enough to cause the disease [26]. Of note in this respect is the fact that many individuals may carry prominent genetic risk factors for $S A D$, as well as express profound $A \beta$ and tau pathology, yet never develop the disease [27]. Indeed, even monozygotic twins can have indefinite SAD outcomes [28]. Thus, there is currently no single genetic model that explains the mode of disease transmission and it has been estimated that only half of the phenotypic variance in SAD is attributed to solely genetic factors [29]. Altogether, it is now hypothesized that both yet unidentified rare genetic variants with large effect sizes, interactions between different loci, as well as non-genetic factors, contribute to the "missing heritability" of SAD, which emphasizes its complexity.

Various conditions and environmental factors have been identified that either positively or negatively influence the development of SAD. While ageing is known to be the major risk factor, pre-existing conditions such as obesity, diabetes, hypertension and a previous diagnosis of depression have been shown to increase 
the risk of developing the disease [30]. Additionally, exposure to heavy metals, smoking and alcoholism, are also considered as risk factors for SAD development [31, 32]. Physical exercise, cognitive reserve, high levels of education and various nutritional factors, on the other hand, have been depicted as protective factors $[30,31,33]$. Studies have furthermore demonstrated that affective experiences may also play a role in the pathogenesis of the disease. While prolonged and/ or repetitive exposure to stress has been associated with a faster progression of sAD, mental well-being or having a general sense of purpose in life is associated with lower AD pathology [34, 35]. As already addressed earlier, it is known that in AD patients psychopathology occurs (long) before the cognitive symptoms appear, which suggests that adverse psychological experiences could potentially also cause or exacerbate AD-related neuropathology. In fact, affective symptomatology could also represent an early sign, i.e. a prodromal phase, of the disease. All in all, since environmental factors alone also fail to explain SAD development, it is currently becoming more clear that the etiology of the disease likely dependents on the synergistic effects of both genetic and environmental factors [36].

In this latter context, epigenetic processes, which mediate the interaction between the genome and the environment, could provide a mechanistic explanation in view of the etiology of SAD [37]. In addition to an individual's genetic susceptibility, environmental insults could disturb epigenetic mechanisms of gene expression with adverse consequences, concomitant with developing pathology as a result. These epigenetic changes, which can occur throughout life, could evolve into profound $A D$ pathology or interact with the embedded effects of other contributing factors to both induce and influence disease development and progression. The model explained here advocates for a combination of genetics, environmental risk factors and epigenetic mechanisms, where these factors may operate synergistically during the pre-clinical phase of the disease, even decades before the appearance of the first clinical symptoms. In recent years, we have therefore seen a significant increase in studies focusing on the role of epigenetic mechanisms in the development and course of SAD.

\section{Epigenetic mechanisms: DNA methylation and hydroxymethylation}

The term epigenetics, initially formulated by Conrad Hal Waddington in 1942, implies "on top of" or "in addition to" genetics, referring to changes in gene expression that cannot be explained by changes in the DNA sequence [38]. The meaning of the term epigenetics, however, has itself undergone an evolution that resembles our dramatically deepened understanding of the mechanisms that underlie gene regulation [39]. Our present definition has moved from being solely regarded as a regulatory process of gene silencing and activation during development, e.g. in view of cellular differentiation, to a crucial and highly dynamic plethora of 
processes involved in translating exposure to environmental stimuli to changes in gene expression patterns. As such, a unique characteristic that differentiates epigenetic from genetic variation is that epigenetic processes are responsive to the environment in a more dynamic fashion. Accordingly, their manifestation can have deleterious effects, e.g. in response to adverse environmental stimuli, a process which nowadays is thought to contribute to SAD [40]. These changes may be reflected at various stages throughout an individual's life, and even in subsequent generations, through a process termed transgenerational epigenetic inheritance [41, 42].

From a molecular point of view, epigenetic mechanisms consist of DNA modifications, histone modifications and noncoding RNAs, each offering a distinct - though often interdependent - layer of transcriptional control [43]. Collectively, these mechanisms determine the chromatin architecture, the concomitant accessibility of a genetic locus to the transcriptional machinery, while they are often also involved in the regulation of post-transcriptional processes [44-46]. Recent work argues that particularly covalent modifications to the DNA and histones are modifiable throughout the lifespan and that these can be altered by a person's experiences [47]. Alterations in the chemistry of these modifications can bring about chromatin remodeling processes, changing the accessibility of the DNA to the transcriptional machinery, and, thereby, gene expression patterns. Thus, environmental variation possesses means of changing these modifications, although some are more programmed and persistent than others, as seen during e.g. stem cell differentiation [48]. As such, these DNA modifications can be highly stable, as observed in genomic imprinting, or highly dynamic, such as epigenetic changes associated with memory formation [49, 50]. Importantly, whereas genetic alterations usually reflect permanent changes of the DNA sequence, epigenetic changes generally reflect reversible processes, still, with a pivotal role in regulating specific brain functions and related behaviors. Of note, the exact mechanisms by which the environment induces its effects on the epigenetic machinery remains largely unknown.

To date, the most extensively characterized epigenetic DNA modification is methylation, which involves the addition of a methyl group at the 5 position on the pyrimidine ring of a cytosine, i.e. 5-methylcytosine (5-mC) [44, 51]. DNA methylation occurs mainly at cytosine-phosphate-guanine $(\mathrm{CpG})$ positions, although non-CpG cytosine, guanine and adenine methylation have also been reported [43]. CpG sites are primarily packed in so-called CpG islands, which are stretches longer than 200 base pairs that have over $50 \%$ of $\mathrm{CpG}$ content [52]. These islands are often part of regulatory elements, including gene promoters. Historically, DNA methylation in promotor regions has been associated with transcriptional repression, although 
recent findings have also demonstrated that gene expression can be enhanced in a methylation-dependent manner [43, 53, 54]. Overall, the promotor methylation status directly influences the possible binding of transcription factors, which interact with histone-modifying enzymes that regulate chromatin accessibility, hence modulating transcriptional activity $[55,56]$. In addition, DNA methylation within the transcribed portion of a gene is also observed and has been implicated in e.g. alternative splicing [57], although it remains to be further elucidated how gene expression is exactly regulated through gene body methylation. Aside from methylation, several other DNA modifications have also been described, including 5-hydroxymethylcytosine (5-hmC), 5-formylcytosine (5-fC) and 5-carboxylcytosine (5-caC). The derivation of these other modifications generally occurs through the process of active DNA demethylation, which implies the removal of the methyl group from cytosine bases [58]. This process is achieved by the oxidation of the methyl group by ten-eleven translocation (TET) enzymes, leading to the sequential production of each of these modifications, respectively.

Although initially considered solely as an intermediate modification during the process of active demethylation, DNA hydroxymethylation, has also been shown to exist as a stable epigenetic mark, similar to DNA methylation. Interestingly, it has been demonstrated that 5 -hmC is highly enriched in the brain, where, in parallel to $5-\mathrm{mC}$, it is thought to fulfill an important role during neurodevelopment and memory formation, among others [59,60]. Furthermore, studies have shown that 5 -hmC is also important in pluripotent stem cell processes, such as proliferation and neural differentiation [61]. At the DNA level, 5-hmC can be found both at CpG sites and at non-CpG dinucleotides [62]. Genome-wide localization experiments have demonstrated that 5 -hmC can be found in and around promoter regions, enhancers, transcription start sites and in intragenic regions [63, 64]. In addition, it has been suggested that changes in the $5-\mathrm{hmC}$ architecture are involved in chromatin remodeling processes, thereby influencing gene expression patterns [65, 66]. Interestingly, DNA hydroxymethylation is both associated with transcriptional expression and repression [66, 67]. In conclusion, the studies presented above underline the importance of both $5-\mathrm{mC}$ and $5-\mathrm{hmC}$ in transcriptional regulation during healthy brain functioning. Given the importance of these modifications in the human brain, it is reasonable to assume that dysregulation of these marks could contribute to various brain disorders, including SAD.

\section{DNA methylation and hydroxymethylation in sporadic Alzheimer's disease}

Over the last decade, the number of publications on the role of epigenetic dysregulation in SAD has increased significantly. It is now well-accepted that environmental factors affect the epigenome and that epigenetic changes might provide a mechanistic link between adverse environmental exposure and the 
development and course of SAD. The rapidly growing, but still juvenile field of neuroepigenetics is therefore starting to provide novel insights into the underlying pathophysiological mechanisms of this disease. The increasing interest in epigenetic dysregulation is furthermore attributed to the clinical potential of epigenetic marks, as they could be used for the development of biomarker assays, potentially allowing early detection of $\mathrm{SAD}$, as well as providing opportunities for more detailed disease classification. Moreover, the reversible nature of epigenetic modifications makes them suitable candidates for preventive treatment strategies and clinical interventions. For these reasons, the characterization of epigenomic profiles in brain tissue samples, as well as in blood, in both patients and people at risk of developing the disease is currently offering an appealing approach to study SAD.

When looking at the neuroepigenomic studies that have been conducted in SAD so far, one can observe that, over the last years, the field has moved away from the use of (antibody-based) technologies targeting global epigenetic marks [66, 68-73] towards more in-depth epigenome-wide arrays and sequencing-based platforms that provide mechanistic insight with a much broader coverage and higher resolution $[43,74]$. Although a potential role for DNA (hydroxy)methylation in the pathogenesis of SAD became evident from these initial antibody-based studies, their findings have remained inconsistent and inconclusive - most likely attributable to the aspecificity and lack of reliability of the methodology used. Thus, the imperative need for more concise and conclusive data has shifted the field to the use of more novel, robust techniques, including the aforementioned platforms. The continuous advancements of the Illumina microarray, combined with its costeffectiveness, have made this approach the most appealing method in sample cohort studies to date. Especially a combination of the microarray with independent validation using pyrosequencing is developing itself into a golden standard for DNA (hydroxy)methylation studies in SAD. The dawn of these approaches in combination with advanced bioinformatics analysis have led to the current era of epigenomewide association studies (EWAS). Although these EWAS will be discussed in more detail in Chapter 2, it is worth mentioning that initial studies targeting different brain regions and blood samples derived from SAD patients have already identified ADspecific DNA (hydroxy)methylation changes in various genomic loci, emphasizing their importance in the disease $[74,75]$.

In spite of the fact that previous work has indicated a critical role for DNA (hydroxy) methylation changes in the development and course of SAD, many questions related to these marks remain yet unanswered. For instance, it is still unclear what exactly the functional consequences of these alterations are, how they specifically contribute to developing pathology, and whether these marks indeed represent valuable biomarkers. More research is necessary in order to obtain a 
better understanding of their role in SAD, to develop effective therapeutics and diagnostic alternatives, and thus to eventually be able to alleviate the clinical and socioeconomic burden of the disease.

Given the notion that aberrant DNA (hydroxy)methylation patterns could be involved in both the progression, as well as the etiology, of the disease, it will furthermore be necessary to assess whether these marks are dysregulated in regions of the brain affected early in the pathogenesis of AD. Interestingly, such studies have not been performed up until now, as most of the investigations outlined above targeted epigenetic dysregulation in cortical brain regions, or the hippocampus. Even though these areas of the brain are affected by the disease, as mentioned earlier, it is becoming more apparent that pathological changes in these regions particularly occur in later and more advanced stages of SAD. To this end, targeting epigenetic dysregulation in brainstem regions including the DRN and the LC might provide a more suitable strategy for identifying alterations involved in the early pathogenesis of SAD.

Nevertheless, in addition to simply profiling epigenetic marks in these brain regions, the field also requires further advances that could overcome remaining (methodological) challenges, thereby contributing to the production of more conclusive data. On the one hand, there are challenges directly attributed to the complex neurobiology of the disease [80]. First, DNA (hydroxy)methylation changes occur with aging, with different patterns observed depending on the timing, the tissue assessed and the genomic location analyzed [81, 82]. This emphasizes the importance of balancing age across experimental groups and confirms the need of discriminating epigenetic marks associated with the disease from those that occur during normal physiological aging. Second, disease comorbidities in SAD patients are common and could hinder the identification of sAD-specific epigenomic profiles [76]. It is therefore necessary to carefully select experimental groups based on the presence or absence of other pathology. Third, there is the problem of tissue and cell-type specificity of epigenetic modifications. $S A D$ is characterized by a temporospatial pattern of changes, affecting different regions of the brain as the disease progresses. Brain regions therefore need to be selected carefully, as they could be affected differentially across the different disease stages. Of a similar nature, is the issue of cell-type specificity. SAD is characterized by neuronal loss, altering the cell-type composition of diseased samples, which complicates the interpretation of generated data. In fact, the use of heterogeneous tissues could obscure cell-type specific modifications related to $S A D$, as changes in one cell type could negate or distort changes in another [77]. On the other hand, there are various methodological challenges that one should consider. Although some might be overcome based on adapting study designs, it 
will also require technological advances or modifications of existing protocols in order to allow more sophisticated and reliable analyses. Two examples of these include the aspecificity of the epigenetic marks assessed and causal interference. Techniques involving the use of microarrays and pyrosequencing rely on a DNA bisulfite treatment $[78,79]$. However, it has been demonstrated that this treatment does not allow for the discrimination between 5 -mC and 5-hmC [80]. In fact, a bisulfite methylation analysis without prior oxidation yields cumulative measures of both modifications [80]. Future studies will therefore need to adapt current protocols in order to produce more informative data. In addition, it is important to realize that EWAS in blood or brain tissue does not allow discrimination between cause and consequence of epigenetic changes in $A D$, as certain epigenetic marks could also result from $A D$-associated neuropathology and/or its neurobiological and behavioral consequences. Technological advances such as induced pluripotent stem cell (iPSC) models and epigenetic editing techniques, however, are anticipated to aid in overcoming this issue, allowing one to study the effect of epigenetic changes on cellular and molecular responses in vitro [81]. Thus, hopes are placed on such interdisciplinary approaches that will allow for a better understanding on the exact role of epigenetics in SAD in the future.

\section{Advanced models of Alzheimer's disease: induced pluripotent stem cells}

In 2006, a major scientific breakthrough was made with the discovery that mouse fibroblasts could be reprogrammed towards iPSCs by viral delivery of four transcription factors, i.e. OCT4, SOX2, KLF4 and CMYC [82]. These cells demonstrated an expression profile and developmental potential similar to embryonic stem cells (ESCs), including their properties of self-renewal and their ability to differentiate into the three embryonic germ layers. One year later, it was demonstrated that also human fibroblasts could be reprogrammed, allowing one to produce personalized iPSCs from theoretically every individual [83, 84]. These studies were shortly followed by reports demonstrating the successful generation of iPSCs from various other human somatic cells and by alternative methods, such as blood cells and non-integrative, non-viral based techniques, respectively $[85,86]$. After the discovery of the technology, a large proportion of research has moved directly into the establishment of protocols for the differentiation of iPSCs into neural populations. The possibility of creating disease-relevant neural cells derived from patients' iPSCs received substantial interest for the generation of human disease models. In fact, many brain disorders that previously lacked a comprehensive cellular model, including SAD, could now potentially be modeled using patient-derived iPSCs. Consequently, the scientific community has put a lot of effort in developing robust and scalable methods for generating large numbers of differentiated neural cells from iPSCs. 
Over the last years, protocols for differentiating iPSCs towards neural precursor cells (NPCs) and various neuronal subtypes, as well as astrocytes, microglia, and oligodendrocytes, became available [87, 88]. These protocols generally involve the chemical stimulation of iPSCs through patterning cues or the use of ectopic overexpression of lineage-specific transcription factors, which both play a central role during neural development in the early embryo [87]. In addition to the networks of patterning molecules and transcription factors that orchestrate neural differentiation, it is currently well-accepted that epigenetic mechanisms are also key players within these circuits [89]. Recent advances in epigenetic editing and RNA interference therefore offer novel, alternative strategies for neural differentiation in vitro [81, 87]. Aside from directed differentiation of iPSCs, which constrains the differentiation of these cells towards a specific neural subtype, protocols involving the direct reprogramming of somatic cells towards neural cells or NPCs also became available [87]. Even though these methods have demonstrated that functional neurons can be differentiated from iPSCs or fibroblast, the optimization and refinement of these approaches, as well as the establishment of novel methods to produce neural subtypes for which no protocols have been established yet, is currently an ongoing process [87]. Nevertheless, it is anticipated that further efforts in this field will play a significant role in the development of disease-relevant cultures for the establishment of cellular models of $A D$.

The opportunity of developing iPSC-based models based on the approach described above has ushered a new exciting era of scientific research into SAD. Cellular models consisting of neurons and glia derived from patients' iPSCs are hypothesized to harbor and manifest disease hallmarks in vitro [90]. As such, these models offer unprecedented opportunities to study these hallmarks in more detail, potentially contributing to the development of a better understanding on the underlying molecular mechanisms of the disease. Furthermore, the availability of patient-derived neural populations provides a personalized platform for highthroughput drugs and toxicity screenings that could aid in the development of novel therapeutics or diagnostic tools [91, 92]. Additionally, the opportunity of creating co-cultures or three-dimensional models incorporating multiple brain cell types offers a new approach towards modeling the complex cellular interactions in integrated neural networks found in the human brain [90, 93]. These procedures are becoming increasingly popular nowadays, given the general advantages that iPSCs have in disease modeling compared with traditional animal and cellular models. Next to their human origin, these advantages include their easy accessibility, expandability, the ability to give rise to almost all major brain cells, relatively less ethical concerns when compared to work with human ESCs or animal models, and their potential for personalized medicine [94]. 
Besides these general advantages, iPSCs also have the potential to overcome challenges specifically related to $S A D$ research. To date, $A D$ studies have mainly relied on transgenic mouse models, immortalized cell lines and post-mortem brain tissues. Although various animal models carrying fAD mutations have displayed certain neuropathological and behavioral deficits, they are mainly modeling fAD and, furthermore, do not develop all phenotypic aspects characteristic of human $A D$, such as neuronal death with distinct neurofibrillary tangle formation [95]. In addition, the effort of generating mice carrying the human $A P O E$ gene has not led to a model that truly mimics SAD development [96]. Post-mortem brain tissues and immortalized cell lines on the other hand, have offered opportunities to study SAD in human cells. However, these tissues are limited for studying the earlier stages of the disease and do not allow one to study the causeeffect relationships between molecular hallmarks and developing pathology [97]. Although immortalized cell lines provide an unlimited resource for $A D$ research, these models do not harbor the unique genomes of SAD patients. The characteristics of iPSCs, however, provide a unique platform to detect early-disease phenotypes, which may point towards underlying pathogenic mechanisms of SAD later in life [98]. In addition, since human iPSCs can be derived from individuals with $S A D$, they contain a patient-specific pathogenic background [99]. Furthermore, since these cells are cultivated in laboratory conditions, it allows for direct manipulations of various molecular mechanisms, as well as their microenvironment, allowing more mechanistic studies on causeeffect relationships.

In order to achieve this potential, neurons from patients' iPSCs should naturally manifest pathological mechanisms in vitro. Indeed, pioneering studies using iPSCs from fAD patients have found the presence of disease hallmarks in differentiated neural cells, including altered APP processing, elevated levels of neurotoxic A $\beta$ peptides, increased levels of hyperphosphorylated tau protein and activation of GSK3 $\beta$, a physiological kinase of tau $[99,100]$. As such, these model systems have proven to be instrumental in modelling molecular alterations associated with genetic mutations in AD-causing genes, such as APP and PSEN1. Although SAD is more prevalent, only a limited number of iPSC-based models have been developed for this form of the disease [99, 100]. In very few cases, iPSC-derived neurons from SAD patients were studied in parallel to lines obtained from fAD patients, with the main goal of seeking to find $A D$-associated cellular phenotypes for the validation of their potential for SAD modeling. Interestingly, these initial SAD models have shown a high degree of variability and inconsistencies in terms of disease hallmarks, which might be explained by the heterogeneous nature of $S A D$ as observed in patients. On the other hand, given that these studies represent initial attempts of developing disease-relevant model systems, various methodological 
factors are likely contributing to these lacking hallmarks [99]. Further improving these models to facilitate more meaningful studies for SAD will therefore be a major goal in the upcoming years.

While it is well-established that epigenetic alterations contribute to SAD, detailed characterization of epigenomic profiles in iPSC-derived neural cells has not been performed yet. The complex and interconnected network of epigenetic changes, including DNA (hydroxy)methylation, has previously only been studied in animal models and in human postmortem brain samples. In future studies, it will therefore be vital to profile e.g. the methylome and hydroxymethylome and their downstream effects on the transcriptome in neural cells differentiated from patients' iPSCs. Furthermore, it will be crucial to compare these profiles with the same omics profiles in identical cells found in their brains in order to further validate the model for epigenomic and transcriptomic research. With the advent of iPSC technology, it can be expected that patient-derived neural models represent valid tools to explore epigenetic changes involved in SAD. Based on the availability of epigenetic editing [81] nowadays, iPSC-derived neuronal populations generated from SAD patients can be epigenetically probed and exposed to adverse environmental factors, as well as treated with drug libraries, to investigate their effects on molecular and cellular responses. Moreover, epigenetic-editing might even represent an interesting (proof-of-concept) therapeutic intervention on itself that could be explored further using these iPSC-based models. Along similar lines, probing the genomes of SAD patients and studying their interaction with AD-associated environmental factors could potentially contribute to a better understanding of complex gene-environment interactions, as well as the involvement of epigenetic mechanisms. To conclude, although the field of iPSC technology is still in its early development, it is anticipated that patient-derived neural populations differentiated from iPSCs could bridge the gap between animal models and clinical studies, which will ultimately contribute to a better understanding of the disease that could aid in establishing therapeutic interventions.

\section{Overview of the thesis}

The research that is compiled in this thesis can be divided in two parts. The first part, consisting of four chapters, is centered around the role of epigenetic dysregulation in the etiopathophysiology of SAD. In addition to providing insights into the most recent developments in neuroepigenomic studies of this disease, the first part of the thesis also touches upon remaining challenges, and provides a future outlook on possible developments in the field. The second part, which includes three more chapters, is focused on the application of iPSC-based disease models for the study of $A D$, including but not limited to mechanistic studies on epigenetic dysregulation using this platform. Aside from outlining the research that 
has been conducted using iPSC-based models for SAD to date, the second part of the thesis also provides insights into the acquisition of disease-relevant neural cultures based on directed differentiation of iPSCs, and furthermore includes an experimental approach for the establishment of such a model system.

The general introduction only briefly described epigenetic dysregulation, and specifically alterations in DNA methylation and hydroxymethylation, in relation to SAD. Chapter 2 therefore offers a more in-depth perspective on EWAS in $S A D$. Among others, the degree of dysregulation in both DNA methylation and hydroxymethylation in various brain tissues and blood samples derived from patients are summarized. Furthermore, relevant caveats in relation to these studies that could affect correct interpretation of the obtained results are discussed and future outlooks on possible solutions, as well as developments, to overcome these challenges are provided.

Following on that notion, Chapter 3 represents a methodological research article offering a possible solution to the issue of modification specificity in neuroepigenomic studies of SAD. In more detail, an approach for the targeted detection of unmodified cytosine, $5-\mathrm{mC}$ and $5-\mathrm{hmC}$ levels at single $\mathrm{CpG}$ sites by oxidative bisulfite pyrosequencing is provided. For this purpose, three spike-in pyrosequencing controls that can be added to a given DNA target sample were developed in order to assess the successful application of the described method. By applying these spike-in controls, as well as by analyzing both brain tissue and iPSC samples, the efficacy of the oxidative bisulfite pyrosequencing approach for the detection of the aforementioned cytosine states in the context of single CpG sites is demonstrated.

Chapter 4 introduces a possible solution to overcome the issue of cellular heterogeneity in neuroepigenomic studies of SAD. In this chapter, it is demonstrated, for the first time, that limiting dilution bisulfite pyrosequencing (LDBSP) can be successfully applied on pools of individually isolated post-mortem neurons using laser capture microdissection (LCM). The general workflow of LDBSP for the detection of DNA bisulfite methylation in multiple target genes on single alleles derived from these neurons is provided. Furthermore, a novel approach for data correction when applying this technique is being described, aimed at reducing bias and allowing the user to more accurately estimate the DNA methylation status of target genes using this method.

In Chapter 5, the body of EWAS reviewed in previous chapters, is complemented with the most recent findings on epigenetic dysregulation in the brainstem in AD. More specifically, this chapter provides data on the role of DNA methylation and 
hydroxymethylation in the DRN and LC of SAD patients. Common and distinct epigenetic signatures in both brainstem nuclei were identified and validated by a subsequent bisulfite pyrosequencing analysis. The EWAS presented here is furthermore complemented by a highly innovative cell subtype-specific analysis in the DRN, which was based on the LCM-LDBSP approach described in the previous chapter.

Chapter 6 moves away from profiling epigenetic patterns in post-mortem tissues or cells and introduces the use of iPSC-based model systems in SAD research. This chapter reviews iPSC-based studies that have been conducted in SAD to date. In addition, important sources of variability related to such model systems, next to those that might be explained by the heterogeneous nature of SAD, are being discussed.

In the development of iPSC-based models for SAD, Chapter 7 summarizes the progress that has been made in generating various neuronal subtypes from both stem cells and somatic cells. This review dissects the directed- and direct differentiation protocols for the generation of disease-relevant neural cultures with special emphasis on chemically defined systems, transcription factor-mediated reprogramming and epigenetic-based approaches. Furthermore, efforts that are being made to increase the efficiency of current protocols are discussed and the potential for the use of these cells in disease modelling, drug discovery and regenerative medicine are being addressed.

The final study presented in Chapter 8 comprises an exploratory approach, in which the establishment and characterization of a human neuronal in vitro model system for AD using iPSCs is being described. Based on the step-wise application of neural patterning factors, iPSCs were differentiated towards NPCs and, subsequently, cortical neurons, which represents a cellular population known to be affected in the pathophysiology of $A D$. In order to further explore the potential of this in vitro model for future $A D$ studies, the expression of $A D$-associated proteins were assessed in the differentiated neuronal cells.

All in all, the research collected in this thesis covers a multitude of studies in which state-of-the-art techniques, brain tissue samples and model systems are being applied in order to study the role of epigenetic dysregulation in the etiopathogenesis of (s)AD. 


\section{References}

1. Vatanabe IP, Manzine PR, Cominetti MR. Historic concepts of dementia and Alzheimer's disease: From ancient times to the present. Rev Neurol (Paris). 2019.

2. Maurer K, Volk S, Gerbaldo H. Auguste D and Alzheimer's disease. The Lancet. 1997;349(9064):1546-9.

3. Prince MJ. World Alzheimer Report 2015: the global impact of dementia: an analysis of prevalence, incidence, cost and trends: Alzheimer's Disease International; 2015.

4. Silva MVF, Loures CdMG, Alves LCV, de Souza LC, Borges KBG, das Graças Carvalho M. Alzheimer's disease: risk factors and potentially protective measures. Journal of biomedical science. 2019;26(1):33.

5. Cerejeira J, Lagarto L, Mukaetova-Ladinska E. Behavioral and psychological symptoms of dementia. Frontiers in neurology. 2012;3:73.

6. Huang $\mathrm{H}-\mathrm{C}$, Jiang Z-F. Accumulated amyloid- $\beta$ peptide and hyperphosphorylated tau protein: relationship and links in Alzheimer's disease. Journal of Alzheimer's disease. 2009;16(1):15-27.

7. Scheltens P, Blennow K, Breteler MM, de Strooper B, Frisoni GB, Salloway S, et al. Alzheimer's disease. Lancet. 2016;388(10043):505-17.

8. Mohandas E, Rajmohan V, Raghunath B. Neurobiology of Alzheimer's disease. Indian journal of psychiatry. 2009;51(1):55.

9. Tönnies E, Trushina E. Oxidative stress, synaptic dysfunction, and Alzheimer's disease. Journal of Alzheimer's Disease. 2017;57(4):1105-21.

10. Van Leeuwen FW, de Kleijn DP, van den Hurk HH, Neubauer A, Sonnemans MA, Sluijs JA, et al. Frameshift mutants of $\beta$ amyloid precursor protein and ubiquitin-B in Alzheimer's and Down patients. Science. 1998;279(5348):242-7.

11. Kumar K, Kumar A, Keegan RM, Deshmukh R. Recent advances in the neurobiology and neuropharmacology of Alzheimer's disease. Biomedicine \& pharmacotherapy. 2018;98:297-307.

12. Beason-Held LL, Goh JO, An Y, Kraut MA, O'Brien RJ, Ferrucci L, et al. Changes in brain function occur years before the onset of cognitive impairment. Journal of Neuroscience. 2013;33(46):18008-14.

13. Braak H, Braak E. Neuropathological stageing of Alzheimer-related changes. Acta neuropathologica. 1991;82(4):239-59.

14. Grinberg L, Rüb U, Ferretti R, Nitrini R, Farfel J, Polichiso L, et al. The dorsal raphe nucleus shows phospho-tau neurofibrillary changes before the transentorhinal region in Alzheimer's disease. A precocious onset? Neuropathology and applied neurobiology. 2009;35(4):406-16.

15. Braak H, Thal DR, Ghebremedhin E, Del Tredici K. Stages of the pathologic process in Alzheimer disease: age categories from 1 to 100 years. Journal of Neuropathology \& Experimental Neurology. 2011;70(11):960-9.

16. Tanzi RE. The genetics of Alzheimer disease. Cold Spring Harbor perspectives in medicine. 2012;2(10):a006296.

17. Stozicka Z, Zilka N, Novak M. Risk and protective factors for sporadic Alzheimer's disease. Acta virologica. 2007;51(4):205-22.

18. Hollingworth P, Harold D, Sims R, Gerrish A, Lambert J-C, Carrasquillo MM, et al. Common variants at $A B C A 7, M S 4 A 6 A / M S 4 A 4 E, E P H A 1, C D 33$ and CD2AP are associated with Alzheimer's disease. Nature genetics. 2011;43(5):429.

19. Naj AC, Jun G, Beecham GW, Wang L-S, Vardarajan BN, Buros J, et al. Common variants at MS4A4/MS4A6E, CD2AP, CD33 and EPHA1 are associated with late-onset Alzheimer's disease. Nature genetics. 2011;43(5):436-41. 
20. Guerreiro R, Wojtas A, Bras J, Carrasquillo M, Rogaeva E, Majounie E, et al. TREM2 variants in Alzheimer's disease. New England Journal of Medicine. 2013;368(2):117-27.

21. Lambert JC, Heath S, Even G, Campion D, Sleegers K, Hiltunen M, et al. Genome-wide association study identifies variants at CLU and CR1 associated with Alzheimer's disease. Nat Genet. 2009;41(10):1094-9.

22. Harold D, Abraham R, Hollingworth P, Sims R, Gerrish A, Hamshere ML, et al. Genome-wide association study identifies variants at CLU and PICALM associated with Alzheimer's disease. Nature genetics. 2009;41(10):1088.

23. Seshadri S, Fitzpatrick AL, Ikram MA, DeStefano AL, Gudnason V, Boada M, et al. Genome-wide analysis of genetic loci associated with Alzheimer disease. Jama. 2010;303(18):1832-40.

24. Lambert J-C, Ibrahim-Verbaas CA, Harold D, Naj AC, Sims R, Bellenguez C, et al. Meta-analysis of 74,046 individuals identifies 11 new susceptibility loci for Alzheimer's disease. Nature genetics. 2013;45(12):1452.

25. Cuyvers E, Sleegers K. Genetic variations underlying Alzheimer's disease: evidence from genome-wide association studies and beyond. The Lancet Neurology. 2016;15(8):857-68.

26. Yamazaki Y, Zhao N, Caulfield TR, Liu C-C, Bu G. Apolipoprotein E and Alzheimer disease: pathobiology and targeting strategies. Nature Reviews Neurology. 2019;15(9):501-18.

27. Liu C-C, Kanekiyo T, Xu H, Bu G. Apolipoprotein E and Alzheimer disease: risk, mechanisms and therapy. Nature Reviews Neurology. 2013;9(2):106.

28. Creasey H, Jorm A, Longley W, Broe G, Henderson A. Monozygotic twins discordant for Alzheimer's disease. Neurology. 1989;39(11):1474-.

29. Ridge PG, Mukherjee S, Crane PK, Kauwe JS, Consortium AsDG. Alzheimer's disease: analyzing the missing heritability. PloS one. 2013;8(11).

30. Edwards III GA, Gamez N, Escobedo Jr G, Calderon O, Moreno-Gonzalez I. Modifiable risk factors for Alzheimer's disease. Frontiers in aging neuroscience. 2019;11.

31. Killin LO, Starr JM, Shiue IJ, Russ TC. Environmental risk factors for dementia: a systematic review. BMC geriatrics. 2016;16(1):1-28.

32. Bisht K, Sharma K, Tremblay M-Ė. Chronic stress as a risk factor for Alzheimer's disease: Roles of microglia-mediated synaptic remodeling, inflammation, and oxidative stress. Neurobiology of stress. 2018;9:9-21.

33. Sharp ES, Gatz M. The relationship between education and dementia an updated systematic review. Alzheimer disease and associated disorders. 2011;25(4):289.

34. Boyle PA, Buchman AS, Wilson RS, Yu L, Schneider JA, Bennett DA. Effect of purpose in life on the relation between Alzheimer disease pathologic changes on cognitive function in advanced age. Archives of general psychiatry. 2012;69(5):499-504.

35. Chen ST, Siddarth P, Saito NY, Rueda F, Haight T, Ercoli LM, et al. Psychological well-being and regional brain amyloid and tau in mild cognitive impairment. The American Journal of Geriatric Psychiatry. 2014;22(4):362-9.

36. Lesuis SL, Hoeijmakers L, Korosi A, de Rooij SR, Swaab DF, Kessels HW, et al. Vulnerability and resilience to Alzheimer's disease: early life conditions modulate neuropathology and determine cognitive reserve. Alzheimer's research \& therapy. 2018;10(1):1-20.

37. Lahiri DK, Maloney B, Zawia NH. The LEARn model: an epigenetic explanation for idiopathic neurobiological diseases. Molecular psychiatry. 2009;14(11):992-1003.

38. Choudhuri S. From Waddington's epigenetic landscape to small noncoding RNA: some important milestones in the history of epigenetics research. Toxicology mechanisms and methods. 2011;21(4):252-74.

39. Felsenfeld G. A brief history of epigenetics. Cold Spring Harbor perspectives in biology. 2014;6(1):a018200. 
40. Liu X, Jiao B, Shen L. The epigenetics of Alzheimer's disease: Factors and therapeutic implications. Frontiers in genetics. 2018;9:579.

41. Heard E, Martienssen RA. Transgenerational epigenetic inheritance: myths and mechanisms. Cell. 2014;157(1):95-109.

42. Guerrero-Bosagna C, Skinner MK. Environmentally induced epigenetic transgenerational inheritance of phenotype and disease. Molecular and cellular endocrinology. 2012;354(1-2):3-8.

43. Lardenoije R, latrou A, Kenis G, Kompotis K, Steinbusch HW, Mastroeni D, et al. The epigenetics of aging and neurodegeneration. Prog Neurobiol. 2015;131:21-64.

44. Klose RJ, Bird AP. Genomic DNA methylation: the mark and its mediators. Trends in biochemical sciences. 2006;31(2):89-97.

45. Jonas S, Izaurralde E. Towards a molecular understanding of microRNA-mediated gene silencing. Nature reviews genetics. 2015;16(7):421-33.

46. Mattick JS. The central role of RNA in human development and cognition. FEBS letters. 2011;585(11):1600-16.

47. Roth TL, David Sweatt J. Annual research review: epigenetic mechanisms and environmental shaping of the brain during sensitive periods of development. Journal of Child Psychology and Psychiatry. 2011;52(4):398-408.

48. Atlasi $Y$, Stunnenberg HG. The interplay of epigenetic marks during stem cell differentiation and development. Nature Reviews Genetics. 2017;18(11):643-58.

49. Reik W, Walter J. Genomic imprinting: parental influence on the genome. Nature Reviews Genetics. 2001;2(1):21-32.

50. Woldemichael BT, Bohacek J, Gapp K, Mansuy IM. Epigenetics of memory and plasticity. Progress in molecular biology and translational science. 122: Elsevier; 2014. p. 305-40.

51. Kumar S, Chinnusamy V, Mohapatra T. Epigenetics of modified DNA bases: 5-methylcytosine and beyond. Frontiers in genetics. 2018;9:640.

52. Cross SH, Bird AP. CpG islands and genes. Current opinion in genetics \& development. 1995;5(3):309-14.

53. Silva PNO, Gigek CO, Leal MF, Bertolucci PHF, de Labio RW, Payao SLM, et al. Promoter methylation analysis of SIRT3, SMARCA5, HTERT and CDH1 genes in aging and Alzheimer's disease. Journal of Alzheimer's Disease. 2008;13(2):173-6.

54. Guilleret I, Benhattar J. Unusual distribution of DNA methylation within the hTERT CpG island in tissues and cell lines. Biochemical and biophysical research communications. 2004;325(3):1037-43.

55. Yin Y, Morgunova E, Jolma A, Kaasinen E, Sahu B, Khund-Sayeed S, et al. Impact of cytosine methylation on DNA binding specificities of human transcription factors. Science. 2017;356(6337):eaaj2239.

56. Portela A, Esteller M. Epigenetic modifications and human disease. Nature biotechnology. 2010;28(10):1057.

57. Flores K, Wolschin F, Corneveaux JJ, Allen AN, Huentelman MJ, Amdam GV. Genome-wide association between DNA methylation and alternative splicing in an invertebrate. BMC genomics. 2012;13(1):480.

58. Ito S, Shen L, Dai Q, Wu SC, Collins LB, Swenberg JA, et al. Tet proteins can convert 5-methylcytosine to 5-formylcytosine and 5-carboxylcytosine. Science. 2011;333(6047):1300-3.

59. Sun W, Zang L, Shu Q, Li X. From development to diseases: the role of 5-hmC in brain. Genomics. 2014;104(5):347-51.

60. Globisch D, Münzel M, Müller M, Michalakis S, Wagner M, Koch S, et al. Tissue distribution of 5-hydroxymethylcytosine and search for active demethylation intermediates. PloS one. 2010;5(12).

61. Cheng Y, Xie N, Jin P, Wang T. DNA methylation and hydroxymethylation in stem cells. Cell biochemistry and function. 2015;33(4):161-73. 
62. Ficz G, Branco MR, Seisenberger S, Santos F, Krueger F, Hore TA, et al. Dynamic regulation of 5-hydroxymethylcytosine in mouse ES cells and during differentiation. Nature. 2011;473(7347):398402.

63. Williams K, Christensen J, Pedersen MT, Johansen JV, Cloos PA, Rappsilber J, et al. TET1 and hydroxymethylcytosine in transcription and DNA methylation fidelity. Nature. 2011;473(7347):343-8.

64. Pastor WA, Pape UJ, Huang Y, Henderson HR, Lister R, Ko M, et al. Genome-wide mapping of 5-hydroxymethylcytosine in embryonic stem cells. Nature. 2011;473(7347):394-7.

65. Valinluck V, Tsai H-H, Rogstad DK, Burdzy A, Bird A, Sowers LC. Oxidative damage to methyl$\mathrm{CpG}$ sequences inhibits the binding of the methyl-CpG binding domain (MBD) of methyl-CpG binding protein 2 (MeCP2). Nucleic acids research. 2004;32(14):4100-8.

66. Coppieters N, Dragunow M. Epigenetics in Alzheimer's disease: a focus on DNA modifications. Current pharmaceutical design. 2011;17(31):3398-412.

67. Wu H, D'Alessio AC, Ito S, Wang Z, Cui K, Zhao K, et al. Genome-wide analysis of 5 -hydroxymethylcytosine distribution reveals its dual function in transcriptional regulation in mouse embryonic stem cells. Genes \& development. 2011;25(7):679-84.

68. Bihaqi S, Schumacher A, Maloney B, Lahiri D, Zawia N. Do epigenetic pathways initiate late onset Alzheimer disease: towards a new paradigm. 2012.

69. Mastroeni D, McKee A, Grover A, Rogers J, Coleman PD. Epigenetic differences in cortical neurons from a pair of monozygotic twins discordant for Alzheimer's disease. PloS one. 2009;4(8).

70. Mastroeni D, Grover A, Delvaux E, Whiteside C, Coleman PD, Rogers J. Epigenetic changes in Alzheimer's disease: decrements in DNA methylation. Neurobiology of aging. 2010;31(12):2025-37.

71. Chouliaras L, Mastroeni D, Delvaux E, Grover A, Kenis G, Hof PR, et al. Consistent decrease in global DNA methylation and hydroxymethylation in the hippocampus of Alzheimer's disease patients. Neurobiology of aging. 2013;34(9):2091-9.

72. Bradley-Whitman M, Lovell M. Epigenetic changes in the progression of Alzheimer's disease. Mechanisms of ageing and development. 2013;134(10):486-95.

73. Coppieters N, Dieriks BV, Lill C, Faull RL, Curtis MA, Dragunow M. Global changes in DNA methylation and hydroxymethylation in Alzheimer's disease human brain. Neurobiology of aging. 2014;35(6):1334-44.

74. Roubroeks JAY, Smith RG, van den Hove DLA, Lunnon K. Epigenetics and DNA methylomic profiling in Alzheimer's disease and other neurodegenerative diseases. J Neurochem. 2017; 143(2):158-70.

75. Smith R, Pishva E, Shireby G, Smith AR, Roubroeks JA, Hannon E, et al. Meta-analysis of epigenome-wide association studies in Alzheime's disease highlights 220 differentially methylated loci across cortex. BioRxiv. 2020.

76. Haaksma ML, Vilela LR, Marengoni A, Calderón-Larrañaga A, Leoutsakos J-MS, Rikkert MGO, et al. Comorbidity and progression of late onset Alzheimer's disease: a systematic review. PLoS One. 2017;12(5).

77. Blalock EM, Buechel HM, Popovic J, Geddes JW, Landfield PW. Microarray analyses of lasercaptured hippocampus reveal distinct gray and white matter signatures associated with incipient Alzheimer's disease. J Chem Neuroanat. 2011;42(2):118-26.

78. Carless MA. Determination of DNA methylation levels using Illumina HumanMethylation450 BeadChips. Chromatin Protocols: Springer; 2015. p. 143-92.

79. Bassil CF, Huang Z, Murphy SK. Bisulfite pyrosequencing. Ovarian Cancer: Springer; 2013. p. 95-107.

80. Booth MJ, Ost TW, Beraldi D, Bell NM, Branco MR, Reik W, et al. Oxidative bisulfite sequencing of 5-methylcytosine and 5-hydroxymethylcytosine. Nat Protoc. 2013;8(10):1841-51. 
81. Thakore PI, Black JB, Hilton IB, Gersbach CA. Editing the epigenome: technologies for programmable transcription and epigenetic modulation. Nature methods. 2016;13(2):127.

82. Takahashi K, Yamanaka S. Induction of pluripotent stem cells from mouse embryonic and adult fibroblast cultures by defined factors. cell. 2006;126(4):663-76.

83. Takahashi K, Tanabe K, Ohnuki M, Narita M, Ichisaka T, Tomoda K, et al. Induction of pluripotent stem cells from adult human fibroblasts by defined factors. cell. 2007;131(5):861-72.

84. Yu J, Vodyanik MA, Smuga-Otto K, Antosiewicz-Bourget J, Frane JL, Tian S, et al. Induced pluripotent stem cell lines derived from human somatic cells. science. 2007;318(5858):1917-20.

85. Raab S, Klingenstein M, Liebau S, Linta L. A comparative view on human somatic cell sources for iPSC generation. Stem cells international. 2014;2014.

86. Malik N, Rao MS. A review of the methods for human iPSC derivation. Pluripotent stem cells: Springer; 2013. p. 23-33.

87. Riemens RJM, van den Hove DLA, Esteller M, Delgado-Morales R. Directing neuronal cell fate in vitro: achievements and challenges. Progress in neurobiology. 2018;168:42-68.

88. Zheng W, Li Q, Zhao C, Da Y, Zhang H-L, Chen Z. Differentiation of glial cells from hiPSCs: potential applications in neurological diseases and cell replacement therapy. Frontiers in cellular neuroscience. 2018;12:239.

89. Boland MJ, Nazor KL, Loring JF. Epigenetic regulation of pluripotency and differentiation. Circulation research. 2014;115(2):311-24.

90. Penney J, Ralvenius WT, Tsai L-H. Modeling Alzheimer's disease with iPSC-derived brain cells. Molecular Psychiatry. 2019:1-20.

91. Haston KM, Finkbeiner S. Clinical Trials in a Dish: The Potential of Pluripotent Stem Cells to Develop Therapies for Neurodegenerative Diseases. Annu Rev Pharmacol Toxicol. 2015.

92. Ross CA, Akimov SS. Human-induced pluripotent stem cells: potential for neurodegenerative diseases. Hum Mol Genet. 2014;23(R1):R17-26.

93. Zhang D, Pekkanen-Mattila M, Shahsavani M, Falk A, Teixeira Al, Herland A. A 3D Alzheimer's disease culture model and the induction of P21-activated kinase mediated sensing in iPSC derived neurons. Biomaterials. 2014;35(5):1420-8.

94. Shi Y, Inoue H, Wu JC, Yamanaka S. Induced pluripotent stem cell technology: a decade of progress. Nat Rev Drug Discov. 2016.

95. Drummond E, Wisniewski T. Alzheimer's disease: experimental models and reality. Acta neuropathologica. 2017;133(2):155-75.

96. Williams T, Borchelt DR, Chakrabarty P. Therapeutic approaches targeting Apolipoprotein E function in Alzheimer's disease. Molecular Neurodegeneration. 2020;15(1):8.

97. van den Hove DLA, Riemens RJM, Koulousakis P, Pishva E. Epigenome-wide association studies in Alzheimer's disease; Achievements and challenges. Brain Pathology. 2020.

98. Yang J, Li S, He XB, Cheng C, Le W. Induced pluripotent stem cells in Alzheimer's disease: applications for disease modeling and cell-replacement therapy. Mol Neurodegener. 2016;11(1):39.

99. Riemens R, Kenis G, van den Beucken T. Human-induced pluripotent stem cells as a model for studying sporadic Alzheimer's disease. Neurobiology of learning and memory. 2020:107318.

100. Riemens RJM, Soares ES, Esteller M, Delgado-Morales R. Stem Cell Technology for (Epi) genetic Brain Disorders. Neuroepigenomics in Aging and Disease: Springer; 2017. p. 443-75. 

Chapter 2

\section{Epigenome-wide association studies in Alzheimer's disease; Achievements and challenges}

Renzo J.M. Riemens ${ }^{1,3 *}$, Daniël L.A. van den Hove ${ }^{1,2 *}$, Philippos Koulousakis ${ }^{1 *}$, Ehsan Pishva ${ }^{1,4}$

${ }^{1}$ Department of Psychiatry and Neuropsychology, School for Mental Health and Neuroscience (MHeNs), Maastricht University, Maastricht, the Netherlands.

${ }^{2}$ Division of Molecular Psychiatry, Laboratory of Translational Neuroscience, Center of Mental Health, Department of Psychiatry, University of Würzburg, Würzburg, Germany.

IInstitute of Human Genetics, Julius Maximilians University, Würzburg, Germany.

${ }^{4}$ College of Medicine and Health, University of Exeter Medical School, Exeter University, Exeter, UK.

${ }^{*}$ Authors contributed equally to this work.

Brain Pathology. 2020 Sep;30(5):978-983.

Doi: 10.1111/bpa.12880. 



\begin{abstract}
Alzheimer's disease (AD) represents a devastating progressive neurodegenerative disease with a complex pathophysiology, affecting millions of people worldwide. Recent epigenome-wide association studies suggest a key role for epigenetic mechanisms in its development and course. Despite the fact that current evidence on the role of epigenetic dysregulation in aging and $A D$ is convincing, the pioneering field of neuroepigenetics is still facing many challenges that need to be addressed to fundamentally increase our understanding about the underlying mechanisms of this neurodegenerative disorder. This perspective paper describes the current state of play for epigenetic research into $A D$ and discusses how new methodological advances in the field of epigenetics and related data science disciplines could further spur the development of novel therapeutic agents and biomarker assays.
\end{abstract}

\title{
Keywords
}

Alzheimer's disease; DNA methylation; epigenetics; methylome-wide association studies. 


\section{Epigenome-scale studies in Alzheimer's disease}

Alzheimer's disease (AD) is a chronic neurodegenerative disorder characterized by the deposition of amyloid-beta (A $\beta$ ) plaques, neurofibrillary tangles (NFTs) of hyperphosphorylated tau protein, which ultimately lead to neuronal cell death. Genetic research exploring the etiology of $A D$ has helped elucidate some of the pathogenic mechanisms underlying the disorder. Mutations in the APP, PSEN1 and PSEN2 genes are key players in developing early onset familial AD (fAD), whereas APOE was discovered as the most important genetic risk factor for late onset sporadic AD (SAD) [1]. Genome-wide association studies (GWAS) have helped identify several AD loci that are common in the general population, but show small risk effects (the most recent genetic studies of $A D$ are summarized elsewhere in this mini-symposium series [2]). However, as only one third of $A D$ cases can be explained by common variants or missing heritability, it is highly likely that nongenetic factors play a substantial role in the development and course of the disorder. As such, the multitude of nongenetic risk factors for developing sAD suggests the involvement of epigenetic mechanisms [3].

Epigenetic mechanisms refer to processes that regulate gene expression without altering the DNA sequence. Changes in the epigenome are acquired throughout life and are subject to alterations based on the environment that the cell or organism is exposed to. There are various types of epigenetic modifications, which include DNA modifications, histone modifications, as well as noncoding RNAs (ncRNAs) [4]. This perspective particularly focuses on the DNA modification of 5-methylcytosine (5-mC) as it has been the best characterized in the context of $A D$, discussing recent scientific achievements and challenges in the context of AD. Over the last decade, major technological advances have allowed the first epigenome-wide association studies (EWAS) of DNA methylation (herein termed methylome-wide association studies (MWAS)) in AD brain tissue (for a timely review, see [5]; in addition, see [6]). The first MWAS on AD made use of Illumina Infinium Human Methylation 27K arrays to study more than 27.000 CpG sites in 14.475 genes within the frontal cortex [7] (see Table 1). The most significant AD-associated differentially methylated cytosine-phosphate-guanine $(\mathrm{CpG})$ site was located within the TMEM59 gene, and displayed DNA hypomethylation when comparing 12 AD patients with 12 age- and gendermatched controls. A similar 27K MWAS on 15 AD patients and 5 non-demented controls identified Braak-associated DNA methylation alterations in four loci, including two residing in DUSP22, displaying DNA hypermethylation, concomitant with decreased DUSP22 RNA expression, with increasing Braak stage [8]. Subsequent approaches made use of the Illumina Infinium Human Methylation 450K array, which interrogates more than $485.000 \mathrm{CpG}$ sites. Several larger sample-size $450 \mathrm{~K}$ studies ( $\mathrm{N}=68-740$ depending on study), primarily focusing on cortical brain tissue, provided converging evidence for numerous genes displaying differential methylation in $A D$, that is, ANK1, C10orf105, CDH23, DIP2A, LOC100507547, PPT2, PPT2-EGFL8, PRDM16, PRRT1, RHBDF2, RNF39, RPL13, SERPINF1 and SERPINF2 [9-12]. 
Table 1. Summary of findings from AD MWAS.

\begin{tabular}{|c|c|c|c|c|c|c|}
\hline Author & Year & $\begin{array}{l}\text { Sample } \\
\text { size }\end{array}$ & Method & Tissue type & $\begin{array}{l}\text { DNA treatment } \\
\text { and modification } \\
\text { identified }\end{array}$ & $\begin{array}{l}\text { Top differentially } \\
\text { methylated loci }\end{array}$ \\
\hline $\begin{array}{l}\text { Bakulski } \\
\text { et al. [7] }\end{array}$ & 2012 & 24 & $\begin{array}{l}\text { Illumina } \\
\text { Methylation } \\
\text { 27K array }\end{array}$ & Frontal cortex & $\begin{array}{l}\text { Bisulfite treatment } \\
\text { (total DNA } \\
\text { modifications }[5-\mathrm{mC}+5- \\
\mathrm{hmC}] \text { ) }\end{array}$ & TMEM59 \\
\hline $\begin{array}{l}\text { Sanchez-Mut } \\
\text { et al. [8] }\end{array}$ & 2014 & 20 & $\begin{array}{l}\text { Illumina } \\
\text { Methylation } \\
27 \mathrm{~K} \text { array }\end{array}$ & Hippocampus & $\begin{array}{l}\text { Bisulfite treatment } \\
\text { (total DNA } \\
\text { modifications [5-mC+5- } \\
\mathrm{hmC}] \text { ) }\end{array}$ & DUSP22 \\
\hline $\begin{array}{l}\text { Lunnon } \\
\text { et. al [6] }\end{array}$ & 2014 & 122 & $\begin{array}{l}\text { Illumina } \\
\text { Methylation } \\
\text { 450K array }\end{array}$ & $\begin{array}{l}\text { Superior temporal } \\
\text { gyrus, prefrontal } \\
\text { cortex, cerebellum, } \\
\text { blood }\end{array}$ & $\begin{array}{l}\text { Bisulfite treatment } \\
\text { (total DNA } \\
\text { modifications } \\
{[5-\mathrm{mC}+5-\mathrm{hmC}] \text { ) }}\end{array}$ & ANK1 \\
\hline $\begin{array}{l}\text { De Jaeger } \\
\text { et al. [9] }\end{array}$ & 2014 & 708 & $\begin{array}{l}\text { Illumina } \\
\text { Methylation } \\
\text { 450K array }\end{array}$ & $\begin{array}{l}\text { Dorsolateral } \\
\text { prefrontal cortex }\end{array}$ & $\begin{array}{l}\text { Bisulfite treatment } \\
\text { (total DNA } \\
\text { modifications } \\
{[5-\mathrm{mC}+5-\mathrm{hmC}] \text { ) }}\end{array}$ & $\begin{array}{l}\text { ABCA7, BIN1, } \\
\text { ANK1, CDH23, } \\
\text { DIP2A, RHBDF2, } \\
\text { RPL13, SERPINF1, } \\
\text { SERPINF2 }\end{array}$ \\
\hline $\begin{array}{l}\text { Watson } \\
\text { et al. [11] }\end{array}$ & 2016 & 68 & $\begin{array}{l}\text { Illumina } \\
\text { Methylation } \\
450 \mathrm{~K} \text { array }\end{array}$ & $\begin{array}{l}\text { Superior temporal } \\
\text { gyrus }\end{array}$ & $\begin{array}{l}\text { Bisulfite treatment } \\
\text { (total DNA } \\
\text { modifications } \\
{[5-\mathrm{mC}+5-\mathrm{hmC}] \text { ) }}\end{array}$ & $\begin{array}{l}\text { MOV10L1, B3GALT4, } \\
\text { DUSP6, TBX15, OXT }\end{array}$ \\
\hline $\begin{array}{l}\text { Gasparoni } \\
\text { et al. [14] }\end{array}$ & 2018 & 128 & $\begin{array}{l}\text { Illumina } \\
\text { Methylation } \\
\text { 450K array }\end{array}$ & $\begin{array}{l}\text { Bulk brain tissue } \\
\text { (Frontal + temporal } \\
\text { cortex) }\end{array}$ & $\begin{array}{l}\text { Bisulfite treatment } \\
\text { (total DNA } \\
\text { modifications } \\
{[5-\mathrm{mC}+5-\mathrm{hmC}] \text { ) }}\end{array}$ & $\begin{array}{l}\text { CLU, SYNJ2, } \\
\text { NCOR2, RAI1, } \\
\text { CXXC5, INPP5A, } \\
\text { MCF2L, ANK1, } \\
\text { MAP2, LRRC8B, } \\
\text { STK32C, S100B }\end{array}$ \\
\hline $\begin{array}{l}\text { Smith } \\
\text { et al. [34] }\end{array}$ & 2018 & 147 & $\begin{array}{l}\text { Illumina } \\
\text { Methylation } \\
450 \mathrm{~K} \text { array }\end{array}$ & $\begin{array}{l}\text { Prefrontal cortex, } \\
\text { superior temporal } \\
\text { gyrus, hippocampus }\end{array}$ & $\begin{array}{l}\text { Bisulfite treatment } \\
\text { (total DNA } \\
\text { modifications } \\
{[5-\mathrm{mC}+5-\mathrm{hmC}] \text { ) }}\end{array}$ & HOXA3 \\
\hline $\begin{array}{l}\text { Semick } \\
\text { et al. [12] }\end{array}$ & 2019 & 73 & $\begin{array}{l}\text { Illumina } \\
\text { Methylation } \\
\text { 450K array }\end{array}$ & $\begin{array}{l}\text { Hippocampus, } \\
\text { entorhinal cortex, } \\
\text { dorsolateral } \\
\text { prefrontal cortex, } \\
\text { cerebellum }\end{array}$ & $\begin{array}{l}\text { Bisulfite treatment } \\
\text { (total DNA } \\
\text { modifications } \\
{[5-\mathrm{mC}+5-\mathrm{hmC}] \text { ) }}\end{array}$ & $\begin{array}{l}\text { ANK1, ANKRD30B, } \\
\text { WDR81, SERPINF2, } \\
\text { MYO1C }\end{array}$ \\
\hline $\begin{array}{l}\text { Smith } \\
\text { et al. [13] }\end{array}$ & 2019 & 96 & $\begin{array}{l}\text { Illumina } \\
\text { Methylation } \\
450 \mathrm{~K} \text { array }\end{array}$ & Entorhinal cortex & $\begin{array}{l}\text { Oxidative-bisulfite } \\
\text { treatment } \\
\text { (5-mC, 5-hmC and } \\
5-\mathrm{uC} \text { independently }\end{array}$ & $\begin{array}{l}\text { WNT5B, FBXL16, } \\
\text { ANK1, ARID5B, } \\
\text { ALLC }\end{array}$ \\
\hline $\begin{array}{l}\text { Lardenoije } \\
\text { et al. [24] }\end{array}$ & 2019 & 80 & $\begin{array}{l}\text { Illumina } \\
\text { Methylation } \\
450 \mathrm{~K} \text { array }\end{array}$ & $\begin{array}{l}\text { Middle temporal } \\
\text { gyrus, blood }\end{array}$ & $\begin{array}{l}\text { Oxidative-bisulfite } \\
\text { treatment } \\
\text { (5-mC, 5-hmC and } \\
5-\mathrm{uC} \text { independently) }\end{array}$ & $\begin{array}{l}\text { OXT, CHRNB1, } \\
\text { RHBDF2, C3 }\end{array}$ \\
\hline
\end{tabular}

Abbreviations: $5-\mathrm{hmC}=$ hydroxymethylation; $5-\mathrm{mC}=$ methylation $; 5-\mathrm{uC}=$ unmodified cytosine. 
Interestingly, of the genes identified, ANK1, CHD23, DIP2A, RHBDF2, RPL13, SERPINF1 and SERPINF2 were also found to display significant AD-associated gene expression changes, supporting a potential functional role for DNA methylation in AD [10]. Differential methylation of $A N K 1$ has been established in five studies $[6,9,12-14]$. Furthermore, two genes of the dual specificity phosphatase (DUSP) family of proteins were shown to be differentially methylated in two independent studies as well $[8,11]$. An overview of key findings and study designs for all MWAS published to date can be found in Table 1. Notably, a recent cross-cortical metaanalysis performed on nine previously published MWAS data sets consisting of 1408 donors, identified 220 CpGs significantly associated with AD neuropathology. These probes were annotated to 121 unique genes, of which 96 had not been previously reported in AD MWAS [15].

Although these MWAS findings have been highly valuable for improving our understanding of the molecular etiology of $A D$, there are a number of limitations to these approaches. Such challenges would need to be addressed properly in future endeavors to allow for appropriate reliable interpretation of generated data in order to significantly increase our understanding about the disease processes underlying $A D$. In this mini-symposium article, we showcase DNA methylation EWAS in AD, addressing several achievements and challenges in this respect in more detail. While this particular perspective article focuses on DNA methylation studies, there is also a substantial body of literature on other levels of epigenetic regulation, including ncRNAs such as microRNAs (miRNAs). For a timely review, in the context of $A D$, see [16]. Notably, many of the considerations referred to below do apply to other epigenetic processes as well.

\section{Genomic coverage}

Current state-of-the-art EWAS platforms used for studying DNA methylation alterations in $A D$ have mostly been microarray based. This approach has been largely successful owing to their sensitivity and relative ease of execution in terms of analysis and standardization of protocols, allowing for a reliable comparison between studies. Ultimately, the common platform used across the MWAS to date would also lend itself well to meta-analyses, the first of which has just been undertaken in the case of $A D$ [15]. However, despite a significant increase in CpG coverage on the Illumina microarrays over recent years, current EWAS platforms still interrogate only a small proportion of $\mathrm{CpG}$ sites and predominantly assess CpG-rich promoter regulatory regions, hence, missing a large proportion of the human DNA methylome. As an example, even though the latest Illumina Infinium Methylation EPIC BeadChip array, which allows for the interrogation of over $850.000 \mathrm{CpG}$ sites across the genome, shows improved coverage of regulatory elements, such as enhancers, when compared to its predecessors, even now, only 
a limited proportion of distal and proximal regulatory elements are represented [17]. For a carefully annotated description of CpG coverage on Illumina arrays, see [18] and [19]. These EWAS microarray platforms further lack the possibility to thoroughly assess DNA methylation at non-CpG (cytosine and adenine) sites. The use of (more expensive) next-generation sequencing-based approaches allowing for methylation assessment across the entire genome shows great promise in this respect, although these come with other major challenges as well, including those related to power (requiring larger sample sizes in view of multiple testing; see below), sequencing depth and associated costs.

\section{Statistical power}

Existing MWAS findings should be interpreted with caution, as most published studies seem (severely) underpowered. Power calculations for EPIC-based DNA methylation studies demonstrate that existing studies with data on 1000 samples are adequately powered to detect small differences at the majority of sites [20]. Furthermore, for future endeavors that may use next generation sequencing approaches to increase coverage, increased samples sizes will be required. Evidently, when taking into account the intrinsic methylation susceptibility of specific CpG sites (i.e. likelihood of an individual CpG to be methylated, and the nucleotide distance between neighboring $\mathrm{CpG}$ sites [21]), or when assessing differentially methylated regions (DMRs) consisting of adjacent differentially methylated positions (DMPs), smaller sample sizes are sufficient to reach genomewide significance. In this respect, Bonferroni correction for the number of probes on the array is often presumed to be too conservative as DNA methylation values at neighboring probes are generally known to be correlated. As such, in addition to false-discovery rate (FDR) correction, recent endeavors attempt to estimate the number of independent tests performed in an EWAS and adjust the significance level accordingly [20,22]. Altogether, although it is important to consider sample size when interpreting EWAS results, it is worth noting that different CpG sites show different variance and they will, therefore, be differently powered at the same sample size.

\section{Specificity of the epigenetic marks assessed}

Accumulating evidence suggests an important role for other types of cytosine modifications such as 5-hydroxymethylcytosine (5-hmC) in the human brain [23]. However, the great majority of MWAS on AD published to date have made use of sodium bisulfite-treated DNA, which is unable to discriminate between 5-mC and its first oxidation product, 5-hmC. Recently, the first EWAS simultaneously assessing DNA methylation and hydroxymethylation in isolation in AD by making use of oxidative-bisulfite DNA treatment was published [13]. This study highlighted different levels of $5-\mathrm{mC}, 5-\mathrm{hmC}$ or unmodified cytosine $(5-\mathrm{uC})$ in a number of 
different genes, including WNT5B, ANK1, ARID5B, FBXL16, ALLC and JAG2. More specifically, in view of the earlier MWAS findings on $A N K 1$, this study demonstrated that ANK1 DNA modification alterations in AD are as a result of DNA hypermethylation and hypohydroxymethylation, suggestive of a loss of active DNA demethylation of ANK1 in AD. Recently, a similar approach was used in AD and control individuals using cortical tissue from the middle temporal gyrus and peripheral whole blood samples and led to the identification of a common DMR associated with $A D$, close to the transcription start site of OXT [24]. The study suggested OXT 5-mC and 5-hmC levels change in opposite directions within the middle temporal gyrus in AD. Interestingly, the detection of a differentially methylated region near OXT is in line with a recent report of a nearly identical AD-associated OXT DMR in the superior temporal gyrus, which is located directly above the middle temporal gyrus [11].

While earlier studies on DNA modification changes have primarily focused on 5-mC and 5-hmC, other marks such as 5-formylcytosine (5-fC) and 5-carboxylcytosine (5-caC), which both represent oxidized derivatives of 5-hmC, are heavily understudied. Little is known about the frequency or functionality of these modifications in the healthy or diseased human brain. Further studies are therefore required to develop a better understanding on their potential role in the development and course of AD. Alternative, more specific technologies such as DNA-immunoprecipitation (IP) sequencing approaches with DNA being captured by specific antibodies to distinct DNA modifications hold great promise for assessing these epigenetic marks. In addition, direct DNA sequencing using novel third generation sequencing platforms, such as the Oxford Nanopore Technologies (ONT) minion/ promethion or the Pacific Biosciences (Pacbio) Sequel, allows calling of both single nucleotide polymorphisms (SNPs) and a range of different DNA modifications by sequencing native DNA without prior amplification via PCR or labeling the sample chemically, which represents another promising approach [25]. Evidently, these more advanced sequencing approaches do still require distinct, more challenging data science investments for calling of different DNA modifications.

\section{Cell-type specificity and composition}

A major issue that challenges the field of neuroepigenetics is that of tissue and celltype specificity of epigenetic modifications. $A D$ is characterized by neuronal loss, and activation of glia cells, concomitant with alterations in the cell-type composition of brain samples, which challenges the correct interpretation of results when making use of heterogeneous bulk tissue samples. As such, cell-type specific modifications in one cell-type could, for example, mask changes in another [26]. Aside from cell-type specific changes in activity or changes in cellular proportions that can occur during the 
development and course of $A D$, differences in cell-type compositions between samples derived from different individuals that arise as a result of tissue sampling is also a consideration. A workable, though often expensive and tedious, solution to this issue could be specifically profiling cell-type specific samples, to be acquired via, for example, laser capture microdissection (LCM), fluorescence-activated cell sorting (FACS) or magnetic-activated cell sorting (MACS). This could even exploit single-cell sequencing technologies to investigate cell-specific epigenomes [27]. Alternatively, one can use advanced bioinformatic approaches to correct for celltype composition in MWAS data generated in bulk (unsorted) tissue. As an example, one can estimate neuronglia proportions based on DNA methylation values in EWAS data and this approach has been adopted by many in the AD epigenetics field [2830]. Akin to the issues associated with studying epigenetic changes in the brain, cell type specificity issues also apply to studies making use of whole blood samples and these can be addressed in similar ways, for example, by adjusting data for cell-type specific DNA methylation markers [20, 21].

\section{Causal inference}

At present, one of the major limitations of current endeavors investigating the role of epigenetic dysregulation in aging and $A D$, is the issue of causality. Alterations in DNA modifications could be either causal in the disease process or could themselves arise as a direct or indirect result of pathological or secondary behavioral and psychological changes associated with disease. Similarly, age, comorbidity or the use of therapeutic agents may have a profound impact on the epigenome, both in the brain and blood. In addition, epigenetic signatures identified in advanced $A D$ cases, particularly in brain regions affected relatively late in the disease process, may provide limited information on causality. An interesting notion in this regard is the study of epigenomic changes with respect to the spatiotemporal spread of pathology in AD. For example, utilizing postmortem $A D$ brain tissue derived from donors at different disease stages and comparing the epigenome of brain regions implicated in the early stages of $A D$ (i.e. brainstem) to those regions affected in more advanced phases (i.e. cortical regions) in the same individuals, could allow the identification of the relative, spatiotemporal contribution of potentially causal, region-specific epigenetic alterations in the development and course of AD. Similarly, when assessing the blood epigenome, longitudinal studies capturing early stages of cognitive decline, and preferably also exposure to environmental factors throughout life (which may evidently also impact on the blood epigenome), are needed [31]. Alternatively, rodent models of AD could also be used to explore causal epigenetic mechanism in $A D$, by studying alterations prior to the onset of pathology. However, one limitation in this regard is that these murine systems are models of familial $A D$, bearing autosomal dominant mutations and are not a true model of sporadic disease. Another approach to elucidate 
causal epigenetic mechanisms in $A D$ would be through the use of epigenetic editing. The use of novel editing constructs based on the catalytically deactivated clustered regularly interspaced short palindromic repeats (CRISPR) associated protein 9 (i.e. CRISPR-dCas9) fused to chromatin modifying enzymes such as DNA methyltransferase 3a (DNMT3A) or TET1, also known as EpiEffectors [32], allows adding, or removing, of methyl groups to specific DNA loci, respectively [33]. The use of this system in vitro in, for example induced pluripotent stem cells (iPSCs) could ultimately allow the field to determine the functional implications of epigenetic signatures identified in MWAS and to establish whether these represent a cause, a consequence, or both, of the disease process. Further information on this concept can be found elsewhere in this mini-symposium series [35]. As epigenetic changes are often reversible, the identification of causative epigenetic signatures may provide promising targets for future therapeutic interventions.

\section{Multi-omics approaches}

Importantly, the ability to identify specific and early signatures of $A D$ is hindered by the substantial clinical heterogeneity among patients, for instance there is considerable variability in disease duration and cognitive decline rate between patients, which likely reflects inter-individual variation in genetics, exposure to environmental factors and underlying neuropathology. In recent years, several "big data" approaches have allowed the identification of robust genomic, epigenomic and transcriptomic changes in AD. However, these studies have largely been restricted to correlating a single layer of molecular information with respect to a single measure of disease severity, in a single tissue, and it is not yet possible to identify the exact mechanisms involved in the pathophysiology of AD or to predict a patient's disease trajectory with current peripheral biomarkers. The recent development of powerful computational frameworks now offers multilayer inter-regulatory approaches to understand the development and course of $A D$, while accounting for inter-individual differences in genotype and exposure to environmental risk factors. As such, multi-omics approaches - that include an integrative analysis of various layers of epigenetic regulation - and associated data science tools show great promise in the development of novel diagnostic tools and treatment strategies for $A D$, with further details on this approach provided elsewhere in this mini-symposium [36].

\section{Concluding remarks}

There is increasing interest in the role of epigenetic dysregulation in aging and $A D$, with the primary focus of most EWAS studies being on DNA methylation. Platforms such as the Illumina Infinium Methylation 450K and EPIC BeadChip arrays have enabled cost-efficient, high-throughput profiling of methylomic variation across large numbers of samples. EWAS results should be interpreted with caution though, particularly when dealing with low sample-size studies. Future 
endeavors should aim for specific, adequately powered approaches, with large sample sizes and well-characterized environmental, medication and ante- and/or postmortem data, while considering tissue and cellular heterogeneity. Moreover, candidate signatures should be functionally validated to determine whether they could represent novel causal mechanisms.

\section{Acknowledgments}

Funds have been provided by the Joint Program - Neurodegenerative Disease Research (JPND) for the EPI-AD consortium (http://www.neurodegeneration research.eu/wpcontent/uploads/2015/10/Factsheet_EPI-AD.pdf). The project is supported through the following funding organizations under the aegis of JPND; the Netherlands, The Netherlands Organization for Health Research and Development (ZonMw); United Kingdom, Medical Research Council; Germany, German Federal ministry of Education and Research (BMBF); Luxembourg, National Research Fund (FNR). This project has received funding from the European Union's Horizon 2020 research and innovation program under Grant Agreement No. 643417.

\section{References}

1. Bertram L, Lill CM, Tanzi RE. The genetics of Alzheimer disease: back to the future. Neuron. 2010;68(2):270-81.

2. Bertram L, Tanzi RE. Genomic mechanisms in Alzheimer's disease. Brain Pathology. 2020;30(5):966-77.

3. Liu X, Jiao B, Shen L. The epigenetics of Alzheimer's disease: Factors and therapeutic implications. Frontiers in genetics. 2018;9:579.

4. Chouliaras L, Rutten BP, Kenis G, Peerbooms O, Visser PJ, Verhey F, et al. Epigenetic regulation in the pathophysiology of Alzheimer's disease. Progress in neurobiology. 2010;90(4):498-510.

5. Lardenoije R, Pishva E, Lunnon K, van den Hove DL. Neuroepigenetics of Aging and Age-Related Neurodegenerative Disorders. Prog Mol Biol Transl Sci. 2018;158:49-82.

6. Lunnon K, Mill J. Epigenetic studies in Alzheimer's disease: current findings, caveats, and considerations for future studies. American journal of medical genetics Part B, Neuropsychiatric genetics : the official publication of the International Society of Psychiatric Genetics. 2013;162B(8):789-99.

7. Bakulski KM, Dolinoy DC, Sartor MA, Paulson HL, Konen JR, Lieberman AP, et al. Genome-wide DNA methylation differences between late-onset Alzheimer's disease and cognitively normal controls in human frontal cortex. Journal of Alzheimer's Disease. 2012;29(3):571-88.

8. Sanchez-Mut JV, Aso E, Heyn H, Matsuda T, Bock C, Ferrer I, et al. Promoter hypermethylation of the phosphatase DUSP22 mediates PKA-dependent TAU phosphorylation and CREB activation in Alzheimer's disease. Hippocampus. 2014;24(4):363-8.

9. De Jager PL, Srivastava G, Lunnon K, Burgess J, Schalkwyk LC, Yu L, et al. Alzheimer's disease: early alterations in brain DNA methylation at ANK1, BIN1, RHBDF2 and other loci. Nat Neurosci. 2014;17(9):1156-63.

10. Lunnon K, Smith R, Hannon E, De Jager PL, Srivastava G, Volta M, et al. Methylomic profiling implicates cortical deregulation of ANK1 in Alzheimer's disease. Nat Neurosci. 2014;17(9):1164-70.

11. Watson CT, Roussos P, Garg P, Ho DJ, Azam N, Katsel PL, et al. Genome-wide DNA methylation profiling in the superior temporal gyrus reveals epigenetic signatures associated with Alzheimer's disease. Genome medicine. 2016;8(1):1-14.

12. Semick SA, Bharadwaj RA, Collado-Torres L, Tao R, Shin JH, Deep-Soboslay A, et al. Integrated 
DNA methylation and gene expression profiling across multiple brain regions implicate novel genes in Alzheimer's disease. Acta neuropathologica. 2019;137(4):557-69.

13. Smith AR, Smith RG, Pishva E, Hannon E, Roubroeks JAY, Burrage J, et al. Parallel profiling of DNA methylation and hydroxymethylation highlights neuropathology-associated epigenetic variation in Alzheimer's disease. Clin Epigenetics. 2019;11(1):52.

14. Gasparoni G, Bultmann S, Lutsik P, Kraus TF, Sordon S, Vlcek J, et al. DNA methylation analysis on purified neurons and glia dissects age and Alzheimer's disease-specific changes in the human cortex. Epigenetics \& chromatin. 2018;11(1):41.

15. Smith R, Pishva E, Shireby G, Smith AR, Roubroeks JA, Hannon E, et al. Meta-analysis of epigenomewide association studies in Alzheime's disease highlights 220 differentially methylated loci across cortex. BioRxiv. 2020.

16. Van den Hove DL, Kompotis K, Lardenoije R, Kenis G, Mill J, Steinbusch HW, et al. Epigenetically regulated microRNAs in Alzheimer's disease. Neurobiology of aging. 2014;35(4):731-45.

17. Pidsley R, Zotenko E, Peters TJ, Lawrence MG, Risbridger GP, Molloy P, et al. Critical evaluation of the Illumina MethylationEPIC BeadChip microarray for whole-genome DNA methylation profiling. Genome biology. 2016;17(1):1-17.

18. Sandoval J, Heyn H, Moran S, Serra-Musach J, Pujana MA, Bibikova M, et al. Validation of a DNA methylation microarray for 450,000 CpG sites in the human genome. Epigenetics. 2011;6(6):692-702.

19. Moran S, Arribas C, Esteller M. Validation of a DNA methylation microarray for $850,000 \mathrm{CpG}$ sites of the human genome enriched in enhancer sequences. Epigenomics. 2016;8(3):389-99.

20. Mansell G, Gorrie-Stone TJ, Bao Y, Kumari M, Schalkwyk LS, Mill J, et al. Guidance for DNA methylation studies: statistical insights from the Illumina EPIC array. BMC genomics. 2019;20(1):1-15.

21. Affinito $O$, Palumbo D, Fierro A, Cuomo M, De Riso G, Monticelli A, et al. Nucleotide distance influences co-methylation between nearby CpG sites. Genomics. 2020;112(1):144-50.

22. Saffari A, Silver MJ, Zavattari P, Moi L, Columbano A, Meaburn EL, et al. Estimation of a significance threshold for epigenome-wide association studies. Genetic epidemiology. 2018;42(1):20-33.

23. Cheng Y, Bernstein A, Chen D, Jin P. 5-Hydroxymethylcytosine: a new player in brain disorders? Experimental neurology. 2015;268:3-9.

24. Lardenoije R, Roubroeks J, Pishva E, Leber M, Wagner H, latrou A, et al. T40. Alzheimer's Disease DNA (Hydroxy) Methylome in the Brain and Blood: Evidence for OXT Methylation as a Preclinical Marker. Biological psychiatry. 2019;85(10):S144.

25. Jain M, Olsen HE, Paten B, Akeson M. The Oxford Nanopore MinION: delivery of nanopore sequencing to the genomics community. Genome biology. 2016;17(1):1-11.

26. Blalock EM, Buechel HM, Popovic J, Geddes JW, Landfield PW. Microarray analyses of laser-captured hippocampus reveal distinct gray and white matter signatures associated with incipient Alzheimer's disease. J Chem Neuroanat. 2011;42(2):118-26.

27. Bheda P, Schneider R. Epigenetics reloaded: the single-cell revolution. Trends in cell biology. 2014;24(11):712-23.

28. Guintivano J, Aryee MJ, Kaminsky ZA. A cell epigenotype specific model for the correction of brain cellular heterogeneity bias and its application to age, brain region and major depression. Epigenetics. 2013;8(3):290-302.

29. Houseman EA, Accomando WP, Koestler DC, Christensen BC, Marsit CJ, Nelson HH, et al. DNA methylation arrays as surrogate measures of cell mixture distribution. BMC bioinformatics. 2012;13(1):86.

30. Horvath S. DNA methylation age of human tissues and cell types. Genome biology. 2013;14(10):3156.

31. van den Hove DL, Kenis G, Rutten BP. Epigenetic dysregulation in Alzheimer's disease: cause or consequence? Epigenomics. 2014;6(1):9-11. 
32. Kungulovski G, Jeltsch A. Epigenome Editing: State of the Art, Concepts, and Perspectives. Trends in Genetics. 2016;32(2):101-13.

33. Liu XS, Wu H, Ji X, Stelzer Y, Wu X, Czauderna S, et al. Editing DNA Methylation in the Mammalian Genome. Cell. 2016;167(1):233-47 e17.

34. Smith RG, Hannon E, De Jager PL, Chibnik L, Lott SJ, Condliffe D, et al. Elevated DNA methylation across a 48-kb region spanning the HOXA gene cluster is associated with Alzheimer's disease neuropathology. Alzheimer's \& Dementia. 2018;14(12):1580-8.

35. Schrauben M, Dempster E, Lunnon K. Applying gene-editing technology to elucidate the functional consequence of genetic and epigenetic variation in Alzheimer's disease. Brain Pathology. 2020;30(5):992-1004.

36. Ma Y, Klein HU, De Jager PL. Considerations for integrative multi-omic approaches to explore Alzheimer's disease mechanisms. Brain Pathology. 2020;30(5):984-91. 



\section{Chapter 3}

\section{Targeted detection of unmodified cytosine, 5-methylcytosine and 5-hydroxymethylcytosine levels at single CpG sites by oxidative bisulfite pyrosequencing}

Re zo J Riemens ${ }^{1,2}$, Gunter Kenis ${ }^{1}$, Diego Mastroeni ${ }^{3}$, Thomas Haaf², Danto A. v? den Hove . $^{1,4}$

${ }^{1}$ Department of Psychiatry and Neuropsychology, School for Mental Health and Neuroscience (MHeNs), Maastricht University, Maastricht, the Netherlands.

${ }^{2}$ Institute of Human Genetics, Julius Maximilians University, Wuerzburg, Germany.

${ }^{3}$ L.J. Roberts Alzheimer's Disease Center, Banner Sun Health Research Institute, Sun City, Arizona, United States of America.

${ }^{4}$ Laboratory of Translational Neuroscience, Department of Psychiatry, Psychosomatics and Psychotherapy, University of Wuerzburg, Wuerzburg, Germany. 
Chapter 4

Targeted methylation profiling of single
laser-capture microdissected post-
mortem brain cells by adapted limiting
dilution bisulfite pyrosequencing (LDBSP)

Renzo J.M. Riemens ${ }^{1,2}$, Gunter Kenis ${ }^{1}$, Jennifer Nolz ${ }^{3}$, Sonia C. Susano Chaves ${ }^{1}$, Diane Duroux ${ }^{4}$, Ehsan Pishva ${ }^{1}$, Diego Mastroeni ${ }^{3}$, Kristel van Steen ${ }^{4,5}$, Thomas Haaf ${ }^{2}$, Daniël L.A. van den Hove ${ }^{1,6}$

'Department of Psychiatry and Neuropsychology, School for Mental Health and Neuroscience (MHeNs), Maastricht University, Maastricht, the Netherlands.

${ }^{2}$ Institute of Human Genetics, Julius Maximilians University, Wuerzburg, Germany.

${ }^{3}$ Biodesign Institute, Neurodegenerative Disease Research Center, Arizona State University, Tempe, Arizona, United States of America.

${ }^{4}$ WELBIO, GIGA-R Medical Genomics - BIO3, University of Liège, Liège, Belgium; Department of Human Genetics - Systems Medicine, University of Leuven, Leuven, Belgium.

${ }^{5}$ Department of Human Genetics - Systems Medicine, University of Leuven, Leuven, Belgium.

${ }^{6}$ Laboratory of Translational Neuroscience, Department of Psychiatry, Psychosomatics and Psychotherapy, University of Wuerzburg, Wuerzburg, Germany. 



\begin{abstract}
A reoccurring issue in neuroepigenomic studies, especially in the context of neurodegenerative disease, is the use of (heterogeneous) bulk tissue, which generates noise during epigenetic profiling. A workable solution to this issue is to quantify epigenetic patterns in individually isolated neuronal cells using laser capture microdissection (LCM). For this reason, we established a novel approach for targeted DNA methylation profiling of individual genes that relies on a combination of LCM and limiting dilution bisulfite pyrosequencing (LDBSP). Using this approach, we determined cytosine-phosphate-guanine (CpG) methylation rates in a multi-targeted manner on single alleles derived from 50 neurons that were isolated from unfixed post-mortem brain tissue. In the present manuscript, we describe the general pipeline and, as a showcase, demonstrate how targeted methylation analysis of various genes, in this case DNAJB13, PGLYRP1, RHBDF2, $C 3, L M X 1 B$ and $O X T$, can be performed simultaneously. By doing so, we describe an adapted data analysis pipeline for LDBSP, allowing one to include and correct CpG methylation rates derived from multi-allele reactions. In addition, we show that the efficiency of LDBSP on 50 LCM neurons is similar to the efficiency obtained in previously published studies using this technique on other isolated cell types. Overall, the method described here provides the user with a more accurate estimation of the DNA methylation status of each target gene in the analyzed cell pools, thereby adding further validity to this approach.
\end{abstract}

\title{
Keywords
}

Limiting dilution bisulfite pyrosequencing, laser-capture microdissection, DNA methylation, single cell, epigenetics. 


\section{Introduction}

An increasing number of studies has implicated a central role for epigenetic mechanisms such as DNA methylation in the pathophysiology of neurodegenerative disorders, including Alzheimer's disease (AD) [1, 2]. The field of neuroepigenomics, however, still faces many challenges that impede attempts to disentangle the exact contribution of DNA methylation alterations in the development and course of disorders like AD. A central issue is the cellular heterogeneity of the studied bulk tissue samples, which represents a major source of noise in epigenetic profiling [3]. Next to drastically decreasing the signal-to-noise ratio, the use of bulk tissue samples does not allow one to conclude whether differences found between e.g. patient and control brains are related to disease status rather than changes in cell type composition [1]. In fact, neurodegenerative diseases like AD are characterized by neuronal loss, concomitant with alterations in the brain cellular architecture [2]. Thus, differences in cellular composition between tissue samples from patients and control cases can lead to misinterpretation of the acquired epigenetic data. Aside from disease-related changes in cellular proportions, differences in cell type composition between different samples that arise from tissue sampling represents another issue introducing bias. Moreover, even in the case of comparable cellular proportions, heterogeneous tissues could mask cell-type specific modifications related to the disease, as changes in one cell type could dilute or oppose changes in another, thereby obscuring important cell subtype-specific aberrations when analyzed together [4].

In order to overcome the issue of cellular heterogeneity in methylome-wide studies, it is possible correct for cell type composition using advanced bioinformatics approaches [5-7]. However, there is an ongoing dispute concerning the validity of this approach $[3,8,9]$. A more accurate alternative, and the only option in the case of targeted candidate gene-based approaches, is to profile epigenetic patterns in individually isolated cells that were selected e.g. based on a cell typespecific marker. This latter approach does not only avoid noise that is induced by differences in cell type composition of the studied bulk tissue samples, but it also provides a more detailed overview of epigenetic profiles in individual cell types. In recent years, limiting dilution bisulfite pyrosequencing (LDBSP) has emerged as a cost-effective approach, allowing targeted bisulfite methylation profiling at a single cytosine-phosphate-guanine $(\mathrm{CpG})$ site resolution in a single or a few cells [10]. The principle of this technique relies on an excessive dilution of the bisulfite-treated target DNA obtained from a single cell so that maximum one allele (or none) is present in each of the downstream reactions. Subsequently, each individual DNA molecule is separately amplified by (semi-) nested polymerase chain reactions (PCRs) and analyzed by means of pyrosequencing. Thus, when applied in conjunction with a highly selective isolation procedure, such as laser- 
capture microdissection (LCM) based on the immunoreactivity of a cell-specific marker, this technique offers an appealing approach for the detection of methylation patterns in individual cellular populations of the brain.

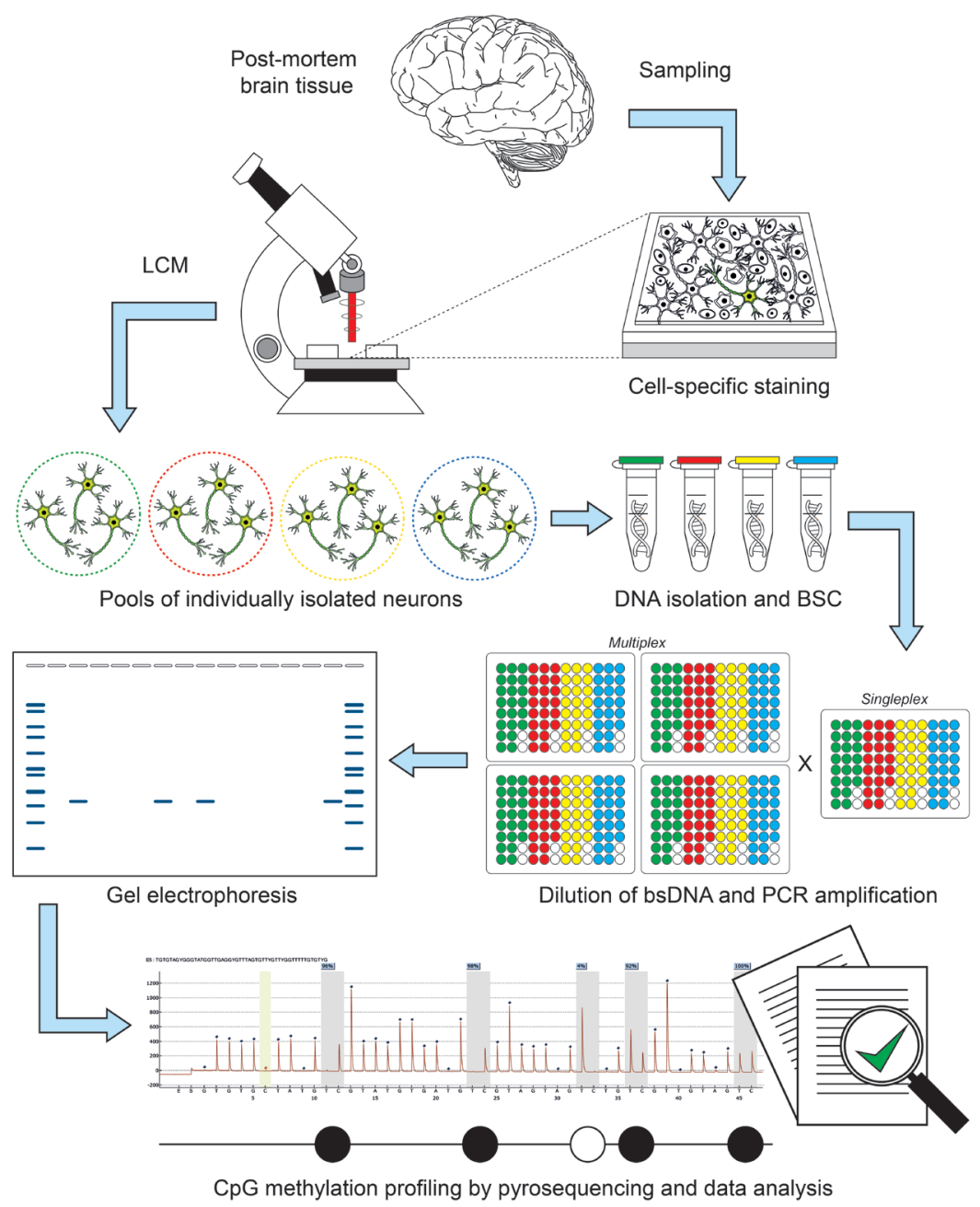

Figure 1. Overview of the limiting dilution bisulfite pyrosequencing (LDBPS) procedure for pools of individually isolated neurons using laser capture microdissection (LCM). Tissue from post-mortem brains is sampled and stained using a cell type-specific marker. Neurons are then isolated and divided in small cell pools. DNA derived from these neurons then undergoes a bisulfite conversion (BSC) treatment and is subsequently diluted to a single allele level. The bisulfite converted DNA (bsDNA) is then amplified twice by means of a (semi-)nested polymerase chain reaction (PCR) in multiplex-singleplex formation. Product-yielding reactions are then visualized on an agarose gel and the cytosine-phosphate-guanine $(\mathrm{CpG})$ methylation status of these products is then profiled by means of pyrosequencing. 
Although LDBSP has been shown to be compatible for individual 2-16 cell embryos, single germinal vesicle oocytes and haploid sperms [10,11], to date, this technique has not been applied on ex vivo brain cells. In the present study, we therefore demonstrate, for the first time, that LDBSP can be successfully applied on pools of 50 neurons, isolated by LCM from unfixed post-mortem brain tissue (Figure 1). Here, we describe the general adapted LCM-LDBSP pipeline and, as a showcase, demonstrate how a targeted methylation analysis can be performed in multiple genes (DNAJB13, PGLYRP1, RHBDF2, C3, LMX1B and OXT) simultaneously. Importantly, diluting a DNA target sample derived from 50 neurons to a singleallele-level will occasionally render reactions with more than one allele, a scenario that does not regularly occur when conducting LDBSP using one or just a few cells. We therefore describe an adapted data analysis pipeline specifically designed for assessing pools of LCM neurons that allow to include and correct the methylation data for multi-allele reactions. This novel approach, which compromises a CpGsite calling procedure, combined with an integrated in-depth analysis of the raw $\mathrm{CpG}$ methylation rates, aims at avoiding unintentionally induced bias due to the blunt exclusion of reactions that suggest to contain more than one allele. Overall, the method described here allows the user to more accurately determine the DNA methylation status of the target genes in the analyzed samples, thereby adding further validity to the experimental data acquired. In addition, we show that the efficiency of LDBSP on 50 neurons isolated with LCM from post-mortem brain tissues is similar to the efficiency achieved in previously published studies using this technique on other isolated cell types.

\section{Materials and methods}

A step-wise overview of the LDBSP protocol is available in the Supplementary material.

\section{Ethics statement}

Written informed consent for brain autopsies was obtained in compliance with institutional guidelines of the Banner Sun Health Research Institute (BSHRI, Sun City, Arizona, US). The Banner Sun Health Research Institute Review Board approved the entire study, including the recruitment, enrollment and autopsy procedures. Each individual and their respective relative(s) consented to brain autopsy for the purpose of scientific research as part of the BSHRI Brain and Body Donation Program (BBDP). The human brain tissue used in this study was derived from routine autopsies, fully qualifying for $4 \mathrm{C}$ exemption by the National Institute of Health $(\mathrm{NIH})$ guidelines [12]. All samples were analyzed anonymously throughout the experimental procedures. 


\section{Sample collection}

Frozen unfixed dorsal raphe nuclei (DRN) tissue from 24 individuals (12 female $A D$ and 12 female age-matched non-demented control cases) were collected at the BSHRI. Brain samples were frozen and stored at $-80^{\circ} \mathrm{C}$ after autopsy, with an average post-mortem interval (PMI) of $2.69 \pm 0.82$ hours. A final diagnosis of $A D$ or non-demented healthy control was made based on the NIH AD Center criteria [12]. Comorbidity with any other type of dementia, mild cognitive impairment, cerebrovascular disorders and presence of non-microscopic infarcts were applied as exclusion criteria. For demographic and other relevant information about the studied samples, please refer to Supplementary Table 1.

\section{Immunohistochemistry}

Frozen DRN tissue sections of $10 \mu \mathrm{m}$ from were mounted onto polyethylene naphthalate (PEN) slides and fixed in ice-cold $50 \%$ acetone $/ 50 \%$ ethanol solution for 5 minutes on ice. Sections were washed in ice cold phosphate buffered saline (PBS), blocked in 1\% hydrogen peroxide for 2 minutes, followed by 3 quick submersions in ice-cold PBS. Sections were then placed in a dilution of primary antibody against serotonin (5-HT; Abcam, ab66047) in PBS for 10 minutes at room temperature. After the incubation, sections were washed three times in PBS and incubated with avidin-biotin complex in PBS for 10 minutes at room temperature. Next, sections were washed three times in $50 \mathrm{mM}$ Tris buffer and immersed in 3.3'-diaminobenzidine (DAB) solution $(9.3 \mathrm{ml} 50 \mathrm{mM}$ Tris; $200 \mu \mathrm{l} \mathrm{DAB}(5 \mathrm{mg} /$ $\mathrm{ml}$ ); $500 \mu \mathrm{l}$ saturated nickel; and $4 \mu \mathrm{l}$ of $1 \%$ hydrogen peroxidase) for 5 minutes, followed by two quick rinses in $50 \mathrm{mM}$ Tris to stop the reaction. All sections were stored at $-80^{\circ} \mathrm{C}$ until further processing.

\section{Laser-capture microdissection}

$5-\mathrm{HT}$ is a monoamine neurotransmitter that is specifically expressed by serotonergic neurons [13]. For this reason, LCM of serotonergic neurons from the DRN sections was performed based on 5-HT-immunoreactivity. In brief, sections were dipped in $100 \%$ ethanol, allowed to dry, and loaded onto a Leica AS-LMD LCM microscope (Leica, Wetzlar, Germany). Single serotonergic neurons were cut and then dropped into an inverted microcentrifuge cap containing $10 \mu \mathrm{l}$ of TrisEDTA (TE) buffer. Per individual subject, 150 serotonergic neurons were captured at 20X magnification and divided in small pools of 50 cells per microcentrifuge tube, i.e. three pools of 50 neurons per subject. All isolated cells were stored at $-80^{\circ} \mathrm{C}$ until further processing.

\section{DNA isolation and sodium bisulfite treatment}

Genomic DNA from a pool of 50 neurons was isolated and bisulfite-converted using the EZ DNA Methylation-Direct Kit (Zymo Research, Irvine, CA, USA) with 
the following adjustments. In brief, $1 \mu \mathrm{l}$ of proteinase $\mathrm{K}(20 \mu \mathrm{g} / \mu \mathrm{l})$ and $11 \mu \mathrm{l}$ of M-Digestion buffer $(2 \mathrm{X})$ were added to a microcentrifuge tube containing the cells and incubated overnight at $50^{\circ} \mathrm{C}$. Subsequently, the complete lysate was transferred to a PCR tube and $143 \mu \mathrm{l}$ of bisulfite conversion reagent was used to wash out the digestion tube before adding it to the sample. Bisulfite conversion was performed in a thermal cycler running at $98^{\circ} \mathrm{C}$ for 8 minutes and then at $64^{\circ} \mathrm{C}$ for 3.5 hours. A volume of $200 \mu$ binding buffer was added to the spin column before loading the bisulfite-converted sample. The PCR tube used for bisulfite conversion was washed out twice by first adding $200 \mu \mathrm{l}$ of binding buffer to the tube and then by transferring this volume to the sample-containing column. After centrifugation $(10,000 \times \mathrm{g} ; 30$ seconds), the column was washed with $100 \mu \mathrm{l}$ washing buffer, incubated for 15 minutes with $200 \mu$ desulfonation buffer and washed twice again with $200 \mu \mathrm{l}$ washing buffer. The bisulfite-converted DNA was eluted in a single Eppendorf tube by running $20 \mu \mathrm{l}$ of elution buffer through the column twice (Two times at 10,000 x g; 30 seconds). Eppendorf LoBind microcentrifuge tubes (Merck KGaA, Darmstadt, Germany) and TipOne Low Retention Tips (STARLAB, Hamburg, Germany) with low affinity for DNA were used throughout the whole procedure. Multiplex PCR amplifications were performed directly after elution of the bisulfite-converted DNA.

\section{Multiplex polymerase chain reaction}

All assays were based on a (semi-)nested PCR design and amplified in multiplexsingleplex formation. Primers were designed with the PyroMark Assay Design 2.0 software (Qiagen, Hilden, Germany; see Supplementary Table 2). Bisulfite-treated DNA derived from a pool of 50 neurons was diluted to a single allele level by adding a multiplex PCR mixture with a capacity of 22 individual reactions to the sample (determined empirically). Each individual multiplex PCR reaction made use of $2.5 \mu \mathrm{l} \mathrm{PCR}$ buffer (10X) with $20 \mathrm{mM} \mathrm{MgCl} 2,0.5 \mu \mathrm{l} 10 \mathrm{mM}$ dNTP mix, $1 \mu \mathrm{l}$ of each primer $\left(10 \mu \mathrm{M}\right.$ stock) and $0.2 \mu \mathrm{l}(5 \mathrm{U} / \mu \mathrm{l})$ FastStart ${ }^{\mathrm{TM}}$ Taq DNA Polymerase (Roche Diagnostics $\mathrm{GmbH}$, Mannheim, Germany) in a total volume of $25 \mu \mathrm{l}$. After adding the bisulfite DNA to the complete mixture, the sample was pipetted up-anddown in order to homogeneously disperse all bisulfite DNA molecules throughout the solution and fractions of $25 \mu \mathrm{l}$ were divided over 22 wells of a microtiter plate. Multiplex PCRs were then performed with an initial denaturation step at $95^{\circ} \mathrm{C}$ for 5 minutes, followed by 43 cycles with denaturation at $95^{\circ} \mathrm{C}$ for 30 seconds, annealing at $56^{\circ} \mathrm{C}$ for 30 seconds and extension at $72^{\circ} \mathrm{C}$ for 1 minute, with a final extension step at $72^{\circ} \mathrm{C}$ for 7 minutes.

\section{Singleplex polymerase chain reaction}

For each individual singleplex PCR reaction, $1 \mu \mathrm{l}$ of the multiplex product was used as a template. In addition, every single singleplex PCR reaction made use 
of $2.5 \mu \mathrm{l} \mathrm{PCR}$ buffer (10X) with $20 \mathrm{mM} \mathrm{MgCl} 2,0.5 \mu \mathrm{l} 10 \mathrm{mM}$ dNTP mix, $1 \mu \mathrm{l}$ of each primer $\left(10 \mu \mathrm{M}\right.$ stock) and $0.2 \mu \mathrm{l}(5 \mathrm{U} / \mu \mathrm{l})$ FastStart $^{\mathrm{TM}}$ Taq DNA Polymerase (Roche Diagnostics $\mathrm{GmbH}$, Mannheim, Germany) in a total volume of $25 \mu \mathrm{l}$. Amplifications for each of the target genes were then performed with an initial denaturation step at $95^{\circ} \mathrm{C}$ for 5 minutes, followed by 45 cycles with denaturation at $95^{\circ} \mathrm{C}$ for 30 seconds, annealing at $58^{\circ} \mathrm{C}$ for 30 seconds and extension at $72^{\circ} \mathrm{C}$ for 1 minute, with a final extension step at $72^{\circ} \mathrm{C}$ for 7 minutes. Reactions that yielded a singleplex PCR product- were identified on an agarose gel and $3 \mu \mathrm{l}$ of the product was utilized per assay for bisulfite pyrosequencing.

\section{Bisulfite pyrosequencing}

The PyroMark Q96 MD pyrosequencing system (Qiagen) with the PyroMark Gold Q96 CDT reagent kit (Qiagen, Hilden, Germany) were used according to the manufacturer's instructions. Methylation levels at a single $\mathrm{CpG}$ resolution were quantified with the Pyro Q-CpG 1.0.9 software (Qiagen). All assays were tested for their sensitivity using the EpiTect PCR Control DNA Set (Qiagen). For further details on the pyrosequencing assays and sequencing primers, please refer to Supplementary Table 2.

\section{Statistical analysis}

All statistical analyses were performed with the IBM SPSS Statistics software version 25. In order to determine whether the estimated CpG methylation rates were significantly different between the novel and the traditional LDBSP data analysis pipeline, a one-way repeated measures multivariate analysis of variance (MANOVA) was performed for each target gene. The multivariate analysis was performed in order to determine whether there was a combined difference in CpG methylation rates measured over all $\mathrm{CpG}$ sites, which was followed by a univariate test for each individual site. A $p$ value of $<0.05$ was considered as statistically significant.

\section{Results}

\section{LDBSP allows for methylation profiling of pools of 50 LCM-collected neurons} In order to assess the compatibility of LDBSP on neurons that were individually isolated from unfixed post-mortem brain tissue using LCM, batches consisting of $1,5,10$ and 50 cells were initially processed following the working procedures described in the present manuscript. Following LDBSP, only pools of 50 cells yielded a favorable amount of sequenceable PCR products for each gene, whereas lower cell numbers generally yielded little to no amplicons (data not shown). Next, 150 neurons per individual from 24 donors were isolated, divided over three pools of 50 cells each and processed for further analysis. In order to ensure adequate dilution to a single-allele-level, the dilution factor that is applied 
when conducting LDBSP should be larger than the maximum number of DNA molecules in the starting sample. For pragmatic reasons, at least half of the reactions should therefore not contain a PCR product after the procedure [11]. In our hands, on average, 4.46 PCR products were obtained per gene per pool of 50 neurons, hence a dilution factor of 22 was used. Of note, we did not observe any striking differences in the number of acquired products when increasing the number of dilutions (up to 96x; data not shown). Overall, for DNAJB13, PGLYRP1, RHBDF2, C3, LMX1B and OXT, respectively, 416, 233, 315, 189, 532 and 241 PCR products were obtained (Table 1). All of these were then successfully taken forward for methylation profiling using pyrosequencing.

Table 1. Overview of the limiting dilution bisulfite pyrosequencing (LDBSP) parameters

\begin{tabular}{|c|c|c|c|c|c|c|c|}
\hline Singleplex PCR & DNAJB13 & PGLYRP1 & RHBDF2 & C3 & LMX1B & OXT & Mean \\
\hline $\begin{array}{l}\text { Number (+ percentage) } \\
\text { of reactions with PCR product }\end{array}$ & $\begin{array}{l}416 \\
(26.26)\end{array}$ & $\begin{array}{l}233 \\
(14.71)\end{array}$ & $\begin{array}{l}315 \\
(19.89)\end{array}$ & $\begin{array}{l}189 \\
(11.93)\end{array}$ & $\begin{array}{l}532 \\
(33.59)\end{array}$ & $\begin{array}{l}241 \\
(15.21)\end{array}$ & $\begin{array}{l}321 \\
(20.27)\end{array}$ \\
\hline \multicolumn{8}{|l|}{ Allele estimation traditional $L D B S P$ criteria } \\
\hline $\begin{array}{l}\text { Number (+ percentage) } \\
\text { of included reactions } \\
\text { (1 allele) }\end{array}$ & $\begin{array}{l}346 \\
(83.17)\end{array}$ & $\begin{array}{l}205 \\
(87.98)\end{array}$ & $\begin{array}{l}298 \\
(94.60)\end{array}$ & $\begin{array}{l}186 \\
(98.41)\end{array}$ & $\begin{array}{l}474 \\
(89.10)\end{array}$ & $\begin{array}{l}221 \\
(91.70)\end{array}$ & $\begin{array}{l}288.33 \\
(89.82)\end{array}$ \\
\hline $\begin{array}{l}\text { Number (+ percentage) } \\
\text { of excluded reactions (multi-allele/ } \\
\text { artifact) }\end{array}$ & $\begin{array}{l}70 \\
(16.83)\end{array}$ & $\begin{array}{l}28 \\
(12.02)\end{array}$ & $\begin{array}{l}17 \\
(5.40)\end{array}$ & $\begin{array}{l}3 \\
(1.59)\end{array}$ & $\begin{array}{l}58 \\
(10.90)\end{array}$ & $\begin{array}{l}20 \\
(8.30)\end{array}$ & $\begin{array}{l}32.67 \\
(10.18)\end{array}$ \\
\hline \multicolumn{8}{|l|}{ Allele estimation novel LDBSP criteria } \\
\hline $\begin{array}{l}\text { Number (+ percentage) } \\
\text { of reactions with } 1 \text { allele }\end{array}$ & $\begin{array}{l}356 \\
(85.58)\end{array}$ & $\begin{array}{l}208 \\
(89.27)\end{array}$ & $\begin{array}{l}300 \\
(95.24)\end{array}$ & $\begin{array}{l}186 \\
(98.41)\end{array}$ & $\begin{array}{l}475 \\
(89.29)\end{array}$ & $\begin{array}{l}232 \\
(96.27)\end{array}$ & $\begin{array}{l}292.83 \\
(91.23)\end{array}$ \\
\hline $\begin{array}{l}\text { Number (+ percentage) } \\
\text { of reactions with } 2 \text { alleles }\end{array}$ & $\begin{array}{l}23 \\
(5.53)\end{array}$ & $\begin{array}{l}4 \\
(1.72)\end{array}$ & $\begin{array}{l}7 \\
(2.22)\end{array}$ & $\begin{array}{l}0 \\
(0.00)\end{array}$ & $\begin{array}{l}21 \\
(3.95)\end{array}$ & $\begin{array}{l}3 \\
(1.24)\end{array}$ & $\begin{array}{l}9.67 \\
(3.01)\end{array}$ \\
\hline $\begin{array}{l}\text { Number (+ percentage) } \\
\text { of reactions with } 3 \text { alleles }\end{array}$ & $\begin{array}{l}37 \\
(8.89)\end{array}$ & $\begin{array}{l}21 \\
(9.01)\end{array}$ & $\begin{array}{l}8 \\
(2.54)\end{array}$ & $\begin{array}{l}3 \\
(1.59)\end{array}$ & $\begin{array}{l}36 \\
(6.77)\end{array}$ & $\begin{array}{l}6 \\
(2.49)\end{array}$ & $\begin{array}{l}18.50 \\
(5.76)\end{array}$ \\
\hline $\begin{array}{l}\text { Number (+ percentage) } \\
\text { of multi-allele reactions }\end{array}$ & $\begin{array}{l}60 \\
(14.42)\end{array}$ & $\begin{array}{l}25 \\
(10.73)\end{array}$ & $\begin{array}{l}15 \\
(4.76)\end{array}$ & $\begin{array}{l}3 \\
(1.59)\end{array}$ & $\begin{array}{l}57 \\
(10.71)\end{array}$ & $\begin{array}{l}9 \\
(3.73)\end{array}$ & $\begin{array}{l}28.17 \\
(8.77)\end{array}$ \\
\hline $\begin{array}{l}\text { Number (+ percentage) } \\
\text { of recovered alleles }\end{array}$ & $\begin{array}{l}513 \\
(7.13)\end{array}$ & $\begin{array}{l}279 \\
(3.88)\end{array}$ & $\begin{array}{l}338 \\
(4.69)\end{array}$ & $\begin{array}{l}195 \\
(2.71)\end{array}$ & $\begin{array}{l}625 \\
(8.68)\end{array}$ & $\begin{array}{l}256 \\
(3.56)\end{array}$ & $\begin{array}{l}367.67 \\
(5.11)\end{array}$ \\
\hline
\end{tabular}

A summary of all the LDBSP parameters for pools of 50 neurons isolated from unfixed post-mortem brain tissue using laser capture microdissection (LCM). Displayed are the total number of obtained singleplex polymerase chain reaction (PCR) products for each gene, the total amount of included and excluded products according to the traditional LDBSP method for the downstream data analysis, as well as the total amount of estimated alleles according to the novel LDBSP data analysis approach. Please refer to the written text for further specifications on both allele estimation methods and criteria. 


\section{LDBSP on pools of $\mathbf{5 0}$ neurons occasionally renders reactions with more than one target allele}

As the principle of LDBSP is based on the seclusion of individual alleles, the vast majority of PCR products should theoretically represent an amplicon derived from one DNA molecule [10]. As a given CpG site on a single allele is simply methylated or not, pyrosequencing of these PCR products should result in obtaining binary $\mathrm{CpG}$ methylation readouts, i.e. the percentage of methylation should approach $0 \%$ for unmethylated and $100 \%$ for methylated CpG sites. Because the quantitative measurement of a $\mathrm{CpG}$ site by pyrosequencing does not only depend on its methylation status, but also on the sensitivity of the assay and other technological factors, earlier studies have considered CpG methylation values of $<20 \%$ and $>80 \%$ indicative of unmethylated and methylated $\mathrm{CpG}$ sites, respectively [10, 11, 14]. Moreover, products that contained at least one $\mathrm{CpG}$ methylation value between $20 \%$ and $80 \%$ were excluded from further downstream analyses, as these were considered to represent measures of multiple target alleles in a single reaction or from other technological artifacts. Importantly, as previous studies have mainly applied LDBSP on a single or a few cells, the occurrence of multi-allele reactions has often been negligible [11].

When applying LDBSP on pools containing a larger number of cells, i.e. 50 neurons in our case, one can observe that a substantial proportion of the obtained products do not survive the traditional LDBSP inclusion criteria. Evidently, processing DNA isolated from a pool of 50 neurons increases the chance of allele clumping when compared to conducting LDBSP on a single or just a few cells. As such, when two different alleles with an opposite methylation status for at least one $\mathrm{CpG}$ site end up in the same reaction, the methylation value obtained for specifically this site should approach $\sim 50 \%$. Along similar lines, when three alleles end up in the same reaction with a different methylation status for at least one CpG site, then the methylation value obtained for specifically this site should approach either $\sim 33.33 \%$ or $\sim 66.66 \%$. From all the PCR products of DNAJB13, PGLYRP1, RHBDF2, C3, LMX1B and OXT, respectively, $16.83 \%, 12.02 \%, 5.40 \%, 1.59 \%$, $10.90 \%$ and $8.30 \%$ would have been omitted from further downstream analyses, as at least one $\mathrm{CpG}$ site in these products demonstrated a value between $20 \%$ and $80 \%$ (Table 1). A thorough inspection of these deviating CpG methylation values revealed that the vast majority approached either $50 \%$, or $33 \% / 67 \%$, indicative of the presence of two or three alleles. Thus, based on these typical patterns of CpG methylation values, we concluded that a considerable amount of the LDBSP data could be attributed to reactions that contained more than one target allele, hence suggesting an adapted protocol for estimating methylation rates using this approach is required. Evidently, one can also obtain methylation values of $0 \%$ or $100 \%$ in the case of two or three allele-reactions, which demands for a reliable determination of the number of alleles in each reaction. 

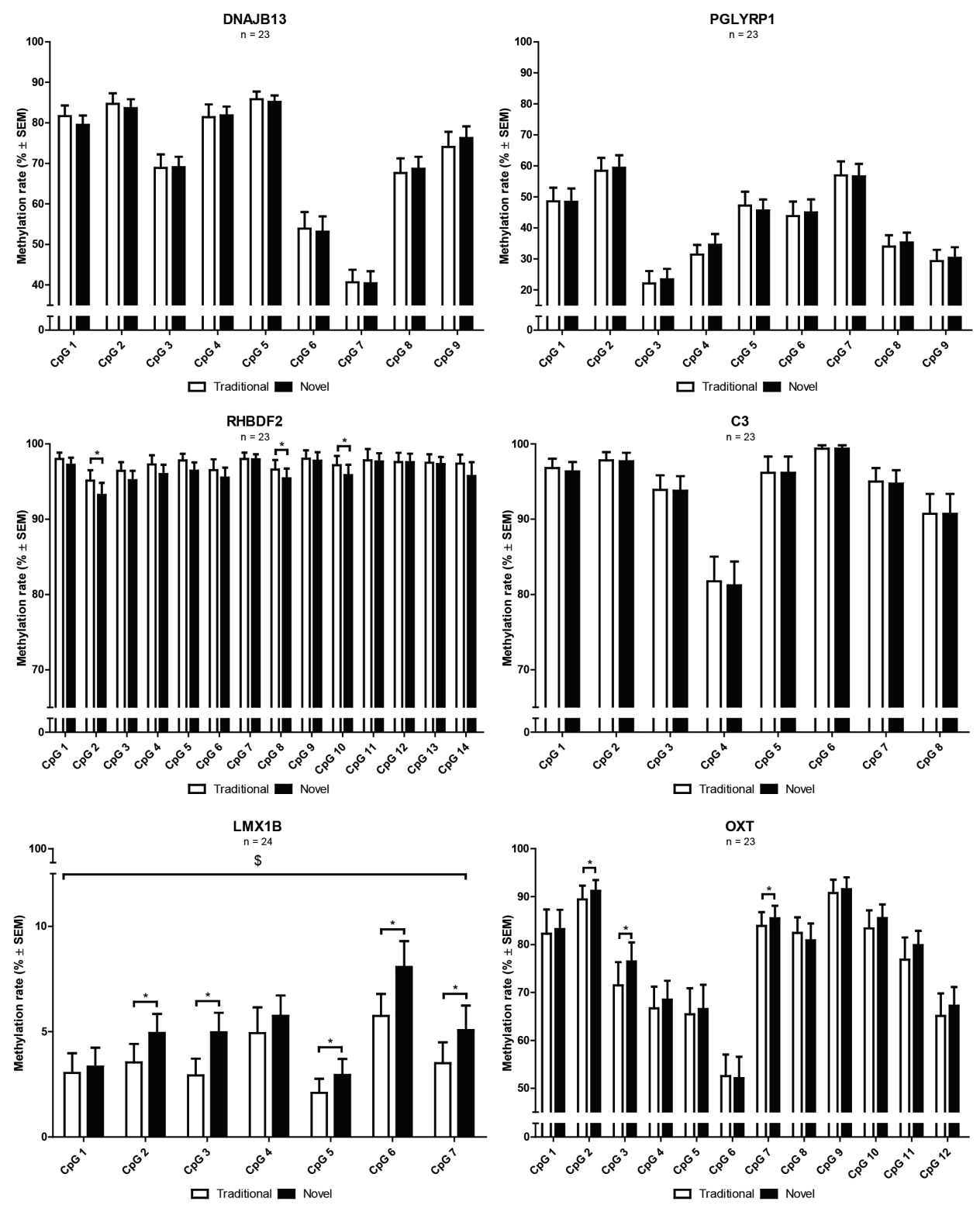

Figure 2. Displayed for each target gene are the paired cytosine-phosphate-guanine ( $\mathrm{CpG}$ ) methylation rates (mean percentage \pm standard error of the mean (SEM)) estimated by both the traditional and novel downstream data analysis for limiting dilution bisulfite pyrosequencing (LDBSP) on pools of 50 neurons. A one-way repeated measures multivariate analysis of variance (MANOVA) was performed in order to determine whether there was a combined significant difference in the $\mathrm{CpG}$ methylation rates estimated by both methods. Subsequently a univariate analysis was performed in order to identify differences between the methylation rates for each individual $\mathrm{CpG}$ site. Significant findings for the multivariate and univariate tests are indicated with $\$$ and ${ }^{*}$, respectively. A $p$ value of $<0.05$ was considered as statistically significant. 


\section{An integrated analysis of CpG readouts for LDBSP data with correction for multi-allele reactions}

In order to correct for the number of estimated alleles present in each of the PCR reactions, we established a novel method for the downstream data analysis. For this purpose, thresholds of (1) $\leq 8.33 \%$ and $\geq 91.33 \%$, (2) $50 \pm 8.33 \%$, and (3) $33.33 \pm 8.33 \%$ and $66.66 \pm 8.33 \%$, were set for the $\mathrm{CpG}$ methylation values, enabling assessing both 1-, 2- and 3-allele reactions, respectively. Accordingly, individual CpG sites that fell within the first, second and third threshold range were called and considered indicative for the potential presence of one, two and three alleles, respectively. A definitive (total) allele score for each individual product was then assigned following a multi-step filtering process that was based on the criteria described hereafter. All products solely displaying binary $\mathrm{CpG}$ methylation patterns (i.e. when every $\mathrm{CpG}$ site within an amplicon displays methylation levels within the first threshold range) were directly considered to be derived from single allele reactions, as the $\mathrm{CpG}$ methylation profiles for these products displayed a strong, typical binary pattern that is expected for a single DNA molecule. Similarly, and based on the assumption described in the previous section, all products displaying $\mathrm{CpG}$ methylation values that fell only in the first and second, but not third, or only in the first and third, but not second, threshold ranges were scored as two or three alleles, respectively.

Subsequently, products containing $\mathrm{CpG}$ methylation values that fell outside of the assigned threshold ranges, i.e. between $8.33 \%-25 \%$ and $75 \%-91.33 \%$, and/or products with values that were indicative of both two and three alleles simultaneously, were assessed. The observation of such more ambiguous products displaying small deviations from the assigned threshold values are likely to be caused by technical variation induced during the PCR and pyrosequencing procedure and the more stringent thresholds used in the present study in this respect $(8.33 \%$ versus $20 \%$ in previous studies; see above). All of these products were therefore thoroughly inspected by two investigators that were blinded to the experimental conditions, and a decision on the total number of alleles present in each individual reaction, i.e. one, two or three alleles, was made independently, while taking into account a combination of different factors. These included, but were not limited to, small technological variation that was previously observed during sensitivity testing of the assays, the directionality and methylation status of other CpG sites in the same product and the total number of dominant allele indicators, i.e. whether a product demonstrated more or less suggestive $\mathrm{CpG}$ sites for either two or three alleles. Furthermore, a likelihood estimation for each $\mathrm{CpG}$ site was made by taking into account the methylation status frequency on other gene-specific products obtained from the same individual, as well as from identical products obtained from other individuals. A cross comparison between the independent score sheets was then performed (98.38\% overlap per gene on average) and reactions with a deviating 
score between the first two investigators were assessed by a third (blinded) investigator. A final allele number was then assigned for these reactions based on the overlap between the score sheets of the third and first two investigators, i.e. when two out of the three investigators assigned the same score then this allele number was used for the respective reaction.

Table 2. One-way repeated measures multivariate analysis of variance (MANOVA)

\begin{tabular}{|c|c|c|c|c|c|c|}
\hline Multivariate test & RHBDF2 & OXT & DNAJB13 & PGLYRP1 & C3 & LMX1B \\
\hline Combined effect & 0.390 & 0.474 & 0.078 & 0.392 & 0.385 & 0.012 \\
\hline \multicolumn{7}{|l|}{ Univariate tests } \\
\hline Cpg 1 & 0.163 & 0.544 & 0.194 & 0.962 & 0.328 & 0.435 \\
\hline Cpg 2 & 0.027 & 0.048 & 0.354 & 0.493 & 0.328 & 0.010 \\
\hline Cpg 3 & 0.066 & 0.005 & 0.905 & 0.527 & 0.328 & 0.009 \\
\hline Cpg 4 & 0.100 & 0.407 & 0.799 & 0.090 & 0.312 & 0.314 \\
\hline Cpg 5 & 0.062 & 0.581 & 0.560 & 0.519 & 1,000 & 0.040 \\
\hline Cpg 6 & 0.205 & 0.692 & 0.662 & 0.517 & 1,000 & 0.001 \\
\hline Cpg 7 & 0.936 & 0.033 & 0.859 & 0.863 & 0.328 & 0.006 \\
\hline Cpg 8 & 0.026 & 0.349 & 0.567 & 0.544 & 1,000 & - \\
\hline CpG 9 & 0.328 & 0.381 & 0.117 & 0.857 & - & - \\
\hline CpG 10 & 0.033 & 0.087 & - & - & - & - \\
\hline Cpg 11 & 0.810 & 0.228 & - & - & - & - \\
\hline Cpg 12 & 0.963 & 0.394 & - & - & - & - \\
\hline Cpg 13 & 0.779 & - & - & - & - & - \\
\hline CpG 14 & 0.054 & - & - & - & - & - \\
\hline
\end{tabular}

Displayed are the $p$-values for both the multivariate test of the combined cytosine-phosphate-guanine $(C p G)$ effects and the univariate tests for each individual $\mathrm{CpG}$ site per target gene. A p-value of $<0.05$ was considered as stastically significant.

Overall, we estimated that from the PCR products of DNAJB13, PGLYRP1, RHBDF2, C3, LMX1B and OXT, 85.58\%, 89.27\%, 95.24\%, 98.41\%, 89.29\% and $96.27 \%$, respectively, were derived from single alleles, whereas $5.53 \%, 1.72 \%$, $2.22 \%, 0.00 \%, 3.95 \%$ and $1.24 \%$, respectively, were derived from two alleles, and $8.89 \%, 9.01 \%, 2.54 \%, 1.59 \%, 6.77 \%$ and $2.49 \%$, respectively, were derived from three alleles (Table 1). By taking into account these multi-allele reactions, we therefore estimated that in total 513, 279, 338, 195, 625 and 256 alleles were recovered for DNAJB13, PGLYRP1, RHBDF2, C3, LMX1B and OXT, respectively, with an average recovery rate of $5.11 \%$. It has previously been demonstrated that 
shorter assays generally have a higher recovery rate for LDBSP [11], something that could also be observed in our data set. In fact, the highest number of alleles were recovered for the shortest assay (LMX1B, 625 alleles; multiplex, 257 basepairs (bp); singleplex, $249 \mathrm{bp}$ ), while the lowest number was recovered for the longest assay (C3, 195 alleles; multiplex 441 bp; singleplex, 364 bp). Accordingly, a Pearson's correlation test revealed a strong negative correlation $(r=-0.866, p$ $=0.026$ ) between the number of recovered alleles and multiplex amplicon length, thereby confirming previous observation.

Based on the final allele estimations above, the CpG methylation rates, representing the percentage of methylated $\mathrm{CpG}$ sites from the total number of recovered alleles per gene, were calculated per individual. An overview of the average $\mathrm{CpG}$ methylation rates can be found in Figure 2 for each target gene. In addition to estimating these rates by the novel method described above, we also quantified them according to the traditional LDBSP approach that is based on excluding multi-allele reactions.

\section{Gene specific changes in the CpG methylation data based on the novel integrated analysis}

Next, for the CpG site methylation rates of each gene, a one-way repeated measures MANOVA was performed in order to determine whether there was a combined significant difference in the CpG methylation rates estimated by the traditional and novel LDBSP data analysis approach described above (Table 2). A significant effect $(p=0.012)$ was identified for $L M X 1 B$, demonstrating that, for this gene, the estimated $\mathrm{CpG}$ methylation rates are different depending on the applied calling procedure. A subsequent univariate analysis for each individual $\mathrm{CpG}$ site, indicated that the estimated methylation rates for 5 out of 7 sites were significantly different between the two approaches (CpG 2, $p=0.010$; CpG 3, $p=0.009$; CpG $5, p=0.040 ; \mathrm{CpG} 6, p=0.001 ; \mathrm{CpG} 7, p=0.006$ ). While no significant combined effect was identified for any of the other target genes, the univariate analyses did reveal that the estimated methylation rates for two CpG sites in both RHBDF2 and OXT were different between the traditional and novel method. While for RHBDF2 CpG site $8(p=0.026)$ and $10(p=0.033)$ were significantly different, CpG site 3 $(p=0.005)$ and $7(p=0.033)$ of OXT differed between the two approaches. All together, these findings demonstrate that the obtained methylation rates can be significantly affected by the method that is applied for downstream data analysis when conducting LDBSP on pools of 50 neurons. In other words, when excluding reactions that suggest to contain multiple alleles, instead of correcting the derived methylation rates based on the number of estimated alleles present in the reaction, the eventual LDBSP data can differ significantly. For this reason, and in order to prevent potential bias in the experimental outcomes, we strongly suggest to apply 
this adapted approach for LDBSP when analyzing pools including larger numbers of cells. Overall, this novel pipeline approach provides a closer estimate of the true $\mathrm{CpG}$ methylation rates for a target gene.

\section{Discussion}

In the present study, we demonstrated, for the first time, that LDBSP can be successfully applied on LCM neurons derived from unfixed post-mortem brains. In brief, brain tissue sections were stained for a neuronal subtype-specific marker (i.e. 5-HT). Immuno-positive cells were then individually isolated using LCM and subsequently divided in small pools of 50 neurons. Next, the bisulfite-converted DNA isolated from a pool of neurons was diluted to a single allele-level and then amplified using (semi-)nested PCRs in multiplex-singleplex formation, targeting DNAJB13, PGLYRP1, OXT, RHBDF2, C3 and LMX1B simultaneously. Finally, the methylation status of the target genes was then quantified using bisulfite pyrosequencing.

In contrast to most previous studies [10-15], LDBSP on pools of 50 neurons renders a considerable degree $(8.77 \%$ on average per gene) of the downstream PCR reactions containing more than one target allele. The presence of these multiple alleles could be identified by the observed 'aberrant' CpG methylation values obtained from the respective reactions. Pyrosequencing of single alleles normally results in the derivation of a binary $\mathrm{CpG}$ methylation readout, that is the methylation values approach $0 \%$ for unmethylated and $100 \%$ for methylated sites [10]. Although in the vast majority of reactions a binary $\mathrm{CpG}$ methylation pattern could be detected (91.23\%), for $3.01 \%$ and $5.76 \%$ of the sequenced products, respectively, we obtained values that approached $50 \%$ or $33 / 67 \%$ for at least one $\mathrm{CpG}$ site. As such, we discovered that these reactions contained either two or three alleles, in which at least one DNA molecule had an opposite CpG methylation status as compared to the other allele(s) present in the same reaction. Traditionally, thresholds of $<20 \%$ and $>80 \%$ have been applied in order to define the methylation status of $\mathrm{CpG}$ sites, and all products displaying more intermediate values $(20-80 \%)$ suggesting the presence of more than one allele, were excluded from further downstream analysis [10-15]. However, we argued that, particularly when the degree of these reactions is more substantial, exclusion might actually influence the data negatively, either by inducing bias or by reducing or reinforcing effect sizes.

We therefore established a novel data analysis pipeline that allows one to include and correct the LDBSP data for these multi-allele reactions, hence providing a more accurate estimation of the $\mathrm{CpG}$ methylation rates. For this purpose, novel $\mathrm{CpG}$ site thresholds of (1) $\leq 8.33 \%$ and $\geq 91.33 \%$, (2) $50 \pm 8.33 \%$, and (3) $33.33 \pm 8.33 \%$ and 
$66.66 \pm 8.33 \%$, were applied in order to identify reactions that contained one, two or three alleles, respectively. Next, a CpG site calling procedure was performed. First products displaying $\mathrm{CpG}$ methylation values that fell within the assigned threshold ranges were categorized as either one, two or three alleles. Remaining products containing ambiguous CpG sites, i.e. i) products with multiple CpG sites showing presence of either two or three alleles, or ii) methylation values falling outside of the threshold values, were subsequently inspected by three researchers blinded to the experimental conditions. An allele score was then assigned independently taking into account factors that could explain variability in the CpG methylation data, such as known differences in the sensitivity of the sequencing assays that were assessed before conducting the experiment using DNA methylation standards. Additional measures such as the directionality and methylation status of other $\mathrm{CpG}$ sites in the same product or the total number of dominant allele indicators in a single product were also considered. Moreover, a likelihood estimation was performed for each CpG site taking into account the methylation status frequency for other gene-specific products obtained from the same individual, as well as from identical products obtained from other individuals. A cross comparison between the independent score sheets was then performed (98.38\% overlap per gene on average), reactions with a deviating score between the first two investigators were assessed by a third (blinded) investigator and a final allele number was then assigned for these reactions based on the overlap between the score sheets of the third and first two investigators.

For each gene, we then compared the CpG methylation rates estimated both by the traditional ( $<20 \%$ and $>80 \%$ cut-offs) and novel data analysis approach described above. Strikingly, significant differences in the combined $\mathrm{CpG}$ effects for $L M X 1 B$, as well as for individual $C p G$ sites in $L M X 1 B, R H B D F 2$ and OXT were identified. Thus, these findings emphasized that the derived LDBSP methylation data can significantly differ depending on the method that is being applied for the downstream data analysis. Importantly, this will especially affect loci that for biological reasons display a varying methylation status over all the recovered allele, that is when part of them is fully methylated and part of them fully unmethylated. In such a scenario, the average methylation rates will likely fall in the intermediate range of $20-80 \%$ and, hence, the chances of obtaining a multi-allele reactions with values outside of the traditional threshold will therefore be higher. Therefore, the probability of detecting differences between both methods is also dependent on the average methylation scores. In either way, when LDBSP renders reactions with more than one target allele, independent of the cell types that are analyzed, we strongly suggest to correct the $\mathrm{CpG}$ methylation rates based on the number of target alleles present in a single reaction. Overall, we strongly believe that estimating the $\mathrm{CpG}$ methylation rates based on the $\mathrm{CpG}$ methylation values 
provides a more accurate representation of the true methylation rates obtained for a specific target gene, hence adding more validity to the data.

One important consideration, is that dependent on the design of an individual experiment, potentially more than three alleles might be present in an individual reaction, which would mean the estimation of allele numbers in a single reaction based on CpG methylation values becomes too ambiguous, as one also needs to consider potential technical variation that might affect these read-outs. In our hands, the proportion of reactions that contain more than three alleles seemed to be very little or even negligible, and these reactions therefore hardly affect the estimated methylation data. Even when present, the method proposed in the present manuscript will most likely classify these reactions as three alleles and, hence, the methylation data obtained from these reactions has been partially corrected when compared to the traditional analysis pipeline. Evidently, when ending up with a high degree of $(>3)$ multi-allele reactions, it is advised to dilute the DNA more.

Another important consideration is that LDBSP does not allow the user to detect reactions that appear to contain only one allele, which in reality contain 2 or more alleles with an identical methylation pattern. In such a scenario, the methylation profile of a PCR product would appear binary, hence suggesting the presence of only one target allele. Indeed, this represents an issue independent of the downstream data analysis that is used for the analysis of the $\mathrm{CpG}$ methylation rates, i.e. the traditional or novel approach proposed here. Overall, this becomes a bigger challenge when due to biological reasons the $\mathrm{CpG}$ methylation rates of a target gene approach either 0 or $100 \%$, meaning that most of the recovered alleles will display either fully unmethylated or methylated $\mathrm{CpG}$ sites, respectively. In our study, this might therefore have affected genes such as $\angle M X 1 B, C 3$ and $R H B D F 2$, although we often observed that at least one of the CpG sites in these gene-derived products displayed an opposite methylation status compared to the other sites derived from the same product. In other words, the methylation status of all $\mathrm{CpG}$ sites in a target gene were not always identical and often displayed a deviating methylation state for one site when compared to the others. For this reason, the occurrence of these opposite methylation patterns at single CpG sites still allowed us to detect the presence of multiple alleles when such molecules end up in a reaction with other alleles showing a more homogenous pattern of CpG methylation. It is therefore advisable to always target a substantial number of CpG sites per target gene, e.g. 7-14 in the present manuscript, in order to increase the chance of obtaining, and hence detecting, at least one site with a 'non-binary' methylation status on one of the target alleles. As such, a readout of such a reaction would demonstrate an intermediate methylation value, e.g. $\sim 50 \%$ 
and $\sim 33 \%$ or $\sim 66 \%$, for specifically this CpG site. When targeting only a few or even a single $\mathrm{CpG}$ site, further diluting the DNA may be advisory.

Undoubtedly, when conducting LDBSP, it is paramount to ensure proper dilution of the isolated target DNA a priori, in order to minimize the occurrence of multiallele reactions, even before one ought to correct the derived methylation values as proposed in the present manuscript. LDBSP is thought to follow a Poisson distribution, meaning that with increasing dilution the chances of obtaining multiallele reactions should become smaller. As a benchmark, it is therefore advised to use more dilutions than the number of target alleles present in the reactions, whilst taking into account potential loss due to handling. Adequate dilution to a single allele-level can be assessed by the ratio between the number of productyielding and non-product yielding reactions, of which the latter should occur more often than the former. Although in the present study the number of productyielding reactions was substantially lower $(20.27 \%$ on average) compared to the downstream reactions that did not contain a product $(79.73 \%$ on average), we still obtained reactions with more than one target allele, suggesting that other technological factors, or biochemical or biophysical properties of the DNA, play a role as well. Nevertheless, in a scenario where the DNA has been diluted properly and where multi-allele reactions are still encountered it will likely remain more appropriate to correct methylation values for these reactions as compared to excluding them from the data analysis. Of note, simply further diluting the DNA will be on the expense of throughput and comes along with additional costs.

Finally, in order to demonstrate the overall efficiency of the approach, we made a final estimation on the total number of alleles that were recovered after correcting for the number of alleles present in a single reaction. Assuming a 100\% DNA recovery starting from three pools of 50 neurons, we estimated that the allele recovery rate of LDBSP on pools of 50 neurons isolated with LCM is $5.11 \%$ per gene of the total number of alleles. In comparison, previous studies have estimated recovery rates ranging from $0.3 \%$ up to $25 \%$ using intact cell pools consisting of 10 oocytes, two-cell embryos and 16 -cell embryos $[11,13,14]$. The recovery rate achieved in the present study on the pools of 50 neurons is therefore similar to other studies using these other cell types, despite the fact that a higher amount of starting material is needed when conducting the technique on neurons isolated with LCM. Nevertheless, one should not overlook the differences in sample collection procedures that were applied in these studies. In fact, the success of extracting DNA from cells isolated with LCM is dependent on several critical factors, e.g. cutting procedures, thickness of the sections, staining procedures, exposure to heat produced by the laser, and the DNA isolation process, which all could affect the eventual quantity of DNA that is recovered from the cells. Furthermore, the 
degradation and low complexity of bisulfite-converted DNA opposes another challenge for the methylation analysis of small amounts of DNA from only few cells. As such, a previous study demonstrated that shorter assays generally have a higher recovery rate for LDBSP [11]. Interestingly, also in our study the highest number of alleles were recovered for the shortest assay $(L M X 1 B, 625$ alleles; multiplex, 257 basepairs (bp); singleplex, 249 bp), while the lowest number was recovered for the longest assay (C3, 195 alleles; multiplex 441 bp; singleplex, 364 bp). Accordingly, a strong negative correlation between the number of recovered alleles and multiplex amplicon length was identified, thereby confirming previous observation.

In conclusion, the approach described here, relying on a combination of LDBSP with LCM, offers a novel and alternative strategy to single cell bisulfite sequencing techniques that can be applied for the study of DNA methylation marks in the human brain. Moreover, the approach offers a workable solution for the challenge of tissue and cell-type specificity as encountered in the field of neuroepigenomics. In fact, LDBSP on pools of neuronal populations allows one to determine DNA methylation profiles in a multi-targeted and cell subtype-specific manner, hence avoiding potential noise in epigenetic data that is induced by analyzing heterogeneous tissue samples. Aside from allowing the identification of methylation marks in individual neuronal cells, we expect that similar strategies using other isolation techniques and other cell subtypes in combination with LDBSP will be increasingly valuable for future neuroepigenomic studies.

\section{Acknowledgments}

Funds have been provided by the Joint Program - Neurodegenerative Disease Research (JPND) for the EPI-AD consortium (http://www.epi-ad.eu/). The project is supported through the following funding organizations under the aegis of JPND; the Netherlands, The Netherlands Organization for Health Research and Development (ZonMw); United Kingdom, Medical Research Council; Germany, German Federal ministry of Education and Research (BMBF); Luxembourg, National Research Fund (FNR). This project has received funding from the European Union's Horizon 2020 research and innovation program under Grant Agreement No. 643417. 


\section{References}

1. van den Hove DL, Riemens RJ, Koulousakis P, Pishva E. Epigenome-wide association studies in Alzheimer's disease; Achievements and challenges. Brain Pathology. 2020.

2. Lardenoije R, latrou A, Kenis G, Kompotis K, Steinbusch HW, Mastroeni D, et al. The epigenetics of aging and neurodegeneration. Prog Neurobiol. 2015;131:21-64.

3. Gasparoni G, Bultmann S, Lutsik P, Kraus TF, Sordon S, VIcek J, et al. DNA methylation analysis on purified neurons and glia dissects age and Alzheimer's disease-specific changes in the human cortex. Epigenetics \& chromatin. 2018;11(1):41.

4. Roubroeks JAY, Smith RG, van den Hove DLA, Lunnon K. Epigenetics and DNA methylomic profiling in Alzheimer's disease and other neurodegenerative diseases. J Neurochem. 2017;143(2):158-70.

5. Houseman EA, Accomando WP, Koestler DC, Christensen BC, Marsit CJ, Nelson HH, et al. DNA methylation arrays as surrogate measures of cell mixture distribution. BMC bioinformatics. 2012;13(1):86.

6. Houseman EA, Molitor J, Marsit CJ. Reference-free cell mixture adjustments in analysis of DNA methylation data. Bioinformatics. 2014;30(10):1431-9.

7. Rahmani E, Zaitlen N, Baran Y, Eng C, Hu D, Galanter J, et al. Sparse PCA corrects for cell type heterogeneity in epigenome-wide association studies. Nature methods. 2016;13(5):443.

8. Rahmani E, Zaitlen N, Baran Y, Eng C, Hu D, Galanter J, et al. Correcting for cell-type heterogeneity in DNA methylation: a comprehensive evaluation. Nature methods. 2017;14(3):218-9.

9. Zheng SC, Beck S, Jaffe AE, Koestler DC, Hansen KD, Houseman AE, et al. Correcting for cell-type heterogeneity in epigenome-wide association studies: revisiting previous analyses. Nature methods. 2017;14(3):216-7.

10. Hajj NE, Kuhtz J, Haaf T. Limiting Dilution Bisulfite Pyrosequencing(R): A Method for Methylation Analysis of Individual DNA Molecules in a Single or a Few Cells. Methods Mol Biol. 2015;1315:221 39.

11. El Hajj N, Trapphoff T, Linke M, May A, Hansmann T, Kuhtz J, et al. Limiting dilution bisulfite (pyro) sequencing reveals parent-specific methylation patterns in single early mouse embryos and bovine oocytes. Epigenetics. 2011;6(10):1176-88.

12. Beach TG, Sue LI, Walker DG, Roher AE, Lue L, Vedders L, et al. The Sun Health Research Institute Brain Donation Program: description and experience, 1987-2007. Cell Tissue Bank. 2008;9(3):22945.

13. Charnay Y, Léger L. Brain serotonergic circuitries. Dialogues in clinical neuroscience. 2010;12(4):471.

14. Mattern F, Heinzmann J, Herrmann D, Lucas-Hahn A, Haaf T, Niemann H. Gene-specific profiling of DNA methylation and mRNA expression in bovine oocytes derived from follicles of different size categories. Reprod Fertil Dev. 2017;29(10):2040-51.

15. Trapphoff T, El Hajj N, Zechner U, Haaf T, Eichenlaub-Ritter U. DNA integrity, growth pattern, spindle formation, chromosomal constitution and imprinting patterns of mouse oocytes from vitrified preantral follicles. Human reproduction. 2010;25(12):3025-42.

16. Heinzmann J, Hansmann T, Herrmann D, Wrenzycki C, Zechner U, Haaf T, et al. Epigenetic profile of developmentally important genes in bovine oocytes. Molecular reproduction and development. 2011;78(3):188-201.

17. Diederich M, Hansmann T, Heinzmann J, Barg-Kues B, Herrmann D, Aldag P, et al. DNA methylation and mRNA expression profiles in bovine oocytes derived from prepubertal and adult donors. Reproduction. 2012;144(3):319. 


\section{Supplementary material}

\section{Limiting dilution bisulfite pyrosequencing protocol for $\mathbf{5 0}$ laser capture microdissected cells \\ Reagents}

- Laser capture microdissected cells (pools of 50 cells) in $8 \mu$ Tris- Ethylenediaminetetraacetic acid (TE) buffer or phosphate buffer saline (PBS)

- FastStart ${ }^{\mathrm{TM}}$ Taq DNA Polymerase (Sigma Aldrich, cat. no. 12032929001)

- EZ DNA Methylation-Direct Kit (Zymo Research, cat. no. D5020)

- Agarose

- DNA loading dye, e.g. Orange G DNA Loading Dye (ThermoFisher Scientific, cat. no. R0631)

- Nucleic acid gel stain, e.g. GelsGelStar ${ }^{\mathrm{TM}}$ Nucleic Acid Gel Stain (Lonza, cat. no. 50535)

- DNA 100 bp ladder, e.g. 100 bp DNA Ladder (ThermoFisher Scientific, cat. no. 15628050)

- PyroMark Gold Q96 CDT reagent kit (Qiagen, cat. no. 972824)

\section{Equipment}

- Manual pipettes

- Multichannel pipette

- Pipette filtered tips

- Eppendorf LoBind microcentrifuge tubes (Merck KGaA, Darmstadt, Germany, cat. no. 022431021)

- TipOne Low Retention Tips (STARLAB, Hamburg, Germany, cat. no. S1180-8810)

- Eppendorf®ThermoMixer® F1.5 (Sigma Aldrich, cat. no. EP5384000012)

- Microcentrifuge with PCR tube adaptors

- Thermal cycler

- Thin-walled PCR tubes, $0.2 \mathrm{ml}$

- PyroMark Q96 MD pyrosequencing system (Qiagen)

- Gel electrophoresis equipment

\section{Reagents preperations}

CT Conversion Reagent (EZ DNA Methylation-DirectTM Kit; D5020)

The CT Conversion Reagent provided with the EZ DNA Methylation-DirectTM Kit (D5020) is a solid mixture and must be prepared prior to first use. Please refer to the protocol provided with the kit for further details.

1. Add $790 \mu \mathrm{L}$ of M-Solubilization Buffer to a tube of CT Conversion Reagent

2. Add $300 \mu \mathrm{L}$ of M-Dilution Buffer to the tube of CT Conversion Reagent

3. Mix at room temperature with frequent vortexing or shaking for 10 minutes

4. Add $160 \mu \mathrm{L}$ of M-Reaction Buffer to the tube of CT Conversion Reagent

5. Mix an additional 1 minute 


\section{M-Wash Buffer}

The M-Wash Buffer provided with the EZ DNA Methylation-DirectTM Kit (D5020) must be prepared prior to first use. Please refer to the protocol provided with the kit for further details.

1. Add $24 \mathrm{~mL}$ of $100 \%$ ethanol to the $6 \mathrm{~mL}$ M-Wash Buffer concentrate (D5020)

\section{Procedure}

\section{DNA isolation (EZ DNA Methylation-DirectTM Kit; D5020)}

Laser capture microdissected cells in pools of 50 should be collected in $8 \mu \mathrm{TE}$ buffer or PBS, as this is the optimal working volume for the EZ DNA MethylationDirect Kit. In our case, cells were collected in $10 \mu \mathrm{l}$, hence volumes of the CT Conversion Reagent were adjusted accordingly in order to maintain the suggested reagent concentrations during the bisulfite conversion. For the original reagent volumes using laser capture microdissected cells in a total volume of $8 \mu l$, please refer to the protocol provided with the kit. Further details on LDBSP sample handling can be find below.

1. Briefly centrifuge the tube with laser capture microdissected cells

2. Add $11 \mu \mathrm{l} \mathrm{M}$-Digestion Buffer $(2 \mathrm{X})$ to the sample

3. Add $1 \mu \mathrm{l}$ of Proteinase $\mathrm{K}$ to the sample

4. Briefly vortex and centrifuge the tube

5. Incubate the sample in a heating block at $50^{\circ} \mathrm{C}$ for overnight

\section{Bisulfite conversion of DNA (EZ DNA Methylation-DirectTM Kit: D5020)}

When pipetting the sample, always use TipOne Low Retention Tips in order to minimize loss of DNA due to handling. Prepared CT Conversion Reagent from the EZ DNA Methylation-Direct Kit should be divided in $143 \mu \mathrm{l}$ ready-to-use aliquots in separate Eppendorf tubes, as this will allow one to use the same pipet tip per sample throughout the entire procedure. Similarly, the M-Binding Buffer was prepared in $600 \mu \mathrm{L}$ ready-to-use aliquots per sample. Please find further details on handling below.

1. After incubation, transfer the digested sample to a PCR tube

2. By using the same pipet tip, take $143 \mu \mathrm{l}$ CT Conversion Reagent

3. Use the CT Conversion Reagent to wash out the digestion tube

4. Add the CT Conversion Reagent to the sample containing PCR tube

5. Briefly vortex and centrifuge the tube

6. Place the PCR tube in a thermal cycler and perform the following steps:
a. $98^{\circ} \mathrm{C}$ for 8 minutes
b. $64^{\circ} \mathrm{C}$ for 3.5 hours
c. $4^{\circ} \mathrm{C}$ storage

7. Add $200 \mu \mathrm{L}$ of M-Binding Buffer into a Zymo-Spin ${ }^{\mathrm{TM}} \mathrm{IC}$ Column and place the column into a provided Collection Tube 
8. Pipet the bisulfite converted sample into the Zymo-Spin ${ }^{\mathrm{TM}} \mathrm{IC}$ Column

9. By using the same pipet tip, take $200 \mu \mathrm{L}$ of M-Binding Buffer

10. Use the M-Binding Buffer to wash out the PCR tube used for bisulfite conversion

11. Add the $200 \mu \mathrm{L}$ of M-Binding Buffer to the sample containing Zymo-Spin ${ }^{\mathrm{TM}} \mathrm{IC}$ Column

12. Repeat step 9-11 once more

13. Close the cap and mix by inverting the column several times

14. Centrifuge at full speed $(>10,000 \times \mathrm{g})$ for 30 seconds

15. Discard the flow-through

16. Add $100 \mu \mathrm{l}$ of M-Wash Buffer to the column

17. Centrifuge at full speed $(>10,000 \times \mathrm{g})$ for 30 seconds

18. Add $200 \mu \mathrm{l}$ of M-Desulphonation Buffer to the column and let stand at room temperature $\left(20-30^{\circ} \mathrm{C}\right)$ for $15-20$ minutes

19. Centrifuge at full speed $(>10,000 \times \mathrm{g})$ for 30 seconds

20. Add $200 \mu \mathrm{l}$ of M-Wash Buffer to the column

21. Centrifuge at full speed $(>10,000 \times \mathrm{g})$ for 30 seconds

22. Add $200 \mu \mathrm{l}$ of M-Wash Buffer to the column

23. Centrifuge at full speed $(>10,000 \times \mathrm{g})$ for 30 seconds

24. Place the column into a $1.5 \mathrm{ml}$ LoBind Eppendorf tube

25. Add $10 \mu \mathrm{l}$ of M-Elution Buffer directly to the column matrix

26. Centrifuge for 30 seconds at full speed $(>10,000 \times \mathrm{g})$ to elute the DNA

27. Add $10 \mu \mathrm{l}$ of M-Elution Buffer directly to the column matrix

28. Centrifuge for 30 seconds at full speed $(>10,000 \times \mathrm{g})$ to elute the DNA

NOTE: We strongly advice to process the samples directly for PCR amplification without intermediate storage by freezing in order to avoid potential loss of bisulfiteconverted DNA due to free-thaw-cycles.

\section{Multiplex PCR}

The volumes for the Multiplex PCR can vary depending on the number of dilutions and target genes used for the LDBSP analysis. For illustrative purposes, we here provide an example based on 6 target genes and 22 dilutions, using a total volume of $25 \mu l$ for each individual PCR reaction. Overall, the content of each individual $P C R$ reaction in relation to the buffer, dNTPs and Taq DNA polymerase should be identical, whereas volumes of $\mathrm{H}_{2} \mathrm{O}$ vary depending on the number of target genes. Furthermore, always prepare several extra reactions for negative and positive controls, as well as for counteracting pipetting errors. The calculation demonstrated here is based on 22 dilutions (or reactions) for the samples, 2 control reactions and 1 extra reaction for pipetting errors, giving a total of 25 reactions. 
6. Prepare a single PCR mix containing the following reagents:

a. $2.5 \mu \mathrm{L}$ PCR buffer (10X) with $20 \mathrm{mM} \mathrm{MgCl} 2$ per reaction

$\rightarrow 25 \times 2.5 \mu \mathrm{L}=62.5 \mu \mathrm{L}$

b. $0.5 \mu \mathrm{L} 10 \mathrm{mM} \mathrm{dNTP}$ mix per reaction

$\rightarrow 25 \times 0.5 \mu L=12.5 \mu L$

c. $1 \mu \mathrm{L}$ of the forward primer $(10 \mu \mathrm{M}$ stock) per gene per reaction

$\rightarrow 25 \times 6$ genes $\times 1 \mu \mathrm{L}=150 \mu \mathrm{L}$

d. $1 \mu \mathrm{L}$ of the reverse primer ( $10 \mu \mathrm{M}$ stock) per gene per reaction

$\rightarrow 25 \times 6$ genes $\times 1 \mu \mathrm{L}=150 \mu \mathrm{L}$

e. $0.2 \mu \mathrm{L}(5 \mathrm{U} / \mu \mathrm{L})$ FastStart ${ }^{\mathrm{TM}}$ Taq DNA Polymerase per reaction

$\rightarrow 0.2 * 25=5 \mu L$

$\rightarrow$ Components 'a'-'e'; total volume $=380 \mu \mathrm{l}$

7. Mix by shaking and then centrifuge the PCR mix

8. Divide the PCR mix in two separate Eppendorf tubes according to the necessary volumes

a. Tube 1,22 reactions: $334.4 \mu \mathrm{l} \mathrm{PCR}$ mix

$\rightarrow 380 / 25 \times 22=334.4 \mu \mathrm{l}$

b. Tube 2, 2 reactions: $30.4 \mu \mathrm{l} \mathrm{PCR} \mathrm{mix}$

$\rightarrow 380 / 25 \times 2=30.4 \mu l$

9. Add a required volume of $\mathrm{H}_{2} \mathrm{O}$ to both the PCR mixes (See details below)

a. Tube 1, 22 reactions: $195.6 \mu \mathrm{l} \mathrm{H}_{2} \mathrm{O}$

$\rightarrow 22$ reactions $\times 25 \mu$ l total volume $=550 \mu \mathrm{l}$

$\rightarrow$ Minus $334.4 \mu \mathrm{l} \mathrm{PCR} \mathrm{mix}=215.6 \mu \mathrm{l}$

$\rightarrow$ Minus $20 \mu$ l bisulfite converted DNA $=195.6 \mu \mathrm{l}$

b. Tube 2, 2 reactions: $17.6 \mu \mathrm{l} \mathrm{H}_{2} \mathrm{O}$

$\rightarrow 2$ reactions $\times 25 \mu$ lotal volume $=50 \mu \mathrm{l}$

$\rightarrow$ Minus $30.4 \mu \mathrm{l} \mathrm{PCR}$ mix $=19.6 \mu \mathrm{l}$

$\rightarrow$ Minus $2 \times 1 \mu$ l of template (Control DNA or $\mathrm{H}_{2} \mathrm{O}$ ) $=17.6 \mu \mathrm{l}$

10. Mix by shaking and then centrifuge the PCR mixes

11. Add the complete PCR mix from 'Tube 1 ' to the $20 \mu$ of bisulfite converted DNA

12. Mix thoroughly by pipetting up-and-down

13. By using the same pipet tip, dispense $25 \mu$ of the mixture over 22 wells of a micro-titer plate (mix thoroughly inbetween dispensation steps)

14. Dispense 24 microliter of the PCR mix from 'Tube 2 ' into 2 wells of a micro-titer plate

15. Add the necessary control templates to the latter 2 wells (e.g. positive and negative)

16. Seal the micro-titer plate

17. Place the micro-titer plate in a thermal cycler and perform the following steps:

a. $95^{\circ} \mathrm{C}$ for 5 minutes

b. $95^{\circ} \mathrm{C}$ for 30 seconds

$56^{\circ} \mathrm{C}$ for 30 seconds

$72^{\circ} \mathrm{C}$ for 1 minute

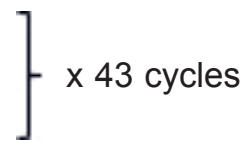



c. $72^{\circ} \mathrm{C}$ for 7 minutes
d. $4^{\circ} \mathrm{C}$ storage

NOTE: The optimum annealing temperature and required number of cycles should be tested a priori, both independently, as well as in multiplex formation. For the multiplex PCR amplifications, all primers need to be compatible for the same cycler conditions. The program displayed here was applied in the present study.

\section{Singleplex PCR}

Prepare a total volume of singleplex PCR mix that is sufficient for the number of sample containing reactions (i.e. 22 in this case), negative controls and some extra to counteract pipetting errors.

1. Each PCR reaction should contain the following reagents per tube (Total volume of $25 \mu \mathrm{L}$ ):
a. $1 \mu \mathrm{l}$ of Multiplex PCR product
b. $2.5 \mu \mathrm{L}$ PCR buffer (10X) with $20 \mathrm{mM} \mathrm{MgCl} 2$
c. $0.5 \mu \mathrm{L} 10 \mathrm{mM}$ dNTP mix
d. $1 \mu \mathrm{L}$ of the forward primer (10 $\mu \mathrm{M}$ stock)
e. $1 \mu \mathrm{L}$ of the reverse primer $(10 \mu \mathrm{M}$ stock)
f. $0.2 \mu \mathrm{L}(5 \mathrm{U} / \mu \mathrm{L})$ FastStart ${ }^{\mathrm{TM}}$ Taq DNA Polymerase
g. $18.8 \mu \mathrm{l}$ of $\mathrm{H}_{2} \mathrm{O}$

2. Place the micro-titer plate in a thermal cycler and perform the following steps:
a. $95^{\circ} \mathrm{C}$ for 5 minutes
b. $95^{\circ} \mathrm{C}$ for 30 seconds $58^{\circ} \mathrm{C}$ for 30 seconds $72^{\circ} \mathrm{C}$ for 1 minute
c. $72^{\circ} \mathrm{C}$ for 7 minutes
d. $4^{\circ} \mathrm{C}$ storage

3. Visualize the PCR products on a $2 \%$ agarose gel, using the Orange G DNA Loading Dye, the GelsGelStar ${ }^{\mathrm{TM}}$ Nucleic Acid Gel Stain and a 100 bp ladder according to the manufacturer's instructions.

4. Identify which of the reactions yield a gene-specific PCR product

5. Use the required amount of PCR product as suggested by the manufacturer's instructions for subsequent pyrosequencing analysis.

6. Please refer to the manufacturer's instructions for details on bisulfite pyrosequencing

NOTE: The optimum annealing temperature and required number of cycles should be tested a priori for each target gene, using both bisulfite converted DNA, as well as multiplex product. The program displayed here was applied in the present study. 
Supplementary Table 1. Cohort demographics

\begin{tabular}{lll}
\hline & AD patients & Non-demented controls \\
\hline N & 12 & 12 \\
Age of death; Mean (SD) & Female & Female \\
PMI; Mean (SD) & $84.5(5.04)$ & $77.92(13.00)$ \\
Plaque total; Mean (SD) & $2.89(0.96)$ & $2.48(0.62)$ \\
Tangle total; Mean (SD) & $13.69(1.71)$ & $2.16(3.54)$ \\
Braak stage; Range & $14.0(1.36)$ & $3.33(2.38)$ \\
\hline
\end{tabular}

Dorsal raphe nucleus (DRN) tissue obtained from the Banner Sun Health Research Institute (BSHRI, Sun City, Arizona, US) as part of the Brain and Body Donation Program (BBDP). Displayed is the number of samples in each group, the sex of the subjects and the mean plus standard deviation (SD) of their age of death, postmortem interval (PMI, in hours), total plaque load and total tangle load, as well as their range of Braak stage. 
Supplementary Table 2. Primer overview

\begin{tabular}{|c|c|c|c|c|}
\hline \multicolumn{5}{|l|}{ PCR primers } \\
\hline Gene & Forward primer (5'-3') & Reverze primer (5'-3') & Target region (GRCh37) & $\begin{array}{l}\text { Product } \\
\text { size (bp) }\end{array}$ \\
\hline $\begin{array}{l}\text { DNAJB13 } \\
\text { (Multiplex) }\end{array}$ & $\begin{array}{l}\text { GGTATTTTGGGAGGATGA } \\
\text { GTTA }\end{array}$ & $\begin{array}{l}\text { CCСTTCAAAAACAAAC } \\
\text { CAACTAAT }\end{array}$ & $11: 73668561-73668825$ & 265 \\
\hline $\begin{array}{l}\text { DNAJB13 } \\
\text { (Singleplex) }\end{array}$ & $\begin{array}{l}\text { GTATTTTGGGAGGATGA } \\
\text { GTTATAATTG }\end{array}$ & $\begin{array}{l}\text { (Bio-) CTTCAAAAACAAAC } \\
\text { CAACTAATAC }\end{array}$ & $11: 73668563-73668824$ & 262 \\
\hline $\begin{array}{l}\text { PGLYRP1 } \\
\text { (Multiplex) }\end{array}$ & $\begin{array}{l}\text { GTTGGGGAGAGTTTAG } \\
\text { GTAAG }\end{array}$ & $\begin{array}{l}\text { ATTCCTATTAAATTATTCAAT } \\
\text { ATTCСАСT }\end{array}$ & 19:46526238-46526634 & 397 \\
\hline $\begin{array}{l}\text { PGLYRP1 } \\
\text { (Singleplex) }\end{array}$ & $\begin{array}{l}\text { TGGGGAGAGTTTAGGT } \\
\text { AAGTA }\end{array}$ & $\begin{array}{l}\text { (Bio-) } \\
\text { TCCTATTAAATTATTCAAT } \\
\text { ATTCCACTAA }\end{array}$ & $19: 46526240-46526632$ & 393 \\
\hline $\begin{array}{l}\text { LMX1B } \\
\text { (Multiplex) }\end{array}$ & $\begin{array}{l}\text { TTTTTTAAGGGGGTGG } \\
\text { AGTAGAG }\end{array}$ & $\begin{array}{l}\text { CCTCACCTCCСCAAACT } \\
\text { TCCTAACATT }\end{array}$ & 9:129375928-129376184 & 257 \\
\hline $\begin{array}{l}\text { LMX1B } \\
\text { (Singleplex) }\end{array}$ & $\begin{array}{l}\text { GGGGGTGGAGTAGAG } \\
\text { GGA }\end{array}$ & $\begin{array}{l}\text { (Bio-) } \\
\text { CCTCACСTCCCCAAACT } \\
\text { TCСТAАСАTT }\end{array}$ & $9: 129375936-129376184$ & 249 \\
\hline $\begin{array}{l}\text { OXT } \\
\text { (Multiplex) }\end{array}$ & $\begin{array}{l}\text { GTTTAGGTTTTGTTAAT } \\
\text { GAAGAGGAA }\end{array}$ & $\begin{array}{l}\text { ACCAAAACTAAAAACTC } \\
\text { ACCTTAC }\end{array}$ & $20: 3052117-3052440$ & 324 \\
\hline $\begin{array}{l}\text { OXT } \\
\text { (Singleplex) }\end{array}$ & $\begin{array}{l}\text { GTTTAGGTTTTGTTTAAT } \\
\text { GAAGAGGAA }\end{array}$ & $\begin{array}{l}\text { (Bio-) TCTTACCTCC } \\
\text { CAAAAAACAATTC }\end{array}$ & $20: 3052117-3052392$ & 276 \\
\hline $\begin{array}{l}\text { C3 } \\
\text { (Multiplex) }\end{array}$ & $\begin{array}{l}\text { GATTGGGTTTTATTTGAGTG } \\
\text { TAAGAT }\end{array}$ & $\begin{array}{l}\text { CCAACAATAAATTAAAAA } \\
\text { CTCCAATCTTC }\end{array}$ & $19: 6713187-6713627$ & 441 \\
\hline $\begin{array}{l}\text { C3 } \\
\text { (Singleplex) }\end{array}$ & $\begin{array}{l}\text { GATTGGGTTTTATTTGAGTG } \\
\text { TAAGAT }\end{array}$ & $\begin{array}{l}\text { (Bio-) AACCTAAAACCCT } \\
\text { CCTTATCTAT }\end{array}$ & 19:6713187-6713550 & 364 \\
\hline $\begin{array}{l}\text { RHBDF2 } \\
\text { (Multiplex) }\end{array}$ & AGGGTTTTGGGGATTTAGTG & $\begin{array}{l}\text { CCAAAAACCTCACAAAC } \\
\text { AAATC }\end{array}$ & $17: 74475178-74475497$ & 320 \\
\hline $\begin{array}{l}\text { RHBDF2 } \\
\text { (Singleplex) }\end{array}$ & AGGGTTTTGGGGATTTAGTG & $\begin{array}{l}\text { (Bio-) CATCACСССТTCC } \\
\text { АСАСАСТСАA }\end{array}$ & $17: 74475178-74475435$ & 258 \\
\hline
\end{tabular}

\begin{tabular}{|c|c|c|c|c|c|}
\hline \multicolumn{6}{|c|}{ Pyrosequencing primers } \\
\hline Gene & Sequencing primer ( $\left.5^{\prime}-3^{\prime}\right)$ & $\begin{array}{l}\text { Number } \\
\text { of CpGs }\end{array}$ & $\begin{array}{l}\text { PyroMark } \\
\text { Orientation }\end{array}$ & $\begin{array}{l}\text { Target region } \\
\text { (GRCh37) }\end{array}$ & Primer \\
\hline DNAJB13 & GGATGAGTTATAATTGGG & 5 & $\begin{array}{l}\text { Lower strand } \\
\left(5^{\prime}-3^{\prime}\right)\end{array}$ & $11: 73668745-73668795$ & 1 \\
\hline DNAJB13 & TTTTGTTATTTGTTTGAAAA & 4 & $\begin{array}{l}\text { Lower strand } \\
\left(5^{\prime}-3^{\prime}\right)\end{array}$ & $11: 73668618-73668673$ & 2 \\
\hline PGLYRP1 & TTTTTTTTTGGTTGGGTTAG & 1 & $\begin{array}{l}\text { Upper strand } \\
\left(5^{\prime}-3^{\prime}\right)\end{array}$ & $19: 46526451-46526454$ & 1 \\
\hline PGLYRP1 & GAGGGATGTATTGTGG & 3 & $\begin{array}{l}\text { Upper strand } \\
\left(5^{\prime}-3^{\prime}\right)\end{array}$ & $19: 46526468-46526509$ & 2 \\
\hline PGLYRP1 & GTTTAGTAGGGAGGG & 5 & $\begin{array}{l}\text { Upper strand } \\
\left(5^{\prime}-3^{\prime}\right)\end{array}$ & $19: 46526524-46526555$ & 3 \\
\hline LMX1B & $\begin{array}{l}\text { GTTTAGAAGAAGATTAAAA } \\
\text { TTTTTG }\end{array}$ & 7 & $\begin{array}{l}\text { Upper strand } \\
\left(5^{\prime}-3^{\prime}\right)\end{array}$ & 9:129375982-129376016 & 1 \\
\hline OXT & GGTTTTGTTAATGAAGAGGAA & 5 & $\begin{array}{l}\text { Upper strand } \\
\left(5^{\prime}-3^{\prime}\right)\end{array}$ & $20: 3052143-3052173$ & 1 \\
\hline
\end{tabular}


Supplementary Table 2. (Continued)

\begin{tabular}{|c|c|c|c|c|c|}
\hline \multicolumn{6}{|c|}{ Pyrosequencing primers } \\
\hline Gene & Sequencing primer ( $\left.5^{\prime}-3^{\prime}\right)$ & $\begin{array}{l}\text { Number of } \\
\text { CpGs }\end{array}$ & $\begin{array}{l}\text { PyroMark } \\
\text { Orientation }\end{array}$ & $\begin{array}{l}\text { Target region } \\
\text { (GRCh37) }\end{array}$ & Primer \\
\hline OXT & ATATTATTAATTTTTTAAAATAGAG & 2 & $\begin{array}{l}\text { Upper strand } \\
\left(5^{\prime}-3^{\prime}\right)\end{array}$ & $20: 3052215-3052224$ & 2 \\
\hline OXT & AATGTTTAGGTATAAAAAGGT & 5 & $\begin{array}{l}\text { Upper strand } \\
\left(5^{\prime}-3^{\prime}\right)\end{array}$ & $20: 3052248-3052297$ & 3 \\
\hline $\mathrm{C} 3$ & TTTTAATTTGAGAAGGGAGA & 2 & $\begin{array}{l}\text { Upper strand } \\
\left(5^{\prime}-3^{\prime}\right)\end{array}$ & 19:6713340-6713376 & 1 \\
\hline C3 & TGGGGTAGGGATTAAA & 4 & $\begin{array}{l}\text { Upper strand } \\
\left(5^{\prime}-3^{\prime}\right)\end{array}$ & 19:6713398-6713426 & 2 \\
\hline C3 & ATTTAGGTAGGGAAA & 2 & $\begin{array}{l}\text { Upper strand } \\
\left(5^{\prime}-3^{\prime}\right)\end{array}$ & 19:6713450-6713475 & 3 \\
\hline RHBDF2 & TTTTTGGTTGGGGAG & 5 & $\begin{array}{l}\text { Upper strand } \\
\left(5^{\prime}-3^{\prime}\right)\end{array}$ & $17: 74475230-74475271$ & 1 \\
\hline RHBDF2 & GTATGTTGTAGTGGTG & 5 & $\begin{array}{l}\text { Upper strand } \\
\left(5^{\prime}-3^{\prime}\right)\end{array}$ & $17: 74475287-74475332$ & 2 \\
\hline RHBDF2 & GATTTTAAATTATTGGGTTG & 4 & $\begin{array}{l}\text { Upper strand } \\
\left(5^{\prime}-3^{\prime}\right)\end{array}$ & $17: 74475355-74475403$ & 3 \\
\hline
\end{tabular}

Displayed is an overview of the polymerase chain reaction (PCR) and pyrosequencing primers that were used in this study. Provided for each target gene are the multiplex and singleplex primer sequences (Bio, biotinylated), as well as corresponding genomic coordinates (Ensembl GRCh37 assembly) and amplicon sizes in base pairs (bp). For the pyrosequencing primers, the total number of $\mathrm{CpG}$ sites, the targeted region for sequencing and the orientation of the assay in the PyroMark assay design software 2.0 are listed per gene. 



\title{
Chapter 5
}

\section{Brain-region- and cell type-specific epigenetic profiling strongly implicates a role for dysregulation of $T N X B$ and other loci in the brainstem in Alzheimer's disease}

\author{
Renzo J.M. Riemens ${ }^{1,2 *}$, Ehsan Pishva ${ }^{1,3 *}$, Artemis latrou ${ }^{4}$, Janou Roubroeks ${ }^{3}$, \\ Jennifer Nolz ${ }^{5}$, Roy Lardenoije ${ }^{1,6}$, Muhammad Ali ${ }^{1,7}$, Antonio Del Sol7, \\ Raul Delgado-Morales ${ }^{1,8}$, Manel Esteller ${ }^{9}$, Gunter Kenis ${ }^{1}$, Bart P.F. Rutten ${ }^{1}$, \\ Klaus-Peter Lesch ${ }^{10}$, Jonathan Mill ${ }^{3}$, Diego Mastroeni ${ }^{1,5,11}$, Alfredo Ramirez ${ }^{12}$, \\ Thomas Haaf ${ }^{2}$, Katie Lunnon ${ }^{3 \dagger}$, Daniël L.A. van den Hove Lit10 $^{1,1}$ \\ 'Department of Psychiatry and Neuropsychology, School for Mental Health and Neuroscience (MHeNs), \\ Maastricht University, Maastricht, the Netherlands. \\ ${ }^{2}$ Institute of Human Genetics, Julius Maximilians University, Wuerzburg, Germany. \\ ${ }^{3}$ University of Exeter Medical School, University of Exeter, Exeter, United Kingdom. \\ ${ }^{4}$ Rush Alzheimer's Neurodisease Center, Rush University Medical Center, Chicago, Illinois, United \\ States of America.
}

${ }^{5}$ Biodesign Institute, Neurodegenerative Disease Research Center, Arizona State University, Tempe, Arizona, United States of America.

${ }^{6}$ Department of Psychiatry and Psychotherapy, University Medical Center Göttingen, Göttingen, Germany.

${ }^{7}$ Luxembourg Centre for Systems Biomedicine (LCSB), University of Luxembourg, Esch-sur-Alzette, Luxembourg.

${ }^{8}$ Cancer Epigenetics and Biology Program (PEBC), Bellvitge Biomedical Research Institute - IDIBELL, L'Hospitalet del Llobregat, Barcelona, Catalonia, Spain.

${ }^{9}$ Josep Carreras Leukaemia Research Institute (IJC), Badalona, Barcelona, Spain; Centro de Investigacion Biomedica en Red Cancer (CIBERONC), Madrid, Spain; Institucio Catalana de Recerca I Estudis Avançats (ICREA), Barcelona, Catalonia, Spain; Physiological Sciences Department, School of Medicine and Health Sciences, University of Barcelona (UB), Barcelona, Catalonia, Spain.

${ }^{10}$ Laboratory of Translational Neuroscience, Department of Psychiatry, Psychosomatics and Psychotherapy, University of Wuerzburg, Wuerzburg, Germany.

${ }^{11}$ L.J. Roberts Center for Alzheimer's Research Banner Sun Health Research Institute, Sun City, Arizona, United States of America.

${ }^{12}$ Division for Neurogenetics and Molecular Psychiatry, Department of Psychiatry and Psychotherapy, Medical Faculty, University of Cologne, Cologne, Germany; Department for Neurodegenerative Diseases and Geriatric Psychiatry, University of Bonn, Bonn, Germany.

\footnotetext{
* Authors contributed equally to this work

+ Authors contributed equally to this work
} 



\section{Abstract}

Increasing evidence suggests that dysregulation within brainstem nuclei such as the dorsal raphe nuclei (DRN) and the locus coeruleus (LC) represents one of the first steps in the pathogenesis of Alzheimer's disease (AD). Furthermore, recent epigenome-wide association studies (EWAS) have implicated a central role for epigenetic alterations in both the development and course of the disease. However, the extent of disease-specific deviant epigenetic signatures in the brainstem have not been investigated yet. The present study represents the first large-scale epigenetic analysis in the brainstem of AD, targeting both the DRN and the LC in parallel. In bulk tissues derived from both brainstem nuclei, differentially modified cytosine-phosphate-guanine $(\mathrm{CpG})$ sites and regions were quantified at the level of three cytosine states, i.e. 5-methylcytosine (5-hmC), 5-hydroxymethylcytosine (5$\mathrm{mC}$ ) and unmodified cytosine (5-uC), utilizing the Illumina Infinium MethylationEPIC BeadChip array and making use of both customary bisulfite and innovative oxidative bisulfite treatment. Following methodological validation in a subset of patients from the same cohort using bisulfite pyrosequencing, we then targeted the bisulfite methylation signatures of our discovery findings in an independent patient cohort, where we profiled single laser capture microdissected serotonergic and non-serotonergic cells isolated from the DRN by means of modified limiting dilution bisulfite pyrosequencing (LDBSP). Overall, within both brainstem regions assessed, our EWAS revealed a strong overlapping dysregulation in the Tenascin XB (TNXB) gene, next to the identification of both previously identified and novel epigenetic loci that we hypothesize to play a pivotal role in the early development of $A D$. Furthermore, we demonstrated, for the first time, that the methylation profile of TNXB in the DRN is dependent on both the disease phenotype and the cell type analyzed, which warrants the need for future cell type-specific neuroepigenetic studies in AD.

\section{Keywords}

Alzheimer's disease, brainstem, dorsal raphe nucleus, locus coeruleus, DNA methylation (5-mC), DNA hydroxymethylation (5-hmC), Infinium MethylationEPIC BeadChip Kit, epigenome-wide association study (EWAS), single cell. 


\section{Introduction}

Over the last years, prime sites of early interrogation of Alzheimer's disease (AD) have been extended beyond brain regions such as the hippocampus and the entorhinal cortex, since increasing evidence suggests that also the brainstem is among the first regions affected in the disease [1-3]. In fact, recent studies have demonstrated that neurofibrillary tangles in the brains of $A D$ patients can already be observed in brainstem nuclei such as the dorsal raphe nuclei (DRN) and the locus coeruleus (LC), even before the manifestations of the first clinical symptoms [1-4]. In view of this notion, it is currently hypothesized that from these brainstem nuclei pathology could spread to subcortical areas, including the hippocampus, and, subsequently, to areas of the neocortex, marking the clinical stages of this neurodegenerative disease. Other evidence for a central role of the brainstem comes from observations using magnetic resonance imaging, indicating that this brain region is subject to both significant volume reductions and structural deformations in AD patients [5, 6]. Moreover, the occurrence of various non-cognitive behavioral and neuropsychological symptoms in $A D$, particularly in prodromal stages, including depression, disturbances in mood, emotions, appetite, respiratory and circadian rhythm, suggests the involvement of the DRN and the LC in the disorder [2].

Research aimed at exploring the etiopathophysiology of AD has furthermore implicated a crucial role for epigenetic mechanisms, in addition to both independent and interdependent genetic, environmental and life-style factors that influence the development and course of the disease [7, 8]. For this reason, the characterization of epigenetic profiles in brain tissue samples derived from AD patients and people at risk of developing $A D$ is currently offering a highly attractive approach to study this disease. As such, several recent epigenome-wide association studies (EWAS) have identified robust differences in DNA methylation and hydroxymethylation in the AD cortex across independent patient cohorts [9-20]. Aside from studies targeting the brain, other EWAS using peripheral blood samples derived from patients have also identified dysregulated loci that harbor biomarker potential towards predicting the development and/or course of AD [15, 21]. Despite the increasing number of EWAS that offer novel insights into its pathophysiology, examining the brainstem for potential disease-specific deviant epigenetic signatures, indicative of the more incipient stages of $A D$, has, however, not been performed yet.

In the present study, we therefore conducted the first large-scale EWAS in the brainstem of $A D$ to date. Our analytical approach consisted of two stages and focused on identifying alterations in cytosine-phosphate-guanine $(\mathrm{CpG})$ modifications associated with Braak stage at the level of unmodified cytosine (5-uC), 5-methylcytosine (5$\mathrm{mC}$; reflecting DNA methylation) and 5-hydroxymethylcytosine (5-hmC; reflecting 
DNA hydroxymethylation). First, in DNA derived from both DRN and LC bulk tissues, differentially modified positions and regions for each of the aforementioned modifications were quantified by using Illumina Infinium MethylationEPIC BeadChip arrays in combination with customary bisulfite and novel oxidative bisulfite treatment. These discovery findings were subsequently confirmed in a subset of patients from the same cohort using bisulfite pyrosequencing. Second, we followed up our EWAS by a validation study in an independent patient cohort targeting bisulfite methylation signatures in serotonergic and non-serotonergic cells isolated from the DRN by laser capture microdissection (LCM). Overall, within both brainstem regions assessed, our EWAS revealed a strong overlapping dysregulation in a locus of Tenascin XB ( $T N X B$ ), next to highlighting both previously identified and novel epigenetic loci that we hypothesize to play a pivotal role in the early development of AD. Furthermore, we demonstrated, for the first time, that $A D$-associated dysregulation of bisulfite methylation patterns in the DRN, including that of TNXB, is dependent on both the disease phenotype and the cell type analyzed, which emphasizes the need for future cell type-specific neuroepigenetic studies in AD.

\section{Materials and methods}

\section{Subjects and samples}

\section{EWAS discovery and pyrosequencing validation cohorts}

The post-mortem DRN and LC bulk tissue samples that were used in the discovery EWAS and pyrosequencing validation analysis were obtained from the MRC London Brain bank for Neurodegenerative Disease (London, UK). Samples were provided with informed consent according to the Declaration of Helsinki (1991) and ethical approval for the study was provided by the NHS South East London REC 3. In total, 94 DRN samples and $81 \mathrm{LC}$ samples from both $\mathrm{AD}$ patients and neurologically healthy controls were obtained and used for the EWAS. These two cohorts were derived from a total number of 109 individual subjects, meaning that 66 (60.55\%) of them had matched tissue samples for both brainstem regions, thereby contributing to both cohorts. Upon screening for $A D$ neurofibrillary pathology at autopsy, subjects were distributed to the relevant Braak stage ranging from Braak stage 0 to Braak stage VI. The tangle burden is described in a range from no AD pathology (Braak 0 ), to primary pathology in early-affected regions such as the brainstem (Braak I-II), to mid-stage subcortical pathology (Braak III-IV) and to severe widespread AD pathology (Braak V-VI). All samples were dissected by trained specialists, snap frozen and stored at $-80^{\circ} \mathrm{C}$ up until further processing. For each sample, genomic DNA (gDNA) was extracted from $\sim 50 \mathrm{mg}$ of tissue using a standard phenol-chloroform extraction method. For validation purposes, 62 and 52 identical isolated gDNA samples from a subset of these DRN and LC samples, respectively, were used for bisulfite pyrosequencing analysis of TNXB. Demographic and relevant information about the samples and cohorts are provided in Table 1. 


\section{Cell-specific DRN validation cohort}

The post-mortem DRN tissue samples that were used in the cell-specific replication analysis were obtained from the Banner Sun Health Research Institute (Sun City, Arizona, USA). Age-matched DRN tissue samples from 12 female subjects without(Control cases) and 12 female subjects with a clinical diagnosis of $A D$ ( $A D$ cases) were collected. Each subject and her respective relative(s) consented to brain autopsy for the purpose of scientific research as part of the Banner Sun Health Research Institute Brain and Body Donation Program (BBDP) [22]. Written informed consent for autopsy was obtained in compliance with institutional guidelines. The institutional review board approved the entire study, including the recruitment, enrollment and autopsy procedures. A final diagnosis of AD at autopsy was made by following the National Institutes of Health (NIH) AD Center criteria [22]. Comorbidity with any other type of dementia, mild cognitive impairment, cerebrovascular disorders and presence of nonmicroscopic infarcts were applied as exclusion criteria. After autopsy, brain samples were snap frozen and stored at $-80^{\circ} \mathrm{C}$ up until further processing. For demographic and relevant information about the studied samples, please refer to Table 1.

\section{EWAS discovery analysis}

\section{Bisulfite and oxidative-bisulfite treatment}

The TrueMethyITM 24 Kit version 2.0 by CEGXTM (Cambridge Epigenetix Limited, Cambridge, UK) was used for bisulfite and oxidative bisulfite conversion of gDNA isolated from the DRN and LC bulk tissues. All laboratory procedures were performed at GenomeScan (GenomeScan B.V., Leiden, the Netherlands) and according to the manufacturer's instructions. Prior to the conversion, high molecular weight gDNA was quantified using a PicoGreen assay (Invitrogen, Carlsbad, California, USA) and the gDNA quality was assessed by gel-electrophoresis, confirming that all samples were of sufficient quantity and quality. From each subject and brainstem region, 1 $\mu \mathrm{g}$ of gDNA was used, which, after purification and denaturation, was split up into two samples that underwent either the DNA oxidation or a mock DNA oxidation, for the oxidative bisulfite and bisulfite treated samples, respectively. Subsequently, all samples were bisulfite converted and the yields of the (oxidative) bisulfite DNA were assessed by a Qubit ssDNA assay (Invitrogen). An additional restriction quality control was performed for a qualitative assessment of 5-hmC oxidation and bisulfite conversion using the Fragment Analyzer.

\section{IIlumina Infinium MethylationEPIC BeadArray}

From each bisulfite- and oxidative bisulfite-treated DNA sample, $8 \mu \mathrm{L}$ was amplified and hybridized on the Infinium MehtylationEPIC BeadChip (EPIC array; Illumina, Inc., San Diego, CA, U.S.A.). All samples were randomized with respect to sex and Braak stage in order to avoid batch effects and were processed in batches of 8 per 
BeadChip. Bisulfite- and oxidative bisulfite-treated samples from the same individuals were run on the same chip to avoid batch effects. Illumina iScan was used for imaging of the array. Sample preparation, hybridization, and washing steps were performed according to the manufacturer's instructions.

\section{Pyrosequencing validation analysis}

\section{Bisulfite treatment}

The EZ-96 DNA Methylation-Gold Kit (D5008; Irvine, CA, USA) was applied for bisulfite conversion of an identical subset of gDNA samples used in the EWAS (i.e. pyrosequencing validation cohort), which were isolated from both the DRN and LC bulk tissues. Per sample, $200 \mathrm{ng}$ of gDNA was processed according to the manufacturer's instructions and all samples were bisulfite converted simultaneously per brainstem region in order to avoid batch effects. Finally, bisulfite-converted samples were eluted in $20 \mu \mathrm{l}$ of elution buffer and $1 \mu \mathrm{l}$ was used for polymerase chain reaction (PCR) amplifications, and subsequent bisulfite pyrosequencing.

\section{Bisulfite pyrosequencing}

Pyrosequencing was used to quantify the bisulfite methylation $(5-\mathrm{mC}+5-\mathrm{hmC})$ levels across eight individual TNXB CpG sites, including cg10365886, cg14188106 and cg07524919, spanning from 32063869 to 32063940 within chromosome 6 (Ensembl GRCh37 assembly). A single amplicon of 209 bp (6:32063788-32063996) was PCR amplified using primers designed with the PyroMark Assay Design software 2.0 (Qiagen, Hilden, Germany) (Supplementary Table 13). PCRs were performed with an initial denaturation step at $95^{\circ} \mathrm{C}$ for 5 minutes, followed by 45 cycles at $95^{\circ} \mathrm{C}$ for 30 seconds, $62^{\circ} \mathrm{C}$ for 30 seconds and $72^{\circ} \mathrm{C}$ for 30 seconds, with a final extension step at $72^{\circ} \mathrm{C}$ for 1 minute. All PCR reactions, contained $2.5 \mu \mathrm{l} \mathrm{PCR}$ buffer (10X) with $20 \mathrm{mM}$ $\mathrm{MgCl} 2,0.5 \mu \mathrm{l} 10 \mathrm{mM}$ dNTP mix, $1 \mu \mathrm{l}$ of each primer $(5 \mu \mathrm{M}$ stock) and $0.2 \mu \mathrm{l}(5 \mathrm{U} / \mu \mathrm{l})$ FastStart ${ }^{\mathrm{TM}}$ Taq DNA Polymerase (Roche Diagnostics $\mathrm{GmbH}$, Mannheim, Germany) in a total volume of $25 \mu$. Pyrosequencing of the amplicon was performed using a single sequencing primer covering the 72-bp region. DNA bisulfite methylation was quantified in the validation cohort using the PyroMark Q48 Autoprep system (Qiagen) and the Pyro Q48 Autoprep 2.4.2 software following the manufacturer's instructions. The sensitivity of the assay was initially assessed using methylated and unmethylated DNA standards from the EpiTect PCR Control DNA Set (Qiagen).

\section{Cell-specific DRN validation analysis}

\section{Laser capture microdissection}

Frozen DRN tissue sections of $10 \mu \mathrm{m}$ from were mounted onto polyethylene naphthalate (PEN) slides and fixed in ice-cold $50 \%$ acetone $/ 50 \%$ ethanol solution for 5 minutes on ice. Sections were washed in ice cold phosphate buffered saline (PBS), 
blocked in $1 \%$ hydrogen peroxide for 2 minutes, followed by 3 quick submersions in ice-cold PBS. Sections were then placed in a dilution of primary antibody against serotonin (5-HT; Abcam, ab66047) in PBS for 10 minutes at room temperature. After the incubation, sections were washed three times in PBS and incubated with avidinbiotin complex in PBS for 10 minutes at room temperature. Next, sections were washed three times in $50 \mathrm{mM}$ Tris buffer and immersed in 3.3'-diaminobenzidine (DAB) solution ( $9.3 \mathrm{ml} 50 \mathrm{mM}$ Tris; $200 \mu \mathrm{LAB}(5 \mathrm{mg} / \mathrm{ml}) ; 500 \mu \mathrm{l}$ saturated nickel; and $4 \mu \mathrm{l}$ of $1 \%$ hydrogen peroxidase) for 5 minutes, followed by two quick rinses in $50 \mathrm{mM}$ Tris to stop the reaction. All sections were stored at $-80^{\circ} \mathrm{C}$ until further processing. 5-HT is a monoamine neurotransmitter that is specifically expressed by serotonergic neurons [23]. For this reason, LCM of serotonergic neurons from the DRN sections was performed based on 5-HT-immunoreactivity. In brief, sections were dipped in $100 \%$ ethanol, allowed to dry, and loaded onto a Leica AS-LMD LCM microscope (Leica, Wetzlar, Germany). Single serotonergic neurons were cut and then dropped into an inverted microcentrifuge cap containing $10 \mu$ of Tris-EDTA (TE) buffer. Per individual subject, 150 serotonergic neurons were captured at 20X magnification and divided in small pools of 50 cells per microcentrifuge tube, i.e. three pools of 50 neurons per subject. For the non-serotonergic cells, 50 regions of similar single cell-size were dissected in areas free of 5-HT-immunoreactivity and collected as described above in $10 \mu \mathrm{l}$ of TE buffer. In each of the experimental groups, two sections rendered unusable for additional isolation of non-serotonergic cells and were therefore excluded from further analysis (Non-serotonergic cells: $A D$, $n=10$; Control, $n=10$ ). All cells isolated with $L C M$ were stored at $-80^{\circ} \mathrm{C}$ until further processing.

\section{DNA isolation and bisulfite treatment}

Genomic DNA from a pool of serotonergic neurons or non-serotonergic cells was isolated and bisulfite-converted using the EZ DNA Methylation-Direct Kit (Zymo Research, Irvine, CA, USA) with the following adjustments. In brief, $1 \mu$ l of proteinase $\mathrm{K}(20 \mu \mathrm{g} / \mu \mathrm{l})$ and $11 \mu \mathrm{l}$ of M-Digestion buffer $(2 \mathrm{X})$ were added to a microcentrifuge tube containing the cells and incubated overnight at $50^{\circ} \mathrm{C}$. Subsequently, the complete lysate was transferred to a PCR tube and $143 \mu$ of bisulfite conversion reagent was used to wash out the digestion tube before adding it to the sample. Bisulfite conversion was performed in a thermal cycler running at $98^{\circ} \mathrm{C}$ for 8 minutes and then at $64^{\circ} \mathrm{C}$ for 3.5 hours. A volume of $200 \mu \mathrm{l}$ binding buffer was added to the spin column before loading the bisulfite-converted sample. The PCR tube used for bisulfite conversion was washed out twice by first adding $200 \mu$ of binding buffer to the tube and then by transferring this volume to the sample-containing column. After centrifugation $(10,000$ $\mathrm{x} \mathrm{g} ; 30 \mathrm{sec}$ ), the column was washed with $100 \mu \mathrm{l}$ washing buffer, incubated for $15 \mathrm{~min}$ with $200 \mu \mathrm{l}$ desulfonation buffer and washed twice again with $200 \mu \mathrm{l}$ washing buffer. The bisulfite-converted DNA was eluted in a single Eppendorf tube by running $20 \mu \mathrm{l}$ 
of elution buffer through the column twice (2 times at 10,000 x g; $30 \mathrm{sec}$ ). Eppendorf LoBind microcentrifuge tubes (Merck KGaA, Darmstadt, Germany) and TipOne Low Retention Tips (STARLAB, Hamburg, Germany) with low affinity for DNA were used throughout the whole procedure. PCR amplifications were performed directly after elution of the bisulfite-converted DNA.

\section{Polymerase chain reaction}

The targeted bisulfite pyrosequencing assays for TNXB was based on a (semi-) nested PCR. Primers were designed with the PyroMark Assay Design 2.0 software (Qiagen), based on the Ensemble GRCh37 assembly (See Supplementary Table 13). Only for the LDBSP analysis, bisulfite-treated DNA from a pool of serotonergic cells was diluted to a single allele level by adding a PCR mixture with a capacity of 22 individual reactions to the sample (determined empirically). Each individual PCR reaction made use of $2.5 \mu \mathrm{l} \mathrm{PCR}$ buffer (10X) with $20 \mathrm{mM} \mathrm{MgCl} 2,0.5 \mu \mathrm{l} 10 \mathrm{mM}$ dNTP mix, $1 \mu$ l of each primer ( $10 \mu \mathrm{M}$ stock) and $0.2 \mu \mathrm{l}(5 \mathrm{U} / \mu \mathrm{l})$ FastStart TM Taq DNA Polymerase (Roche Diagnostics $\mathrm{GmbH}$, Mannheim, Germany) in a total volume of $25 \mu \mathrm{l}$. After adding the bisulfite DNA to the complete mixture, the sample was pipetted up-and-down in order to homogeneously disperse all bisulfite-converted DNA molecules throughout the solution and fractions of $25 \mu \mathrm{l}$ were divided over 22 wells of a microtiter plate. A single amplicon of 440 bp (6:32063558-32063997) using the outer primers for $T N X B$ was amplified based on an initial denaturation step at $95^{\circ} \mathrm{C}$ for 5 minutes, followed by 43 cycles at $95^{\circ} \mathrm{C}$ for 30 seconds, $56^{\circ} \mathrm{C}$ for 30 seconds and $72^{\circ} \mathrm{C}$ for 1 minute, with a final extension step at $72^{\circ} \mathrm{C}$ for 7 minutes. For each individual singleplex PCR reaction, $1 \mu \mathrm{l}$ of the multiplex product was used as a template. A single amplicon of 432 bp (6:32063566-32063997) was amplified for TNXB using the inner primers using the same PCR compound concentrations and under similar thermocycler conditions as described above, although with 45 cycles and an annealing temperature of $58^{\circ} \mathrm{C}$. Bisulfite-treated DNA derived from pools of non-serotonergic cells was also amplified using (semi-) nested PCRs (i.e. extended pre-amplification); although with omitting the step of over-diluting the bisulfite converted DNA pool to single alleles. All PCR settings, as well as subsequent bisulfite pyrosequencing procedures, were performed under the exact same conditions for both of the analyzed cell types.

\section{Bisulfite pyrosequencing}

Pyrosequencing was used in order to determine the bisulfite methylation $(5-\mathrm{mC}+5-$ $\mathrm{hmC}$ ) status of the Braak-associated region in TNXB in both the serotonergic neurons and non-serotonergic cells of the DRN. For TNXB, the DNA bisulfite methylation status was quantified over a region spanning from 32063774 to 32063913 within chromosome 6 (Ensemble GRCh37 assembly), including six Illumina probes: cg10365886, cg07524919, cg14188106, cg26266427, cg01337207 
and cg02989255. The PyroMark Q96 MD pyrosequencing system (Qiagen) with the PyroMark Gold Q96 CDT reagent kit (Qiagen) were used according to the manufacturer's instructions. Methylation levels at a single $\mathrm{CpG}$ site resolution were quantified with the Pyro Q-CpG 1.0.9 software (Qiagen). The sensitivity of both assays was tested as described earlier using (un)methylated DNA standards from the EpiTect PCR Control DNA Set (Qiagen). The assay for TNXB was based on three pyrosequencing primers in order to maximize $\mathrm{CpG}$ site coverage over the targeted region (See Supplementary Table 13).

\section{Data processing and statistical analyses}

\section{EWAS quality control and data processing}

All computations and statistical analyses were performed using $R$ version 3.3.2 [24] and Bioconductor version 3.5 [25]. Raw signal intensities generated from both bisulfite (BS) and oxidative bisulfite (oxBS) treated samples for each brain region were used to construct MethylumiSet object using 'readEPIC' function in wateRmelon package [26] and RGChanne/Set objects using 'read.metharray.exp' function in minfi package [27]. We confirmed that samples that were treated for bisulfite and oxidative bisulfite conversion from the same identifiers were sourced from the same DNA samples using 59 single nucleotide polymorphism (SNP) probes on the array. Probes with common (minor allele frequency (MAF) $>5 \%$ ) SNPs in the $C G$ or single base extension position or cross-reactive probes were flagged and discarded. A principal component analysis (PCA)-based approach was used to examine a potential mismatch between reported and predicted sex. Using the 'pfilter' function within the wateRmelon package samples with a detection $p>0.05$ in more than $5 \%$ of probes, probes with more than three beadcount in $5 \%$ of the samples and probes having $1 \%$ of samples with a detection $p$ value $>0.05$ were identified and removed. Next the BS and oxBS datasets were split, and by using 'preprocessRaw' function the red and green channels for an Illumina methylation array were converted into methylation signals followed by a Noob background correction method with dye-bias normalization [28]. In order to estimate the proportion of DNA modifications, the maximum likelihood (ML) method described by Qu et al. [29] was used. The MLML function within the MLML2R package [30] was applied, which uses combined methylated and unmethylated signals from BS and oxBS arrays as an input, and returns the estimated proportion of 5-uC, 5-mC, and 5 -hmC for each $\mathrm{CpG}$ site. Only probes with a mean beta value $>0.1$ on both the bisulfite- and oxidative-bisulfite EPIC arrays were included in the MLML method. Finally, for 5-hmC, we only used sites in our analysis when 5 -hmC was present in more than half of the sample population. 


\section{Differentially modified position analyses}

In the DRN and LC EWAS, differentially methylated, hydroxymethylated, and unmodified cytosine positions (i.e. DUPS, DMPs and DHPs) associated with Braak stage were identified using linear regression models for each individual $\mathrm{CpG}$ probes. All analyses were adjusted for age, sex, and surrogate variables. Surrogate variable analysis (SVA) was performed in order to determine and estimate variation stemming from unknown sources including the cell type compositions. The identified surrogate variables (SVs) were correlated with variables known to affect methylation status, i.e. age, sex, post mortem interval, and the first five surrogate variables were included as covariates in the model along with age and sex. The $p$ values abstained from EWAS were assessed using inflation index lambda $(\lambda)$ value and the respective $Q Q$ plot. The $p$ values were adjusted for multiple testing using the Benjamin-Hochberg, false discovery rate (FDR) procedure. Probes with nominal $p$ values $<1.0 \mathrm{E}-3$ were ordered based on a combined $p$ values and regression estimate ranking. Illumina EPIC array probes were annotated using the Illumina UCSC gene annotation (Ensemble GRCh37 assembly).

\section{Differentially modified region analyses}

In order to identify differentially methylated, hydroxymethylated and unmodified regions (DMR, DHR, DUR) in our EWAS data, comb-p tool was used [31] with a distance of 500bp and a seeded $p$ value of $1.0 \mathrm{E}-3$.

\section{Gene ontology enrichment analysis}

For the top-ranked DUPs, DMPs and DHPs in DRN and LC, underlying biological processes and pathways were examined using a Gene Ontology (GO) enrichment analysis. Analysis were performed using the missMethyl package [32], which takes into account the different number of probes per gene on the array.

\section{Pyrosequencing validation data analysis}

For the pyrosequencing validation analysis of $T N X B, C p G$ bisulfite methylation percentages that passed quality control were exported from the Q48 Autoprep 2.4.2 software to the $\mathrm{R}$ statistical environment. Subsequently, a linear regression analysis per brainstem region was performed with the bisulfite methylation signals per $\mathrm{CpG}$ site as outcome, Braak Stage as predictor, and with age and sex added as covariates in the model. Cases with missing $\mathrm{CpG}$ bisulfite methylation values after pyrosequencing (DRN, $n=2 ; L C, n=2$ ) were excluded pairwise. In addition, a Pearson's correlation analysis was performed between the bisulfite methylation values obtained by bisulfite pyrosequencing and the EPIC array for each of the three Illumina probes in TNXB, i.e. cg10365886, cg14188106 and cg07524919. For cg07524919 in the LC, one case was excluded due to missing bisulfite methylation values for this $\mathrm{CpG}$ site after pyrosequencing. 


\section{Limiting dilution bisulfite pyrosequencing}

For LDBSP on the serotonergic neurons derived from the DRN, sequenceable amplicon-yielding reactions for TNXB after the second PCR step were assessed for their representation of single-, two or three-alleles based on the CpG methylation readouts, as described previously [33]. In brief, thresholds of (1) $\leq 8.33 \%$ and $\geq 91.33 \%$, (2) $50 \pm 8.33 \%$, and (3) $33.33 \pm 8.33 \%$ and $66.66 \pm 8.33 \%$, were set for the CpG methylation values. Accordingly, individual $\mathrm{CpG}$ sites that fell within the first, second and third threshold range were called and considered indicative for the potential presence of one, two and three alleles, respectively. A definitive (total) allele score for each individual product was then assigned following a multi-step filtering process that was based on the following criteria. All products solely displaying binary $\mathrm{CpG}$ methylation patterns, i.e. when every $\mathrm{CpG}$ site within an amplicon displays methylation levels within the first threshold range, were directly considered to be derived from single allele reactions, as the $\mathrm{CpG}$ methylation profiles for these products displayed a strong, typical binary pattern that is expected for a single DNA molecule [33, 34]. All products displaying $\mathrm{CpG}$ methylation values that fell only in the first and second, but not third, or only in the first and third, but not second, threshold ranges were scored as two or three alleles, respectively. Subsequently, products containing $\mathrm{CpG}$ methylation values that fell outside of the assigned threshold ranges, i.e. between $8.33 \%-25 \%$ and $75 \%-91.33 \%$, and/or products with values that were indicative of both two and three alleles simultaneously, were assessed. All of these products were therefore thoroughly inspected by two investigators that were blinded to the experimental conditions, and a decision on the total number of alleles present in each individual reaction, i.e. one, two or three alleles, was made independently, while taking into account a combination of different factors. These included, but were not limited to, small technological variation that was previously observed during sensitivity testing of the assays, the directionality and methylation status of other $\mathrm{CpG}$ sites in the same product and the total number of dominant allele indicators, i.e. whether a product demonstrated more or less suggestive $\mathrm{CpG}$ sites for either two or three alleles. Furthermore, a likelihood estimation for each $\mathrm{CpG}$ site was made by taking into account the methylation status frequency on other gene-specific products obtained from the same individual, as well as from identical products obtained from other individuals. A cross comparison between the independent score sheets was then performed ( $98.31 \%$ overlap in scoring) and reactions with a deviating score between the first two investigators were assessed by a third (blinded) investigator. A final allele number was then assigned for these reactions based on the overlap between the score sheets of the third and first two investigators, i.e. when two out of the three investigators assigned the same score then this allele number was used for the respective reaction. Overall, it was estimated that from the $237(C=104$, $A D=133)$ PCR products, $91.56 \%(C=93.27 \% ; A D=90.23 \%)$ were derived from a single allele, whereas $5.49 \%(C=4.81 \% ; A D=6.02 \%)$ were derived from two alleles, and $2.95 \%(C=1.92 \% ; A D=3.76 \%)$ from three alleles (Supplementary Table 14 ). 
By taking into account these multi-allele reactions, it was therefore estimated that in total 264 alleles $(C=113 ; A D=151)$ were recovered with a recovery rate of $3.67 \%$ ( $C$ $=3.14 \% ; A D=4.19 \%)$. For each subject, the methylation rate for each $\mathrm{CpG}$ site was determined by expressing the number of methylated $\mathrm{CpG}$ sites as a percentage of the total number of $\mathrm{CpG}$ sites over the estimated alleles, whilst correcting for the number of alleles present in each of the reactions, i.e. one, two or three alleles.

\section{General linear model}

For the cell-specific DRN validation analysis, average bisulfite methylation levels across the targeted region of $T N X B$ were calculated in both the serotonergic neurons and non-serotonergic cells. In the serotonergic neurons, the bisulfite methylation rates obtained for the targeted $\mathrm{CpG}$ sites in both genes were first averaged over the region per subject and subsequently over the experimental groups. For the non-serotonergic cells, only cases for which all CpG sites surpassed quality control by the Pyro Q-CpG 1.0.9 software were included and averaged per subject, as well as the experimental groups. One AD case and one control case were excluded from the analysis for TNXB (Nonserotonergic cells, $T N X B ; A D, n=9 ; C, n=9$ ), as these did not met the quality control criteria. A general linear model with experimental condition (AD- and control cases) as between-subject factor and cell type (Serotonergic neurons and non-serotonergic cells) as within-subject factor was conducted in order to test for interaction and main effects. A $p$ value $<0.05$ was considered as statistically significant.

Table 1. Demographics of studied cohorts

\begin{tabular}{|c|c|c|c|c|c|c|}
\hline & \multicolumn{2}{|c|}{$\begin{array}{l}\text { EWAS } \\
\text { (Discovery) }\end{array}$} & \multicolumn{2}{|c|}{$\begin{array}{l}\text { Pyrosequencing } \\
\text { (Validation) }\end{array}$} & \multicolumn{2}{|c|}{$\begin{array}{l}\text { LDBSP and Pyrosequencing } \\
\text { (Replication) }\end{array}$} \\
\hline & DRN & LC & DRN & LC & $5-\mathrm{HT}+$ & 5-HT- \\
\hline $\mathbf{N}$ & 94 & 81 & 62 & 52 & $12 \mathrm{AD} ; 12 \mathrm{C}$ & $10 \mathrm{AD} ; 10 \mathrm{C}$ \\
\hline $\operatorname{Sex}(F / M)$ & $53 / 40^{*}$ & $46 / 35$ & $33 / 29$ & $30 / 22$ & $24 / 0$ & $20 / 0$ \\
\hline $\begin{array}{l}\text { Age of death; Mean } \\
( \pm \text { SD) }\end{array}$ & $\begin{array}{l}83.85 \\
(9.04)\end{array}$ & $\begin{array}{l}83.96 \\
(9.53)\end{array}$ & $\begin{array}{l}84.90 \\
(9.16)\end{array}$ & $\begin{array}{l}84.50 \\
(10.13)\end{array}$ & $\begin{array}{l}81.21 \\
(10.21)\end{array}$ & $\begin{array}{l}81.35 \\
(11.31)\end{array}$ \\
\hline $\begin{array}{l}\text { PMI; } \\
\text { Mean ( } \pm \text { SD) }\end{array}$ & $\begin{array}{l}32.66 \\
(20.04)\end{array}$ & $\begin{array}{l}34.69 \\
(22.94)\end{array}$ & $\begin{array}{l}43.87 \\
(89.68)\end{array}$ & $\begin{array}{l}34.69 \\
(22.94)\end{array}$ & $\begin{array}{l}2.69 \\
(0.82)\end{array}$ & $\begin{array}{l}2.77 \\
(0.87)\end{array}$ \\
\hline $\begin{array}{l}\text { Braak Stage; Range } \\
\text { (Median) }\end{array}$ & $\begin{array}{l}0-\mathrm{VI} \\
(\mathrm{V})\end{array}$ & $\begin{array}{l}0-\mathrm{VI} \\
(\mathrm{V})\end{array}$ & $\begin{array}{l}0-\mathrm{VI} \\
(\mathrm{V})\end{array}$ & $\begin{array}{l}0-\mathrm{VI} \\
(\mathrm{V})\end{array}$ & $\begin{array}{l}\text { I-VI } \\
(\mathrm{IV})\end{array}$ & $\begin{array}{l}\text { I-VI } \\
(\mathrm{IV})\end{array}$ \\
\hline
\end{tabular}

Displayed are the demographics of the patient cohorts that were used in the present study. Dorsal raphe nuclei (DRN) and locus coerulues (LC) bulk tissues used in the discovery epigenome-wide association study (EWAS) and pyrosequencing validation study were obtained from the Medical Research Council (MRC) London Brainbank for Neurodegenerative Disease (London, UK). Serotonergic (5-HT+) neurons and non-serotonergic (5-HT-) cells isolated from the DRN by means of laser capture microdissection (LCM) used in the replication study were obtained from the Banner Sun Health Research Institute (BSHRI; Sun City, Arizona, USA) Brain and Body Donation Program (BBDP). For all cohorts, the number of samples in each group, the distributions of sex ( $F$, female; $M$, male), age of death (mean \pm standard deviation (SD)), postmortem interval (PMI; in hours) and Braak stage are shown. ${ }^{*}=$ From one individual sex was not specified. 


\section{Results}

Differentially modified positions associated with Braak stage in the DRN and LC

In the first stage of our analysis, epigenetic profiles at the level of 5-uC, 5-mC and 5-hmC were quantified in the DRN and LC in over 850.000 single CpG sites. For this purpose, we used largely matched bulk tissue samples of the DRN and the LC from 94 and 81 donors, respectively, which were collected from the Medical Research Council (MRC) London Brain bank for Neurodegenerative Disease (see Table 1 for the demographics of our studied cohorts). Our discovery cohorts in both brainstem regions represented the entire spectrum of AD pathology defined by Braak stage, which is a standardized measure of neurofibrillary tangle burden determined at autopsy [3]. In these subjects, we focused at identifying Braak stage-associated epigenetic alterations. As such, we aimed at capturing alterations that might be associated with both the early development and the course of AD. Thus, the acquired array data derived from both brainstem regions was pre-processed, normalized and subjected to quality control, after which linear regression analyses were conducted using either the 5-uC, 5-mC or 5-hmC signals as outcome and Braak stage as predictor. All analyses were performed whilst controlling for sex and age, with surrogate variables added to the model as appropriate to correct for unmeasured confounding factors (see Materials and methods).

For the DRN, the top-ranked differentially unmodified positions (DUPS), differentially methylated positions (DMPs) and differentially hydroxymethylated positions (DHPs) are shown in Supplementary Tables 1-3, respectively. None of the $\mathrm{CpG}$ sites identified for each of the three cytosine states reached the false discovery rate (FDR) threshold for experiment-wide significance that has been established for the Illumina Infinium MethylationEPIC BeadChip array, i.e. $p$ $<9.0$ E-08 [35]. The displayed nominally significant probes with a cut-off of $p<$ 0.001 were ranked based on a combined $p$ value and regression estimate ranking, resulting in 1029 DUPs, 647 DMPs and 831 DHPs in the DRN. For the LC, the top-ranked Braak-associated DUPs $(n=611)$, DMPs $(n=777)$ and DHPs $(n=741)$ are provided in Supplementary Tables 4-6, respectively. Similar to the DRN, none of the individual CpG sites identified in the LC at the level of 5-uC, 5-mC and 5-hmC surpassed the FDR threshold for experiment-wide significance.

Most of the top-ranked CpG sites identified in the DRN and the LC represented unique gene-associated probes for each of the three cytosine states. However, we observed numerous nominally significant DUPs in both brainstem regions that were annotated to TNXB. The six probes in the DRN, i.e. cg19455923 (regression estimate $(\mathrm{RE})=-110.1127, p=1.74 \mathrm{E}-04), \operatorname{cg} 17832639(\mathrm{RE}=34.49144887, p$ 
$=3.25 \mathrm{E}-04), \operatorname{cg} 14196170(\mathrm{RE}=14.71958219, p=2.93 \mathrm{E}-05), \mathrm{cg} 27387193(\mathrm{RE}$

$=11.28174141, p=2.76 \mathrm{E}-04), \operatorname{cg} 05473289(\mathrm{RE}=17.93969959, p=4.94 \mathrm{E}-04)$ and cg16385684 (RE = -23.52117157, $p=8.32 \mathrm{E}-04)$, were dispersed throughout the gene. The six DUPs in the LC however, i.e. cg27387193 (RE = 10.54119093, $p=2.51 \mathrm{E}-04), \mathrm{cg} 10923662(\mathrm{RE}=27.81154951, p=5.66 \mathrm{E}-04), \mathrm{cg} 10890302$ $(\mathrm{RE}=27.80155724, p=8.13 \mathrm{E}-04), \mathrm{cg} 00525277(\mathrm{RE}=11.73343449, p=$ 5.99E-04), $\mathrm{cg} 04753078(\mathrm{RE}=15.07520956, p=8.34 \mathrm{E}-04)$ and $\mathrm{cg} 19267551$ $(\mathrm{RE}=11.55809141, p=9.16 \mathrm{E}-04)$, were clustered within a 639 base pair (bp) window (6:32063619-32064258) in the gene body of TNXB. Interestingly, cg27387193, which overlapped in both brainstem regions, also demonstrated a similar RE, while cg14196170, which was identified in the DRN only, just lays 24 bp upstream (6:32063595) to the six probes identified in the LC. Moreover, the $\mathrm{RE}$ for each of the aforementioned DUPs that were identified within this region of TNXB (6:32063595-32064258) was positively associated with progressing Braak stage, suggesting that an increase in 5-uC in these loci is related to more advanced AD neuropathology. In the LC, we furthermore found cg04753078 (RE $=-10.15576914 ; p=7.05 \mathrm{E}-04)$ and $\operatorname{cg} 14196170(\mathrm{RE}=-10.00801167 ; p=6.38 \mathrm{E}-$ 05) listed among the top-ranked DMPs, demonstrating a negative association with Braak stage. When looking at the DHPs in the LC, also cg14196170 (RE $=12.14700315 ; p=1.72 \mathrm{E}-04)$ showed up as top hit, displaying a positive association of 5-hmC with increasing AD neuropathology. Altogether, these findings suggest that active DNA demethylation of $T N X B$ is typical with increasing Braak stage in both brainstem regions. 
Table 2. Differentially modified regions in the dorsal raphe nuclei (DRN)

\begin{tabular}{|c|c|c|c|c|c|c|c|c|c|c|}
\hline \multicolumn{11}{|c|}{ DURs (5-uC) } \\
\hline Rank & $\begin{array}{l}\text { USCS } \\
\text { annot. }\end{array}$ & Chr. & Start & End & Probes & $p$ value & $\begin{array}{l}\text { Šidák } p \\
\text { value }\end{array}$ & $\begin{array}{l}\text { Gene } \\
\text { feature }\end{array}$ & $\begin{array}{l}\text { Cpg } \\
\text { island } \\
\text { feature }\end{array}$ & $\begin{array}{l}\text { As- } \\
\text { soc. }\end{array}$ \\
\hline 1 & PGLYRP1 & 19 & 46526321 & 46526652 & 9 & $3.19 \mathrm{E}-13$ & $7.24 \mathrm{E}-10$ & $\begin{array}{l}\text { TSS; } \\
\text { 5'UTR; } \\
\text { CDS }\end{array}$ & Island & $\uparrow$ \\
\hline 2 & TNXB & 6 & 32063607 & 32064033 & 11 & $2.63 E-12$ & 4.64E-09 & CDS & Island & $\uparrow$ \\
\hline 3 & CAST & 5 & 95997186 & 95997356 & 4 & 3.35E-09 & $1.48 \mathrm{E}-05$ & Intergenic & Shore & $\uparrow$ \\
\hline 4 & GNAT1 & 3 & 50230792 & 50230886 & 3 & $1.14 \mathrm{E}-08$ & 9.09E-05 & $\begin{array}{l}\text { Intron; } \\
\text { CDS }\end{array}$ & Island & $\uparrow$ \\
\hline 5 & RBMXL2 & 11 & 7110074 & 7110149 & 5 & 1.07E-08 & 1.07E-04 & Intergenic & Island & $\uparrow$ \\
\hline \multicolumn{11}{|c|}{ DMR (5-mC) } \\
\hline Rank & $\begin{array}{l}\text { USCS } \\
\text { annot. }\end{array}$ & Chr. & Start & End & Probes & $p$ value & $\begin{array}{l}\text { Šidák } p \\
\text { value }\end{array}$ & $\begin{array}{l}\text { Gene } \\
\text { feature }\end{array}$ & $\begin{array}{l}\text { CpG } \\
\text { island } \\
\text { feature }\end{array}$ & $\begin{array}{l}\text { As- } \\
\text { soc. }\end{array}$ \\
\hline 1 & MALAT1 & 11 & 65266482 & 65266562 & 4 & 7.03E-09 & 6.61E-05 & NC exon & Shore & $\uparrow$ \\
\hline
\end{tabular}

Displayed are the differentially unmodifed regions (DURs) and the differentially methylated region (DMR) associated with Braak stage in the dorsal raphe nuclei (DRN). For each region, the Illumina gene annotation (UCSC annotation), the chromosomal position and coordinates (Ensembl GRCh37 assembly), the number of probes, the $p$ value, the multiple testing corrected Šidák $p$ value, the gene feature (TSS, transcription start site; 5'UTR, 5'untranslated region; CDS, coding sequence; NC, noncoding), the cytosine-phosphate-guanine (CpG) island feature and the association with increasing Braak stage are shown. All regions are ranked based on their Šidák $p$ value with Šidák $p<0.05$ as a cut-off.

\section{Differentially modified regions associated with Braak stage in the DRN and LC}

Subsequently, a regional analysis, looking at the spatial correlation of adjacent modified positions, was performed, using comb-p [31], in order to identify regions containing three or more neighboring DUPs, DMPs or DHPs that displayed a Šidák-corrected $p$ value $<0.05$ within a 500 -bp sliding window. In the DRN, we identified five significant differentially unmethylated regions (DURs) and one differentially methylated region (DMR), which are listed in Table 2. For the LC, we identified three DURs and two DMRs, which are listed in Table 3. We then examined whether any of these hits were shared between the two brainstem regions. Strikingly, we observed a strong overlap in our findings within the coding sequence of $T N X B$. While the second most significant DUR in the DRN (Šidák-corrected $p=4.64 \mathrm{E}-09$ ) consisted of a region spanning 426 bp (6:32063607-32064033) and 11 probes, in the LC, a larger but overlapping region in TNXB (6:32063726-32064259) spanning 533 bp and covering 18 probes showed up as most significant DUR (Šidák-corrected $p=1.39 \mathrm{E}-17$ ). Furthermore, in the LC, a nearly identical region (6:32063607-32064259) that consisted of 18 probes within a 652-bp window was identified as most significant DMR (Šidák-corrected $p=7.47 \mathrm{E}$ 20). All other significant DURs, DMRs and DHRs that were discovered in our regional analysis were specific to the individual brainstem regions. 
Table 3. Differentially modified regions in the locus coeruleus (LC)

\begin{tabular}{|c|c|c|c|c|c|c|c|c|c|c|}
\hline \multicolumn{11}{|c|}{ DURs (5-uC) } \\
\hline Rank & $\begin{array}{l}\text { USCS } \\
\text { annot. }\end{array}$ & Chr. & Start & End & Probes & $p$ value & $\begin{array}{l}\text { Šidák } p \\
\text { value }\end{array}$ & $\begin{array}{l}\text { Gene } \\
\text { feature }\end{array}$ & $\begin{array}{l}\text { CpG island } \\
\text { feature }\end{array}$ & Assoc. \\
\hline 1 & TNXB & 6 & 32063726 & 32064259 & 18 & 1.39E-17 & $2.02 \mathrm{E}-14$ & CDS & Island & $\uparrow$ \\
\hline 2 & ZFYVE28 & 4 & 2366555 & 2366673 & 4 & 1.65E-09 & $1.08 \mathrm{E}-05$ & Intron & Island & $\uparrow$ \\
\hline 3 & ANKRD2 & 10 & 99338056 & 99338189 & 3 & $3.03 \mathrm{E}-08$ & 1.76E-04 & $\begin{array}{l}\text { Intron; } \\
\text { CDS }\end{array}$ & Island & $\uparrow$ \\
\hline \multicolumn{11}{|c|}{ DMRs (5-mC) } \\
\hline Rank & $\begin{array}{l}\text { USCS } \\
\text { annot. }\end{array}$ & Chr. & Start & End & Probes & $p$ value & $\begin{array}{l}\text { Šidák } p \\
\text { value }\end{array}$ & $\begin{array}{l}\text { Gene } \\
\text { feature }\end{array}$ & $\begin{array}{l}\text { CpG island } \\
\text { feature }\end{array}$ & Assoc. \\
\hline 1 & TNXB & 6 & 32063607 & 32064259 & 18 & 7.47E-20 & 8.86E-17 & CDS & Island & $\downarrow$ \\
\hline 2 & $\begin{array}{l}\text { LOC105377777; } \\
\text { DLGAP2;DLGAP2 }\end{array}$ & 8 & 833012 & 833090 & 3 & 4.45E-06 & 4.32E-02 & $\begin{array}{l}\text { NC exon; } \\
\text { NC intron; } \\
\text { Intron }\end{array}$ & Shore & \\
\hline
\end{tabular}

Displayed are the differentially unmodified regions (DURs) and differentially methylated regions (DURs) associated with Braak stage in the locus coeruleus (LC). For each region, the lllumina gene annotation (UCSC annotation), the chromosomal position and coordinates (Ensembl GRCh37 assembly), the number of probes, the $p$ value, the multiple testing corrected Šidák $p$ value, the gene feature (CDS, coding sequence; NC, non-coding), the cytosine-phosphate-guanine (CpG) island feature and the association with increasing Braak stage are shown. All regions are ranked based on their Šidák $p$ value with Šidák $p<0.05$ as a cut-off.

We then explored whether any of these differentially modified regions in our array data have been nominated in previous EWAS or whether these represented novel $A D$-associated hits. For the $D R N$, similar regions as identified for the DURs in TNXB and PGLYRP1 have been previously annotated in EWAS targeting various regions of the $A D$ cortex, including the entorhinal cortex, the superior temporal gyrus and the prefrontal cortex [11, 19]. The remaining DURs in GNAT1, CAST and RBMXL2, as well as the DMR in MALAT1, represented novel Braak stage-associated regional hits. For the LC, similar regions as the DUR and DMR in TNXB, as well as the DUR in ZFYVE28, have been previously associated with $A D$ in the aforementioned cortical tissues [11], while the DUR in ANKRD2 and the DMR in LOC105377777IDLGAP2 have not been previously identified in other AD EWAS. Overall, based on the substantial overlap in both the DRN and the LC, as well as previous associations in the AD cortex, our findings strongly suggest that epigenetic dysregulation in TNXB may play an important role in both the early development and course of AD. Furthermore, our brainstem EWAS highlighted several other previously identified loci, as well as novel epigenetic signatures, that might play a pivotal role in both the early pathogenesis and progression of $A D$. 


\section{Structural and functional genomic annotation enrichment analysis}

Next, we were interested to identify biological, cellular and molecular pathways in the DRN and LC that were enriched among Braak stage-associated probes. For this purpose, we performed a Gene Ontology (GO) enrichment analysis in each brainstem region using the top-ranked DUPs, DMPs and DHPs. The top 20 enriched GO terms for each cytosine state in the DRN and the LC are displayed in Supplementary Figures $1 \mathrm{~A}$ and 1B, respectively. Additionally, a more elaborated overview of the top 50 ranked GO terms, as well as corresponding identifiers, ontologies, the number of (differentially modified) genes, $p$ values and FDR-adjusted $p$ values for 5-uC, 5-mC and 5-hmC in both the DRN and LC can be found in Supplementary Tables 7-12.

For the DRN, we identified two pathways related to the DHPs that surpassed the FDR threshold for multiple testing correction (FDR-adjusted $p<0.05$ ). The most significant $\mathrm{GO}$ term in our 5 -hmC enrichment analyses was found in 'homophilic cell adhesion via plasma membrane adhesion molecules' (FDR adjusted $p=1.16 \mathrm{E}-03$ ), demonstrating that from the 167 annotated genes, 27 were differentially modified. The second significantly enriched $\mathrm{GO}$ term that was associated with our 5-hmC data from the DRN-EWAS was found for 'calcium ion binding' (FDR-adjusted $p=1.09 \mathrm{E}$ 02), revealing that from the 690 genes, 56 were altered. All other GO terms identified in the DRN at the level of each cytosine state did not surpass the FDR threshold for significance.

In the LC, we identified four pathways that were significantly enriched among our DMPs after multiple testing correction. The most significant enriched GO term was identified in 'homophilic cell adhesion via plasma membrane adhesion molecules' (FDR-adjusted $p=7.25 \mathrm{E}-11$ ), in which 35 of the 161 genes were differentially modified. The second most significant $\mathrm{GO}$ term was found for 'cellcell adhesion via plasma-membrane adhesion molecules' (FDR-adjusted $p=$ $1.19 \mathrm{E}-06$ ) with 35 out of 161 genes that were altered. The third most significantly enriched pathway in our 5-mC LC-EWAS data was found for 'calcium ion binding' (FDR-adjusted $p=1.17 \mathrm{E}-03$ ), revealing that the modification levels of 53 genes from a total of 167 were affected. Finally, the fourth significantly enriched GO term concerned 'cell-cell adhesion' (FDR adjusted $p=2.75 \mathrm{E}-03$ ), in which 57 of 782 genes were differentially modified. The remaining GO terms identified in the LC for the DUPs, DMPs and DHPs did not survive the FDR threshold for multiple testing correction. 
Table 4. Regression analysis during the validation of the TNXB Braak-associated region in the dorsal raphe nuclei (DRN).

\begin{tabular}{lllllll}
\hline \multicolumn{7}{c}{ DRN } \\
\hline CpG & Probe ID & RE & SE & $t$ value & $p$ value & \\
\hline 1 & cg10365886 & -0.10599 & 0.02909 & $-3,644$ & $5.75 \mathrm{E}-04$ & $* * *$ \\
2 & - & -0.10048 & 0.02656 & $-3,783$ & $3.69 \mathrm{E}-04$ & $* * *$ \\
3 & cg14188106 & -0.12461 & 0.03575 & $-3,486$ & $9.43 \mathrm{E}-04$ & $* * *$ \\
4 & cg07524919 & -0.12121 & 0.03006 & $-4,032$ & $1.63 \mathrm{E}-04$ & $* * *$ \\
5 & - & -0.12586 & 0.03256 & $-3,866$ & $2.82 \mathrm{E}-04$ & $* *$ \\
6 & - & -0.09968 & 0.03033 & $-3,286$ & $1.73 \mathrm{E}-03$ & $*$ \\
7 & - & -0.12871 & 0.03687 & $-3,491$ & $9.36 \mathrm{E}-04$ & $*$ \\
8 & - & -0.12202 & 0.03827 & $-3,188$ & $2.34 \mathrm{E}-03$ & $*$ \\
\hline
\end{tabular}

The DNA bisulfite methylation patterns quantified by pyrosequencing over eight cytosine-phosphateguanine (CpG) sites in TNXB, including cg10365886, cg14188106 and cg07524919, were all significantly associated with Braak stage in the DRN. Displayed for each CpG site is their respective number in the sequence, the Illumina probe identifier (probe ID - if applicable), the regression estimate (RE) for the Braak stage-associated analysis, the standard error (SE), the $t$-statistics and accompanying $p$ values. * $=p<0.05 ;{ }^{* * *}=p<0.001$

Table 5. Regression analysis during the validation of the TNXB Braak-associated region in the locus coeruleus (LC).

\begin{tabular}{llllll}
\hline \multicolumn{5}{c}{ LC } \\
\hline 1 & Probe ID & RE & SE & $\boldsymbol{t}$ value & $\boldsymbol{p}$ value \\
\hline 2 & - & -0.0415 & 0.03159 & $-1,314$ & $1.95 \mathrm{E}-01$ \\
3 & $\operatorname{cg} 14188106$ & -0.04263 & 0.04395 & -0.97 & $3.37 \mathrm{E}-01$ \\
4 & cg07524919 & -0.05173 & 0.03744 & $-1,382$ & $1.74 \mathrm{E}-01$ \\
5 & - & -0.04597 & 0.04051 & $-1,135$ & $2.62 \mathrm{E}-01$ \\
6 & - & -0.05208 & 0.03993 & $-1,304$ & $1.99 \mathrm{E}-01$ \\
7 & - & -0.08598 & 0.04086 & $-2,104$ & $4.08 \mathrm{E}-02$
\end{tabular}

The DNA bisulfite methylation pattern quantified by pyrosequencing for each of the eight cytosinephosphate-guanine (CpG) sites in TNXB, including cg10365886, cg14188106 and cg07524919, was significantly associated with Braak stage for CpG site 7 in the LC. Displayed for each CpG site is their respective number in the sequence, the Illumina probe identifier (probe ID - if applicable), the regression estimate (RE) for the Braak stage-associated analysis, the standard error (SE), the $t$-statistics and accompanying $p$ values. ${ }^{*}=p<0.05$ 


\section{Pyrosequencing validation of TNXB in the DRN and LC}

In order to validate our discovery findings from the brainstem EWAS, we compared our EWAS-derived data sets to bisulfite pyrosequencing data generated from a subset of the DNA samples obtained from the DRN and LC bulk tissues (Table 1). In these samples, the DNA bisulfite methylation status of TNXB was quantified across a region of 72 bp (6:32063869-32063940), spanning eight CpG sites. The targeted region overlapped with the DUR in the DRN, and with both the DUR and DMR identified in the LC, including three Illumina probes that were part of these identified Braak-associated regions, i.e. cg10365886, cg14188106 and cg07524919. Importantly, at this stage of the analysis, we aimed at a technical validation of the observed Braak-associated alterations by assessing the level of bisulfite methylation, i.e. the cumulative measure of $5-\mathrm{mC}$ and 5-hmC, which is inversely proportional to the measurements of 5-uC [29]. All the acquired pyrosequencing data were adjusted for sex and age, similar to the EWAS described above. In addition to the performed linear regression analyses in both individual brainstem regions, we furthermore conducted Pearson's correlation analyses between the bisulfite methylation values estimated by the two technologies; i.e. by the Illumina Infinium MethylationEPIC BeadChip array and by bisulfite pyrosequencing. For this validation analyses, a $p$ value $<0.05$ was considered statistically significant.

The results from the linear regression analyses on the $T N X B$ pyrosequencing data derived from the DRN and the LC are provided in Tables 4 and 5, respectively. We identified a strong significant association with Braak stage for all eight $\mathrm{CpG}$ sites in the DRN (Lowest $p=1.63 \mathrm{E}-04$ ) and for one CpG site in the LC (CpG site number 7, $p$ $=4.08 \mathrm{E}-02$ ). All analyzed $T N X B$ CpG sites in both brainstem regions demonstrated hypomethylation with increasing AD neuropathology, which was indicated by the negative REs. The results from the correlation analyses in both the DRN and the LC are shown in Figure 1. The pattern of DNA bisulfite methylation in TNXB quantified during the EWAS of the DRN and the LC was identical to the pyrosequencing data in each of the brainstem regions, with a highly significant and strong correlation between the values estimated by the two independent technologies for all three analyzed probes (lowest $p=2.2 \mathrm{E}-16$ with highest $r=0.84$ ). Thus, despite the use of a smaller sample size for our validation approach (for reasons of limited tissue availability), we observed a robust validation of our EWAS discovery findings in this Braak-associated region of TNXB. In addition to the analyzed Illumina probes, we furthermore demonstrated that adjacent $\mathrm{CpG}$ sites in this gene support the notion of DNA hypomethylation with increasing Braak stage, both in the DRN, as well as in the LC. Altogether, these data provide compelling evidence for an association between active DNA demethylation in this TNXB locus and an increase of ADrelated neuropathological features in both brainstem regions assessed. 


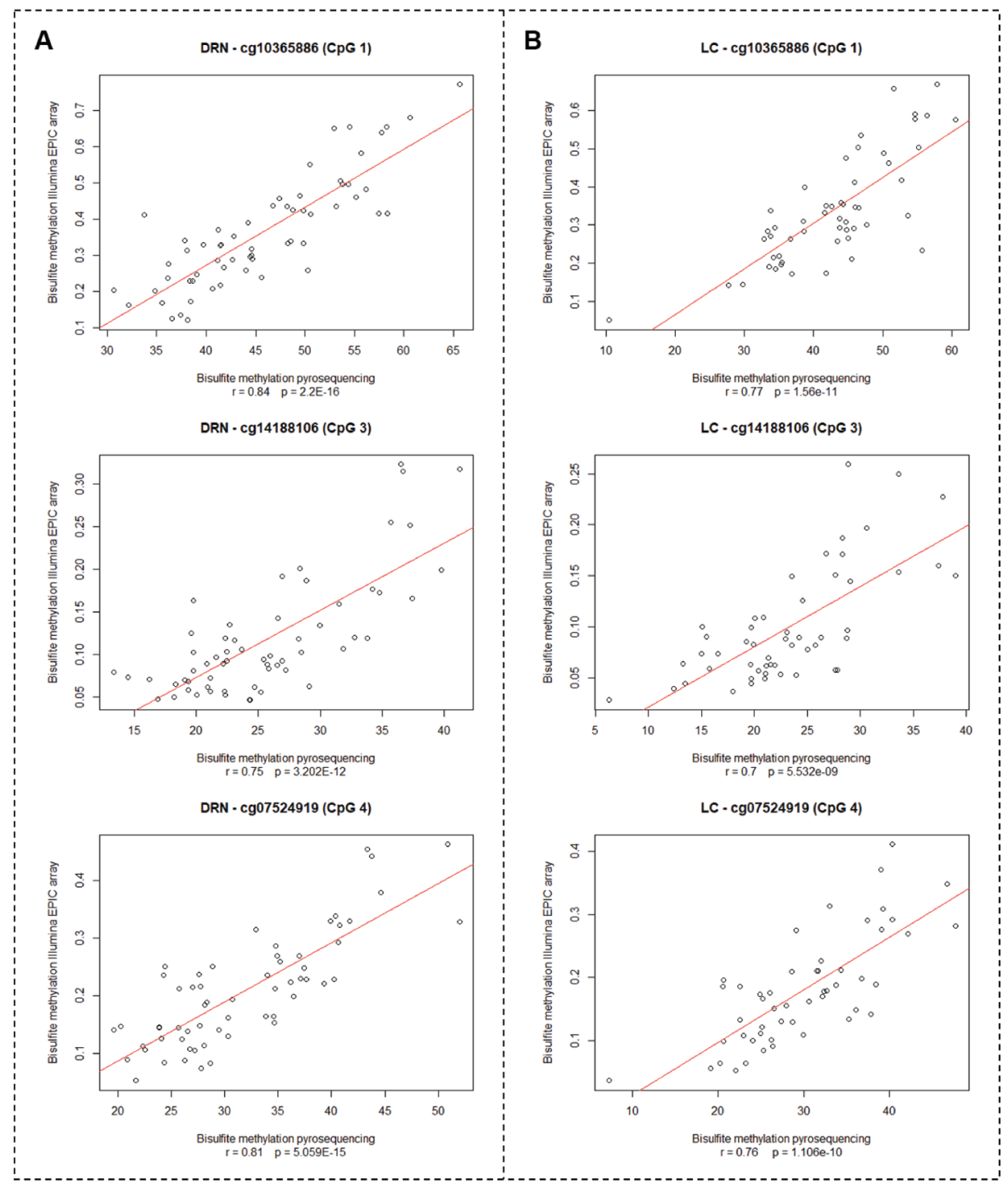

Figure 1. Correlation analysis during the validation study of the Tenascin XB (TNXB) Braak-associated region. DNA bisulfite methylation patterns quantified by the Illumina Infinium MethylationEPIC BeadChip array and pyrosequencing in TNXB were highly significantly and strongly correlated for cg10365886, cg14188106 and cg07524919 in both the dorsal raphe nuclei (DRN) and the locus coeruleus (LC) (A and $\mathbf{B}$, respectively).

\section{Cell type-specific analysis of TNXB in serotonergic and non-serotonergic cells in the DRN}

In the second stage of our analysis, we aimed at validating our EWAS findings of $T N X B$ in an independent patient cohort, an attempt which we combined with 
a novel cell type-specific and targeted epigenetic analysis in single DRN cells isolated by LCM. This targeted approach was performed with the aim to define the cellular origin of the identified epigenetic signature. Given that bulk tissues were used in the DRN EWAS described above, it is expected that various cellular populations residing in this brainstem region, including serotonergic neurons and other parenchymal cells such as glia, contribute to the obtained epigenetic signals. As such, the cellular heterogeneity of these bulk tissue samples constitutes a major source of noise in epigenetic profiling approaches that represents a reoccurring challenge in AD EWAS [36]. Moreover, since AD is characterized by neuronal loss and increased microglia activation, differences in cellular composition between (brainstem) tissue samples obtained at varying stages of the disease can interfere with the interpretation of the acquired epigenetic data. In order to tackle this issue and based on the hypothesized role of the serotonergic neurotransmitter system in mediating affective symptoms linked to $A D$, we therefore first isolated single serotonergic neurons from the DRN by means of LCM, using an immunohistochemical labeling of serotonin, i.e. 5-HT-positive $(5-\mathrm{HT}+)$ cells . For this purpose, post-mortem DRN tissue derived from 12 subjects without- (Control cases; Braak I-IV) and 12 subjects with a clinical diagnosis of $A D$ (AD cases; Braak V-VI) were utilized (Table 1), which were all part of the Banner Sun Health Research Institute Brain and Body Donation Program (BBDP; [22]).

Per individual, a total of 150 serotonergic cells divided in three pools of 50 cells were isolated with LCM from the DRN tissue. Limiting dilution bisulfite pyrosequencing (LDBSP) was then applied on each pool of cells to analyze the bisulfite methylation status of the Braak-associated region in TNXB on single alleles [34]. This type of adapted pyrosequencing allows for assessing DNA methylation patterns in only a single or a few cells and hence is very suited for low cell numbers as acquired with LCM. As such, the DNA bisulfite methylation status in TNXB was quantified in the serotonergic neurons across a region of 140 bp (6:32063774-32063913) spanning eleven CpG sites. This region overlaps with the DUR identified in the DRN, both the DUR and DMR identified in the LC, as well as the region targeted during our EWAS pyrosequencing validation analysis. From the eleven $\mathrm{CpG}$ sites in the sequenced region, six represented Illumina probes that were also part of the Braak-associated differentially modified regions, i.e. cg10365886, cg07524919, cg14188106, cg26266427, cg01337207 and cg02989255. Considering the smaller sample size and the lack of access to multiple Braak 0, III and IV cases, the LDBSP-derived CpG methylation data were analyzed using a case-control model based on clinical diagnosis, rather than looking for an association with Braak stage. 


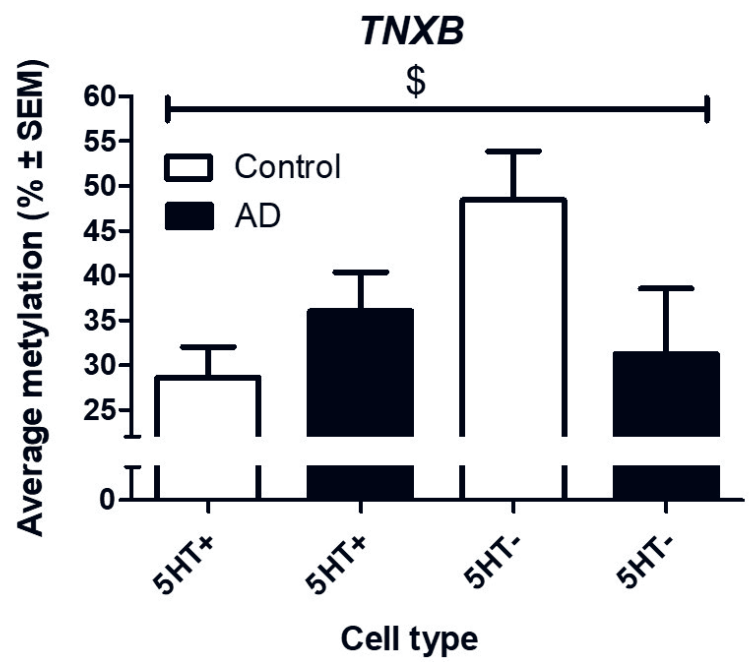

Figure 2. General linear model during the cell type-specific validation study in the dorsal raphe nuclei (DRN) of the Braak-associated region in Tenascin XB (TNXB). Average DNA bisulfite methylation levels quantified in both the serotonergic (5-HT+) neurons and non-serotonergic (5-HT-) cells derived from the DRN of Alzheimer's disease (AD) patients and healthy controls (Control) are shown for TNXB. A general linear model with experimental condition as a between-subject factor (AD/Control) and cell type (5-HT+/5-HT-) as a within-subject factor revealed a significant interaction effect between cell type and experimental condition. The AD-associated bisulfite methylation profiles were exactly opposite in the serotonergic neurons when compared to the non-serotonergic cells, the latter which resembled our EWAS data in the DRN. For the interaction effect: $\$=p<0.05$.

Remarkably, by applying LDBSP, we observed a trend towards AD-associated bisulfite hypermethylation in the serotonergic neurons, i.e. a pattern opposite to that identified in the EWAS of the DRN (Figure 2). Accordingly, we then hypothesized that the EWAS-associated TNXB hypomethylation should originate from other, i.e. non-serotonergic, cells in the DRN bulk tissue. In order to confirm this hypothesis, we isolated additional, i.e. 5-HT-negative (5-HT-), cells by LCM from the same BBDP DRN tissue samples used in the 5-HT+ LDBSP analysis (AD = 10; $C=10)$, but now isolating tissue that was free of $5-\mathrm{HT}$ immunoreactivity. As such, this isolated tissue most likely consisted of non-serotonergic parenchymal cellular populations, including glia cells, glutamatergic, GABAergic and dopaminergic neurons, amongst others [37]. Importantly, isolation of these cells based on the absence of 5-HT immunoreactivity in a limited amount of leftover DRN tissue sections did not allow us to determine the cell numbers that were isolated and, therefore, the average bisulfite methylation status over all residing non-serotonergic cells in the exact same region of $T N X B$ was quantified by using regular bisulfite pyrosequencing with an extended pre-amplification protocol (see Materials and methods). 
Subsequently, average TNXB bisulfite methylation levels were then compared between both experimental groups using a general linear model with experimental condition (AD/Control) as a between-subject and cell type (5-HT+/5-HT-) as a withinsubject factor. Strikingly, when comparing the bisulfite methylation levels of TNXB in individually isolated serotonergic neurons with those of non-serotonergic cells in the DRN, we found a significant interaction effect between cell type and experimental condition ( $p=0.046$; Figure 2), with AD-associated bisulfite methylation profiles being exactly opposite in serotonergic neurons when compared to non-serotonergic cells. More specifically, while bisulfite methylation levels tended to be increased in serotonergic neurons derived from $A D$ patients, bisulfite hypomethylation was observed in non-serotonergic cells from $A D$ patients when compared to healthy controls - the latter of which resembled the initially acquired EWAS data in the DRN bulk tissue. Overall, these data corroborated our previous findings in the DRN-EWAS, indicating that epigenetic dysregulation in $T N X B$ in $A D$ is likely attributed to nonserotonergic cells within this brainstem nucleus, a finding that is indirectly supported by the notion that the LC, which is free of serotonergic cells, also displayed TNXB hypomethylation with advancing Braak stage.

\section{Discussion}

The present study reflects the first comprehensive epigenetic analysis in the brainstem of AD to date, targeting both the DRN and the LC simultaneously. In our AD discovery cohort, two independent EWAS on bulk tissue derived from both brainstem regions were performed, with the aim of examining potential epigenetic differences at the level of 5-mC, 5-hmC and 5-uC accompanying ADassociated pathology. In total, 1029 nominal significant DUPs, 647 DMPs and 831 DHPs were identified in the DRN, whereas for the LC, 611 DUPs, 777 DMPs and 741 DHPs were found. In a subsequent regional analysis we then identified significant overlapping, both previously annotated and novel, differentially modified regions that were associated with Braak stage in both the DRN and LC. We furthermore followed up the EWAS in both brainstem regions with a technical validation of the acquired array data using bisulfite pyrosequencing in a subset of samples derived from the same patient cohort. Moreover, our brainstem discovery EWAS was complemented with a novel cell type-specific and targeted analysis in the DRN, assessing methylation signatures in lasercaptured serotonergic neurons and non-serotonergic cells derived from an independent $A D$ patient cohort. As such, we aimed at validating our EWAS data and, simultaneously, to unravel potential cell-type specific contributions of the identified epigenetic signatures within the DRN.

The regional analysis in the DRN and the LC revealed a strong overlapping dysregulation at the level of $5-\mathrm{uC}$ and $5-\mathrm{mC}$ in a gene-coding region of TNXB. 
Subsequent bisulfite pyrosequencing of this locus in the same patient cohort confirmed the notion that active DNA demethylation in TNXB was associated with progressing AD neuropathology in both brainstem regions assessed. Interestingly, dysregulation at the level of DNA methylation in a similar region of TNXB has previously also been annotated in an AD EWAS targeting cortical tissues, including the entorhinal cortex, superior temporal gyrus and prefrontal cortex, which were derived from the same patient cohort as used in the present study [11]. Next to epigenetic variation, genetic variation in TNXB has also been directly associated with a risk of developing $A D$ [38-40], as well as with progressive supranuclear palsy [41], which is a tau-related disorder. TNXB expresses a glycoprotein with anti-adhesive properties, but its exact physiological role in the brain is not yet clear [42]. In addition to AD, epigenetic dysregulation in a similar locus of TNXB has also been identified in EWAS targeting peripheral blood samples of patients and people at risk of developing several stress-related disorders, including anorexia nervosa, schizophrenia, remitted major depressive disorder and bipolar disorder [43-45]. These are interesting observations, given the established link between stress-related pathology and $A D$, especially when considering the physiological role of the DRN and the LC in the stress response. Of note, we also performed a methylation quantitative trait locus (mQTL) analysis in order to examine whether the previously identified genetic variation could explain the observed methylation differences in our EWAS, but could not identify such an effect (data not shown), suggesting that the observed alterations represent a true epigenetic ADassociated signature. Evidently, more research on the exact function of TNXB, the interplay between genetic and epigenetic variation in $T N X B$, and exploring the exact cause-effect relationship underlying this $A D$ neuropathology-associated epigenetic signature, is vital in order to develop a better understanding of the exact role of this gene in the disease.

In addition to $T N X B$, a DUR in the DRN that has previously been nominated in other AD EWAS was identified for PGLYRP1. The same gene displayed differential methylation in the aforementioned cross-cortex analysis using the same $A D$ cohort [11]. Furthermore, a similar locus has been reported to demonstrate differential methylation in peripheral blood samples derived from individuals with down syndrome, a patient population known to be at increased risk of developing AD [46]. PGLYRP1 encodes an innate immunity protein that is a known activator of TREM-1 [15]. Interestingly, studies have demonstrated that overexpression of TREM-1 in APP/PSEN1 mice, a mouse model for AD, facilitates microglial-mediated amyloid beta clearance and restores AD-related cognitive impairment, emphasizing the importance of the PGLYRP1-TREM-1 interaction in the pathophysiology of AD [16]. However, whether there is a functional connection between the reported epigenetic alterations in PGLYRP1 
and TREM-1 efficacy remains to be elucidated. For the LC, a DMR was identified in ZFYVE28 in a region that has previously been associated with $A D$ in cortical tissue [11]. ZFYVE28 is a regulator of epidermal growth factor receptor (EGFR) signaling that acts by promoting EGFR degradation in endosomes when not mono-ubiquitinated [47]. Interestingly, several recent studies have demonstrated a beneficial effect of EGFR inhibitors in reducing amyloid beta peptides and improving memory performance both in in vitro and/or in vivo AD models [48, 49], pointing towards a crucial role for EGFR regulation in the disease. Whether there is a mechanistic link between epigenetic changes in ZFYVE28 and altered EGFR signaling in AD remains to be investigated.

Next to the overlapping loci that were identified in previous reports, our brainstem EWAS also revealed novel genes displaying changes associated with $A D$ pathology at the level of 5-uC and 5-mC. Interestingly, while representing new associations, three of these genes have been functionally linked to $A D$ before, supporting the relevance of the current findings. These include CAST and MALAT1, for which we identified a DRN-specific DUR and DMR, respectively, as well as LOC105377777;DLGAP2;DLGAP, for which we identified a DMR within the LC. Calpastatin, the protein expressed by CAST, is known to protect against neuronal death induced by amyloid beta $[50,51]$. Furthermore, CAST depletion has been shown to act upstream of calpains to activate a calpain-dependent cascade of protein kinase activation, cytoskeletal protein hyperphosphorylation, cytoskeletal proteolysis and neurodegeneration [52]. In another study using APP/PSEN1 mice, CAST downregulation was linked to hyperglycemia and the promotion AD pathological hallmarks, the former which is typically observed in diabetes, which is a known risk factor for developing AD [53]. The long noncoding RNA MALAT1 on the other hand, has recently been shown to convey neuroprotective effects in $A D$ by inhibiting apoptosis and inflammation while promoting neurite outgrowth [54, 55]. For LOC105377777;DLGAP2;DLGAP2, lower cortical DLGAP2 expression has been observed in $A D$, associated with more plaques and tangles at autopsy and faster cognitive decline [56]. Furthermore, an association for this locus at the level of genetic variation, gene expression and protein expression, as well as altered methylation levels, have been associated with the disease [56, 57]. Further research is necessary to assess whether the identified epigenetic alterations in these loci also have functional implications and hence could mediate the effects observed in the aforementioned studies. For GNAT1, RBMXL2 and ANKRD2, no direct or indirect association or functional connection has been identified with $A D$ to date. Interestingly, however, is that other ankyrin repeat containing proteins, such as $A N K 1$, have previously been associated with $A D$ neuropathology in other EWAS $[11,12,16]$. 
By conducting a GO term pathway analyses, we furthermore identified altered biological mechanisms in the DRN and LC, which were related to our top-ranked DUPs, DMPs and DHPs. Strikingly, genes related to 'homophilic cell adhesion via plasma membrane adhesion molecules' and 'calcium ion binding' were overrepresented in both brainstem regions. In the LC, we furthermore discovered two more pathways that were related to cell-cell interactions, i.e. 'cell-cell adhesion via plasma-membrane adhesion molecules' and 'cell-cell adhesion'. Interestingly, the cell adhesion molecules annotated to these aforementioned GO-terms have a major function in dendrite development, synaptic connectivity and neural circuit formation [58, 59], closely linked to key neuropathological features of AD. In the context of 'calcium ion binding', an increasing numbers of studies suggests that disruption of intracellular calcium ion homeostasis plays important roles in orchestrating the dynamic of the neuropathology of $A D$ and associated memory loss, and cognitive dysfunction [60]. In fact, calcium dysregulation may even play an important role in the pathogenesis of $A D$, by inducing synaptic deficits and promoting the accumulation of amyloid beta plaques and neurofibrillary tangles [61]. All in all, these findings confirmed that the identified differentially modified genes are strongly linked to well-known AD-associated neuropathological processes, which further supports the relevance of epigenetic dysregulation in both brainstem regions in AD.

Finally, in the cell-type specific validation study using an independent patient cohort, where we targeted both laser-captured serotonergic and non-serotonergic cells derived from the DRN, we showed that epigenetic signatures in TNXB within this brainstem nucleus are strongly dependent upon the cell type analyzed. Whereas $A D$-associated hypermethylation was found for TNXB within serotonergic neurons, hypomethylation was identified in the patient-derived non-serotonergic cells. As such, these data suggest that epigenetic dysregulation in TNXB in AD is likely attributable to the non-serotonergic cells within the DRN, as the identified patterns in these cells overlap with the previously obtained bulk tissue EWAS data. This finding is further supported by the notion that the LC, which is free of serotonergic cells, also displayed TNXB hypomethylation with advancing Braak stage. These cell-specific findings also illustrate that a potential loss of serotonergic neurons, as commonly observed in $A D$ [2,62], possibly resulting in a different proportion of various cell types within the bulk tissues examined, is not able to explain our bulk tissue EWAS data, indicating a true epigenetic AD-specific signature. Thus, our results support the notion that epigenetic data derived from heterogeneous postmortem bulk tissue should be interpreted with caution, as changes in one cell type could negate or mask changes in another. Overall, this has crucial implications for future planned epigenetic studies in $A D$, as it warrants the need for single cell (-type) neuroepigenetic analyses, opposite to the more common bulk tissue analyses that have been performed to date. Evidently, interrogation of epigenetic 
marks is most informative when studied at a single-cell level, where intercellular differences can be dissected leading to a more refined understanding of their contribution to the disease. Taken together, the present study strongly implicates a role for $T N X B$ dysregulation in the brainstem of $A D$ in the development and course of the disease, and highlights potential cell-specific effects regarding this locus that emphasizes the need for future single cell(-type) neuroepigenetic studies in AD.

\section{Acknowledgments}

Funds have been provided by the Joint Program - Neurodegenerative Disease Research (JPND) for the EPI-AD consortium (http://www.epi-ad.eu/). The project is supported through the following funding organizations under the aegis of JPND; the Netherlands, The Netherlands Organization for Health Research and Development (ZonMw); United Kingdom, Medical Research Council; Germany, German Federal ministry of Education and Research (BMBF); Luxembourg, National Research Fund (FNR). This project has received funding from the European Union's Horizon 2020 research and innovation program under Grant Agreement No. 643417.

\section{References}

1. Simic G, Stanic G, Mladinov M, Jovanov-Milosevic N, Kostovic I, Hof PR. Does Alzheimer's disease begin in the brainstem? Neuropathology and applied neurobiology. 2009;35(6):532-54.

2. latrou A, Kenis G, Rutten BP, Lunnon K, van den Hove DL. Epigenetic dysregulation of brainstem nuclei in the pathogenesis of Alzheimer's disease: looking in the correct place at the right time? Cell Mol Life Sci. 2017;74(3):509-23.

3. Braak H, Thal DR, Ghebremedhin E, Del Tredici K. Stages of the pathologic process in Alzheimer disease: age categories from 1 to 100 years. Journal of Neuropathology \& Experimental Neurology. 2011;70(11):960-9.

4. Grinberg L, Rüb U, Ferretti R, Nitrini R, Farfel J, Polichiso L, et al. The dorsal raphe nucleus shows phospho-tau neurofibrillary changes before the transentorhinal region in Alzheimer's disease. A precocious onset? Neuropathology and applied neurobiology. 2009;35(4):406-16.

5. Lee JH, Ryan J, Andreescu C, Aizenstein H, Lim HK. Brainstem morphological changes in Alzheimer's disease. Neuroreport. 2015;26(7):411.

6. Ji X, Wang H, Zhu M, He Y, Zhang H, Chen X, et al. Brainstem atrophy in the early stage of Alzheimer's disease: a voxel-based morphometry study. Brain Imaging and Behavior. 2021;15(1):49-59.

7. Lardenoije R, latrou A, Kenis G, Kompotis K, Steinbusch HW, Mastroeni D, et al. The epigenetics of aging and neurodegeneration. Prog Neurobiol. 2015;131:21-64.

8. Lardenoije R, Pishva E, Lunnon K, van den Hove DL. Neuroepigenetics of Aging and Age-Related Neurodegenerative Disorders. Prog Mol Biol TransI Sci. 2018;158:49-82.

9. Bakulski KM, Dolinoy DC, Sartor MA, Paulson HL, Konen JR, Lieberman AP, et al. Genome-wide DNA methylation differences between late-onset Alzheimer's disease and cognitively normal controls in human frontal cortex. Journal of Alzheimer's Disease. 2012;29(3):571-88.

10. Sanchez-Mut JV, Aso E, Heyn H, Matsuda T, Bock C, Ferrer I, et al. Promoter hypermethylation of the phosphatase DUSP22 mediates PKA-dependent TAU phosphorylation and CREB activation in Alzheimer's disease. Hippocampus. 2014;24(4):363-8. 
11. Lunnon K, Smith R, Hannon E, De Jager PL, Srivastava G, Volta M, et al. Methylomic profiling implicates cortical deregulation of ANK1 in Alzheimer's disease. Nat Neurosci. 2014;17(9):1164-70.

12. De Jager PL, Srivastava G, Lunnon K, Burgess J, Schalkwyk LC, Yu L, et al. Alzheimer's disease: early alterations in brain DNA methylation at ANK1, BIN1, RHBDF2 and other loci. Nat Neurosci. 2014;17(9):1156-63.

13. Smith AR, Smith RG, Condliffe D, Hannon E, Schalkwyk L, Mill J, et al. Increased DNA methylation near TREM2 is consistently seen in the superior temporal gyrus in Alzheimer's disease brain. Neurobiology of aging. 2016;47:35-40.

14. Smith AR, Smith RG, Pishva E, Hannon E, Roubroeks JAY, Burrage J, et al. Parallel profiling of DNA methylation and hydroxymethylation highlights neuropathology-associated epigenetic variation in Alzheimer's disease. Clin Epigenetics. 2019;11(1):52.

15. Lardenoije R, Roubroeks JA, Pishva E, Leber M, Wagner H, latrou A, et al. Alzheimer's diseaseassociated (hydroxy) methylomic changes in the brain and blood. Clinical epigenetics. 2019;11(1):164.

16. Smith R, Pishva E, Shireby G, Smith AR, Roubroeks JA, Hannon E, et al. Meta-analysis of epigenomewide association studies in Alzheime's disease highlights 220 differentially methylated loci across cortex. BioRxiv. 2020.

17. Watson CT, Roussos P, Garg P, Ho DJ, Azam N, Katsel PL, et al. Genome-wide DNA methylation profiling in the superior temporal gyrus reveals epigenetic signatures associated with Alzheimer's disease. Genome medicine. 2016;8(1):1-14.

18. Gasparoni G, Bultmann S, Lutsik P, Kraus TF, Sordon S, VIcek J, et al. DNA methylation analysis on purified neurons and glia dissects age and Alzheimer's disease-specific changes in the human cortex. Epigenetics \& chromatin. 2018;11(1):41.

19. Smith RG, Hannon E, De Jager PL, Chibnik L, Lott SJ, Condliffe D, et al. Elevated DNA methylation across a 48-kb region spanning the HOXA gene cluster is associated with Alzheimer's disease neuropathology. Alzheimer's \& Dementia. 2018;14(12):1580-8.

20. Semick SA, Bharadwaj RA, Collado-Torres L, Tao R, Shin JH, Deep-Soboslay A, et al. Integrated DNA methylation and gene expression profiling across multiple brain regions implicate novel genes in Alzheimer's disease. Acta neuropathologica. 2019;137(4):557-69.

21. Roubroeks JA, Smith AR, Smith RG, Pishva E, Ibrahim Z, Sattlecker M, et al. An epigenome-wide association study of Alzheimer's disease blood highlights robust DNA hypermethylation in the HOXB6 gene. Neurobiology of Aging. 2020.

22. Beach TG, Sue LI, Walker DG, Roher AE, Lue L, Vedders L, et al. The Sun Health Research Institute Brain Donation Program: description and experience, 1987-2007. Cell Tissue Bank. 2008;9(3):229-45.

23. Charnay $Y$, Léger L. Brain serotonergic circuitries. Dialogues in clinical neuroscience. 2010;12(4):471.

24. Team RC. R: A language and environment for statistical computing. Vienna, Austria; 2013.

25. Gentleman RC, Carey VJ, Bates DM, Bolstad B, Dettling M, Dudoit S, et al. Bioconductor: open software development for computational biology and bioinformatics. Genome biology. 2004;5(10):R80.

26. Pidsley R, Wong CC, Volta M, Lunnon K, Mill J, Schalkwyk LC. A data-driven approach to preprocessing Illumina 450K methylation array data. BMC genomics. 2013;14(1):1-10.

27. Aryee MJ, Jaffe AE, Corrada-Bravo H, Ladd-Acosta C, Feinberg AP, Hansen KD, et al. Minfi: a flexible and comprehensive Bioconductor package for the analysis of Infinium DNA methylation microarrays. Bioinformatics. 2014;30(10):1363-9.

28. Fortin J-P, Triche Jr TJ, Hansen KD. Preprocessing, normalization and integration of the Illumina HumanMethylationEPIC array with minfi. Bioinformatics. 2017;33(4):558-60.

29. Qu J, Zhou M, Song Q, Hong EE, Smith AD. MLML: consistent simultaneous estimates of DNA methylation and hydroxymethylation. Bioinformatics. 2013;29(20):2645-6.

30. Kiihl SF, Martinez-Garrido MJ, Domingo-Relloso A, Bermudez J, Tellez-Plaza M. MLML2R: an R package 
for maximum likelihood estimation of DNA methylation and hydroxymethylation proportions. Statistical applications in genetics and molecular biology. 2019;18(1).

31. Pedersen BS, Schwartz DA, Yang IV, Kechris KJ. Comb-p: software for combining, analyzing, grouping and correcting spatially correlated P-values. Bioinformatics. 2012;28(22):2986-8.

32. Phipson B, Maksimovic J, Oshlack A. missMethyl: an R package for analyzing data from Illumina's HumanMethylation450 platform. Bioinformatics. 2016;32(2):286-8.

33. Riemens RJM, Kenis G, Nolz J, Susano Chaves SC, Duroux D, Pishva E, et al. Targeted methylation profiling of single laser-capture microdissected post-mortem brain cells by adapted limiting dilution bisulfite pyrosequencing (LDBSP). 2021.

34. Hajj NE, Kuhtz J, Haaf T. Limiting Dilution Bisulfite Pyrosequencing(R): AMethod for Methylation Analysis of Individual DNA Molecules in a Single or a Few Cells. Methods Mol Biol. 2015;1315:221-39.

35. Mansell G, Gorrie-Stone TJ, Bao Y, Kumari M, Schalkwyk LS, Mill J, et al. Guidance for DNA methylation studies: statistical insights from the Illumina EPIC array. BMC genomics. 2019;20(1):1-15.

36. van den Hove DL, Riemens RJ, Koulousakis P, Pishva E. Epigenome-wide association studies in Alzheimer's disease; Achievements and challenges. Brain Pathology. 2020.

37. McDevitt RA, Tiran-Cappello A, Shen H, Balderas I, Britt JP, Marino RA, et al. Serotonergic versus nonserotonergic dorsal raphe projection neurons: differential participation in reward circuitry. Cell reports. 2014;8(6):1857-69.

38. Chapuis J, Hot D, Hansmannel F, Kerdraon O, Ferreira S, Hubans C, et al. Transcriptomic and genetic studies identify IL-33 as a candidate gene for Alzheimer's disease. Molecular psychiatry. 2009;14(11):1004-16.

39. Sherva R, Baldwin CT, Inzelberg R, Vardarajan B, Cupples LA, Lunetta K, et al. Identification of novel candidate genes for Alzheimer's disease by autozygosity mapping using genome wide SNP data. Journal of Alzheimer's Disease. 2011;23(2):349-59.

40. Nazarian A, Yashin Al, Kulminski AM. Genome-wide analysis of genetic predisposition to Alzheimer's disease and related sex disparities. Alzheimer's research \& therapy. 2019;11(1):5.

41. Chang T, editor Tau Network Genes in a Genome Wide Association Study of Progressive Supranuclear Palsy. MOVEMENT DISORDERS; 2017: WILEY 111 RIVER ST, HOBOKEN 07030-5774, NJ USA.

42. Valcourt U, Alcaraz LB, Exposito J-Y, Lethias C, Bartholin L. Tenascin-X: beyond the architectural function. Cell adhesion \& migration. 2015;9(1-2):154-65.

43. Booij L, Casey KF, Antunes JM, Szyf M, Joober R, Israël M, et al. DNA methylation in individuals with anorexia nervosa and in matched normal-eater controls: A genome-wide study. International Journal of Eating Disorders. 2015;48(7):874-82.

44. Kesselmeier M, Pütter C, Volckmar A-L, Baurecht H, Grallert H, Illig T, et al. High-throughput DNA methylation analysis in anorexia nervosa confirms TNXB hypermethylation. The World Journal of Biological Psychiatry. 2018;19(3):187-99.

45. McCartney DL, Walker RM, Morris SW, Anderson SM, Duff BJ, Marioni RE, et al. Altered DNA methylation associated with a translocation linked to major mental illness. npj Schizophrenia. 2018;4(1):1-7.

46. Haertle L, Müller T, Lardenoije R, Maierhofer A, Dittrich M, Riemens RJ, et al. Methylomic profiling in trisomy 21 identifies cognition-and Alzheimer's disease-related dysregulation. Clinical epigenetics. 2019;11(1):1-11.

47. Mosesson Y, Chetrit D, Schley L, Berghoff J, Ziv T, Carvalho S, et al. Monoubiquitinylation regulates endosomal localization of Lst2, a negative regulator of EGF receptor signaling. Developmental cell. 2009;16(5):687-98.

48. Tavassoly O, Sato T, Tavassoly I. Inhibition of Brain Epidermal Growth Factor Receptor Activation: ANovel Target in Neurodegenerative Diseases and Brain Injuries. Molecular Pharmacology. 2020;98(1):13-22.

49. Wang L, Chiang H-C, Wu W, Liang B, Xie Z, Yao X, et al. Epidermal growth factor receptor is a preferred 
target for treating Amyloid- $\beta$-induced memory loss. Proceedings of the National Academy of Sciences. 2012;109(41):16743-8.

50. Nixon RA, Saito K-I, Grynspan F, Griffin WR, Katayama S, Honda T, et al. Calcium-activated neutral proteinase (calpain) system in aging and Alzheimer's disease. Annals of the New York Academy of Sciences. 1994;747:77.

51. Nakayama J, Yoshizawa T, Yamamoto $\mathrm{N}$, Arinami T. Mutation analysis of the calpastatin gene (CAST) in patients with Alzheimer's disease. Neuroscience letters. 2002;320(1-2):77-80.

52. Rao MV, Mohan PS, Peterhoff CM, Yang D-S, Schmidt SD, Stavrides PH, et al. Marked calpastatin (CAST) depletion in Alzheimer's disease accelerates cytoskeleton disruption and neurodegeneration: neuroprotection by CAST overexpression. Journal of Neuroscience. 2008;28(47):12241-54.

53. Zhu L, Gong L, Yang T, Xiao X. Calpastatin Mediates Development of Alzheimer's Disease in Diabetes. Journal of Alzheimer's Disease. 2019;68(3):1051-9.

54. Ma P, Li Y, Zhang W, Fang F, Sun J, Liu M, et al. Long Non-coding RNAMALAT1 Inhibits Neuron Apoptosis and Neuroinflammation While Stimulates Neurite Outgrowth and Its Correlation With MiR-125b Mediates PTGS2, CDK5 and FOXQ1 in Alzheimer's Disease. Current Alzheimer Research. 2019;16(7):596-612.

55. Li L, Xu Y, Zhao M, Gao Z. Neuro-protective roles of long non-coding RNA MALAT1 in Alzheimer's disease with the involvement of the microRNA-30b/CNR1 network and the following PI3K/AKT activation. Experimental and Molecular Pathology. 2020;117:104545.

56. Ouellette AR, Neuner SM, Dumitrescu L, Anderson LC, Gatti DM, Mahoney ER, et al. Cross-Species Analyses Identify Dlgap2 as a Regulator of Age-Related Cognitive Decline and Alzheimer's Dementia. Cell reports. 2020;32(9):108091.

57. Chaudhry M, Wang X, Bamne MN, Hasnain S, Demirci FY, Lopez OL, et al. Genetic variation in imprinted genes is associated with risk of late-onset Alzheimer's disease. Journal of Alzheimer's Disease. 2015;44(3):989-94.

58. Friedman LG, Benson DL, Huntley GW. Cadherin-based transsynaptic networks in establishing and modifying neural connectivity. Current topics in developmental biology. 112: Elsevier; 2015. p. 415-65.

59. Peek SL, Mah KM, Weiner JA. Regulation of neural circuit formation by protocadherins. Cellular and molecular life sciences. 2017;74(22):4133-57.

60. Wang Y, Shi Y, Wei H. Calcium dysregulation in Alzheimer's disease: a target for new drug development. Journal of Alzheimer's disease \& Parkinsonism. 2017;7(5).

61. Tong BC-K, Wu AJ, Li M, Cheung K-H. Calcium signaling in Alzheimer's disease \& therapies. Biochimica et Biophysica Acta (BBA)-Molecular Cell Research. 2018;1865(11):1745-60.

62. Theofilas P, Dunlop S, Heinsen H, Grinberg LT. Turning on the light within: subcortical nuclei of the isodentritic core and their role in Alzheimer's disease pathogenesis. Journal of Alzheimer's Disease. 2015;46(1):17-34. 


\section{Supplementary material}

Supplementary Figure 1. Gene Ontology (GO) terms enriched in the dorsal raphe nucleus (DRN) and locus coeruleus LC. Displayed are the top 20 enriched GO terms in the DRN (A) and LC (B) for each cytosine state: unmodified cytosine (5-uC), 5-methylcytosine (5-mC) and 5-hydroxymethylcytosine (5-hmC). The $x$-axis displays the number of differentially modified genes in the pathway.
A

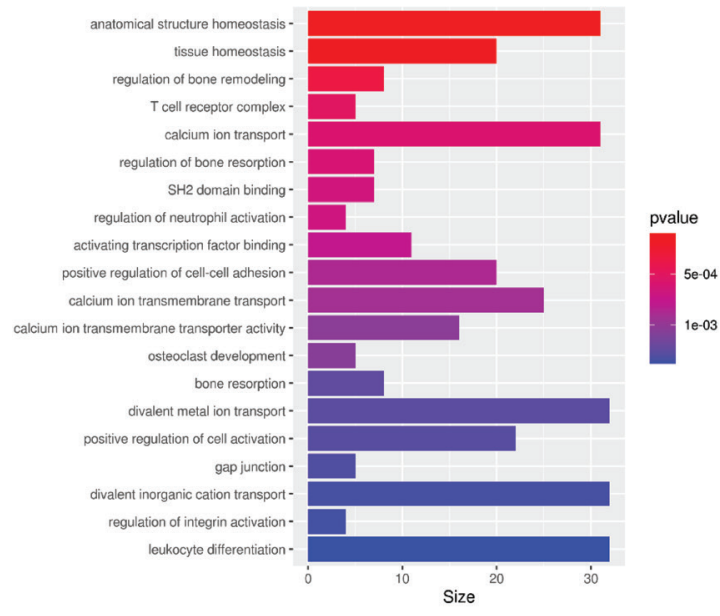

B

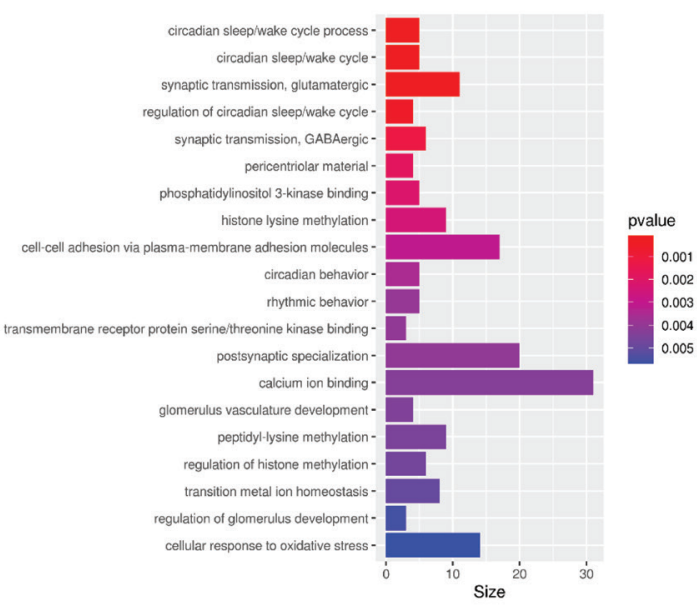



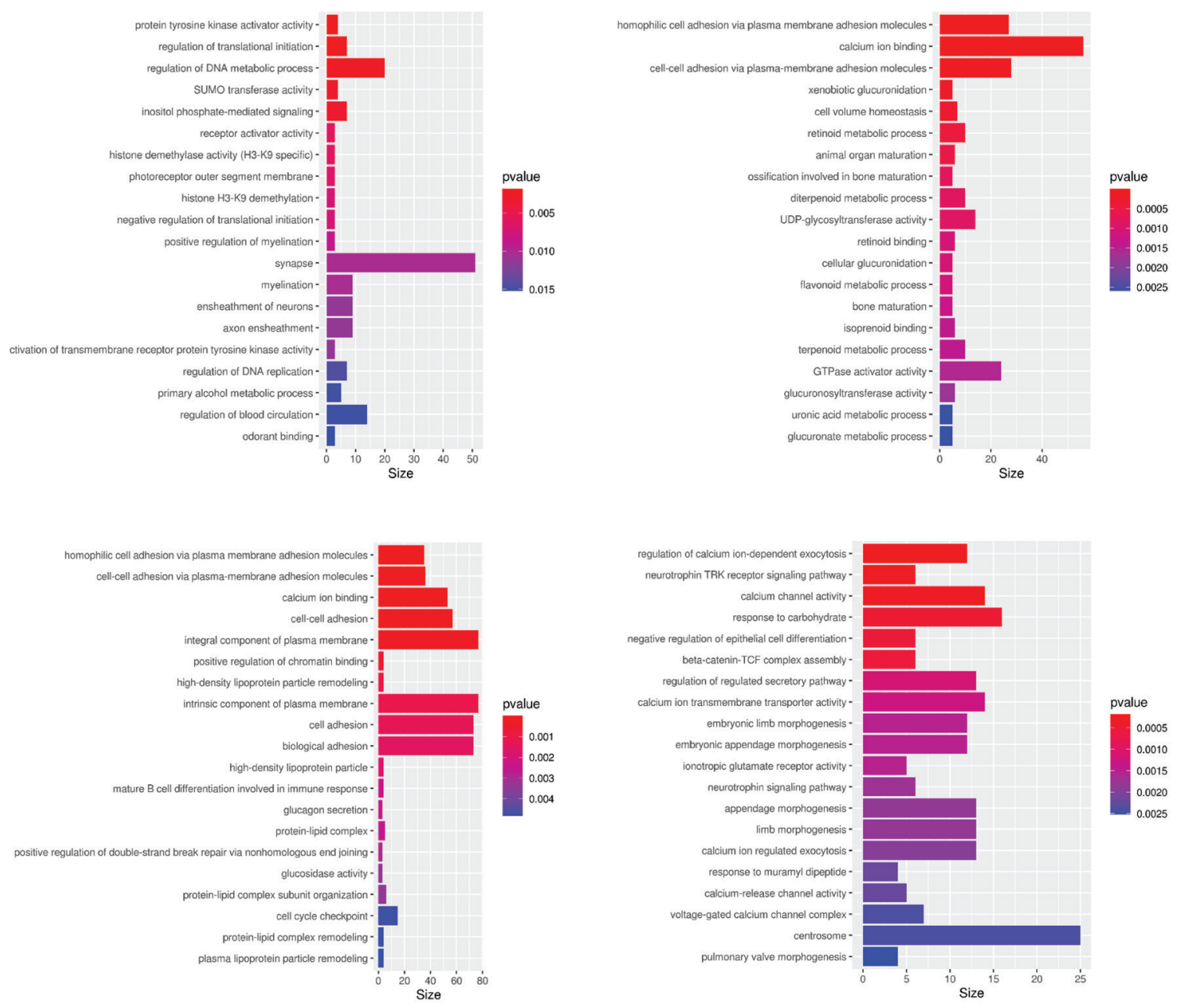
Supplementary Table 1. Top 100 ranked differentially unmodified positions (DUPs) in the dorsal raphe nuclei (DRN)

\begin{tabular}{|c|c|c|c|c|c|c|c|}
\hline Rank & Probe & Chromosome & Position & RE & SE & $t$ & $p$ value \\
\hline 1 & cg24088087 & 10 & 50747319 & 275.5560163 & 58.56392056 & 4.705218053 & 1.08E-05 \\
\hline 2 & cg21146221 & 22 & 30279857 & -127.95102 & 26.66437096 & -4.798576353 & 7.54E-06 \\
\hline 3 & cg23248871 & 19 & 45004792 & -160.4101653 & 36.77468014 & -4.361973093 & $3.90 \mathrm{E}-05$ \\
\hline 4 & cg24066712 & 1 & 20982867 & 99.8026816 & 19.69174422 & 5.068249946 & 2.63E-06 \\
\hline 5 & cg07379335 & 5 & 127211437 & 127.8666408 & 30.43421173 & 4.201411291 & $6.99 \mathrm{E}-05$ \\
\hline 6 & cg16256719 & 1 & 228613082 & -200.9376863 & 48.62737944 & -4.132192369 & 8.96E-05 \\
\hline 7 & cg14225485 & 2 & 14772568 & 90.32632367 & 19.66975559 & 4.592142655 & 1.66E-05 \\
\hline 8 & cg06714901 & 12 & 111283526 & 88.16537314 & 18.74654862 & 4.703018937 & 1.09E-05 \\
\hline 9 & cg00109772 & 19 & 14638399 & 90.76773256 & 20.66696314 & 4.391924054 & 3.49E-05 \\
\hline 10 & $\operatorname{cg} 25456440$ & 5 & 132202607 & -675.8455142 & 167.7148171 & -4.029730502 & 1.29E-04 \\
\hline 11 & cg25459301 & 8 & 10941183 & 84.92135939 & 18.87164507 & 4.499944709 & 2.34E-05 \\
\hline 12 & cg17867860 & 2 & 49054009 & 100.8966953 & 24.09625278 & 4.187235924 & 7.36E-05 \\
\hline 13 & cg19037107 & 11 & 134126323 & 96.55238634 & 23.09082693 & 4.181417436 & 7.51E-05 \\
\hline 14 & cg21968868 & 1 & 203617631 & 108.1014362 & 26.42819217 & 4.09038331 & $1.04 \mathrm{E}-04$ \\
\hline 15 & cg00351957 & 8 & 103571768 & 93.8311369 & 22.48197172 & 4.17361689 & 7.73E-05 \\
\hline 16 & cg03948693 & 16 & 69125843 & 92.46522547 & 22.13797852 & 4.176769138 & 7.64E-05 \\
\hline 17 & cg02938045 & 16 & 48222834 & 104.4106415 & 25.65293161 & 4.070125125 & 1.12E-04 \\
\hline 18 & $\operatorname{cg} 08811130$ & 1 & 110285277 & 78.65990855 & 18.15208081 & 4.333382457 & 4.33E-05 \\
\hline 19 & cg16008609 & 1 & 213123675 & -210.7933164 & 53.15253954 & -3.965818344 & $1.61 \mathrm{E}-04$ \\
\hline 20 & cg10162019 & 5 & 146889701 & -154.992089 & 39.2939016 & -3.944431138 & 1.74E-04 \\
\hline 21 & cg27196940 & 4 & 110736273 & 74.85598275 & 17.70653429 & 4.227590872 & $6.36 \mathrm{E}-05$ \\
\hline 22 & cg22765964 & 11 & 75110494 & 118.4586417 & 30.02188075 & 3.945743528 & $1.73 \mathrm{E}-04$ \\
\hline 23 & cg00258805 & 17 & 29876732 & -205.9814617 & 52.72114233 & -3.906999215 & $1.98 \mathrm{E}-04$ \\
\hline 24 & cg19455923 & 6 & 32016113 & -110.1127 & 27.91765884 & -3.944195343 & 1.74E-04 \\
\hline 25 & cg11688683 & 11 & 120465256 & 116.2912615 & 29.57456197 & 3.932138085 & $1.81 \mathrm{E}-04$ \\
\hline 26 & cg06967232 & 1 & 247148831 & -80.12879142 & 19.6627735 & -4.075152034 & 1.10E-04 \\
\hline 27 & $\operatorname{cg} 00836101$ & 15 & 39876248 & 62.01610249 & 14.21858385 & 4.36162301 & 3.91E-05 \\
\hline 28 & cg27243121 & 11 & 70281584 & 78.39347014 & 19.2108733 & 4.080682275 & $1.08 \mathrm{E}-04$ \\
\hline 29 & cg13121728 & 16 & 10765186 & 87.86434487 & 21.81570605 & 4.027572826 & $1.30 \mathrm{E}-04$ \\
\hline 30 & cg14811321 & 6 & 168084536 & 84.10081364 & 20.86573456 & 4.030570474 & $1.28 \mathrm{E}-04$ \\
\hline 31 & cg00452094 & 21 & 45888218 & 75.82746543 & 18.49115926 & 4.100741568 & $1.00 \mathrm{E}-04$ \\
\hline 32 & cg06273744 & 22 & 40754965 & 89.09795242 & 22.3649374 & 3.983823019 & $1.51 \mathrm{E}-04$ \\
\hline 33 & $\operatorname{cg} 00446758$ & 7 & 132764863 & 106.5970183 & 27.36014775 & 3.896068811 & 2.05E-04 \\
\hline 34 & cg15670836 & 12 & 58936880 & 70.86932485 & 17.4295005 & 4.066055987 & 1.13E-04 \\
\hline 35 & $\operatorname{cg} 00527316$ & 21 & 41356964 & 85.02610483 & 21.48417282 & 3.957615941 & 1.66E-04 \\
\hline 36 & cg18215878 & 11 & 47152876 & 111.0448146 & 28.64147141 & 3.877063891 & 2.19E-04 \\
\hline 37 & cg13496359 & 9 & 109686439 & 74.20294736 & 18.40917329 & 4.030759349 & $1.28 \mathrm{E}-04$ \\
\hline 38 & cg11235259 & 19 & 47526657 & 54.18839896 & 12.43817547 & 4.356619594 & $3.98 \mathrm{E}-05$ \\
\hline 39 & $\operatorname{cg} 00411741$ & 19 & 18208476 & -273.1099286 & 71.23359628 & -3.834004499 & $2.54 \mathrm{E}-04$ \\
\hline 40 & cg15028904 & 5 & 140700449 & -480.8187681 & 125.7546222 & -3.823467954 & 2.63E-04 \\
\hline 41 & cg22391883 & 1 & 16563727 & -724.663516 & 189.7850801 & -3.818337645 & $2.68 \mathrm{E}-04$ \\
\hline 42 & cg23884626 & 6 & 31126149 & -485.6867805 & 127.1112569 & -3.820958051 & 2.66E-04 \\
\hline 43 & cg26199493 & 1 & 226497589 & 87.19071962 & 22.16687096 & 3.933379672 & $1.80 \mathrm{E}-04$ \\
\hline 44 & cg16381797 & 5 & 95997312 & 55.51711834 & 13.1233352 & 4.230412277 & $6.30 \mathrm{E}-05$ \\
\hline 45 & cg09316855 & 6 & 11806488 & 115.6832027 & 30.08550746 & 3.845147132 & $2.45 \mathrm{E}-04$ \\
\hline 46 & cg20218040 & 6 & 14369697 & 88.0586099 & 22.46007184 & 3.920673563 & $1.89 \mathrm{E}-04$ \\
\hline 47 & cg07505669 & 22 & 40754947 & 59.90658516 & 14.44264173 & 4.147896643 & 8.47E-05 \\
\hline 48 & $\operatorname{cg} 07481380$ & 19 & 3834519 & 60.18075473 & 14.54253357 & 4.13825792 & 8.77E-05 \\
\hline 49 & cg26181763 & 17 & 9728527 & 68.52355579 & 16.99527538 & 4.031917945 & $1.28 \mathrm{E}-04$ \\
\hline 50 & cg11535971 & 10 & 33848245 & 58.40561915 & 14.07289439 & 4.150220809 & $8.40 \mathrm{E}-05$ \\
\hline 51 & cg01348938 & 19 & 2806850 & -76.68129399 & 19.37073092 & -3.958616446 & 1.65E-04 \\
\hline 52 & cg13713927 & 3 & 184534541 & 84.92912384 & 21.71081164 & 3.911835506 & $1.94 \mathrm{E}-04$ \\
\hline 53 & cg11921048 & 3 & 156347867 & 94.5600643 & 24.43089211 & 3.870512131 & $2.24 \mathrm{E}-04$ \\
\hline
\end{tabular}




\begin{tabular}{|c|c|c|}
\hline UCSC annotation & Gene feature & $\mathrm{CpG}$ island feature \\
\hline ERCC6; ERCC6-PGBD3; ERCC6-PGBD3 & TSS200; TSS200; 5'UTR & Island \\
\hline MTMR3; MTMR3; MTMR3 & 5'UTR; 5'UTR; 5'UTR & Island \\
\hline ZNF180 & TSS1500 & Island \\
\hline DDOST & Body & \\
\hline HIST3H3 & TSS200 & Island \\
\hline FAM84A & TSS1500 & Island \\
\hline CCDC63; CCDC63; CCDC63 & TSS1500; TSS1500; TSS1500 & \\
\hline UQCRQ; GDF9; GDF9; GDF9; GDF9; GDF9 & Body; TSS200; TSS200; TSS1500; & $\begin{array}{l}\text { Northern shore } \\
\text { Island }\end{array}$ \\
\hline & TSS1500; TSS1500 & \\
\hline XKR6 & Body & \\
\hline ACAD8 & Body & Southern shelf \\
\hline ATP2B4; ATP2B4 & 5'UTR; 5'UTR & \\
\hline ODF1 & Body & \\
\hline ABCC11; ABCC11; ABCC11 & Body; Body; Body & \\
\hline VASH2; VASH2; VASH2 & TSS1500 TSS1500 TSS1500 & $\begin{array}{l}\text { Southern shore } \\
\text { Island }\end{array}$ \\
\hline VASHL, VASHL; VASHL & 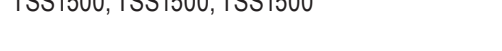 & $\begin{array}{l}\text { ISland } \\
\text { Island }\end{array}$ \\
\hline GAR1; GAR1 & TSS1500; TSS1500 & Northern shore \\
\hline SNORD15A; RPS3 & TSS1500; TSS200 & Island \\
\hline & & Island \\
\hline TNXB & Body & \\
\hline GRIK4; GRIK4; GRIK4 & 5'UTR; 5'UTR; 5'UTR & \\
\hline ZNF695; ZNF695; ZNF695; ZNF670-ZNF695 & 3'UTR; Body; Body; Body & \\
\hline THBS1 & Body & Southern shelf \\
\hline CTTN; CTTN & 3'UTR; 3'UTR & \\
\hline TEKT5 & Body & \\
\hline LOC441178 & TSS200 & \\
\hline ADSL; ADSL & Body; Body & \\
\hline $\mathrm{CHCHD} 3$ & Body & Northern shore \\
\hline C11orf49; C11orf49; C11orf49; C11orf49; C11orf49; C11orf49; C11orf49 & Body; Body; Body; Body; Body; Body; Body & \\
\hline ZNF462; MIR548Q & Body; Body & \\
\hline NPAS1 & Body & Southern shore \\
\hline MAST3 & TSS200 & Northern shore \\
\hline TAF7 & TSS200 & Island \\
\hline C1orf89 & TSS200 & Island \\
\hline CCHCR1; CCHCR1; TCF19; TCF19; CCHCR1 & TSS1500; TSS1500; TSS200; TSS200; TSS200 & Island \\
\hline LIN9 & TSS1500 & Southern shore \\
\hline CAST; CAST & TSS1500; TSS1500 & Northern shore \\
\hline ADSL; ADSL & Body; Body & \\
\hline ZFR2 & Body & Island \\
\hline GLP2R & TSS1500 & \\
\hline THOP1 & Body & Northern shore \\
\hline VPS8; VPS8 & 5'UTR; 5'UTR & \\
\hline
\end{tabular}


Supplementary Table 1. (Continued)

\begin{tabular}{|c|c|c|c|c|c|c|c|}
\hline Rank & Probe & Chromosome & Position & RE & SE & $t$ & $p$ value \\
\hline 54 & cg20933293 & 17 & 56429700 & -217.3370164 & 57.32660668 & -3.791206718 & $2.94 \mathrm{E}-04$ \\
\hline 55 & cg09806242 & 6 & 10303745 & 51.72917379 & 12.30798687 & 4.202894784 & $6.96 \mathrm{E}-05$ \\
\hline 56 & cg09856611 & 3 & 73403300 & 82.40861533 & 21.09117666 & 3.907255469 & 1.97E-04 \\
\hline 57 & cg23404137 & 18 & 36370257 & -46.54148333 & 10.18884378 & -4.567886635 & $1.81 \mathrm{E}-05$ \\
\hline 58 & $\operatorname{cg} 22666350$ & 17 & 4052392 & -56.21297095 & 13.61494022 & -4.128771045 & 9.07E-05 \\
\hline 59 & cg08951005 & 10 & 79803774 & 66.67400258 & 16.71811771 & 3.988128553 & 1.49E-04 \\
\hline 60 & cg16930098 & 20 & 30489015 & 96.53368525 & 25.18198302 & 3.833442552 & $2.54 \mathrm{E}-04$ \\
\hline 61 & cg21339445 & 21 & 45528892 & -83.36058348 & 21.49482222 & -3.87817041 & $2.18 \mathrm{E}-04$ \\
\hline 62 & cg15067802 & 9 & 114860737 & 62.56184631 & 15.67283855 & 3.991736793 & 1.47E-04 \\
\hline 63 & cg07139190 & 6 & 7162285 & -44.87612134 & 10.18445594 & -4.406334672 & 3.31E-05 \\
\hline 64 & cg23326220 & 19 & 51563756 & 42.73344241 & 8.450226732 & 5.057076427 & $2.75 \mathrm{E}-06$ \\
\hline 65 & cg16678626 & 6 & 160513496 & -51.98256763 & 12.71608152 & -4.087939163 & 1.05E-04 \\
\hline 66 & cg18382831 & 3 & 138191900 & -93.39579679 & 24.44405832 & -3.820797495 & 2.66E-04 \\
\hline 67 & cg24985835 & 17 & 79520203 & 108.4714223 & 28.74296816 & 3.773842065 & 3.12E-04 \\
\hline 68 & cg02817292 & 14 & 23532175 & 89.3526646 & 23.35407459 & 3.825998938 & 2.61E-04 \\
\hline 69 & cg21157465 & 1 & 11020631 & -63.12368098 & 16.02234775 & -3.93972731 & 1.76E-04 \\
\hline 70 & cg00312919 & 7 & 38217710 & 124.5972264 & 33.13914857 & 3.759819784 & 3.27E-04 \\
\hline 71 & cg15848350 & 3 & 128326938 & 43.76583376 & 10.19791614 & 4.291644796 & $5.04 \mathrm{E}-05$ \\
\hline 72 & cg03945836 & 16 & 19138344 & 68.4001632 & 17.53029129 & 3.90182696 & 2.01E-04 \\
\hline 73 & cg03381007 & 16 & 88893573 & -48.06168677 & 11.59680307 & -4.144391043 & $8.58 \mathrm{E}-05$ \\
\hline 74 & cg20766090 & 11 & 2159131 & 170.0046649 & 45.50090817 & 3.736291687 & $3.54 \mathrm{E}-04$ \\
\hline 75 & cg22038207 & 4 & 152808142 & 44.81889916 & 10.78927735 & 4.154022341 & $8.29 \mathrm{E}-05$ \\
\hline 76 & cg10758875 & 15 & 56725876 & -74.49128613 & 19.37770894 & -3.844174065 & $2.45 \mathrm{E}-04$ \\
\hline 77 & cg10467427 & 6 & 1633242 & 83.3263001 & 21.82316227 & 3.818250492 & $2.68 \mathrm{E}-04$ \\
\hline 78 & cg23293975 & 20 & 45625848 & -53.95847685 & 13.60248246 & -3.966810984 & $1.61 \mathrm{E}-04$ \\
\hline 79 & cg18165313 & 10 & 109872152 & -41.46865328 & 9.579098863 & -4.329076656 & 4.40E-05 \\
\hline 80 & cg25410105 & 6 & 150945640 & 51.63799732 & 12.95783295 & 3.98507972 & $1.51 \mathrm{E}-04$ \\
\hline 81 & cg07447350 & 4 & 16901824 & 77.08908552 & 20.15383794 & 3.825032521 & $2.62 \mathrm{E}-04$ \\
\hline 82 & $\operatorname{cg} 05935360$ & 19 & 11639865 & -951.585562 & 256.5448461 & -3.709236714 & $3.88 \mathrm{E}-04$ \\
\hline 83 & cg03554286 & 3 & 185497487 & 57.90586759 & 14.76696866 & 3.921310387 & $1.88 \mathrm{E}-04$ \\
\hline 84 & cg08947125 & 3 & 12802515 & -89.44829738 & 23.67033157 & -3.778920339 & 3.06E-04 \\
\hline 85 & cg01987776 & 7 & 138516319 & 47.15762815 & 11.62484985 & 4.056622559 & $1.17 \mathrm{E}-04$ \\
\hline 86 & cg05075268 & 22 & 19718750 & 43.3302499 & 10.43278168 & 4.153278695 & $8.31 \mathrm{E}-05$ \\
\hline 87 & cg05533872 & 2 & 112908757 & 99.3940528 & 26.4913594 & 3.751942333 & 3.36E-04 \\
\hline 88 & cg12807924 & 20 & 36013546 & 47.13650669 & 11.6638908 & 4.041233539 & 1.24E-04 \\
\hline 89 & cg09959585 & 14 & 103570855 & 49.33905839 & 12.35164686 & 3.994532789 & 1.46E-04 \\
\hline 90 & cg03346733 & 19 & 13987364 & 37.72311923 & 8.508473563 & 4.433594222 & $3.00 \mathrm{E}-05$ \\
\hline 91 & cg03265360 & 6 & 16472398 & 69.37749066 & 18.0806776 & 3.837106783 & 2.51E-04 \\
\hline 92 & cg08968069 & 16 & 72903003 & 72.60675705 & 19.01125092 & 3.819146743 & 2.67E-04 \\
\hline 93 & cg27289832 & 3 & 71592784 & 39.08494946 & 9.038586561 & 4.324232467 & $4.48 \mathrm{E}-05$ \\
\hline 94 & cg08931356 & 1 & 237057546 & 60.81153062 & 15.69522343 & 3.874524685 & $2.21 \mathrm{E}-04$ \\
\hline 95 & cg00002531 & 7 & 127721794 & -57.32480574 & 14.71659136 & -3.895250221 & 2.06E-04 \\
\hline 96 & cg24022821 & 6 & 28831886 & -318.4817417 & 86.07149278 & -3.700200048 & 3.99E-04 \\
\hline 97 & cg23439947 & 22 & 28254426 & 103.4331577 & 27.74028526 & 3.728626319 & 3.63E-04 \\
\hline 98 & cg26770421 & 6 & 10735168 & 56.71910629 & 14.56518288 & 3.894156823 & 2.07E-04 \\
\hline 99 & cg07151462 & 2 & 173179124 & 49.39524301 & 12.49516169 & 3.953149567 & 1.68E-04 \\
\hline 100 & cg06562246 & 17 & 34309577 & 49.66977334 & 12.57309363 & 3.950481466 & 1.70E-04 \\
\hline
\end{tabular}

Displayed for each ranked probe is the chromosomal location and position (Ensembl GRCh37 assembly), the regression estimate (RE) for the Braak stage-associated analysis, the standard error (SE), the t-statistics, the accompanying $p$ values, the Illumina gene annotation (UCSC annotation), the gene feature (TSS1500, 200 to 1500 nucleotides (nt) upstream of transcription start site (TSS); TSS200, up to 200 nt upstream of TSS; 5'UTR, 5'untranslated region; Body, gene body; 3'UTR, 3' untranslated region) and the cytosine-phosphate-guanine (CpG) island feature. Probes are ranked based on a combined $p$ value (cut-off $=p<0.001$ ) and regression estimate ranking . 


\begin{tabular}{|c|c|c|}
\hline UCSC annotation & Gene feature & CpG island feature \\
\hline SUPT4H1 & TSS200 & Island \\
\hline CYB5D2; CYB5D2; CYB5D2 & 5'UTR; Body; 5'UTR & \\
\hline RPS24 & Body & \\
\hline TTLL9 & Body & \\
\hline PWP2 & Body & Southern shore \\
\hline SUSD1; SUSD1; SUSD1; MIR3134; SUSD1; SUSD1; SUSD1 & $\begin{array}{l}\text { Exon boundaries; Exon boundaries; } \\
\text { Exon boundaries; Body; Body; Body; Body }\end{array}$ & \\
\hline RREB1; RREB1; RREB1; RREB1 & 5'UTR; 5'UTR; 5'UTR; 5'UTR & \\
\hline KLK13 & Body & \\
\hline LOC729603; IGF2R & TSS1500; Body & Southern shore \\
\hline ESYT3 & Body & \\
\hline C17orf70; C17orf70 & TSS1500; TSS1500 & Southern shore \\
\hline ACIN1; ACIN1; ACIN1; ACIN1; ACIN1 & Body; Body; Body; Body; Body & Southern shelf \\
\hline C1orf127; C1orf127 & Body; Body & \\
\hline STARD3NL & TSS1500 & $\begin{array}{l}\text { Island } \\
\text { Island }\end{array}$ \\
\hline GALNS & Body & Northern shore \\
\hline INS-IGF2; IGF2; IGF2; IGF2 & Body; Body; 5'UTR; 5'UTR & Island \\
\hline MNS1; TEX9 & Body; $3^{\prime}$ UTR & \\
\hline GMDS; GMDS & Body; Body & \\
\hline EYA2; EYA2 & Body; Body & \\
\hline PLEKHG1 & 5'UTR & \\
\hline LDB2; LDB2; LDB2; LDB2; LDB2 & TSS1500; TSS1500; TSS1500; TSS1500; TSS1500 & \\
\hline ECSIT; ECSIT; ECSIT; ECSIT & 5'UTR; TSS200; 5'UTR; 5'UTR & Island \\
\hline IGF2BP2; IGF2BP2 & Body; Body & \\
\hline TMEM40 & Body & \\
\hline \multirow[t]{2}{*}{ KIAA1549; KIAA1549 } & 3'UTR; 3'UTR & \\
\hline & & Island \\
\hline FBLN7; FBLN7 & Body; Body & \\
\hline SRC; SRC & Body; Body & Southern shore \\
\hline C14orf73 & Body & Island \\
\hline NANOS3 & TSS1500 & Northern shore \\
\hline ATXN1; ATXN1 & 5'UTR; 5'UTR & \\
\hline ZFHX3; ZFHX3 & Body; Body & \\
\hline FOXP1; FOXP1; FOXP1; FOXP1; FOXP1 & TSS200; 5'UTR; 5'UTR; 5'UTR; 5'UTR & \\
\hline MTR; MTR; MTR & Body; Body; Body & \\
\hline SND1; MIR593 & Body; TSS200 & \\
\hline & & Island \\
\hline PIIPNB; PIIPNB; PIIPNB & Body; Body; Body & \\
\hline CCL16 & TSS1500 & \\
\hline
\end{tabular}


Supplementary Table 2. Top 100 ranked differentially methylated positions (DMPs) in the dorsal raphe nuclei (DRN)

\begin{tabular}{|c|c|c|c|c|c|c|c|}
\hline Rank & Probe & Chromosome & Position & RE & SE & $t$ & $p$ value \\
\hline 1 & cg03442014 & 16 & 23607821 & 152.1853638 & 34.86627573 & 4.364829928 & $3.72 \mathrm{E}-05$ \\
\hline 2 & cg00339300 & 1 & 61508924 & 463.6149796 & 111.3774606 & 4.162556565 & 7.80E-05 \\
\hline 3 & cg21540765 & 1 & 200008933 & 203.0088517 & 49.27401443 & 4.119998218 & $9.09 E-05$ \\
\hline 4 & cg21336876 & 12 & 100536815 & 350.8025324 & 86.38446546 & 4.060944645 & 1.12E-04 \\
\hline 5 & cg24671939 & 18 & 3593798 & 81.05029979 & 19.03812481 & 4.257262761 & $5.53 \mathrm{E}-05$ \\
\hline 6 & cg02827328 & 9 & 100881403 & 640.1800163 & 163.3235977 & 3.919703124 & $1.84 \mathrm{E}-04$ \\
\hline 7 & cg14182420 & 7 & 135242446 & 69.89612255 & 16.24393268 & 4.302906441 & 4.68E-05 \\
\hline 8 & cg16636721 & 21 & 47920571 & 67.46546117 & 15.77041216 & 4.277977043 & $5.13 \mathrm{E}-05$ \\
\hline 9 & cg11013544 & 22 & 42062791 & 182.3850332 & 45.91988316 & 3.971809609 & $1.54 \mathrm{E}-04$ \\
\hline 10 & cg15647861 & 19 & 50320776 & 245.3163197 & 62.16994693 & 3.945898812 & $1.68 \mathrm{E}-04$ \\
\hline 11 & cg17255342 & 2 & 29181736 & 79.43439409 & 19.05463694 & 4.16876975 & 7.63E-05 \\
\hline 12 & cg23493016 & 10 & 106440778 & 43.86415027 & 9.864989461 & 4.446446745 & $2.75 \mathrm{E}-05$ \\
\hline 13 & cg14702927 & 19 & 46366445 & 112.1124942 & 28.63488895 & 3.915241101 & 1.87E-04 \\
\hline 14 & cg18957501 & 22 & 24246964 & 36.57113877 & 7.689921251 & 4.755723443 & $8.48 \mathrm{E}-06$ \\
\hline 15 & cg10873891 & 20 & 35806913 & 79.68493421 & 19.98882318 & 3.986474516 & $1.46 \mathrm{E}-04$ \\
\hline 16 & ch.6.93211F & 6 & 3310225 & 36.95110859 & 8.25544884 & 4.475966032 & $2.46 \mathrm{E}-05$ \\
\hline 17 & $\operatorname{cg} 13966567$ & 17 & 882342 & -79.69936368 & 20.0321764 & -3.978567385 & $1.50 \mathrm{E}-04$ \\
\hline 18 & cg25298664 & 15 & 74753253 & 187.2760544 & 48.45659852 & 3.864820482 & $2.23 \mathrm{E}-04$ \\
\hline 19 & cg18165313 & 10 & 109872152 & 39.57038389 & 9.375501545 & 4.220615153 & $6.32 \mathrm{E}-05$ \\
\hline 20 & cg18547866 & 7 & 100464803 & 372.2324804 & 97.11222044 & 3.833013792 & 2.49E-04 \\
\hline 21 & cg06768385 & 1 & 214557399 & 39.01433032 & 9.1791741 & 4.250309439 & 5.67E-05 \\
\hline 22 & cg21146221 & 22 & 30279857 & 99.72576701 & 25.61191578 & 3.8937254 & $2.02 \mathrm{E}-04$ \\
\hline 23 & cg00723431 & 1 & 234743527 & 43.06059566 & 10.33512297 & 4.166432832 & 7.69E-05 \\
\hline 24 & cg10348596 & 3 & 128045033 & 34.62400727 & 7.676044373 & 4.510657519 & 2.16E-05 \\
\hline 25 & cg27098574 & 16 & 75285489 & 36.60567415 & 8.476274894 & 4.31860394 & 4.42E-05 \\
\hline 26 & $\operatorname{cg} 00683895$ & 19 & 58739944 & 137.7404989 & 35.8319653 & 3.844067657 & $2.40 \mathrm{E}-04$ \\
\hline 27 & $\operatorname{cg} 24654765$ & 5 & 1887193 & 373.581911 & 97.88310553 & 3.816612775 & 2.63E-04 \\
\hline 28 & ch.11.991049F & 11 & 47494477 & 67.15339201 & 17.03399975 & 3.942314958 & 1.70E-04 \\
\hline 29 & $\operatorname{cg} 25017194$ & 12 & 6566966 & 36.8227229 & 8.776647222 & 4.195534122 & $6.92 \mathrm{E}-05$ \\
\hline 30 & cg21655969 & 15 & 90792609 & 33.71533815 & 7.65162803 & 4.406296021 & 3.19E-05 \\
\hline 31 & cg09618400 & 12 & 6723242 & 74.62394869 & 19.05984643 & 3.915243965 & 1.87E-04 \\
\hline 32 & cg21943376 & 11 & 118800953 & -267.0819365 & 70.01489024 & -3.814644793 & 2.65E-04 \\
\hline 33 & cg18397528 & 17 & 7816510 & 37.67267269 & 9.052912955 & 4.161386824 & 7.83E-05 \\
\hline 34 & cg22985466 & 12 & 57992676 & -31.2626363 & 6.593505247 & -4.741428895 & $8.96 \mathrm{E}-06$ \\
\hline 35 & cg20420007 & 1 & 173399241 & 55.01190558 & 13.88665799 & 3.961493515 & 1.59E-04 \\
\hline 36 & cg01021976 & 12 & 58424937 & -31.3314203 & 6.775418073 & -4.624278526 & $1.41 \mathrm{E}-05$ \\
\hline 37 & cg03498048 & 14 & 99733089 & 131.8760599 & 34.54060866 & 3.818000463 & $2.62 \mathrm{E}-04$ \\
\hline 38 & ch.9.2787281F & 9 & 2797281 & 36.63973261 & 8.825941689 & 4.151368081 & 8.12E-05 \\
\hline 39 & cg26856965 & 10 & 102802736 & 54.35432138 & 13.87078317 & 3.918619497 & 1.85E-04 \\
\hline 40 & cg04378603 & 11 & 65266494 & 47.83080887 & 12.10651624 & 3.950831759 & 1.65E-04 \\
\hline 41 & cg18174834 & 1 & 212740460 & 64.13793797 & 16.53423815 & 3.879098475 & 2.12E-04 \\
\hline 42 & $\operatorname{cg} 05423144$ & 4 & 139792229 & 38.55556721 & 9.677648381 & 3.983980993 & 1.47E-04 \\
\hline 43 & ch.12.1175666F & 12 & 56599430 & 34.99294825 & 8.579612914 & 4.078616204 & $1.05 \mathrm{E}-04$ \\
\hline 44 & $\operatorname{cg} 04563422$ & 14 & 101532902 & -28.38472525 & 6.425042179 & -4.417827068 & $3.06 \mathrm{E}-05$ \\
\hline 45 & cg05393131 & 5 & 156755714 & 35.44503864 & 8.889427546 & 3.987325219 & 1.46E-04 \\
\hline 46 & cg03286076 & 16 & 1264599 & -28.12710604 & 6.48051822 & -4.340255684 & $4.08 \mathrm{E}-05$ \\
\hline 47 & cg27000511 & 9 & 96216109 & 42.94419276 & 11.05766048 & 3.883659914 & 2.09E-04 \\
\hline 48 & cg23602181 & 18 & 9913801 & 509.6958059 & 136.8458696 & 3.724597662 & $3.60 \mathrm{E}-04$ \\
\hline 49 & cg09635586 & 16 & 11836025 & 117.1990439 & 31.21537548 & 3.754529368 & $3.26 \mathrm{E}-04$ \\
\hline 50 & cg04141970 & 18 & 3594060 & 85.9012493 & 22.77358175 & 3.771969217 & 3.07E-04 \\
\hline
\end{tabular}




\begin{tabular}{|c|c|c|}
\hline UCSC annotation & Gene feature & CpG island feature \\
\hline NDUFAB1 & TSS200 & $\begin{array}{l}\text { Island } \\
\text { Island }\end{array}$ \\
\hline NR5A2; NR5A2 & Body; Body & Island \\
\hline UHRF1BP1L; UHRF1BP1L & TSS200; TSS200 & Southern shore \\
\hline FLJ35776; DLGAP1; DLGAP1 & TSS1500; Body; Body & \\
\hline TRIM14; TRIM14; TRIM14; TRIM14 & 1st exon; 1st exon; 1st exon; 1st exon & Island \\
\hline NUP205 & TSS1500 & \\
\hline DIP2A; DIP2A; DIP2A; DIP2A; DIP2A; DIP2A; DIP2A & Body; Body; Body; Body; Body; Body; Body & $\begin{array}{l}\text { Southern shore } \\
\text { Island }\end{array}$ \\
\hline MED25 & TSS1500 & Northern shore \\
\hline SORCS3 & Body & \\
\hline FOXA3; SYMPK; SYMPK & TSS1500; 5'UTR; 1st exon & Island \\
\hline $\begin{array}{l}\text { RPN2; RPN2; MROH8; MROH8; MROH8 } \\
\text { SLC22A23; SLC22A23 }\end{array}$ & $\begin{array}{l}\text { TSS1500; TSS1500; Body; Body; Body } \\
\text { Body; Body }\end{array}$ & Northern shore \\
\hline NXN & Body & Island \\
\hline $\begin{array}{l}\text { UBL7; UBL7; UBL7; UBL7; UBL7-AS1; UBL7-AS1; } \\
\text { UBL7-AS1; UBL7; UBL7; UBL7 }\end{array}$ & $\begin{array}{l}\text { 5'UTR; 5'UTR; 5'UTR; 5'UTR; TSS1500; } \\
\text { TSS1500; TSS1500; 1st exon; 5'UTR; 5'UTR }\end{array}$ & Northern shore \\
\hline MIR6875; TRIP6 & TSS1500; TSS200 & Southern shore \\
\hline PTPN14 & Body & Island \\
\hline MTMR3; MTMR3; MTMR3 & 5'UTR; 5'UTR; 5'UTR & Island \\
\hline IRF2BP2; IRF2BP2 & Body; Body & Northern shore \\
\hline EEFSEC & Body & \\
\hline $\begin{array}{l}\text { BCAR1; BCAR1; BCAR1; BCAR1; BCAR1; BCAR1; } \\
\text { BCAR1; BCAR1; BCAR1 }\end{array}$ & $\begin{array}{l}\text { Body; 5'UTR; 1st exon; Body; Body; 5'UTR; } \\
\text { Body; Body; 1st exon }\end{array}$ & Island \\
\hline ZNF544 & TSS200 & Island \\
\hline CTD-2194D22.4; IRX4; IRX4; IRX4; IRX4; IRX4; IRX4 & $\begin{array}{l}\text { TSS1500; TSS200; TSS200; 1st exon; 1st exon; } \\
\text { 5'UTR; 5'UTR }\end{array}$ & Island \\
\hline CUGBP1; CUGBP1; CUGBP1 & Body; Body; Body & \\
\hline TAPBPL & Body & \\
\hline \multirow[t]{2}{*}{ TTLL13 } & TSS200 & Island \\
\hline & & Northern shelf \\
\hline PIP4K2C; PIP4K2C; PIP4K2C; PIP4K2C & Body; Body; Body; Body & \\
\hline \multirow[t]{2}{*}{ BCL11B; BCL11B } & Body; Body & Northern shelf \\
\hline & & Island \\
\hline MALAT1 & Body & Southern shore \\
\hline ATF3 & 5'UTR & \\
\hline RNF41; RNF41; RNF41 & 3'UTR; 3'UTR; 3'UTR & \\
\hline MIR656 & TSS200 & Southern shore \\
\hline CYFIP2; CYFIP2; CYFIP2; CYFIP2 & Body; Body; Body; Body & \\
\hline CACNA1H; CACNA1H & Body; Body & Northern shore \\
\hline FAM120AOS; FAM120A; FAM120A; FAM120A; FAM120A & TSS1500; Body; Body; Body; Body & Southern shore \\
\hline VAPA; VAPA & TSS200; TSS200 & Island \\
\hline TXNDC11 & Body & Northern shore \\
\hline DLGAP1-AS1; DLGAP1; DLGAP1; DLGAP1; DLGAP1; & TSS200; Body; Body; Body; Body; Body; Body; & \\
\hline DLGAP1; DLGAP1; DLGAP1; DLGAP1; DLGAP1 & Body; Body; Body & \\
\hline
\end{tabular}


Supplementary Table 2. (Continued)

\begin{tabular}{|c|c|c|c|c|c|c|c|}
\hline Rank & Probe & Chromosome & Position & $\mathrm{RE}$ & SE & $t$ & $p$ value \\
\hline 51 & $\operatorname{cg} 00782260$ & 1 & 16142290 & 48.09635946 & 12.53029112 & 3.838407184 & 2.44E-04 \\
\hline 52 & cg25256175 & 22 & 30828305 & -28.05954413 & 6.710663983 & -4.181336481 & $7.29 \mathrm{E}-05$ \\
\hline 53 & cg21638110 & 10 & 126430141 & 84.15061473 & 22.34248259 & 3.766395 & $3.13 \mathrm{E}-04$ \\
\hline 54 & cg27180153 & 13 & 25320559 & 28.45169113 & 6.900294915 & 4.123257264 & $8.98 \mathrm{E}-05$ \\
\hline 55 & cg17780956 & 4 & 156297616 & 446.4218229 & 120.3289059 & 3.710013146 & 3.79E-04 \\
\hline 56 & cg02796548 & 1 & 92372096 & 36.02741566 & 9.224692226 & 3.905541212 & $1.94 \mathrm{E}-04$ \\
\hline 57 & cg24506767 & 6 & 52264762 & 49.40710329 & 12.94496458 & 3.816704401 & $2.63 \mathrm{E}-04$ \\
\hline 58 & cg03500459 & 6 & 2876783 & 117.0590911 & 31.34792252 & 3.734189755 & $3.49 \mathrm{E}-04$ \\
\hline 59 & cg22268510 & 6 & 32118420 & -22.67295459 & 4.82899409 & -4.695171328 & $1.07 \mathrm{E}-05$ \\
\hline 60 & cg10487619 & 16 & 81238825 & 55.08978757 & 14.57953091 & 3.778570648 & $3.00 \mathrm{E}-04$ \\
\hline 61 & cg24754949 & 9 & 36555992 & 91.36845698 & 24.48622543 & 3.731422683 & $3.52 \mathrm{E}-04$ \\
\hline 62 & cg25531478 & 6 & 111279493 & 48.05566253 & 12.66050881 & 3.795713366 & $2.83 \mathrm{E}-04$ \\
\hline 63 & cg04031129 & 1 & 43638029 & 788.5918523 & 215.0072816 & 3.6677444861 & 4.36E-04 \\
\hline 64 & cg07362810 & 10 & 131525915 & 36.21010635 & 9.411936759 & 3.84725347 & 2.37E-04 \\
\hline 65 & cg25809301 & 3 & 71179811 & 98.34103026 & 26.4392203 & 3.719513252 & $3.67 \mathrm{E}-04$ \\
\hline 66 & cg23420697 & 3 & 58038212 & 39.36234094 & 10.33816143 & 3.807479814 & $2.72 \mathrm{E}-04$ \\
\hline 67 & cg20080247 & 8 & 133887910 & 48.05603662 & 12.73050224 & 3.774873584 & $3.04 \mathrm{E}-04$ \\
\hline 68 & cg10851774 & 8 & 20113188 & -22.16864687 & 5.047526899 & -4.391981918 & 3.37E-05 \\
\hline 69 & cg09861346 & 5 & 176037510 & -61.01543644 & 16.29153172 & -3.745224051 & $3.36 \mathrm{E}-04$ \\
\hline 70 & cg12565250 & 10 & 72165370 & 30.49527024 & 7.842399139 & 3.888512902 & $2.06 \mathrm{E}-04$ \\
\hline 71 & cg07141231 & 21 & 26980614 & 85.20797302 & 22.93540065 & 3.715129041 & $3.72 \mathrm{E}-04$ \\
\hline 72 & $\operatorname{cg} 12210890$ & 8 & 110988071 & -79.63693624 & 21.3853459 & -3.723902181 & $3.61 \mathrm{E}-04$ \\
\hline 73 & cg07214473 & 17 & 46048181 & 150.1196675 & 40.73978692 & 3.684841744 & 4.12E-04 \\
\hline 74 & cg24012595 & 7 & 99150098 & 80.11085462 & 21.54370486 & 3.718527297 & $3.68 \mathrm{E}-04$ \\
\hline 75 & cg14336803 & 9 & 19046431 & 28.21685175 & 7.196913137 & 3.920688109 & 1.84E-04 \\
\hline 76 & cg10310275 & 19 & 16296904 & 94.97975814 & 25.66221484 & 3.701152014 & $3.90 \mathrm{E}-04$ \\
\hline 77 & cg05229927 & 7 & 3278961 & -22.02421653 & 5.23065052 & -4.210607542 & $6.55 \mathrm{E}-05$ \\
\hline 78 & cg09989688 & 11 & 45929369 & 21.04627539 & 4.771390095 & 4.41093161 & $3.14 \mathrm{E}-05$ \\
\hline 79 & cg05491695 & 11 & 65266512 & 36.90968286 & 9.749004103 & 3.785995212 & 2.93E-04 \\
\hline 80 & cg00340102 & 20 & 34129456 & -80.45830404 & 21.71169071 & -3.705759496 & $3.84 \mathrm{E}-04$ \\
\hline 81 & cg11267619 & 7 & 29604021 & 425.1724341 & 116.680822 & 3.64389303 & $4.73 \mathrm{E}-04$ \\
\hline 82 & cg22387174 & 7 & 155302770 & 80.39647644 & 21.69975937 & 3.704947833 & $3.85 \mathrm{E}-04$ \\
\hline 83 & cg10840389 & 10 & 72141924 & 214.8809623 & 58.86160571 & 3.650613328 & $4.62 \mathrm{E}-04$ \\
\hline 84 & cg00924357 & 7 & 131012552 & 629.4688207 & 173.4674714 & 3.6287427 & 4.97E-04 \\
\hline 85 & cg25280938 & 7 & 6543803 & 31.10601753 & 8.121998129 & 3.829847907 & $2.52 \mathrm{E}-04$ \\
\hline 86 & cg03260785 & 9 & 100395621 & 517.7361213 & 142.6715692 & 3.628866805 & 4.97E-04 \\
\hline 87 & cg10488854 & 1 & 37078857 & -23.03226696 & 5.686554111 & -4.050302963 & $1.17 \mathrm{E}-04$ \\
\hline 88 & cg17581200 & 17 & 80040464 & -19.59343406 & 4.246315422 & -4.614220119 & $1.46 \mathrm{E}-05$ \\
\hline 89 & cg19632574 & 1 & 26324332 & 90.98432801 & 24.7440935 & 3.67701197 & $4.23 \mathrm{E}-04$ \\
\hline 90 & cg07822469 & 1 & 182585393 & 49.39184414 & 13.25277755 & 3.726905092 & $3.58 \mathrm{E}-04$ \\
\hline 91 & cg27532721 & 8 & 17534102 & 26.72290135 & 6.8684157 & 3.890693651 & 2.04E-04 \\
\hline 92 & cg03062389 & 22 & 42606263 & 19.51902066 & 4.246595496 & 4.596392729 & $1.56 \mathrm{E}-05$ \\
\hline 93 & cg06245023 & 9 & 130635140 & 21.19490644 & 5.06361794 & 4.185723861 & 7.17E-05 \\
\hline 94 & cg08047546 & 7 & 151170250 & -24.81417581 & 6.305280809 & -3.93545927 & $1.75 \mathrm{E}-04$ \\
\hline 95 & cg14080129 & 9 & 90439478 & -37.13297659 & 9.90828275 & -3.74767026 & $3.33 \mathrm{E}-04$ \\
\hline 96 & cg04972065 & 12 & 53591766 & 32.06649437 & 8.476723514 & 3.782887848 & 2.96E-04 \\
\hline 97 & cg23571456 & 6 & 26383374 & 37.39747851 & 9.990448631 & 3.743323237 & $3.38 \mathrm{E}-04$ \\
\hline 98 & cg08891047 & 11 & 56309888 & -41.94519342 & 11.26349893 & -3.723993198 & 3.61E-04 \\
\hline 99 & cg16307912 & 17 & 32078405 & 30.18433579 & 7.97194747 & 3.786318951 & $2.92 \mathrm{E}-04$ \\
\hline 100 & cg08960352 & 12 & 68043561 & 190.7151927 & 52.78633164 & 3.612965456 & $5.24 \mathrm{E}-04$ \\
\hline
\end{tabular}

Displayed for each ranked probe is the chromosomal location and position (Ensembl GRCh37 assembly), the regression estimate (RE) for the Braak stage-associated analysis, the standard error (SE), the t-statistics, the accompanying $p$ values, the Illumina gene annotation (UCSC annotation), the gene feature (TSS1500, 200 to 1500 nucleotides (nt) upstream of transcription start site (TSS); TSS200, up to $200 \mathrm{nt}$ upstream of TSS; $5^{\prime}$ UTR, $5^{\prime}$ untranslated region; Body, gene body; $3^{\prime} U T R, 3^{\prime}$ untranslated region) and the cytosine-phosphate-guanine (CpG) island feature. Probes are ranked based on a combined $p$ value (cut-off $=p<0.001)$ and regression estimate ranking . 


\begin{tabular}{|c|c|c|}
\hline FAM53B & 5'UTR & $\begin{array}{l}\text { Northern shore } \\
\text { Island }\end{array}$ \\
\hline MAP9 & 5'UTR & Island \\
\hline TGFBR3 & TSS1500 & \\
\hline PAQR8 & 5'UTR & Northern shelf \\
\hline SERPINB9P1 & TSS200 & Southern shore \\
\hline PRRT1 & Body & Island \\
\hline PKD1L2; PKD1L2; PKD1L2 & Body; Body; Body & \\
\hline GTF3C6 & TSS1500 & Northern shore \\
\hline WDR65; WDR65; EBNA1BP2; EBNA1BP2; WDR65; WDR65; & 5'UTR; 1st exon; TSS200; 1st exon; 1st exon; & Island \\
\hline WDR65; WDR65; WDR65 & 5'UTR; Body; 1st exon; 5'UTR & \\
\hline MGMT & Body & \\
\hline FOXP1 & Body & \\
\hline FLNB; FLNB; FLNB; FLNB & Body; Body; Body; Body & \\
\hline TG & Body & \\
\hline LZTS1 & TSS1500 & \\
\hline GPRIN1 & TSS1500 & Island \\
\hline EIF4EBP2 & Body & Southern shore \\
\hline MRPL39; MRPL39 & TSS1500; TSS1500 & Southern shore \\
\hline KCNV1 & TSS1500 & Island \\
\hline CDK5RAP3; CDK5RAP3; CDK5RAP3; CDK5RAP3; CDK5RAP3 & TSS200; TSS200; TSS200; TSS200; Body & Island \\
\hline C7orf38 & TSS1500 & $\begin{array}{l}\text { Southern shore } \\
\text { Northern shelf }\end{array}$ \\
\hline FAM32A & Body & Southern shore \\
\hline C11orf94 & TSS1500 & \\
\hline MALAT1 & Body & Southern shore \\
\hline ERGIC3; ERGIC3 & TSS1500; TSS1500 & Northern shore \\
\hline PRR15 & 5'UTR & Island \\
\hline CNPY1 & 5'UTR & Island \\
\hline LRRC20; LRRC20; LRRC20 & 5'UTR; TSS1500; 5'UTR & Island \\
\hline MKLN1; MKLN1 & Body; TSS200 & Island \\
\hline GRID2IP & Body & Island \\
\hline TSTD2; NCBP1; TSTD2 & 5'UTR; TSS200; 1st exon & Island \\
\hline FASN & Body & Northern shore \\
\hline PAFAH2 & 5'UTR & $\begin{array}{l}\text { Northern shore } \\
\text { Southern shore }\end{array}$ \\
\hline MTUS1; MTUS1; MTUS1; MTUS1; MTUS1 & Body; Body; Body; Body; Body & \\
\hline TCF20; TCF20 & 1st exon; 1st exon & \\
\hline AK1; AK1 & Body; Exon boundaries & \\
\hline RHEB & Body & \\
\hline ITGB7 & Body & $\begin{array}{l}\text { Northern shore } \\
\text { Island }\end{array}$ \\
\hline BTN2A2; BTN2A2; BTN2A2; BTN2A2 & 5'UTR; 1st exon; 1st exon; 5'UTR & \\
\hline OR8U8; OR5M11 & Body; 1st exon & \\
\hline ACCN1 & Body & \\
\hline DYRK2; DYRK2 & Body; 5'UTR & Island \\
\hline
\end{tabular}


Supplementary Table 3. Top 100 ranked differentially hydroxymethylated positions (DHPs) in the dorsal raphe nuclei (DRN)

\begin{tabular}{|c|c|c|c|c|c|c|c|}
\hline Rank & Probe & Chromosome & Position & RE & SE & $t$ & $p$ value \\
\hline 1 & cg03442014 & 16 & 23607821 & 152.1853638 & 34.86627573 & 4.364829928 & 3.72E-05 \\
\hline 2 & cg00339300 & 1 & 61508924 & 463.6149796 & 111.3774606 & 4.162556565 & 7.80E-05 \\
\hline 3 & cg21540765 & 1 & 200008933 & 203.0088517 & 49.27401443 & 4.119998218 & $9.09 \mathrm{E}-05$ \\
\hline 4 & cg21336876 & 12 & 100536815 & 350.8025324 & 86.38446546 & 4.060944645 & 1.12E-04 \\
\hline 5 & cg24671939 & 18 & 3593798 & 81.05029979 & 19.03812481 & 4.257262761 & 5.53E-05 \\
\hline 6 & cg02827328 & 9 & 100881403 & 640.1800163 & 163.3235977 & 3.919703124 & 1.84E-04 \\
\hline 7 & cg14182420 & 7 & 135242446 & 69.89612255 & 16.24393268 & 4.302906441 & 4.68E-05 \\
\hline 8 & cg16636721 & 21 & 47920571 & 67.46546117 & 15.77041216 & 4.277977043 & $5.13 \mathrm{E}-05$ \\
\hline 9 & cg11013544 & 22 & 42062791 & 182.3850332 & 45.91988316 & 3.971809609 & $1.54 \mathrm{E}-04$ \\
\hline 10 & cg15647861 & 19 & 50320776 & 245.3163197 & 62.16994693 & 3.945898812 & $1.68 \mathrm{E}-04$ \\
\hline 11 & cg17255342 & 2 & 29181736 & 79.43439409 & 19.05463694 & 4.16876975 & 7.63E-05 \\
\hline 12 & cg23493016 & 10 & 106440778 & 43.86415027 & 9.864989461 & 4.446446745 & $2.75 \mathrm{E}-05$ \\
\hline 13 & cg14702927 & 19 & 46366445 & 112.1124942 & 28.63488895 & 3.915241101 & 1.87E-04 \\
\hline 14 & cg18957501 & 22 & 24246964 & 36.57113877 & 7.689921251 & 4.755723443 & $8.48 \mathrm{E}-06$ \\
\hline 15 & cg10873891 & 20 & 35806913 & 79.68493421 & 19.98882318 & 3.986474516 & 1.46E-04 \\
\hline 16 & ch.6.93211F & 6 & 3310225 & 36.95110859 & 8.25544884 & 4.475966032 & $2.46 \mathrm{E}-05$ \\
\hline 17 & cg13966567 & 17 & 882342 & -79.69936368 & 20.0321764 & -3.978567385 & $1.50 \mathrm{E}-04$ \\
\hline 18 & cg25298664 & 15 & 74753253 & 187.2760544 & 48.45659852 & 3.864820482 & $2.23 \mathrm{E}-04$ \\
\hline 19 & cg18165313 & 10 & 109872152 & 39.57038389 & 9.375501545 & 4.220615153 & $6.32 \mathrm{E}-05$ \\
\hline 20 & cg18547866 & 7 & 100464803 & 372.2324804 & 97.11222044 & 3.833013792 & $2.49 \mathrm{E}-04$ \\
\hline 21 & cg06768385 & 1 & 214557399 & 39.01433032 & 9.1791741 & 4.250309439 & 5.67E-05 \\
\hline 22 & cg21146221 & 22 & 30279857 & 99.72576701 & 25.61191578 & 3.8937254 & $2.02 \mathrm{E}-04$ \\
\hline 23 & cg00723431 & 1 & 234743527 & 43.06059566 & 10.33512297 & 4.166432832 & 7.69E-05 \\
\hline 24 & cg10348596 & 3 & 128045033 & 34.62400727 & 7.676044373 & 4.510657519 & $2.16 \mathrm{E}-05$ \\
\hline 25 & cg27098574 & 16 & 75285489 & 36.60567415 & 8.476274894 & 4.31860394 & 4.42E-05 \\
\hline 26 & cg00683895 & 19 & 58739944 & 137.7404989 & 35.8319653 & 3.844067657 & $2.40 \mathrm{E}-04$ \\
\hline 27 & cg24654765 & 5 & 1887193 & 373.581911 & 97.88310553 & 3.816612775 & 2.63E-04 \\
\hline 28 & ch.11.991049F & 11 & 47494477 & 67.15339201 & 17.03399975 & 3.942314958 & 1.70E-04 \\
\hline 29 & cg25017194 & 12 & 6566966 & 36.8227229 & 8.776647222 & 4.195534122 & $6.92 \mathrm{E}-05$ \\
\hline 30 & cg21655969 & 15 & 90792609 & 33.71533815 & 7.65162803 & 4.406296021 & 3.19E-05 \\
\hline 31 & $\operatorname{cg} 09618400$ & 12 & 6723242 & 74.62394869 & 19.05984643 & 3.915243965 & 1.87E-04 \\
\hline 32 & cg21943376 & 11 & 118800953 & -267.0819365 & 70.01489024 & -3.814644793 & $2.65 \mathrm{E}-04$ \\
\hline 33 & cg18397528 & 17 & 7816510 & 37.67267269 & 9.052912955 & 4.161386824 & 7.83E-05 \\
\hline 34 & cg22985466 & 12 & 57992676 & -31.2626363 & 6.593505247 & -4.741428895 & 8.96E-06 \\
\hline 35 & cg20420007 & 1 & 173399241 & 55.01190558 & 13.88665799 & 3.961493515 & 1.59E-04 \\
\hline 36 & cg01021976 & 12 & 58424937 & -31.3314203 & 6.775418073 & -4.624278526 & 1.41E-05 \\
\hline 37 & cg03498048 & 14 & 99733089 & 131.8760599 & 34.54060866 & 3.818000463 & $2.62 \mathrm{E}-04$ \\
\hline 38 & ch.9.2787281F & 9 & 2797281 & 36.63973261 & 8.825941689 & 4.151368081 & 8.12E-05 \\
\hline 39 & cg26856965 & 10 & 102802736 & 54.35432138 & 13.87078317 & 3.918619497 & 1.85E-04 \\
\hline 40 & $\operatorname{cg} 04378603$ & 11 & 65266494 & 47.83080887 & 12.10651624 & 3.950831759 & $1.65 \mathrm{E}-04$ \\
\hline 41 & cg18174834 & 1 & 212740460 & 64.13793797 & 16.53423815 & 3.879098475 & 2.12E-04 \\
\hline 42 & cg05423144 & 4 & 139792229 & 38.55556721 & 9.677648381 & 3.983980993 & 1.47E-04 \\
\hline 43 & ch.12.1175666F & 12 & 56599430 & 34.99294825 & 8.579612914 & 4.078616204 & 1.05E-04 \\
\hline 44 & $\operatorname{cg} 04563422$ & 14 & 101532902 & -28.38472525 & 6.425042179 & -4.417827068 & 3.06E-05 \\
\hline 45 & $\operatorname{cg} 05393131$ & 5 & 156755714 & 35.44503864 & 8.889427546 & 3.987325219 & 1.46E-04 \\
\hline 46 & cg03286076 & 16 & 1264599 & -28.12710604 & 6.48051822 & -4.340255684 & 4.08E-05 \\
\hline 47 & cg27000511 & 9 & 96216109 & 42.94419276 & 11.05766048 & 3.883659914 & $2.09 \mathrm{E}-04$ \\
\hline 48 & cg23602181 & 18 & 9913801 & 509.6958059 & 136.8458696 & 3.724597662 & $3.60 \mathrm{E}-04$ \\
\hline 49 & cg09635586 & 16 & 11836025 & 117.1990439 & 31.21537548 & 3.754529368 & $3.26 \mathrm{E}-04$ \\
\hline 50 & cg04141970 & 18 & 3594060 & 85.9012493 & 22.77358175 & 3.771969217 & 3.07E-04 \\
\hline
\end{tabular}




\begin{tabular}{|c|c|c|}
\hline UCSC annotation & Gene feature & $\mathrm{CpG}$ island feature \\
\hline NDUFAB1 & TSS200 & $\begin{array}{l}\text { Island } \\
\text { Island }\end{array}$ \\
\hline NR5A2; NR5A2 & Body; Body & Island \\
\hline UHRF1BP1L; UHRF1BP1L & TSS200; TSS200 & Southern shore \\
\hline FLJ35776; DLGAP1; DLGAP1 & TSS1500; Body; Body & \\
\hline TRIM14; TRIM14; TRIM14; TRIM14 & 1stexon; 1st exon; 1st exon; 1st exon & Island \\
\hline NUP205 & TSS1500 & \\
\hline DIP2A; DIP2A; DIP2A; DIP2A; DIP2A; DIP2A; DIP2A & Body; Body; Body; Body; Body; Body; Body & $\begin{array}{l}\text { Southern shore } \\
\text { Island }\end{array}$ \\
\hline MED25 & TSS1500 & Northern shore \\
\hline SORCS3 & Body & \\
\hline FOXA3; SYMPK; SYMPK & TSS1500; 5'UTR; 1st exon & Island \\
\hline RPN2; RPN2; MROH8; MROH8; MROH8 & TSS1500; TSS1500; Body; Body; Body & Northern shore \\
\hline SLC22A23; SLC22A23 & Body; Body & \\
\hline NXN & Body & Island \\
\hline $\begin{array}{l}\text { UBL7; UBL7; UBL7; UBL7; UBL7-AS1; UBL7-AS1; UBL7-AS1; } \\
\text { UBL7; UBL7; UBL7 }\end{array}$ & $\begin{array}{l}\text { 5'UTR; 5'UTR; 5'UTR; 5'UTR; TSS1500; TSS1500; } \\
\text { TSS1500; 1st exon; 5'UTR; 5'UTR }\end{array}$ & Northern shore \\
\hline MIR6875; TRIP6 & TSS1500; TSS200 & Southern shore \\
\hline PTPN14 & Body & Island \\
\hline MTMR3; MTMR3; MTMR3 & 5'UTR; 5'UTR; 5'UTR & Island \\
\hline IRF2BP2; IRF2BP2 & Body; Body & Northern shore \\
\hline EEFSEC & Body & \\
\hline $\begin{array}{l}\text { BCAR1; BCAR1; BCAR1; BCAR1; BCAR1; BCAR1; } \\
\text { BCAR1; BCAR1; BCAR1 }\end{array}$ & $\begin{array}{l}\text { Body; 5'UTR; 1st exon; Body; Body; 5'UTR; } \\
\text { Body; Body: 1st exon }\end{array}$ & Island \\
\hline ZNF544 & TSS200 & Island \\
\hline CTD-2194D22.4; IRX4; IRX4; IRX4; IRX4; IRX4; IRX4 & $\begin{array}{l}\text { TSS1500; TSS200; TSS200; 1st exon; 1st exon; } \\
\text { 5'UTR; 5'UTR }\end{array}$ & Island \\
\hline CUGBP1; CUGBP1; CUGBP1 & Body; Body; Body & \\
\hline TAPBPL & Body & \\
\hline \multirow[t]{2}{*}{ TTLL13 } & TSS200 & Island \\
\hline & & Northern shelf \\
\hline PIP4K2C; PIP4K2C; PIP4K2C; PIP4K2C & Body; Body; Body; Body & \\
\hline \multirow[t]{2}{*}{ BCL11B; BCL11B } & Body; Body & Northern shelf \\
\hline & & Island \\
\hline MALAT1 & Body & Southern shore \\
\hline ATF3 & 5'UTR & \\
\hline RNF41; RNF41; RNF41 & 3'UTR; 3'UTR; 3'UTR & \\
\hline MIR656 & TSS200 & Southern shore \\
\hline CYFIP2; CYFIP2; CYFIP2; CYFIP2 & Body; Body; Body; Body & \\
\hline CACNA1H; CACNA1H & Body; Body & Northern shore \\
\hline FAM120AOS; FAM120A; FAM120A; FAM120A; FAM120A & TSS1500; Body; Body; Body; Body & Southern shore \\
\hline VAPA; VAPA & TSS200; TSS200 & Island \\
\hline TXNDC11 & Body & Northern shore \\
\hline DLGAP1-AS1; DLGAP1; DLGAP1; DLGAP1; DLGAP1; DLGAP1; & TSS200; Body; Body; Body; Body; Body; & \\
\hline DLGAP1; DLGAP1; DLGAP1; DLGAP1 & Body; Body; Body; Body & \\
\hline
\end{tabular}


Supplementary Table 3. (Continued)

\begin{tabular}{|c|c|c|c|c|c|c|c|}
\hline Rank & Probe & Chromosome & Position & $\mathrm{RE}$ & SE & $t$ & $p$ value \\
\hline 51 & cg00782260 & 1 & 16142290 & 48.09635946 & 12.53029112 & 3.838407184 & 2.44E-04 \\
\hline 52 & cg25256175 & 22 & 30828305 & -28.05954413 & 6.710663983 & -4.181336481 & $7.29 \mathrm{E}-05$ \\
\hline 53 & cg21638110 & 10 & 126430141 & 84.15061473 & 22.34248259 & 3.766395 & $3.13 \mathrm{E}-04$ \\
\hline 54 & cg27180153 & 13 & 25320559 & 28.45169113 & 6.900294915 & 4.123257264 & $8.98 \mathrm{E}-05$ \\
\hline 55 & cg17780956 & 4 & 156297616 & 446.4218229 & 120.3289059 & 3.710013146 & 3.79E-04 \\
\hline 56 & cg02796548 & 1 & 92372096 & 36.02741566 & 9.224692226 & 3.905541212 & $1.94 \mathrm{E}-04$ \\
\hline 57 & cg24506767 & 6 & 52264762 & 49.40710329 & 12.94496458 & 3.816704401 & $2.63 \mathrm{E}-04$ \\
\hline 58 & cg03500459 & 6 & 2876783 & 117.0590911 & 31.34792252 & 3.734189755 & $3.49 \mathrm{E}-04$ \\
\hline 59 & cg22268510 & 6 & 32118420 & -22.67295459 & 4.82899409 & -4.695171328 & 1.07E-05 \\
\hline 60 & cg10487619 & 16 & 81238825 & 55.08978757 & 14.57953091 & 3.778570648 & $3.00 \mathrm{E}-04$ \\
\hline 61 & cg24754949 & 9 & 36555992 & 91.36845698 & 24.48622543 & 3.731422683 & $3.52 \mathrm{E}-04$ \\
\hline 62 & cg25531478 & 6 & 111279493 & 48.05566253 & 12.66050881 & 3.795713366 & $2.83 \mathrm{E}-04$ \\
\hline 63 & cg04031129 & 1 & 43638029 & 788.5918523 & 215.0072816 & 3.6677444861 & 4.36E-04 \\
\hline 64 & cg07362810 & 10 & 131525915 & 36.21010635 & 9.411936759 & 3.84725347 & 2.37E-04 \\
\hline 65 & cg25809301 & 3 & 71179811 & 98.34103026 & 26.4392203 & 3.719513252 & $3.67 \mathrm{E}-04$ \\
\hline 66 & cg23420697 & 3 & 58038212 & 39.36234094 & 10.33816143 & 3.807479814 & $2.72 \mathrm{E}-04$ \\
\hline 67 & cg20080247 & 8 & 133887910 & 48.05603662 & 12.73050224 & 3.774873584 & $3.04 \mathrm{E}-04$ \\
\hline 68 & cg10851774 & 8 & 20113188 & -22.16864687 & 5.047526899 & -4.391981918 & $3.37 \mathrm{E}-05$ \\
\hline 69 & cg09861346 & 5 & 176037510 & -61.01543644 & 16.29153172 & -3.745224051 & $3.36 \mathrm{E}-04$ \\
\hline 70 & cg12565250 & 10 & 72165370 & 30.49527024 & 7.842399139 & 3.888512902 & $2.06 \mathrm{E}-04$ \\
\hline 71 & cg07141231 & 21 & 26980614 & 85.20797302 & 22.93540065 & 3.715129041 & $3.72 \mathrm{E}-04$ \\
\hline 72 & $\operatorname{cg} 12210890$ & 8 & 110988071 & -79.63693624 & 21.3853459 & -3.723902181 & $3.61 \mathrm{E}-04$ \\
\hline 73 & $\operatorname{cg} 07214473$ & 17 & 46048181 & 150.1196675 & 40.73978692 & 3.684841744 & $4.12 \mathrm{E}-04$ \\
\hline 74 & cg24012595 & 7 & 99150098 & 80.11085462 & 21.54370486 & 3.718527297 & $3.68 \mathrm{E}-04$ \\
\hline 75 & cg14336803 & 9 & 19046431 & 28.21685175 & 7.196913137 & 3.920688109 & 1.84E-04 \\
\hline 76 & cg10310275 & 19 & 16296904 & 94.97975814 & 25.66221484 & 3.701152014 & $3.90 \mathrm{E}-04$ \\
\hline 77 & cg05229927 & 7 & 3278961 & -22.02421653 & 5.23065052 & -4.210607542 & $6.55 \mathrm{E}-05$ \\
\hline 78 & cg09989688 & 11 & 45929369 & 21.04627539 & 4.771390095 & 4.41093161 & $3.14 \mathrm{E}-05$ \\
\hline 79 & cg05491695 & 11 & 65266512 & 36.90968286 & 9.749004103 & 3.785995212 & 2.93E-04 \\
\hline 80 & cg00340102 & 20 & 34129456 & -80.45830404 & 21.71169071 & -3.705759496 & $3.84 \mathrm{E}-04$ \\
\hline 81 & cg11267619 & 7 & 29604021 & 425.1724341 & 116.680822 & 3.64389303 & $4.73 \mathrm{E}-04$ \\
\hline 82 & cg22387174 & 7 & 155302770 & 80.39647644 & 21.69975937 & 3.704947833 & $3.85 \mathrm{E}-04$ \\
\hline 83 & cg10840389 & 10 & 72141924 & 214.8809623 & 58.86160571 & 3.650613328 & $4.62 \mathrm{E}-04$ \\
\hline 84 & cg00924357 & 7 & 131012552 & 629.4688207 & 173.4674714 & 3.6287427 & 4.97E-04 \\
\hline 85 & cg25280938 & 7 & 6543803 & 31.10601753 & 8.121998129 & 3.829847907 & $2.52 \mathrm{E}-04$ \\
\hline 86 & cg03260785 & 9 & 100395621 & 517.7361213 & 142.6715692 & 3.628866805 & 4.97E-04 \\
\hline 87 & cg10488854 & 1 & 37078857 & -23.03226696 & 5.686554111 & -4.050302963 & $1.17 \mathrm{E}-04$ \\
\hline 88 & cg17581200 & 17 & 80040464 & -19.59343406 & 4.246315422 & -4.614220119 & 1.46E-05 \\
\hline 89 & cg19632574 & 1 & 26324332 & 90.98432801 & 24.7440935 & 3.67701197 & $4.23 \mathrm{E}-04$ \\
\hline 90 & $\operatorname{cg} 07822469$ & 1 & 182585393 & 49.39184414 & 13.25277755 & 3.726905092 & $3.58 \mathrm{E}-04$ \\
\hline 91 & cg27532721 & 8 & 17534102 & 26.72290135 & 6.8684157 & 3.890693651 & 2.04E-04 \\
\hline 92 & cg03062389 & 22 & 42606263 & 19.51902066 & 4.246595496 & 4.596392729 & $1.56 \mathrm{E}-05$ \\
\hline 93 & $\operatorname{cg} 06245023$ & 9 & 130635140 & 21.19490644 & 5.06361794 & 4.185723861 & 7.17E-05 \\
\hline 94 & cg08047546 & 7 & 151170250 & -24.81417581 & 6.305280809 & -3.93545927 & $1.75 \mathrm{E}-04$ \\
\hline 95 & cg14080129 & 9 & 90439478 & -37.13297659 & 9.90828275 & -3.74767026 & $3.33 \mathrm{E}-04$ \\
\hline 96 & cg04972065 & 12 & 53591766 & 32.06649437 & 8.476723514 & 3.782887848 & $2.96 \mathrm{E}-04$ \\
\hline 97 & cg23571456 & 6 & 26383374 & 37.39747851 & 9.990448631 & 3.743323237 & $3.38 \mathrm{E}-04$ \\
\hline 98 & cg08891047 & 11 & 56309888 & -41.94519342 & 11.26349893 & -3.723993198 & 3.61E-04 \\
\hline 99 & cg16307912 & 17 & 32078405 & 30.18433579 & 7.97194747 & 3.786318951 & $2.92 \mathrm{E}-04$ \\
\hline 100 & cg08960352 & 12 & 68043561 & 190.7151927 & 52.78633164 & 3.612965456 & $5.24 \mathrm{E}-04$ \\
\hline
\end{tabular}

Displayed for each ranked probe is the chromosomal location and position (Ensembl GRCh37 assembly), the regression estimate (RE) for the Braak stage-associated analysis, the standard error (SE), the t-statistics, the accompanying $p$ values, the Illumina gene annotation (UCSC annotation), the gene feature (TSS1500, 200 to 1500 nucleotides (nt) upstream of transcription start site (TSS); TSS200, up to $200 \mathrm{nt}$ upstream of TSS; 5'UTR, 5'untranslated region; Body, gene body; $3^{\prime}$ UTR, 3' untranslated region) and the cytosine-phosphate-guanine ( $\mathrm{CpG}$ ) island feature. Probes are ranked based on a combined $p$ value (cut-off $=p<0.001$ ) and regression estimate ranking. 


\begin{tabular}{|c|c|c|}
\hline UCSC annotation & Gene feature & $\mathrm{CpG}$ island feature \\
\hline FAM53B & 5'UTR & $\begin{array}{l}\text { Northern shore } \\
\text { Island }\end{array}$ \\
\hline MAP9 & 5'UTR & Island \\
\hline TGFBR3 & TSS1500 & \\
\hline PAQR8 & 5'UTR & Northern shelf \\
\hline SERPINB9P1 & TSS200 & Southern shore \\
\hline PRRT1 & Body & Island \\
\hline PKD1L2; PKD1L2; PKD1L2 & Body; Body; Body & \\
\hline GTF3C6 & TSS1500 & Northern shore \\
\hline WDR65; WDR65; EBNA1BP2; EBNA1BP2; WDR65; WDR65; WDR65; & 5'UTR; 1st exon; TSS200; 1st exon; 1st exon; & Island \\
\hline WDR65; WDR65 & 5'UTR; Body; 1st exon; 5'UTR & \\
\hline MGMT & Body & \\
\hline FOXP1 & Body & \\
\hline FLNB; FLNB; FLNB; FLNB & Body; Body; Body; Body & \\
\hline TG & Body & \\
\hline LZTS1 & TSS1500 & \\
\hline GPRIN1 & TSS1500 & Island \\
\hline EIF4EBP2 & Body & Southern shore \\
\hline MRPL39; MRPL39 & TSS1500; TSS1500 & Southern shore \\
\hline KCNV1 & TSS1500 & Island \\
\hline CDK5RAP3; CDK5RAP3; CDK5RAP3; CDK5RAP3; CDK5RAP3 & TSS200; TSS200; TSS200; TSS200; Body & Island \\
\hline C7orf38 & TSS1500 & $\begin{array}{l}\text { Southern shore } \\
\text { Northern shelf }\end{array}$ \\
\hline FAM32A & Body & Southern shore \\
\hline C11orf94 & TSS1500 & \\
\hline MALAT1 & Body & Southern shore \\
\hline ERGIC3; ERGIC3 & TSS1500; TSS1500 & Northern shore \\
\hline PRR15 & 5'UTR & Island \\
\hline CNPY1 & 5'UTR & Island \\
\hline LRRC20; LRRC20; LRRC20 & 5'UTR; TSS1500; 5'UTR & Island \\
\hline MKLN1; MKLN1 & Body; TSS200 & Island \\
\hline GRID2IP & Body & Island \\
\hline TSTD2; NCBP1; TSTD2 & 5'UTR; TSS200; 1st exon & Island \\
\hline FASN & Body & Northern shore \\
\hline PAFAH2 & 5'UTR & $\begin{array}{l}\text { Northern shore } \\
\text { Southern shore }\end{array}$ \\
\hline MTUS1; MTUS1; MTUS1; MTUS1; MTUS1 & Body; Body; Body; Body; Body & \\
\hline TCF20; TCF20 & 1st exon; 1st exon & \\
\hline AK1;AK1 & Body; Exon boundaries & \\
\hline RHEB & Body & \\
\hline & & Northern shore \\
\hline ITGB7 & Body & Island \\
\hline BTN2A2; BTN2A2; BTN2A2; BTN2A2 & 5'UTR; 1st exon; 1st exon; 5'UTR & \\
\hline OR8U8; OR5M11 & Body; 1st exon & \\
\hline ACCN1 & Body & \\
\hline DYRK2; DYRK2 & Body; 5'UTR & Island \\
\hline
\end{tabular}


Supplementary Table 4. Top 100 ranked differentially unmodified positions (DUPs)

in the locus coeruleus (LC)

\begin{tabular}{|c|c|c|c|c|c|c|c|}
\hline Rank & Probe & Chromosome & Position & RE & SE & $t$ & $p$ value \\
\hline 1 & cg05775895 & 3 & 12838266 & 277.1749287 & 61.90650359 & 4.477315186 & 2.91E-05 \\
\hline 2 & cg05224975 & 20 & 57049253 & -117.2800978 & 23.19068893 & -5.057206288 & 3.35E-06 \\
\hline 3 & cg11267619 & 7 & 29604021 & 491.3782777 & 116.4528028 & 4.219548742 & 7.32E-05 \\
\hline 4 & cg11959007 & 13 & 76432094 & -160.2558812 & 37.57580802 & -4.264868533 & $6.24 \mathrm{E}-05$ \\
\hline 5 & $\operatorname{cg} 01486300$ & 11 & 71885949 & -115.0633189 & 26.33007239 & -4.370034279 & 4.29E-05 \\
\hline 6 & cg15531185 & 3 & 167415859 & -91.81059444 & 19.25808254 & -4.76737984 & $1.00 \mathrm{E}-05$ \\
\hline 7 & cg22068764 & 1 & 170501645 & -253.1292187 & 60.80758413 & -4.162790257 & 8.93E-05 \\
\hline 8 & cg00604202 & 6 & 99272987 & 85.02374214 & 15.77736084 & 5.388971135 & $9.30 \mathrm{E}-07$ \\
\hline 9 & cg07330172 & 12 & 59932731 & -125.2908759 & 30.42753168 & -4.117681223 & $1.05 \mathrm{E}-04$ \\
\hline 10 & cg07209547 & 7 & 16873191 & -79.45576479 & 15.75975264 & -5.041688574 & $3.56 \mathrm{E}-06$ \\
\hline 11 & $\operatorname{cg} 04582364$ & 5 & 52778441 & -109.2618288 & 26.41805025 & -4.135877848 & $9.81 \mathrm{E}-05$ \\
\hline 12 & cg05254590 & 8 & 55382640 & -166.06681 & 40.88034884 & -4.062264994 & 1.27E-04 \\
\hline 13 & cg01061283 & 8 & 63147364 & -109.2061951 & 26.44831182 & -4.129042181 & $1.00 \mathrm{E}-04$ \\
\hline 14 & cg06793461 & 12 & 103987955 & -79.82935514 & 17.56174358 & -4.54563949 & 2.27E-05 \\
\hline 15 & cg04988171 & 7 & 86847564 & -87.931112 & 20.29767409 & -4.332078228 & 4.91E-05 \\
\hline 16 & cg10312966 & 6 & 121717017 & -99.8590694 & 23.88837359 & -4.180237262 & $8.40 \mathrm{E}-05$ \\
\hline 17 & cg21610556 & 4 & 181452599 & -104.6540147 & 25.51927941 & -4.100978443 & 1.11E-04 \\
\hline 18 & $\operatorname{cg} 02450124$ & 15 & 58429751 & -118.4688347 & 29.52053808 & -4.013098759 & $1.50 \mathrm{E}-04$ \\
\hline 19 & $\operatorname{cg} 16965936$ & 21 & 43255477 & 69.06390774 & 14.1476788 & 4.881642334 & $6.53 \mathrm{E}-06$ \\
\hline 20 & cg21839449 & 7 & 157956042 & -76.43803553 & 17.37056591 & -4.400434385 & 3.84E-05 \\
\hline 21 & cg12413852 & 18 & 55820185 & -105.8824946 & 26.24354546 & -4.034610901 & $1.39 \mathrm{E}-04$ \\
\hline 22 & cg00050133 & 4 & 178167583 & -74.14812874 & 16.82202973 & -4.407799174 & $3.74 \mathrm{E}-05$ \\
\hline 23 & cg12866769 & 15 & 67041828 & -68.96777812 & 15.03064302 & -4.588478218 & $1.94 \mathrm{E}-05$ \\
\hline 24 & cg21373732 & 14 & 99886511 & -90.52854458 & 22.106226 & -4.095160548 & 1.13E-04 \\
\hline 25 & cg18675840 & 1 & 245703471 & 79.07185283 & 19.01162987 & 4.159130668 & $9.05 \mathrm{E}-05$ \\
\hline 26 & cg19712963 & 1 & 24069298 & -86.28693736 & 21.5032579 & -4.012737872 & $1.50 \mathrm{E}-04$ \\
\hline 27 & ch.17.2065773F & 17 & 76200219 & -57.63521382 & 12.1632779 & -4.73846066 & $1.12 \mathrm{E}-05$ \\
\hline 28 & cg13482990 & 2 & 140582839 & -62.95580061 & 14.70937482 & -4.279978001 & 5.91E-05 \\
\hline 29 & cg12746706 & 6 & 169276508 & 71.52095017 & 17.41755883 & 4.106255697 & 1.09E-04 \\
\hline 30 & cg11383275 & 2 & 67623023 & -57.42414781 & 12.91303668 & -4.446990218 & $3.25 \mathrm{E}-05$ \\
\hline 31 & cg17690975 & 6 & 76564689 & -84.5572518 & 21.22702405 & -3.983471804 & $1.66 \mathrm{E}-04$ \\
\hline 32 & $\operatorname{cg} 06538616$ & 14 & 36402316 & -61.97513982 & 14.69834924 & -4.216469402 & 7.40E-05 \\
\hline 33 & cg03550821 & 6 & 143474125 & -100.6463181 & 25.79086158 & -3.902402321 & 2.19E-04 \\
\hline 34 & $\operatorname{cg} 00875467$ & 3 & 76875348 & -90.08720841 & 22.94001344 & -3.927077403 & 2.01E-04 \\
\hline 35 & cg25113483 & 8 & 28321280 & 55.31737607 & 12.56368615 & 4.402957494 & 3.81E-05 \\
\hline 36 & cg06587475 & 8 & 26279631 & 108.4225369 & 27.98107071 & 3.874853038 & $2.40 \mathrm{E}-04$ \\
\hline 37 & cg23106652 & 13 & 47368091 & -82.50245959 & 20.92656325 & -3.942475341 & $1.91 \mathrm{E}-04$ \\
\hline 38 & cg27163126 & 8 & 103953809 & -76.18693736 & 19.16915885 & -3.974453858 & $1.71 \mathrm{E}-04$ \\
\hline 39 & cg04340928 & 16 & 55067613 & -229.9522895 & 60.14561477 & -3.82325944 & $2.85 \mathrm{E}-04$ \\
\hline 40 & cg04169380 & 6 & 161516638 & -65.4055128 & 16.24186798 & -4.026969858 & $1.43 \mathrm{E}-04$ \\
\hline 41 & cg13851625 & 7 & 26676500 & -91.05879487 & 23.32289206 & -3.904266874 & 2.17E-04 \\
\hline 42 & $\operatorname{cg} 00733324$ & 14 & 67845244 & -90.26208611 & 23.15314562 & -3.898480474 & 2.22E-04 \\
\hline 43 & cg01907875 & 18 & 41650428 & -50.80036739 & 11.42383769 & -4.446874052 & $3.25 \mathrm{E}-05$ \\
\hline 44 & cg22665099 & 10 & 97055150 & -63.47384816 & 15.83203598 & -4.009203126 & $1.52 \mathrm{E}-04$ \\
\hline 45 & cg21524032 & 13 & 50030794 & -84.7167459 & 21.68803867 & -3.906150629 & $2.16 \mathrm{E}-04$ \\
\hline 46 & cg12545993 & 8 & 119964636 & 84.35871185 & 21.58487097 & 3.908233316 & $2.14 \mathrm{E}-04$ \\
\hline 47 & cg00335757 & 8 & 80767166 & -50.17742896 & 11.39872657 & -4.402020579 & $3.82 \mathrm{E}-05$ \\
\hline 48 & cg15057359 & 2 & 77750866 & -58.13929618 & 14.29377957 & -4.06745437 & $1.24 \mathrm{E}-04$ \\
\hline 49 & cg14947281 & 10 & 101501169 & -93.56885004 & 24.28207497 & -3.853412452 & $2.58 \mathrm{E}-04$ \\
\hline 50 & cg25664353 & 6 & 114019348 & 69.73878629 & 17.78683879 & 3.920808364 & 2.06E-04 \\
\hline 51 & cg08854791 & 5 & 179407590 & -88.8828497 & 23.10663808 & -3.846637031 & 2.64E-04 \\
\hline 52 & cg09406933 & 16 & 75254017 & 75.04587899 & 19.30226984 & 3.88793026 & 2.30E-04 \\
\hline
\end{tabular}




\begin{tabular}{|c|c|c|}
\hline UCSC annotation & Gene feature & CpG island feature \\
\hline CAND2; CAND2 & 1stexon; 1stexon & Island \\
\hline APCDD1L; APCDD1L; APCDD1L & Body; Body; Body & \\
\hline PRR15 & 5'UTR & Island \\
\hline LMO7; LMO7 & Body; 3'UTR & \\
\hline \multirow{2}{*}{$\begin{array}{l}\text { PDCD10; PDCD10; PDCD10 } \\
\text { GORAB; GORAB; GORAB }\end{array}$} & Body; Body; Body & \\
\hline & Body; Body; Body & Jelond \\
\hline FST;FST & Body; Body & $\begin{array}{l}\text { Southern shore } \\
\text { Island }\end{array}$ \\
\hline STAB2 & Body & \\
\hline TMEM243 & Body & Northern shore \\
\hline AQP9 & TSS1500 & \\
\hline PRDM15; PRDM15 & Body; Body & Island \\
\hline PTPRN2; PTPRN2; PTPRN2 & Body; Body; Body & Northern shore \\
\hline NEDD4L; NEDD4L; NEDD4L; NEDD4L; NEDD4L; NEDD4L; & Body; Body; Body; & \\
\hline NEDD4L; NEDD4L & 5'UTR; 5'UTR; 5'UTR; Body; Body & \\
\hline SMAD6; SMAD6; SMAD6 & Body; Body; Body & \\
\hline SETD3; SETD3 & Body; Body & \\
\hline KIF26B & Body & \\
\hline TCEB3 & TSS1500 & Northern shore \\
\hline AFMID; AFMID & Body; Body & \\
\hline ETAA1 & TSS1500 & Northern shore \\
\hline MYO6; MYO6 & Body; Body & \\
\hline AIG1 & Body & \\
\hline FBX016; FBX016 & Body; Body & \\
\hline ESD & 5'UTR & Northern shelf \\
\hline AZIN1-AS1; AZIN1-AS1 & Body; Body & \\
\hline MAP3K4; MAP3K4 & Body; Body & \\
\hline C7orf71 & TSS1500 & \\
\hline \multirow[t]{2}{*}{ EIF2S1 } & Body & \\
\hline & & Island \\
\hline SETDB2; SETDB2 & Body; Body & \\
\hline TNFRSF11B & TSS1500 & Island \\
\hline LOC101927040 & Body & \\
\hline LRRTM4; LRRTM4 & TSS1500; TSS1500 & \\
\hline CUTC & Body & \\
\hline RNF130; RNF130 & Body; Body & \\
\hline CTRB1 & Body & \\
\hline
\end{tabular}


Supplementary Table 4. (Continued)

\begin{tabular}{|c|c|c|c|c|c|c|c|}
\hline Rank & Probe & Chromosome & Position & RE & SE & $t$ & $p$ value \\
\hline 53 & cg21735115 & 12 & 9800259 & 67.48839544 & 17.24501171 & 3.913502441 & 2.11E-04 \\
\hline 54 & cg06126581 & 17 & 16229132 & -94.55382406 & 24.81114161 & -3.810942098 & 2.97E-04 \\
\hline 55 & cg12299771 & 14 & 50012007 & -66.08017425 & 16.86871037 & -3.917322238 & 2.08E-04 \\
\hline 56 & cg07331152 & 5 & 7983756 & -99.83838214 & 26.42823866 & -3.777716079 & 3.32E-04 \\
\hline 57 & $\operatorname{cg} 13503413$ & 10 & 60937257 & -56.23722473 & 14.07702682 & -3.994964666 & $1.60 \mathrm{E}-04$ \\
\hline 58 & cg11030597 & 9 & 7537075 & -52.43686252 & 12.96970653 & -4.043026138 & 1.35E-04 \\
\hline 59 & cg03304589 & 7 & 63734144 & 81.79287041 & 21.34492253 & 3.831959113 & 2.77E-04 \\
\hline 60 & cg21339170 & 3 & 176763896 & -43.80496222 & 9.834346548 & -4.454282956 & $3.17 \mathrm{E}-05$ \\
\hline 61 & cg06346857 & 1 & 21877579 & -132.1273416 & 35.4614307 & -3.725945035 & 3.94E-04 \\
\hline 62 & cg17428843 & 1 & 221845800 & -54.21065635 & 13.52522309 & -4.008115504 & 1.53E-04 \\
\hline 63 & cg20066716 & 7 & 49815181 & 107.8203954 & 28.79033256 & 3.745020841 & $3.70 \mathrm{E}-04$ \\
\hline 64 & cg07267974 & 8 & 62539945 & -86.51643464 & 22.83793272 & -3.788277849 & $3.21 \mathrm{E}-04$ \\
\hline 65 & cg03783777 & 12 & 2822034 & 56.88671872 & 14.43537675 & 3.940785177 & $1.92 \mathrm{E}-04$ \\
\hline 66 & cg01603981 & 5 & 80043300 & 63.72244227 & 16.40009695 & 3.885491804 & $2.32 \mathrm{E}-04$ \\
\hline 67 & cg19688999 & 10 & 59961765 & -91.63601944 & 24.35863463 & -3.761952212 & $3.50 \mathrm{E}-04$ \\
\hline 68 & cg22071224 & 2 & 171572348 & -76.97768465 & 20.17434666 & -3.815622184 & $2.93 \mathrm{E}-04$ \\
\hline 69 & cg07715503 & 4 & 170121522 & -43.66400595 & 10.24622022 & -4.261474475 & $6.31 \mathrm{E}-05$ \\
\hline 70 & cg03882355 & 2 & 30939430 & -48.0056749 & 11.82659959 & -4.05912744 & $1.28 \mathrm{E}-04$ \\
\hline 71 & cg00632007 & 6 & 1598984 & -79.77615041 & 21.0988738 & -3.78106202 & 3.29E-04 \\
\hline 72 & cg09445207 & 22 & 18341142 & -62.07136883 & 16.02901612 & -3.872437857 & 2.42E-04 \\
\hline 73 & cg04724720 & 11 & 65640939 & -107.1747446 & 28.83984975 & -3.716203292 & 4.07E-04 \\
\hline 74 & cg00213182 & 4 & 36718783 & 43.93339074 & 10.47226617 & 4.195213338 & 7.97E-05 \\
\hline 75 & cg23276399 & 14 & 102427771 & 40.15556584 & 8.58706961 & 4.676282791 & $1.41 \mathrm{E}-05$ \\
\hline 76 & cg13065537 & 3 & 193853752 & 193.2726711 & 52.42873304 & 3.686388357 & 4.49E-04 \\
\hline 77 & cg15006851 & 6 & 134830131 & -42.69305687 & 10.05008621 & -4.248028919 & $6.62 \mathrm{E}-05$ \\
\hline 78 & cg23228887 & 2 & 190543482 & 54.48384069 & 13.84150759 & 3.936264915 & $1.95 \mathrm{E}-04$ \\
\hline 79 & cg07016184 & 15 & 96884187 & 361.2182177 & 98.47901323 & 3.667971539 & 4.77E-04 \\
\hline 80 & cg22598496 & 3 & 190980644 & -41.36553681 & 9.538835854 & -4.336539327 & 4.83E-05 \\
\hline 81 & cg11162637 & 15 & 101818008 & -67.38524497 & 17.63315699 & -3.821507685 & 2.87E-04 \\
\hline 82 & cg19830670 & 4 & 75097154 & -38.57295556 & 8.104093787 & -4.759687705 & $1.03 \mathrm{E}-05$ \\
\hline 83 & cg24284976 & 3 & 73862941 & -45.64727152 & 11.26959354 & -4.050480736 & $1.32 \mathrm{E}-04$ \\
\hline 84 & cg11932767 & 6 & 32864607 & -87.2345496 & 23.35711691 & -3.73481667 & 3.83E-04 \\
\hline 85 & cg25969802 & 1 & 98088314 & -42.40963059 & 10.09823013 & -4.199709262 & 7.85E-05 \\
\hline 86 & cg00913912 & 2 & 153282173 & -49.48829303 & 12.49126144 & -3.961833099 & 1.79E-04 \\
\hline 87 & cg26545307 & 2 & 141958753 & -117.4407146 & 31.9717525 & -3.67326485 & 4.69E-04 \\
\hline 88 & cg16596716 & 2 & 75825456 & 65.37845586 & 17.20697215 & 3.799532846 & 3.09E-04 \\
\hline 89 & cg03122917 & 3 & 130073646 & -40.10117636 & 9.356188932 & -4.286058848 & 5.78E-05 \\
\hline 90 & cg07522285 & 3 & 14929139 & -47.46746089 & 11.93946621 & -3.975676974 & $1.71 \mathrm{E}-04$ \\
\hline 91 & cg26033238 & 2 & 121231363 & -37.2249777 & 7.906584494 & -4.708098387 & $1.25 \mathrm{E}-05$ \\
\hline 92 & cg12914151 & 17 & 62309147 & -72.954197 & 19.39169508 & -3.762136147 & $3.50 \mathrm{E}-04$ \\
\hline 93 & cg01039664 & 16 & 69027363 & 60.69854053 & 15.8090411 & 3.839482744 & $2.70 \mathrm{E}-04$ \\
\hline 94 & cg11976592 & 6 & 76078187 & -42.50241174 & 10.32768232 & -4.115387209 & 1.05E-04 \\
\hline 95 & cg23501964 & 20 & 19866810 & -47.76817827 & 12.05803804 & -3.961521609 & 1.79E-04 \\
\hline 96 & cg11375836 & 3 & 43896529 & 65.62270014 & 17.40865841 & 3.769543786 & $3.41 \mathrm{E}-04$ \\
\hline 97 & cg07274676 & 6 & 28232729 & -58.94546216 & 15.39157061 & -3.829723662 & $2.79 \mathrm{E}-04$ \\
\hline 98 & $\operatorname{cg} 15865113$ & 14 & 85853478 & -45.55454077 & 11.43277763 & -3.984555832 & 1.65E-04 \\
\hline 99 & cg06698686 & 13 & 49796489 & -61.54837155 & 16.1837455 & -3.803098087 & 3.05E-04 \\
\hline 100 & cg03964554 & 17 & 59521084 & -41.31063879 & 9.970708664 & -4.143199865 & $9.56 \mathrm{E}-05$ \\
\hline
\end{tabular}

Displayed for each ranked probe is the chromosomal location and position (Ensembl GRCh37 assembly), the regression estimate (RE) for the Braak stage-associated analysis, the standard error (SE), the t-statistics, the accompanying $p$ values, the Illumina gene annotation (UCSC annotation), the gene feature (TSS1500, 200 to 1500 nucleotides (nt) upstream of transcription start site (TSS); TSS200, up to 200 nt upstream of TSS; 5'UTR, 5'untranslated region; Body, gene body; 3'UTR, 3' untranslated region) and the cytosine-phosphate-guanine ( $\mathrm{CpG})$ island feature. Probes are ranked based on a combined $p$ value (cut-off $=p<0.001$ ) and regression estimate ranking. 


\begin{tabular}{|c|c|c|}
\hline UCSC annotation & Gene feature & $\mathrm{CpG}$ island feature \\
\hline LOC374443 & TSS1500 & Northern shore \\
\hline PIGL & Body & \\
\hline PHYHIPL; PHYHIPL; PHYHIPL & 5'UTR; Body; 1st exon & Southern shore \\
\hline TBL1XR1 & Body & \\
\hline ALPL;ALPL & 5'UTR; TSS1500 & \\
\hline VWC2 & Body & Island \\
\hline ASPH; ASPH; ASPH; ASPH; ASPH; ASPH; ASPH; ASPH; ASPH & Body; Body; Body; Body; Body; Body; Body; Body; Bod & \\
\hline MSH3 & Body & \\
\hline IPMK & Body & \\
\hline LOC440925; SP5 & TSS1500; Body & Island \\
\hline \multirow[t]{2}{*}{ SH3RF1 } & Body & \\
\hline & & Northern shore \\
\hline MICAL3; MICAL3 & Body; Body & \\
\hline \multirow[t]{2}{*}{ EFEMP2 } & TSS1500 & Island \\
\hline & & Northern shelf \\
\hline HES1 & TSS200 & Northern shore \\
\hline ANKAR & Body & $\begin{array}{l}\text { Southern shelf } \\
\text { Island }\end{array}$ \\
\hline SELS; SELS & TSS1500; TSS1500 & Southern shore \\
\hline MTHFD2L & Body & \\
\hline LOC100294145; LOC100294145 & Body; Body & Southern shore \\
\hline DPYD & Body & \\
\hline FMNL2 & Body & \\
\hline LRP1B & Body & \\
\hline COL6A5; COL6A5; COL6A5 & 5'UTR; 5'UTR; Body & \\
\hline FGD5 & Body & \\
\hline TEX2; TEX2; TEX2 & 5'UTR; 5'UTR; TSS1500 & \\
\hline TANGO6 & Body & \\
\hline \multirow[t]{2}{*}{ FILIP1 } & Body & \\
\hline & & Northern shelf \\
\hline MLNR & Body & Southern shore \\
\hline
\end{tabular}


Supplementary Table 5. Top 100 ranked differentially methylated positions (DMPs)

in the locus coeruleus (LC)

\begin{tabular}{|c|c|c|c|c|c|c|c|}
\hline Rank & Probe & Chromosome & Position & RE & SE & $t$ & $p$ value \\
\hline 1 & cg19265143 & 1 & 28907637 & -235.7018337 & 46.86845394 & -5.029008083 & $3.74 \mathrm{E}-06$ \\
\hline 2 & cg06793267 & 3 & 57993846 & -367.1921366 & 80.20683065 & -4.578065654 & 2.02E-05 \\
\hline 3 & cg27254871 & 2 & 206750458 & -193.7961418 & 41.95441751 & -4.619207065 & $1.73 \mathrm{E}-05$ \\
\hline 4 & cg08946575 & 1 & 85931072 & -84.90253179 & 18.79217286 & -4.517973116 & 2.51E-05 \\
\hline 5 & cg19108372 & 1 & 183605267 & -224.8127272 & 53.59715679 & -4.194489795 & 7.99E-05 \\
\hline 6 & $\operatorname{cg} 04845871$ & 1 & 207996319 & -64.61917125 & 14.19566479 & -4.552035582 & 2.22E-05 \\
\hline 7 & cg00930873 & 15 & 58357973 & -64.81381417 & 14.51794997 & -4.464391619 & 3.05E-05 \\
\hline 8 & cg01805869 & 2 & 26915772 & -172.2016798 & 41.58645181 & -4.140812025 & 9.64E-05 \\
\hline 9 & cg27277034 & 3 & 40575533 & -59.99136991 & 13.45651713 & -4.45816472 & $3.12 \mathrm{E}-05$ \\
\hline 10 & cg20670037 & 19 & 58639808 & -62.11879931 & 14.39166923 & -4.316302598 & 5.19E-05 \\
\hline 11 & cg09279544 & 19 & 8674388 & -279.4581039 & 69.29420427 & -4.032921755 & 1.40E-04 \\
\hline 12 & cg24396624 & 3 & 25469860 & -66.56853228 & 15.8708946 & -4.19437807 & 7.99E-05 \\
\hline 13 & ch.17.2065773F & 17 & 76200219 & 54.81949247 & 12.70372863 & 4.315228548 & $5.21 \mathrm{E}-05$ \\
\hline 14 & cg07499372 & 19 & 57631232 & 53.70540771 & 12.50503602 & 4.294702358 & $5.61 \mathrm{E}-05$ \\
\hline 15 & cg25376657 & 17 & 16229039 & 54.96947487 & 12.99957845 & 4.228558264 & 7.09E-05 \\
\hline 16 & cg12862167 & 14 & 91752171 & 49.77703955 & 11.53716524 & 4.31449481 & 5.23E-05 \\
\hline 17 & $\operatorname{cg} 16474684$ & 4 & 2470049 & 70.66200586 & 17.17760787 & 4.113611533 & $1.06 \mathrm{E}-04$ \\
\hline 18 & cg15054586 & 19 & 50194696 & -329.2565526 & 84.40657547 & -3.90084008 & $2.20 \mathrm{E}-04$ \\
\hline 19 & cg12790874 & 13 & 100310405 & -211.1199738 & 53.73915257 & -3.928606308 & $2.00 \mathrm{E}-04$ \\
\hline 20 & cg23968184 & 2 & 196438113 & 67.56868886 & 16.71459583 & 4.042496125 & 1.36E-04 \\
\hline 21 & cg00558156 & 8 & 23261618 & -53.37163384 & 12.90505376 & -4.135715732 & 9.82E-05 \\
\hline 22 & cg00785478 & 1 & 120604399 & -40.41689339 & 9.457338232 & -4.273601345 & $6.05 \mathrm{E}-05$ \\
\hline 23 & cg09732027 & 6 & 29984811 & 62.57170533 & 15.38955171 & 4.06585627 & $1.25 \mathrm{E}-04$ \\
\hline 24 & $\operatorname{cg} 22541679$ & 13 & 78493666 & -322.4609998 & 83.45555127 & -3.863865194 & $2.49 \mathrm{E}-04$ \\
\hline 25 & cg06059849 & 1 & 109656930 & -92.65139737 & 23.5783908 & -3.929504695 & $2.00 \mathrm{E}-04$ \\
\hline 26 & cg18643191 & 17 & 20465437 & 55.24290442 & 13.70432539 & 4.031056098 & $1.41 \mathrm{E}-04$ \\
\hline 27 & cg18572198 & 5 & 5050553 & 70.39127589 & 17.80383805 & 3.953713559 & $1.84 \mathrm{E}-04$ \\
\hline 28 & cg21295467 & 11 & 123986166 & -294.3159433 & 76.5772297 & -3.843387184 & 2.67E-04 \\
\hline 29 & cg08410015 & 10 & 119862094 & -55.33454187 & 13.77927176 & -4.015781302 & 1.49E-04 \\
\hline 30 & cg02184543 & 10 & 7226492 & -32.95182337 & 7.299555856 & -4.51422306 & $2.55 \mathrm{E}-05$ \\
\hline 31 & cg02759617 & 8 & 88746918 & 53.88857599 & 13.42575357 & 4.013821325 & $1.50 \mathrm{E}-04$ \\
\hline 32 & cg00242449 & 17 & 7340872 & -33.75850906 & 7.795128071 & -4.330718976 & 4.93E-05 \\
\hline 33 & cg21484213 & 2 & 142888868 & -98.14687522 & 25.23726021 & -3.888967123 & $2.29 \mathrm{E}-04$ \\
\hline 34 & cg16739342 & 10 & 12221327 & -66.21527713 & 16.80114786 & -3.941116267 & 1.92E-04 \\
\hline 35 & cg00573948 & 7 & 101330867 & -32.8866926 & 7.491535447 & -4.389846759 & $3.99 \mathrm{E}-05$ \\
\hline 36 & cg23128263 & 1 & 66797473 & -69.74425212 & 17.84475114 & -3.908390293 & $2.14 \mathrm{E}-04$ \\
\hline 37 & cg13497069 & 6 & 29426119 & 36.02036865 & 8.650016905 & 4.16419633 & 8.89E-05 \\
\hline 38 & cg01072259 & 2 & 37946508 & 47.18252359 & 11.79960913 & 3.998651401 & $1.58 \mathrm{E}-04$ \\
\hline 39 & cg25533997 & 18 & 35144259 & -29.25234088 & 6.447789117 & -4.53680174 & $2.35 \mathrm{E}-05$ \\
\hline 40 & cg21211367 & 2 & 162094118 & -143.3632098 & 37.53075096 & -3.819886522 & $2.89 \mathrm{E}-04$ \\
\hline 41 & cg25699851 & 15 & 68624713 & 28.10427811 & 5.69458373 & 4.935264707 & 5.33E-06 \\
\hline 42 & cg14417711 & 11 & 6478126 & 184.2694812 & 48.45989271 & 3.802515252 & 3.06E-04 \\
\hline 43 & cg21032945 & 5 & 5652728 & 56.07724425 & 14.32439628 & 3.914806821 & $2.10 \mathrm{E}-04$ \\
\hline 44 & cg10960253 & 1 & 92296137 & -47.43755101 & 11.97918937 & -3.959996754 & $1.80 \mathrm{E}-04$ \\
\hline 45 & cg25565383 & 2 & 17699773 & -311.8402925 & 82.43573141 & -3.782829207 & 3.27E-04 \\
\hline 46 & cg14678430 & 7 & 128494150 & 36.45977698 & 8.930030378 & 4.082827878 & $1.18 \mathrm{E}-04$ \\
\hline 47 & cg02091786 & 11 & 62415525 & 29.13592149 & 6.650703005 & 4.380878453 & 4.12E-05 \\
\hline 48 & cg27408178 & 4 & 108974899 & 35.17052961 & 8.524264859 & 4.125931114 & $1.02 \mathrm{E}-04$ \\
\hline 49 & cg04331204 & 8 & 143835180 & -38.0223288 & 9.38773439 & -4.050213525 & 1.32E-04 \\
\hline 50 & cg25840838 & 11 & 47488535 & -32.2135785 & 7.689176559 & -4.189470518 & 8.13E-05 \\
\hline 51 & cg00298218 & 1 & 83955790 & 27.65290416 & 5.605105241 & 4.933520956 & 5.37E-06 \\
\hline 52 & cg10516989 & 16 & 2868780 & 50.4374971 & 12.88219073 & 3.915288801 & $2.09 \mathrm{E}-04$ \\
\hline
\end{tabular}




\begin{tabular}{|c|c|c|}
\hline UCSC annotation & Gene feature & $\mathrm{CpG}$ island feature \\
\hline SNHG12; SNORA44; SNORA16A; SNORA61 & Body; TSS1500; TSS200; TSS1500 & Northern shore \\
\hline FLNB; FLNB; FLNB; FLNB & TSS1500; TSS1500; TSS1500; TSS1500 & Island \\
\hline DDAH1; DDAH1 & TSS200; 5'UTR & Island \\
\hline ARPC5; ARPC5; RGL1; RGL1; RGL1; RGL1; RGL1; RGL1 & $\begin{array}{l}\text { TSS200; TSS200; 1st exon; 1st exon; 1st exon; } \\
\text { 5'UTR; 5'UTR; 5'UTR }\end{array}$ & Island \\
\hline ALDH1A2; ALDH1A2 & TSS200; TSS200 & Island \\
\hline KCNK3 & 1stexon & Island \\
\hline ZNF621; ZNF621 & 3'UTR; 3'UTR & \\
\hline ZNF329 & Body & \\
\hline ADAMTS10 & 5'UTR & Island \\
\hline RARB; RARB; RARB; RARB & 5'UTR; 1st exon; 1st exon; 5'UTR & \\
\hline AFMID; AFMID & Body; Body & \\
\hline USP29 & TSS1500 & Southern shore \\
\hline PIGL & Body & \\
\hline CCDC88C & Body & Northern shelf \\
\hline RNF4 & TSS1500 & Northern shore \\
\hline СРT1C; СРT1C; СРT1C; CPT1C; СPT1C & 5'UTR; 5'UTR; 5'UTR; 5'UTR; Body & Island \\
\hline CLYBL & Body & Island \\
\hline LOXL2; LOXL2 & 5'UTR; 1stexon & Island \\
\hline NOTCH2; NOTCH2 & Body; Body & \\
\hline NCRNA00171 & Body & \\
\hline EDNRB; EDNRB; EDNRB & TSS1500; 5'UTR; TSS1500 & Southern shore \\
\hline KIAA1324; C1orf194 & 1stexon; TSS1500 & Southern shore \\
\hline LINC01020 & Body & \\
\hline VWA5A; VWA5A; VWA5A; VWA5A; VWA5A; VWA5A & 1st exon; 5'UTR; 1st exon; 5'UTR; 5'UTR; 1st exon & \\
\hline CASC2; CASC2 & Body; Body & \\
\hline SFMBT2; SFMBT2 & Body; Body & \\
\hline FGF11; TMEM102; FGF11 & TSS1500; 3'UTR; TSS1500 & Island \\
\hline LRP1B; LRP1B & 5'UTR; 1stexon & Southern shore \\
\hline NUDT5 & Body & \\
\hline & & Northern shore \\
\hline PDE4B; PDE4B; PDE4B; PDE4B; PDE4B; PDE4B & TSS1500; Body; Body; Body; Body; Body & \\
\hline $\mathrm{OR} 2 \mathrm{H} 1$ & TSS200 & \\
\hline BRUNOL4; BRUNOL4; BRUNOL4; BRUNOL4 & Body; Body; Body; Body & Northern shore \\
\hline & & Northern shore \\
\hline ITGA11 & Body & \\
\hline TRIM3; TRIM3 & Body; Body & Island \\
\hline $\begin{array}{l}\text { TGFBR3; TGFBR3; TGFBR3; TGFBR3 } \\
\text { RAD51AP2 }\end{array}$ & $\begin{array}{l}\text { Body; Body; Body; Body } \\
\text { TSS200 }\end{array}$ & \\
\hline FLNC; FLNC & Body; Body & Island \\
\hline GANAB; GANAB; INTS5 & TSS1500; TSS1500; Body & Southern shore \\
\hline LEF1; LEF1; LEF1; LEF1 & 3'UTR; 3'UTR; 3'UTR; Body & \\
\hline LYPD2 & TSS1500 & \\
\hline CELF1; CELF1; CELF1; CELF1; CELF1 & 3'UTR; 3'UTR; 3'UTR; 3'UTR; 3'UTR & \\
\hline PRSS21; PRSS21; PRSS21; PRSS21; PRSS21 & Body; Body; Body; Body; Body & Southern shore \\
\hline
\end{tabular}


Supplementary Table 5. (Continued)

\begin{tabular}{|c|c|c|c|c|c|c|c|}
\hline Rank & Probe & Chromosome & Position & $\mathrm{RE}$ & SE & $t$ & $p$ value \\
\hline 53 & cg17191367 & 1 & 50570612 & 28.94258485 & 6.656804725 & 4.347819419 & 4.64E-05 \\
\hline 54 & cg17085352 & 12 & 54332026 & -62.96593443 & 16.33749869 & -3.854074337 & $2.57 \mathrm{E}-04$ \\
\hline 55 & cg09484243 & 5 & 134463743 & 43.387776 & 10.97064548 & 3.954897283 & $1.83 \mathrm{E}-04$ \\
\hline 56 & cg14881567 & 19 & 16583117 & 129.2161761 & 34.0487605 & 3.795033187 & 3.14E-04 \\
\hline 57 & cg12816995 & 18 & 76699288 & 27.70430241 & 6.102116927 & 4.540113331 & 2.32E-05 \\
\hline 58 & cg02853268 & 11 & 1969721 & -31.42722217 & 7.518185669 & -4.180160421 & $8.40 \mathrm{E}-05$ \\
\hline 59 & cg16871328 & 16 & 57622905 & -36.33740557 & 9.009604709 & -4.033185333 & 1.40E-04 \\
\hline 60 & $\operatorname{cg} 09377423$ & 6 & 37751308 & -57.32538259 & 14.87334707 & -3.854235522 & $2.57 \mathrm{E}-04$ \\
\hline 61 & cg00171785 & 11 & 6044373 & -109.3159005 & 28.79988089 & -3.795706687 & 3.13E-04 \\
\hline 62 & cg01645899 & 12 & 2996093 & -27.647868999 & 6.080748878 & -4.546786842 & $2.26 \mathrm{E}-05$ \\
\hline 63 & $\operatorname{cg} 00789116$ & 10 & 120761718 & 27.17464154 & 5.720713246 & 4.750219138 & $1.07 \mathrm{E}-05$ \\
\hline 64 & cg10639981 & 19 & 50762343 & -28.40608855 & 6.540712145 & -4.342965708 & 4.72E-05 \\
\hline 65 & cg01961069 & 16 & 67189629 & -241.9642251 & 64.3185657 & -3.761965499 & 3.50E-04 \\
\hline 66 & cg25813748 & 13 & 103518224 & 34.72788897 & 8.537891523 & 4.06750178 & 1.24E-04 \\
\hline 67 & cg21931174 & 14 & 20977318 & 36.06546074 & 8.952903048 & 4.02835377 & 1.42E-04 \\
\hline 68 & cg03783039 & 4 & 184401720 & -39.36036334 & 9.9391724 & -3.960124823 & $1.80 \mathrm{E}-04$ \\
\hline 69 & cg05106686 & 12 & 68738010 & -31.83845792 & 7.709244681 & -4.129906267 & $1.00 \mathrm{E}-04$ \\
\hline 70 & cg20740028 & 7 & 28450406 & -264.5634476 & 70.82354554 & -3.735529555 & 3.82E-04 \\
\hline 71 & cg08351753 & 20 & 43408177 & 32.70812488 & 8.003467729 & 4.086744145 & 1.16E-04 \\
\hline 72 & cg18371836 & 10 & 122553700 & -25.81279625 & 4.673452504 & -5.523282033 & $5.48 \mathrm{E}-07$ \\
\hline 73 & cg05889864 & 6 & 122972445 & 33.22023588 & 8.159772074 & 4.071221056 & $1.23 \mathrm{E}-04$ \\
\hline 74 & cg09591921 & 16 & 24126487 & 26.60000141 & 5.82456101 & 4.566868021 & $2.10 \mathrm{E}-05$ \\
\hline 75 & cg14695920 & 9 & 94997513 & -44.76674805 & 11.50936992 & -3.889591554 & $2.28 \mathrm{E}-04$ \\
\hline 76 & cg18537222 & 3 & 12435731 & 35.10770195 & 8.79050441 & 3.993821095 & $1.60 \mathrm{E}-04$ \\
\hline 77 & cg09295258 & 11 & 119274583 & 27.03969421 & 6.224598626 & 4.344006069 & $4.71 \mathrm{E}-05$ \\
\hline 78 & $\operatorname{cg} 08114611$ & 5 & 94172463 & 48.2995411 & 12.5810682 & 3.839065201 & $2.71 \mathrm{E}-04$ \\
\hline 79 & cg04393637 & 17 & 75790091 & 33.39864697 & 8.333363452 & 4.007823151 & 1.53E-04 \\
\hline 80 & cg16093863 & 3 & 144393272 & 37.7990054 & 9.686043352 & 3.902419597 & $2.19 \mathrm{E}-04$ \\
\hline 81 & cg07615572 & 17 & 7405889 & -30.85115139 & 7.587855595 & -4.065859057 & 1.25E-04 \\
\hline 82 & cg18754985 & 3 & 98237750 & 24.79688456 & 5.168837552 & 4.79738129 & 8.96E-06 \\
\hline 83 & cg23845609 & 11 & 47609357 & -25.42658417 & 5.674457124 & -4.480884007 & 2.87E-05 \\
\hline 84 & cg12289867 & 10 & 1623710 & -25.42325971 & 5.673408822 & -4.481125988 & 2.87E-05 \\
\hline 85 & cg10434137 & 13 & 44032630 & 27.7377438 & 6.66412008 & 4.1622515 & 8.95E-05 \\
\hline 86 & cg13063967 & 12 & 12418943 & -98.24067657 & 26.41868415 & -3.718605969 & 4.04E-04 \\
\hline 87 & cg26911948 & 12 & 2157278 & 66.72515328 & 17.72825916 & 3.76377357 & $3.48 \mathrm{E}-04$ \\
\hline 88 & cg25046661 & 3 & 151839485 & 24.50125959 & 4.935566696 & 4.964224191 & $4.78 \mathrm{E}-06$ \\
\hline 89 & cg03002059 & 8 & 11402647 & -30.9327288 & 7.717597682 & -4.008077393 & $1.53 \mathrm{E}-04$ \\
\hline 90 & cg13221907 & 6 & 170755442 & 36.24851567 & 9.313280975 & 3.892131652 & 2.26E-04 \\
\hline 91 & cg17587467 & 11 & 64652643 & 41.45384106 & 10.80850257 & 3.83529918 & $2.74 \mathrm{E}-04$ \\
\hline 92 & cg04572695 & 6 & 100441722 & -166.5267694 & 45.06486369 & -3.69526846 & $4.36 \mathrm{E}-04$ \\
\hline 93 & cg22917346 & 13 & 44453235 & -224.195738 & 60.77127164 & -3.689173057 & $4.45 \mathrm{E}-04$ \\
\hline 94 & cg15052158 & 10 & 131570747 & -28.36596078 & 6.955801982 & -4.078028796 & 1.20E-04 \\
\hline 95 & cg18451156 & 12 & 133424373 & -24.3256532 & 5.241503931 & -4.640968225 & $1.60 \mathrm{E}-05$ \\
\hline 96 & cg25396488 & 6 & 29641118 & 33.03594372 & 8.394943278 & 3.935219408 & $1.96 \mathrm{E}-04$ \\
\hline 97 & cg21737243 & 7 & 99012691 & -26.66290258 & 6.366083733 & -4.188273938 & 8.17E-05 \\
\hline 98 & cg23164993 & 1 & 65613294 & -408.3883075 & 111.4238386 & -3.665178948 & 4.81E-04 \\
\hline 99 & cg12898370 & 12 & 24737779 & 31.58801994 & 8.005029959 & 3.946021452 & $1.89 \mathrm{E}-04$ \\
\hline 100 & $\operatorname{cg} 08554039$ & 15 & 40262416 & -25.67337457 & 6.074517404 & -4.226405632 & 7.14E-05 \\
\hline
\end{tabular}

Displayed for each ranked probe is the chromosomal location and position (Ensembl GRCh37 assembly), the regression estimate (RE) for the Braak stage-associated analysis, the standard error (SE), the t-statistics, the accompanying $p$ values, the Illumina gene annotation (UCSC annotation), the gene feature (TSS1500, 200 to 1500 nucleotides (nt) upstream of transcription start site (TSS); TSS200, up to 200 nt upstream of TSS; 5 'UTR, 5'untranslated region; Body, gene body; 3'UTR, 3' untranslated region) and the cytosine-phosphate-guanine (CpG) island feature. Probes are ranked based on a combined $p$ value (cut-off $=p<0.001$ ) and regression estimate ranking. 


\begin{tabular}{|c|c|c|}
\hline UCSC annotation & Gene feature & CpG island feature \\
\hline ELAVL4; ELAVL4; ELAVL4 & TSS1500; Body; Body & \\
\hline HOXC13 & TSS1500 & Northern shore \\
\hline C5orf66 & 5'UTR & \\
\hline EPS15L1 & TSS1500 & Island \\
\hline MRPL23 & Body & Southern shore \\
\hline RHNO1; RHN01; RHNO1; RHN01; RHN01; RHNO1; RHNO1 & Body; Body; Body; Body; Body; Body; Body & Northern shelf \\
\hline MYH14; MYH14; MYH14 & Body; Body; Body & Island \\
\hline TRADD & Body & Island \\
\hline BIVM-ERCC5; ERCC5 & Body; Body & \\
\hline \multirow[t]{2}{*}{ RNASE10 } & TSS1500 & \\
\hline & & Northern shelf \\
\hline CREB5 & 5'UTR & Southern shore \\
\hline RIMS4; RIMS4 & Body; Body & \\
\hline PKIB; PKIB; PKIB; PKIB; PKIB; PKIB & TSS1500; 5'UTR; 5'UTR; 5'UTR; 5'UTR; 5'UTR & \\
\hline PRKCB; PRKCB & Body; Body & \\
\hline IARS; IARS; IARS & Body; Body; Body & \\
\hline PPARG; PPARG; PPARG; PPARG & Body; Body; Body; Body & \\
\hline USP2-AS1 & Body & \\
\hline \multirow[t]{2}{*}{ MCTP1; MCTP1; MCTP1 } & Body; Body; Body & \\
\hline & & Southern shore \\
\hline POLR2A & Body & \\
\hline CLDND1; CLDND1; CLDND1; CLDND1; CLDND1; CLDND1 & Body; Body; Body; Body; Body; Body & Northern shelf \\
\hline FAM180B & Body & Northern shore \\
\hline ADARB2 & Body & \\
\hline ENOX1-AS2; ENOX1; ENOX1; ENOX1 & Body; 5'UTR; 5'UTR; 5'UTR & \\
\hline LRP6 & Body & Northern shore \\
\hline CACNA1C-IT2 & TSS1500 & \\
\hline \multirow[t]{2}{*}{ BLK } & Body & \\
\hline & & Northern shore \\
\hline MCHR2-AS1; MCHR2; MCHR2 & TSS200; 5'UTR; 5'UTR & Island \\
\hline CCDC122; C13orf31; C13orf31 & 5'UTR; TSS200; TSS1500 & Island \\
\hline CHFR; CHFR; CHFR; CHFR; CHFR & Body; Body; Body; Body; Body & \\
\hline ZFP57 & Body & \\
\hline BUD31 & Body & \\
\hline AK3L1; AK3L1; AK3L1; AK3L1 & 5'UTR; TSS1500; TSS1500; 1st exon & Island \\
\hline C12orf67 & TSS1500 & \\
\hline EIF2AK4 & Body & \\
\hline
\end{tabular}


Supplementary Table 6. Top 100 ranked differentially hydroxymethylated positions (DHPs) in the locus coeruleus (LC)

\begin{tabular}{|c|c|c|c|c|c|c|c|}
\hline Rank & Probe & Chromosome & Position & RE & SE & $t$ & $p$ value \\
\hline 1 & $\operatorname{cg} 00053361$ & 8 & 127250180 & -62.14971407 & 14.05520712 & -4.421828406 & $3.56 \mathrm{E}-05$ \\
\hline 2 & cg23968184 & 2 & 196438113 & -66.51323794 & 15.31426896 & -4.343219914 & 4.72E-05 \\
\hline 3 & cg19591003 & 14 & 94392757 & -47.05792749 & 9.696387631 & -4.853140085 & 7.27E-06 \\
\hline 4 & $\operatorname{cg} 04994405$ & 1 & 65731836 & 57.21702512 & 13.038981 & 4.388151584 & 4.02E-05 \\
\hline 5 & cg00347713 & 15 & 81680130 & -45.2902628 & 9.862030362 & -4.592387281 & $1.91 \mathrm{E}-05$ \\
\hline 6 & cg13020390 & 1 & 88837555 & 64.27111371 & 15.11960286 & 4.250846686 & $6.55 \mathrm{E}-05$ \\
\hline 7 & cg13137032 & 4 & 5904897 & 61.45395872 & 14.65213362 & 4.194198629 & $8.00 \mathrm{E}-05$ \\
\hline 8 & cg18133284 & 3 & 63032108 & -44.69904089 & 10.10122842 & -4.425109404 & 3.52E-05 \\
\hline 9 & cg27408178 & 4 & 108974899 & -40.90539965 & 8.854027427 & -4.619976613 & $1.73 \mathrm{E}-05$ \\
\hline 10 & cg03872724 & 17 & 60881608 & 40.38068035 & 8.723718777 & 4.628837929 & 1.67E-05 \\
\hline 11 & cg18643191 & 17 & 20465437 & -50.04115337 & 11.99579853 & -4.171556671 & 8.66E-05 \\
\hline 12 & cg08351753 & 20 & 43408177 & -41.20634115 & 9.629172143 & -4.279323346 & 5.92E-05 \\
\hline 13 & cg18308985 & 5 & 5239937 & 51.71313803 & 12.69859474 & 4.07235124 & $1.22 \mathrm{E}-04$ \\
\hline 14 & cg06715134 & 8 & 62357092 & 60.08016235 & 15.01863943 & 4.000373178 & 1.57E-04 \\
\hline 15 & cg25913233 & 5 & 151066683 & 39.54313266 & 9.468754256 & 4.176170549 & 8.52E-05 \\
\hline 16 & cg07049329 & 19 & 52209605 & 43.56052162 & 10.77715067 & 4.041933064 & 1.36E-04 \\
\hline 17 & cg17709286 & 8 & 32852907 & -39.83607065 & 9.737896245 & -4.090829235 & 1.15E-04 \\
\hline 18 & cg04191452 & 10 & 29528675 & -36.50578834 & 8.70631771 & -4.193022763 & 8.03E-05 \\
\hline 19 & cg02184543 & 10 & 7226492 & 32.40413226 & 7.232208286 & 4.480530839 & 2.88E-05 \\
\hline 20 & cg24911283 & 4 & 24780369 & -37.7015075 & 9.225220948 & -4.086786399 & 1.16E-04 \\
\hline 21 & cg08706907 & 11 & 46696158 & 33.60668714 & 8.036142247 & 4.181942791 & 8.35E-05 \\
\hline 22 & cg08410015 & 10 & 119862094 & 44.90897724 & 11.33739371 & 3.961137665 & 1.79E-04 \\
\hline 23 & cg00034101 & 1 & 40098595 & 30.40626474 & 7.01380785 & 4.335200706 & 4.86E-05 \\
\hline 24 & cg23036065 & 15 & 55972380 & 34.36221073 & 8.393401393 & 4.093955374 & $1.13 \mathrm{E}-04$ \\
\hline 25 & cg14695920 & 9 & 94997513 & 40.30693139 & 10.11286175 & 3.985709723 & 1.65E-04 \\
\hline 26 & cg12588208 & 3 & 119547934 & 28.62084821 & 5.96043224 & 4.801807496 & 8.81E-06 \\
\hline 27 & cg16502726 & 16 & 75282608 & 40.85075805 & 10.29850026 & 3.966670585 & 1.76E-04 \\
\hline 28 & cg01420298 & 5 & 88324824 & -32.09452702 & 7.774233964 & -4.128320188 & $1.01 \mathrm{E}-04$ \\
\hline 29 & cg02475777 & 4 & 1388615 & 45.81881161 & 11.71131765 & 3.912353246 & $2.11 \mathrm{E}-04$ \\
\hline 30 & cg19258310 & 11 & 13951237 & 42.16608493 & 10.67024838 & 3.951743524 & $1.85 \mathrm{E}-04$ \\
\hline 31 & cg26746027 & 4 & 94124976 & 28.36632269 & 5.95462526 & 4.763746071 & $1.02 E-05$ \\
\hline 32 & cg12289867 & 10 & 1623710 & 30.56563658 & 7.306479245 & 4.183360488 & 8.31E-05 \\
\hline 33 & cg03879902 & 19 & 46171872 & 59.86239595 & 15.46346373 & 3.871215208 & 2.43E-04 \\
\hline 34 & cg01890546 & 7 & 884588 & -30.90224935 & 7.488220324 & -4.126781533 & $1.01 \mathrm{E}-04$ \\
\hline 35 & cg05693680 & 19 & 3022636 & 48.39310715 & 12.43730292 & 3.890964743 & 2.27E-04 \\
\hline 36 & cg02031359 & 5 & 178367423 & 28.87092462 & 6.644622264 & 4.345006152 & 4.69E-05 \\
\hline 37 & cg01624229 & 2 & 153252785 & -29.58032349 & 7.028077393 & -4.20887845 & 7.60E-05 \\
\hline 38 & cg11750696 & 1 & 7074500 & -28.09926793 & 6.18275001 & -4.544784745 & $2.28 \mathrm{E}-05$ \\
\hline 39 & cg02600434 & 2 & 30924426 & 43.96823488 & 11.27687382 & 3.898973739 & $2.21 \mathrm{E}-04$ \\
\hline 40 & $\operatorname{cg} 23417011$ & 5 & 93077375 & -34.12626414 & 8.502965648 & -4.013454312 & $1.50 \mathrm{E}-04$ \\
\hline 41 & cg21737243 & 7 & 99012691 & 27.25505045 & 5.827143062 & 4.677257819 & $1.40 \mathrm{E}-05$ \\
\hline 42 & cg06428091 & 16 & 3340853 & -41.94721416 & 10.77411988 & -3.893330928 & $2.26 \mathrm{E}-04$ \\
\hline 43 & cg13083079 & 5 & 55075960 & 28.23617338 & 6.580469458 & 4.29090562 & 5.69E-05 \\
\hline 44 & cg07499372 & 19 & 57631232 & -55.63457183 & 14.61732749 & -3.806070013 & 3.02E-04 \\
\hline 45 & cg15924582 & 14 & 102356845 & -26.59707986 & 5.852220919 & -4.544783976 & $2.28 \mathrm{E}-05$ \\
\hline 46 & cg00318643 & 16 & 89105494 & -29.05324115 & 7.038288921 & -4.127884132 & $1.01 \mathrm{E}-04$ \\
\hline 47 & cg17232722 & 19 & 3178607 & 27.10900971 & 6.201796245 & 4.371154524 & 4.27E-05 \\
\hline 48 & cg05380156 & 17 & 76141571 & 27.45102034 & 6.363901012 & 4.313552377 & $5.25 \mathrm{E}-05$ \\
\hline 49 & cg20774846 & 8 & 105479420 & 25.59855616 & 5.021375655 & 5.09791697 & 2.87E-06 \\
\hline 50 & cg04950623 & 22 & 28102227 & 34.1688803 & 8.622267932 & 3.962864593 & $1.78 \mathrm{E}-04$ \\
\hline 51 & cg07615572 & 17 & 7405889 & 28.99391814 & 7.080709016 & 4.094776112 & 1.13E-04 \\
\hline 52 & cg03002059 & 8 & 11402647 & 38.2502259 & 9.829393519 & 3.891412611 & 2.27E-04 \\
\hline 53 & cg14596205 & 11 & 19232715 & -34.06238384 & 8.617291264 & -3.95279477 & $1.84 \mathrm{E}-04$ \\
\hline
\end{tabular}


FAM181A; C14orf86

DNAJC6

TMC3-AS1

LEF1; LEF1; LEF1; LEF1

MARCH10; MARCH10

RIMS4; RIMS4

ADAMTS16

CLVS1

SPARC

SFMBT2; SFMBT2

KIAA0652; KIAA0652; KIAA0652; KIAA0652; KIAA0652

CASC2; CASC2

HEYL

PRTG

IARS; IARS; IARS

GSK3B; GSK3B

BCAR1; BCAR1; BCAR1; BCAR1; BCAR1; BCAR1; BCAR1; BCAR1 MEF2C-AS1

CRIPAK

GRID2; GRID2

ADARB2

GIPR

UNC84A; UNC84A

TLE2; TLE2; TLE2; TLE2

ZNF454

FMNL2

CAMTA1

FAM172A; FAM172A; POU5F2; FAM172A; FAM172A

BUD31

ZNF263

DDX4; DDX4; DDX4; DDX4

USP29

PPP2R5C; PPP2R5C; PPP2R5C; PPP2R5C; PPP2R5C

\section{S1PR4}

C17orf99

DPYS

POLR2A

BLK

CSRP3
Body; TSS200

Body

Body

Island

3'UTR; 3'UTR; 3'UTR; Body

5'UTR; 5'UTR

Northern shelf

Body; Body

Body

Body

TSS200

Southern shore

Body; Body

Body; 3'UTR; Body; Body; 3'UTR

Body; Body

Body

Body

Body; Body; Body

Body; Body

TSS1500; Body; Body; Body; Body; Body; Body; $\quad$ Northern shelf Body; Body

1stexon

Island

Body; Body

Body

5'UTR

Body; Body

Body; Body; Body; Body

TSS1500

Body

Body

Body; Body; TSS200; Body; Body

Body

3'UTR

Body; Body; Body; Body

TSS1500

Body; Body; Body; Body; Body

Southern shore Northern shore Northern shelf Northern shore

TSS200

TSS1500

TSS200

Southern shore
Body

Body

TSS1500 
Supplementary Table 6. (Continued)

\begin{tabular}{|c|c|c|c|c|c|c|c|}
\hline Rank & Probe & Chromosome & Position & RE & SE & $t$ & $p$ value \\
\hline 54 & cg25183883 & 13 & 70654280 & 31.42976161 & 7.891096091 & 3.982939917 & 1.66E-04 \\
\hline 55 & $\operatorname{cg} 04224811$ & 3 & 55709442 & 29.07370373 & 7.156614987 & 4.06249376 & 1.27E-04 \\
\hline 56 & cg08904079 & 9 & 126181029 & 27.46584665 & 6.614950015 & 4.152086801 & 9.27E-05 \\
\hline 57 & cg06614044 & 18 & 42324631 & 44.07209995 & 11.58015661 & 3.805829355 & 3.03E-04 \\
\hline 58 & cg02162815 & 14 & 89305821 & 80.51471431 & 21.43933125 & 3.75546762 & $3.58 \mathrm{E}-04$ \\
\hline 59 & $\operatorname{cg} 10269110$ & 19 & 55873515 & 31.062356 & 7.830237617 & 3.966974889 & $1.76 \mathrm{E}-04$ \\
\hline 60 & cg09362918 & 7 & 147704677 & 31.95881912 & 8.0874878 & 3.951637382 & $1.85 \mathrm{E}-04$ \\
\hline 61 & cg23326197 & 7 & 99382370 & -28.22927427 & 6.919401279 & -4.079727873 & 1.19E-04 \\
\hline 62 & cg08935914 & 14 & 69387818 & 26.17251357 & 6.130816553 & 4.269009412 & $6.14 \mathrm{E}-05$ \\
\hline 63 & cg09225071 & 17 & 67980864 & 55.74253259 & 14.83106253 & 3.758498925 & $3.54 \mathrm{E}-04$ \\
\hline 64 & cg05871135 & 3 & 151159930 & -40.03770931 & 10.47061321 & -3.823817049 & $2.85 \mathrm{E}-04$ \\
\hline 65 & cg17250812 & 3 & 141460046 & 28.51927466 & 7.054347302 & 4.042794243 & 1.35E-04 \\
\hline 66 & cg18371836 & 10 & 122553700 & 24.22751075 & 4.568081571 & 5.303651079 & 1.30E-06 \\
\hline 67 & cg25699851 & 15 & 68624713 & -24.79701928 & 5.705778277 & -4.345948629 & 4.67E-05 \\
\hline 68 & cg02313829 & 11 & 75136574 & -65.49407814 & 17.56923038 & -3.727771607 & $3.92 \mathrm{E}-04$ \\
\hline 69 & $\operatorname{cg} 16009352$ & 6 & 126912363 & 65.02458051 & 17.44575751 & 3.727243169 & $3.93 \mathrm{E}-04$ \\
\hline 70 & cg03238482 & 17 & 75418673 & -27.53293062 & 6.82710136 & -4.032887337 & 1.40E-04 \\
\hline 71 & cg05131483 & 16 & 23706242 & -35.25830208 & 9.218119597 & -3.824890935 & $2.84 \mathrm{E}-04$ \\
\hline 72 & cg26684226 & 22 & 46858421 & 30.28447474 & 7.737291781 & 3.914092372 & $2.10 \mathrm{E}-04$ \\
\hline 73 & cg19880831 & 15 & 33159516 & 28.21903344 & 7.083255908 & 3.983907091 & $1.66 \mathrm{E}-04$ \\
\hline 74 & cg04399083 & 6 & 82461423 & 40.73365998 & 10.82162284 & 3.764099025 & $3.48 \mathrm{E}-04$ \\
\hline 75 & cg08365892 & 10 & 5693584 & 42.11976815 & 11.21931419 & 3.754219503 & $3.59 \mathrm{E}-04$ \\
\hline 76 & cg25376657 & 17 & 16229039 & -55.21371718 & 14.84015476 & -3.720562088 & 4.01E-04 \\
\hline 77 & cg21248322 & 14 & 97645376 & -24.22809232 & 5.635945149 & -4.298851688 & 5.53E-05 \\
\hline 78 & cg13907034 & 2 & 67487793 & -29.74326805 & 7.608306194 & -3.909315331 & 2.14E-04 \\
\hline 79 & cg26121193 & 5 & 135328777 & 36.20919474 & 9.531532236 & 3.79888499 & 3.10E-04 \\
\hline 80 & cg10079875 & 7 & 77304699 & -23.57108862 & 5.264004865 & -4.477786253 & 2.91E-05 \\
\hline 81 & cg08542751 & 5 & 170221963 & -27.97488997 & 7.052409287 & -3.966713903 & 1.76E-04 \\
\hline 82 & cg20120218 & 3 & 85071786 & -34.1232843 & 8.965406169 & -3.806105787 & $3.02 \mathrm{E}-04$ \\
\hline 83 & cg05702638 & 6 & 43034789 & -35.68319919 & 9.429670289 & -3.784140706 & $3.25 \mathrm{E}-04$ \\
\hline 84 & cg06185703 & 10 & 104874603 & 23.34719258 & 5.307936165 & 4.398544342 & 3.87E-05 \\
\hline 85 & cg16992440 & 12 & 53777383 & -28.5830216 & 7.280979014 & -3.925711302 & $2.02 \mathrm{E}-04$ \\
\hline 86 & cg22821677 & 15 & 21941178 & -25.47128569 & 6.299856707 & -4.043153182 & 1.35E-04 \\
\hline 87 & cg24639246 & 13 & 29390312 & -36.9606517 & 9.82428817 & -3.762170965 & $3.50 \mathrm{E}-04$ \\
\hline 88 & cg02061596 & 6 & 168351335 & -24.3252482 & 5.847737972 & -4.159770549 & $9.02 E-05$ \\
\hline 89 & cg27473061 & 2 & 127793088 & 49.13915865 & 13.24120014 & 3.711080426 & 4.14E-04 \\
\hline 90 & cg11535648 & 11 & 45744794 & -27.14975668 & 6.840459275 & -3.968996173 & 1.74E-04 \\
\hline 91 & cg09417399 & 11 & 11971576 & 36.12749774 & 9.586741905 & 3.768485487 & $3.43 \mathrm{E}-04$ \\
\hline 92 & cg25264393 & 11 & 134176598 & -23.24809644 & 5.394599414 & -4.309513024 & $5.32 \mathrm{E}-05$ \\
\hline 93 & cg01501775 & 12 & 54095918 & -25.6172033 & 6.405309747 & -3.999369947 & 1.57E-04 \\
\hline 94 & cg14251442 & 5 & 167864068 & 30.63794458 & 8.009466804 & 3.825216501 & $2.84 \mathrm{E}-04$ \\
\hline 95 & cg16604801 & 17 & 2718310 & 34.57163757 & 9.167183109 & 3.771238903 & 3.39E-04 \\
\hline 96 & cg13604697 & 18 & 18700867 & 33.94605263 & 8.987566151 & 3.7770017 & 3.33E-04 \\
\hline 97 & cg08762290 & 8 & 123711460 & 27.45568681 & 6.993080368 & 3.926122019 & $2.02 \mathrm{E}-04$ \\
\hline 98 & cg24155515 & 6 & 15387512 & 22.49029681 & 4.993715482 & 4.503720104 & $2.65 \mathrm{E}-05$ \\
\hline 99 & cg07622648 & 8 & 94039594 & -37.9979702 & 10.17744972 & -3.733545363 & $3.85 \mathrm{E}-04$ \\
\hline 100 & cg01072259 & 2 & 37946508 & -44.88264092 & 12.11445408 & -3.704883491 & 4.23E-04 \\
\hline
\end{tabular}

Displayed for each ranked probe is the chromosomal location and position (Ensembl GRCh37 assembly), the regression estimate (RE) for the Braak stage-associated analysis, the standard error (SE), the t-statistics, the accompanying $p$ values, the Illumina gene annotation (UCSC annotation), the gene feature (TSS1500, 200 to 1500 nucleotides (nt) upstream of transcription start site (TSS); TSS200, up to $200 \mathrm{nt}$ upstream of TSS; 5'UTR, 5'untranslated region; Body, gene body; $3^{\prime} U T R, 3^{\prime}$ untranslated region) and the cytosine-phosphate-guanine (CpG) island feature. Probes are ranked based on a combined $p$ value (cut-off $=p<0.001$ ) and regression estimate ranking . 


\begin{tabular}{|c|c|c|}
\hline UCSC annotation & Gene feature & CpG island feature \\
\hline KLHL1; KLHL1 & Body; Body & \\
\hline ERC2 & 3'UTR & \\
\hline DENND1A; DENND1A & Body; Body & \\
\hline SETBP1; SETBP1 & Body; Body & Island \\
\hline TTC8; TTC8; TTC8; TTC8; TTC8; TTC8 & 5'UTR; 5'UTR; Body; Body; Body; Body & \\
\hline FAM71E2 & Body & Northern shore \\
\hline CNTNAP2; MIR548T & Body; Body & \\
\hline CYP3A4 & TSS1500 & \\
\hline ACTN1; ACTN1; ACTN1 & Body; Body; Body & \\
\hline IGSF10; IGSF10; IGSF10 & Body; TSS1500; TSS1500 & \\
\hline RNF7; RNF7 & Body; Body & Southern shelf \\
\hline ITGA11 & Body & \\
\hline KLHL35 & Body & Island \\
\hline SEPT9; SEPT9; SEPT9; SEPT9; SEPT9; SEPT9; SEPT9 & Body; Body; Body; Body; Body; Body; Body & \\
\hline ERN2 & Body & \\
\hline CELSR1 & Body & Northern shore \\
\hline FMN1; FMN1 & Body; Body & \\
\hline FAM46A & Body & Island \\
\hline ASB13; ASB13; ASB13 & Body; Body; Body & \\
\hline PIGL & Body & \\
\hline LOC102800447 & Body & \\
\hline GABRP & Body & \\
\hline CADM2; CADM2 & Body; Body & \\
\hline KLC4; KLC4; KLC4; KLC4; KLC4; KLC4 & Body; Body; Body; Body; Body; Body & \\
\hline NT5C2; NT5C2 & Body; Body & \\
\hline SP1; SP1; SP1 & Body; Body; Body & Southern shelf \\
\hline LOC646214 & TSS1500 & \\
\hline & & Northern shelf \\
\hline MLLT4; MLLT4; MLLT4 & Body; Body; Body & Northern shore \\
\hline LOC100507384 & Body & \\
\hline USP47; USP47 & Body; Body & \\
\hline GLB1L3 & Body & \\
\hline WWC1; WWC1; WWC1 & Body; Body; Body & \\
\hline RAP1GAP2; RAP1GAP2 & Body; Body & \\
\hline JARID2; JARID2 & Body; 5'UTR & \\
\hline
\end{tabular}


Supplementary Table 7. Top 50 enriched Gene Ontology (GO) terms for unmodified cytosine (5-uC) in the dorsal raphe nucleus (DRN)

\begin{tabular}{|c|c|c|c|c|c|c|}
\hline Rank & GO term & ID & Ontology & $\begin{array}{l}\text { Number } \\
\text { of genes }\end{array}$ & $\begin{array}{l}\text { Differentially } \\
\text { modified }\end{array}$ & $p$ value \\
\hline 1 & Anatomical structure homeostasis & G0:0060249 & Biological process & 397 & 31 & $1.01 \mathrm{E}-04$ \\
\hline 2 & Tissue homeostasis & G0:0001894 & Biological process & 209 & 20 & $1.29 \mathrm{E}-04$ \\
\hline 3 & Regulation of bone remodeling & GO:0046850 & Biological process & 43 & 8 & $3.50 \mathrm{E}-04$ \\
\hline 4 & T cell receptor complex & GO:0042101 & Cellular component & 17 & 5 & 5.09E-04 \\
\hline 5 & Calcium ion transport & G0:0006816 & Biological process & 396 & 31 & 5.84E-04 \\
\hline 6 & Regulation of bone resorption & GO:0045124 & Biological process & 37 & 7 & $6.12 \mathrm{E}-04$ \\
\hline 7 & SH2 domain binding & GO:0042169 & Molecular function & 32 & 7 & $6.70 \mathrm{E}-04$ \\
\hline 8 & Regulation of neutrophil activation & G0:1902563 & Biological process & 11 & 4 & $6.85 \mathrm{E}-04$ \\
\hline 9 & Activating transcription factor binding & G0:0033613 & Molecular function & 74 & 11 & 7.73E-04 \\
\hline 10 & Positive regulation of cell-cell adhesion & G0:0022409 & Biological process & 242 & 20 & 8.79E-04 \\
\hline 11 & Calcium ion transmembrane transport & G0:0070588 & Biological process & 289 & 25 & $9.24 \mathrm{E}-04$ \\
\hline 12 & Calcium ion transmembrane transporter activity & G0:0015085 & Molecular function & 133 & 16 & $1.04 \mathrm{E}-03$ \\
\hline 13 & Osteoclast development & GO:0036035 & Biological process & 18 & 5 & 1.06E-03 \\
\hline 14 & Bone resorption & GO:0045453 & Biological process & 57 & 8 & $1.25 \mathrm{E}-03$ \\
\hline 15 & Divalent metal ion transport & G0:0070838 & Biological process & 440 & 32 & $1.27 \mathrm{E}-03$ \\
\hline 16 & Positive regulation of cell activation & G0:0050867 & Biological process & 301 & 22 & $1.29 \mathrm{E}-03$ \\
\hline 17 & Gapjunction & G0:0005921 & Cellular component & 30 & 5 & $1.31 \mathrm{E}-03$ \\
\hline 18 & Divalent inorganic cation transport & GO:0072511 & Biological process & 443 & 32 & 1.34E-03 \\
\hline 19 & Regulation of integrin activation & GO:0033623 & Biological process & 12 & 4 & $1.35 \mathrm{E}-03$ \\
\hline 20 & Leukocyte differentiation & GO:0002521 & Biological process & 479 & 32 & $1.38 \mathrm{E}-03$ \\
\hline 21 & Positive regulation of leukocyte cell-cell adhesion & GO:1903039 & Biological process & 205 & 17 & $1.40 \mathrm{E}-03$ \\
\hline 22 & Integrin activation & GO:0033622 & Biological process & 21 & 5 & $1.57 \mathrm{E}-03$ \\
\hline 23 & Positive regulation of leukocyte activation & G0:0002696 & Biological process & 290 & 21 & $1.83 \mathrm{E}-03$ \\
\hline 24 & Positive regulation of T cell proliferation & GO:0042102 & Biological process & 92 & 9 & 1.94E-03 \\
\hline 25 & Regulation of leukocyte differentiation & G0:1902105 & Biological process & 257 & 20 & $1.96 \mathrm{E}-03$ \\
\hline 26 & Detection of light stimulus & G0:0009583 & Biological process & 65 & 8 & 2.04E-03 \\
\hline 27 & Protein kinase binding & GO:0019901 & Molecular function & 616 & 42 & 2.21E-03 \\
\hline 28 & Regulation of cytokine production & G0:0001817 & Biological process & 619 & 35 & $2.21 \mathrm{E}-03$ \\
\hline 29 & Zinc ion binding & GO:0008270 & Molecular function & 764 & 45 & 2.22E-03 \\
\hline
\end{tabular}




\section{FDR adjusted $p$ Genes}

5.61E-01 CCT4; USH1G; CNGB1; COL2A1; PARP1; CTSH; ADRB2; NXNL2; EPAS1; ERCC1; ERCC6; FEN1; NCS1; WWTR1; STK39; SIGLEC15; ANKRD11; INPP5D; CLDN18; GAR1; FGGY; PRKCA; MAP2K7; BBS4; SRC; SYK; TFRC; XRCC5; CACNB2; HIST3H3; LDB2

5.61E-01 USH1G; CNGB1; COL2A1; CTSH; ADRB2; NXNL2; EPAS1; ERCC6; WWTR1; STK39; SIGLEC15; ANKRD11; INPP5D; CLDN18; PRKCA; BBS4; SRC; SYK; TFRC; LDB2

5.78E-01 SIGLEC15; INPP5D; CLDN18; PRKCA; SRC; SYK; TFRC; SYT7

5.78E-01 SYK;ZAP70; SKAP1; CD6; CD8A

$5.78 \mathrm{E}-01$ LILRB1; PHB2; TPCN2; NCS1; STAC3; ABL1; NALCN; ATP2C1; GRIN2C; GRM6; HRC; LETM1; TRPM1; ATP2B4; P2RX5; PDE4D; PIK3CG; PLCG2; PSEN2; CACHD1; CXCL12; TRPM2; FAM155A; CACNA1C; CACNA1E; CACNB2; CACNB4; ORAI2; CUL5; CAMK2B; CASQ1

5.78E-01 SIGLEC15; INPP5D; CLDN18; PRKCA; SRC; SYK; TFRC

$5.78 \mathrm{E}-01$ $5.78 \mathrm{E}-01$ $5.78 \mathrm{E}-01$ $5.78 \mathrm{E}-01$ LILRB1; ABL1; SRC; SYK; RUFY1; SKAP1; SQSTM1 ITGAM; ITGB2; PTAFR; SYK NEK6; ATF2; HIPK2; IFI27; SMAD2; CIITA; PRDM16; LDB2; NCOR1; ZNF516; HDAC4 FSTL3; CXCL13; LILRB1; CYLD; CD55; EPB49; IGF2; IL15; CD46; PRKCZ; PTAFR; RPS3; SRC; SYK; TFRC; ZAP70; RUNX1; SKAP1; IL1RL2; CD6

5.78E-01 PHB2; TPCN2; NCS1; STAC3; ABL1; NALCN; ATP2C1; GRIN2C; HRC; LETM1; TRPM1; ATP2B4; PDE4D; PIK3CG; PLCG2; CACHD1; TRPM2; FAM155A; CACNA1C; CACNA1E; CACNB2; CACNB4; ORAI2; CUL5; CASQ1

5.78E-01 TPCN2; NCS1; ATP2C1; GRIN2C; LETM1; TRPM1; ATP2B4; CACHD1; TRPM2; FAM155A; CACNA1C; CACNA1E; CACNB2; CACNB4; ORAI2; CUL5

$5.78 \mathrm{E}-01$ $5.78 \mathrm{E}-01$ $5.78 \mathrm{E}-01$

5.78E-01

$5.78 \mathrm{E}-01$ LILRB1; FOXP1; SIGLEC15; CLDN18; SRC ADRB2; SIGLEC15; INPP5D; CLDN18; PRKCA; SRC; SYK; TFRC SLC30A9; LILRB1; PHB2; TPCN2; NCS1; STAC3; ABL1; NALCN; ATP2C1; GRIN2C; GRM6; HRC; LETM1; TRPM1; ATP2B4; P2RX5; PDE4D; PIK3CG; PLCG2; PSEN2; CACHD1; CXCL12; TRPM2; FAM155A; CACNA1C; CACNA1E; CACNB2; CACNB4; ORAI2; CUL5; CAMK2B; CASQ1

5.78E-01 LILRB1; CYLD; CD55; EPB49; IGF2; IL15; INPP5D; ITGAM; ITGB2; CD46; PRKCZ; PTAFR; RPS3; SRC; SYK; TFRC; THBS1; TNFRSF4; ZAP70; RUNX1; IL1RL2; CD6

GJB6; PANX3; GJA5; SGSM3; PANX2

SLC30A9; LILRB1; PHB2; TPCN2; NCS1; STAC3; ABL1; NALCN; ATP2C1; GRIN2C; GRM6; HRC; LETM1; TRPM1; ATP2B4; P2RX5; PDE4D; PIK3CG; PLCG2; PSEN2; CACHD1; CXCL12; TRPM2; FAM155A; CACNA1C; CACNA1E; CACNB2; CACNB4; ORAI2; CUL5; CAMK2B; CASQ1

$5.78 \mathrm{E}-01$ CDH17; CXCL13; SRC; SKAP1

$5.78 \mathrm{E}-01$

CDH17; FSTL3; LILRB1; PARP1; CYLD; ADGRG3; ACIN1; ABL1; FOXP1; SIGLEC15; TMEM176B; IL15; INPP5D; LFNG; CD46; CLDN18; PLCG2; POU2F2; PRKCA; PRKCZ; SRC; SYK; TFRC; TRPM2; ZAP70; ZC3H12A; LY6D; RUNX1;a IL1RL2; PGLYRP1; CD8A; HDAC4

5.78E-01 LILRB1; CYLD; CD55; IGF2; IL15; CD46; PRKCZ; PTAFR; RPS3; SRC; SYK; TFRC; ZAP70; RUNX1; SKAP1; IL1RL2; CD6 $6.21 \mathrm{E}-01$ $6.48 \mathrm{E}-01$ CDH17; CXCL13; CXCL12; SRC; SKAP1 LILRB1; CYLD; CD55; IGF2; IL15; INPP5D; ITGAM; ITGB2; CD46; PRKCZ; PTAFR; RPS3; SRC; SYK; TFRC; THBS1; TNFRSF4; ZAP70; RUNX1; IL1RL2; CD6

6.48E-01 CD55; IGF2; IL15; CD46; RPS3; SYK; TFRC; ZAP70; CD6

6.48E-01 FSTL3; LILRB1; CYLD; ACIN1; ABL1; FOXP1; SIGLEC15; TMEM176B; IL15; INPP5D; CD46; CLDN18; PRKCA; PRKCZ; SYK; ZAP70; ZC3H12A; RUNX1; IL1RL2; PGLYRP1

6.48E-01 CNGB1; GNAT1; GRK4; GRM6; GUCY2D; EYS; ACCN1; ARRB1

6.48E-01 NEK6; FRS2; NISCH; ATF2; PARP1; CYLD; DNM2; TPCN2; MAPRE3; NCS1; ABL1; NBEA; STK39; GNAT1; NR3C1; RACGAP1; ITGB2; ARRB1; ATP2B4; TPRKB; PRKAR1B; CENPJ; PRKCZ; MAP2K7; PTAFR; RPTOR; PTPRR; RPS3; SRC; SYK; TRAF3; PRKRIP1; SIKE1; CDK5RAP3; PITPNM3; FAM83A; CADPS; SKAP1; SQSTM1; CD8A; MAPKAPK2; HDAC4

6.48E-01 SPON2; IGF2BP2; LILRB1; PANX3; ATF2; CYLD; LILRA4; ABL1; FOXP1; IL15; INPP5D; LTBP1; ARRB1; CD46; PDE4D; PLCG2; RNF216; PRKCZ; PANX2; PTAFR; SIGIRR; RPS3; SRC; SYK; THBS1; TRAF3; TNFRSF4; XRCC5; ZC3H12A; RUNX1; IL1RL2; PGLYRP1; CD6; MAPKAPK2; IL27RA

6.48E-01 SEC23A; PITRM1; CPXM2; PARP1; PRICKLE1; CYLD; PIKFYVE; MYT1L; TRIM35; ZZEF1; ZFR2; TRIM2; UBR4; BHMT2; ALPI; NR5A1; NR3C1; NANOS3; MDM2; MMP1; MNAT1; MTR; ZFHX3; PEX10; ZCCHC10; ZCWPW1; PRKCA; GATAD2B; RXRA; RXRB; ZSWIM4; SUPT4H1; TCEA3; THRB; TNP2; TRAF3; ZNF22; NEIL1; ADAM33; PDXK; SQSTM1; PGLYRP1; CPA5; GDA; HDAC4 
Supplementary Table 7. (Continued)

\begin{tabular}{|c|c|c|c|c|c|c|}
\hline Rank & GO term & ID & Ontology & $\begin{array}{l}\text { Number } \\
\text { of genes }\end{array}$ & $\begin{array}{l}\text { Differentially } \\
\text { modified }\end{array}$ & $p$ value \\
\hline 30 & Regulation of tissue remodeling & GO:0034103 & Biological process & 82 & 9 & $2.36 \mathrm{E}-03$ \\
\hline 31 & $\begin{array}{l}\text { Lymphocyte activation involved in } \\
\text { immune response }\end{array}$ & GO:0002285 & Biological process & 161 & 13 & $2.42 \mathrm{E}-03$ \\
\hline 32 & Supramolecular fiber & GO:0099512 & Cellular component & 903 & 51 & $2.60 \mathrm{E}-03$ \\
\hline 33 & Cytokine production & GO:0001816 & Biological process & 687 & 38 & $2.61 \mathrm{E}-03$ \\
\hline 34 & Positive regulation of cell adhesion & GO:0045785 & Biological process & 384 & 28 & $2.71 \mathrm{E}-03$ \\
\hline 35 & Adaptive immune response & GO:0002250 & Biological process & 368 & 22 & $2.73 \mathrm{E}-03$ \\
\hline 36 & $\begin{array}{l}\text { Detection of light stimulus involved } \\
\text { in visual perception }\end{array}$ & GO:0050908 & Biological process & 15 & 4 & $2.84 \mathrm{E}-03$ \\
\hline 37 & $\begin{array}{l}\text { Detection of light stimulus involved } \\
\text { in sensory perception }\end{array}$ & GO:0050962 & Biological process & 15 & 4 & $2.84 \mathrm{E}-03$ \\
\hline 38 & Supramolecular polymer & GO:0099081 & Cellular component & 908 & 51 & $3.10 \mathrm{E}-03$ \\
\hline 39 & Transcriptional repressor complex & GO:0017053 & Cellular component & 78 & 10 & $3.11 \mathrm{E}-03$ \\
\hline 40 & Lymphocyte activation & GO:0046649 & Biological process & 616 & 36 & $3.18 \mathrm{E}-03$ \\
\hline 41 & Supramolecular complex & GO:0099080 & Cellular component & 909 & 51 & $3.19 \mathrm{E}-03$ \\
\hline 42 & $\begin{array}{l}\text { Desensitization of } g \text { protein- } \\
\text { coupled receptor signaling pathway }\end{array}$ & GO:0002029 & Biological process & 16 & 4 & $3.22 \mathrm{E}-03$ \\
\hline 43 & $\begin{array}{l}\text { Negative adaptation of signaling } \\
\text { pathway }\end{array}$ & GO:0022401 & Biological process & 16 & 4 & $3.22 \mathrm{E}-03$ \\
\hline 44 & $\begin{array}{l}\text { G protein-coupled receptor } \\
\text { signaling pathway involved in } \\
\text { heart process }\end{array}$ & GO:0086103 & Biological process & 16 & 4 & $3.28 \mathrm{E}-03$ \\
\hline 45 & Microtubule plus-end & GO:0035371 & Cellular component & 20 & 5 & 3.67E-03 \\
\hline 46 & Adaptation of signaling pathway & GO:0023058 & Biological process & 17 & 4 & $3.80 \mathrm{E}-03$ \\
\hline 47 & Kinase binding & GO:0019900 & Molecular function & 693 & 45 & $3.92 \mathrm{E}-03$ \\
\hline 48 & T cell proliferation & GO:0042098 & Biological process & 170 & 13 & 4.09E-03 \\
\hline 49 & Calcium ion transport into cytosol & GO:0060402 & Biological process & 145 & 14 & 4.11E-03 \\
\hline 50 & Bone remodeling & GO:0046849 & Biological process & 82 & 9 & 4.28E-03 \\
\hline
\end{tabular}

Displayed for each ranked $G O$ term is the identifier (ID), the related ontology, the total number of genes belonging to the $G O$ term, the number of genes that are differentially modified, the $p$ value, the false discovery rate (FDR) adjusted $p$ value and the names of the differentially modified genes. The GO terms are ranked based on their $p$ value. 


\section{FDR adjusted $p$ Genes}

\begin{tabular}{|c|c|}
\hline $\begin{array}{l}6.48 \mathrm{E}-01 \\
6.48 \mathrm{E}-01\end{array}$ & $\begin{array}{l}\text { SIGLEC15; IL15; INPP5D; CLDN18; PRKCA; SRC; SYK; TFRC; SYT7 } \\
\text { CDH17; LILRB1; ERCC1; ABL1; FOXP1; LFNG; CD46; PLCG2; PRKCZ; TFRC; ZC3H12A; PGLYRP1; IL27RA }\end{array}$ \\
\hline $6.48 \mathrm{E}-01$ & $\begin{array}{l}\text { KRTAP16-1; CCT4; NEK6; KIF3A; AKAP13; IFFO2; MYOM3; COL2A1; DNAH2; CYLD; TTLL9; DNAH8; DNM2; CTTN; } \\
\text { EPB49; MAPRE3; NINL; PALLD; TTLL5; CLASP1; KRT23; STAU2; TEKT2; IGSF22; RACGAP1; HRC; NCKAP5; KRTAP10-9; } \\
\text { KRTAP12-4; KRTAP10-12; LMNB1; LTBP1; ATP2B4; ODF1; MNS1; CENPJ; PSEN2; MTUS1; SLAIN2; SHROOM3; SRC; } \\
\text { CACNA1C; CDK5RAP3; DNAL1; OBSCN; CASQ1; GAS7; SKAP1; SQSTM1; NEXN; HDAC4 }\end{array}$ \\
\hline $6.48 \mathrm{E}-01$ & $\begin{array}{l}\text { SPON2; IGF2BP2; LILRB1; PANX3; ATF2; CYLD; CD55; PLA2R1; LILRA4; ABL1; FOXP1; IL15; INPP5D; LTBP1; ARRB1; CD46; } \\
\text { PDE4D; PIK3CG; PLCG2; RNF216; PRKCZ; PANX2; PTAFR; SIGIRR; RPS3; SRC; SYK; THBS1; TRAF3; TNFRSF4; XRCC5; } \\
\text { ZC3H12A; RUNX1; IL1RL2; PGLYRP1; CD6; MAPKAPK2; IL27RA }\end{array}$ \\
\hline $6.48 \mathrm{E}-01$ & $\begin{array}{l}\text { FSTL3; AGR2; CXCL13; LILRB1; TRIOBP; CYLD; CD55; DNM2; EPB49; ABL1; IGF2; IL15; CD46; ZFHX3; PRKCA; PRKCZ; } \\
\text { PTAFR; RPS3; RREB1; CXCL12; SRC; SYK; TFRC; ZAP70; RUNX1; SKAP1; IL1RL2; CD6 }\end{array}$ \\
\hline $6.48 \mathrm{E}-01$ & $\begin{array}{l}\text { CXCL13; LILRB1; CTSH; CD55; ERCC1; LAMP3; INPP5D; CD46; PIK3CG; POU2F2; PRKCZ; SYK; TAP1; TFRC; ZAP70; } \\
\text { ZC3H12A; STX7; SKAP1; CD6; CD7; CD8A; IL27RA }\end{array}$ \\
\hline $6.48 \mathrm{E}-01$ & CNGB1; GNAT1; GRM6; EYS \\
\hline $6.48 \mathrm{E}-01$ & CNGB1; GNAT1; GRM6; EYS \\
\hline $6.48 \mathrm{E}-01$ & $\begin{array}{l}\text { KRTAP16-1; CCT4; NEK6; KIF3A; AKAP13; IFFO2; MYOM3; COL2A1; DNAH2; CYLD; TTLL9; DNAH8; DNM2; CTTN; } \\
\text { EPB49; MAPRE3; NINL; PALLD; TTLL5; CLASP1; KRT23; STAU2; TEKT2; IGSF22; RACGAP1; HRC; NCKAP5; KRTAP10-9; } \\
\text { KRTAP12-4; KRTAP10-12; LMNB1; LTBP1; ATP2B4; ODF1; MNS1; CENPJ; PSEN2; MTUS1; SLAIN2; SHROOM3; SRC; } \\
\text { CACNA1C; CDK5RAP3; DNAL1; OBSCN; CASQ1; GAS7; SKAP1; SQSTM1; NEXN; HDAC4 }\end{array}$ \\
\hline $6.48 \mathrm{E}-01$ & CHD5; LIN9; DEPDC1; RCOR3; GATAD2B; PHF12; PRDM16; TBX15; NCOR1; HDAC4 \\
\hline $6.48 \mathrm{E}-01$ & $\begin{array}{l}\text { CDH17; LILRB1; CYLD; CD55; DDOST; ERCC1; ADGRG3; ABL1; FOXP1; IGF2; IL15; INPP5D; ITGB2; LFNG; CD46; IL21R; } \\
\text { PIK3CG; PLCG2; POU2F2; PRKCZ; RPS3; SRC; SYK; TFRC; TNFRSF4; ZAP70; ZC3H12A; LY6D; RUNX1; IL1RL2; PGLYRP1; } \\
\text { CD6; CD7; CD8A; IL27RA; HDAC4 }\end{array}$ \\
\hline $6.48 \mathrm{E}-01$ & $\begin{array}{l}\text { KRTAP16-1; CCT4; NEK6; KIF3A; AKAP13; IFFO2; MYOM3; COL2A1; DNAH2; CYLD; TTLL9; DNAH8; DNM2; CTTN; } \\
\text { EPB49; MAPRE3; NINL; PALLD; TTLL5; CLASP1; KRT23; STAU2; TEKT2; IGSF22; RACGAP1; HRC; NCKAP5; KRTAP10-9; } \\
\text { KRTAP12-4; KRTAP10-12; LMNB1; LTBP1; ATP2B4; ODF1; MNS1; CENPJ; PSEN2; MTUS1; SLAIN2; SHROOM3; SRC; } \\
\text { CACNA1C; CDK5RAP3; DNAL1; OBSCN; CASQ1; GAS7; SKAP1; SQSTM1; NEXN; HDAC4 }\end{array}$ \\
\hline $6.48 \mathrm{E}-01$ & ADRB2; DNM2; GRK4; ARRB1 \\
\hline $6.48 \mathrm{E}-01$ & ADRB2; DNM2; GRK4; ARRB1 \\
\hline $6.48 \mathrm{E}-01$ & AKAP13; ATP2B4; PDE4D; SRC \\
\hline $7.08 \mathrm{E}-01$ & MAPRE3; CLASP1; NCKAP5; SLAIN2; SKAP1 \\
\hline 7.19E-01 & ADRB2; DNM2; GRK4; ARRB1 \\
\hline $7.26 \mathrm{E}-01$ & $\begin{array}{l}\text { NEK6; FRS2; NISCH; AKAP13; ATF2; PARP1; CYLD; DNM2; TPCN2; MAPRE3; NCS1; ABL1; NBEA; STK39; GNAT1; NR3C1; } \\
\text { RACGAP1; ITGB2; ARRB1; ATP2B4; TPRKB; PFKL; TOLLIP; PRKAR1B; CENPJ; PRKCZ; MAP2K7; PTAFR; RPTOR; PTPRR; } \\
\text { RPS3; SRC; SYK; TRAF3; PRKRIP1; SIKE1; CDK5RAP3; PITPNM3; FAM83A; CADPS; SKAP1; SQSTM1; CD8A; MAPKAPK2; } \\
\text { HDAC4 }\end{array}$ \\
\hline $7.29 \mathrm{E}-01$ & LILRB1; CD55; ABL1; IGF2; IL15; CD46; PIK3CG; RPS3; SYK; TFRC; TNFRSF4;ZAP70; CD6 \\
\hline $7.29 \mathrm{E}-01$ & TPCN2; ABL1; GRIN2C; HRC; LETM1; TRPM1; ATP2B4; P2RX5; PDE4D; PLCG2; TRPM2; FAM155A; CACNA1C; CASQ1 \\
\hline 7.34E-01 & ADRB2; SIGLEC15; INPP5D; CLDN18; PRKCA; SRC; SYK; TFRC; SYT7 \\
\hline
\end{tabular}


Supplementary Table 8. Top 50 enriched Gene Ontology (GO) terms for 5-methylcytosine (5-mC) in the dorsal raphe nucleus (DRN)

\begin{tabular}{|c|c|c|c|c|c|c|}
\hline Rar & GO term & ID & Ontology & $\begin{array}{l}\text { Number } \\
\text { of genes }\end{array}$ & $\begin{array}{l}\text { Differentially } \\
\text { modified }\end{array}$ & $p$ value \\
\hline 1 & Protein tyrosine kinase activator activity & GO:0030296 & Molecular function & 16 & 4 & $1.85 \mathrm{E}-03$ \\
\hline 2 & Regulation of translational initiation & GO:0006446 & Biological process & 71 & 7 & 2.00E-03 \\
\hline 3 & Regulation of DNA metabolic process & GO:0051052 & Biological process & 389 & 20 & $2.80 \mathrm{E}-03$ \\
\hline 4 & Sumo transferase activity & GO:0019789 & Molecular function & 23 & 4 & $3.20 \mathrm{E}-03$ \\
\hline 5 & Inositol phosphate-mediated signaling & GO:0048016 & Biological process & 56 & 7 & 3.25E-03 \\
\hline 6 & Receptor activator activity & GO:0030546 & Molecular function & 10 & 3 & $6.05 \mathrm{E}-03$ \\
\hline 7 & Histone demethylase activity (H3-K9 specific) & GO:0032454 & Molecular function & 12 & 3 & $6.08 \mathrm{E}-03$ \\
\hline 8 & Photoreceptor outer segment membrane & GO:0042622 & Cellular component & 14 & 3 & $6.53 \mathrm{E}-03$ \\
\hline 9 & Histone H3-K9 demethylation & GO:0033169 & Biological process & 13 & 3 & 7.39E-03 \\
\hline 10 & Negative regulation of translational initiation & GO:0045947 & Biological process & 17 & 3 & $7.40 \mathrm{E}-03$ \\
\hline 11 & Positive regulation of myelination & GO:0031643 & Biological process & 13 & 3 & $8.72 \mathrm{E}-03$ \\
\hline 12 & Synapse & GO:0045202 & Cellular component & 1102 & 51 & $1.01 \mathrm{E}-02$ \\
\hline
\end{tabular}

Myelination

Ensheathment of neurons

Axon ensheathment

Activation of transmembrane receptor protein tyrosine kinase activity

Regulation of DNA replication

Primary alcohol metabolic process

Regulation of blood circulation

Odorant binding

Ion channel inhibitor activity

Regulation of ossification

Action potential

Oxidoreductase activity, acting on $\mathrm{NAD}(\mathrm{P}) \mathrm{H}$,

quinone or similar compound as acceptor

Organellar large ribosomal subunit

Mitochondrial large ribosomal subunit

Channel inhibitor activity

Nucleoside monophosphate biosynthetic process

Plasma membrane raft

Adherens junction assembly

Transcription elongation from

RNA polymerase ii promoter

Synaptic vesicle endocytosis

Presynaptic endocytosis

Heart contraction

Regulation of membrane repolarization

Calcineurin-mediated signaling

Tertiary granule membrane

Response to starvation

Negative regulation of DNA metabolic process

Tertiary granule

Epithelial cell morphogenesis

Regulation of cell shape

Positive regulation of plasma membrane

bounded cell projection assembly

Dendrite extension

5'-nucleotidase activity

Positive regulation of DNA repair

Regulation of myelination

Modulation by virus of host process

Surfactant homeostasis

Heart process
GO:0042552
GO:0007272
GO:0008366
GO:0007171
GO:0006275
GO:0034308
GO:1903522
GO:0005549
GO:0008200
GO:0030278
GO:0001508
GO:0016655
GO:0000315
GO:0005762
GO:0016248
GO:0009124
GO:0044853
GO:0034333
GO:0006368
GO:0048488
GO:0140238
GO:0060047
GO:0060306
GO:0097720
GO:0070821
GO:0042594
GO:0051053
GO:0070820
GO:0003382
GO:0008360
GO:0120034

G0:0097484

GO:0008253

GO:0045739

GO:0031641

GO:0019054

GO:0043129

G0:0003015

\begin{tabular}{|c|c|c|c|}
\hline Biological process & 111 & 9 & 1.01E-02 \\
\hline Biological process & 113 & 9 & $1.12 \mathrm{E}-02$ \\
\hline Biological process & 113 & 9 & $1.12 \mathrm{E}-02$ \\
\hline Biological process & 13 & 3 & $1.13 \mathrm{E}-02$ \\
\hline Biological process & 103 & 7 & $1.44 \mathrm{E}-02$ \\
\hline Biological process & 73 & 5 & $1.50 \mathrm{E}-02$ \\
\hline Biological process & 250 & 14 & $1.51 \mathrm{E}-02$ \\
\hline Molecular function & 82 & 3 & $1.52 \mathrm{E}-02$ \\
\hline Molecular function & 36 & 4 & $1.55 \mathrm{E}-02$ \\
\hline Biological process & 185 & 11 & 1.61E-02 \\
\hline Biological process & 127 & 9 & 1.65E-02 \\
\hline Molecular function & 52 & 4 & 1.67E-02 \\
\hline Cellular component & 57 & 4 & 1.79E-02 \\
\hline Cellular component & 57 & 4 & 1.79E-02 \\
\hline Molecular function & 37 & 4 & 1.89E-02 \\
\hline Biological process & 153 & 9 & 1.94E-02 \\
\hline Cellular component & 101 & 8 & 1.94E-02 \\
\hline Biological process & 86 & 8 & 1.95E-02 \\
\hline Biological process & 83 & 6 & 1.96E-02 \\
\hline Biological process & 48 & 5 & 2.02E-02 \\
\hline Biological process & 48 & 5 & $2.02 \mathrm{E}-02$ \\
\hline Biological process & 233 & 13 & 2.09E-02 \\
\hline Biological process & 35 & 4 & $2.15 \mathrm{E}-02$ \\
\hline Biological process & 46 & 5 & 2.19E-02 \\
\hline Cellular component & 69 & 5 & $2.22 \mathrm{E}-02$ \\
\hline Biological process & 176 & 10 & 2.30E-02 \\
\hline Biological process & 139 & 8 & $2.30 \mathrm{E}-02$ \\
\hline Cellular component & 158 & 8 & 2.42E-02 \\
\hline Biological process & 30 & 4 & $2.44 \mathrm{E}-02$ \\
\hline Biological process & 145 & 10 & $2.45 \mathrm{E}-02$ \\
\hline Biological process & 95 & 7 & 2.61E-02 \\
\hline Biological process & 31 & 4 & 2.63E-02 \\
\hline Molecular function & 12 & 2 & $2.66 \mathrm{E}-02$ \\
\hline Biological process & 57 & 5 & 2.67E-02 \\
\hline Biological process & 34 & 4 & $2.78 \mathrm{E}-02$ \\
\hline Biological process & 22 & 3 & $2.90 \mathrm{E}-02$ \\
\hline Biological process & 11 & 2 & 2.94E-02 \\
\hline Biological process & 241 & 13 & 3.01E-02 \\
\hline
\end{tabular}

$1.12 \mathrm{E}-02$

$1.12 \mathrm{E}-02$

44E-02

$1.50 \mathrm{E}-02$

$1.52 \mathrm{E}-02$

$1.55 \mathrm{E}-02$

$1.65 \mathrm{E}-02$

$67 \mathrm{E}-02$

$89 \mathrm{E}-02$

95E-02

02F-02

2.02E-02

$15 \mathrm{E}-02$

2.19E-02

$30 \mathrm{E}-02$

2.30E-02

$2.42 \mathrm{E}-02$

$2.45 \mathrm{E}-02$

2.61E-02

2.63E-02

$2.78 \mathrm{E}-02$

2.94E-02

3.01E-02

Displayed for each ranked GO term is the identifier (ID), the related ontology, the total number of genes belonging to the $G 0$ term, the number of genes that are differentially modified, the $p$ value, the false discovery rate (FDR) adjusted $p$ value and the names of the differentially modified genes. The GO terms are ranked based on their $p$ value. 


\section{FDR adjusted $p$ Genes}

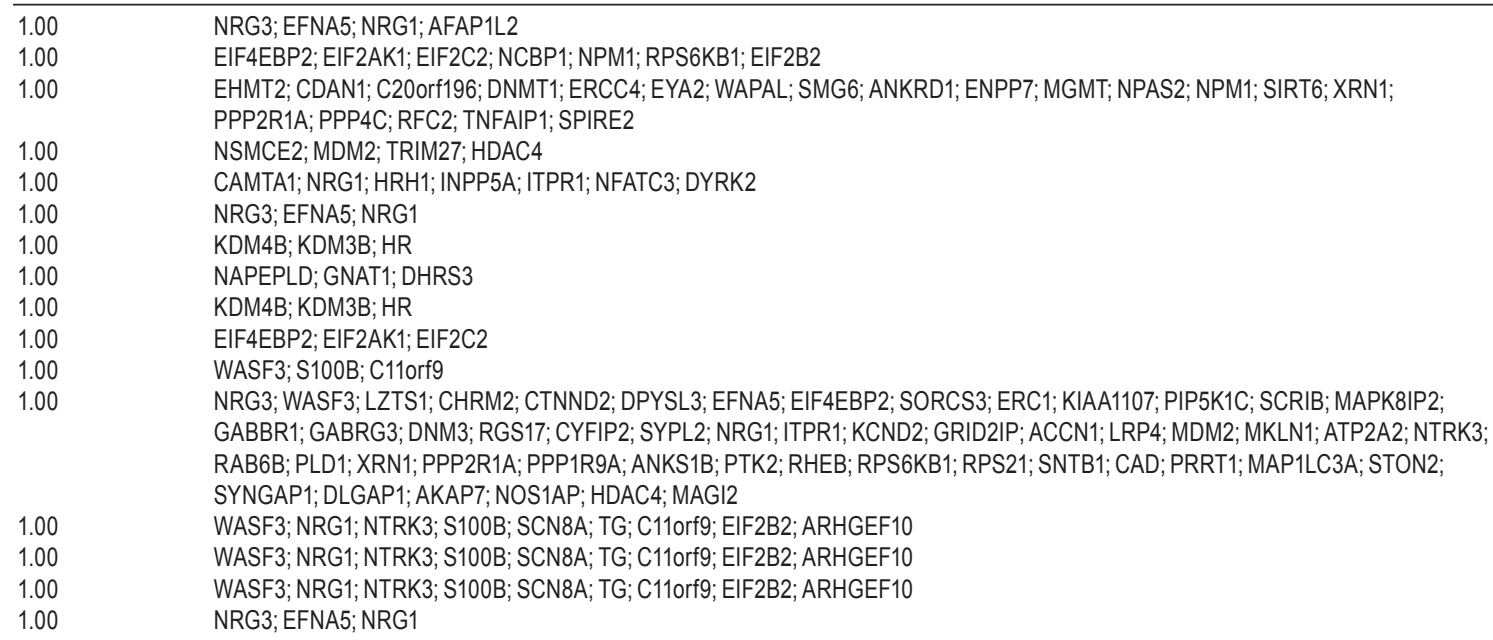
WASF3; NRG1; NTRK3; S100B; SCN8A; TG; C11orf9; EIF2B2; ARHGEF10 WASF3; NRG1; NTRK3; S100B; SCN8A; TG; C11orf9; EIF2B2; ARHGEF10 WASF3; NRG1; NTRK3; S100B; SCN8A; TG; C11orf9; EIF2B2; ARHGEF10 NRG3; EFNA5; NRG1

EHMT2; CDAN1; WAPAL; ENPP7; PPP2R1A; RFC2; TNFAIP1

AKR1C4; NAPEPLD; ALDH1A2; CACNA1H; DHRS3

CHRM2; SMTNL1; HRH1; ITPR1; KCNJ12; ACCN1; MDM2; ATP2A2; SPTBN4; SCN4B; TNNT2; CACNA1H; NOS1AP; HDAC4 OR5M11; OR8K1; OR8U8 NEDD4L; KCNV1; ITPR1; STX8 NPNT; LRP4; DDR2; ATRAID; ANKH; FAM20C; PTK2; ZBTB16; LIMD1; DHRS3; HDAC4 NEDD4L; KCND2; ATP2A2; NTRK3; SCN4B; SCN8A; CACNB3; CACNA1H; NOS1AP AKR1C4; NDUFA5; NDUFAB1; CBR1

MRPL22; NDUFAB1; MRPL30; MRPL39 MRPL22; NDUFAB1; MRPL30; MRPL39 NEDD4L; KCNV1; ITPR1; STX8 DUT; AK1; AKD1; ENTPD8; SIRT6; ATP5G1; PFKP; CAD; HDAC4 EFNA5; MYOF; ITGAM; KCND2; LRP4; MYO1D; CD177; NOS1AP EFNA5; LIMCH1; PIP5K1C; BCAS3; PTK2; TBCD; TRIP6; PHLDB2 TCEB3C; MLLT1; NCBP1; POLR2C; ELP2; TCEB1

KIAA1107; PIP5K1C; SCRIB; DNM3; STON2 KIAA1107; PIP5K1C; SCRIB; DNM3; STON2 CHRM2; NEDD4L; ITPR1; KCNJ12; MDM2; ATP2A2; SPTBN4; SCN4B; SGCD; TNNT2; CACNA1H; NOS1AP; HDAC4 NEDD4L; SCN4B; CACNB3; NOS1AP CAMTA1; NR5A2; NRG1; NFATC3; DYRK2 MCEMP1; ITGAM; PLD1; CD177; CD59 EHMT2; EIF2AK1; FOXA3; ATF3; BCAS3; RPTOR; SSTR3; CAD; MAP1LC3A; MTMR3 CDAN1; C20orf196; ERCC4; WAPAL; SMG6; ANKRD1; ENPP7; XRN1 MCEMP1; ITGAM; PLD1; CD177; STXBP2; TIMP2; DOK3; CD59 FLNB; NOTCH4; BCL11B; COL23A1 WASF3; ARHGEF18; GNA12; MKLN1; PLXNA1; PLXNA2; PTK2; SHROOM3; S100B; LIMD1 DPYSL3; COBL; DNM3; AUTS2; BCAS3; MARK4; HDAC4

NEDD4L; AUTS2; CYFIP2; CPNE6

NT5C3L; NT5DC1

C20orf196; EYA2; MGMT; NPAS2; SPIRE2

WASF3; S100B; TG; C11orf9

SCRIB; NTRK3; VAPA

SFTPA1; LPCAT1

CHRM2; NEDD4L; ITPR1; KCNJ12; MDM2; ATP2A2; SPTBN4; SCN4B; SGCD; TNNT2; CACNA1H; NOS1AP; HDAC4 
Supplementary Table 9. Top 50 enriched Gene Ontology (GO) terms for 5-hydroxymethylcytosine (5-hmC) in the dorsal raphe nucleus (DRN)

\begin{tabular}{llllll}
\hline Rank GO term & ID & Ontology & $\begin{array}{l}\text { Number } \\
\text { of genes }\end{array}$ & $\begin{array}{l}\text { Differentially } \\
\text { modified }\end{array}$ & $p$ value \\
\hline $\begin{array}{l}\text { Homophilic cell adhesion via plasma membrane } \\
\text { adhesion molecules }\end{array}$ & GO:0007156 & Biological process & 167 & 27 & $1.31 \mathrm{E}-07$ \\
$2 \quad$ Calcium ion binding & G0:0005509 & Molecular function & 690 & 56 & $2.47 \mathrm{E}-06$
\end{tabular}

Cell-cell adhesion via plasma-membrane

$\overline{\mathrm{GO}} 00098742$ adhesion molecules

Xenobiotic glucuronidation

5 Cell volume homeostasis

6 Retinoid metabolic process

7 Animal organ maturation

8 Ossification involved in bone maturation

9 Diterpenoid metabolic process

10 UDP-glycosyltransferase activity

11 Retinoid binding

12 Cellular glucuronidation

13 Flavonoid metabolic process

14 Bone maturation

15 Isoprenoid binding

16 Terpenoid metabolic process

17 GTPase activator activity

18 Glucuronosyltransferase activity

19 Uronic acid metabolic process

20 Glucuronate metabolic process

21 Regulation of catecholamine secretion

22 Cardiac conduction

23 Positive regulation of mitochondrial translation

24 Primary alcohol metabolic process

25 Isoprenoid metabolic process

26 Catecholamine secretion

$27 \quad$ SH2 domain binding

28 Gtpase regulator activity

29 Calcium ion import across plasma membrane

30 Calcium ion import into cytosol

31 Positive regulation of epidermal growth factor receptor signaling pathway

32 Cell-cell adhesion

33 Nucleoside-triphosphatase regulator activity

34 Positive regulation of ERBB signaling pathway
GO:0052697

GO:0006884

GO:0001523

GO:0048799

GO:0043931

GO:0016101

GO:0008194

G0:0005501

GO:0009812

GO:0070977

GO:0019840

GO:0006721

GO:0005096

GO:0015020

GO:0006063

GO:0019585

GO:0050433

GO:0061337

GO:0070131

GO:0034308

GO:0006720

GO:0050432

GO:0042169

GO:0030695

GO:0098703

GO:1902656

GO:0045742

GO:0098609
GO:1901186
GO:0052695

\section{Biological process}

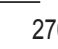

Biological process

Biological process

Biological process

Biological process

Biological process

Biological process

Molecular function

Molecular function

Biological process

Biological process

Biological process

Molecular function

Biological process

Molecular function

Molecular function

Biological process

Biological process

Biological process

Biological process

Biological process

Biological process

Biological process

Biological process

Molecular function

Molecular function

Biological process

Biological process

Biological process

Biological process

49

4.94E-03

7.01E-05

2.62E-04

3.17E-04

4.53E-04

5.31E-04

7.90E-04

8.22E-04

8.62E-04

1.06E-03

1.06E-03

1.10E-03

$1.26 \mathrm{E}-03$

1.42E-03

$1.42 \mathrm{E}-03$

$1.53 \mathrm{E}-03$

1.75E-03

2.59E-03

$2.59 \mathrm{E}-03$

$2.65 \mathrm{E}-03$

$2.76 \mathrm{E}-03$

2.83E-03

$3.11 \mathrm{E}-03$

3.55E-03

$3.81 \mathrm{E}-03$

$3.96 \mathrm{E}-03$

4.73E-03

4.82E-03

4.82E-03

$4.92 \mathrm{E}-03$

4

5

25

6.31E-03

Biological process
347

33
7.40E-03 


\section{FDR adjusted $p \quad$ Genes}

1.16E-03

1.09E-02

2.07E-01

5.62E-01

5.62E-01

6.68E-01

$6.72 \mathrm{E}-01$

7.50E-01

7.50E-01

7.50E-01

7.50E-01

7.50E-01

7.50E-01

7.88E-01

7.88E-01

7.88E-01

7.96E-01

8.61E-01

1.00

1.00

1.00

1.00

1.00

1.00

1.00

1.00

1.00

1.00

1.00

1.00

1.00

1.00

1.00

1.00

CDH7; CDH15; FAT1; PALLD; DCHS2; PCDHGB7; PCDHGB6; PCDHGB5; PCDHGB3; PCDHGB2; PCDHGB1; PCDHGA11; PCDHGA10; PCDHGA9; PCDHGA7; PCDHGA6; PCDHGA5; PCDHGA4; PCDHGA3; PCDHGA2; PCDHGA1; DSCAML1; PVRL4; PCDHGB4; CELSR1; PCDHGA8; CLSTN3

CDH7; CDH15; CGREF1; FBLN7; NCAN; DGKB; FBLN1; FAT1; KCNIP3; EHD4; ANXA11; HPCAL1; ITPR1; AGRN; LRP1; LTBP3; PLA2G2A; DCHS2; PRF1; STAB2; PCDHGB7; PCDHGB6; PCDHGB5; PCDHGB3; PCDHGB2; PCDHGB1; PCDHGA11; PCDHGA10; PCDHGA9; PCDHGA7; PCDHGA6; PCDHGA5; PCDHGA4; PCDHGA3; PCDHGA2; PCDHGA1; PLSCR3; MAN1C1; HEG1; SYT13; EPS15L1; RYR1; SLIT3; DST; SPARC; TPD52; COLEC11; CUBN; ACTN4; DYSF; SYT3; PCDHGB4; CELSR1; PCDHGA8; RAB11FIP3; CLSTN3

CDH7; CDH15; FAT1; PALLD; CLDN18; DCHS2; PCDHGB7; PCDHGB6; PCDHGB5; PCDHGB3; PCDHGB2; PCDHGB1; PCDHGA11; PCDHGA10; PCDHGA9; PCDHGA7; PCDHGA6; PCDHGA5; PCDHGA4; PCDHGA3; PCDHGA2; PCDHGA1; DSCAML1; PVRL4; PCDHGB4; CELSR1; PCDHGA8; CLSTN3

UGT1A10; UGT1A8; UGT1A7; UGT1A6; UGT1A9

ANO6; LRRC8B; KCNMA1; LRRC8D; SLC12A9; SLC12A4; SLC12A8

NAPEPLD; AGRN; LRP1; UGT1A8; UGT1A7; UGT1A9; SDC1; STRA6; ALDH1A2; DHRS3

ANO6; IGF1; RHOA; RYR1; XYLT1; ALDH1A2

ANO6; IGF1; RHOA; RYR1; XYLT1

NAPEPLD; AGRN; LRP1; UGT1A8; UGT1A7; UGT1A9; SDC1; STRA6; ALDH1A2; DHRS3

MGAT4B; MGAT4A; MGAT5B; C3orf21; ALG14; EXT1; GALNT2; B4GALNT3; UGT1A10; UGT1A8; UGT1A7; UGT1A6; UGT1A9; XYLT1

IGF2R; UGT1A8; UGT1A7; UGT1A9; STRA6; ALDH1A2

UGT1A10; UGT1A8; UGT1A7; UGT1A6; UGT1A9

UGT1A10; UGT1A8; UGT1A7; UGT1A6; UGT1A9

ANO6; IGF1; RHOA; RYR1; XYLT1

IGF2R; UGT1A8; UGT1A7; UGT1A9; STRA6; ALDH1A2

NAPEPLD; AGRN; LRP1; UGT1A8; UGT1A7; UGT1A9; SDC1; STRA6; ALDH1A2; DHRS3

FAM13A; ADAP1; AGAP2; AGAP1; DAB2IP; DOCK2; PGAM5; ARHGAP27; RASA3; ARHGEF12; ACAP2; TBC1D22A; ABR;

GRLF1; AGFG2; C20orf95; ASAP1; RIN2; ARHGAP15; RASA1; SMAP2; SYNGAP1; TRIP10; SRGAP3

EXT1; UGT1A10; UGT1A8; UGT1A7; UGT1A6; UGT1A9

UGT1A10; UGT1A8; UGT1A7; UGT1A6; UGT1A9

UGT1A10; UGT1A8; UGT1A7; UGT1A6; UGT1A9

ADRA2A; SYT13; CXCL12; P2RY12; SNCG; VIP; SYT3

GJC1; CTNNA3; ITPR1; KCNK1; KCNQ1; MIR328; ATP2B2; ATP2B4; RYR1; TRPC1; CACNA1C; CACNA2D1

MRPS27; CCDC56; NSUN4

ALDH2; NAPEPLD; DKK3; IGF1; ALDH1A2; CACNA1H; DHRS3

NAPEPLD; AGRN; LRP1; UGT1A8; UGT1A7; UGT1A9; SDC1; STRA6; ALDH1A2; DHRS3

ADRA2A; SYT13; CXCL12; P2RY12; SNCG; VIP; SYT3

KHDRBS2; SH3PXD2B; LAX1; SRC; AFAP1L2; SQSTM1

FAM13A; ADAP1; AGAP2; AGAP1; DAB2IP; DOCK2; PGAM5; ARHGAP27; RASA3; ARHGEF12; ACAP2; TBC1D22A; ABR; GRLF1; AGFG2; C20orf95; ASAP1; RIN2; ARHGAP15; RASA1; SMAP2; SYNGAP1; TRIP10; SRGAP3

ATP2B4; TRPV6; FAM155A; CACNA2D1

ATP2B4; TRPV6; FAM155A; CACNA2D1

ADRA2A; DOK1; HIP1; PDE6G; AFAP1L2

DNAJB6; CDH7; CDH15; FAT1; PALLD; CTNNA3; HLA-DMB; IGF1; ID01; ITPKB; RHOA; NPHP1; CLDN18; FAM49B; DCHS2; LAX1; SIRPG; PRKAR1A; PCDHGB7; PCDHGB6; PCDHGB5; PCDHGB3; PCDHGB2; PCDHGB1; PCDHGA11; PCDHGA10; PCDHGA9; PCDHGA7; PCDHGA6; PCDHGA5; PCDHGA4; PCDHGA3; PCDHGA2; PCDHGA1; DSCAML1; IL21; CXCL12; P2RY12; SRC; TNR; TSTA3; WNT1; PDCD1LG2; PVRL4; PCDHGB4; CELSR1; CD58; PCDHGA8; CLSTN3 DNAJB6; FAM13A; ADAP1; AGAP2; AGAP1; DAB2IP; DOCK2; PGAM5; ARHGAP27; RASA3; ARHGEF12; ACAP2; TBC1D22A; ABR; GRLF1; AGFG2; C20orf95; ASAP1; RIN2; ARHGAP15; RASA1; SMAP2; SYNGAP1; TRIP10; SRGAP3 ADRA2A; DOK1; HIP1; PDE6G; AFAP1L2 
Supplementary Table 9. (Continued)

\begin{tabular}{|c|c|c|c|c|c|c|}
\hline Rank & GO term & ID & Ontology & $\begin{array}{l}\text { Number } \\
\text { of genes }\end{array}$ & $\begin{array}{l}\text { Differentially } \\
\text { modified }\end{array}$ & $p$ value \\
\hline 35 & Positive regulation of GTPase activity & GO:0043547 & Biological process & 399 & 28 & $7.52 \mathrm{E}-03$ \\
\hline 36 & Inorganic anion transmembrane transporter activity & y G0:0015103 & Molecular function & 157 & 13 & $7.62 \mathrm{E}-03$ \\
\hline 37 & Transferase activity, transferring glycosyl groups & GO:0016757 & Molecular function & 270 & 17 & $8.18 \mathrm{E}-03$ \\
\hline 38 & Retinoic acid binding & GO:0001972 & Molecular function & 19 & 4 & $8.24 \mathrm{E}-03$ \\
\hline 39 & Retinoic acid metabolic process & GO:0042573 & Biological process & 22 & 4 & $8.30 \mathrm{E}-03$ \\
\hline 40 & Negative regulation of cell-substrate adhesion & GO:0010812 & Biological process & 66 & 8 & $8.60 \mathrm{E}-03$ \\
\hline 41 & Negative regulation of alcohol biosynthetic process & GO:1902931 & Biological process & 17 & 3 & $8.62 \mathrm{E}-03$ \\
\hline 42 & Protein ADP-ribosylation & GO:0006471 & Biological process & 31 & 4 & $8.63 \mathrm{E}-03$ \\
\hline 43 & Acetylglucosaminyltransferase activity & GO:0008375 & Molecular function & 52 & 6 & $9.05 \mathrm{E}-03$ \\
\hline 44 & Enzyme activator activity & GO:0008047 & Molecular function & 520 & 31 & $9.22 \mathrm{E}-03$ \\
\hline 45 & Negative regulation of steroid metabolic process & GO:0045939 & Biological process & 30 & 4 & $9.28 \mathrm{E}-03$ \\
\hline 46 & Bleb assembly & GO:0032060 & Biological process & 11 & 3 & $9.29 \mathrm{E}-03$ \\
\hline 47 & Positive regulation of podosome assembly & GO:0071803 & Biological process & 11 & 3 & $9.55 \mathrm{E}-03$ \\
\hline 48 & $\begin{array}{l}\text { Positive regulation of voltage-gated calcium } \\
\text { channel activity }\end{array}$ & GO:1901387 & Biological process & 12 & 3 & $9.83 \mathrm{E}-03$ \\
\hline 49 & Podosome assembly & GO:0071800 & Biological process & 19 & 4 & $9.86 \mathrm{E}-03$ \\
\hline 50 & Transmembrane transporter activity & GO:0022857 & Molecular function & 1044 & 53 & $9.98 \mathrm{E}-03$ \\
\hline
\end{tabular}

Displayed for each ranked $\mathrm{GO}$ term is the identifier (ID), the related ontology, the total number of genes belonging to the $\mathrm{GO}$ term, the number of genes that are differentially modified, the $p$ value, the false discovery rate (FDR) adjusted $p$ value and the names of the differentially modified genes. The GO terms are ranked based on their $p$ value and those that are significantly enriched after multiple testing correction (FDR adjusted $p<0.05$ ) are seperated from the other terms by a dashed line. 


\section{FDR adjusted $p$ Genes}

\begin{tabular}{|c|c|}
\hline 1.00 & $\begin{array}{l}\text { FAM13A; ADAP1; AGAP2; AGAP1; DAB2IP; DOCK2; PGAM5; ARHGAP27; RASA3; ARHGEF12; ACAP2; TBC1D22A; } \\
\text { ABR; GRLF1; AGFG2; C20orf95; AGRN; ASAP1; RIN2; BCAS3; ARHGAP15; GRHL3; RASA1; SMAP2; SYNGAP1; TRIP10; } \\
\text { ARHGEF10; SRGAP3 }\end{array}$ \\
\hline 1.00 & ABCC5; SLC26A7; ABCC2; ANO6; LRRC8B; SLC26A4; LRRC8D; SLC12A9; ANO2; SLC12A4; BEST1; SLC12A8; ABCC11 \\
\hline 1.00 & $\begin{array}{l}\text { MGAT4B; MGAT4A; MGAT5B; C3orf21; PARP15; ALG14; EXT1; GALNT2; B4GALNT3; ART3; UGT1A10; UGT1A8; UGT1A7; } \\
\text { UGT1A6; UGT1A9; XYLT1; TNKS }\end{array}$ \\
\hline 1.00 & IGF2R; UGT1A8; UGT1A7; UGT1A9 \\
\hline 1.00 & UGT1A8; UGT1A7; UGT1A9; ALDH1A2 \\
\hline 1.00 & FBLN1; RHOA; BCAS3; AJAP1; RASA1; SRC; WNT1; ACTN4 \\
\hline 1.00 & SCAP; DKK3; NFKB1 \\
\hline 1.00 & PARP15; ART3; XRCC1; TNKS \\
\hline 1.00 & MGAT4B; MGAT4A; MGAT5B; ALG14; EXT1;XYLT1 \\
\hline 1.00 & $\begin{array}{l}\text { DNAJB6; FAM13A; ADAP1; AGAP2; AGAP1; DAB2IP; DOCK2; PGAM5; ARHGAP27; FBLN1; RASA3; ARHGEF12; ACAP2; } \\
\text { TBC1D22A; SH3PXD2B; ABR; GRLF1; GUCA2A; AGFG2; C20orf95; ASAP1; RIN2; BCAS3; ARHGAP15; GPRC5C; RASA1; } \\
\text { SMAP2; AFAP1L2; SYNGAP1; TRIP10; SRGAP3 }\end{array}$ \\
\hline 1.00 & SCAP; DKK3; NFKB1; UGT1A8 \\
\hline 1.00 & ANO6; EMP2; MYLK \\
\hline 1.00 & RHOA; ASAP1; SRC \\
\hline 1.00 & STAC3; STAC2; CACNA2D1 \\
\hline 1.00 & SH3PXD2B; RHOA; ASAP1; SRC \\
\hline 1.00 & $\begin{array}{l}\text { GJC1; ABCC5; SLC26A7; SLC5A11; ABCC2; SLC7A13; ANO6; SLC29A1; RASA3; SLC35A3; SLC44A1; ABCB9; LRRC8B; NNT; } \\
\text { GRIK1; KCNIP3; ITPR1; KCNK1; KCNMA1; KCNQ1; ATP2B2; ATP2B4; SLC45A1; ATP5G2; SLC26A4; LRRC8D; SLC38A7; } \\
\text { TRPV6; PRF1; SLC39A4; PANX2; SLC12A9; ANO2; SLC44A2; JPH3; RYR1; STRA6; SLC12A4; SLC14A1; SLC22A2; SLC22A5; } \\
\text { TRPC1; FAM155A; BEST1; CACNA1C; CACNA2D1; SLC2A10; SLC14A2; SLC12A8; ABCC11; CACNA1H; SLC28A1; CACNA2D4 }\end{array}$ \\
\hline
\end{tabular}


Supplementary Table 10. Top 50 enriched Gene Ontology (GO) terms for unmodified cytosine $(5-u C)$ in the locus coeruleus (LC)

\begin{tabular}{|c|c|c|c|c|c|c|}
\hline Rank & GO term & ID & Ontology & $\begin{array}{l}\text { Number } \\
\text { of genes }\end{array}$ & $\begin{array}{l}\text { Differentially } \\
\text { modified }\end{array}$ & $p$ value \\
\hline 1 & Circadian sleep/wake cycle process & GO:0022410 & Biological process & 23 & 5 & $9.36 \mathrm{E}-05$ \\
\hline 2 & Circadian sleep/wake cycle & GO:0042745 & Biological process & 25 & 5 & $1.50 \mathrm{E}-04$ \\
\hline 3 & Synaptic transmission, glutamatergic & GO:0035249 & Biological process & 88 & 11 & 1.67E-04 \\
\hline 4 & Regulation of circadian sleep/wake cycle & GO:0042749 & Biological process & 20 & 4 & $5.60 \mathrm{E}-04$ \\
\hline 5 & Synaptic transmission, GABAergic & GO:0051932 & Biological process & 43 & 6 & $1.20 \mathrm{E}-03$ \\
\hline 6 & Pericentriolar material & GO:0000242 & Cellular component & 21 & 4 & $1.80 \mathrm{E}-03$ \\
\hline 7 & Phosphatidylinositol 3-kinase binding & GO:0043548 & Molecular function & 30 & 5 & $2.15 \mathrm{E}-03$ \\
\hline 8 & Histone lysine methylation & GO:0034968 & Biological process & 104 & 9 & 2.41E-03 \\
\hline 9 & $\begin{array}{l}\text { Cell-cell adhesion via plasma-membrane } \\
\text { adhesion molecules }\end{array}$ & G0:0098742 & Biological process & 259 & 17 & 3.13E-03 \\
\hline 10 & Circadian behavior & GO:0048512 & Biological process & 41 & 5 & $3.52 \mathrm{E}-03$ \\
\hline 11 & Rhythmic behavior & GO:0007622 & Biological process & 42 & 5 & $3.95 \mathrm{E}-03$ \\
\hline 12 & $\begin{array}{l}\text { Transmembrane receptor protein } \\
\text { serine/threonine kinase binding }\end{array}$ & G0:0070696 & Molecular function & 14 & 3 & 4.06E-03 \\
\hline 13 & Postsynaptic specialization & G0:0099572 & Cellular component & 324 & 20 & $4.08 \mathrm{E}-03$ \\
\hline 14 & Calcium ion binding & G0:0005509 & Molecular function & 672 & 31 & $4.30 \mathrm{E}-03$ \\
\hline 15 & Glomerulus vasculature development & G0:0072012 & Biological process & 22 & 4 & $4.42 \mathrm{E}-03$ \\
\hline 16 & Peptidyl-lysine methylation & GO:0018022 & Biological process & 118 & 9 & $4.53 \mathrm{E}-03$ \\
\hline 17 & Regulation of histone methylation & G0:0031060 & Biological process & 59 & 6 & 4.69E-03 \\
\hline 18 & Transition metal ion homeostasis & GO:0055076 & Biological process & 127 & 8 & $4.95 \mathrm{E}-03$ \\
\hline 19 & Regulation of glomerulus development & G0:0090192 & Biological process & 12 & 3 & $5.55 \mathrm{E}-03$ \\
\hline 20 & Cellular response to oxidative stress & GO:0034599 & Biological process & 276 & 14 & 5.67E-03 \\
\hline 21 & $\begin{array}{l}\text { Regulation of synaptic transmission, } \\
\text { glutamatergic }\end{array}$ & GO:0051966 & Biological process & 65 & 7 & $6.05 \mathrm{E}-03$ \\
\hline 22 & Renal system vasculature development & G0:0061437 & Biological process & 24 & 4 & $6.27 \mathrm{E}-03$ \\
\hline 23 & Kidney vasculature development & GO:0061440 & Biological process & 24 & 4 & $6.27 \mathrm{E}-03$ \\
\hline 24 & $\begin{array}{l}\text { Regulation of cell proliferation involved } \\
\text { in kidney development }\end{array}$ & GO:1901722 & Biological process & 12 & 3 & $6.42 \mathrm{E}-03$ \\
\hline 25 & Heparan sulfate proteoglycan binding & GO:0043395 & Molecular function & 14 & 3 & $7.29 \mathrm{E}-03$ \\
\hline 26 & Adult locomotory behavior & G0:0008344 & Biological process & 77 & 7 & 7.37E-03 \\
\hline 27 & Ubiquitin-specific protease binding & GO:1990381 & Molecular function & 15 & 3 & 7.83E-03 \\
\hline 28 & Histone methylation & GO:0016571 & Biological process & 129 & 9 & $8.28 \mathrm{E}-03$ \\
\hline 29 & $\begin{array}{l}\text { Homophilic cell adhesion via plasma } \\
\text { membrane adhesion molecules }\end{array}$ & GO:0007156 & Biological process & 161 & 12 & 8.79E-03 \\
\hline 30 & Receptor serine/threonine kinase binding & G0:0033612 & Molecular function & 20 & 3 & $8.85 \mathrm{E}-03$ \\
\hline 31 & Lamin binding & G0:0005521 & Molecular function & 15 & 3 & 8.89E-03 \\
\hline 32 & Glomerular mesangium development & GO:0072109 & Biological process & 13 & 3 & $8.90 \mathrm{E}-03$ \\
\hline 33 & Cell adhesion & GO:0007155 & Biological process & 1337 & 52 & $9.28 \mathrm{E}-03$ \\
\hline
\end{tabular}




\title{
FDR adjusted $p$ Genes
}

\author{
4.84E-01 BTBD9; ADORA1; NLGN1; HCRTR2; NPS \\ 4.84E-01 BTBD9; ADORA1; NLGN1; HCRTR2; NPS \\ 4.84E-01 ADORA1; NLGN1; GRID1; GRIN1; GRM3; GRM4; PARK2; NPS; DGKI; NRXN1; CLSTN3 \\ 1.00 ADORA1; NLGN1; HCRTR2; NPS \\ $1.00 \quad$ ADORA1; NLGN1; GABRA1; NPS; BAIAP3; CLSTN3 \\ $1.00 \quad$ CEP152; LCK; NEK1; TNKS \\ $1.00 \quad$ DAB1; RASD2; LCK; $A X L ; C B L$ \\ ADORA1; CTNND2; DAB1; NLGN1; GABRA1; DISC1; GRID1; GRIN1; GRM3; ITPR1; ANKS1B; PTPRS; RAPSN; SPTBN1; PKP4; \\ MPDZ; LIN7A; DGKI; CLSTN3; MAGI2 \\ PCDHA7; PCDHA6; PCDHA5; PCDHA4; PCDHA3; PCDHA2; PCDHA1; PLSCR2; TRPM2; CBL; DLK1; BAIAP3; NRXN1; PLCH2; \\ CLSTN3; PCDHA9 \\ HES1; BMP7; PDGFD; CFLAR \\ KDM4C; AUTS2; WHSC1L1; KDM4D; PRDM16; RDBP; SETDB2; SETD3; EED \\ KDM4C; AUTS2; WHSC1L1; KDM4D; RDBP; EED \\ BTBD9; LCK; SLC11A2; PARK2; CUTC; SLC3OA5; SFXN5; ABCG2 \\ BMP7; PDGFD; CFLAR \\ PDCD10; EIF2S1; ANKRD2; ARNT; PARK2; AXL; SELS; BMP7; STAT6; TPM1; TRPM2; VRK2; PDGFD; CFLAR \\ ADORA1; NLGN1; GRM3; GRM4; NPS; DGKI; NRXN1 \\ HES1; BMP7; PDGFD; CFLAR \\ HES1; BMP7; PDGFD; CFLAR \\ BMP7; PDGFD; CFLAR \\ FST; PTPRC; PTPRS \\ BTBD9; DAB1; FGF12; HIPK2; GRIN1; PARK2; PUM1 \\ MARCH6; PARK2; SELS \\ KDM4C; AUTS2; WHSC1L1; KDM4D; PRDM16; RDBP; SETDB2; SETD3; EED \\ PALLD; PCDH7; PCDHA8; PCDHA7; PCDHA6; PCDHA5; PCDHA4; PCDHA3; PCDHA2; PCDHA1; CLSTN3; PCDHA9 \\ SMAD6; BMP7; MAGI2 \\ TMEM201; PLCB1; LBR \\ BMP7; PDGFD; CFLAR \\ EDIL3; TESK2; SPINK5; LILRB4; COL5A1; RC3H1; CTNND2; DAB1; NLGN1; PALLD; COL29A1; ODZ4; SIGLEC8; HES1; VWC2; \\ LCK; LMO7; SMAD6; MLLT4; MUC4; MYO10; PCDH7; KIF26B; STAB2; LRRC16A; PRKCA; AXL; PCDHA8; PCDHA7; PCDHA6; \\ PCDHA5; PCDHA4; PCDHA3; PCDHA2; PCDHA1; PTK2; PTPRC; PTPRS; TNN; BMP7; TECTA; TNXB; TPM1; PKP4; ADAM19; \\ MPDZ; AIMP1; ADGRG1; NRXN3; NRXN1; CLSTN3; PCDHA9 \\ NLGN1; GABRA1; GRID1; GRIN1; PTPRS; RAPSN; DGKI; CLSTN3 \\ EDIL3; TESK2; SPINK5; LILRB4; COL5A1; RC3H1; CTNND2; DAB1; NLGN1; PALLD; COL29A1; ODZ4; SIGLEC8; HES1; VWC2; \\ LCK; LMO7; SMAD6; MLLT4; MUC4; MYO10; PCDH7; KIF26B; STAB2; LRRC16A; PRKCA; AXL; PCDHA8; PCDHA7; PCDHA6; \\ PCDHA5; PCDHA4; PCDHA3; PCDHA2; PCDHA1; PTK2; PTPRC; PTPRS; TNN; BMP7; TECTA; TNXB; TPM1; PKP4; ADAM19; \\ MPDZ; AIMP1; ADGRG1; NRXN3; NRXN1; CLSTN3; PCDHA9 \\ WHSC1L1; PRDM16; SETDB2; SETD3; EED \\ ADORA1; CTNND2; DAB1; NLGN1; DISC1; GRID1; GRIN1; GRM3; ITPR1; ANKS1B; PTPRS; SPTBN1; PKP4; MPDZ; LIN7A; \\ DGKI; CLSTN3; MAGI2
}


Supplementary Table 10. (Continued)

\begin{tabular}{|c|c|c|c|c|c|c|}
\hline Rank & GO term & ID & Ontology & $\begin{array}{l}\text { Number } \\
\text { of genes }\end{array}$ & $\begin{array}{l}\text { Differentially } \\
\text { modified }\end{array}$ & $p$ value \\
\hline 38 & Regulation of embryonic development & GO:0045995 & Biological process & 123 & 9 & $1.07 \mathrm{E}-02$ \\
\hline 39 & $\begin{array}{l}\text { Regulation of antigen receptor-mediated signaling } \\
\text { pathway }\end{array}$ & GO:0050854 & Biological process & 56 & 5 & 1.09E-02 \\
\hline 40 & Bmp signaling pathway & GO:0030509 & Biological process & 141 & 9 & 1.11E-02 \\
\hline 41 & Neuron to neuron synapse & GO:0098984 & Cellular component & 326 & 19 & $1.14 \mathrm{E}-02$ \\
\hline 42 & Positive T cell selection & G0:0043368 & Biological process & 34 & 4 & $1.17 \mathrm{E}-02$ \\
\hline 43 & Chaperone cofactor-dependent protein refolding & GO:0051085 & Biological process & 29 & 3 & $1.17 \mathrm{E}-02$ \\
\hline 44 & Asymmetric synapse & GO:0032279 & Cellular component & 305 & 18 & $1.18 \mathrm{E}-02$ \\
\hline 45 & Activation of mapkk activity & GO:0000186 & Biological process & 49 & 5 & $1.21 \mathrm{E}-02$ \\
\hline 46 & Proteoglycan binding & GO:0043394 & Molecular function & 32 & 4 & 1.23E-02 \\
\hline 47 & Social behavior & G0:0035176 & Biological process & 47 & 5 & $1.25 \mathrm{E}-02$ \\
\hline 48 & Intraspecies interaction between organisms & GO:0051703 & Biological process & 47 & 5 & 1.25E-02 \\
\hline 49 & Glomerulus development & GO:0032835 & Biological process & 58 & 6 & 1.25E-02 \\
\hline 50 & Regulation of behavior & GO:0050795 & Biological process & 64 & 5 & $1.26 \mathrm{E}-02$ \\
\hline
\end{tabular}

Displayed for each ranked $\mathrm{GO}$ term is the identifier (ID), the related ontology, the total number of genes belonging to the $\mathrm{GO}$ term, the number of genes that are differentially modified, the $p$ value, the false discovery rate (FDR) adjusted $p$ value and the names of the differentially modified genes. The GO terms are ranked based on their $p$ value. 


\section{FDR adjusted $p$ Genes}

\begin{tabular}{ll}
\hline 1.00 & COL5A1; PLCB1; NIPBL; ODZ4; CRB2; HES1; BMP7; GORAB; SEPT7 \\
1.00 & LILRB4; RC3H1; ELF2; LCK; PTPRC \\
& \\
1.00 & GDF1; PDCD4; CRB2; HIPK2; HES1; VWC2; SMAD6; SFRP4; BMP7 \\
1.00 & ADORA1; CTNND2; DAB1; NLGN1; DISC1; GRID1; GRIN1; GRM3; ITPR1; PRKAR1B; ANKS1B; PTPRS; SPTBN1; PKP4; MPDZ; \\
& LIN7A; DGKI; CLSTN3; MAGI2 \\
1.00 & DOCK2; PTPRC; BCL11B; STAT6 \\
1.00 & GAK; HSPA9; DNAJB13 \\
1.00 & ADORA1; CTNND2; DAB1; NLGN1; DISC1; GRID1; GRIN1; GRM3; ITPR1; ANKS1B; PTPRS; SPTBN1; PKP4; MPDZ; LIN7A; \\
& DGKI; CLSTN3; MAGI2 \\
1.00 & FRS2; ADORA1; MAP3K4; ZAK; MAP3K14 \\
1.00 & FST; COL5A1; PTPRC; PTPRS \\
1.00 & GRID1; GRIN1; ATXN1; NRXN3; NRXN1 \\
1.00 & GRID1; GRIN1; ATXN1; NRXN3; NRXN1 \\
1.00 & HES1; BMP7; PDGFD;CFLAR; PROM1; MAGI2 \\
1.00 & ADORA1; NLGN1; HCRTR2; NPS; NRXN1 \\
\hline
\end{tabular}


Supplementary Table 11. Top 50 enriched Gene Ontology (GO) terms for 5-methylcytosine (5-mC) in the locus coeruleus (LC)

\begin{tabular}{|c|c|c|c|c|c|c|}
\hline Rank & GO term & ID & Ontology & $\begin{array}{l}\text { Number } \\
\text { of genes }\end{array}$ & $\begin{array}{l}\text { Differentially } \\
\text { modified }\end{array}$ & $p$ value \\
\hline 1 & $\begin{array}{l}\text { Homophilic cell adhesion via plasma membrane } \\
\text { adhesion molecules }\end{array}$ & GO:0007156 & Biological process & 161 & 35 & $8.34 \mathrm{E}-15$ \\
\hline 2 & $\begin{array}{l}\text { Cell-cell adhesion via plasma-membrane } \\
\text { adhesion molecules }\end{array}$ & GO:0098742 & Biological process & 259 & 36 & $2.74 \mathrm{E}-10$ \\
\hline 3 & Calcium ion binding & GO:0005509 & Molecular function & 672 & 53 & 4.04E-07 \\
\hline 4 & Cell-cell adhesion & GO:0098609 & Biological process & 782 & 57 & $1.26 \mathrm{E}-06$ \\
\hline 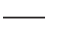 & ---- & - - & $-\quad-$ & - & - & - - - \\
\hline 5 & Integral component of plasma membrane & GO:0005887 & Cellular component & 1521 & 77 & $2.99 \mathrm{E}-04$ \\
\hline 6 & Positive regulation of chromatin binding & GO:0035563 & Biological process & 13 & 4 & 5.97E-04 \\
\hline 7 & High-density lipoprotein particle remodeling & GO:0034375 & Biological process & 17 & 4 & 9.87E-04 \\
\hline 8 & Intrinsic component of plasma membrane & GO:0031226 & Cellular component & 1594 & 77 & $1.13 \mathrm{E}-03$ \\
\hline 9 & Cell adhesion & GO:0007155 & Biological process & 1337 & 73 & $1.33 \mathrm{E}-03$ \\
\hline 10 & Biological adhesion & GO:0022610 & Biological process & 1344 & 73 & $1.51 \mathrm{E}-03$ \\
\hline 11 & High-density lipoprotein particle & GO:0034364 & Cellular component & 25 & 4 & $1.68 \mathrm{E}-03$ \\
\hline 12 & $\begin{array}{l}\text { Mature } B \text { cell differentiation involved in } \\
\text { immune response }\end{array}$ & GO:0002313 & Biological process & 17 & 4 & $2.32 \mathrm{E}-03$ \\
\hline 13 & Glucagon secretion & GO:0070091 & Biological process & 10 & 3 & $2.42 \mathrm{E}-03$ \\
\hline 14 & Protein-lipid complex & GO:0032994 & Cellular component & 39 & 5 & 2.53E-03 \\
\hline 15 & $\begin{array}{l}\text { Positive regulation of double-strand break repair } \\
\text { via nonhomologous end joining }\end{array}$ & GO:2001034 & Biological process & 10 & 3 & $2.83 \mathrm{E}-03$ \\
\hline 16 & Glucosidase activity & GO:0015926 & Molecular function & 12 & 3 & $3.33 \mathrm{E}-03$ \\
\hline 17 & Protein-lipid complex subunit organization & GO:0071825 & Biological process & 49 & 6 & $3.40 \mathrm{E}-03$ \\
\hline
\end{tabular}




\section{FDR adjusted $p$ Genes}

7.25E-11 $\quad$ CDH2; CDH13; SDK1; PLXNB2; CDH20; DCHS2; PCDHGB3; PCDHGB2; PCDHGB1; PCDHGA7; PCDHGA6; PCDHGA5; PCDHGA4; PCDHGA3; PCDHGA2; PCDHGA1; PCDHAC2; PCDHAC1; PCDHA13; PCDHA12; PCDHA11; PCDHA10; PCDHA8; PCDHA7; PCDHA6; PCDHA5; PCDHA4; PCDHA3; PCDHA2; PCDHA1; KIRREL3; PCDHGB4; CELSR1; PCDHGA8; PCDHA9 1.19E-06 CDH2; CDH13; SDK1; PLXNB2; CLDN15; CDH20; DCHS2; PCDHGB3; PCDHGB2; PCDHGB1; PCDHGA7; PCDHGA6; PCDHGA5; PCDHGA4; PCDHGA3; PCDHGA2; PCDHGA1; PCDHAC2; PCDHAC1; PCDHA13; PCDHA12; PCDHA11; PCDHA10; PCDHA8; PCDHA7; PCDHA6; PCDHA5; PCDHA4; PCDHA3; PCDHA2; PCDHA1; KIRREL3; PCDHGB4; CELSR1; PCDHGA8; PCDHA9

1.17E-03 CDH2; CDH13; MYL9; CAPN11; MEGF6; NINL; CDH20; EHD4; LOXL2; MYO5A; NOTCH2; NOTCH3; NIN; LRP1B; DCHS2; PPP3R2; PCDHGB3; PCDHGB2; PCDHGB1; PCDHGA7; PCDHGA6; PCDHGA5; PCDHGA4; PCDHGA3; PCDHGA2; PCDHGA1; PCDHAC2; PCDHAC1; PCDHA13; PCDHA12; PCDHA11; PCDHA10; PCDHA8; PCDHA7; PCDHA6; PCDHA5; PCDHA4; PCDHA3; PCDHA2; PCDHA1; CD248; EPS15L1; ELTD1; MCTP1; PITPNM3; NCALD; RAB11FIP4; PCDHGB4; DLK1; SYT7; CELSR1; PCDHGA8; PCDHA9

2.75E-03 CDH2; CDH13; MYL9; MAP3K8; CTNND1; DLG1; SDK1; PLXNB2; CLDN15; ALOX15; GNAS; CDH20; RNASE10; LAG3; LCK; LRP6; MLLT4; LEF1; PDCD1; PIK3R1; DCHS2; PCDHGB3; PCDHGB2; PCDHGB1; PCDHGA7; PCDHGA6; PCDHGA5; PCDHGA4; PCDHGA3; PCDHGA2; PCDHGA1; PCDHAC2; PCDHAC1; PCDHA13; PCDHA12; PCDHA11; PCDHA10; PCDHA8; PCDHA7; PCDHA6; PCDHA5; PCDHA4; PCDHA3; PCDHA2; PCDHA1; METTL3; ZMIZ1; PTK2; TRPV4; MAD1L1; KIRREL3; SKAP1; RUNX3; PCDHGB4; CELSR1; PCDHGA8; PCDHA9

CDH2; B3GNT3; TSPAN9; GRIN3A; TMC1; OLFM3; CPT1C; CNTFR; CSPG4; DLG1; GPR183; EDNRB; ITGA11; PLXNB2; GABRP; EPHA6; GRIK5; TRHDE; IGF1R; IL11RA; TMPRSS9; KCNK3; SHISA6; LIFR; NOTCH2; PCDHGB3; PCDHGB2; PCDHGB1; PCDHGA7; PCDHGA6; PCDHGA5; PCDHGA4; PCDHGA3; PCDHGA2; PCDHGA1; PCDHAC2; PCDHAC1; PCDHA13; PCDHA12; PCDHA11; PCDHA10; PCDHA8; PCDHA7; PCDHA6; PCDHA5; PCDHA4; PCDHA3; PCDHA2; PCDHA1; SEMA3G; TRPC7; SLC24A3; BAI1; KIAA1324; TRPV4; SCNN1G; ELTD1; SEMA4A; HHIP; SLC1A2; SLC6A12; SLC14A1; TACR1; TGFBR3; TRAF5; GPR172A; LRRC8C; MCHR2; CNTNAP4; PCDHGB4; SYT7; SLC28A1; KL; STX8; CELSR1; PCDHGA8; PCDHA9

8.64E-01 DTX3L; CDT1; MED25; PARP9 ABCA5; APOC1; LIPC; APOM CDH2; B3GNT3; TSPAN9; GRIN3A; TMC1; OLFM3; CPT1C; CNTFR; CSPG4; DLG1; GPR183; EDNRB; ITGA11; PLXNB2; GABRP; EPHA6; GRIK5; TRHDE; IGF1R; IL11RA; TMPRSS9; KCNK3; SHISA6; LIFR; NOTCH2; PCDHGB3; PCDHGB2; PCDHGB1; PCDHGA7; PCDHGA6; PCDHGA5; PCDHGA4; PCDHGA3; PCDHGA2; PCDHGA1; PCDHAC2; PCDHAC1; PCDHA13; PCDHA12; PCDHA11; PCDHA10; PCDHA8; PCDHA7; PCDHA6; PCDHA5; PCDHA4; PCDHA3; PCDHA2; PCDHA1; SEMA3G; TRPC7; SLC24A3; BAI1; KIAA1324; TRPV4; SCNN1G; ELTD1; SEMA4A; HHIP; SLC1A2; SLC6A12; SLC14A1; TACR1; TGFBR3; TRAF5; GPR172A; LRRC8C; MCHR2; CNTNAP4; PCDHGB4; SYT7; SLC28A1; KL; STX8; CELSR1; PCDHGA8; PCDHA9 CDH2; CDH13; MYL9; COL6A2; COL12A1; MAP3K8; CTNND1; DLG1; ENG; SDK1; ITGA11; PLXNB2; CLDN15; ALOX15; CADM2; AATF; GNAS; CDH20; TMEM102; GSK3B; RHOD; APLP1; RNASE10; LAG3; LCK; LOXL2; LRP6; MLLT4; NTM; LEF1; PDCD1; PIK3R1; DCHS2; PCDHGB3; PCDHGB2; PCDHGB1; PCDHGA7; PCDHGA6; PCDHGA5; PCDHGA4; PCDHGA3; PCDHGA2; PCDHGA1; PCDHAC2; PCDHAC1; PCDHA13; PCDHA12; PCDHA11; PCDHA10; PCDHA8; PCDHA7; PCDHA6; PCDHA5; PCDHA4; PCDHA3; PCDHA2; PCDHA1; METTL3; ZMIZ1; PTK2; BAI1; TRPV4; TNXB; C4orf31; MAD1L1; KIRREL3; CNTNAP4; SKAP1; RUNX3; PCDHGB4; CELSR1; PCDHGA8; PCDHA9 CDH2; CDH13; MYL9; COL6A2; COL12A1; MAP3K8; CTNND1; DLG1; ENG; SDK1; ITGA11; PLXNB2; CLDN15; ALOX15; CADM2; AATF; GNAS; CDH20; TMEM102; GSK3B; RHOD; APLP1; RNASE10; LAG3; LCK; LOXL2; LRP6; MLLT4; NTM; LEF1; PDCD1; PIK3R1; DCHS2; PCDHGB3; PCDHGB2; PCDHGB1; PCDHGA7; PCDHGA6; PCDHGA5; PCDHGA4; PCDHGA3; PCDHGA2; PCDHGA1; PCDHAC2; PCDHAC1; PCDHA13; PCDHA12; PCDHA11; PCDHA10; PCDHA8; PCDHA7; PCDHA6; PCDHA5; PCDHA4; PCDHA3; PCDHA2; PCDHA1; METTL3; ZMIZ1; PTK2; BAI1; TRPV4; TNXB; C4orf31; MAD1L1; KIRREL3; CNTNAP4; SKAP1; RUNX3; PCDHGB4; CELSR1; PCDHGA8; PCDHA9 HDLBP; APOC1; LIPC; APOM C17orf99; GPR183; NOTCH2; ITFG2

PASK; UCN; SYT7 BIN1; HDLBP; APOC1; LIPC; APOM DTX3L; SETMAR; PARP9 
Supplementary Table 11. (Continued)

\begin{tabular}{|c|c|c|c|c|c|c|}
\hline Rank & GO term & ID & Ontology & $\begin{array}{l}\text { Number } \\
\text { of genes }\end{array}$ & $\begin{array}{l}\text { Differentially } \\
\text { modified }\end{array}$ & $p$ value \\
\hline 18 & Cell cycle checkpoint & GO:0000075 & Biological process & 213 & 15 & $4.78 \mathrm{E}-03$ \\
\hline 19 & Protein-lipid complex remodeling & GO:0034368 & Biological process & 28 & 4 & $4.82 \mathrm{E}-03$ \\
\hline 20 & Plasma lipoprotein particle remodeling & GO:0034369 & Biological process & 28 & 4 & $4.82 \mathrm{E}-03$ \\
\hline 21 & Nucleotide-sugar metabolic process & GO:0009225 & Biological process & 36 & 5 & $5.84 \mathrm{E}-03$ \\
\hline 22 & Protein-containing complex remodeling & GO:0034367 & Biological process & 30 & 4 & 6.07E-03 \\
\hline 23 & Sterol transport & GO:0015918 & Biological process & 107 & 9 & $6.29 \mathrm{E}-03$ \\
\hline 24 & Protein autoubiquitination & GO:0051865 & Biological process & 62 & 7 & $6.82 \mathrm{E}-03$ \\
\hline 25 & Mature B cell differentiation & GO:0002335 & Biological process & 21 & 4 & $6.87 \mathrm{E}-03$ \\
\hline 26 & Regulation of chromatin binding & GO:0035561 & Biological process & 23 & 4 & $6.90 \mathrm{E}-03$ \\
\hline 27 & $\begin{array}{l}\text { Negative regulation of lipoprotein particle } \\
\text { clearance }\end{array}$ & GO:0010985 & Biological process & 21 & 3 & $6.92 \mathrm{E}-03$ \\
\hline 28 & Cholesterol transport & G0:0030301 & Biological process & 97 & 8 & $7.00 \mathrm{E}-03$ \\
\hline 29 & Regulation of $\mathrm{G} 0$ to $\mathrm{G} 1$ transition & GO:0070316 & Biological process & 38 & 5 & $8.95 \mathrm{E}-03$ \\
\hline 30 & Negative regulation of cell cycle & GO:0045786 & Biological process & 566 & 29 & $9.00 \mathrm{E}-03$ \\
\hline 31 & Radial glial cell differentiation & G0:0060019 & Biological process & 12 & 3 & $9.39 \mathrm{E}-03$ \\
\hline 32 & $\mathrm{G} 0$ to $\mathrm{G} 1$ transition & GO:0045023 & Biological process & 40 & 5 & $1.02 \mathrm{E}-02$ \\
\hline 33 & Plasma lipoprotein particle & GO:0034358 & Cellular component & 37 & 4 & $1.08 \mathrm{E}-02$ \\
\hline 34 & Lipoprotein particle & GO:1990777 & Cellular component & 37 & 4 & $1.08 \mathrm{E}-02$ \\
\hline 35 & Aldehyde dehydrogenase (NAD) activity & GO:0004029 & Molecular function & 14 & 3 & 1.15E-02 \\
\hline 36 & Plasma lipoprotein particle clearance & GO:0034381 & Biological process & 71 & 6 & $1.17 \mathrm{E}-02$ \\
\hline 37 & $\begin{array}{l}\text { Intrinsic apoptotic signaling pathway by } \\
\text { p53 class mediator }\end{array}$ & GO:0072332 & Biological process & 74 & 7 & 1.27E-02 \\
\hline 38 & $\begin{array}{l}\text { Positive regulation of heterotypic } \\
\text { cell-cell adhesion }\end{array}$ & GO:0034116 & Biological process & 15 & 3 & $1.33 \mathrm{E}-02$ \\
\hline 39 & $\begin{array}{l}\text { Intrinsic apoptotic signaling pathway } \\
\text { in response to dna damage by p53 class mediator }\end{array}$ & GO:0042771 & Biological process & 42 & 5 & $1.41 \mathrm{E}-02$ \\
\hline 40 & Base conversion or substitution editing & G0:0016553 & Biological process & 20 & 3 & $1.41 \mathrm{E}-02$ \\
\hline 41 & Regulation of blood pressure & GO:0008217 & Biological process & 165 & 11 & 1.44E-02 \\
\hline 42 & Triglyceride lipase activity & GO:0004806 & Molecular function & 23 & 3 & $1.48 \mathrm{E}-02$ \\
\hline 43 & $B$ cell activation involved in immune response & G0:0002312 & Biological process & 67 & 6 & $1.50 \mathrm{E}-02$ \\
\hline 44 & Protein-lipid complex assembly & GO:0065005 & Biological process & 32 & 4 & $1.57 \mathrm{E}-02$ \\
\hline 45 & Retinoid X receptor binding & GO:0046965 & Molecular function & 15 & 3 & $1.63 \mathrm{E}-02$ \\
\hline 46 & SNAP receptor activity & GO:0005484 & Molecular function & 29 & 4 & $1.67 \mathrm{E}-02$ \\
\hline 47 & Reverse cholesterol transport & G0:0043691 & Biological process & 19 & 3 & $1.70 \mathrm{E}-02$ \\
\hline 48 & Very-low-density lipoprotein particle clearance & GO:0034447 & Biological process & 10 & 2 & $1.77 \mathrm{E}-02$ \\
\hline 49 & Secondary palate development & GO:0062009 & Biological process & 24 & 4 & $1.92 \mathrm{E}-02$ \\
\hline 50 & Synaptic vesicle exocytosis & GO:0016079 & Biological process & 118 & 10 & 2.01E-02 \\
\hline
\end{tabular}

Displayed for each ranked GO term is the identifier (ID), the related ontology, the total number of genes belonging to the GO term, the number of genes that are differentially modified, the $p$ value, the false discovery rate (FDR) adjusted $p$ value and the names of the differentially modified genes. The GO terms are ranked based on their $p$ value and those that are significantly enriched after multiple testing correction (FDR adjusted $p<0.05$ ) are seperated from the other terms by a dashed line. 


\section{FDR adjusted $p$ Genes}

1

FOXN3; RAD9B; DLG1; EIF2AK4; MSH2; ORC1L; NSUN2; CHFR; USP28; KIAA1967; SETMAR; XRCC3; CDT1; C12orf32; MAD1L1

ABCA5; APOC1; LIPC; APOM

ABCA5; APOC1; LIPC; APOM

GNPDA1; GUK1; PMM2; CSGALNACT1; GFPT2

ABCA5; APOC1; LIPC; APOM

ABCA5; STX12; APOC1; LIPC; LRP6; PPARG; APOM; SYT7; OSBPL2

DTX3L; RNF133; SASH1; UHRF1; RNF4; UBE3A; MTA1

C17orf99; GPR183; NOTCH2; ITFG2

DTX3L; CDT1; MED25; PARP9

MYLIP; APOC1; LRPAP1

ABCA5; STX12; APOC1; LIPC; LRP6; PPARG; APOM; SYT7

E2F6; CBX5; APAF1; EHMT1; C12orf32

HEXIM1; FOXN3; RAD9B; DLG1; E2F6; CBX5; GAS2; BIN1; APAF1; EIF2AK4; MSH2; NOTCH2; ORC1L; NSUN2; USP47; CHFR; RPTOR; USP28; KIAA1967; SETMAR; XRCC3; EHMT1; CDT1; MED25; C12orf32; MAD1L1; SLC25A33; RUNX3; KAT2B CDH2; LEF1; METTL3

E2F6; CBX5; APAF1; EHMT1; C12orf32

HDLBP; APOC1; LIPC; APOM

HDLBP; APOC1; LIPC; APOM

ALDH2; ALDH1A3; ALDH1A2

MYLIP; HDLBP; APOC1; LIPC; LRPAP1; APOM

ANKRD2; HIPK2; MSH2; WWOX; USP28; AEN; BCL2L12

ALOX15; LCK; SKAP1

HIPK2; MSH2; USP28; AEN; BCL2L12

ADARB2; RBM47; METTL3

CORIN; CPA3; CYP11B1; EDNRB; DDAH1; ERAP1; PPARG; UCN; NCALD; NAV2; RASL10B

CES5A; CES8; LIPC

C17orf99; GPR183; CD180; MSH2; NOTCH2; ITFG2

BIN1; APOC1; APOM; PCDHGA3

PPARG; RARB; MED25

VTI1A; STX2; STX12; STX8

ABCA5; LIPC; APOM

APOC1; LRPAP1

DLG1; LEF1; MMP25; TGFBR3

TRIM9; GRIN3A; RIMS4; STX2; GRIK5; GSK3B; PRKCB; CADPS; SYT7; CADPS2 
Supplementary Table 12. Top 50 enriched Gene Ontology (GO) terms for 5-hydroxymethylcytosine $(5-\mathrm{hmC})$ in the locus coeruleus (LC)

\begin{tabular}{|c|c|c|c|c|c|c|}
\hline Rank & GO term & ID & Ontology & $\begin{array}{l}\text { Number } \\
\text { of genes }\end{array}$ & $\begin{array}{l}\text { Differentially } \\
\text { modified }\end{array}$ & $p$ value \\
\hline 1 & Regulation of calcium ion-dependent exocytosis & G0:0017158 & Biological process & 104 & 12 & 1.83E-04 \\
\hline 2 & Neurotrophin TRK receptor signaling pathway & GO:0048011 & Biological process & 31 & 6 & $2.78 \mathrm{E}-04$ \\
\hline 3 & Calcium channel activity & GO:0005262 & Molecular function & 122 & 14 & $3.00 \mathrm{E}-04$ \\
\hline 4 & Response to carbohydrate & GO:0009743 & Biological process & 222 & 16 & 4.86E-04 \\
\hline 5 & $\begin{array}{l}\text { Negative regulation of epithelial cell } \\
\text { differentiation }\end{array}$ & G0:0030857 & Biological process & 46 & 6 & $5.36 \mathrm{E}-04$ \\
\hline 6 & Beta-catenin-TCF complex assembly & G0:1904837 & Biological process & 30 & 6 & $5.68 \mathrm{E}-04$ \\
\hline 7 & Regulation of regulated secretory pathway & GO:1903305 & Biological process & 147 & 13 & 1.13E-03 \\
\hline 8 & Calcium ion transmembrane transporter activity & GO:0015085 & Molecular function & 138 & 14 & $1.26 \mathrm{E}-03$ \\
\hline 9 & Embryonic limb morphogenesis & GO:0030326 & Biological process & 125 & 12 & $1.52 \mathrm{E}-03$ \\
\hline 10 & Embryonic appendage morphogenesis & GO:0035113 & Biological process & 125 & 12 & $1.52 \mathrm{E}-03$ \\
\hline 11 & Ionotropic glutamate receptor activity & GO:0004970 & Molecular function & 19 & 5 & $1.52 \mathrm{E}-03$ \\
\hline 12 & Neurotrophin signaling pathway & GO:0038179 & Biological process & 39 & 6 & $1.73 \mathrm{E}-03$ \\
\hline 13 & Appendage morphogenesis & GO:0035107 & Biological process & 146 & 13 & $1.83 \mathrm{E}-03$ \\
\hline 14 & Limb morphogenesis & GO:0035108 & Biological process & 146 & 13 & $1.83 \mathrm{E}-03$ \\
\hline 15 & Calcium ion regulated exocytosis & GO:0017156 & Biological process & 152 & 13 & 1.94E-03 \\
\hline 16 & Response to muramyl dipeptide & GO:0032495 & Biological process & 20 & 4 & $2.18 \mathrm{E}-03$ \\
\hline 17 & Calcium-release channel activity & GO:0015278 & Molecular function & 21 & 5 & $2.20 \mathrm{E}-03$ \\
\hline 18 & Voltage-gated calcium channel complex & GO:0005891 & Cellular component & 43 & 7 & $2.40 \mathrm{E}-03$ \\
\hline 19 & Centrosome & G0:0005813 & Cellular component & 510 & 25 & $2.41 \mathrm{E}-03$ \\
\hline 20 & Pulmonary valve morphogenesis & G0:0003184 & Biological process & 16 & 4 & $2.52 \mathrm{E}-03$ \\
\hline 21 & Forelimb morphogenesis & G0:0035136 & Biological process & 41 & 6 & 2.59E-03 \\
\hline 22 & Nephron tubule development & GO:0072080 & Biological process & 93 & 9 & 2.83E-03 \\
\hline 23 & Voltage-gated calcium channel activity & GO:0005245 & Molecular function & 47 & 7 & $3.17 \mathrm{E}-03$ \\
\hline 24 & Synaptic vesicle cycle & G0:0099504 & Biological process & 195 & 15 & 3.23E-03 \\
\hline 25 & Radial glial cell differentiation & G0:0060019 & Biological process & 12 & 3 & 3.31E-03 \\
\hline 26 & Renal tubule development & GO:0061326 & Biological process & 95 & 9 & $3.56 \mathrm{E}-03$ \\
\hline 27 & Z disc & G0:0030018 & Cellular component & 126 & 12 & $3.59 \mathrm{E}-03$ \\
\hline 28 & Response to monosaccharide & GO:0034284 & Biological process & 200 & 13 & 3.73E-03 \\
\hline 29 & Synaptic vesicle localization & GO:0097479 & Biological process & 165 & 13 & $3.89 \mathrm{E}-03$ \\
\hline 30 & Foregut morphogenesis & GO:0007440 & Biological process & 10 & 3 & $3.95 \mathrm{E}-03$ \\
\hline 31 & Ionotropic glutamate receptor signaling pathway & GO:0035235 & Biological process & 25 & 5 & $4.00 \mathrm{E}-03$ \\
\hline 32 & $\mathrm{SH} 3 / \mathrm{SH} 2$ adaptor activity & GO:0005070 & Molecular function & 54 & 7 & 4.17E-03 \\
\hline 33 & Ligand-gated ion channel activity & GO:0015276 & Molecular function & 144 & 12 & $4.35 \mathrm{E}-03$ \\
\hline 34 & Ligand-gated channel activity & GO:0022834 & Molecular function & 144 & 12 & 4.35E-03 \\
\hline 35 & $\begin{array}{l}\text { Regulation of translational initiation } \\
\text { in response to stress }\end{array}$ & G0:0043558 & Biological process & 14 & 3 & 4.39E-03 \\
\hline 36 & Cell cycle & G0:0007049 & Biological process & 1768 & 62 & 4.47E-03 \\
\hline
\end{tabular}

\footnotetext{
37 Calcium channel complex

38 Embryonic forelimb morphogenesis

39 Positive regulation of neuroblast proliferation

40 Labyrinthine layer morphogenesis
}

GO:0034704

GO:0035115

GO:0002052

GO:0060713
Cellular component

Biological process

Biological process

Biological process
65

33

22

21
$4.48 \mathrm{E}-03$

$4.48 \mathrm{E}-03$

$4.58 \mathrm{E}-03$

4.60E-03 
8.38E-01 8.38E-01 8.38E-01

8.38E-01

8.38E-01

8.38E-01

9.11E-01

9.11E-01

9.11E-01

9.11E-01

9.11E-01

9.11E-01

9.11E-01

9.11E-01

9.11E-01

9.11E-01

9.11E-01

9.11E-01

9.11E-01

9.11E-01

9.11E-01

9.11E-01

9.11E-01

9.11E-01

9.11E-01

9.11E-01

9.11E-01

9.11E-01

9.11E-01

9.11E-01

9.11E-01

9.11E-01

9.11E-01

9.11E-01

9.11E-01

9.11E-01

9.11E-01

9.11E-01

9.11E-01

9.11E-01

CPLX1; CHRM2; TRIM9; GRIN3A; RIMS4; GRIK5; GSK3B; NOTCH1; PRKCB; WNT7A; CACNA1I; CACNA1H GRB2; NDN; CASP3; WASF1; HAP1; BCAR1

GRIN3A; CACNG4; ITPR1; CATSPER4; CACNA2D3; JPH1; JPH3; RYR3; TRPV1; CACNA1C; CACNB4; ORAI2; CACNA1I; CACNA1H

COL6A2; EIF2B1; PTK2B; GIPR; GRIK5; LEP; PKLR; PRKCB; APOM; MAPK13; PTPRN2; SMARCA4; SPARC; CDT1; CASP3; BRSK2

YAP1; CTNNB1; JAG1; GSK3B; NOTCH1; GRHL2

CTNNB1; LEF1; SMARCA4; TERT; TLE2; TCF7L1

CPLX1; CHRM2; TRIM9; GRIN3A; RIMS4; ABR; GRIK5; GSK3B; NOTCH1; PRKCB; WNT7A; CACNA11; CACNA1H

GRIN3A; CACNG4; ITPR1; CATSPER4; CACNA2D3; JPH1; JPH3; RYR3; TRPV1; CACNA1C; CACNB4; ORAI2; CACNA1I;

CACNA1H

CTNNB1; NOTCH1; CHST11; LEF1; SKI; C5orf42; TFAP2A; WNT7A; CACNA1C; GRHL2; FRAS1; ALDH1A2

CTNNB1; NOTCH1; CHST11; LEF1; SKI; C5orf42; TFAP2A; WNT7A; CACNA1C; GRHL2; FRAS1; ALDH1A2

GRIN3A; PTK2B; GRID2; GRIK4; GRIK5

GRB2; NDN; CASP3; WASF1; HAP1; BCAR1

CTNNB1; FMN1; NOTCH1; CHST11; LEF1; SKI; C5orf42; TFAP2A; WNT7A; CACNA1C; GRHL2; FRAS1; ALDH1A2

CTNNB1; FMN1; NOTCH1; CHST11; LEF1; SKI; C5orf42; TFAP2A; WNT7A; CACNA1C; GRHL2; FRAS1; ALDH1A2

CPLX1; CHRM2; TRIM9; GRIN3A; RIMS4; ERC2; GRIK5; GSK3B; NOTCH1; PRKCB; WNT7A; CACNA1l; CACNA1H

JAG1; NOTCH1; CARD9; TRIM41

ITPR1; JPH1; JPH3; RYR3; TRPV1

CACNG4; CATSPER4; CACNA2D3; CACNA1C; CACNB4; CACNA1I; CACNA1H

NUBP2; TUBGCP2; TTC8; CTNNB1; RAP1GAP2; ZFYVE26; HEPACAM2; DISC1; GNAI1; GSK3B; NDN; NPM1; KIAA1377; SKI; TBCD; TFAP2A; TTC26; DYNLRB1; MAD1L1; NLRC5; FBF1; HAP1; BRSK2; KIAA0101; CDC25B

JAG1; HEYL; NOTCH1; ROBO2

CTNNB1; FMN1; TFAP2A; WNT7A; CACNA1C; ALDH1A2

YAP1; CTNNB1; ADAMTS16; DLG1; JAG1; HEYL; FMN1; NOTCH1; KIF26B

CACNG4; CATSPER4; CACNA2D3; CACNA1C; CACNB4; CACNA1I; CACNA1H

CDH2; CPLX1; CHRM2; TRIM9; GRIN3A; RIMS4; CTNNB1; ERC2; GRIK5; GSK3B; PRKCB; DENND1A; WNT7A; BRSK2;

DNAJC6

CDH2; LEF1; METTL3

YAP1; CTNNB1; ADAMTS16; DLG1; JAG1; HEYL; FMN1; NOTCH1; KIF26B

CTNNB1; BIN1; ANK3; PARVB; MURC; NRAP; JPH1; RYR3; CACNA1C; CSRP3; SORBS2; ACTN1

COL6A2; EIF2B1; PTK2B; GIPR; GRIK5; LEP; PKLR; APOM; PTPRN2; SMARCA4; SPARC; CASP3; BRSK2

CDH2; CPLX1; CHRM2; TRIM9; GRIN3A; RIMS4; CTNNB1; ERC2; GRIK5; GSK3B; PRKCB; WNT7A; BRSK2

CTNNB1; SMAD3; NOTCH1

GRIN3A; PTK2B; GRID2; GRIK4; GRIK5

CLNK; GRB2; BLNK; SHB; TP53BP2; SKAP1; GRAP2

GRIN3A; DLG1; PTK2B; GABRP; GRID2; GRIK4; GRIK5; ITPR1; JPH1; JPH3; RYR3; TRPV1

GRIN3A; DLG1; PTK2B; GABRP; GRID2; GRIK4; GRIK5; ITPR1; JPH1; JPH3; RYR3; TRPV1

EIF2AK4; NPM1; PPP1CA

ERN2; CGREF1; SEPT9; TUBGCP2; POLS; CTNNB1; DLG1; E2F3; WAPAL; UNC84A; ZFYVE26; HEPACAM2; SGSM3; BIN1; GNAI1; LIN9; ANK3; NR3C1; UHRF1; HELLS; KIF22; LEP; SMAD3; MAX; EIF2AK4; TRIM37; MYH9; NOTCH1; NPM1; MOV10L1; DDX4; PPP1CA; USP47; PPP2R2A; PPP6C; CHFR; PRKCB; MAPK13; METTL3; PCBP4; STARD9; MICAL3; KIAA1377; USP29; MRPL41; BOLL; TBCD; TERT; TP53BP2; XRCC3; CCNJL; WDR76; CDT1; CASP3; C12orf32; MAD1L1; AFAP1L2; RAE1; BRSK2; BRE; KIAA0101; CDC25B

CACNG4; CATSPER4; CACNA2D3; RYR3; CACNA1C; CACNB4; CACNA1I; CACNA1H

CTNNB1; TFAP2A; WNT7A; CACNA1C; ALDH1A2

CTNNB1; DCT; DISC1; NOTCH1

GRB2; LEF1; GRHL2; NCOA1 
Supplementary Table 12. (Continued)

\begin{tabular}{|c|c|c|c|c|c|c|}
\hline Rank & GO term & ID & Ontology & $\begin{array}{l}\text { Number } \\
\text { of genes }\end{array}$ & $\begin{array}{l}\text { Differentially } \\
\text { modified }\end{array}$ & $p$ value \\
\hline 41 & $\begin{array}{l}\text { Nucleobase-containing compound } \\
\text { catabolic process }\end{array}$ & GO:0034655 & Biological process & 546 & 23 & $4.61 \mathrm{E}-03$ \\
\hline 42 & ncRNA metabolic process & GO:0034660 & Biological process & 537 & 20 & 4.63E-03 \\
\hline 43 & Establishment of blood-brain barrier & GO:0060856 & Biological process & 10 & 3 & 4.93E-03 \\
\hline 44 & Ionotropic glutamate receptor complex & GO:0008328 & Cellular component & 51 & 7 & 4.96E-03 \\
\hline 45 & Response to UV & GO:0009411 & Biological process & 135 & 9 & $5.05 \mathrm{E}-03$ \\
\hline 46 & Glutamatergic synapse & GO:0098978 & Cellular component & 350 & 23 & $5.13 \mathrm{E}-03$ \\
\hline 47 & DNA metabolic process & GO:0006259 & Biological process & 987 & 36 & $5.15 \mathrm{E}-03$ \\
\hline 48 & Regulation of exocytosis & GO:0017157 & Biological process & 203 & 14 & $5.26 \mathrm{E}-03$ \\
\hline 49 & Semi-lunar valve development & GO:1905314 & Biological process & 32 & 5 & $5.36 \mathrm{E}-03$ \\
\hline 50 & Protein-DNA complex & GO:0032993 & Cellular component & 189 & 9 & 0.00551393 \\
\hline
\end{tabular}

Displayed for each ranked $\mathrm{GO}$ term is the identifier (ID), the related ontology, the total number of genes belonging to the $\mathrm{GO}$ term, the number of genes that are differentially modified, the $p$ value, the false discovery rate (FDR) adjusted $p$ value and the names of the differentially modified genes. The $G 0$ terms are ranked based on their $p$ value. 


\section{FDR adjusted $p \quad$ Genes}

\begin{tabular}{ll}
\hline $9.11 E-01$ & LRPPRC; ERN2; POLS; ACOT7; DPYS; XRN2; NT5C2; TNRC6B; SMG6; KPNA1; NPM1; CECR1; PKLR; PPP2R2A; METTL3; \\
& PCBP4; RNASEL; RPL4; ERI3; ADPGK; CASP3; KHSRP; ENTPD1 \\
$9.11 E-01$ & NPM3; ERN2; TBL3; POLS; XRN2; RPRD2; IARS; SMAD3; TYW1B; RRP15; POLR2A; MOV10L1; DDX4; METTL3; RNASEL; \\
& SMARCA4; SP1; ERI3; METTL8; KHSRP \\
$9.11 E-01$ & CTNNB1; TRPV1; WNT7A \\
$9.11 E-01$ & GRIN3A; DLG1; PTK2B; CACNG4; GRID2; GRIK4; GRIK5 \\
$9.11 E-01$ & ERCC5; EIF2AK4; NPM1; USP47; MAPK13; METTL3; CASP3; C12orf32; KIAA0101 \\
$9.11 E-01$ & WASF3; CPLX1; CHRM2; GRIN3A; DLG1;PTK2B; ABLIM3; ERC2; CACNG4; GRID2; ABR; GRIK5; GSK3B; HIP1; KPNA1; \\
& PPP1CA; PPP2R2A; ARHGAP22; CLSTN2; SPARC; WNT7A; GPR123; ACTN1 \\
$9.11 E-01$ & MCRS1; POLS; CTNNB1; RNF168; ERCC5; PTK2B; XRN2; WAPAL; SMG6; ZFYVE26; SHPRH; LIN9; UHRF1; GTF2H3; HELLS; \\
& KIF22; KPNA1; NPM1; LEF1; POLR2A; MOV10L1; DDX4; USP47; NEIL3; FAM111A; COPS7B; TERT; XRCC3; ERI3; GRHL2; \\
& CDT1;CASP3; C12Orf32; TOP3B; BRE; KIAA0101 \\
$9.11 E-01$ & CPLX1; CHRM2; TRIM9; GRIN3A; RIMS4; ABR; GRIK5; GSK3B; NOTCH1; PRKCB; WNT7A; CACNA1I; CACNA1H; HAP1 \\
$9.11 E-01$ & JAG1; ELN; HEYL; NOTCH1; ROBO2 \\
0.910798446 & CTNNB1; ERCC5; SHPRH; GTF2H3; MAX; NPM1; LEF1; SP1; TERT \\
\end{tabular}


Supplementary Table 13. Primer overview Tenascin XB (TNXB)

\begin{tabular}{|c|c|c|c|}
\hline \multicolumn{4}{|l|}{ PCR primers } \\
\hline Gene & Forward primer ( $\left.5^{\prime}-3^{\prime}\right)$ & \multicolumn{2}{|c|}{ Reverze primer (5'-3') } \\
\hline$T N X B$ & $\begin{array}{l}\text { (Bio-)GTGAAGTAGGTTATT } \\
\text { TAGGGGAAGAT }\end{array}$ & \multicolumn{2}{|c|}{$\begin{array}{l}\text { СТСАСТСАССТААССТТСССАА } \\
\text { САААТ }\end{array}$} \\
\hline TNXB (Multiplex) & TGTGAAGTAGGTTATTTAGGGGAAGA & \multicolumn{2}{|c|}{$\begin{array}{l}\text { ССТССАAАACAAACССТАACTAA } \\
\text { АААСТСТ }\end{array}$} \\
\hline TNXB (Singleplex) & TGTGAAGTAGGTTATTTAGGGGAAGA & \multicolumn{2}{|c|}{$\begin{array}{l}\text { (Bio-)ACAAACCCTAACTAAA } \\
\text { AACTCTTCCTACC }\end{array}$} \\
\hline DNAJB13 (Multiplex) & GGTATTTTGGGAGGATGAGTTA & \multicolumn{2}{|c|}{ СССТTСАAАAАСАAАССААСТААТ } \\
\hline DNAJB13 (Singleplex) & GTATTTTGGGAGGATGAGTTATAATTG & \multicolumn{2}{|c|}{$\begin{array}{l}\text { (Bio-)CTTCAAAAACAAACC } \\
\text { AACTAATAC }\end{array}$} \\
\hline \multicolumn{4}{|c|}{ Pyrosequencing primers } \\
\hline Gene & Sequencing primer ( $\left.5^{\prime}-3^{\prime}\right)$ & $\begin{array}{l}\text { Number } \\
\text { of CpGs }\end{array}$ & Target region (GRCh37) \\
\hline$T N X B$ & СААТСАТТССААСАСТАССТ & 8 & $6: 32063869-32063940$ \\
\hline TNXB (Primer 1) & GGTTAGTGTTTAGATGGG & 5 & $6: 32063874-32063913$ \\
\hline TNXB (Primer 2) & AGGATTGAGGTGTGA & 4 & $6: 32063823-32063867$ \\
\hline TNXB (Primer 3) & GGTGTGTGTATTTGTTG & 2 & 6:32063774-32063806 \\
\hline DNAJB13 (Primer 1) & GGATGAGTTATAATTGGG & 5 & $11: 73668745-73668795$ \\
\hline DNAJB13 (Primer 2) & TTTTGTTATTTGTTTGAAAA & 4 & $11: 73668618-73668673$ \\
\hline
\end{tabular}

Displayed is an overview of the polymerase chain reaction (PCR) and pyrosequencing primers for TNXB and DNAJB13 that were used in this study. Provided for each of the target genes are the primer sequences (Bio, biotinylated), as well as corresponding genomic coordinates (Ensembl GRCh37 assembly), amplicon sizes in base pairs (bp) and the analysis in which the primers have been applied (Pyrosequencing validation or cell-specific dorsal raphe nuclei (DRN) validation). For the pyrosequencing primers, the total number of $\mathrm{CpG}$ sites, the targeted region for sequencing, the orientation of the assay in the PyroMark assay design software 2.0 and the analysis in which the primers have been applied are listed per gene. 


\begin{tabular}{lll}
\hline Target region (GRCh37) & Product size (bp) & Analysis \\
\hline 6:32063788-32063996 & 209 & Pyroseq. validation \\
6:32063558-32063997 & 440 & Cell-specific DRN validation \\
6:32063566-32063997 & 432 & Cell-specific DRN validation \\
11:73668561-73668825 & 265 & Cell-specific DRN validation \\
11:73668563-73668824 & 262 & Cell-specific DRN validation \\
\hline & & \\
\hline PyroMark Orientation & & Analysis \\
\hline Lower strand (5'-3') & & Pyroseq. validation \\
Lower strand (5'-3') & Cell-specific DRN validation \\
Lower strand (5'-3') & Cell-specific DRN validation \\
Lower strand (5'-3') & Cell-specific DRN validation \\
Lower strand (5'-3') & Cell-specific DRN validation \\
Lower strand (5'-3') & Cell-specific DRN validation \\
\hline
\end{tabular}


Supplementary Table 14. Overview of the limiting dilution bisulfite pyrosequencing (LDBSP) parameters for Tenaxcin XB (TNXB)

\begin{tabular}{llll}
\hline Singleplex PCR & Control & AD & Total \\
\hline Number (+ percentage) of reactions with PCR product & $104(13.13)$ & $133(16.79)$ & $237(14.96)$ \\
\hline Allele estimation & & & \\
\hline Number (+ percentage) of reactions with 1 allele & $97(93.27)$ & $120(90.23)$ & $217(91.56)$ \\
Number (+ percentage) of reactions with 2 alleles & $5(4.81)$ & $8(6.02)$ & $13(5.49)$ \\
Number (+ percentage) of reactions with 3 alleles & $2(1.92)$ & $5(3.76)$ & $7(2.95)$ \\
Number (+ percentage) of multi-allele reactions & $7(6.73)$ & $13(9.77)$ & $20(8.44)$ \\
Number (+ percentage) of recovered alleles & $113(3.14)$ & $151(4.19)$ & $264(3.67)$ \\
\hline A
\end{tabular}

A summary of all the LDBSP parameters for pools of 50 serotonergic neurons isolated from unfixed post-mortem brain tissue using laser capture microdissection (LCM). Displayed are the total number of obtained polymerase chain reaction (PCR) products per experimental groups and the total amount of estimated alleles. Please refer to the written text for further specifications on the allele estimation method and criteria. 


\section{Chapter 6}

\section{Human-induced pluripotent stem cells as a model for studying sporadic Alzheimer's disease}

Renzo J.M. Riemens ${ }^{1,2}$, Gunter Kenis ${ }^{1}$, Twan van den Beucken ${ }^{3}$

1'Department of Psychiatry \& Neuropsychology, Graduate School MHeNS (School for Mental Health and Neuroscience), allocated with the Faculty Health Medicine and Life Sciences of Maastricht University, Maastricht, the Netherlands.

${ }^{2}$ Institute of Human Genetics, Julius Maximilian University, Wuerzburg, Germany.

${ }^{3}$ Department of Toxicogenomics, Graduate School GROW (Research School for Oncology and Developmental Biology), allocated with the Faculty Health Medicine and Life Sciences of Maastricht University, Maastricht, the Netherlands.

Neurobiology of Learning and Memory. 2020 Sep 22;107318.

Doi: 10.1016/j.nlm.2020.107318. 



\begin{abstract}
The discovery of induced pluripotent stem cell (iPSC) technology has the potential to accelerate scientific research for Alzheimer's disease (AD). iPSCs are therefore increasingly considered for AD modeling and drug development. Nevertheless, most of the work conducted so far has mainly focused on iPSC models from patients with familial $A D$ (fAD), while actually sporadic $A D$ (sAD) is more prevalent and represents over $90 \%$ of the $A D$ cases in the population. The development of more SAD models is therefore key for studying this multifactorial disorder. In fact, probing the unique genomes of SAD patients and their interaction with ADassociated environmental factors could contribute to a better understanding of this disease. However, initial iPSC-based models for SAD have shown a high degree of variability and inconsistencies in terms of $A D$ hallmarks. In this review, we provide an overview of the studies that have been conducted for SAD so far. In addition, we critically assess important sources of variability related to the model in addition to those that might be explained by the heterogeneous nature of SAD. These considerations might aid in developing more consistent iPSC models of sAD, which could help in developing a better understanding of the molecular mechanisms underlying the disease.
\end{abstract}

\title{
Keywords
}

Alzheimer's Disease, induced pluripotent stem cells, somatic mosaicism. 


\section{Introduction}

With the increasing life span of the general population, Alzheimer's disease (AD), which is an age-related neurodegenerative disorder, is becoming a major disease burden and socio-economic challenge worldwide. It was estimated in 2015 that 46.8 million people in the world are living with AD or a related form of dementia and it is expected that this number will double by 2040 [1]. The vast majority, i.e. more than $95 \%$, of the $A D$ cases are characterized by a late-onset form and sporadic development of the disease (that is sporadic AD (SAD)). Its origin is multifactorial, having both genetic and environmental factors, as well as their complex interactions, contributing to the onset and course of the disease. On the other hand, familial $A D$ ( $f A D$ ), which represent the early-onset form, is known to arise from mutations in genes such as amyloid precursor protein (APP), presenilin 1 (PSEN1) and presenilin 2 (PSEN2) [2]. Although distinguishable based on their time of onset and mode of transmission, both types of AD appear identical in light of clinical features and neuropathological hallmarks. Lesions of accumulated amyloid beta $(A \beta)$ protein and neurofibrillary tangles consisting of hyperphosphorylated tau protein are typically observed in AD brains, and are known to contribute to the neuronal cell death and immune activation, leading to region-specific atrophy. The loss of neural integrity in regional neurotransmitter systems is thought to underlie the clinical symptoms, such as cognitive decline and learning deficits, which are typically observed in AD patients.

In the past decades, our knowledge on the pathophysiology that underlies $A D$ has advanced tremendously. In particular, the neurotoxicity of aberrant $A \beta$ and neurofibrillary tangles are now much better understood. However, efforts to translate these insights into clinical success have floundered. Although researchers have conducted a large number of clinical trials for potential treatments for AD, almost none of the drugs have been brought to the market. Despite the blame being placed on a variety of factors, one of the main concerns has been the animal and cellular models that were used in the initial stages of drug development [3]. Most of these have relied on transgenic models harboring genetic mutations associated with fAD. Despite the fact that these have proven to be instrumental for basic research, they only partially reflect $A D$ phenotypes and have thus far have been unable to explain the complex and heterogeneous nature of SAD. It has therefore been debated that more accurate models for SAD harboring the enormous amounts of individual genetic backgrounds within the patient population are required to further improve our insights into SAD development and progression. Moreover, it has even been hypothesized that such models would potentially be able to aid in the identification of novel biomarkers for early detection of SAD, as well as in the discovery of new and much needed therapeutics. 
With the foundation of human induced pluripotent stem cell (iPSCs) over a decade ago, new doors for AD-research have been opened [4]. Since their discovery, human iPSCs have been offering a promising avenue to fill the gap between animal and human research, providing a new platform for disease modelling and drug testing that has the potential to broaden our knowledge on the underlying pathophysiology. Pluripotent stem cell technology is currently providing an unlimited source of patient-derived cells that can be differentiated into disease-relevant cells, including neurons and glia (reviewed in $[5,6])$. The majority of the conducted work so far in AD has, however, used iPSC-cells derived from patients with $F A D$ where, similar to previous animal studies, specific genetic mutations were studied that drive the disease onset. The establishment of more iPSCbased models from SAD patients is therefore necessary and could potentially benefit research into disease etiology and development of therapeutic strategies. However, developing iPSC models of SAD remains challenging due to the multifactorial nature of the disease, the live-long disease progression and the high degree of inter-individual heterogeneity that might be reflected in cells derived from SAD patients. Furthermore, aside from the variability associated with the disease, several technological challenges currently remain that could further impact on the variability that is observed in these SAD-iPSC models. In this review, we therefore provide an overview of the studies that have been conducted thus far and we critically assess important sources of variability related to the model in addition to those that might be explained by the heterogeneous nature of the disease.

\section{iPSC-derived models for SAD}

To date, a limited number of studies has been performed with iPSC-derived neurons from SAD patients (Table 1). Modeling AD using patient-derived iPSCs was initiated from $f A D$ cases with known mutations in disease-causing genes, such as APP, PSEN1 and PSEN2. iPSC-derived neurons from SAD patients in these studies were often studied in parallel, with the main goal of seeking to find AD-associated cellular phenotypes for the validation of their potential for SAD modeling. For this reason, many of these reports specifically look into the presence of AD-associated hallmarks such as amyloid beta, hyperphosphorylated tau and elevated levels of GSK-3 $\beta$. The very first study in 2012 , which is a comparative study using iPSCderived neurons from fAD and SAD patients [7], demonstrated increased levels of $A \beta(1-40)$ that were found to be secreted from SAD neurons in comparison to non$A D$ controls. These findings in the SAD neurons were in line with the observations in neurons from fAD patients. However, this was only observed using iPSCderived neurons from one out of two SAD patients. Secreted levels of $A \beta(1-40)$ using iPSC-derived neurons from a second patients were similar to levels obtained using iPSC-derived neurons from two non-demented control subjects. Also other hallmarks of AD, i.e. phosphorylated tau and active GSK-3 $\beta$, were elevated in the iPSC-derived neurons that exhibited elevated $A \beta(1-40)$ secretion, while the levels 
of these markers for the other SAD patient-derived neurons were again close to those observed in non-demented controls. Overall, this study suggests that iPSCderived neurons from SAD patients can display similar hallmarks to fAD lines, but that these are not evident for every SAD patient. Interestingly, this heterogeneity corresponds with the complexity of $S A D$ and our general understanding on disease causation and progression. In a later study, Kondo and colleagues reported that iPSC-derived neurons from 2 distinct SAD patients did not show elevated secretion of $A \beta(1-40)$ and $A \beta(1-42)$ compared to iPSC-derived neurons obtained from 3 control subjects [8]. Instead, neurons derived from one SAD patient displayed elevated levels of intracellular $A \beta$ fragments, while this was not observed for the second SAD patient line. Finally, Foveau et al. reported the generation of 3 iPSCderived neural models from SAD patients and 3 control lines from non-cognitively impaired individuals [9]. Secreted levels of $A \beta(1-40)$ and $A \beta(1-42)$ tended to be lower in $S A D$ derived neural models although this was not statistically significant. No changes in the ratio $A \beta(1-42) / A \beta(1-40)$ were observed for $S A D$ derived neural models compared to controls

In addition, several studies have also directly focused on patient-derived iPSC lines that harbor known SAD genetic risk factors. In the study by Duan and coworkers, iPSC-derived neurons were generated from $3 \mathrm{SAD}$ patients all carrying the $A P O E 3 / E 4$ genotype [10]. In this study, increased extracellular levels of $A \beta(1-42)$ were reported for two out of three patient-derived neural models, while $A \beta(1-40)$ levels remained unchanged compared to a panel of iPSC-derived neurons from control subjects. Ochalek and coworkers found increased extracellular levels of both $A \beta(1-40)$ and $A \beta(1-42)$ in neurons derived from 4 sAD patients compared to control neurons, while the ratio $A \beta(1-42) / A \beta(1-40)$ did not change relative to controls [11]. In addition, hyperphosphorylation of tau, increased GSK3 $\beta$ activity, APP synthesis and APP C-terminal cleavage was detected in neurons from SAD compared to neurons obtained from iPSC control lines. The study performed by Young et al. focused on SORL1, which is an endocytic trafficking factor whose levels modulate the processing of APP to $A \beta$ and other proteolytic products implicated in SAD [12]. Loss of SORL1 expression has been documented in SAD cases and has been associated with SAD in both candidate gene and GWAS analyses. By studying patient iPSC-derived neurons, this latter study confirmed the importance of the SORL1/APP pathway in SAD, and their findings corroborated previous studies in cell and animal models [12]. In another study by Hossini et al., SAD IPSC-derived neurons were analyzed to assess their reflection of disease phenotype in gene expression patterns and the expression of typical AD proteins [13]. The differentiated neurons reflected SAD phenotypes due to the presence of phosphorylated tau proteins and the upregulation of GSK-3 $\beta$. Further analysis of the neurons revealed significant changes in the expression of other genes 
associated with $A D$, including subunits of the proteasome complex. Moreover, a disease-specific protein association network that models $A D$ pathology on the transcriptome level could be generated from the AD-iPSC lines.

Taken together, these studies have demonstrated that SAD patients' iPSC-derived neurons are able to recapitulate neuropathological processes of the disease, which represent critical first steps in assessing the potential of using iPSCs in SAD research. Simultaneously, these studies have also proven that a high degree of variability in terms of disease hallmarks in iPSC-derived neurons from SAD patients is common. Variability between cell lines in terms of the $A \beta$ type, i.e. $A \beta(1-42)$ or $A \beta(1-40)$, which is altered in SAD derived neurons, as well as variability between iPSC-derived neurons from different SAD patients within a study (Table 1). While some of this might be explained due to heterogeneous nature of $s A D$, it is likely that other source of variability related to the model play a role in this as well.

Until now, most of the described work on SAD models has been conducted using iPSC-derived neurons, mainly because protocols to generate human iPSC-derived microglia and astrocytes were absent or laborious. However, over recent years, several protocols have been published for differentiating iPSC into microglia [1417]. This has been a breakthrough for studying the function of microglia in human disease as human microglia appear very distinct from its murine counterpart especially in regards to immune activation $[18,19]$. Human microglia were found to have higher expression of genes involved in pathways related to longevity and anti-inflammatory responses compared to rodent microglia [19]. This suggests that the function of human microglia may not be well recapitulated in murine $A D$ models.

Applying iPSC-derived microglia, Lin et al. investigated the role of the most significate $A D$ risk gene, $A P O E 4$ on human microglia function and gene expression [20]. For this an $A P O E 3 / 3$ iPSC line was edited using CRISPR/Cas9 technology to create an isogenic APOE4/4 line. This modification was found to result in reduced phagocytic activity of the microglia and altered gene expression levels of over 1,000 genes. In a reciprocal approach, gene editing of a patient-derived APOE4/4 bearing iPSC line into an $A P O E 3 / 3$ genotype restored the impaired phagocytic phenotype. 
Table 1. Overview of published studies using induced pluripotent stem cell (iPSC)-derived neurons from sporadic Alzheimer's disease (SAD) patients.

\begin{tabular}{|c|c|c|c|c|c|c|c|c|c|}
\hline Study & ID & $A \beta(1-40)$ & $A \beta(1-42)$ & $A \beta 42 / 40$ & int. $A \beta$ & p-TAU & aGSK3 & 3 Sex & $\begin{array}{l}\text { \#iPCS lines } \\
\text { per patient }\end{array}$ \\
\hline \multirow{2}{*}{$\begin{array}{l}\text { Israel } \\
\text { et al. (2012) }\end{array}$} & sAD1 & - & N.R. & N.R. & N.R. & - & - & $\mathrm{F}$ & 3 \\
\hline & sAD2 & $\uparrow$ & N.R. & N.R. & N.R. & $\uparrow$ & $\uparrow$ & M & 5 \\
\hline \multirow[t]{2}{*}{ Kondo et al. (2013) } & AD3E211 & - & - & - & - & N.R. & N.R. & M & 1 \\
\hline & AD8K213 & - & - & - & $\uparrow$ & N.R. & N.R. & M & 1 \\
\hline \multirow{3}{*}{$\begin{array}{l}\text { Duan } \\
\text { et al. (2014) }\end{array}$} & AG04402 & N.R. & $\uparrow$ & $\uparrow$ & N.R. & N.R. & N.R. & M & 1 \\
\hline & AG11414 & N.R. & $\uparrow$ & $\uparrow$ & N.R. & N.R. & N.R. & M & 1 \\
\hline & AG05810 & N.R. & - & - & N.R. & N.R. & N.R. & $\mathrm{F}$ & 1 \\
\hline \multirow[t]{4}{*}{ Ochalek et al. (2017) } & BIOT-0904-LOAD & $\uparrow$ & $\uparrow$ & - & N.R. & $\uparrow$ & $\uparrow$ & $\mathrm{F}$ & 2 \\
\hline & BIOT-0630-LOAD & $\uparrow$ & $\uparrow$ & - & N.R. & $\uparrow$ & $\uparrow$ & M & 1 \\
\hline & BIOT-4828-LOAD & $\uparrow$ & $\uparrow$ & - & N.R. & $\uparrow$ & $\uparrow$ & $\mathrm{F}$ & 2 \\
\hline & BIOT-0726-LOAD & $\uparrow$ & $\uparrow$ & - & N.R. & $\uparrow$ & $\uparrow$ & $\mathrm{F}$ & 1 \\
\hline \multirow[t]{3}{*}{ Foveau et al. (2019) } & CQ2 & - & - & - & N.D. & N.R. & N.R. & $\mathrm{F}$ & 2 \\
\hline & CQ3 & - & - & - & N.R. & N.R. & N.R. & M & 2 \\
\hline & CQ6 & - & - & - & N.R. & N.R. & N.R. & M & 2 \\
\hline
\end{tabular}

ID is identifier, in.A $\beta$ is intracellular A $\beta, p-T A U$ is phosphorylated TAU, aGSK3 $\beta$ is GSK3 $\beta$ activity, N.R. is not reported, N.D. is not detected, $F$ is female, $M$ is male, - is no change, $\uparrow$ is increased.

A series of studies illustrates the importance of studying another $A D$ risk gene, triggering receptor expressed on myeloid cells-2 (TREM2) in a human microglia model. In humans a heterozygous TREM2 R47H mutation confers increased $\mathrm{AD}$ risk, almost to a similar degree as a single APOE4 e4 allele [21, 22]. Mice bearing a heterozygous TREM R47H mutation exhibited aberrant TREM2 mRNA splicing and reduced expression of TREM2 mRNA and protein [23]. Strikingly, TREM2 mRNA levels and splicing were normal in human iPSC-derived microglia carrying the TREM2 R47H mutation. In line with this, Claes and colleagues found that heterozygous TREM2 $\mathrm{H} 47 \mathrm{H}$ iPSC-derived human microglia displayed normal phagocytosis capacity in contrast to TREM2 heterozygous and homozygous knockout microglia [24]. This indicates that TREM2 R47H phenotypes found in murine models cannot be translated to humans, and highlights the need for adequate human microglia models to study SAD.

Differentiation protocols to generate human iPSC-derived astrocytes from iPSC lines have been published nearly a decade ago $[8,25]$. However, these protocols were laborious and yielded only a low percentage of differentiated astrocytes. 
Nevertheless, using this method it was found that astrocytes differentiated from SAD and $A A D$ patient-derived IPSC lines displayed $A \beta$ accumulation [8]. Using a slightly modified protocol, Oksanen et al. generated astrocytes using iPSC lines from three fAD patients with PSEN1 exon 9 deletion [26]. The astrocyte cultures from fAD patients showed elevated secretion of $A \beta(1-42)$, while $A \beta(1-40)$ secretion was unaltered in comparison to astrocyte cultures obtained from healthy control lines or gene-corrected isogenic controls. In addition, the iPSC-derived astrocytes from fAD patients showed increased oxidative stress and altered metabolism. Finally, Jones et al. described a protocol that can generate enriched populations of mature cortical astrocytes (>95\%), within 30 days of induction starting from NPCs [27]. A $\beta$ levels were not investigated in this study, however, iPSC-derived astrocytes from both SAD and fAD patients displayed altered morphological appearance, aberrant localization of astrocyte markers and an overall atrophic profiles.

Differentiation of neurons, astrocytes and microglia from patient-derived iPSC offers a powerful toolbox for studying SAD. Nevertheless, in vitro cultures of these cell types often lack essential aspects from their natural environment including cell-cell contact between multiple cell types, which negatively impacts on the maturity and functionality of these cells. To overcome these limitations researchers have been largely using two strategies; growing iPSC-derived cells in 3 dimensional (3D) structures, including spheres and organoid models or by transplanting iPSC-derived cells into the brain of mice. For instance, astrocyte maturation can be greatly improved by cultivating iPSC-derived astrocytes in 3D human cortical spheroids (hCSs) [28] in direct contact with neurons for prolonged periods of time [29]. Astrocytes in long-term cultures (over 20 months) display gene expression patterns and functional properties of matured astrocytes [29]. Also microglia function can be enhanced by allowing interactions from a 3D brain environment (reviewed in [30]). For example, Abud and coworkers showed that culturing human iPSC-derived microglia (iMGs) on hiPSC 3D brain organoids (BORGs) results in migration and engraftment of the iMGs [14]. Integrated iMGs display a more mature and ramified morphology and respond to injury similar as microglia in brain tissue. Trujillo et al. describe the generation of functional cortical organoids from NPCs [31]. By applying single-cell transcriptional profiling and functional validation over a 10-month period of time, the authors could demonstrate that long-term development of cortical organoids is a highly dynamic process involving a range of different cell types. In addition to cellular maturation, the cortical organoids displayed increased electrophysiological activity. Eventually the organoids displayed electrophysiological network activity that was reminiscent of the spontaneous and synchronized activity in developing human neocortex [31]. 


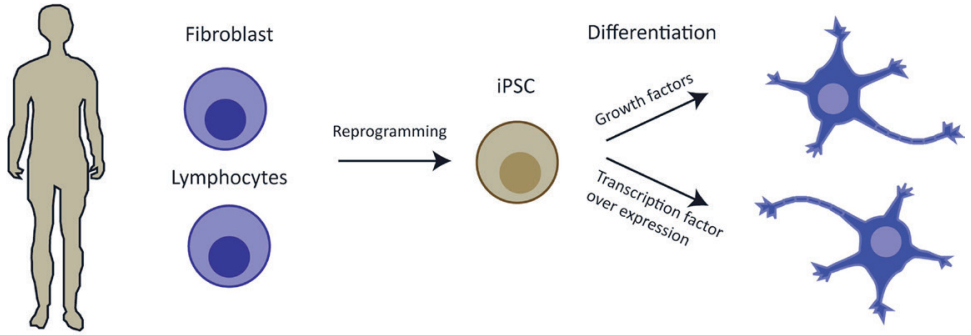

B

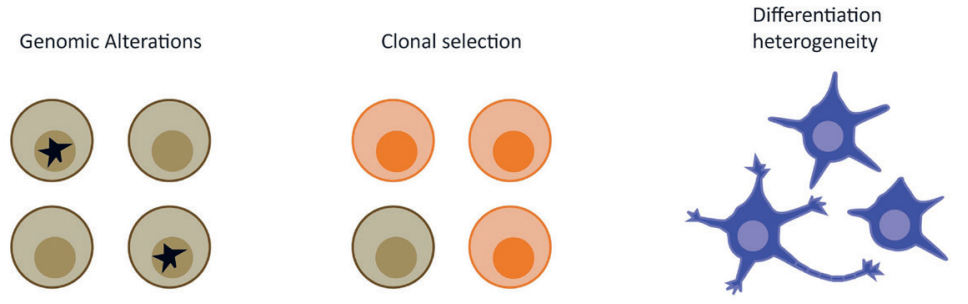

C
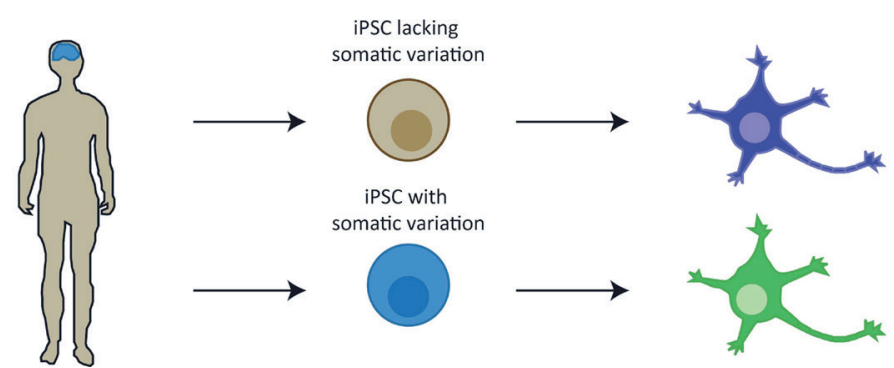

Figure 1. Generation of human induced pluripotent stem cell (iPSC)-derived cell models for sporadic Alzheimer's disease (SAD). A. Somatic cells can be reprogrammed to iPSCs. Skin fibroblasts or peripheral blood lymphocytes (PBLs) are frequently used as source of somatic cells. Final neuronal cell types of interest can be generated through directed differentiation of iPSCs using signaling molecules or by exogenous expression of lineage-specific transcription factors. B. Potential sources of variability in iPSC-derived cell models. Reprogramming can induce genomic alterations that might stay undetected in case of small alterations (Left panel). Human somatic cells might not be as equipotent as previously assumed. Selection of superior iPSC clones can bias the ultimate disease model (middle panel). Long and complex differentiation schemes can influence robustness of the ultimate disease model. Variability can occur in terms of the maturity of the desired cellular phenotype, the percentage of cells that obtained the correct phenotype and the percentage of unwanted phenotypes created during differentiation (left panel). C. Somatic mosaicism is frequently detected in brains of patients with neurological diseases like AD (indicated in blue). Generated iPSC models from fibroblasts or PBLs might lack these unique genotypes that could be essential for an accurate SAD iPSC-derived cell model. (For interpretation of the references to colour in this figure legend, the reader is referred to the web version of this article.) 
These examples illustrate how the 3D microenvironment in organoid structures can affect maturity and functionality of cells of the CNS. For a more comprehensive review on brain organoids, please refer to [32].

A powerful alternative way to grow iPSC-derived cells within the context of a CNS microenvironment relies on transplanting cells into the brain of mice. For example, Espuny-Camacho et al. transplanted human iPSC-derived cortical neuronal precursors into the brain of a murine $A D$ model and could show that these precursor cells differentiate into mature human neurons that are integrated into the murine brain [33]. These neurons express 3R/4R tau splice forms, display aberrant changes in phosphorylation and conformational of tau, and undergo neurodegeneration. Hasselmann and colleagues, transplanted hiPSC-derived hematopoietic progenitor cells into the postnatal brain of humanized, immunecompromised mice [34]. This resulted in context-dependent differentiation of the HPCs into microglia and other CNS macrophages. Transcriptome analysis revealed a high degree of clustering between human microglia obtained through transplantation in mice and ex vivo human microglia. This suggest that the murine brain environment promotes differentiation of HPCs into human microglia. Human microglia surrounding $A \beta$ plaques in transplanted mice display robust transcriptional response that are partly distinct from murine microglia. Linaro et al. found that cortical pyramidal neurons derived from human ESC line H9 can integrate as single cells in neonatal mouse cortex upon transplantation [35]. In particular, the transplanted neurons displayed robust maturation at the single-cell level and strong interactions with the host neurons. Moreover, sensory stimulation resulted in physiological responses with good resemblance to that of native cortical neurons.

All together, these studies show great potential for chimeric xenograft models for studying diseases like SAD. Transplation of patient-derived and/ or genetically modified cell types and assessing their in vivo function in a natural microenvironment may lead to a more complete understanding of SAD.

\section{Sources of variability in iPSC-derived neural models for SAD}

The ability to generate iPSC-derived cell models of SAD patients harbors great potential for studying the etiology of $S A D$ as well as for the development of biomarkers or therapeutic targets for SAD (Figure 1A). More specifically, this platform provides a valuable tool in exploring the complex heterogeneous nature in the etiology of SAD through the interrogation of functional effects of genetic, epigenetic and transcriptional variants linked to risk, as well as protective environmental factors. However, there are several issues associated with iPSC models that require further investigation before the actual potential of this 
technology in the context of SAD becomes more evident. Here we will discuss for several of these issues, and how these could impact the interpretation of the findings in relation to SAD.

\section{iPSC generation and differentiation}

Generation of iPSCs from SAD patients and differentiation into disease relevant neural lineages with high reproducibility is a key factor for developing robust SAD models. However, iPSC models and derived neurons have their limitations and using them to study SAD is particularly challenging. First of all, it is well known that reprogramming of somatic cells into iPSC can give rise to random genomic alterations including copy number variants, indels and karyotypic aberations that were not present in the parental somatic cells (Figure 1B) (reviewed in [36]). While it is relatively easy to select iPSC lines with normal karyotypes it is less straightforward to avoid smaller rearrangements or indels. Evidently, any alteration that is not present in somatic cells is undesirable and could potentially affect study outcome.

Second, evidence supports that not all iPSC lines have equal potency to differentiate into the desired mature phenotype (Figure 1B) [37, 38]. Hu et al. report reduced and more variable neural differentiation efficiencies using a panel of human iPSC lines [37]. This was not related to technical or cell culture related issues since various human embryonic stem cells used in parallel revealed robust and efficient differentiation. All tested iPSC lines in this study were generated by reprogramming of fibroblasts. In addition, the tissue of origin from which iPSC lines are derived can also affect differentiation potential. Profound differences in differentiation efficiency were observed for iPSC lines that originated from umbilical cord blood cells (CB-iPSCs) and foreskin keratinocytes (K-iPSCs) [38]. While CBiPSCs were more efficient in generating hematopoietic cell types than K-iPSCs, K-iPSCs differentiated more efficiently into keratinocytes compared to CB-iPSCs. This study suggests that iPSCs can retain epigenetic marks from the original tissue the iPSCs were derived from. It will therefore be necessary to assess how variability between different neuronal lines derived from donor cells of distinct developmental lineages could affect the interpretation of disease phenotypes that might be observed in SAD lines.

Third, reprogramming somatic cells into iPSCs has been associated with loss of aging traits such as certain epigenetic chromatin marks, telomerase shortening and altered mitochondrial metabolism [39, 40]. These aging hallmarks are likely of high relevance for the development of an age-related disease like SAD. Certain phenotypes associated with SAD might not be discovered in the absence of aging. Brain cells derived from iPSCs might therefore not be the most optimal 
model for studying $S A D$, but this requires further investigation. Efforts to create neurons that have preserved the aging signature have led to direct reprogramming of fibroblasts into neurons. However, it is unclear how other neural traits are affected by direct reprogramming or what the impact of direct reprogramming is on genomic integrity. In addition, limited neurons can be produced since there's no cell with self-renewing capacity [40, 41]. Whether neurons with a preserved aging signature presents a better in vitro cell model for studying SAD compared to iPSC-derived neurons remains to be investigated. For this reason, a wealth of research in currently focused on developing physiologically complete models of accelerating aging and defining methods for the concept of age-preservation during reprogramming. In this regard, one could also think of age accelerating compounds, prolonged cultures times or the production of cellular intermediates that allow expansion before differentiation, while maintaining their aging hallmarks [42]. As such, these efforts might provide a suitable solution to this issue.

Fourth, aside from the fact that the current differentiation protocols remain limited in the generation of all neural cell types found in the brain (reviewed in [6]), great variability in the final neuronal phenotype is introduced by the extents and complexity of the differentiation protocols themselves (Figure 1B). A variety of protocols have been developed to generate neuronal cells from iPSCs (reviewed in [6, 43]) and can be obtained by directed differentiation using signaling molecules or by artificial overexpression of lineage-specific transcription factors. The lack of consensus in differentiation methodology complicates study comparisons. However, this does not account for all variability that is introduced by culturing practices of iPSC models. A multicenter study investigated the transcriptome and proteome of two human iPSC lines at two time points during differentiation into cortical projection neurons using the same standard operating procedure in three independent inductions [44]. The authors report poor reproducibility in differential gene expression signatures between these two lines across the five test sites. These results illustrate that even when intra-laboratory variability is acceptable, huge inter-laboratory variability can exist. Despite the use of a standard operating procedure and exactly the same iPSC lines, very subtle differences in culturing conditions between laboratories can lead to large differences in phenotypic outcome.

\section{Control cells}

A completely distinct problem that complicates interpretation of studies using iPSC $S A D$ lines besides the issues already described above lies in the identification of adequate control cell lines. It is not trivial to define a healthy or non-diseased control. Most studies utilizing iPSC-derived disease models apply iPSC lines from unrelated healthy donors as controls, since isogenic non-diseased controls are not available or impossible to produce. A genome-wide association studies performed 
by the 1000 genomes project consortium revealed that a set of control cell lines obtained from multiple individuals contained 50 to 100 disease-associated genetic variants [45]. This illustrates the problem we face in finding good reference models. Other initiatives have shown that the most prominent source of variability in a reprogrammed iPSC is related to its own genetic background [46-50]. Studying a sufficiently large reference panel may help to reduce the noise that results from the heterogeneity in the genetic background from individuals. Ultimately, this will be beneficial for the discovery of subtle, but genuine disease causing factors.

\section{Somatic mosaicism}

In the recent years, multiple studies have found proof for the presence of de novo somatic mutations in neurological disease (reviewed in [51]). Somatic mutations can arise postzygotically during embryonic development or during postnatal life. The impact of this so-called somatic mosaicism is depending on the time in life and the cell type in which these somatic mutations occur. Somatic mutations that take place early in life will be present in the majority of cells in the human body. Whereas somatic mutations that occur late will only be present in a small subset of cells and may be restricted to a single tissue. It has been found that somatic mutations with alternate allele frequencies as little as $1 \%$ can cause disease [52]. If indeed somatic mutations in the human brain play an important role in onset and progression of SAD than it is important that the IPSC also carry these somatic mutations. In a study by Bushman and coworkers it was reported that neurons isolated from SAD patients displayed increases in DNA contents and elevated APP gene copy numbers (up to 12 copies per neuron) that were not detectable in lymphocytes from the same patients [53]. Later the same group found that $A P P$ mosaicism in the brains of $S A D$ patients was caused by integration of APP genomic complementary DNAs into the genome [54]. Both wild-type sequences as well as smaller, exon lacking variants were found to be incorporated in the genome of SAD neurons. Some of these APP mRNA variants encoded proteins can cause neurotoxicity. It remains to be investigated whether APP mosaicism in the brains of SAD patients is a cause or an effect of AD.

However, in order to generate adequate disease models for SAD it is important to determine whether somatic mosaicism in the brains of SAD patients plays a causal role or whether this occurs as consequence of $A D$. In case that somatic mosaicism is driving $S A D$ development then the iPSC-derived disease model should also carry this genetic variation. If somatic mosaicism occurs as a result of $A D$ then the genetic variation is not essential in the iPSC-derived disease model (Figure 1C). Whether it is possible to generate an iPSC line bearing such a somatic mosaicism is highly questionable for several reasons. First, the somatic mosaicism needs to be present in the tissue from which iPSC are generated. This can be problematic, 
as the study by Bushman and coworkers showed that somatic variants in the brain were absent in lymphocytes [53]. Second, such relatively large genetic alteration may be tolerable in post-mitotic neurons, but is likely not compatible with cell proliferation.

Generating iPSCs from patients with somatic mosaicism can in certain situation also be advantageous. In cases where the disease causing somatic mutations are known, iPSC lines can be generated for mutant and wild-type from the same patient [55]. This offers the unique opportunity to generate an isogenic pair of iPSC clones, thereby reducing some of the noise associated with generic iPSC control lines.

\section{Reprogramming capacity}

For a long time it was thought that all individual somatic cells had the same reprogramming capacity or so called clonal equipotency [56]. However, a recent study revealed that a small subset of "elite" clones dominates reprogramming of somatic mouse cells intro pluripotent stem cells [57] (Figure 1B). Expression of Wnt1 was identified as a key hallmark of elite cells. If this phenomenon also applies for humans, than this will have important consequences for the iPSCderived cell models generated with these selected "elite" clones. This raises the question to what degree the selected "elite" clones can capture the diseaseassociated genotype and thus how representative these models are for the disease. Potentially, somatic mosaicism could affect reprogramming capacity, and lead to counter selection against the desired iPSC genotype. Worst-case scenario is that the disease-associated genotype cannot be captured by reprogramming somatic cells into iPSCs. This is crucially important for accurate disease modelling and drug screening studies.

\section{Conclusions and future perspectives}

The development of iPSC technology has great potential for studying complex multifactorial diseases like SAD. Although hallmarks of $A D$ can be detected in iPSC-derived cell models, there is a high degree of variability in terms of their presence and severity between different SAD lines. This variability can mask important, but subtle genetic differences between individuals. As the number of studies using iPSC-derived SAD models increases, it will be important to ensure highly reproducible and informative data. The compilation of a large reference panel representing non-demented control subjects will be instrumental for adequate interpretation of genomic variants found in SAD patients. It will be important to better understand clonal selection during reprogramming, and the relevance of somatic mosaicism and aging in SAD, to ensure representative iPSCs-derived cell models of SAD. 
In order to increase translatability of pre-clinical findings it will be important to continue to develop and improve SAD models that reflect the brains natural environment. Sophisticated iPSC-derived cell models should consist of multiple cell types, in 3D conformations allowing cell-cell interactions. Further development of more accurate models for studying SAD like long-term organoid cultures and chimeric xenograft models will be essential in the coming years.

While it is well established that genetic alterations contribute to the pathophysiology of SAD, detailed multi-omics characterization of iPSC-based models and the role of environmental factors associated with $S A D$ pathophysiology remain currently underexplored. It is recognized that in the aging brain, environmental risk factors together with complex gene-environment interactions, are playing crucial roles in reinforcing SAD pathogenesis. Recent studies have shown that exposure to these risk factors can bring about epigenomic, transcriptomic, proteomic and metabolomic changes related to $A D$, which can bring about sustained alterations in molecular processes leading into the manifestation of the full-blown disease [58]. Furthermore, many $A D$ researchers tend to suggest that these environmental risk factors operate during the pre-clinical phase of $A D$, even decades before the appearance of the first clinical symptoms. In either way, there is overwhelming support for environmental and extra-genetic risk factors as inducers of SAD pathogenesis. Nevertheless, the exact molecular nature of these interactions, as well as their temporal relationship with the development of the disease, remain largely unknown. Further research from experimental and epidemiological studies that focus on the interaction of these factors with the aforementioned multi-omics modalities therefore remains necessary to develop a better understanding about their contribution to the course and development of the disease. In this regard, iPSC-derived systems might provide exciting opportunities for modelling these interactions, by allowing one to expose cells derived from healthy individuals and SAD patients with different genetic backgrounds to environmental insults and study their cellular and molecular responses in an in vitro setting.

\section{Acknowledgments}

This research was made possible by BReIN (Brightlands e-infrastructure for Neurohealth), an initiative which is co-funded by the Province of Limburg, Maastricht University and Maastricht University Medical Centre + in the Netherlands. 


\section{References}

1. Clay E, Zhou J, Yi Z-M, Zhai S, Toumi M. Economic burden for Alzheimer's disease in China from 2010 to 2050: a modelling study. Journal of Market Access \& Health Policy. 2019;7(1):1667195.

2. Chakrabarti S, Khemka VK, Banerjee A, Chatterjee G, Ganguly A, Biswas A. Metabolic risk factors of sporadic Alzheimer's disease: implications in the pathology, pathogenesis and treatment. Aging and disease. 2015;6(4):282.

3. Laurijssens B, Aujard F, Rahman A. Animal models of Alzheimer's disease and drug development. Drug Discovery Today: Technologies. 2013;10(3):e319-e27.

4. Takahashi K, Yamanaka S. Induction of pluripotent stem cells from mouse embryonic and adult fibroblast cultures by defined factors. cell. 2006;126(4):663-76.

5. Shi Y, Inoue H, Wu JC, Yamanaka S. Induced pluripotent stem cell technology: a decade of progress. Nature reviews Drug discovery. 2017;16(2):115-30.

6. Riemens RJ, van den Hove DL, Esteller M, Delgado-Morales R. Directing neuronal cell fate in vitro: achievements and challenges. Progress in neurobiology. 2018;168:42-68.

7. Israel MA, Yuan SH, Bardy C, Reyna SM, Mu Y, Herrera C, et al. Probing sporadic and familial Alzheimer's disease using induced pluripotent stem cells. Nature. 2012;482(7384):216-20.

8. Kondo T, Asai M, Tsukita K, Kutoku Y, Ohsawa Y, Sunada Y, et al. Modeling Alzheimer's disease with iPSCs reveals stress phenotypes associated with intracellular Abeta and differential drug responsiveness. Cell Stem Cell. 2013;12(4):487-96.

9. Foveau B, Correia AS, Hébert SS, Rainone S, Potvin O, Kergoat M-J, et al. Stem cell-derived neurons as cellular models of sporadic Alzheimer's disease. Journal of Alzheimer's Disease. 2019;67(3):893-910.

10. Duan L, Bhattacharyya BJ, Belmadani A, Pan L, Miller RJ, Kessler JA. Stem cell derived basal forebrain cholinergic neurons from Alzheimer's disease patients are more susceptible to cell death. Mol Neurodegener. 2014;9:3.

11. Ochalek A, Mihalik B, Avci HX, Chandrasekaran A, Téglási A, Bock I, et al. Neurons derived from sporadic Alzheimer's disease iPSCs reveal elevated TAU hyperphosphorylation, increased amyloid levels, and GSK3B activation. Alzheimer's research \& therapy. 2017;9(1):1-19.

12. Young JE, Boulanger-Weill J, Williams DA, Woodruff G, Buen F, Revilla AC, et al. Elucidating molecular phenotypes caused by the SORL1 Alzheimer's disease genetic risk factor using human induced pluripotent stem cells. Cell Stem Cell. 2015;16(4):373-85.

13. Hossini AM, Megges M, Prigione A, Lichtner B, Toliat MR, Wruck W, et al. Induced pluripotent stem cell-derived neuronal cells from a sporadic Alzheimer's disease donor as a model for investigating AD-associated gene regulatory networks. BMC Genomics. 2015;16:84.

14. Abud EM, Ramirez RN, Martinez ES, Healy LM, Nguyen CH, Newman SA, et al. iPSC-derived human microglia-like cells to study neurological diseases. Neuron. 2017;94(2):278-93. e9.

15. Haenseler W, Sansom SN, Buchrieser J, Newey SE, Moore CS, Nicholls FJ, et al. A highly efficient human pluripotent stem cell microglia model displays a neuronal-co-culture-specific expression profile and inflammatory response. Stem cell reports. 2017;8(6):1727-42.

16. Muffat J, Li Y, Yuan B, Mitalipova M, Omer A, Corcoran S, et al. Efficient derivation of microglia-like cells from human pluripotent stem cells. Nature medicine. 2016;22(11):1358-67.

17. Pandya $\mathrm{H}$, Shen MJ, Ichikawa DM, SedlockAB, Choi Y, Johnson KR, et al. Differentiation of human and murine induced pluripotent stem cells to microglia-like cells. Nature neuroscience. 2017;20(5):753.

18. Friedman BA, Srinivasan K, Ayalon G, Meilandt WJ, Lin H, Huntley MA, et al. Diverse brain myeloid expression profiles reveal distinct microglial activation states and aspects of Alzheimer's disease not evident in mouse models. Cell reports. 2018;22(3):832-47.

19. Geirsdottir L, David E, Keren-Shaul H, Weiner A, Bohlen SC, Neuber J, et al. Cross-species single-cell analysis reveals divergence of the primate microglia program. Cell. 2019;179(7):1609-22. e16. 
20. Lin Y-T, Seo J, Gao F, Feldman HM, Wen H-L, Penney J, et al. APOE4 causes widespread molecular and cellular alterations associated with Alzheimer's disease phenotypes in human iPSC-derived brain cell types. Neuron. 2018;98(6):1141-54. e7.

21. Guerreiro R, Bilgic B, Guven G, Brás J, Rohrer J, Lohmann E, et al. A novel compound heterozygous mutation in TREM2 found in a Turkish frontotemporal dementia-like family. Neurobiology of aging. 2013;34(12):2890. e1-. e5.

22. Jonsson T, Stefansson H, Steinberg S, Jonsdottir I, Jonsson PV, Snaedal J, et al. Variant of TREM2 associated with the risk of Alzheimer's disease. New England Journal of Medicine. 2013;368(2):107-16.

23. Xiang X, Piers TM, Wefers B, Zhu K, Mallach A, Brunner B, et al. The Trem2 R47H Alzheimer's risk variant impairs splicing and reduces Trem2 mRNA and protein in mice but not in humans. Molecular neurodegeneration. 2018;13(1):49.

24. Claes C, Van Den Daele J, Boon R, Schouteden S, Colombo A, Monasor LS, et al. Human stem cell-derived monocytes and microglia-like cells reveal impaired amyloid plaque clearance upon heterozygous or homozygous loss of TREM2. Alzheimer's \& Dementia. 2019;15(3):453-64.

25. Krencik R, Zhang S-C. Directed differentiation of functional astroglial subtypes from human pluripotent stem cells. Nature protocols. 2011;6(11):1710-7.

26. Oksanen M, Petersen AJ, Naumenko N, Puttonen K, Lehtonen Š, Olivé MG, et al. PSEN1 mutant iPSC-derived model reveals severe astrocyte pathology in Alzheimer's disease. Stem cell reports. 2017;9(6):1885-97.

27. Jones VC, Atkinson-Dell R, Verkhratsky A, Mohamet L. Aberrant iPSC-derived human astrocytes in Alzheimer's disease. Cell death \& disease. 2017;8(3):e2696-e.

28. Paşca AM, Sloan SA, Clarke LE, Tian Y, Makinson CD, Huber N, et al. Functional cortical neurons and astrocytes from human pluripotent stem cells in 3D culture. Nature methods. 2015;12(7):671-8.

29. Sloan SA, Darmanis S, Huber N, Khan TA, Birey F, Caneda C, et al. Human astrocyte maturation captured in $3 \mathrm{D}$ cerebral cortical spheroids derived from pluripotent stem cells. Neuron. 2017;95(4):779-90. e6.

30. Claes C, Van den Daele J, Verfaillie CM. Generating tissue-resident macrophages from pluripotent stem cells: Lessons learned from microglia. Cellular Immunology. 2018;330:60-7.

31. Trujillo CA, Gao R, Negraes PD, Gu J, Buchanan J, Preissl S, et al. Complex oscillatory waves emerging from cortical organoids model early human brain network development. Cell stem cell. 2019;25(4):558-69. e7.

32. Benito-Kwiecinski S, Lancaster MA. Brain organoids: Human neurodevelopment in a dish. Cold Spring Harbor perspectives in biology. 2020;12(8):a035709.

33. Espuny-Camacho I, Arranz AM, Fiers M, Snellinx A, Ando K, Munck S, et al. Hallmarks of Alzheimer's disease in stem-cell-derived human neurons transplanted into mouse brain. Neuron. 2017;93(5):1066-81. e8.

34. Hasselmann J, Coburn MA, England W, Velez DXF, Shabestari SK, Tu CH, et al. Development of a chimeric model to study and manipulate human microglia in vivo. Neuron. 2019;103(6):1016-33. e10.

35. Linaro D, Vermaercke B, Iwata R, Ramaswamy A, Libé-Philippot B, Boubakar L, et al. Xenotransplanted human cortical neurons reveal species-specific development and functional integration into mouse visual circuits. Neuron. 2019;104(5):972-86. e6.

36. Assou S, Bouckenheimer J, De Vos J. Concise review: assessing the genome integrity of human induced pluripotent stem cells: what quality control metrics? Stem Cells. 2018;36(6):814-21.

37. Hu B-Y, Weick JP, Yu J, Ma L-X, Zhang X-Q, Thomson JA, et al. Neural differentiation of human induced pluripotent stem cells follows developmental principles but with variable potency. Proceedings of the National Academy of Sciences. 2010;107(9):4335-40.

38. Kim K, Zhao R, Doi A, Ng K, Unternaehrer J, Cahan P, et al. Donor cell type can influence 
the epigenome and differentiation potential of human induced pluripotent stem cells. Nature biotechnology. 2011;29(12):1117-9.

39. Lapasset L, Milhavet O, Prieur A, Besnard E, Babled A, Aït-Hamou N, et al. Rejuvenating senescent and centenarian human cells by reprogramming through the pluripotent state. Genes \& development. 2011;25(21):2248-53.

40. Mertens J, Paquola ACM, Ku M, Hatch E, Bohnke L, Ladjevardi S, et al. Directly Reprogrammed Human Neurons Retain Aging-Associated Transcriptomic Signatures and Reveal Age-Related Nucleocytoplasmic Defects. Cell Stem Cell. 2015;17(6):705-18.

41. Hu W, Qiu B, Guan W, Wang Q, Wang M, Li W, et al. Direct conversion of normal and Alzheimer's disease human fibroblasts into neuronal cells by small molecules. Cell stem cell. 2015;17(2):204-12.

42. Mertens J, Reid D, Lau S, Kim Y, Gage FH. Aging in a dish: iPSC-derived and directly induced neurons for studying brain aging and age-related neurodegenerative diseases. Annual review of genetics. 2018;52:271-93.

43. McCaughey-Chapman A, Connor B. Human cortical neuron generation using cell reprogramming: a review of recent advances. Stem cells and development. 2018;27(24):1674-92.

44. Volpato V, Smith J, Sandor C, Ried JS, Baud A, Handel A, et al. Reproducibility of molecular phenotypes after long-term differentiation to human iPSC-derived neurons: a multi-site omics study. Stem cell reports. 2018;11(4):897-911.

45. Consortium GP. A map of human genome variation from population-scale sequencing. Nature. 2010;467(7319):1061.

46. Carcamo-Orive I, Hoffman GE, Cundiff P, Beckmann ND, D'Souza SL, Knowles JW, et al. Analysis of transcriptional variability in a large human iPSC library reveals genetic and non-genetic determinants of heterogeneity. Cell stem cell. 2017;20(4):518-32. e9.

47. Warren CR, O'Sullivan JF, Friesen M, Becker CE, Zhang X, Liu P, et al. Induced pluripotent stem cell differentiation enables functional validation of GWAS variants in metabolic disease. Cell Stem Cell. 2017;20(4):547-57. e7.

48. Pashos EE, Park Y, Wang X, Raghavan A, Yang W, Abbey D, et al. Large, diverse population cohorts of hiPSCs and derived hepatocyte-like cells reveal functional genetic variation at blood lipid-associated loci. Cell stem cell. 2017;20(4):558-70. e10.

49. DeBoever C, Li H, Jakubosky D, Benaglio P, Reyna J, Olson KM, et al. Large-scale profiling reveals the influence of genetic variation on gene expression in human induced pluripotent stem cells. Cell stem cell. 2017;20(4):533-46. e7.

50. Kilpinen H, Goncalves A, Leha A, Afzal V, Alasoo K, Ashford S, et al. Common genetic variation drives molecular heterogeneity in human iPSCs. Nature. 2017;546(7658):370-5.

51. D'Gama AM, Walsh CA. Somatic mosaicism and neurodevelopmental disease. Nature neuroscience. 2018;21(11):1504-14.

52. D'Gama AM, Woodworth MB, Hossain AA, Bizzotto S, Hatem NE, LaCoursiere CM, et al. Somatic mutations activating the mTOR pathway in dorsal telencephalic progenitors cause a continuum of cortical dysplasias. Cell reports. 2017;21(13):3754-66.

53. Bushman DM, Kaeser GE, Siddoway B, Westra JW, Rivera RR, Rehen SK, et al. Genomic mosaicism with increased amyloid precursor protein (APP) gene copy number in single neurons from sporadic Alzheimer's disease brains. Elife. 2015;4:e05116.

54. Lee M-H, Siddoway B, Kaeser GE, Segota I, Rivera R, Romanow WJ, et al. Somatic APP gene recombination in Alzheimer's disease and normal neurons. Nature. 2018;563(7733):639-45.

55. van der Wal E, Den Hamer B, van der Vliet PJ, Tok M, Brands T, Eussen B, et al. Generation of genetically matched hiPSC lines from two mosaic facioscapulohumeral dystrophy type 1 patients. Stem cell research. 2019;40:101560. 
56. Hanna J, Saha K, Pando B, Van Zon J, Lengner CJ, Creyghton MP, et al. Direct cell reprogramming is a stochastic process amenable to acceleration. Nature. 2009;462(7273):595-601.

57. Shakiba N, Fahmy A, Jayakumaran G, McGibbon S, David L, Trcka D, et al. Cell competition during reprogramming gives rise to dominant clones. Science. 2019;364(6438).

58. Lahiri DK, Maloney B, Zawia NH. The LEARn model: an epigenetic explanation for idiopathic neurobiological diseases. Molecular psychiatry. 2009;14(11):992-1003. 


\section{Chapter 7}

\section{Directing neuronal cell fate in vitro: Achievements and challenges}

Renzo J.M. Riemens ${ }^{1,2,3}$, Daniël L.A. van den Hove ${ }^{3,4}$ Manel Esteller ${ }^{1,5,6}$, Raul Delgado-Morales ${ }^{1,3}$

${ }^{1}$ Cancer Epigenetics and Biology Program (PEBC), Bellvitge Biomedical Research Institute - IDIBELL, L'Hospitalet del Llobregat, Barcelona, Catalonia, Spain.

${ }^{2}$ Institute of Human Genetics, Julius Maximilians University, Wuerzburg, Germany.

${ }^{3}$ Department of Psychiatry and Neuropsychology, School for Mental Health and Neuroscience (MHeNs), Maastricht University, Maastricht, the Netherlands.

${ }^{4}$ Laboratory of Translational Neuroscience, Department of Psychiatry, Psychosomatics and Psychotherapy, University of Wuerzburg, Wuerzburg, Germany.

${ }^{5}$ Department of Physiological Sciences II, School of Medicine, University of Barcelona, Barcelona, Catalonia, Spain.

${ }^{6}$ Institucio Catalana de Recerca i Estudis Avançats (ICREA), Barcelona, Catalonia, Spain.

Progress in Neurobiology. 2018 Sep;168:42-68.

Doi: 10.1016/j.pneurobio.2018.04.003. 



\begin{abstract}
Human pluripotent stem cell (PSC) technology and direct somatic cell reprogramming have opened up a promising new avenue in the field of neuroscience. These recent advances allow researchers to obtain virtually any cell type found in the human brain, making it possible to produce and study functional neurons in laboratory conditions for both scientific and medical purposes. Although distinct approaches have shown to be successful in directing neuronal cell fate in vitro, their refinement and optimization, as well as the search for alternative approaches, remains necessary to help realize the full potential of the eventually derived neuronal populations. Furthermore, we are currently limited in the number of neuronal subtypes whose induction is fully established, and different cultivation protocols for each subtype exist, making it challenging to increase the reproducibility and decrease the variances that are observed between different protocols. In this review, we summarize the progress that has been made in generating various neuronal subtypes from PSCs and somatic cells, with special emphasis on chemically defined systems, transcription factor-mediated reprogramming and epigenetic-based approaches. We also discuss the efforts that are being made to increase the efficiency of current protocols and address the potential for the use of these cells in disease modelling, drug discovery and regenerative medicine.
\end{abstract}

\title{
Keywords
}

Brain disorders, cellular reprogramming, directed differentiation, disease modelling, epigenetics, neuronal differentiation, pluripotent stem cells, somatic cells, transdifferentiation.

\section{Highlights}

- Directed differentiation and reprogramming of stem cells and somatic cells allow induction of human neurons in vitro.

- Chemical stimulation for in vitro differentiation recapitulates the neurodevelopmental patterning principles in the embryo.

- Overexpression of transcription factors induces dramatic changes in transcriptional networks that drive cellular conversions.

- Epigenetic manipulation allows induction of profound epigenome remodelling processes that underlie cell fate switches.

- The availability of human neurons in vitro has critical implications for future brain-related studies and drug development. 


\section{Introduction}

Over the last decades, our general knowledge on human brain functions has grown exceedingly thanks to the availability of animal models and human brain tissue. Although their utility is undeniable, both are challenged with limitations that have been impeding progress in gaining complete mechanistic insights, as well as in the development of therapeutic interventions for many brain disorders [1]. The numerous transgenic animal models that have been established mimic pathological mechanisms of the human brain to some extent, but they do not yet satisfactorily capture human disease phenotypes completely [1]. An animal is obviously not a human being and interspecies differences might, therefore, be critical factors underlying the failure of translating a wealth of preclinical findings into clinical implementations [2, 3]. Aside from the distinct (epi)genetic backgrounds, there are major physiological differences that could affect the development of disease phenotypes or differentially affect drug mechanisms, leading to misinterpretation of experimental findings. Human brain tissue samples, on the other hand, can generally only be obtained post-mortem, which complicates the study of disease aetiology and progression, since they simply do not allow to discriminate between cause and consequence of the disorder [4]. Cellular in vitro model systems, however, have the potential to overcome this latter challenge, owing to the possibility of manipulating the (epi)genetic architecture, as well as the environmental exposome, in culture conditions, which allows to study cause-effect relationships of pathological hallmarks in a controlled setting. Unfortunately, many early in vitro model systems have heavily relied on a combination of non-neuronal human cell lines, primary rodent cultures and transgenic rodent cell lines, which, similar to animal models, also all exclude the human neuronal (epi)genomic and phenotypic context [5].

In view of the aforementioned limitations and the unmet necessity of finding therapeutic interventions for brain disorders, the recent availability of human pluripotent stem cell (PSC) technology and somatic cell reprogramming has offered new opportunities for human brain-related studies [6, 7]. PSC technology is an umbrella term that encompasses both embryonic stem cells (ESCs) and induced PSCs (iPSCs) [8]. Aside from being derived from humans, the distinct advantages that these cells have are their unlimited proliferative capacity and the ability to differentiate towards virtually any cell type, including specific neuronal subtypes [9]. These characteristics equip PSCs with the unique feature to provide a theoretically inexhaustible and replenishable source of cells in vitro. Somatic cells on the other hand, albeit restricted by their limited proliferative potential, can be obtained relatively easy from healthy individuals and patients, and can then be directly reprogrammed towards desirable neuronal subtypes in a comparative, but faster framework [10]. Consequently, PSCs, somatic cells and their differentiated 
progeny can nowadays be used to model disease mechanisms in a humanizedsetting, where they allow for the investigation of unique human cellular and molecular features in a cell-specific and personalised matter [11]. In fact, we are currently in the middle of an exciting era where human PSC and somatic cell reprogramming studies are contributing to the understanding of underlying neurobiological processes, as well as the consequences of personal molecular variations on the development and course of brain disorders [10, 12]. In addition, iPSC- and somatic cell-derived neuronal populations provide a platform for highthroughput drug screening and toxicity testing in an upcoming epoch of personalised medicine, which assists the production of therapeutic interventions and might at the same time provide cues for therapeutic resistance [11]. Furthermore, such neuronal populations also harbour therapeutic potential in the field of regenerative medicine, since they might be used for transplant therapies [11].

Given the heterogeneity of neuronal cells found in the human brain and the complex interactions between them, one of the main opportunities PSC technology, directed differentiation and somatic cell reprogramming are offering, is the study of multicellular neural cultures organized in a manner reminiscent to what is seen in distinct anatomical structures of the brain. Such a method offers a promising approach to study higher-order neuronal networks during development and allows studying single neuron connectivity, as well as various other cellular interactions found in the neuronal niche, such as those between glial cells and endothelial cells $[13,14]$. Another major focus in the scientific community that emerges from the unique contributions each of the neuronal subtypes have in specific brain functions and disease, has been to obtain and study pure populations of neurons free of other subtypes. Derivation of such homogenous populations is especially important for the study of disease-associated neurons, where confounding effects of other subtypes should be avoided, such as in transcriptomic- and epigenetic profiling for example. Furthermore, the acquisition of protocols that can produce highly pure populations of neurons can in turn be utilized to customize the features of a multicellular culture. Successfully directing differentiation and cellular reprogramming in vitro has, therefore, been an area of intense research during the past two decades [15]. Many strategies have been explored utilizing mouse and human PSCs, as well as somatic cells, to generate anatomically specified neural precursor cells (NPCs) and differentiated neuronal subtypes (See Fig. 1). As a definition used here, NPCs refer to the mixed population of cells consisting of all undifferentiated progeny from neural stem cells, therefore including both neural stem cells and neural progenitor cells. Altogether, these differentiation methods try to recapitulate the multistep processes of neural development that occur in the early embryo [15]. Thus, an improved understanding of developmental signalling pathways and gene regulatory networks has guided the design of neuronal 
differentiation and cellular reprogramming strategies [8]. Although significant advances have been made, in vitro neuronal differentiation is actually not a process that is fully disciplined yet, as very often other cell types are produced in parallel to what was first intended by the researcher. Furthermore, differentiation protocols for multiple neuronal subtypes remain either unestablished or unstable, or they do not allow to obtain the terminally differentiated neuron with its entire functional maturated characteristics $[16,17]$.

To date, the majority of directed neuronal differentiation and direct somatic cell reprogramming protocols involve chemical stimulation through patterning cues or the use of ectopic overexpression of lineagespecific transcription factors that are known for their genuine involvement during neural development [18-20]. Although it is currently accepted that these networks of patterning molecules and transcription factors orchestrate neuronal induction and differentiation, it is becoming more and more evident that cell-intrinsic mechanisms are also key players within these circuits [21-23]. Neuronal induction and differentiation generally rely on the interplay of activation and inhibition on multiple developmental signalling pathways tightly controlled by the epigenetic machinery $[8,24]$. Within these networks, the epigenetic machinery is essential for fine-tuning genetic programs that coordinate distinct developmental processes, as well as shaping the neuronal identities at a phenotypic resolution [25, 26]. With the current advances in epigenetic editing [27-29] and RNA interference (RNAi) [30, 31], an alternative strategy for directed neuronal differentiation and direct somatic cell reprogramming has become available. Such approaches allow the design and construction of novel specific artificial epigenetic pathways or the redesign of existing endogenous molecular systems, in order to intentionally change epigenetic information at desired loci [32]. Those systems can now be used as an additional tool to guide neuronal cell fate in vitro and also allow to address fundamental questions concerning the role of epigenetics in assigning neuronal cell fates.

In the present review, we will provide an overview on the progress made in generating various neuronal subtypes from PSCs and somatic cells in vitro. We will dissect the current used chemically defined systems, transcription factor-mediated reprogramming methods and epigenetic-based approaches. Furthermore, we will discuss the efforts that are being made to increase the efficiency of current protocols and highlight the potential for the use of this platform in disease modelling, drug discovery and regenerative medicine. 


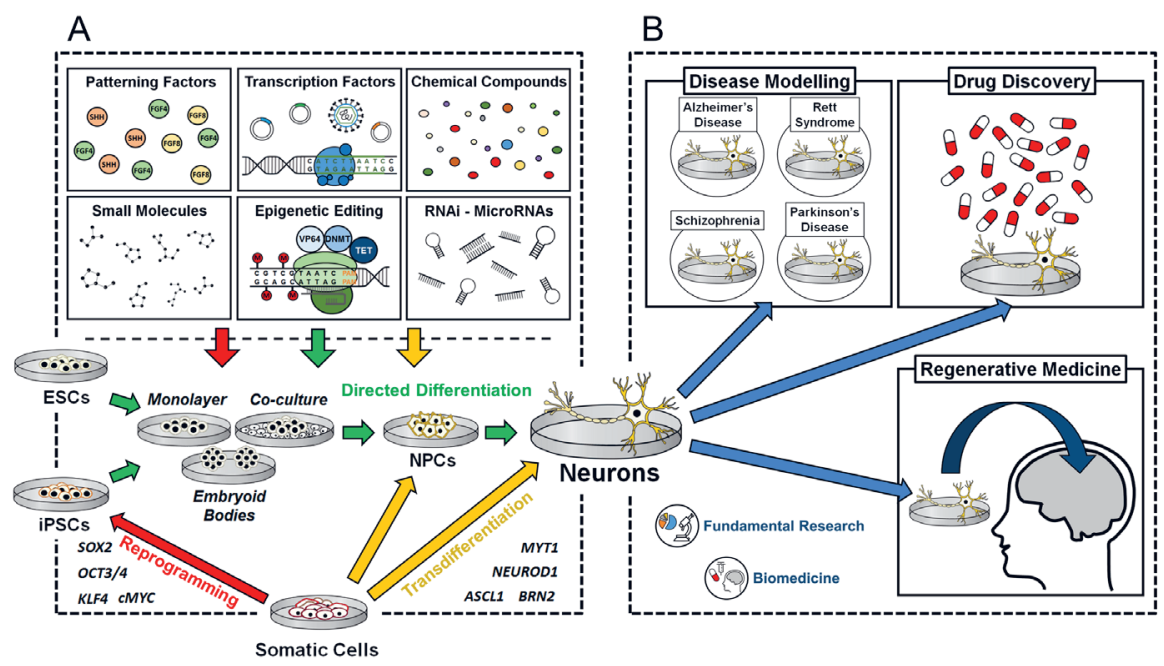

Figure 1. Overview of methods and applications for neurons obtained by directed differentiation and (direct) cellular reprogramming in embryonic stem cells (ESCs), induced pluripotent stem cells (iPSCs) and somatic cells. A. The figure shows the complete directed differentiation and (direct) cellular reprogramming toolkit currently available, including the use of patterning factors, chemical compounds, small molecules, transcription factors, epigenetic editing and RNA interference (RNAi) with the use of microRNAs (miRNAs) as example. The neurodevelopmental transitions starting from PSCs towards neural precursor cells (NPCs) and eventually mature neurons are depicted. Directed differentiation protocols can generally be divided into three methods: monolayer methods, co-culture methods and embryoid body (EB) methods. Reprogramming of somatic cells towards iPSCs can be achieved by increased expression of transcription factors, such as Oct3/4, Sox2, Klf4 and Myc (OSKM, Yamanaka factors). Direct reprogramming of somatic cells towards specific neuronal subtypes can be achieved by co-expression of the pan-neuronal factors Brn2, Ascl1, Myt1 (BAM) and Neurod1 among others, and neuronal subtype-specific transcription factors. B. The direct applications of the obtained neurons in fundamental research and biomedicine, including the use for disease modelling, drug discovery and regenerative medicine, are shown.

\section{Chemically defined systems}

\section{Neural induction and differentiation}

Research to develop protocols for the differentiation of PSCs into clinically relevant cell subtypes has progressed at a rapid pace. Hundreds of protocols have become available that allow to obtain early NPCs and eventually the derivation of desired neuronal populations [33-38]. In the classical embryoid body (EB) method, scientists let PSC form self-assembling aggregates from dissociated suspension cultures, known as EBs $[39,40]$. This method initially aimed to spontaneously differentiate the PSCs into mixed cellular populations encompassing the three germ-layers [41]. As PSCs readily differentiate into many different cell types, the efficiency of neuronal conversion is limited and culture media that enhances neuronal production, as well as further selection procedures, are usually necessary to increase the homogeneity 
of a specific neuronal subpopulation [42]. The second method to reconstitute neural commitment in vitro and to achieve efficient neural induction from PSCs has relied upon adherent monolayer culture differentiation, which eliminates the use of multicellular aggregations [33, 37, 38]. In this method, PSCs are dissociated into single cells and further cultured with conditioned media that also enhances neuronal conversion. Various aspects of the monolayer differentiation protocol have been extensively studied, adapted and optimized recently by different research groups, currently allowing neural induction within a few days [43, 44]. The third method to promote neural induction from PSCs can be achieved by co-cultivation of PSCs with stromal cell feeder layers [37, 45]. Co-culturing PSCs with other cell types is based on the idea that these surrounding cells provide cues that assign cell fates along the neural lineage. The use of stromal feeder layers is, however, only an efficient PSC differentiation strategy for certain neuronal subtypes, such as dopaminergic neurons [46]. Additional efforts on optimising previous methods have recently also lead to the establishment of a combinatory approach for PSC differentiation that is characterised by a chemically transitional EB-like state [47]. A low density monolayer culture on a feeder is differentiated under appropriate culture conditions and induces intermediate progenitor cells with the capability of differentiating into the three germ layers. The main advantage of this approach is that it harbours a reduced innate differentiation propensity of PSCs, even if the PSCs are known for their unfavourable differentiation [47].

Although different strategies for neuronal conversion in PSCs have been explored, the general trend moves towards stromal-free methods combined with chemically defined culture systems. These chemically defined systems utilize culture media that are supplemented with patterning molecules, such as morphogens and growth factors, which force enrichment of the desired neuronal cells. Additionally, an increasing number of studies have also illustrated the use of small molecules and emphasized their significant benefits for neural induction and differentiation in PSCs, as well as for somatic cell reprogramming [48]. Aside from providing positive signals to induce neuronal fates, many of these factors also inhibit signalling pathways that control the differentiation into other germ layers then the ectoderm. Of note, just as in vivo embryonic development can be broken down into distinct stages where distinct patterning molecules are required to induce neuronal cell fates, so too can the in vitro specification of differentiating neuronal population from PSCs [49]. The compositions of the chemically defined media, as well as the compound concentrations at any given time point, can, therefore, significantly redirect the anatomical and functional identity of the differentiating cells. 
Table 1. Chemically defined differentiation systems for neural induction and differentiation in vitro per neuronal phenotype.

\begin{tabular}{|c|c|c|c|c|c|c|}
\hline \multicolumn{7}{|c|}{ Chemically defined systems } \\
\hline Phenotypes & Species & Starting cell types & Culture methods & Chemical driving factors & Phenotypic markers ( $\%$ cells) & References \\
\hline \multirow[t]{15}{*}{$\begin{array}{l}\text { Glutamatergic } \\
\text { neurons }\end{array}$} & Mouse & ESCs & $\mathrm{EB}$ & RA & $\begin{array}{l} \pm 85 \% \text { TUJ1+ } \\
93 \pm 4.7 \% \text { VGLUT } 1+/ \text { TUJ } 1+\end{array}$ & Bibel et al. (2008) \\
\hline & & ESCs (TAU::GFP) & Monolayer & Cyclopamine & $\begin{array}{l} \pm 70 \% \text { TUJ1+ } \\
\pm 70 \% \text { VGLUT1+/TUJ1+ } \\
\pm 13 \% \text { VGLUT2+/TUJ1+ } \\
<50 \% \text { TBR1+/TUJ1+ } \\
<35 \% \text { CTIP2+/TUJ1+ }\end{array}$ & Gaspard et al. 2008 \\
\hline & $\begin{array}{l}\text { Mouse } \\
\text { and human }\end{array}$ & $\begin{array}{l}\text { ESCs (Sox1::GFP and } \\
\text { Bf1::Venus) }\end{array}$ & $\mathrm{EB}$ & DKK1, Lefty-1 and SB431542 & Mouse & Eiraku et al. (2008) \\
\hline & & & & & $83 \%$ VGLUT1+/TUJ1+ & \\
\hline & Human & ESCs & EB & None & $\begin{array}{l} \pm 79 \% \text { TBR } 1+/ \text { TUJ } 1+ \\
\pm 57 \% \text { CTIP2+ }\end{array}$ & Li et al. (2009) \\
\hline & & $\begin{array}{l}\text { ESCs (ACTB::GFP) } \\
\text { and iPSCs }\end{array}$ & Monolayer & NOG & ESCs & $\begin{array}{l}\text { Espuny-Camacho } \\
\text { _et al. (2013) }\end{array}$ \\
\hline & & & & & $\begin{array}{l}<65 \% \text { TUJ1+ } \\
\pm 60 \% \text { VGLUT1+/TUJ1+ } \\
<75 \% \text { TBR1+/TUJ1+ } \\
<72 \% \text { CTIP2+/TUJ1+ } \\
<18 \% \text { CTIP2+/TBR1+/TUJ1+ }\end{array}$ & \\
\hline & & ESCs and iPSCs & (Spin) EB & DOR, DKK1, EGF, FGF2 and NOG & iPSCs (8011) & Kim et al. (2011c) \\
\hline & & & & & $\begin{array}{l}94.7 \pm 2.5 \% \text { VGLUT1+/MAP2+ } \\
9.9 \pm 4.7 \% \text { GABA+/MAP2+ }\end{array}$ & \\
\hline & & & & & iPSCs (BJiPS\#1) & \\
\hline & & & & & $\begin{array}{l}32.8 \pm 8.1 \% \text { VGLUT1+/MAP2+ } \\
71.1 \pm 5.5 \% \text { GABA+/MAP2 }\end{array}$ & \\
\hline & & & $\begin{array}{l}\text { Monolayer (Dual SMAD } \\
\text { inhibition) }\end{array}$ & $\begin{array}{l}\text { DOR, FGF2, NOG, SB431542 and } \\
\text { Vitamin A }\end{array}$ & ESCs & $\begin{array}{l}\text { Shi et al. (2012a, } \\
\text {-2012b) }\end{array}$ \\
\hline & & & & & $\begin{array}{l} \pm 27 \% \text { TBR } 1+ \\
\pm 28 \% \text { CTIP } 2+ \\
\pm 34 \% \text { BRN2+ }\end{array}$ & \\
\hline & & & & & iPSCs & \\
\hline & & & & & $\begin{array}{l}22-29 \% \text { TBR1+ } \\
25-30 \% \text { CTIP2+ } \\
28-36 \% \text { BRN2+ }\end{array}$ & \\
\hline \multirow{2}{*}{$\begin{array}{l}\text { GABAergic } \\
\text { neurons }\end{array}$} & Mouse & ESCs & Co-culture (MS-5) & FGF2, FGF8 and SHH & $\pm 68 \%$ GABA+/TUJ1+ & Barberi et al. (2003) \\
\hline & & ESCs $(L h \times 6:: G F P)$ & EB & FGF2, NOG and SHH-C25II & $\begin{array}{l}91.6 \pm 4.4 \% \text { BF1+/Lxh6-GFP+ } \\
70.5 \pm 7.7 \% \text { DLX2+/Lxh6-GFP+ } \\
97.7 \pm 3.1 \% \text { LHX6+/Lxh6-GFP+ } \\
94.6 \pm 3.7 \% \text { GABA+/Lxh6-GFP+ }\end{array}$ & Maroof et al. (2010) \\
\hline
\end{tabular}


Table 1. (Continued)

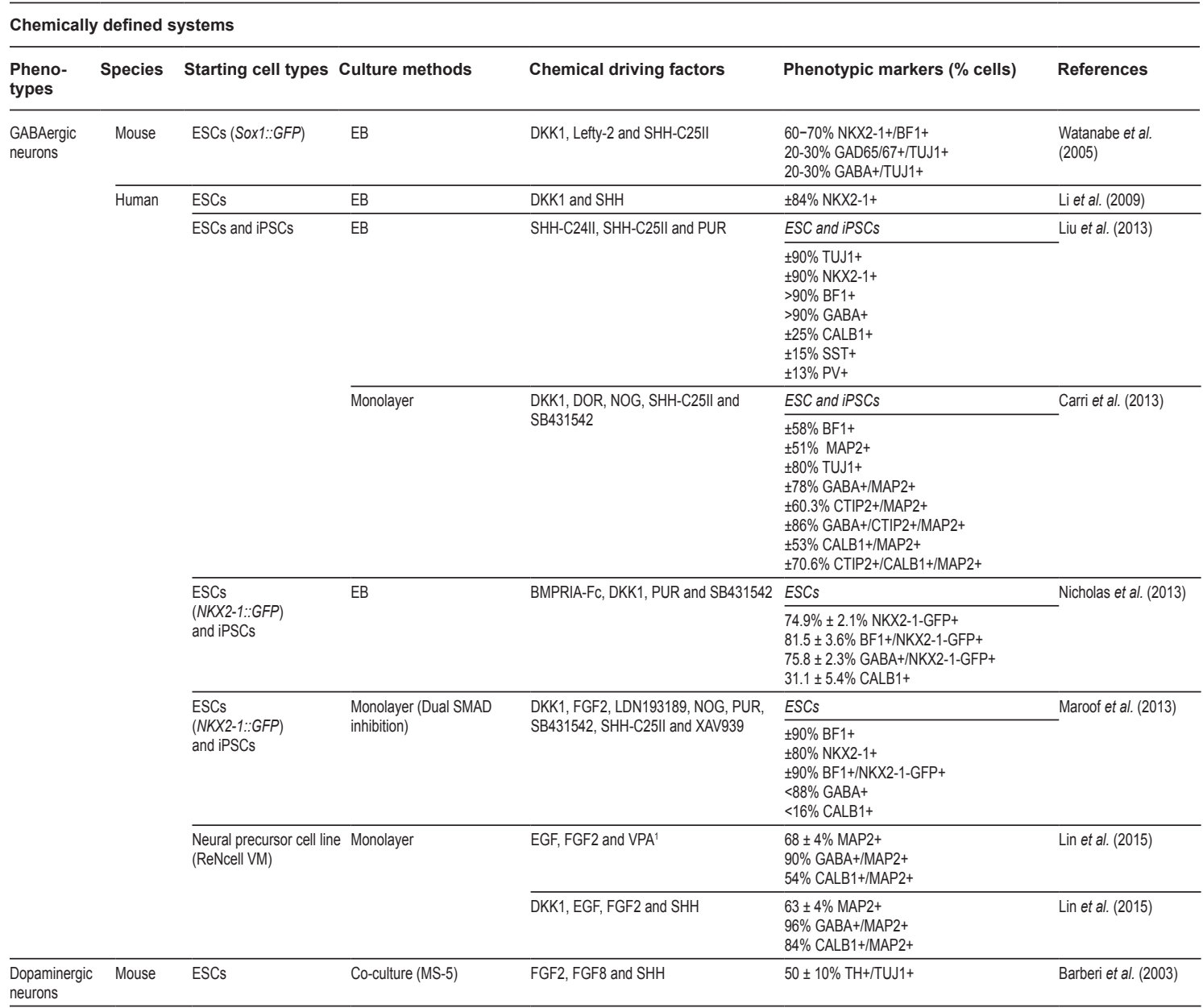

1 Epigenetic factor

Neuronal phenotypes, species, starting cell types, culture methods, chemical driving factors and representative phenotypic markers that have been used to assess the differentiation efficiency and culture homogeneity are broadly summarized. + indicates the percentage of cells in the population that stained positive for a certain marker. Abbreviations: 5-HT, serotonin (5-hydroxytryptamine); A83-01, TGF- $\beta$ kinase/activin receptor-like kinase inhibitor; ACTB, actin beta/beta-actin; BF1, brain factor 1/forkhead box protein G1 (FOXG1); BIO, GSK3 $\beta$ inhibitor 6-bromoindirubin-3'-oxime; BMPRIA-Fc, bone morphogenetic protein receptor 1a-fragment crystallizable; BRN2, brain-specific homeobox/POU domain protein 2 (POU3F2);; CALB1, calbindin 1; CHAT, choline o-acetyltransferase; CHIR99021, GSK3ß inhibitor; CTIP2, b-cell CLL/lymphoma 11b (BCL11B)/ COUP-TF-interacting protein 2 (COUP-TFII); DKK1, dickkopf-1; DLX2, distal-less homeobox 2; DMH-1, dorsomorphin homolog 1; DOR, Dorsomorphin; dsRed, discosoma sp. red fluorescent protein; EB, embryoid body; EGF, epidermal growth factor; EGFP, enhanced green fluorescent protein; ePet, enhancer of the mouse Pet-1 (human FEV) gene; ESCs, embryonic stem cells; FGF10, fibroblast growth factor 10; FGF2, fibroblast growth factor $2 /$ /basic fibroblast growth factor (bFGF); FGF4, fibroblast growth factor 4; FGF8, fibroblast growth factor 8; FGF8b, fibroblast growth factor 8 isoform b; FOXA2, forkhead box protein A2; GABA, $y$-aminobutyric acid; GAD65/67, glutamic acid decarboxylase isoform 65/67 (GAD2/1); GFP, green fluorescent protein; GIRK2, G protein-activated inward rectifier potassium channel 2 (KCNJ6); HB9, homeobox HB9/motor neuron and pancrease homeobox 1 (MNX1); Hh-Ag1.3, small molecule agonist of SHH signalling; iPSCs, induced pluripotent stem cells; ISL1, ISL LIM homeobox 1; ISL1/2, ISL LIM homeobox 1/2; LDN193189, selective BMP signalling inhibitor; Lefty-1, left-right 
Table 1. (Continued)

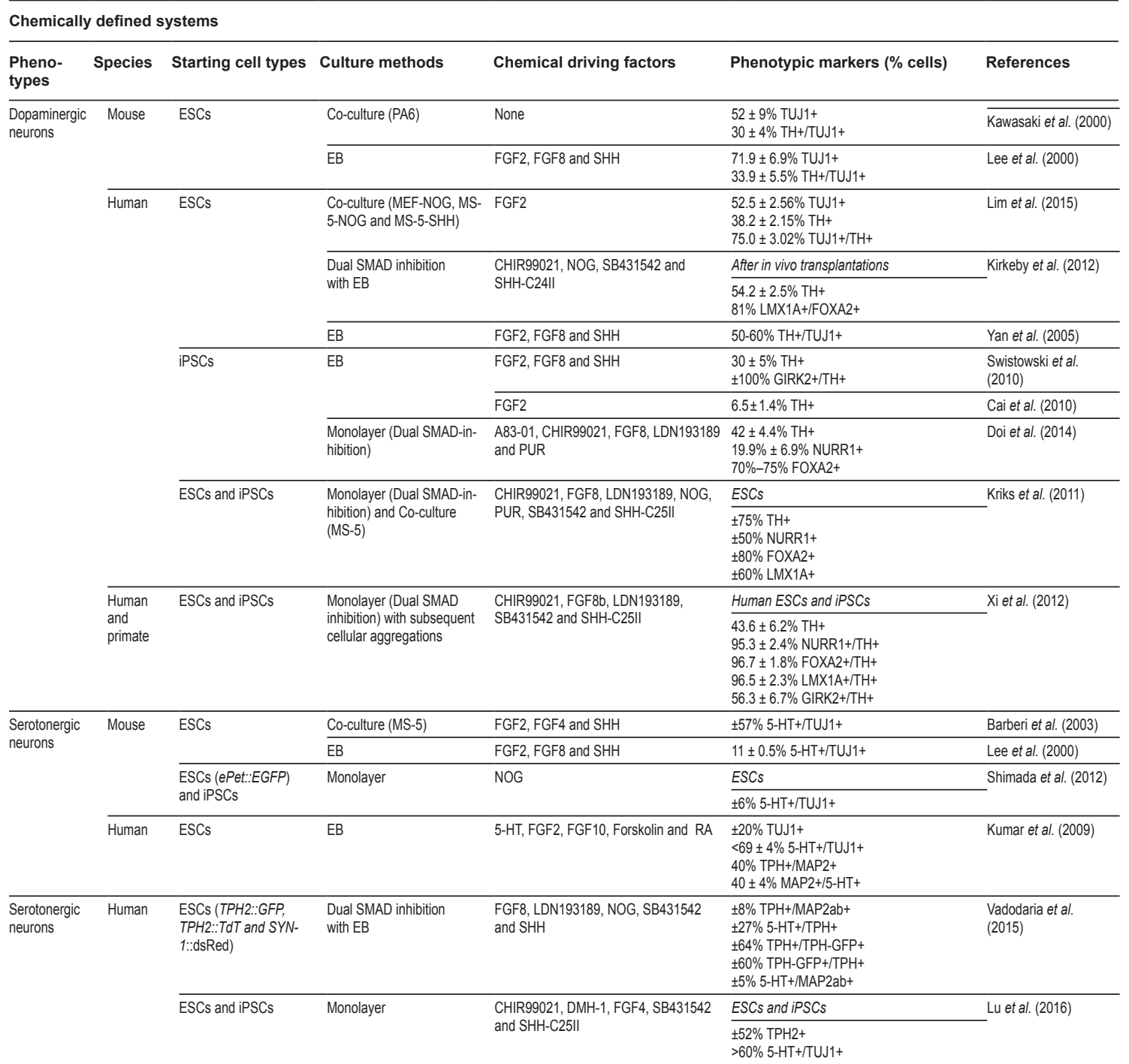

determination factor 1; Lefty-2, left-right determination factor 2; LHX6, LIM homeobox 6; LMX1A, LIM homeobox transcription factor 1 alpha; MAP2, microtubule-associated protein 2; MAP2ab, microtubule-associated protein 2ab; MEF, mouse embryonic fibroblast; MS-5, stromal cell line derived from irradiated murine bone marrow cultures; NKX2-1, NK2 homeobox 1; NOG, Noggin; NURR1, nuclear receptor

related 1 protein;OLIG2, oligodendrocyte transcription factor 2; PA6, stromal cell line derived from newborn calvaria tissue of the C57BL/6 mice; PUR, purmorphamine; PV, parvalbumin; RA, retinoic acid; RUES1-EGFP, human ESC line expressing EGFP; SAG, smoothened agonist; SB431542, transforming growth factor beta inhibitor; SHH, sonic hedgehog; SHH-C24II, recombinant human SHH; SHH-C25II, recombinant mouse SHH; SMAD, transcription factor and member of the BMP and TGF- $\beta$ signalling pathways; Sox1, SRY box 1; SOX3, SRY box 3; SST, somatostatin; SYN1, synapsin 1; TAU, microtubule-associated protein tau (MAPT); TBR1, T-box brain 1; TdT, tandem dimer tomato red fluorescent protein; TH, tyrosine hydroxylase; TPH, tryptophan hydroxylase; TPH2, tryptophan hydroxylase 2; TUJ1, neuron-specific class III beta-tubulin (TUBB3); VGLUT1, vesicular glutamate transporter 1; VGLUT2, vesicular glutamate transporter 2; VPA, valproic acid; XAV939, WNT/B-catenin inhibitor. 
Table 1. (Continued)

\begin{tabular}{|c|c|c|c|c|c|c|}
\hline $\begin{array}{l}\text { Pheno- } \\
\text { types }\end{array}$ & Species & Starting cell types & Culture methods & Chemical driving factors & Phenotypic markers (\% cells) & References \\
\hline \multirow{14}{*}{$\begin{array}{l}\text { Cholinergic } \\
\text { motor neurons }\end{array}$} & \multirow{2}{*}{ Souse } & ESCs & Co-culture (MS-5) & FGF2, RA and SHH & $\pm 60 \%$ HB9+/TUJ1+ & Barberi et al. (2003) \\
\hline & & $\mathrm{ESCs}(H b 9:: G F P)$ & $E B$ & Hh-Ag1.3, RA and SHH & $\begin{array}{l}25 \% \pm 5 \% \text { HB9+ } \\
25 \% \pm 5 \% \text { HBg-GFP+ } \\
>70 \% \text { ISL } 1+/ \text { HBg+ }\end{array}$ & Wichterle et al. (2002) \\
\hline & \multirow[t]{12}{*}{ Human } & ESCs & $\mathrm{EB}$ & FGF2, RA and SHH & $\begin{array}{l}>50 \% \text { ISL1+/TUJ1+/MAP2+ } \\
\pm 50 \% \text { HBg+//SL1/2+ } \\
\pm 21 \% \mathrm{HBg}+\end{array}$ & Li et al. (2005) \\
\hline & & ESCs $(H b 9:: E G F P)$ & $\mathrm{EB}$ & $\mathrm{RA}$ and $\mathrm{SHH}$ & $\begin{array}{l}35.3 \pm 24.9 \text { TUJ1+ } \\
56.1 \pm 9.9 \% \text { ISL1+/CHAT+/TUJ1+ } \\
37.4 \pm 3.3 \% \text { HB9+/TUJ1+ } \\
96.3 \pm 12.5 \% \text { HB9+/Hb9-GFP+ } \\
88.7 \pm 7.4 \% \text { TUJ1+/Hb9-GFP+ }\end{array}$ & $\begin{array}{l}\text { Singh Roy et al. } \\
(2005)\end{array}$ \\
\hline & & \multirow[t]{6}{*}{ ESCs and iPSCs } & \multirow[t]{2}{*}{$\mathrm{EB}$} & \multirow[t]{2}{*}{ RA and SHH agonist } & iPSCs & \multirow[t]{2}{*}{ _Dimos et al. (2008) } \\
\hline & & & & & $\begin{array}{l}20 \% \text { HB9+ } \\
>90 \% \text { ISL } 1 / 2+/ / \text { HB9+ } \\
>50 \% \text { CHAT+/ISL } 1 / 2+/ \text { HB9+ }\end{array}$ & \\
\hline & & & \multirow[t]{4}{*}{ Monolayer } & \multirow[t]{4}{*}{$\mathrm{DOR}, \mathrm{RA}$ and $\mathrm{SHH}$} & ESCs & \multirow[t]{4}{*}{ Q Qu et al. (2014) } \\
\hline & & & & & $\begin{array}{l}69.5 \pm 11.2 \% \text { HB9+/CHAT+/TUJ1+ } \\
78.3 \pm 3.5 \% \text { ISL1+ }\end{array}$ & \\
\hline & & & & & iPSCs & \\
\hline & & & & & $\begin{array}{l}51.6 \pm 5.7 \% \text { HB9+/TUJ1+ } \\
65.4 \pm 5.1 \% \text { ISL1+ }\end{array}$ & \\
\hline & & \multirow{4}{*}{$\begin{array}{l}\text { ESCs and iPSCs } \\
(H b 9::(E) G F P)\end{array}$} & \multirow[t]{4}{*}{$E B$} & \multirow[t]{4}{*}{ PUR and RA } & ESCs & \multirow{4}{*}{$\begin{array}{l}\text { Karumbayaram et al. } \\
\text {-(2009) }\end{array}$} \\
\hline & & & & & $\begin{array}{l}59.1 \pm 7.07 \% \text { OLIG } 2+/ S O X 3+ \\
28.2 \pm 5.7 \% \text { ISL } 1+/ \text { TUJ1+ }\end{array}$ & \\
\hline & & & & & iPSCS & \\
\hline & & & & & $\begin{array}{l}57.6 \% \pm 9.88 \% \text { OLIG2+/SOX3+ } \\
33.6 \% \pm 12 \% \text { ISL1+/TUJ1+ }\end{array}$ & \\
\hline \multirow{10}{*}{$\begin{array}{l}\text { Cholinergic } \\
\text { motor neurons }\end{array}$} & \multirow[t]{6}{*}{ Human } & \multirow{2}{*}{$\begin{array}{l}\text { ESCs and iPSCs } \\
(H b 9: .(E) G F P)\end{array}$} & \multirow{2}{*}{$\begin{array}{l}\text { Dual SMAD inhibition } \\
\text { with EB }\end{array}$} & \multirow{2}{*}{$\begin{array}{l}\text { LDN193189, PUR, RA, SAG, SB435142 } \\
\text { and SHH-C25II }\end{array}$} & ESCs & \multirow[t]{2}{*}{ _ Amoroso et al. (2013) } \\
\hline & & & & & $\begin{array}{l}83 \pm 1 \% \text { TUJ1+ } \\
<29 \pm 4 \% \text { HBg-GFP+ } \\
98 \pm 0 \% \text { HB9-GFP+/TUJ1+ } \\
30 \pm 6 \% \text { ISL1+/HB9-GFP+ } \\
16 \pm 5 \% \text { HB9+/HB9-GFP+ } \\
37 \pm 2 \% \text { ISL1+/HB9+/HBg-GFP+ }\end{array}$ & \\
\hline & & \multirow{4}{*}{$\begin{array}{l}\text { ESCs and iPSCs } \\
\text { (HB9::Venus) }\end{array}$} & \multirow{4}{*}{$\begin{array}{l}\text { Dual SMAD inhibition } \\
\text { with EB }\end{array}$} & \multirow{4}{*}{$\begin{array}{l}\text { BIO, CHIR99021, DOR, LDN193189, } \\
\text { PUR, RA and SB431542 }\end{array}$} & ESCs & \multirow[t]{4}{*}{ _Shimojo et al. (2015) } \\
\hline & & & & & $\begin{array}{l}\text { 50-58\% HB9+ } \\
45-50 \% \text { ISL1+ } \\
43-68 \% \text { CHAT+ } \\
84.1 \pm 2.4 \% \text { HB9+/HBg-Venus }+ \\
39.5 \pm 13.2 \% \text { ISL } 1+/ \text { /HB9-Venus }+ \\
83.4 \pm 1.7 \% \text { CHAT+/HB9-Venus }+\end{array}$ & \\
\hline & & & & & iPSCs & \\
\hline & & & & & $\begin{array}{l}42-48 \% \text { HB9+ } \\
37-48 \% \text { ISL1+ }\end{array}$ & \\
\hline & \multirow{4}{*}{$\begin{array}{l}\text { Human } \\
\text { and } \\
\text { primate }\end{array}$} & \multirow{4}{*}{$\begin{array}{l}\text { ESCs } \\
\text { (RUES1-EGFP) }\end{array}$} & \multirow[t]{4}{*}{ Co-culture (MS-5) } & \multirow[t]{4}{*}{ NOG, RA and SHH } & Human & \multirow[t]{4}{*}{ Lee et al. (2007) } \\
\hline & & & & & $\begin{array}{l}20 \% \text { HB9+ } \\
\pm 26 \% \text { CHAT }+\end{array}$ & \\
\hline & & & & & Primate & \\
\hline & & & & & $\begin{array}{l}43 \% \mathrm{HB} 9+ \\
65 \% \mathrm{HB} 9+/ \mathrm{TUJ} 1+\end{array}$ & \\
\hline
\end{tabular}


Neuronal conversion follows the neural induction principle that is first initiated by removing medium components that promote self-renewal, which on itself is sufficient to trigger differentiation towards all the three embryonic germ layers [50]. Inhibition of extraembryonic and meso-endoderm differentiation can be further enhanced by culturing the cells in serum-free medium and by the actions of early patterning molecules, through which the PSCs progressively start restricting their differentiation potential towards the neural lineage to form early NPCs [50, 51]. Many neural induction protocols include the simultaneous inhibition of transforming growth factor beta (TGF- $\beta$ )/Activin/Nodal and bone morphogenic protein (BMP) signalling pathways, i.e. dual SMAD inhibition, which is similar to what is observed in vivo $[33,52]$. Inhibition of the TGF- $\beta$ and BMP pathways is thought to promote differentiation of PSCs along the neural lineage primarily through inhibition of PSC self-renewal, as well as by blocking differentiation towards alternative cellular lineages [49]. Consequently, signalling molecules such as Noggin (NOG), leftright determination factor (Lefty)-1, Lefty-2, Dickkopf-1 (DKK1), TGF- $\beta$ inhibitor SB431542, glycogen synthase kinase 3 beta (GSK3 $\beta$ ) inhibitor CHIR99021 and BMP inhibitor dorsomorphin homolog 1 (DMH-1) have all been used to promote neural induction from PSCs $[53,54]$. Other pathways, including epidermal growth factor (EGF), fibroblast growth factor (FGF) and wingless-type MMTV integration site family (WNT) signalling, have been described to regulate neuronal differentiation by promoting induction and survival of the NPCs $[49,55]$. In particular, FGF2 has been shown to enhance the neural induction phase and to increase the number of NPCs, whereas omitting it during subsequent stages promotes their differentiation into mature neurons [56].

After neural induction, second series of lineage-specific patterning molecule cocktails have been used to direct the further differentiation of the NPCs towards mature neuronal subtypes. Although the availability of protocols for subtypes within specific neurotransmitter classes is limited and different cultivation conditions for each of these classes exist, protocols for glutamatergic, dopaminergic, $\mathrm{y}$-aminobutyric acid (GABA)-ergic, serotonergic, and cholinergic motor neurons have become available over the years (See Table 1) [53, 57-60]. Specification of the NPCs takes place both along the rostral-caudal and the dorsal-ventral axes of the brain, coordinated by the synergistic actions of temporally and spatially available patterning molecules [61]. The presence and concentrations of these molecules define the transcriptional code and, hence, the identity of the NPCs in a particular domain along both axes. NPCs are generally specified first in the head region and extend caudally, meaning that they become committed to an anterior forebrain fate by a default programme [50]. Correspondingly, NPCs differentiated from PSCs, independent of the differentiation method, carry a rostral identity that is free of caudal markers [50]. Indeed, this anterior phenotype is transient 
and NPCs will take on a definitive regional identity depending on further cues. Treatment with increasing concentrations of sonic hedgehog $(\mathrm{SHH})$ has shown to promote ventralization of the NPCs, while addition of retinoic acid (RA) promotes caudal fates [49] and activation of WNT signalling exerts a dose-dependent effect where increasing concentrations are patterning the NPCs to forebrain, midbrain, hindbrain and anterior spinal cord identities, respectively [49, 50,62]. Although it remains largely unknown how all the patterned NPCs acquire functional and anatomical specificity, it is this regional patterning principle recapitulating in vivo morphogenesis that guides PSC neuronal differentiation in vitro (See Fig. 2) [63]. Moreover, a specific neurotransmitter subtype can often be generated in different parts of the human brain and at different stages, demonstrating that different spatiotemporal cues can likely converge on the same terminal selectors and thereby induce a similar terminal fate [64].

The anatomically directed differentiation processes seen in these neuronal differentiation protocols are characterized by the same expression and temporal regulation of lineage-specific transcription factors as observed in vivo [49]. For instance, NPCs differentiated towards forebrain neurons carry an anterior identity by expressing PAX6 and OTX2, but not more caudal markers like EN1, GBX2 or $\operatorname{HOX}[33,50,65]$. Thus, expression levels of these transcription factors represent useful markers that are widely used to assess the differentiation status of the cellular population (See Table 1). Furthermore, extensive gene expression analysis, electrophysiological characterization, biochemical assessments, and in vivo transplantation into rodent brains have been applied to examine the population characteristics, as well as quality and efficacy of the differentiation protocols (See Table S1). However, with many of these protocols, in vitro-directed differentiation in PSCs still results in highly variable neuronal populations with fluctuating yields of neuronal cells and remarkable differences in efficiency [66]. This variability emerges from clear differences between the protocols, such as the neuronal induction method used, the chemical compositions of the media, the compound concentrations and the chemical exposure times that were used, as well as more undefined differences, including the culture densities and the passage number for example [49].

\section{Glutamatergic neurons}

Despite the differences in developmental principles that underlie the specification of their subpopulations, excitatory glutamatergic neurons can be found throughout the whole central nervous system, such as in the cerebral cortex [67], as well as in subcortical regions like the thalamus [68], and even in the spinal cord among others [69]. To date, strategies that have been established to successfully generate glutamatergic neurons from PSCs (See Table 1) are mainly based on the 
derivation of cortical glutamatergic neurons of which the vast majority originates from dorsal telencephalic regions [70]. As mentioned earlier, forebrain identity is a default programme for neuronal differentiation of PSCs, and existing protocols yield neurons with a glutamatergic identity without the need of an extra second series of patterning molecules. However, attributed to endogenous $\mathrm{SHH}$ signalling, mouse ESCs have been shown to differentiate into anterior NPCs with a ventral phenotype under serum- and morphogen-free culture conditions, resulting in a neuronal population of which only a minority was considered to be glutamatergic [71]. Consequently, inhibition of intrinsic $\mathrm{SHH}$ signalling with the small molecule cyclopamine has been shown to prevent ventralization of the mouse NPCs and significantly increases the derivation of dorsal glutamatergic neurons [71]. In a separate study on the other hand, it has also been demonstrated that mouse ESCs cultured in the presence of retinoic acid (RA) induced highly homogenous neuronal populations, which, similar to previous report, where consistent with an identity of cortical pyramidal neurons [72]. Pyramidal neurons constitute more than $80 \%$ of the cerebral cortex neurons and are further diversified in distinct cortical layers that establish specific patterns of axonal output and dendritic input, providing the essential substrate of cortical circuitry [73]. In a study by Eiraku et al. [57] for example, it was also demonstrated that human ESC-derived cortical neurons stained positive for transcription factors corresponding to the development of the cortical layers in a temporal manner, including RELN, TBR1, CTIP2 and CUX1. However, opposite to the ventral phenotype observed in mouse ESCs, human PSCs have shown to differentiate by a default programme into synchronised populations of NPCs that predominantly express anterior dorsal markers [70, 71]. This dorsal phenotype has been attributed to expression of endogenous WNT ligands, and, as a consequence, inhibition of WNT signalling or activation of SHH signalling has shown to almost completely convert primitive dorsal telencephalic NPCs to ventral progenitors at the expense of glutamatergic neuron identity [70].

Various studies have also addressed the potential of using the derived glutamatergic cultures for fundamental research. Kim et al. [74] suggested an approach for efficient differentiation of human glutamatergic neurons based on a spin EB protocol in ESCs and iPSCs. Interestingly, when co-cultured with human embryonic kidney cells (HEK293T) expressing NLGN3 and NLGN4, but not those containing autism-associated mutations, the iPSC-derived neurons were able to form functional synapses, demonstrating that these neuronal populations are a potential model for the study of synaptic differentiation and function under normal and disorder-associated conditions [74]. Also in their differentiation approach it was demonstrated that the human spin EB-derived NPCs acquired an anterior dorsal forebrain character by a default pathway. While addition of the $\mathrm{SHH}$ agonist purmorphamine (PUR) during the EB stages ventralized the NPCs, inhibition of 
$\mathrm{SHH}$, however, did not enhance expression of dorsal markers as seen in mouse ESCs [74]. Consistent with this finding, a more recent study also showed that cyclopamine treatment was not required for induction of the dorsal telencephalic fate in a human PSC monolayer system [73]. By determining the time of onset for the expression of layer-specific markers during the course of differentiation, it was demonstrated that most of the pyramidal neurons generated here displayed an identity corresponding to deep cortical layers, while upper layer neurons were underrepresented [73]. This contrasts with a more recent report describing a robust culture system in human ESCs for the generation of both electrophysiological active deep- and upper-layer pyramidal neurons in equivalent proportions that were cultured in the presence of retinoids $[75,76]$. In the context of dual SMAD inhibition, they found that vitamin $A$ is crucial for the efficient induction of cortical NPC differentiation and subsequent cortical neurogenesis. These findings agree with previous report [72], which demonstrated that derivatives of retinoids have important roles in the acquisition of NPCs and telencephalic glutamatergic neurons from mouse ESCs. By using this approach, the efficiency of cortical neural induction from PSCs even approaches $100 \%$ [76]. Although various organisations are still missing, such as interactions with glial cells that are essential partners in synaptic functioning, these systems provide the first steps towards functional studies of human cerebral cortex development and the generation of patient-specific cortical networks in vitro. These future applications will be particularly interesting for modelling disorders that are known for their cortical synaptic dysfunctions, including epilepsy, schizophrenia and dementia, and allow high-throughput testing for therapeutic interventions $[75,76]$.

\section{GABAergic neurons}

Various subtypes of inhibitory GABAergic neurons exist in the brain and spinal cord, which can be categorised based on their developmental origins, localization, synaptic connections, co-expression of molecular and neurotransmitter markers and electrophysiological properties [77]. During development, GABA interneurons are synaptically integrated into neuronal networks in the forebrain that originate mostly from the medial ganglionic eminence and, to a lesser extent, from the ventral lateral ganglionic eminence and the anterior dorsal ganglionic eminence [77-79]. These GABAergic NPCs migrate by following radial or tangential pathways, they differentiate into post-mitotic neurons and make connections with local glutamatergic neurons [77, 80, 81]. The vast majority of forebrain GABAergic interneuron progenitors express Nkx2- 1 [82] in addition to the telencephalic transcription factor Bf1, also known as Foxg1 [53], and they can be distinguished from other types of GABAergic neurons, including the striatal GABAergic projection neurons, which originate from the lateral ganglionic eminence [77, 83]. Forebrain GABA interneurons can be divided into many subgroups on the basis 
of molecular markers and their expression of neuropeptides or calciumbinding proteins, including somatostatin (SST), parvalbumin (PV), calretinin, calbindin and neuropeptide $\mathrm{Y}$, although the medial ganglionic eminence progenitors mostly give rise to SST and PV interneurons [77].

The majority of studies on the differentiation of GABAergic neurons in vitro have focused on guiding PSCs toward ventral telencephalic NPCs, primarily defined by the co-expression of Bf1 and Nkx2-1 (See Table 1) [53, 70, 84]. As stated in the previous section of glutamatergic neurons, ventral telencephalic pecursors have been generated from mice ESCs without the need of additional patterning factors, leading to a neuronal population that is enriched in cortical GABAergic interneurons [71].Ventralization of human dorsal telencephalic NPCs on the other hand, has been achieved by addition of concentrated SHH or inhibition of WNT by DKK1 together with low concentrations of $\mathrm{SHH}$, leading to the generation of enriched populations of human cortical GABAergic interneurons [70]. Interestingly, various groups have tried to purify the generated GABAergic NPCs using cellular selection systems. For instance, Maroof et al. [85] described a protocol for the generation of cortical GABAergic interneurons from mouse ESCs based on EB formation and SHH signalling. In this study, a Lhx6-GFP bacterial artificial chromosome reporter construct was used, which allowed for the isolation and enrichment of the newly generated NPCs. Using a similar approach with a previously established NKX2$1::$ GFP human ESC reporter line, two other groups $[16,86]$ have developed a protocol based on the combination of small molecules with the timed activation of SHH signalling. In both studies they showed that the human PSCs develop into GABAergic interneurons with mature physiological properties, both in vitro, as well as after transplantation into rodent brains. Liu et al. [77] on the other hand, have described a protocol without transgenic modification or cell sorting that involves treatment with SHH or its antagonist PUR for directed GABAergic differentiation of human PSCs with high efficiency. After 2 weeks of differentiation, more than $90 \%$ of the neurons were estimated to be GABAergic interneurons, which was confirmed by immunostaining and electrophysiological analysis [77]. 


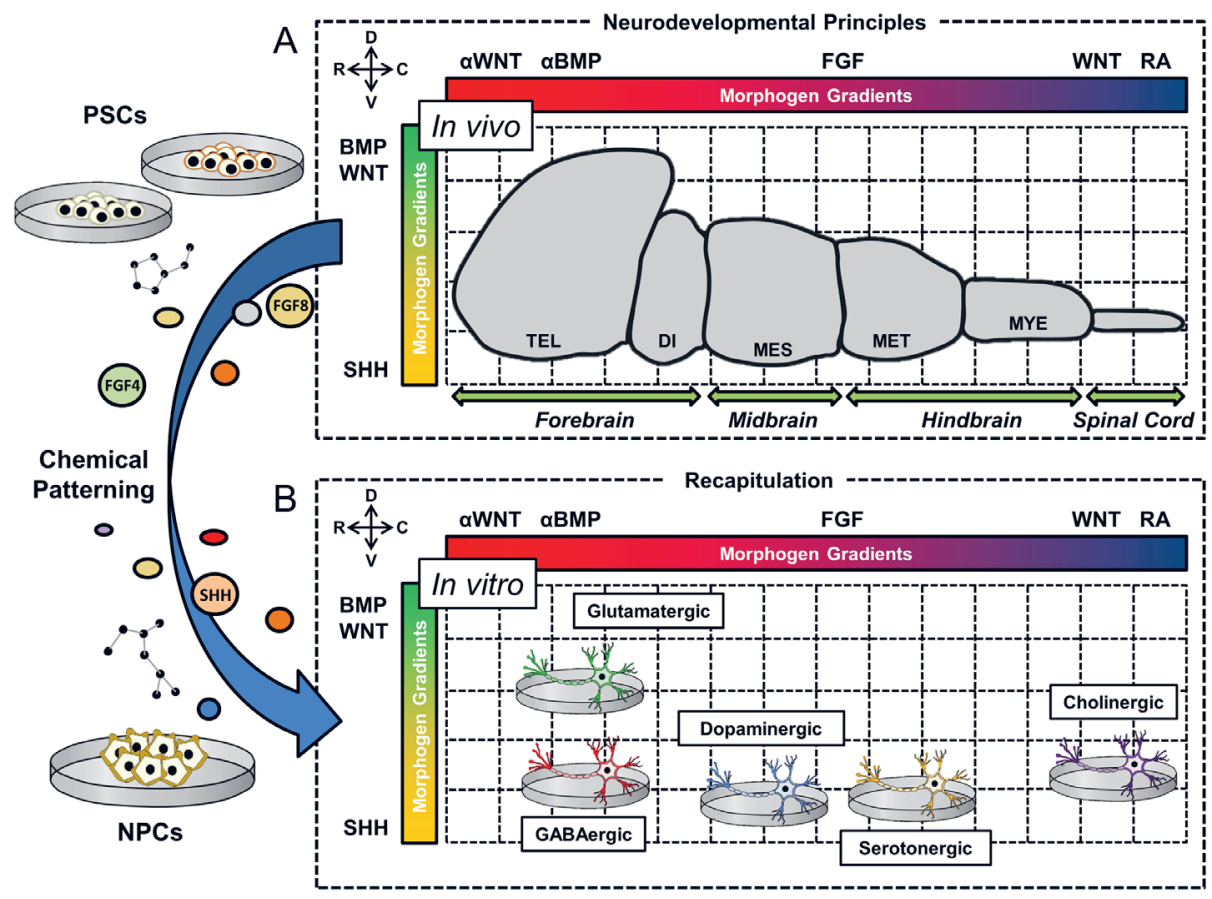

Figure 2. Schematic overview of the working mechanisms of chemically defined neuronal differentiation systems and the underlying neurodevelopmental principles that they recapitulate in vitro. A. The figure shows how various morphogen signalling gradients, including bone morphogenic protein (BMP), wingless-type MMTV integration site family (WNT), fibroblast growth factor (FGF), sonic hedgehog $(\mathrm{SHH})$ and retinoic acid $(\mathrm{RA})$, as well as inhibitors/antagonists $(\alpha)$ of these pathways, pattern the various brain regions during early embryonic development both along the rostral-caudal and dorsal-ventral axes. The depicted brain regions include the telencephalon (TEL), diencephalon (DI), mesencephalon (MES), metencephalon (MET), myelencephalon (MYE) and spinal cord. B. By using the same chemical patterning principles as seen in vivo, pluripotent stem cells (PSCs) and neural precursor cells (NPCs) can be patterned towards neuronal subtypes in vitro, corresponding to the brain regions where they typically originate from. 
Aside from the cortical GABAergic interneurons, several studies have also managed to acquire striatal GABAergic projection neurons, also known as medium spiny neurons [87]. In one of the procedures, neural induction via BMP/ TGF- $\beta$ inhibition was coupled with exposure to SHH and DKK1 to drive ventral telencephalic specification in human ESC and iPSCs, followed by the terminal differentiation towards authentic medium spiny neurons [87]. Authenticity of the resulting neuronal population was monitored by the appearance of BF1/GSX2positive progenitor cells typical for the lateral ganglionic eminence, followed by appearance of CTIP2-, FOXP1- and FOXP2-positive cells. These precursor cells then matured into MAP2/ GABA-positive neurons with $20 \%$ of them coexpressing DARPP-32 and CTIP2, and also carried electrophysiological properties expected for fully functional medium spiny neurons [87]. Most recently, a reliable and simplified two- and three-step protocol to derive striatal GABAergic neurons from immortalized NPCs has also been established, using valproic acid (VPA) or SHH and DKK1, respectively [88]. The differentiated cells expressed appropriate GABAergic markers and responded to ionotropic glutamate receptor stimulation. In accordance, the cells also expressed various glutamate receptor subunits and released GABA upon stimulation [88]. In relation to disease modelling, the derivation of these GABAergic neurons represents a possible critical resource for the study of Huntington's disease and Rett syndrome for example [88, 89].

\section{Dopaminergic neurons}

Dopaminergic neurons are localized in the diencephalon, mesencephalon and the olfactory bulb [90], although the most prominent group resides in the mesencephalon, containing approximately $90 \%$ of the total number of brain dopaminergic neurons [90]. Differentiation protocols for dopaminergic neurons, in particular for the ones originating from the midbrain, have received a lot of attention due to their applicability into regenerative medicine for Parkinson's disease, with numerous differentiation protocols published over the last years (See Table 1) [51, 91, 92]. Studies in mice have shown that dopaminergic midbrain NPCs are specified from the floor plate in the mesencephalon, which is located at the ventral midline of the neural tube [91, 92]. These NPCs are transcriptionally characterised by the expression of Lmx1a, Foxa2, En1 and Otx2, and are controlled by two regulatory feedback loops also involving WNT and SHH signalling [93, 94]. In more detail, WNT1 induces expression of Otx2, which represses Gbx2 to coordinate

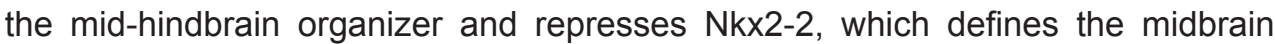
dopaminergic NPC domain from the lateral located progenitors of serotonergic neurons [95]. Thereby, it induces the expression of Lmx1a which either induces the pro-neural gene Ngn2 through Msx1 or inhibits the NPCs from acquiring alternative cell fates by repressing Nkx6-1 [93, 96]. 
It is this developmental principle in mice described above that currently also forms the guideline for differentiating midbrain dopaminergic neurons from human PSC [94]. However, initial reports for the differentiation of dopaminergic neurons have also heavily relied on the use of PA6 and MS- 5 feeder cells [ 45 , 46, 97]. Furthermore, studies have shown the successful differentiation of $\mathrm{TH}$ (tyrosine hydroxylase)- expressing neurons from mouse ESCs and iPSCs based on chemically defined systems that relied on the generation of EBs [98] and the activation of key signalling pathways by SHH and FGF8, a morphogen important for the formation of the isthmus [51, 97-99]. Gradually, studies have tailored and applied these initial studies in primate [94] and human iPSCs [60, $100,101]$, and demonstrated efficient induction of neurons with a dopaminergic phenotype. Importantly, in most of the previous mentioned reports it was actually not conclusively determined whether the obtained $\mathrm{TH}$-positive neurons were really representing midbrain dopaminergic neurons, at least they did not always carry abundant midbrain markers, suggesting that the combination of FGF8 and SHH had the potency to induce the dopaminergic identity but was possibly not sufficient to restrict the neurons to the midbrain fate [94].

A better understanding of essential signalling pathways and transcriptional networks important for dopaminergic neuron midbrain differentiation, as well as their more precise temporal implementation, has improved the protocols over time. Incorporation of WNT/ $\beta$-catenin signalling due to the availability of the GSK3 $\beta$ inhibitor CHIR99021 eventually led to an improved midbrain specification in a reliable and efficient manner [62, 94, 102-105]. This was first demonstrated by Kriks et al. [105], who produced cultures containing around $75 \%$ of floor plate-derived dopaminergic neurons, assessed by immunostaining of markers such as FOXA2 and TH. Further expression analysis in this study also demonstrated abundant co-expression of lineage-specific genes necessary for appropriate dopaminergic neuron specification [105]. Furthermore, the cells were able to be efficiently engraft in rodent brains and survived in vivo without overgrowing, a phenomenon that was previously only observed with very poor performance using human PSC-derived dopaminergic neurons [105]. Their findings were later also confirmed in different human ESC lines, iPSCs, and rhesus monkey iPSCs, showing that a narrow range of $\mathrm{CHIR99021}$ at a particular developmental stage restricts the cells to form midbrain floor plate progenitors which, in the presence of FGF8, acquire a dopaminergic neuron identity [94]. Based on the latter approaches, differentiated dopaminergic populations from Parkinson's disease patient-derived iPSCs have even demonstrated to mimic several pathological mechanisms of the neurological disorder in vitro $[106,107]$. Interestingly, disease phenotypes were only observed in the PSC-derived dopaminergic neurons and not in patients' fibroblasts, which emphasizes the significance of directed dopaminergic differentiation protocols for disease modelling in vitro [107]. 


\section{Serotonergic neurons}

Serotonergic neurons are found in the raphe nuclei that arise from progenitors in the rhombencephalon during development and can be divided into two main clusters: A rostral division located just caudal to the isthmus and a more caudal division situated in the myelencephalon [108]. While the rostral division has widespread innervating projections throughout the brain, the caudal division mainly projects down to the spinal cord [108]. These primary anterior and posterior clusters are further segmented along the rostro-caudal axis according to 9 rhombomeric divisions, where specific transcriptional codes confer positional identities. For instance, the progenitors located at rhombomeric segments 2-3 are distinguished from segment 1 by expression of Hoxa2 but not En1, while progenitors in segment 4 express Phox $2 \mathrm{~b}$, leading into an intervening gap between cluster 3 and 5 , where serotonergic identity is repressed and visceromotoneurons are formed instead [59, 109]. Specification of the distinct segments is thought to be induced by different combinations of morphogen gradients of which the most important ones include SHH, FGF8 and FGF4 [110]. In addition, WNT- and TGF-beta signalling have also been shown to be important for determining boundaries and specifying a hindbrain fate [62, 111, 112]. Developing serotonergic NPCs gradually start expressing Nkx2-2, Ascl1 and Foxa2, which constitutes a primary gene regulatory network for serotonergic specification [108]. Although it is evident that their postmitotic neurons in different regions are transcriptional and functional heterogeneous, this primary genetic cascade activates a secondary network that consists of a set of core transcription factors, including GATA2, GATA3, INSM1, LMX1B and PET1 in mice or FEV in humans, which is key in their terminal specification [108].

Thus far, there have been only few attempts to differentiate serotonergic neurons in chemically defined systems from PSCs (See Table 1). Mice studies have given primary knowledge on the combinations of developmental signals that allow the generation of serotonergic neurons in vitro [110]. One of the first approaches to induce serotonergic neurons from ESCs was based on the formation of EBs in combination with the activation of SHH and FGF8 signalling [98]. The protocol was primarily intended to enrich for dopaminergic neurons, resulting in considerably low yields of serotonergic neurons $( \pm 11 \%)$ compared to the total neuronal population $( \pm 72 \%)$. Mouse ESCs have then also been co-cultured with stromal cells in the presence of SHH and FGF4, yielding a substantially higher proportion $( \pm 57 \%)$ of serotonergic neurons [97]. More recently, a simplified method to generate serotonergic neurons from mouse PSCs in monolayer cultures has also been developed [113]. The cells were cultured on a layer of matrigel in the presence of NOG, a BMP inhibitor, and reached $80 \%$ pure serotonergic cultures after cell sorting [113]. Although their approach increased the homogeneity of serotonergic neurons and appears to be an appealing simpler alternative to culturing mouse 
ESCs on feeder cells, effectively only $\pm 6 \%$ of the initial neuronal cells were considered serotonergic, which remains a relatively low proportion of cells that can be generated from mouse PSCs in vitro.

Studies have consequently demonstrated that the key developmental signalling molecules involved in serotonergic differentiation could also be applied for directing their differentiation utilizing human PSCs. One of the first protocols established in order to obtain serotonergic neurons from human ESCs was based on the induction and enrichment of NPCs that differentiated toward serotonergic neurons under empirically determined culture conditions [114]. In this study, neural differentiation was estimated to be around $20 \%$ with up to $70 \%$ of all neurons staining positive for serotonin $(5-\mathrm{HT})$, generating $14 \%$ of serotonergic neurons in vitro. Interestingly, acidic FGF, which is localized in raphe neurons in rats [115], and 5-HT, which is known to contribute to the development of serotonergic neurons in vivo [116], were added to the growth factor cocktail for differentiation and maturation of the NPCs. Using a differentiation protocol based on FGF8 and SHH, another study [117] found that approximately $8 \%$ of the differentiating human ESCs committed to a serotonergic fate, which was determined by a co-staining of $5-\mathrm{HT}$ and tryptophan hydroxylase (TPH).

Thus, although these first protocols in mice and human PSCs were very promising, they only met limited success considering their low yields, which limits the ability to use these cells for further applications. However, most recently, Lu et al. [59] were the first to develop a robust chemically defined system to induce human iPSC to enriched populations of serotonergic neurons with very high efficiency. Induction of ventral hindbrain NPCs was achieved by maintaining PSCs in medium containing SB431542, DMH-1 and CHIR99021. Following neural induction, the NPCs were exposed to FGF4 together with SHH to promote the acquisition of a serotonergic cell fate, eventually reaching more than $60 \%$ serotonergic neurons [59]. The key aspect here, is the activation of the WNT pathway by CHIR99021 that, within a narrow window of concentrations, gives rise to ventral hindbrain NPCs that further differentiate into serotonergic with a typical identity for rhombomeric divisions 2-3. Interestingly, treatment with the FDA-approved antidepressants tramadol and escitalopram oxalate in this latter study resulted in release or uptake of 5-HT in a dose- and timedependent manner, which emphasized their utility for the evaluation of drug candidates in depression [59]. Dysregulation of the serotonergic system is typical in depression and a common target for antidepressants [118, 119]. 


\section{Cholinergic motor neurons}

Cholinergic motor neurons can be broadly divided into two main groups according to the location of their cell body: (I) Upper motor neurons located in the motor regions of the cerebral cortex, and (II) lower motor neurons, which are located in the brainstem and spinal cord [49]. Upper motor neurons have ascending pathways to lower motor neurons, which project to the musculature, where they control muscle contractions via neuromuscular junctions [49]. Spinal motor NPCs originate from a highly restricted foci in the ventral neural tube in response to RA, FGFs and SHH [52]. These cells express the basic helixloop-helix transcription factor Olig2, which, together with Ngn2, direct the expression of motor neuron fate consolidating genes such as $\mathrm{Hb} 9$ and Isl1. For a more detailed description of the underlying developmental cascade that results in the acquisition of these cells, we would like the reader to refer to other excellent reviews [49, 52]. Motor neurons can be further developmentally allocated to discrete motor columns, which extend along the rostral-caudal neural tube and contain motor neuron pools that are responsible for innervating a single skeletal muscle, each of which is also arranged by an anatomical logic corresponding to their targets [52]. Retinoid signalling plays key roles in the diversification of motor neuron subtypes from the common NPC pool and additionally contributes to spinal cord columnar organisations, which are then again characterized by unique transcriptional codes that define the regional identity of the neuronal subtypes [52].

By recapitulating the developmentally rationalized programme of morphogenic cues, considerable advances using chemically defined systems have primarily been made in differentiating PSCs into lower spinal motor neurons (See Table 1). The desire to regenerate in vitro motor circuitry in the contexts of motor neuron disease and spinal cord injury has been motivating the attempts to produce motor neurons for translational research [49]. Initial studies have outlined methods to derive functional cholinergic motor neurons from mice [97] and human ESCs [58, $120,121]$, while more recent studies have applied similar methods to human iPSC lines [36, 122-124]. The numerous protocols that have been developed utilize various directed differentiation methods, including co-culture with stromal feeders [58, 97], adherent monolayer cultures [123], or the use of EB induction followed by neuralization, cholinergic neuron differentiation and neuronal maturation [36, 124]. In most cases, studies have reported the use of RA treatment with addition of recombinant $\mathrm{SHH}$ or small molecule agonists of the $\mathrm{SHH}$ signalling pathway to induce differentiation of PSCs into cholinergic motor neurons. Patterning NPCs by RA and SHH confers caudal and ventral anatomical identities, respectively, and gives rise to OLIG2 expressing neurons, which in turn begin to express CHAT, HB9 and ISL1 [124]. Cholinergic motor neurons obtained through these methods have been shown to possess numerous characteristics of their in vivo 
equivalents, including electrophysiological properties, the possibility to engraft into the developing spinal cord and the presence of correctly labelled neuromuscular junction complexes, demonstrating the potential ability to form functional muscular junctions in vitro [49]. However, in contrast to the generic developmental principles that allow cholinergic motor neuron specification, the process determining how individual motor neuron subtypes can be generated is relatively less well understood [49]. Nevertheless, in the context of mouse ESC differentiation for example, protocols based on a treatment with RA typically result in spinal motor neurons with a rostral cervical character, as judged by expression of Hoxc5 and Hoxc6, but not Hoxc8 [125]. In the context of human motor neuron differentiation on the other hand, a recent report demonstrated a shift in the proportion of motor neurons expressing the median motor column marker LHX3 or the lateral motor column marker FOXP1, when SHH signalling was activated via a combination of smoothened agonist (SAG) and PUR instead of recombinant SHH [126]. This sensitivity of the differentiating NPCs emphasizes the need for thorough evaluation of the differentiation protocols and also presents the opportunity for the optimization of motor neuron subtype specification [49]. 


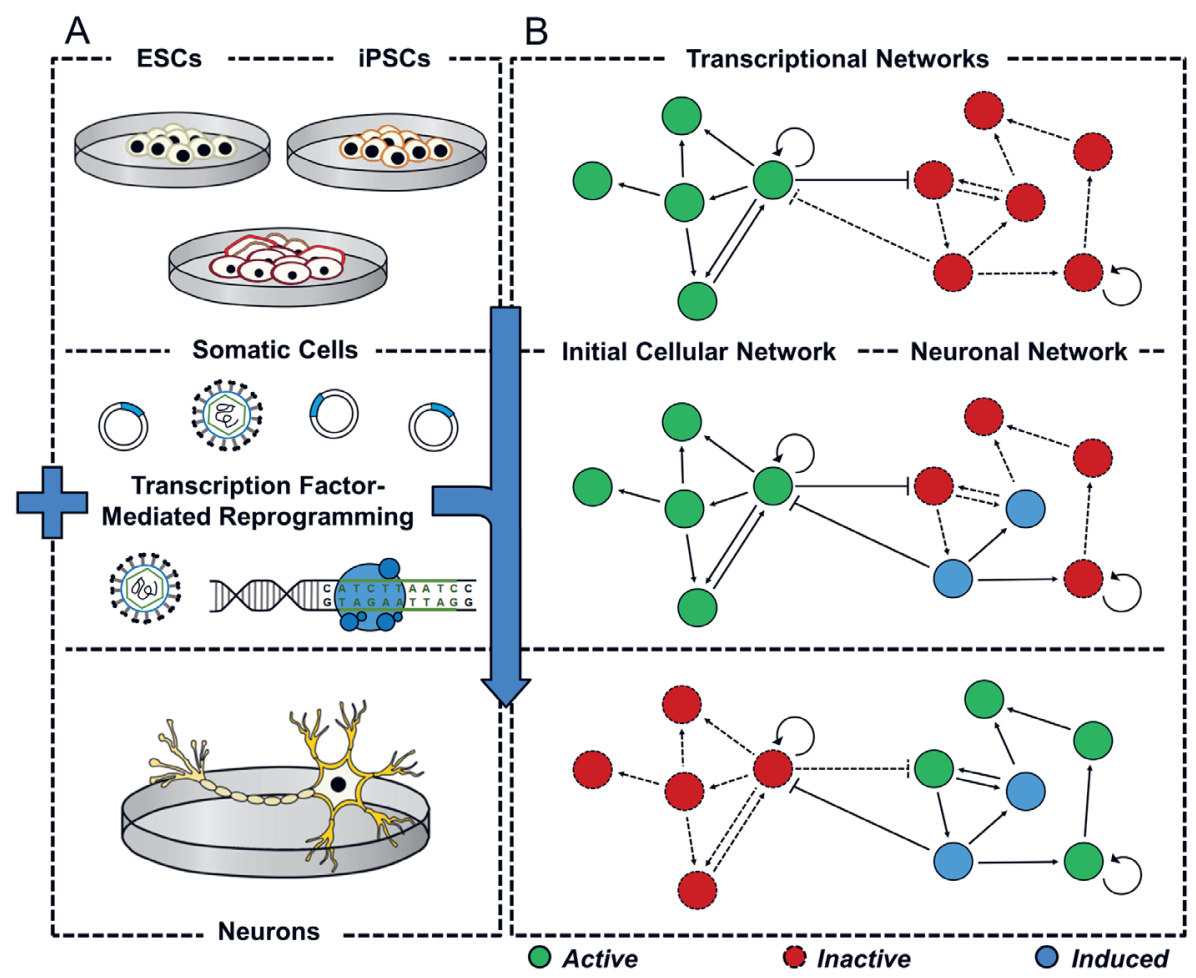

Figure 3. Schematic overview of the working mechanisms of transcription factor-mediated reprogramming along the neural lineage in vitro. A. The figure shows how embryonic stem cells (ESCs), induced pluripotent stem cells (PSCs) and somatic cells can be reprogrammed towards neurons by transcription factor-mediated fate instructions. B. The initial active (in green) transcriptional network that defines and reinforces cellular identity can be perturbed by induction of exogenous key-lineage determinant factors (in blue), leading to the activation (in green) of the neuronal transcription factor network and deactivation (in red) of the initial cellular network, which underlies the cellular fate switch. 
Table 2. Transcription factor-mediated neuronal reprogramming protocols in vitro per neuronal phenotype.

\begin{tabular}{|c|c|c|c|c|}
\hline \multicolumn{5}{|c|}{ Transcription factor-mediated reprogramming } \\
\hline Phenotypes & Species & Starting cell types & Culture methods & Transcription factors \\
\hline \multirow[t]{5}{*}{ Glutamatergic neurons } & Mouse & Fibroblasts (Tau::EGFP) & Monolayer & Ascl1, Brn2 and Myt1l \\
\hline & & Astrocytes & Monolayer & Ngn2 \\
\hline & & ESCs & Monolayer & Ngn2 \\
\hline & Mouse and human & Fibroblasts (Tau::EGFP) and ESCs & Monolayer & Ascl1 \\
\hline & Human & Fibroblasts (Таu::EGFP), ESCs and iPSCs & Monolayer & Ascl1, Brn2, Myt1/ and \\
\hline
\end{tabular}

ESCs and IPSCs Monolayer $\quad$ Ngn2 and Neurod1

\begin{tabular}{llll}
\cline { 2 - 3 } & Fibroblasts (Pitx3::EGFP) & Monolayer & $\begin{array}{l}\text { Ascl1, En1, Foxa2, Lmx1a, Nurr1 } \\
\text { and Pitx3 }\end{array}$ \\
\hline Mouse and human & Mouse fibroblasts (TH::GFP) and human fibroblasts & Monolayer & Asc11, Lmx1a and Nurr1
\end{tabular}

\begin{tabular}{llll}
\hline Human & Fibroblasts & Monolayer & Ascl1, Brn2, Myt1, Foxa2 and Lmx1a \\
\cline { 2 - 4 } & iPSCs & Monolayer & Ascl1, Lmx1a and Nurr1
\end{tabular}




\begin{tabular}{|c|c|c|}
\hline Chemical driving factors & Phenotypic markers ( $\%$ cells) & References \\
\hline DOX & $\begin{array}{l}>20 \% \text { TUJ1+ } \\
53 \% \text { TBR1+/TUJ1+ }\end{array}$ & Vierbuchen et al. (2010) \\
\hline None & $\begin{array}{l}70.2 \pm 6.3 \% \text { TUJ1+ } \\
85.4 \pm 5.0 \% \text { VGLUT1+ } \\
\pm 48.2 \% \text { TBR } 1+\end{array}$ & Heinrich et al. (2010) \\
\hline None & $<40 \%$ TUJ1+ & Thoma et al. (2012) \\
\hline \multirow[t]{2}{*}{ DOX } & Mouse embryonic fibroblasts & \multirow[t]{2}{*}{ Chanda et al. (2014) } \\
\hline & $\pm 45 \%$ VGLUT1+/TAU-EGFP+ & \\
\hline \multirow[t]{4}{*}{ DOX } & Fetal fibroblasts & \multirow[t]{4}{*}{ Pang et al. (2011) } \\
\hline & $\begin{array}{l} \pm 60 \% \text { TUJ1+ } \\
>50 \% \text { VGLUT1+/TUJ1+ } \\
>50 \% \text { VGLUT2+/TUJ1+ } \\
17 \pm 8 \% \text { TBR } 1+/ \text { TUJ1+ }\end{array}$ & \\
\hline & Postnatal fibroblasts & \\
\hline & $81 \pm 17 \%$ TBR $1+/$ TUJ1+ & \\
\hline \multirow[t]{4}{*}{ DOX } & Ngn2- ESCs & \multirow[t]{4}{*}{ Zhang et al. (2013) } \\
\hline & $\pm 80 \% \mathrm{MAP} 2+$ & \\
\hline & Ngn2 - iPSCs & \\
\hline & $\pm 90 \%$ MAP $2+$ & \\
\hline \multirow[t]{2}{*}{ DOX } & Astrocytes & \multirow[t]{2}{*}{ Addis et al. (2011) } \\
\hline & $\begin{array}{l}35.1 \pm 1.5 \% \text { TUJ } 1+ \\
50.9 \pm 3.3 \% \text { TH+/TUJ1+ } \\
18.2 \pm 1.5 \% \text { TH+ }\end{array}$ & \\
\hline \multirow[t]{2}{*}{ DOX } & Fibroblasts & \multirow[t]{2}{*}{ Addis et al. (2011) } \\
\hline & $\begin{array}{l}14.9 \pm 2.3 \% \text { TUJ } 1+ \\
9.1 \pm 0.9 \% \mathrm{TH}+\end{array}$ & \\
\hline DOX, FGF8 and SHH & 9.1\% Pitx3-EGFP+ & Kim et al. (2011b) \\
\hline \multirow[t]{4}{*}{ DOX } & Mouse & \multirow[t]{4}{*}{ Caiazzo et al. (2011) } \\
\hline & $\begin{array}{l} \pm 22 \% \text { TUJ1+ } \\
\pm 17 \% \text { TH+ }\end{array}$ & \\
\hline & Human & \\
\hline & $\begin{array}{l}10 \pm 4 \% \text { TUJ } 1+ \\
6 \pm 2 \% \mathrm{TH}+\end{array}$ & \\
\hline DOX & $\begin{array}{l} \pm 15 \% \text { TUJ1+/MAP2+ } \\
\pm 10 \% \text { TH+/TUJ1+/MAP2+ }\end{array}$ & Pfisterer et al. (2011) \\
\hline \multirow[t]{4}{*}{ DOX } & Fetal fibroblast-derived iPSCs & \multirow[t]{4}{*}{ Theka et al. (2013) } \\
\hline & $\begin{array}{l}51 \pm 4 \% \text { TUJ } 1+ \\
65 \pm 5 \% \text { TH+/TUJ1+ } \\
\pm 30 \% \text { CALB } 1+/ \text { TH }+ \\
\pm 40 \% \text { GIRK2+/TH+ }\end{array}$ & \\
\hline & Parkinson's disease patient-derived iPSCs & \\
\hline & $\begin{array}{l}48 \pm 4 \% \text { TUJ } 1+ \\
26 \pm 3 \% \text { TH+/TUJ } 1+\end{array}$ & \\
\hline None & $<35 \pm 4 \%$ GABA+/TUJ1+ & Wasko (2013) \\
\hline
\end{tabular}


Table 2. (Continued)

\begin{tabular}{|c|c|c|c|c|}
\hline \multicolumn{5}{|c|}{ Transcription factor-mediated reprogramming } \\
\hline Phenotypes & Species & Starting cell types & Culture methods & Transcription factors \\
\hline GABAergic neurons & Mouse & Astrocytes & Monolayer and neurosphere & $D / x 2$ \\
\hline
\end{tabular}

Mouse and human $\quad$ Mouse fibroblasts (GAD67::GFP), human fibroblasts and human iPSCs Monolayer Ascl1, Dlx5, Bf1, Lhx6 and Sox2

\begin{tabular}{|c|c|c|c|c|}
\hline \multirow[t]{2}{*}{ Serotonergic neurons } & \multirow[t]{2}{*}{ Human } & Fibroblasts & Monolayer & $\begin{array}{l}\text { ASCL1, FOXA2, FEV, LMX1B } \\
\text { and hp53shRNA }\end{array}$ \\
\hline & & Fibroblasts (TPH2::GFP and SYN1::dsRed) & Monolayer & $\begin{array}{l}\text { ASCL1, FEV, GATA2, LMX1B, NGN2 } \\
\text { and NKX2-2 }\end{array}$ \\
\hline $\begin{array}{l}\text { Cholinergic motor } \\
\text { neurons }\end{array}$ & Mouse & ESCs & EB & Isl1, Lhx3, Ngn2 and Phox2a \\
\hline \multirow[t]{4}{*}{$\begin{array}{l}\text { Cholinergic motor } \\
\text { neurons }\end{array}$} & Mouse & ESCs & $\mathrm{EB}$ & Isl1, Lhx3, Ngn2 and Phox2a \\
\hline & \multirow[t]{2}{*}{ Mouse and human } & ESCs & Monolayer (mouse) and EB (human) & Phox2a and Phox2b \\
\hline & & Mouse fibroblasts (Hb9::GFP) and human fibroblasts & Monolayer & $\begin{array}{l}\text { Ascl1, Brn2, Myt1l, Lhx3, Isl1, Hb9, Ng } \\
\text { and NEUROD1 }\end{array}$ \\
\hline & Human & ESCs $(H b 9:: G F P)$ and iPSCs & EB & Is/1, Lhx3 and Ngn2 \\
\hline
\end{tabular}

Noradrenergic neurons Mouse and human ESCs Monolayer (mouse) and EB (human) Phox2b

Neuronal phenotypes, species, starting cell types, culture methods, transcription factors, chemical driving factors and representative phenotypic markers that have been used to assess the differentiation efficiency and culture homogeneity are broadly summarized. + indicates the percentage of cells in the population that stained positive for a certain marker. Abbreviations: 5-HT, serotonin (5-hydroxytryptamine); A83-01, TGF-B kinase/activin receptor-like kinase inhibitor; ASCL1, achaete-scute homolog 1 (MASH1/HASH1); Bf1, brain factor 1/forkhead box protein G1 (FOXG1); BMP5, bone morphogenic protein 5; BMP7, bone morphogenic protein 7; Brn2, brain-specific homeobox/POU domain protein 2 (POU3F2); CALB1, calbindin 1; CD4, cluster of differentiation 4; CHAT, choline o-acetyltransferase; CHIR99021, GSK3ß inhibitor; DIx1, distal-less homeobox 1; DIx2, distal-less homeobox 2; DIx5, distal-less homeobox 5; DOX, doxycycline; dsRed, discosoma sp. red fluorescent protein; EGFP, enhanced green fluorescent protein; En1, homeobox protein engrailed 1; ESCs, embryonic stem cells; FEV, ETS transcription factor (PET1); FGF2, fibroblast growth factor 2/basic fibroblast growth factor (bFGF); FGF8, fibroblast growth factor 8; FGF8b, fibroblast growth factor 8 isoform b; FOXA2, forkhead box protein A2; GABA, Y-aminobutyric acid; GAD65/67, glutamic acid decarboxylase isoform 65/67 (GAD2/1); GAD67, glutamic acid decarboxylase isoform 67 (GAD1); GATA2, GATA binding protein 2; GCaMP3, a GFP-based calcium sensor for imaging calcium dynamics; GFP, green fluorescent protein; GIRK2, G protein-activated inward rectifier potassium channel 2 (KCNJ6); HB9, homeobox HB9/motor neuron and pancrease homeobox 1 (MNX1); Hh-Ag1.3, small molecule agonist of SHH signalling; hp53shRNA, human p53 small hairpin RNA; iPSCs, induced pluripotent stem cells; ISL1, ISL LIM homeobox 1; Lbx1, ladybird homeobox 1; LDN193189, selective BMP signalling inhibitor; Lhx1, LIM homeobox 1; Lhx2, LIM homeobox 2; Lhx3, LIM homeobox 3; Lhx6, LIM homeobox 6; 


\begin{tabular}{|c|c|c|}
\hline Chemical driving factors & Phenotypic markers ( $\%$ cells) & References \\
\hline \multirow[t]{2}{*}{ None } & Monolayer & Heinrich et al. (2010) \\
\hline & $\begin{array}{l}35.9 \pm 13.0 \% \text { TUJ } 1+ \\
33.7 \pm 3.6 \% \text { VGAT }+\end{array}$ & \\
\hline \multirow[t]{6}{*}{ DOX } & Mouse fibroblasts & \multirow[t]{6}{*}{ Colasante et al. (2015) } \\
\hline & $\begin{array}{l} \pm 94 \% \text { GAD65/67+/GAD67-GFP+ } \\
\pm 97 \% \text { GABA+/GAD67-GFP+ } \\
\pm 93 \% \text { PV+/GAD67-GFP+ } \\
\pm 3 \% \text { SST+/GAD67-GFP+ }\end{array}$ & \\
\hline & Human fibroblasts & \\
\hline & $\begin{array}{l} \pm 70 \% \text { GABA }+/ \text { TUJ } 1+ \\
\pm 90 \% \text { PV }+/ \text { TUJ } 1+\end{array}$ & \\
\hline & Human iPSCs & \\
\hline & $\begin{array}{l} \pm 50 \% \text { GABA }+/ M A P 2+ \\
\pm 90 \% \text { PV+/MAP2+ } \\
\pm 2 \% \text { SST+/GABA }+\end{array}$ & \\
\hline DOR, DOX, PD0332991 and SB431542 & $\begin{array}{l}<49 \% \text { TUJ } 1+ \\
<23 \% 5-\mathrm{HT}+\end{array}$ & Xu et al. (2015) \\
\hline $\begin{array}{l}\text { A83-01, CHIR99021, DOX, Forskolin, LDN193189, NOG and } \\
\text { SB431542 }\end{array}$ & $\begin{array}{l}58.4 \pm 4.2 \% \text { TUJ1+ } \\
\pm 60 \% \text { MAP2ab+ } \\
61 \pm 15 \% \text { TPH+/MAP2ab+ } \\
38 \pm 2 \% 5-H T+\end{array}$ & Vadodaria et al. (2015) \\
\hline \multirow[t]{2}{*}{ DOX } & Ngn2, Is/1 and Lhx3 & \multirow[t]{2}{*}{ Mazzoni et al. (2013) } \\
\hline & $\begin{array}{l}99.82 \pm 0.17 \% \mathrm{HB} 9+/ / \mathrm{ISL} 1+ \\
0.24 \pm 0.28 \% \mathrm{PHOX} 2 \mathrm{~B}+/ / \mathrm{SL} 1+\end{array}$ & \\
\hline \multirow[t]{2}{*}{ DOX } & Ngn2, Is/1, Phox2a & \multirow[t]{2}{*}{ Mazzoni et al. (2013) } \\
\hline & $\begin{array}{l}0.11 \pm 0.11 \% \mathrm{HB} 9+/ / \mathrm{ISL} 1+ \\
99.03 \pm 0.08 \% \mathrm{PHOX} 2 \mathrm{~B}+/ / \mathrm{ISL} 1+\end{array}$ & \\
\hline \multirow[t]{2}{*}{ FGF2, FGF8b and Hh-Ag1.3 } & Mouse & \multirow[t]{2}{*}{ Mong et al. (2014) } \\
\hline & 61\% PHOX2B+/ISL1+/TUJ1+ & \\
\hline \multirow[t]{2}{*}{ None } & Mouse & \multirow[t]{2}{*}{ Son et al. (2011) } \\
\hline & $\begin{array}{l}5-10 \% \text { Hb9-GFP+ } \\
97.6 \% \text { VACHT+/Hb9-GFP+ }\end{array}$ & \\
\hline \multirow[t]{4}{*}{ Forskolin, RA and SHH } & ESCs & \multirow[t]{4}{*}{ Hester et al. (2011) } \\
\hline & $\begin{array}{l}55 \% \mathrm{Hb9}-\mathrm{GFP}+ \\
\pm 50-62 \% \mathrm{HB}+/ \mathrm{CHAT}+\end{array}$ & \\
\hline & iPSCs & \\
\hline & $49-72 \%$ HB9+/CHAT+ & \\
\hline \multirow[t]{2}{*}{ BMP5, BMP7, Cyclopamine, FGF2 and FGF8b } & Mouse & \multirow[t]{2}{*}{ Mong et al. (2014) } \\
\hline & $17.9 \% \mathrm{TH}+/ \mathrm{PHOX} 2 \mathrm{~A}+/ \mathrm{TUJ} 1+$ & \\
\hline
\end{tabular}

Lmx1a, LIM homeobox transcription factor 1 alpha; LMX1B, LIM Homeobox transcription factor 1 beta; MAP2, microtubule-associated protein 2; MAP2ab, microtubule-associated protein 2ab; Myt11, myelin transcription factor 1 like; NEUROD1, neurogenic differentiation 1; NGN2, neurogenin 2; NKX2-2, NK2 homeobox 2; NOG, Noggin; NURR1, nuclear receptor related 1 protein; Pax2, paired box 2; PD0332991, cyclin-dependent kinase 4/6 inhibitor; Pft1a, pancreas specific transcription factor 1a; PHOX2A, paired-like homeobox 2a; PHOX2B, paired-like homeobox 2b; Pitx2, paired-like homeodomain 2; Pitx3, paired-like homeodomain 3; PV, parvalbumin; RA, retinoic acid; SB431542, transforming growth factor beta inhibitor; SHH, sonic hedgehog; Sox2, SRY box 2; SST, somatostatin; SYN1, synapsin 1; Tau, microtubule-associated protein tau (MAPT); TBR1, T-box brain 1; TH, tyrosine hydroxylase; TPH, tryptophan hydroxylase; TPH2, tryptophan hydroxylase 2; TUJ1, neuron-specific class III beta-tubulin (TUBB3); VACHT, vesicular acetylcholine transporter; VGAT, vesicular GABA transporter; VGLUT1, vesicular glutamate transporter 1; VGLUT2, vesicular glutamate transporter 2. 


\section{Transcription factor-mediated reprogramming Cellular reprogramming and transdifferentiation}

For long it was thought that cellular differentiation and lineage commitment were irreversible processes established during embryonic development [127]. However, the cloning of animals by nuclear transfer demonstrated that maturated molecular mechanisms are reversible and that a nucleus from the most differentiated cell bears the potential to generate an organism [127]. These cell fusion experiments have proved that transcriptional reprogramming can occur by exposing a distinct nucleus to cytoplasmatic components of cells from distinct lineages, although the exact mechanisms underlying these processes remain challenging to address. Substantial interest in transcriptional reprogramming has been rejuvenated upon the discoveries by Dr. Takahashi and Prof. Dr. Yamanaka, who provided the foundation that somatic cells can be reprogrammed to iPSCs. The cells were initially generated by reprogramming fibroblasts via recombinant overexpression of four transcription factors, including Oct3/4, Sox2, Klf4, and Myc (OSKM, Yamanaka factors) [128]. The use of only four transcription factors was sufficient to induce dramatic cell fate changes and to reprogram fully differentiated cells into a more embryonic cell state. The derivation of iPSCs has been substantially adapted and improved by using other sets of transcription factors, including LIN28 and NANOG [129], by introducing non-integrative transgene expression and by using different types of somatic cells [130, 131]. Earlier work on the other hand, has demonstrated that increased activity of a single transcription factor, namely Myod1, is sufficient to directly convert fibroblasts into myocytes by a process known as transdifferentiation [132]. This has supported the notion that cell fate conversions can be direct without the need of precedent dedifferentiation. Consequently, these studies have raised the question whether transcription factormediated reprogramming could also directly induce neuronal fates in somatic cells or even PSCs (See Fig. 3). Notably, and especially important in the context of disease modelling, these direct conversion modalities may prove to be invaluable in the study of late-onset neurodegenerative disorders because the age of somatic cells is maintained in the converted neurons, thus allowing to model the aging process in vitro [133]. In addition, while chemically defined differentiation protocols in PSCs are known for their long multistep protocols, PSCs exposed to specific sets of transcription factors have shown to differentiate much faster without additional culturing steps, therefore providing an appealing, simpler and possibly more effective differentiation strategy for PSCs in vitro [134]. One of the major disadvantages of direct somatic cell reprogramming compared to directed differentiation of PSCs however, is that the former skips the pluripotent state and does not allow expansion of the cells before further applications, thereby limiting the accessibility of cells readily available in vitro. Nevertheless, somatic 
cell reprogramming into an intermediate state that allows substantial proliferation, such as NPCs, has already been considered [135, 136]. For instance, mouse and human fibroblasts have been partially reprogrammed by introducing the four Yamanaka factors, which gave rise to NPCs that were capable of differentiating into both neuronal and glial cells in the presence of leukemia inducible factor (LIF) and FGF2 [137]. The direct differentiation of partially reprogrammed cells may be useful for rapidly preparing high numbers of NPCs that could be expanded before terminal differentiation into target neurons.

\section{Induced glutamatergic neurons}

Starting from a pool of nineteen candidates, Vierbuchen et al. [138] were the first to identify a combination of only three transcription factors, including Brn2, Ascl1, and Myt1I (BAM), which could rapidly and efficiently convert mouse embryonic fibroblasts (MEF) into induced neuronal (iN) cells (See Table 2). These iN cells expressed multiple neuron-specific markers, generated action potentials and were able to form functional synapses [138]. Electrophysiological recordings demonstrated that mainly excitatory postsynaptic potentials could be recorded, providing functional proof that a large majority of the iN cells exhibited a glutamatergic phenotype. Some cells also expressed GABAergic markers at earlier time points, including Gaba and Gad67, suggesting that both neuronal subtypes could be obtained but culture conditions probably favoured the glutamatergic phenotype [127, 138]. These findings in murine somatic cells led to follow-up experiments using human fibroblasts, which eventually led to the successful generation of human iNs with the addition of Neurod1 to the BAM pool [139]. Just like the generation of iPSCs, during that time it was thought that a combination of factors was necessary to fully reprogram iN cells from fibroblasts and the use of a single transcription factor was considered insufficient [140]. However, later it was shown that Ascl1 alone is satisfactory to generate populations of pre-dominantly glutamatergic iN cells from mouse and human fibroblasts, as well as ESCs [140, 141]. A clear hierarchical role of the reprogramming factors has, therefore, been suggested, demonstrating that ASCL1 acts as a key factor to activate the neuronal program, whereas access of BRN2 to the chromatin is apparently more cell-context-dependent and facilitates reprogramming later on [140]. Moreover, although Ascl1 alone is sufficient to generate iNs, endogenous Mytl is subsequently induced during reprogramming, and exogenous Myt1l has, therefore, demonstrated to greatly improve the efficiency of reprogramming and the functional maturity of the resulting iN cells [141]. In fact, it has been shown that MYT1L exerts its proneuronal function by direct repression of many different somatic lineage programs except the neuronal program. This repressive function of MYT1L is mediated via recruitment of a complex containing SIN3B by binding 
to a previously uncharacterized $\mathrm{N}$-terminal domain [141]. In addition, knockdown of Myt1l in primary postmitotic neurons removed the repression of non-neuronal programs and impaired neuronal gene expression and function, indicating that many somatic lineage programs are actively and persistently inhibited by MYT1L to maintain neuronal identity [141]. Aside from fibroblast, for murine astrocytes [142], mouse ESCs [140, 143] and human PSCs [17, 140], it has also been shown that single neurogenic factors, such as Neurod1 and Ngn2, alone are sufficient to rapidly induce the neuronal fate. Although Ascl1-induced iN cells displayed slower maturation kinetics at early developmental stages, their functional properties and neuronal gene-expression profile at later time points are surprisingly similar to that of Ngn2 or BAM iN cells [140].

After the establishment of the BAM pool and the other neurogenic transcription factors, researchers have been attempting to derive other neuronal subtypes by transcription factor-mediated reprogramming and, thanks to that, additional factors have been identified with the ability to induce dopaminergic neurons, GABAergic neurons, serotonergic neurons, cholinergic neurons and adrenergic neurons (See Table 2) [144-148]. Identical to the chemically defined systems, all of these obtained cultures have been subjected to various bioassays and assessments in order to examine their population characteristics (See Table S2). Furthermore, studies have demonstrated that supplementation of chemically defined systems with transcription factor-mediated reprogramming can significantly increase the efficiency of obtaining differentiated neuronal cells, as well as vice versa [145, 149, 150]. Notably, blockade of TGFb/SMAD signalling using Noggin and molecules such as SB431542 and LDN193189, as well as pharmalogical promotion of calcium signalling with CAMP and Forskolin have not only shown to increase iN yield, but have also been used to successfully generate transgene-free iNs [110, $151,152]$. The fast progress in the field of chemically-mediated reprogramming and transdifferentiation provides us new ways to manipulate neuronal fates both in vitro and in vivo. These methods on their own and/or in combination with other approaches may accelerate the eventual applications of patient-specific human neurons generated in vitro, by facilitating the potency and timelines of the protocols, and by aiding in the specification of regional subtypes within neurotransmitter classes.

\section{Induced dopaminergic neurons}

By combining the BAM factors with Lmx1a and Foxa2, which are typically expressed in midbrain dopaminergic NPCs, human fibroblasts have been converted into induced dopaminergic (iDA) neurons [146]. This provided proof-ofprinciple that other subtypes of iN cells can be produced by transcription factor- 
mediated fate instructions. Since then, iDA neurons have been obtained by ectopic overexpression of various combinations of transgenes encoding midbrain-specific transcription factors (See Table 2). For instance, a minimal set of three transcription factors, i.e. Ascl1, Nurr1 and Lmx1a, was sufficient to generate functional iDA neurons from murine and human fibroblasts [153]. The three factors were able to elicit dopaminergic neuronal conversion, resulting in iDA neurons that were highly enriched in genes of the dopaminergic phenotype, released dopamine, exhibited proper electrophysiological profiles, and, in case of the murine iDA neurons, were able to integrate into neonatal mouse brains. Interestingly, the endogenous Th and Vmat2 promoter regions were highly demethylated in the iDA neurons, whereas they were fully methylated in the fibroblasts, indicating their epigenetic reactivation during dopaminergic conversion [153]. In addition to fibroblasts, astrocytes have also been efficiently converted into iDA by using a single polycistronic vector containing ASCL1, LMX1B and NURR1 [154]. The fact that different somatic cells can be reprogrammed towards iDA using similar sets of transcription factors highlights their importance in reprogramming processes and reassigning cell fate. Reprogramming mouse fibroblast based only on Ascl1 and Pitx3 in another study resulted in immature iDA neurons after 4 weeks of culture [150]. However, inclusion of additional factors, such as En1, Foxa2, Lmx1a, and Nurr1, could fully reprogram fibroblasts into iDA neurons that were more similar at the molecular level to bona fide dopaminergic neurons [150]. Furthermore, the murine iDA neurons were able to alleviate symptoms in a mouse model of Parkinson's disease, demonstrating their therapeutic potential for transplant therapies [150]. Although transdifferentiation of fibroblasts in the latter study could be achieved by ectopic overexpression of only 2 factors, the results suggested that additional factors are required to ensure proper maturation of the iDA neurons [150]. Furthermore, by adding SHH and FGF8 to the culture media, iDA neuron reprogramming could be enhanced up to 2 fold when using only 2 factors, and up to 3 fold when using the combination of 6 factors [150]. These findings demonstrate that patterning molecules can act as critical enhancing components in promoting the generation of iDA neurons from fibroblasts. Notably, Theka et al. [155] have established a fast protocol to obtain dopaminergic neurons by overexpressing Ascl1, Nurr1, and Lmx1a in human iPSCs. They were able to generate mature and functional dopaminergic neurons in as few as 21 days, avoiding all the intermediate steps of induction and selection of EBs and NPCs. Strikingly, the resulting neuronal conversion process was very efficient, since approximately $93 \%$ of all the coinfected iPSCs were forced to differentiate into postmitotic iDA neurons [155]. The iPSC-derived neurons expressed all the critical molecular markers of midbrain dopaminergic neurons at the molecular level and exhibited sophisticated functional features, including spontaneous electrical activity and dopamine release [155]. 

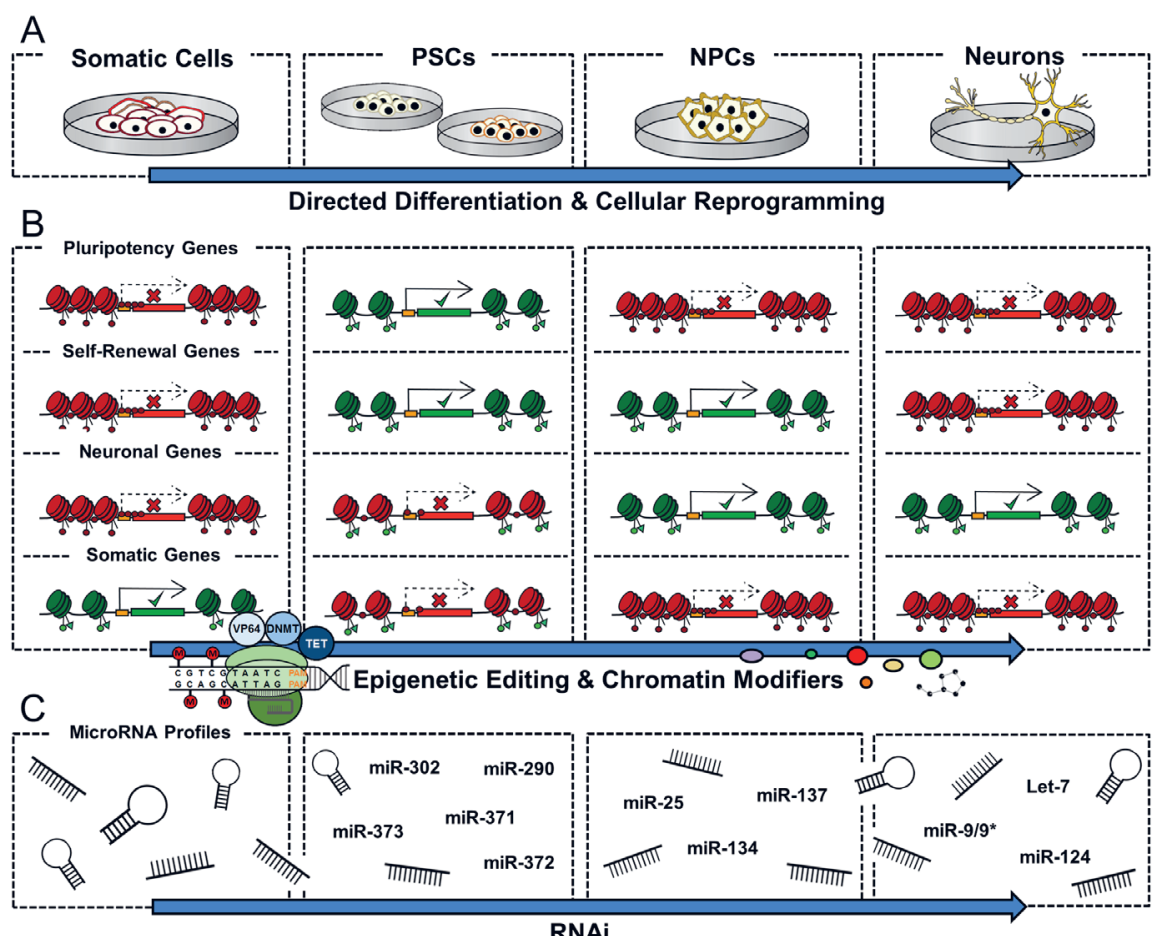

RNAi

Figure 4. Schematic overview of the working mechanisms of epigenetic-based approaches for neuronal differentiation in vitro. A. The figure shows how somatic cells, pluripotent stem cells (PSCs) and neural precursor cells (NPCs) can be differentiated or reprogrammed into neurons. B. In each of these cells, histone and DNA modifications orchestrate transcriptional activity of pluripotency genes, self-renewal genes, neuronal genes and somatic genes, which define their cellular identity. While somatic genes are only active in somatic cells (in green), genes that regulate pluripotency and self-renewal are activated in PSCs (in green), whereas genes that regulate neuronal differentiation are repressed and poised (in red with green modifications) for activation upon further developmental cues. This activation and repression is inverted upon initiation of neural induction and differentiation, leading to the activation of neuronal genes (in green) and repression of PSC genes (in red). By using epigenetic editing and molecules that allow modifying the chromatin structure, activity of genes that redefine and reinforce cellular identity can be altered, offering control over neuronal fate determination in vitro by the natural dynamics of endogenous gene regulation. C. Aside from chromatin modifications, it has been proposed that the cellular microRNA (miRNA) milieu is unique in each cellular subtype and required to facilitate developmental transitions during neuronal differentiation. For PSC regulation, miRNAs such as miR371 and miR-302 are important, while miR-9/9* and miR-124 belong to brain-enriched miRNAs that are activated during neuronal specification. By using RNA interference (RNAi), miRNA profiles that redefine and reinforce cellular identity can be manipulated, leading to changes in transcriptional regulation that could aid neuronal differentiation and specification in vitro. 


\section{Induced GABAergic neurons}

In the first study that achieved to obtain iNs [138], some cells expressed markers of GABAergic differentiation [127]. However, whether enriched populations of induced GABAergic interneurons (iGNs) can be obtained has not been addressed until recently (See Table 2). Wasko [156] was the first to demonstrate that mouse fibroblasts could be directly reprogrammed to iGN-like cells using different pools of transcription factors, including Dlx1, Dlx2, Lbx1, Lhx1, Lhx2, Pax2, Pitx2 and Pft1a in combination with members of the BAM factors. The author states that different groups containing these transcription factors have demonstrated some capacity for the derivation of iGNs, although the most efficient factor combination remains to be determined. Additional experiments will be required to fully characterize the efficiency of the different transcription factor pools, as well as to assess the functional properties and maturity of the eventual derived iGNs. Aside from fibroblasts, iGNs neurons have also been derived by overexpressing the ventral telencephalic fate determinant DIx2 in murine astrocyte cultures [142]. Interestingly, they found that the overall efficiency of DIx2-mediated neuronal reprogramming towards iGNs is much lower compared to Ngn2-mediated reprogramming towards induced glutamatergic neurons, suggesting that cortical astrocytes possess a higher competence to respond to the dorsal telencephalic fate determinant [142]. In addition, five different factors have been identified, including Ascl1, Bf1, Dlx6, Lhx6 and Sox2, which were able to convert mouse and human fibroblast, as well as human iPSCs, into iGNs that possessed characteristics of telencephalic GABAergic interneurons [144]. Molecular profiling showed pronounced activation of forebrain-specific (epi)genetic markers, required for GABAergic fate specification [144]. Furthermore, the iGNs displayed progressively maturing firing patterns comparable to cortical GABAergic interneurons, formed functional synapses, and released GABA upon stimulation [144]. The iGNs also survived and matured upon engraftment into the mouse hippocampus and optogenetic stimulation demonstrated functional integration of the grafted iGNs into the host circuitry, triggering inhibition of host granule neuron activity [144]. This latter study also elegantly demonstrated how human PSCs can be harnessed to generate GABAergic neurons.

\section{Induced serotonergic neurons}

There have only been two studies published that were able to directly obtain induced serotonergic (i5HT) neurons by transcription factor-mediated reprogramming of somatic cells (See Table 2). In the first study [148], human fibroblasts could be directly converted to i5HT neurons by the ectopic expression of ASCL1, FEV, FOXA2, and LMX1B. The transdifferentiation was enhanced by p53 knockdown and appropriate culture conditions, including hypoxia [148]. Addition of the smallmolecule compounds dorsomorphin (DOR), SB431542, ROCK inhibitor Y-27632 
and the CDK4/6 inhibitor PD0332991 improved both the conversion efficiency and morphology of the obtained $\mathrm{i} 5 \mathrm{HT}$ neurons, whereas addition of brain derived neurotrophic factor (BDNF) and glial derived neurotrophic factor (GDNF) slightly improved the morphology but not the conversion efficiency [148]. The i5HT neurons expressed markers for mature serotonergic neurons, had calcium-dependent 5-HT release and selective 5-HT uptake, and exhibited spontaneous action potentials, as well as spontaneous excitatory postsynaptic currents [148]. Moreover, application of 5-HT significantly increased the firing rate of spontaneous action potentials [148]. In the second study, it was demonstrated that overexpression of the transcription factors FEV, GATA2, LMX1B and NKX2-2 in combination with ASCL1 and NGN2 directly and efficiently generated i5HT neurons from human fibroblasts [117]. The i5HT neurons showed increased expression of specific serotonergic genes known to be expressed in the raphe nuclei, displayed spontaneous action potentials, released $5-\mathrm{HT}$ in vitro and functionally responded to selective serotonin reuptake inhibitors (SSRIs) [117].

\section{Induced cholinergic motor neurons}

Transcription factor mediated reprogramming has also been used to derive cholinergic motor neurons (See Table 2). Son et al. [147] reported that the forced expression of the BAM factors, in combination with Hb9, Isl1, Lhx3 and Ngn2 was sufficient to convert mouse fibroblasts into induced motor neurons (iMNs). The iMNs were identified based on an HB9::GFP reporter and exhibited a morphology, gene expression signature, electrophysiological profile, synaptic functionality, in vivo engraftment capacity and sensitivity to degenerative stimuli, similar to ESC-derived cholinergic motor neurons [147]. By adding NEUROD1 to the 7 transcription factors they demonstrated that also human fibroblasts could be converted to iMNs [147]. Other studies have also coupled chemically defined systems with transcription factor-mediated reprogramming in PSCs in order to improve the efficiency and timing to obtain cholinergic motor neurons. For instance, adenoviral delivery of Isl1, Lhx3 and Ngn2 combined with exposure to RA and SHH signalling allowed rapid and efficient (> 55\%) acquisition of electrophysiological active human iMNs within 11 days [149]. In a separate study, the same set of transcription factors were used to sufficiently differentiate mouse ESCs to a cholinergic motor neuron identity [157]. Interestingly, replacement of Lhx3 by Phox2a led to specification of cranial, rather than spinal motor neurons, emphasizing the possibility to direct subtype specification. Phox2a and Phox $2 b$ have also been used to generate both visceral motor neurons and noradrenergic neurons from mouse ESCs in the presence of appropriate patterning molecules [145]. Culturing Phox $2 b$ expressing cells under the signalling influence of FGF8 and BMPs promoted the generation of enriched noradrenergic cultures, while culturing Phox2a or Phox $2 b$ expressing cells with 
FGF8 and SHH generated cholinergic motor neurons instead [145]. The authors demonstrated that the obtained neurons were suitable for drug testing in vitro and, therefore, harbour the potential for the discovery of therapeutic interventions. To conclude, these studies add up to the growing body of protocols, allowing to produce clinically relevant neuronal cells and demonstrate that combinations of distinct methods can work synergistically in obtaining the desired neuronal subtypes.

\section{Epigenetic-based approaches}

\section{Epigenetics in directed differentiation and cellular reprogramming}

Even though every cell in the human brain shares an identical genotype, it consists of many neuronal subtypes with distinct, yet stable, profiles of gene expression patterns. As addressed above, this diverse repertoire of neurons is produced by extrinsic patterning cues and lineage-specific transcription factors that define and reinforce these neuronal subtype-specific expression patterns. The neuronal phenotypes are further stabilized by the epigenetic machinery that maintains their genetic profile over a lifetime [138, 158]. Epigenetics can be defined as stable and heritable modifications on the chromatin that occur without changes in the underlying DNA sequence [159]. It is generally accepted that the epigenetic machinery includes multiple levels of transcriptional control by (re)organizing the chromatin structure and architecture [160, 161]. Histone modifications and DNA methylation are the most predominant examples of chromatin modifications that have been studied over the last years. Both of these modifications play an important role in neuronal cell fate determination and differentiation [24]. In continuously self-renewing ESCs, genes that regulate pluripotency are activated, whereas genes that regulate neuronal differentiation are repressed in a stable and heritable manner over many cell divisions [162-165]. Moreover, this activation and repression needs to be inverted upon initiation of neural induction and differentiation. In addition to these chromatin modifications, noncoding RNAs are other important modulators that regulate gene expression patterns at the posttranscriptional level [161]. A growing body of evidence is revealing that these also represent strong mediators of neuronal cell fate determination [166, 167]. Examples of noncoding RNAs include small interfering RNAs (siRNAs), small modulatory RNAs, ribosomal RNAs, transfer RNAs, natural antisense transcripts, enhancer RNAs and microRNAs (miRNAs), but many other subclasses have been described $[161,168,169]$. Based on the key role of the epigenetic machinery in assigning neuronal fate and identity, it has currently become evident that the underlying mechanisms of both directed differentiation and direct cellular reprogramming encompass epigenetic phenomena $[23,170]$. A significant degree of transcriptional regulation takes place, where epigenetic mechanisms communicate with each other 
in collaboration with the extrinsic patterning cues and transcription factors to guide neuronal cell fate conversions. The profound epigenome remodelling processes in PSCs and somatic cells eventually lead to the acquisition and stabilization of neuronal subtypespecific gene expression profiles, which reinforce their cellular phenotypes. Although the epigenetic machinery has been extensively studied in ESC differentiation along the neural lineage, please refer to Hirabayashi and Gotoh [171], little is known about the extent in which these epigenetic mechanisms are identical in iPSC differentiation. The field is still in its infancy and we are just starting to understand similarities and differences in epigenetic and transcriptional states between iPSCs and ESCs [172-174]. In addition, the underlying epigenetic mechanisms of direct reprogramming in somatic cells are also still incompletely understood and the interactions between transcription factors and the chromatin architecture are currently under investigation [23, 175]. Nevertheless, considering the crucial role of chromatin modifications and transcriptional regulations by non-coding RNAs in modulating neuronal cell fate and identity, (re)programming and interfering with the epigenetic machinery offers an alternative approach for directing neuronal fates in vitro (See Fig. 4, Tables 3 and S3).

\section{Chemical compounds targeting chromatin-remodelling proteins}

First proof-of-concept on epigenetic-based approaches for neuronal differentiation in vitro came from experiments where beneficial effects on direct cellular reprogramming by chemical compounds that target chromatin-remodelling proteins were found (See Table 3). Genome-wide profiling of PSCs and their differentiated progeny has suggested a global, progressive transition from euchromatin to heterochromatin at various loci during differentiation [176]. The epigenetic state of a differentiated cell is, therefore, considered to be more condensed and has significantly less dynamic exchange with transcriptional regulators [176]. However, by targeting remodelling proteins, heterochromatin enriched regions can be altered and recruitment of transcriptional activators can be orchestrated to DNA sites that were previously inaccessible, allowing them to induce dramatic cell fate changes. As a consequence, epigenetic research in PSC differentiation and somatic cell reprogramming has supplied a plethora of potential drug targets to improve neuronal cell fate conversions. For instance, NPCs were induced from mouse fibroblasts by ectopic overexpression of Pax6 and Bf1 [136]. Treatment with a combination of small molecules that inhibit histone deacetylases (HDACs), $\mathrm{H} 3 \mathrm{~K} 27$ methyltransferases, and H3K4me2 demethylases accelerated the direct conversion of fibroblasts into NPCs up to ten times [136]. Remarkably, simultaneous inhibition of BMP- and TGF- $\beta$-signalling almost doubled the frequency of NPCs, again demonstrating that combinations of different approaches can significantly enhance the derivation of the desired cell types in vitro. HDAC inhibitors and 
histone demethylase inhibitors coupled with other appropriate chemical patterning cues have also shown to turn mouse fibroblasts and astrocytes, as well as human urinary cells, into NPCs or neurons under physiological hypoxia conditions and without the need of additional transgene overexpression [177, 178]. Analysis of global gene expression patterns revealed a high degree of similarity between the induced NPCs and control NPCs [178], whereas the neurons induced from cultured astrocytes were electrophysiological active and expressed various subtype specific markers for dopaminergic neurons, GABAergic neurons, glutamatergic neurons and cholinergic motor neurons [177]. The delivery of small molecules or systematic administration of drug cocktails enabling astrocytic-to-neuronal conversions bear the potential for direct induction of desirable cells from resident astrocytes in situ, while bypassing possible adverse effects of genome integrating constructs. Taken together, drugs targeting the chromatin can improve reprogramming efficiency and might function as useful adjuvants in currently used reprogramming protocols, thereby providing a possible alternative strategy to produce patient-specific neuronal cells.

\section{Epigenetic editing}

Epigenetic editing systems [27, 29] offer an alternative tool to supplement, or in some cases even replace components of, current widely used directed differentiation and cellular reprogramming protocols [32]. Making locus-specific alterations to the epigenetic code allows to $(r e)$ shape the mechanistic relationships among chromatin state, gene regulation, and cellular phenotype by the natural dynamics of gene expression [29]. For this reason, these epigenetic editing systems allow probing of signatures responsible for cellular identity and provide intelligent control to direct neuronal cell fates in PSCs and somatic cells [179-182]. DNA-targeting platforms based on the initially established zinc finger proteins (ZFPs), transcription activator-like effector nucleases (TALENs) and the clustered, regularly interspaced, short palindromic repeats (CRISPR)-Cas9 systems, have allowed the recruitment of transcriptional modulators and epigenome-modifying factors to any genomic locus $[27,29,183]$. Virtually any DNA sequence can be targeted with these customizable synthetic epigenetic tools [29]. The direct fusion of transcriptional effector domains to designed DNA-targeting domains can induce transcriptional activation or repression of endogenous key-lineage-determinant genes [29]. Transcriptional effector domains include epigenetic effectors that directly catalyse covalent modifications to DNA or histones, or that recruit other histone modifying enzymes, as well as interfere with chromatin-binding proteins. For instance, epigenetic effectors that directly catalyse covalent modifications to DNA, such as DNA methyltransferases (DNMTs) or ten-eleven translocation (TET) enzymes, can methylate or demethylate $\mathrm{CpGs}$ at non-neuronal and neuronal target 
Table 3. Epigenetic-based neuronal conversion approaches in vitro per neuronal phenotype.

Chemical compounds targeting chromatin remodelling proteins

\begin{tabular}{|c|c|c|c|c|}
\hline Phenotypes & Species & Starting cell types & Culture methods & Epigenetic factors \\
\hline \multirow[t]{2}{*}{ NPCs } & Mouse & $\begin{array}{l}\text { Fibroblasts (Trp53+/+, Trp53-/-, Sox1+/+, } \\
\text { Sox1EGFP/+, Tau+/+ and TauEGFP/+) }\end{array}$ & Monolayer & BIX-01294, t2PCPA and VPA \\
\hline & Mouse and human & Mouse fibroblasts and human urinary cells & Monolayer and neurospheres & $\mathrm{NaB}, \mathrm{TSA}$ and VPA \\
\hline
\end{tabular}

$\begin{array}{lll}\text { Dopaminergic-, } & \text { Mouse Astrocytes (GFAP::GFP and Neurod1::GFP) Monolayer } \\ \text { GABAergic-, glutamatergic- and } & \text { VPA }\end{array}$

GABAergic-, glutamatergic- and

cholinergic motor neurons

\section{Epigenetic editing}

\begin{tabular}{|c|c|c|c|c|}
\hline Phenotypes & Species & Starting cell types & Culture methods & Epigenetic factors \\
\hline \multirow[t]{2}{*}{ Neurons } & Mouse & Fibroblasts (SYN1::RFP) & Monolayer & $\begin{array}{l}\text { VP64-dCas9-VP64 } \\
\text { gRNAs: Brn2, Ascl1, and Myt1I }\end{array}$ \\
\hline & Human & iPSCs & Monolayer & $\begin{array}{l}\text { VP64-p65-Rta-dCas9 } \\
\text { gRNAs: NGN2 and NEUROD1 }\end{array}$ \\
\hline Neurons & Mouse & NPCs & Monolayer and neurospheres & siRNA-REST \\
\hline \multirow[t]{3}{*}{ Dopaminergic neurons } & Mouse & $\operatorname{ESCs}(T H:: G F P)$ & $\begin{array}{l}\text { EB and } \\
\text { co-culture (PA6) }\end{array}$ & miR-132-ASOs \\
\hline & & ESCs & $\mathrm{EB}$ & miR-133b-ASOs \\
\hline & Human & NPCs & Monolayer & $\begin{array}{l}\text { miR-124-inhibitor, miR-125b, miR-125b-mimic, } \\
\text { miR-181a-mimic, miR-181a*-inhibitor and miR-181a/a* }\end{array}$ \\
\hline GABAergic neurons & Human & Fibroblasts (SYN1::EGFP) & Monolayer & $\begin{array}{l}\text { miR-9/9*, } \\
\text { miR-124 } \\
(B c l-x L) \text { and VPA }\end{array}$ \\
\hline
\end{tabular}

\begin{tabular}{|c|c|c|c|c|}
\hline $\begin{array}{l}\text { Glutamatergic- and GABAergic } \\
\text { neurons }\end{array}$ & Human & Fibroblasts & Monolayer & $\begin{array}{l}\text { miR- } 9 / 9^{*} \text {, } \\
\text { miR-124 and VPA }\end{array}$ \\
\hline
\end{tabular}

Glutamatergic- and GABAergic $\quad$ Human
neurons

$\begin{array}{llll}\text { Cholinergic motor neurons } \quad \text { Human } & \text { Fibroblasts } & \text { Monolayer } & \text { miR-124 } \\ & & (B c l-x L) \text { and VPA }\end{array}$

Neuronal phenotypes, species, starting cell types, culture methods, epigenetic factors, transcription factors, chemical driving factors and representative phenotypic markers that have been used to assess the differentiation efficiency and culture homogeneity are broadly summarized. + indicates the percentage of cells in the population that stained positive for a certain marker. Abbreviations: ASCL1, achaete-scute homolog 1 (MASH1/HASH1); ASOs, anti-sense oligonucleotides; Bcl-xL, b-cell lymphoma-extra large; BF1, brain factor 1/forkhead box protein G1 (FOXG1); BIX-01294, histone-lysine methyltransferase inhibitor; BRN2, brain-specific homeobox/POU domain protein 2 (POU3F2); CHAT, choline o-acetyltransferase; CHIR99021, GSK3ß inhibitor; CTIP2, b-cell CLL/lymphoma 11b (BCL11B)/ COUP-TF-interacting protein 2 (COUP-TFII); DCX, doublecortin; DLX1, distal-less homeobox 1; DLX2, distal-less homeobox 2; DOX, doxycycline; EB, embryoid body; EGFP, enhanced green fluorescent protein; ESCs, embryonic stem cells; FGF2 fibroblast growth factor 2/basic fibroblast growth factor (bFGF); FGF8, lithium carbonate; LiCl, lithiumchloride; LIF, leukemia inhibitory factor; MAP2, microtubule-associated protein 2; MYT1L, myelin transcription factor 1 like; NaB, sodium butyrate; NES, nestin; NEUN, neuronal nuclei antigen; NEUROD1, neurogenic differentiation 1; NEUROD2, 


\begin{tabular}{|c|c|c|c|}
\hline Transcription factors & Chemical driving factors & Representative phenotypic markers (\% cells) & References \\
\hline Pax6 and $B f 1$ & DOX, LDN193189 and SB431542 & $30 \%$ Sox1-EGFP+ & Raciti et al. (2013) \\
\hline \multirow[t]{2}{*}{ None } & \multirow{2}{*}{$\begin{array}{l}\text { CHIR99021, LIF, Li2CO3, LiCl, } \\
\text { Repsox, SB431542 and Tranilast }\end{array}$} & Mouse & \multirow[t]{2}{*}{ Cheng et al. (2015b) } \\
\hline & & $\begin{array}{l}<96 \% \text { NES+ } \\
<96 \% \text { SOX2+ } \\
<96 \% \text { PAX6+ } \\
<93 \% \text { NES+/SOX2+ } \\
<93 \% \text { NES+/PAX6+ }\end{array}$ & \\
\hline None & $\begin{array}{l}\text { CHIR99021, FGF2, FGF8, Repsox, } \\
\text { SHH and Tranilast }\end{array}$ & $\begin{array}{l}<13 \% \text { DCX+ } \\
<15 \% \text { NEUN+ } \\
\pm 5 \% \text { GAD } 67+ \\
\pm 4 \% \text { CHAT+ } \\
\pm 3 \% \text { TH+ } \\
\pm 6 \% \text { VGLUT } 1+\end{array}$ & Cheng et al. (2015a) \\
\hline
\end{tabular}

\begin{tabular}{|c|c|c|c|}
\hline Transcription factors & Chemical driving factors & Representative phenotypic markers (\% cells) & References \\
\hline None & CHIR99021, LDN193189 and SB431542 & $\begin{array}{l} \pm 4 \% \text { TUJ1+ } \\
\pm 75 \% \text { MAP2+/TUJ1+ }\end{array}$ & Black et al. (2016) \\
\hline None & DOX & $7 \%$ TUJ1+ & Chavez et al. (2015) \\
\hline None & FGF2 and RA & $\begin{array}{l}<80 \% \text { TUJ1+ } \\
<80 \% \text { MAP2+ }\end{array}$ & Low et al. (2012) \\
\hline None & None & $\begin{array}{l}>25 \% \text { TH+/MAP2+ } \\
70 \% \text { TH+/TH-GFP+ }\end{array}$ & Yang et al. (2012) \\
\hline None & FGF2 & $20 \%$ TH+/PITX3+ & Kim et al. (2007) \\
\hline None & $\begin{array}{l}\text { FGF8b and } \\
\text { SAG }\end{array}$ & $\begin{array}{l}<30 \% \text { TUJ1+ } \\
<15 \% \text { TH+ }\end{array}$ & Stappert et al. (2013) \\
\hline \multirow[t]{4}{*}{ CTIP2, DLX1, DLX2, and MYT1L } & DOX and RA & Postnatal fibroblasts & Victor et al. (2014) \\
\hline & & $\begin{array}{l}87 \% \text { TUJ1+ } \\
90 \% \text { MAP2+ } \\
72.3 \% \text { GABA+/MAP2+ } \\
80 \% \text { BF1+/MAP2+ } \\
60 \% \text { DLX2+/MAP2+ }\end{array}$ & \\
\hline & & Adult fibroblasts & \\
\hline & & $\begin{array}{l}82 \% \text { MAP2+ } \\
86 \% \text { GABA+/MAP2+ }\end{array}$ & \\
\hline ASCL1, MYT1L and NEUROD2 & DOX and FGF2 & $\begin{array}{l}80 \% \text { MAP2+ } \\
\pm 24 \% \text { TBR1+/MAP2+/TUJ1+ } \\
\pm 13 \% \text { CTIP2+/MAP2+/TUJ1+ } \\
\pm 38 \% \text { VGLUT1+/MAP2+/TUJ1+ } \\
\pm 30 \% \text { GAD67+/MAP2+/TUJ1+ } \\
\pm 38 \% \text { DLX1+/MAP2+/TUJ1+ }\end{array}$ & Yoo et al. (2011) \\
\hline \multirow[t]{4}{*}{$B R N 2$ and $M Y T 1 L$} & Cumate, FGF2, NOG and DOX & Postnatal fibroblasts & Ambasudhan et al. (2011) \\
\hline & & $\begin{array}{l}55 \% \text { MAP2+/RFP+ } \\
46 \% \text { NEUN+/RFP+ } \\
8 \% \text { GABA+/RFP+ } \\
12 \% \text { VGAT+/RFP+ }\end{array}$ & \\
\hline & & Adult fibroblasts & \\
\hline & & $28 \%$ RFP+NGLUT1+ & \\
\hline ISL1 and $L H X 3$ & DOX and RA & $\begin{array}{l} \pm 80 \% \text { TUJ1+ } \\
\pm 80 \% \text { MAP2 }+ \\
\pm 80 \% \text { CHAT+/TUJ1+ }\end{array}$ & Abernathy et al. (2017) \\
\hline
\end{tabular}

neuronal differentiation 2; NGN2, neurogenin 2; NPCs, neural precursor cells; PA6, stromal cell line derived from newborn calvaria tissue of the C57BL/6 mice; PAX6, paired box 6; PITX3, paired-like homeodomain 3; RA, retinoic acid; REST, RE1-silencing transcription factor; RFP, red fluorescent protein; SAG, smoothened agonist; SB431542, transforming growth factor beta inhibitor; SHH, sonic hedgehog; siRNAs, small interfering RNAs; Sox1, SRY box 1; SOX2, SRY box 2; SYN1, synapsin 1; t2PCPA, trans-2phenyl-cyclopropylamine hydrochloride; Tau, microtubule-associated protein tau (MAPT); TBR1, T-box brain 1; TH, tyrosine hydroxylase; Trp53, tumor protein p53; TSA, trichostatin A; TUJ1, neuron-specific class III beta-tubulin (TUBB3); VGAT, vesicular GABA transporter; VGLUT1, vesicular glutamate transporter 1; VP64-dCas9-VP64, dCas9 with N-terminal and C-terminal VP64 transactivation domains; VP64-p65-Rta-dCas9, dCas9-based transcriptional activator containing VP64, p65 and Rta; VPA, valproic acid. 
promoters, leading to transcriptional repression or induction, respectively [28, 32]. Other effector domains such as VP64 can recruit histone remodelling factors, leading to increased chromatin accessibility and to the deposition of activating histone modifications at desired neuronal loci $[184,185]$. Alternatively, localization of DNA-targeting domains without an epigenetic effector to promoter regions or regions downstream of the transcription start sites can silence nonneuronal gene expression by steric hindrance of lineage-specific transcription factor binding and RNA polymerase elongation [32, 180]. Thus, custom epigenetic and transcriptional regulation by epigenetic editing-based approaches offer refined control over cell fate decisions, providing an invaluable tool for applications such as directed differentiation and cellular reprogramming along the neural lineage (See Table 3).

Several pioneer studies utilizing these epigenetic editing systems have shown successful and precise deposition or removal of different chromatin modifications to induce directed differentiation and cellular reprogramming for multiple cell types, including iPSCs, myocytes and neurons. Gao et al. [186] used TALEbased transactivators targeting distal enhancers of Oct4 in concert with Sox2, Klf4 and Myc transgene overexpression to generate mouse iPSCs. More recently, a protocol for the direct conversion of mouse fibroblasts to skeletal myocytes using a dCas9-based transactivator targeting the endogenous Myod1 gene has been developed [184]. Other groups have also applied dCas9-based transcriptional regulation to direct the differentiation of human PSCs [180, 187]. In an elegant study, human iPSCs were derived from human skin fibroblast by replacing OCT4 overexpression with dCas9-mediated activation of the endogenous promoter [187]. The authors demonstrated that directed endodermal differentiation of the iPSCs could be achieved by targeting proximal promoters of endodermal and pancreatic keyregulatory transcription factors, including FOXA2, SOX17, GATA4, PDX1, and NKX6-1 [187]. Directed neuronal reprogramming through multiplex endogenous gene activation using an engineered VP64-dCas9-VP64-based transcriptional activator has also been achieved [179]. Induced activation of the BAM factors successfully converted mouse fibroblasts to $\mathrm{iN}$ cells and their expression sustained in high levels during later stages of reprogramming despite the transient delivery of the guide RNAs (gRNAs) [179]. In a separate study, it was demonstrated that rapid and robust neuronal differentiation of human iPSCs could be achieved by targeting NGN2 and NEUROD1 with a VP64-p65-Rta-dCas9-mediated transcriptional activator [180]. Although many of the aforementioned reports include targeted activation of a single gene in combination of concurrent overexpression of multiple transcription factors, these latter examples also demonstrate that multiplex activation with a collection of gRNAs against a set of genes can be used to direct somatic cell reprogramming. 
Previous studies have demonstrated that targeted epigenetic editing of the regulatory elements controlling expression of lineage-specific transcription factors is sufficient for direct conversion between cell types, emphasizing the feasibility and potential advantages of using these synthetic epigenetic systems to direct neuronal cell fate of PSCs and somatic cells in vitro. Moreover, epigenetic editing in isolation has incredible promise as a platform for disease modelling both in vitro, as well as in vivo, whereas using it for transdifferentiation within the native physiological niche of the human brain might provide an alternative strategy to achieve cell fate conversions for applications in regenerative medicine $[28,188,189]$. Several labs have recently pioneered in vivo reprogramming in the brain and spinal cord by converting endogenous glial cells [190192] and NPCs $[193,194]$ into functional neurons. For a more in-depth review on more surpassed studies, please refer to Li and Chen [195] and Srivastava and DeWitt [196]. Epigenetic editing systems applied in a safe and efficient manner that target similar lineage-specific transcription factors, might allow to induce any desirable neuronal subtype in vivo and could eliminate undesired issues in cell transplantations that may arise due to precedent in vitro cultures. To conclude, ground-breaking advances in this field are beginning to yield novel opportunities in the context of inducing neuronal phenotypes and bear excessive potential for many different applications in fundamental research and biomedicine.

\section{RNA interference using miRNAs}

On the one hand, the acquisition of distinct histone and DNA modifications at neuronal genes and non-neuronal genes plays a role in determining neuronal identity. On the other hand, neuronal identity is also determined by synergistic actions of extrinsic cues and the combined expression of transcription factors that are modulated by transcriptional regulators, including non-coding RNAs. Among these, miRNAs have been most extensively studied in relation to cellular identity and although most of the other subclasses are also anticipated to play important roles in regulating neuronal cell fate determination, the exact contribution of many remains elusive. Currently, it is accepted that miRNAs promote the transition from ESC self-renewal to differentiation by either directly suppressing the self-renewal state or by stabilizing the differentiated state [197]. In addition, multiple miRNAs target components or modulators of neural developmental signalling pathways, such as BMP and TGF- $\beta$ signalling, and have been identified to either positively or negatively affect entry along the neural lineage [198]. In mature neurons, it has been proposed that the cellular miRNA milieu might even be unique in each subtype and required to facilitate developmental transitions during differentiation [199]. Some miRNAs even exhibit region-specific expression patterns in the brain, suggesting that neuronal subtypes residing in these regions may express different miRNA profiles [198]. This is exemplified by a study of $\mathrm{He}$ et al. [200], in which they demonstrated substantial differences between the miRNA repertoire expressed in glutamatergic neurons and GABAergic interneurons that co-expressed 
either PV or SST. Specifically, miR-133b and miR-187 were found to be expressed higher in GABAergic interneurons as compared to glutamatergic neurons, where miR-133b was more abundant in PV expressing and miR-187 in SST expressing GABAergic interneurons [200].

Based on the emerging role of miRNAs during neural induction, neuronal differentiation and neuronal subtype specification, techniques such as RNAi using miRNAs holds great promise as an alternative tool to direct neuronal cell fate in vitro (See Table 3) $[30,31]$. RNAi is a post-transcriptional gene silencing technique that has therapeutic opportunities for the treatment of various human disorders and has extensively been employed in translational studies to address fundamental biological questions[30]. The potential of RNAi lies in its capacity to virtually target any RNA molecule of interest, which allows fine-tuning of expression of key-determinant factors in neuronal fate determination. Approaches to downregulate endogenous miRNA expression to influence gene expression opposite of RNAi are also available and could for example be achieved by using anti-sense oligonucleotides (ASOs) (See Table 3) [201]. The temporal control of miRNA regulation might facilitate the induction of neuronal subtype-specific transcriptional networks and aids in recapitulating the natural dynamics of transcriptional regulation during neuronal cell fate commitment. Each miRNA has multiple molecular targets that might play essential roles in the derivation of specific neuronal subtypes and modulating a single miRNA could, therefore, guide entire neural developmental processes. Moreover, successful delivery of miRNAs could be achieved in many different ways depending on the needs of the experiments, as each method has different transfection efficiencies and transgene expression duration. Representative methods that allow expression of a miRNA construct include non-viral delivery systems such as lipid-based transfection, electroporation or the use of microvesicles, or viral delivery systems such as lentiviruses and adenoassociated viruses [202]. The potential of miRNAs to complement current practiced directed neuronal differentiation protocols from PSCs was first demonstrated by Kim et al. [203]. Interestingly, in this study they reported an unexpected negative impact of miR-133b on the generation of dopaminergic neurons from mouse ESCs. MiR-133b was found to be enriched in the human midbrain, while overexpression in this study impaired the generation of TH-positive cells [203]. Inhibition of miR-133b on the other hand, resulted in an increased dopaminergic differentiation. The authors speculated that miR-133b regulates the maturation and function of midbrain dopaminergic neurons within a negative feedback circuit that includes the dopaminergic transcription factor Pitx3. A similar negative impact on the differentiation of dopaminergic neurons from mouse ESCs has been reported in a separate study [204]. Inhibition of miR132 promoted the differentiation of dopaminergic neurons, while ectopic expression of miR- 132 decreased the derivation of TH-positive cells without affecting the total number of neuronal cells. Through a bioinformatics assay they identified Nurr1 as a 
potential molecular target of miR-132, which also represents a key transcription factor of dopaminergic neuron specification. Stappert et al. [31] showed that miR-125b and miR-181a specifically promote the generation of neurons of dopaminergic fate from NPCs derived from human ESCs, whereas miR-181a* inhibits the development of this neurotransmitter subtype. By using a set of miRNAmimics and-inhibitors, they also demonstrated that inhibition of miRNA-124 enhances the development of dopaminergic neurons [31]. Although other studies on additional neuronal subtypes have not been published, these studies demonstrated that time-controlled modulation of specific miRNA activities can contribute to the derivation of defined neuronal cells in vitro.

Recent developments in direct somatic cell reprogramming also highlighted the potential of miRNAs as mediators for transdifferentiation along the neural lineage. The convergence of transcriptional control by miRNAs that leads into direct cellular transitions is exemplified by miR-9/9* and miR-124, which both belong to a set of brain-enriched miRNAs that are activated upon initiation of neurogenesis [205]. It has been shown that overexpression of miR-124 along with BRN2 and MYT1L is able to reprogram human fibroblasts into functional neurons in the absence of other cell types [206]. These iNs exhibited typical neuronal morphology, appropriate electrophysiological properties and were able to form functional synapses between each other [206]. In a separate study, it was reported that the expression of miR-9/9* and miR-124 in human fibroblasts induced their direct conversion into neurons, a process which was enhanced by the addition of several transcription factors, including ASCL1, MYT1L and NEUROD2 [207]. Importantly, they found that the expression of these transcription factors alone without the miRNAs was inefficient to induce a neuronal phenotype, suggesting that this miRNA-induced neuronal state is indulgent to subtype-specific transcription factors that can initiate and advance differentiation towards mature neuronal identities [205]. Co-expression of miR-9/9* and miR-124 with transcription factors enriched in the developing striatum, including CTIP2, DLX1, DLX2, and MYT1L, guided the conversion of human fibroblasts into enriched populations of GABAergic neurons analogous to striatal medium spiny neurons [182].

Synergism between miR-9/9*, miR-124 and two other neuronal subtype-specific transcription factors, i.e. ISL1 and LHX3, has also been shown to be able to induce a highly homogeneous population of spinal cord motor neurons in adult human fibroblasts [205]. Longitudinal analyses of the transcriptome, genome-wide DNAmethylation, and chromatin accessibilities in the latter study revealed that miR-9/9* and miR-124 trigger reconfiguration of the epigenome, including activation of a panneuronal program and the reconfiguration of chromatin accessibilities [205]. Neurons solely induced by miR-9/9*- and miR-124 demonstrated to be functionally excitable and uncommitted toward specific subtypes, but possess open chromatin structures 
at neuronal subtype-specific loci that can be activated upon further instructions [205]. The authors, therefore, also suggested that expression of bot miRNAs in somatic cells initiates gradual but active changes in the activities of multiple chromatin modifiers while simultaneously repressing anti-neuronal genes and activating neuronal genes, resulting in the binary cell fate switch. The fact that pre-existing neuronal loci within the heterochromatic regions opened-up in response to miR-9/9* and miR-124, suggests that miRNA-mediated reprogramming could indeed stem from their ability to induce remodelling of the epigenome. Taken together, these findings demonstrate that miR9/9* and miR-124 control the neurogenic potential of somatic cells and provide a platform for the foundation of subtype-specific neuronal conversions of human cells. For a further in depth description of all other brain-enriched miRNAs, their target genes and exact functions, please refer to excellent reviews by Åkerblom and Jakobsson [208], Coolen et al. [209], Meza-Sosa et al. [210] and Stappert et al. [198].

To conclude, miRNA-mediated neuronal differentiation and transdifferentiation have enlarged our current toolkit for directing neuronal cell fate in vitro and have the potential to widen our understanding on the transcriptional regulations in cell fate decisions. Further insights could in the end be exploited to develop new protocols in order to obtain enriched populations of the complete repertoire of neurons found in the human brain.

\section{Discussion and future directions}

The establishment of efficient stepwise protocols to obtain functional neurons in vitro is highly essential for the study of human brain functions, as well as disease modelling, drugs discovery and regenerative medicine. In this review, we have highlighted the advances that have been made over the last two decades in obtaining neuronal cells from PSCs and somatic cells. Insights from basic research and developmental biology have guided the design of current strategies and numerous protocols for glutamatergic, dopaminergic, GABAergic, serotonergic, and cholinergic/motor neurons have become available. The use of chemically defined systems and ectopic overexpression of key lineage-specific transcription factors have been first-choice to direct neuronal fates in vitro. In the chemically defined systems, PSCs have been exposed to a variety of cocktails containing patterning cues and small molecules that induce differentiation towards early NPCs and eventually specific mature neuronal subtypes. Classical experiments such as nuclear transfer or cell fusion on the other hand, have demonstrated that differentiated cells are not irreversibly committed to their fate. Very recent work has built on these conclusions and discovered that ectopic overexpression of defined transcription factors can directly generate iNs from distinct somatic cell types, as well as from PSCs. Other groups have combined both approaches and have shown successful derivation of neuronal populations and increased protocol efficiencies in both PSCs and somatic cells. 
Scientists have also uncovered the existence of intrinsic mechanisms that influence the responsiveness to patterning cues and transcription factors. As we have outlined above, recent work has demonstrated a significant contribution of the epigenetic machinery to assigning neuronal fate and identity. From cancer studies, it is also becoming apparent that shifts in epigenetic signatures underlie phenotypic changes, and can induce stem cell-like properties in cancer cells due to transcriptional reprogramming [211]. Several pioneering studies have now used this knowledge in combination with the current growing availability of epigenetic editing systems and RNAi, enabling the modification of epigenetic marks at key-determinant loci that allow to direct neuronal fates in vitro. Additionally, lists of potential drugs and drug targets such as histone-modifying enzymes have been suggested to improve neuronal conversions. Such approaches on their own or in combination with others could lead to an accelerated application of the obtained neuronal populations. It is therefore, anticipated that epigenetic editing systems and RNAi will be increasingly involved in PSCdifferentiation and cellular reprogramming in the near future. These epigenomic editing tools even have the potential to become a golden standard for probing interactions among specific chromatin modifications, transcriptional programmes and cellular phenotypes. However, care should be taken in terms of advantages and disadvantages that come together with each of the aforementioned methods. For instance, off-target effects with the use of epigenetic-based strategies are common and should be reduced at all times to prevent experimental bias and undesirable outcomes, especially when one would consider the use of these cells in regenerative medicine. Such systems in combination with genome-integrating techniques can affect the genome in a way that it might lead to adverse changes in the biology of the cell, including changes in its differentiation potential. Genomeintegrating constructs are randomly incorporated into the host genome and the copy number of the exogenous DNA per cell may vary to great extent, since there are often no specific genetic elements or no apparent logic for their integration [212]. Furthermore, integration can occur into various chromatin-regulatory elements and interfere with gene-coding sequences, possibly affecting endogenous transcriptional regulation and three-dimensional chromatin structures. Finally, there is a possibility that transgenes maintain their activity or reactivate in the progeny of the initial targeted cell type. Nevertheless, studies have already shown to circumvent the issue with genomic-integrating techniques by the use of removable constructs, non-integrating systems such as adeno-associated vectors, transfections of mRNAs, transduction of reprogramming proteins and the use of small molecule compounds. Furthermore, promoters controlled by chemical compounds that allow to regulate transgene transcription, such as doxycycline (DOX) for example (See Tables 2 and 3), might provide an alternative to prevent continuously expressed transgenes and, thus, allows better temporal control of transgene activation. 
Despite the achievements in directing neuronal cell fate in vitro, several other challenges also need to be addressed before their full potential in fundamental research and biomedicine can be utilized. Different cultivation protocols for each neuronal subtype currently exist and the majority of these protocols result in heterogeneous neuronal populations with remarkable differences in efficiency. Furthermore, we are currently also unable to enrich for the full repertoire of neurons found in the human brain, especially when considering specific anatomical subtypes within neurotransmitter classes. For these reasons, the refinement and the search for alternative approaches that will allow us to obtain all known neuronal subtypes found in the human brain remains an ongoing demand. Although multicellular cultures will be pivotal for brain-related studies, optimizing the used parameters and devising strategies to enrich for specific neuronal subtypes will also be essential in order to obtain pure populations of neurons, which will allow mechanistic studies and clinical applications in which confounding effects from other cell types can be kept to a minimum. In this respect, one could think of combining different protocols or adding additional factors to existing protocols that may act synergistically in mimicking the complete molecular processes that (re)assign cell fates along the neural lineage. As has been shown in many available approaches, every single driving factor fulfils a crucial role as a part of a bigger network and absence of a single component can completely redirect the terminal differentiation, as well as induce incomplete differentiation or reprogramming processes, leading to the production of immature cells that may not fully recapitulate bona fide neurons. By combining directed differentiation with transcriptional reprogramming and/or epigenetic-based approaches, one might be able to control these cellular conversions in a robust way, which could, in turn, also lead to a greater diversity of neuronal subtypes to be specified in vitro. Another option to enhance culture purity could be achieved by finding more effective ways to isolate the desired cell types in different developmental stages. The use of specific cellular markers or expressing constructs combined with cell-sorting techniques, such as fluorescence-activated cell sorting (FACS) and magnetic-activated cell sorting (MACS) might provide suitable solutions (See Tables S1-3).

The choice of the initial tissue and cell type for generating the neuronal populations also needs considerable attention and could significantly affect the efficiency of a neuronal differentiation protocol. For instance, it is currently recognized that there are epigenetic differences between different PSC lines that can induce lineage differentiation bias [213]. This is exemplified in the study by Kim et al. [74], where they observed that the majority of neurons generated from one iPSC line were glutamatergic, whereas populations generated from another iPSC line mainly consisted of GABAergic neurons, when exposed to the same chemical culture conditions. Scientists have committed to the challenge to find markers that will allow them to predict this bias. The presence of such a marker could reveal which cell line 
has the highest neuronal differentiation capacity, leading to an increased neuronal conversion efficiency later on. It is noted that the origin-dependent epigenetic and transcriptional patterns of the pluripotent state can render iPSC lines with different neuronal differentiation potential. Cell lines that harbour epigenetic signatures, which were maintained and are characteristic of the somatic tissue of origin, have been shown to favour differentiation along lineages related to the donor cell, while restricting alternative cell fates [214]. Furthermore, it has been show that expression levels of other transcriptional regulators in iPSCs, such as miR-371-3, can predict neuronal differentiation propensities [215]. Aside from presenting the concept of epigenetic memory that may influence efforts in directed differentiation in iPSCs, this also emphasizes the role of epigenetic mechanisms in neuronal differentiation and addresses the complexity of the neuronal fate determination that needs the warrant for more comprehensive comparisons between different PSC lines.

Another debate in relation to starting cell choice, but based on direct reprogramming, arises from the initial assumption that developmentally related cells convey a higher conversion efficiency, as compared to cells that originate from distinct germ layers [216]. Indeed, previous studies have shown that cells derived from the same lineage, such as astrocytes and neurons, can be converted with minimal sets of transcription factors, whereas cells of nonectodermal origin require more than one factor or additional chemical stimulation [151]. Even though this is not always the case, as conversion of one neuronal subtype into another is rather difficult and has been achieved only in immature cells $[151,217]$, lineage boundaries established during cellular differentiation and specification might be overcome, depending on the potency of the factors that are employed [216]. However, the questions to what extent direct reprogramming recapitulates the natural dynamics of neuronal differentiation and whether the developmental origin and, more specifically, the epigenetic memory of the starting cell type is negligible when using the proper reprogramming factors remain unanswered. The underlying principle of direct reprogramming is based on the expression of key lineage-specific transcription factors that are essential during development, but their action during direct reprogramming can be rather different, since these factors are operating in a completely different context [216]. Further studies on how factors function in various reprogramming environments may bring new insight that can lead into the establishment of more robust neuronal differentiation protocols in various somatic cell subtypes, as well as in PSCs. Additionally, detailed characterization of the cellular and molecular characteristics involved in guiding PSC-differentiation and somatic cell reprogramming along the neural lineage is expected to contribute, not only to enhance our understanding on the developmental aspects, but also to develop more efficient protocols and rational interventions $[218,219]$. Continuous characterization of patterning cues, small molecules and other driving factors, as well as a comprehensive understanding of the underlying 
molecular pathways that they target will be necessary to achieve a higher efficiency, decrease culture heterogeneity and increase neuronal subtype availability. Single cell analysis and direct comparison of differentiated cells versus undifferentiated counterparts will be crucial in order to find signalling mechanisms, as well as to develop a comprehensive understanding of the epigenetic state and transcriptional programmes involved in neuronal fate specification. Such knowledge can in the end be exploited to manipulate single molecules or even complete molecular networks in the developmental processes and, hence, could aid the production of specific neuronal cells of interest. With the current advances of nextgeneration sequencing technology we are now also able to define genome-wide expression patterns and epigenetic modifications in each cell type. Such approaches have already demonstrated that different cellular subtypes display unique epigenetic signatures that persist as ESCs differentiate into the neuronal lineage. However, how these unique signatures are acquired in specific gene promoters or to what extent they are involved in shaping neuronal fates remains to be elucidated.

A final point of consideration with regard to the use of PSC- and somatic cell-derived neuronal populations in fundamental research and biomedicine, is that the conventional cell culture systems do not fully resemble the in vivo cellular microenvironment, where three-dimensional cell-to-cell interactions form the foundation of the human brain. The simplicity of an in vitro culture system is an advantage, as well as a significant disadvantage, when cellular homogeneity becomes a reliability. Studying pure populations of neuronal subtypes that are in principle part of a more complex integrated cellular network, might lead to under- or overrepresentation of experimental findings depending on the research question. Organoids or three-dimensional culture systems in combination with bioprinting might offer a way to circumvent this issue [220-222]. Several studies have already succeeded in establishing organoids that imitate many features of human cortical development in a precise and complex manner [223225]. Such culture systems derived from human iPSCs hold great potential for the investigation of developmental and evolutionary features of the human brain and provides a useful platform for drug screening and disease modelling [223, 226]. Additionally, the technology of decellularization and recellularization on obtained tissue matrixes to create entire organs in vitro is currently also under development [227]. Unfortunately, despite these advances, the challenge of how to generate organs that cherish the highly integrated cellular complexities like in the human brain persists, and will require collaborative multidisciplinary expertise to overcome. Nevertheless, neuronal in vitro differentiation techniques in combination with these advanced three-dimensional culture systems represent powerful tools for future brain-related studies. The potential to manipulate (epi)genetic and environmental factors in culture conditions, with the possibility to characterize cellular functions, electrophysiological properties and cellular connectivity of various neuronal subtypes in isolation, as well 
as organized in a multi-layered dimension, will be of great utility to enhance our current understanding on brain disorders and will undoubtedly contribute to the development of therapeutic interventions.

\section{Conflict of interest statement}

The authors declare no conflict of interest.

\section{Acknowledgements}

We would like to thank Dr. Sonia Guil and Edilene Siquiera Soares for all the fruitful discussions and insights on this manuscript. We thank CERCA Programme / Generalitat de Catalunya. Renzo J.M. Riemens is supported by Maastricht University (Maastricht, the Netherlands) and Julius Maximilians University (Wuerzburg, Germany). The work of the authors is supported by, among other institutions, the CELLEX Private Foundation, the Secretariat for Universities and Research of the Ministry of Business and Knowledge of the Government of Catalonia, the Health Departments of the Government of Catalonia and the Joint ProgrammeNeurodegenerative Disease Research (JPND) for the EPIAD consortium. The project is supported through the following funding organizations under the aegis of JPND; the Netherlands, The Netherlands Organisation for Health Research and Development (ZonMw); United Kingdom, Medical Research Council; Germany, German Federal ministry of Education and Research (BMBF); Luxembourg, National Research Fund (FNR). This project has received funding from the European Union's Horizon 2020 research and innovation programme under Grant Agreement No. 643417.

\section{References}

1. Nestler EJ, Hyman SE. Animal models of neuropsychiatric disorders. Nature neuroscience. 2010;13(10):1161.

2. AkhtarA. The flaws and human harms of animal experimentation. Cambridge Quarterly of Healthcare Ethics. 2015;24(4):407-19.

3. Mak IW, Evaniew N, Ghert M. Lost in translation: animal models and clinical trials in cancer treatment. American journal of translational research. 2014;6(2):114.

4. Lewis DA. The human brain revisited: opportunities and challenges in postmortem studies of psychiatric disorders. Neuropsychopharmacology. 2002;26(2):143-54.

5. Badger J, Cordero-Llana O, Hartfield E, Wade-Martins R. Parkinson's disease in a dish-using stem cells as a molecular tool. Neuropharmacology. 2014;76:88-96.

6. Paşca SP, Panagiotakos G, Dolmetsch RE. Generating human neurons in vitro and using them to understand neuropsychiatric disease. Annual review of neuroscience. 2014;37:479-501.

7. Shi Y, Inoue H, Wu JC, Yamanaka S. Induced pluripotent stem cell technology: a decade of progress. Nature reviews Drug discovery. 2017;16(2):115-30.

8. Yap MS, Nathan KR, Yeo Y, Lim LW, Poh CL, Richards M, et al. Neural differentiation of human pluripotent stem cells for nontherapeutic applications: toxicology, pharmacology, and in vitro disease modeling. Stem Cells International. 2015;2015.

9. Haston KM, Finkbeiner S. Clinical trials in a dish: the potential of pluripotent stem cells to develop 
therapies for neurodegenerative diseases. Annual review of pharmacology and toxicology. 2016;56:489-510.

10. Hou S, Lu P. Direct reprogramming of somatic cells into neural stem cells or neurons for neurological disorders. Neural Regeneration Research. 2016;11(1):28.

11. Riemens RJ, Soares ES, Esteller M, Delgado-Morales R. Stem Cell Technology for (Epi) genetic Brain Disorders. Neuroepigenomics in Aging and Disease: Springer; 2017. p. 443-75.

12. Young-Pearse TL, Morrow EM. Modeling developmental neuropsychiatric disorders with iPSC technology: challenges and opportunities. Current opinion in neurobiology. 2016;36:66-73.

13. Canfield SG, Stebbins MJ, Morales BS, Asai SW, Vatine GD, Svendsen CN, et al. An isogenic bloodbrain barrier model comprising brain endothelial cells, astrocytes, and neurons derived from human induced pluripotent stem cells. J Neurochem. 2017;140(6):874-88.

14. Kirwan P, Turner-Bridger B, Peter M, Momoh A, Arambepola D, Robinson HP, et al. Development and function of human cerebral cortex neural networks from pluripotent stem cells in vitro. Development. 2015;142(18):3178-87.

15. Mertens J, Marchetto MC, Bardy C, Gage FH. Evaluating cell reprogramming, differentiation and conversion technologies in neuroscience. Nature Reviews Neuroscience. 2016;17(7):424.

16. Maroof AM, Keros S, Tyson JA, Ying S-W, Ganat YM, Merkle FT, et al. Directed differentiation and functional maturation of cortical interneurons from human embryonic stem cells. Cell stem cell. 2013;12(5):559-72.

17. Zhang Y, Pak C, Han Y, Ahlenius H, Zhang Z, Chanda S, et al. Rapid single-step induction of functional neurons from human pluripotent stem cells. Neuron. 2013;78(5):785-98.

18. Denham M, Dottori M. Neural differentiation of induced pluripotent stem cells. Neurodegeneration: Springer; 2011. p. 99-110.

19. Erceg S, Laínez S, Ronaghi M, Stojkovic P, Pérez-Aragó MA, Moreno-Manzano V, et al. Differentiation of human embryonic stem cells to regional specific neural precursors in chemically defined medium conditions. PLoS One. 2008;3(5):e2122.

20. Vierbuchen T, Ostermeier A, Pang ZP, Kokubu Y, Südhof TC, Wernig M. Direct conversion of fibroblasts to functional neurons by defined factors. Nature. 2010;463(7284):1035-41.

21. Gifford CA, Ziller MJ, Gu HC, Trapnell C, Donaghey J, Tsankov A, et al. Transcriptional and Epigenetic Dynamics during Specification of Human Embryonic Stem Cells. Cell. 2013;153(5):1149-63.

22. Lunyak VV, Rosenfeld MG. Epigenetic regulation of stem cell fate. Human molecular genetics. 2008;17(R1):R28-R36.

23. Qin $\mathrm{H}$, Zhao A, Zhang C, Fu X. Epigenetic control of reprogramming and transdifferentiation by histone modifications. Stem Cell Reviews and Reports. 2016;12(6):708-20.

24. Imamura $\mathrm{T}$, Uesaka $\mathrm{M}$, Nakashima K. Epigenetic setting and reprogramming for neural cell fate determination and differentiation. Philos Trans R Soc Lond B Biol Sci. 2014;369(1652).

25. Feng JA, Fouse S, Fan GP. Epigenetic regulation of neural gene expression and neuronal function. Pediatr Res. 2007;61(5):58r-63r.

26. Fitzsimons CP, van Bodegraven E, Schouten M, Lardenoije R, Kompotis K, Kenis G, et al. Epigenetic regulation of adult neural stem cells: implications for Alzheimer's disease. Mol Neurodegener. 2014;9:25.

27. Kungulovski G, Jeltsch A. Epigenome Editing: State of the Art, Concepts, and Perspectives. Trends in Genetics. 2016;32(2):101-13.

28. Liu XS, Wu H, Ji X, Stelzer Y, Wu X, Czauderna S, et al. Editing DNA Methylation in the Mammalian Genome. Cell. 2016;167(1):233-47 e17.

29. Thakore PI, Black JB, Hilton IB, Gersbach CA. Editing the epigenome: technologies for programmable transcription and epigenetic modulation. Nature methods. 2016;13(2):127. 
30. Low WC, Yau WWY, Stanton LW, Marcy G, Goh E, Chew SY. Directing neuronal differentiation of primary neural progenitor cells by gene knockdown approach. DNA and cell biology. 2012;31(7):1148-60.

31. Stappert L, Borghese L, Roese-Koerner B, Weinhold S, Koch P, Terstegge S, et al. MicroRNA-based promotion of human neuronal differentiation and subtype specification. PLoS One. 2013;8(3):e59011.

32. Jurkowski TP, Ravichandran M, Stepper P. Synthetic epigenetics-towards intelligent control of epigenetic states and cell identity. Clinical Epigenetics. 2015;7.

33. Chambers SM, Fasano CA, Papapetrou EP, Tomishima M, Sadelain M, Studer L. Highly efficient neural conversion of human ES and iPS cells by dual inhibition of SMAD signaling. Nat Biotechnol. 2009;27(3):275-80.

34. Erceg S, Ronaghi M, Stojkovic M. Human embryonic stem cell differentiation toward regional specific neural precursors. Stem Cells. 2009;27(1):78-87.

35. Erceg S, Ronaghi M, Zipancic I, Lainez S, Rosello MG, Xiong C, et al. Efficient differentiation of human embryonic stem cells into functional cerebellar-like cells. Stem Cells Dev. 2010;19(11):1745-56.

36. Karumbayaram S, Novitch BG, Patterson M, Umbach JA, Richter L, Lindgren A, et al. Directed differentiation of human-induced pluripotent stem cells generates active motor neurons. Stem Cells. 2009;27(4):806-11.

37. Shi G. Differentiation of human pluripotent stem cells into functional cells. OA Stem Cells. 2013:4.

38. Ying QL, Stavridis M, Griffiths D, Li M, Smith A. Conversion of embryonic stem cells into neuroectodermal precursors in adherent monoculture. Nat Biotechnol. 2003;21(2):183-6.

39. Denham M, Dottori M. Neural differentiation of induced pluripotent stem cells. Methods Mol Biol. 2011;793:99-110.

40. Karanfil I, Bagci-Onder T. Derivation of Neural Stem Cells from Mouse Induced Pluripotent Stem Cells. Methods Mol Biol. 2016;1357:329-38.

41. Itskovitz-Eldor J, Schuldiner M, Karsenti D, Eden A, Yanuka O, Amit M, et al. Differentiation of human embryonic stem cells into embryoid bodies compromising the three embryonic germ layers. Molecular medicine. 2000;6(2):88.

42. Abranches E, Silva M, Pradier L, Schulz H, Hummel O, Henrique D, et al. Neural differentiation of embryonic stem cells in vitro: a road map to neurogenesis in the embryo. PLoS One. 2009;4(7):e6286.

43. Günther K, Appelt-Menzel A, Kwok CK, Walles H, Metzger M, Edenhofer F. Rapid Monolayer Neural Induction of induced Pluripotent Stem Cells Yields Stably Proliferating Neural Stem Cells. Journal of Stem Cell Research \& Therapy. 2016;2016.

44. Yan Y, Shin S, Jha BS, Liu Q, Sheng J, Li F, et al. Efficient and rapid derivation of primitive neural stem cells and generation of brain subtype neurons from human pluripotent stem cells. Stem cells translational medicine. 2013:sctm. 2013-0080.

45. Kawasaki H, Mizuseki K, Nishikawa S, Kaneko S, Kuwana Y, Nakanishi S, et al. Induction of midbrain dopaminergic neurons from ES cells by stromal cell-derived inducing activity. Neuron. 2000;28(1):3140.

46. Lim MS, Shin MS, Lee SY, Minn YK, Hoh JK, Cho YH, et al. Noggin Over-Expressing Mouse Embryonic Fibroblasts and MS5 Stromal Cells Enhance Directed Differentiation of Dopaminergic Neurons from Human Embryonic Stem Cells. PLoS One. 2015;10(9):e0138460.

47. Fujimori K, Matsumoto T, Kisa F, Hattori N, Okano H, Akamatsu W. Escape from Pluripotency via Inhibition of TGF-beta/BMP and Activation of Wnt Signaling Accelerates Differentiation and Aging in hPSC Progeny Cells. Stem cell reports. 2017;9(5):1675-91.

48. Zhang Y, Li W, Laurent T, Ding S. Small molecules, big roles-the chemical manipulation of stem cell fate and somatic cell reprogramming. Journal of cell science. 2012;125(23):5609-20.

49. Davis-Dusenbery BN, Williams LA, Klim JR, Eggan K. How to make spinal motor neurons. Development. 2014;141(3):491-501. 
50. Tao Y, Zhang SC. Neural Subtype Specification from Human Pluripotent Stem Cells. Cell Stem Cell. 2016;19(5):573-86.

51. Pasca SP, Panagiotakos G, Dolmetsch RE. Generating human neurons in vitro and using them to understand neuropsychiatric disease. Annu Rev Neurosci. 2014;37:479-501.

52. Patani R. Generating Diverse Spinal Motor Neuron Subtypes from Human Pluripotent Stem Cells. Stem Cells Int. 2016;2016:1036974.

53. Goulburn AL, Stanley EG, Elefanty AG, Anderson SA. Generating GABAergic cerebral cortical interneurons from mouse and human embryonic stem cells. Stem Cell Res. 2012;8(3):416-26.

54. Li W, Sun W, Zhang Y, Wei W, Ambasudhan R, Xia P, et al. Rapid induction and long-term selfrenewal of primitive neural precursors from human embryonic stem cells by small molecule inhibitors. Proceedings of the National Academy of Sciences of the United States of America. 2011;108(20):8299-304.

55. Dhara SK, Stice SL. Neural differentiation of human embryonic stem cells. Journal of cellular biochemistry. 2008;105(3):633-40.

56. Wilson SI, Graziano E, Harland R, Jessell TM, Edlund T. An early requirement for FGF signalling in the acquisition of neural cell fate in the chick embryo. Curr Biol. 2000;10(8):421-9.

57. Eiraku M, Watanabe K, Matsuo-Takasaki M, Kawada M, Yonemura S, Matsumura M, et al. Selforganized formation of polarized cortical tissues from ESCs and its active manipulation by extrinsic signals. Cell Stem Cell. 2008;3(5):519-32.

58. Lee H, Shamy GA, Elkabetz Y, Schofield CM, Harrsion NL, Panagiotakos G, et al. Directed differentiation and transplantation of human embryonic stem cell-derived motoneurons. Stem Cells. 2007;25(8):1931-9.

59. Lu J, Zhong X, Liu H, Hao L, Huang CT, Sherafat MA, et al. Generation of serotonin neurons from human pluripotent stem cells. Nat Biotechnol. 2016;34(1):89-94.

60. Yan Y, Yang D, Zarnowska ED, Du Z, Werbel B, Valliere C, et al. Directed differentiation of dopaminergic neuronal subtypes from human embryonic stem cells. Stem Cells. 2005;23(6):781-90.

61. Imaizumi K, Sone T, Ibata K, Fujimori K, Yuzaki M, Akamatsu W, et al. Controlling the Regional Identity of hPSC-Derived Neurons to Uncover Neuronal Subtype Specificity of Neurological Disease Phenotypes. Stem cell reports. 2015;5(6):1010-22.

62. Kirkeby A, Grealish S, Wolf DA, Nelander J, Wood J, Lundblad M, et al. Generation of regionally specified neural progenitors and functional neurons from human embryonic stem cells under defined conditions. Cell reports. 2012;1(6):703-14.

63. Liu H, Zhang SC. Specification of neuronal and glial subtypes from human pluripotent stem cells. Cell Mol Life Sci. 2011;68(24):3995-4008.

64. Gabilondo H, Stratmann J, Rubio-Ferrera I, Millan-Crespo I, Contero-Garcia P, Bahrampour S, et al. Neuronal Cell Fate Specification by the Convergence of Different Spatiotemporal Cues on a Common Terminal Selector Cascade. PLoS biology. 2016;14(5):e1002450.

65. Zhang SC, Wernig M, Duncan ID, Brustle O, Thomson JA. In vitro differentiation of transplantable neural precursors from human embryonic stem cells. Nat Biotechnol. 2001;19(12):1129-33.

66. Zhang D, Jiang W. From One-Cell to Tissue: Reprogramming, Cell Differentiation and Tissue Engineering. BioScience. 2015:biv016.

67. Costa MR, Muller U. Specification of excitatory neurons in the developing cerebral cortex: progenitor diversity and environmental influences. Front Cell Neurosci. 2014;8:449.

68. Song H, Lee B, Pyun D, Guimera J, Son Y, Yoon J, et al. Ascl1 and Helt act combinatorially to specify thalamic neuronal identity by repressing DIxs activation. Developmental biology. 2015;398(2):280-91.

69. Thomas Cheng H. Spinal cord mechanisms of chronic pain and clinical implications. Current pain and headache reports. 2010;14(3):213-20. 
70. Li XJ, Zhang X, Johnson MA, Wang ZB, Lavaute T, Zhang SC. Coordination of sonic hedgehog and Wnt signaling determines ventral and dorsal telencephalic neuron types from human embryonic stem cells. Development. 2009;136(23):4055-63.

71. Gaspard N, Bouschet T, Hourez R, Dimidschstein J, Naeije G, van den Ameele J, et al. An intrinsic mechanism of corticogenesis from embryonic stem cells. Nature. 2008;455(7211):351-7.

72. Bibel M, Richter J, Schrenk K, Tucker KL, Staiger V, Korte M, et al. Differentiation of mouse embryonic stem cells into a defined neuronal lineage. Nat Neurosci. 2004;7(9):1003-9.

73. Espuny-Camacho I, Michelsen KA, Gall D, Linaro D, Hasche A, Bonnefont J, et al. Pyramidal neurons derived from human pluripotent stem cells integrate efficiently into mouse brain circuits in vivo. Neuron. 2013;77(3):440-56.

74. Kim JE, O'Sullivan ML, Sanchez CA, Hwang M, Israel MA, Brennand K, et al. Investigating synapse formation and function using human pluripotent stem cell-derived neurons. Proceedings of the National Academy of Sciences of the United States of America. 2011;108(7):3005-10.

75. Shi Y, Kirwan P, Livesey FJ. Directed differentiation of human pluripotent stem cells to cerebral cortex neurons and neural networks. Nat Protoc. 2012;7(10):1836-46.

76. Shi Y, Kirwan P, Smith J, Robinson HP, Livesey FJ. Human cerebral cortex development from pluripotent stem cells to functional excitatory synapses. Nat Neurosci. 2012;15(3):477-86, S1.

77. Liu Y, Liu H, Sauvey C, Yao L, Zarnowska ED, Zhang SC. Directed differentiation of forebrain GABA interneurons from human pluripotent stem cells. Nat Protoc. 2013;8(9):1670-9.

78. Fishell G, Rudy B. Mechanisms of inhibition within the telencephalon:"where the wild things are". Annual review of neuroscience. 2011;34:535-67.

79. Nobrega-Pereira S, Kessaris N, Du T, Kimura S, Anderson SA, Marin O. Postmitotic Nkx2-1 controls the migration of telencephalic interneurons by direct repression of guidance receptors. Neuron. 2008;59(5):733-45.

80. Kriegstein AR, Noctor SC. Patterns of neuronal migration in the embryonic cortex. Trends in neurosciences. 2004;27(7):392-9.

81. Marin O, Rubenstein JL. Cell migration in the forebrain. Annu Rev Neurosci. 2003;26:441-83.

82. Sussel L, Marin O, Kimura S, Rubenstein JL. Loss of Nkx2.1 homeobox gene function results in a ventral to dorsal molecular respecification within the basal telencephalon: evidence for a transformation of the pallidum into the striatum. Development. 1999;126(15):3359-70.

83. Campbell K. Dorsal-ventral patterning in the mammalian telencephalon. Curr Opin Neurobiol. 2003;13(1):50-6.

84. Watanabe K, Kamiya D, NishiyamaA, Katayama T, Nozaki S, Kawasaki H, et al. Directed differentiation of telencephalic precursors from embryonic stem cells. Nature neuroscience. 2005;8(3):288-96.

85. Maroof AM, Brown K, Shi S-H, Studer L, Anderson SA. Prospective isolation of cortical interneuron precursors from mouse embryonic stem cells. The Journal of Neuroscience. 2010;30(13):4667-75.

86. Nicholas CR, Chen J, Tang Y, Southwell DG, Chalmers N, Vogt D, et al. Functional maturation of hPSC-derived forebrain interneurons requires an extended timeline and mimics human neural development. Cell stem cell. 2013;12(5):573-86.

87. Carri AD, Onorati M, Castiglioni V, Faedo A, Camnasio S, Toselli M, et al. Human pluripotent stem cell differentiation into authentic striatal projection neurons. Stem Cell Reviews and Reports. 2013;9(4):461-74.

88. Lin L, Yuan J, Sander B, Golas MM. In Vitro Differentiation of Human Neural Progenitor Cells Into Striatal GABAergic Neurons. Stem Cells Transl Med. 2015;4(7):775-88.

89. Tang Y, Liu L, Li J, Yu L, Wang L, Shi J, et al. Induction and differentiation of human induced pluripotent stem cells into functional cardiomyocytes on a compartmented monolayer of gelatin nanofibers. Nanoscale. 2016;8(30):14530-40. 
90. Chinta SJ, Andersen JK. Dopaminergic neurons. The international journal of biochemistry \& cell biology. 2005;37(5):942-6.

91. Ono Y, Nakatani T, Sakamoto Y, Mizuhara E, Minaki Y, Kumai M, et al. Differences in neurogenic potential in floor plate cells along an anteroposterior location: midbrain dopaminergic neurons originate from mesencephalic floor plate cells. Development. 2007;134(17):3213-25.

92. Placzek M, Briscoe J. The floor plate: multiple cells, multiple signals. Nature reviews Neuroscience. 2005;6(3):230-40.

93. Chung S, Leung A, Han BS, Chang MY, Moon JI, Kim CH, et al. Wnt1-Imx1a forms a novel autoregulatory loop and controls midbrain dopaminergic differentiation synergistically with the $\mathrm{SHH}$ FoxA2 pathway. Cell Stem Cell. 2009;5(6):646-58.

94. Xi J, Liu Y, Liu H, Chen H, Emborg ME, Zhang SC. Specification of midbrain dopamine neurons from primate pluripotent stem cells. Stem Cells. 2012;30(8):1655-63.

95. Prakash N, Brodski C, Naserke T, Puelles E, Gogoi R, Hall A, et al. A Wnt1-regulated genetic network controls the identity and fate of midbrain-dopaminergic progenitors in vivo. Development. 2006;133(1):89-98.

96. Andersson E, Tryggvason U, Deng Q, Friling S, Alekseenko Z, Robert B, et al. Identification of intrinsic determinants of midbrain dopamine neurons. Cell. 2006;124(2):393-405.

97. Barberi T, Klivenyi P, Calingasan NY, Lee H, Kawamata H, Loonam K, et al. Neural subtype specification of fertilization and nuclear transfer embryonic stem cells and application in parkinsonian mice. Nat Biotechnol. 2003;21(10):1200-7.

98. Lee SH, Lumelsky N, Studer L, Auerbach JM, McKay RD. Efficient generation of midbrain and hindbrain neurons from mouse embryonic stem cells. Nat Biotechnol. 2000;18(6):675-9.

99. Ye W, Shimamura K, Rubenstein JL, Hynes MA, Rosenthal A. FGF and Shh signals control dopaminergic and serotonergic cell fate in the anterior neural plate. Cell. 1998;93(5):755-66.

100. Cai J, Yang M, Poremsky E, Kidd S, Schneider JS, lacovitti L. Dopaminergic neurons derived from human induced pluripotent stem cells survive and integrate into 6-OHDA-lesioned rats. Stem Cells Dev. 2010;19(7):1017-23.

101. Swistowski A, Peng J, Liu Q, Mali P, Rao MS, Cheng L, et al. Efficient generation of functional dopaminergic neurons from human induced pluripotent stem cells under defined conditions. Stem cells. 2010;28(10):1893-904.

102. Arenas E, Denham M, Villaescusa JC. How to make a midbrain dopaminergic neuron. Development. 2015;142(11):1918-36.

103. Denham M, Bye C, Leung J, Conley BJ, Thompson LH, Dottori M. Glycogen Synthase Kinase $3 \beta$ and Activin/Nodal Inhibition in Human Embryonic Stem Cells Induces a Pre-Neuroepithelial State That Is Required for Specification to a Floor Plate Cell Lineage. Stem Cells. 2012;30(11):2400-11.

104. Doi D, Samata B, Katsukawa M, Kikuchi T, Morizane A, Ono Y, et al. Isolation of human induced pluripotent stem cell-derived dopaminergic progenitors by cell sorting for successful transplantation. Stem cell reports. 2014;2(3):337-50.

105. Kriks S, Shim JW, Piao J, Ganat YM, Wakeman DR, Xie Z, et al. Dopamine neurons derived from human ES cells efficiently engraft in animal models of Parkinson's disease. Nature. 2011;480(7378):547-51.

106. Fernandez-Santiago R, Carballo-Carbajal I, Castellano G, Torrent R, Richaud Y, Sanchez-Danes A, et al. Aberrant epigenome in iPSC-derived dopaminergic neurons from Parkinson's disease patients. EMBO molecular medicine. 2015.

107. Woodard CM, Campos BA, Kuo SH, Nirenberg MJ, Nestor MW, Zimmer M, et al. iPSC-derived dopamine neurons reveal differences between monozygotic twins discordant for Parkinson's disease. Cell Rep. 2014;9(4):1173-82.

108. Deneris E, Gaspar P. Serotonin neuron development: shaping molecular and structural identities. 
Wiley Interdiscip Rev Dev Biol. 2018;7(1).

109. Alenina N, Bashammakh S, Bader M. Specification and differentiation of serotonergic neurons. Stem Cell Rev. 2006;2(1):5-10.

110. Vadodaria KC, Marchetto MC, Mertens J, Gage FH. Generating human serotonergic neurons in vitro: Methodological advances. BioEssays : news and reviews in molecular, cellular and developmental biology. 2016;38(11):1123-9.

111. Dias JM, Alekseenko Z, Applequist JM, Ericson J. Tgfbeta signaling regulates temporal neurogenesis and potency of neural stem cells in the CNS. Neuron. 2014;84(5):927-39.

112. Rhinn M, Brand M. The midbrain--hindbrain boundary organizer. Curr Opin Neurobiol. 2001;11(1):3442.

113. Shimada T, Takai Y, Shinohara K, Yamasaki A, Tominaga-Yoshino K, Ogura A, et al. A simplified method to generate serotonergic neurons from mouse embryonic stem and induced pluripotent stem cells. J Neurochem. 2012;122(1):81-93.

114. Kumar M, Kaushalya SK, Gressens P, Maiti S, Mani S. Optimized derivation and functional characterization of 5-HT neurons from human embryonic stem cells. Stem Cells Dev. 2009;18(4):615-27.

115. Stock A, Kuzis K, Woodward WR, Nishi R, Eckenstein FP. Localization of acidic fibroblast growth factor in specific subcortical neuronal populations. J Neurosci. 1992;12(12):4688-700.

116. Migliarini S, Pacini G, Pelosi B, Lunardi G, Pasqualetti M. Lack of brain serotonin affects postnatal development and serotonergic neuronal circuitry formation. Mol Psychiatry. 2013;18(10):1106-18.

117. Vadodaria KC, Mertens J, Paquola A, Bardy C, Li X, Jappelli R, et al. Generation of functional human serotonergic neurons from fibroblasts. Mol Psychiatry. 2015.

118. Licinio J, Wong M. Serotonergic neurons derived from induced pluripotent stem cells (iPSCs): a new pathway for research on the biology and pharmacology of major depression. Molecular psychiatry. 2016;21(1):1-2.

119. SierksmaAS, van den Hove DL, Steinbusch HW, Prickaerts J. Major depression, cognitive dysfunction and Alzheimer's disease: is there a link? European journal of pharmacology. 2010;626(1):72-82.

120. Li X-J, Du Z-W, Zarnowska ED, Pankratz M, Hansen LO, Pearce RA, et al. Specification of motoneurons from human embryonic stem cells. Nature biotechnology. 2005;23(2):215-21.

121. Singh Roy N, Nakano T, Xuing L, Kang J, Nedergaard M, Goldman SA. Enhancer-specified GFPbased FACS purification of human spinal motor neurons from embryonic stem cells. Exp Neurol. 2005;196(2):224-34.

122. Dimos JT, Rodolfa KT, Niakan KK, Weisenthal LM, Mitsumoto H, Chung W, et al. Induced pluripotent stem cells generated from patients with ALS can be differentiated into motor neurons. Science. 2008;321(5893):1218-21.

123. Qu Q, Li D, Louis KR, Li X, Yang H, Sun Q, et al. High-efficiency motor neuron differentiation from human pluripotent stem cells and the function of Islet-1. Nature communications. 2014;5:3449.

124. Shimojo D, Onodera K, Doi-Torii Y, Ishihara Y, Hattori C, Miwa Y, et al. Rapid, efficient, and simple motor neuron differentiation from human pluripotent stem cells. Mol Brain. 2015;8(1):79.

125. Wichterle H, Lieberam I, Porter JA, Jessell TM. Directed differentiation of embryonic stem cells into motor neurons. Cell. 2002;110(3):385-97.

126. Amoroso MW, Croft GF, Williams DJ, O'Keeffe S, Carrasco MA, Davis AR, et al. Accelerated high-yield generation of limb-innervating motor neurons from human stem cells. J Neurosci. 2013;33(2):574-86.

127. Vierbuchen T, Wernig M. Direct lineage conversions: unnatural but useful? Nat Biotechnol. 2011;29(10):892-907.

128. Takahashi K, Yamanaka S. Induction of pluripotent stem cells from mouse embryonic and adult fibroblast cultures by defined factors. cell. 2006;126(4):663-76.

129. Yu J, Vodyanik MA, Smuga-Otto K, Antosiewicz-Bourget J, Frane JL, Tian S, et al. Induced pluripotent stem cell lines derived from human somatic cells. Science. 2007;318(5858):1917-20. 
130. Schlaeger TM, Daheron L, Brickler TR, Entwisle S, Chan K, Cianci A, et al. A comparison of nonintegrating reprogramming methods. Nature biotechnology. 2015;33(1):58-63.

131. Takahashi K, Yamanaka S. A decade of transcription factor-mediated reprogramming to pluripotency. Nat Rev Mol Cell Biol. 2016;17(3):183-93.

132. Weintraub H, Tapscott SJ, Davis RL, Thayer MJ, Adam MA, Lassar AB, et al. Activation of musclespecific genes in pigment, nerve, fat, liver, and fibroblast cell lines by forced expression of MyoD. Proceedings of the National Academy of Sciences. 1989;86(14):5434-8.

133. Mertens J, Paquola ACM, Ku M, Hatch E, Bohnke L, Ladjevardi S, et al. Directly Reprogrammed Human Neurons Retain Aging-Associated Transcriptomic Signatures and Reveal Age-Related Nucleocytoplasmic Defects. Cell Stem Cell. 2015;17(6):705-18.

134. Busskamp V, Lewis NE, Guye P, Ng AH, Shipman SL, Byrne SM, et al. Rapid neurogenesis through transcriptional activation in human stem cells. Mol Syst Biol. 2014;10:760.

135. Hou S, Lu P. Direct reprogramming of somatic cells into neural stem cells or neurons for neurological disorders. Neural Regen Res. 2016;11(1):28-31.

136. Raciti M, Granzotto M, Do Duc M, Fimiani C, Cellot G, Cherubini E, et al. Reprogramming fibroblasts to neural-precursor-like cells by structured overexpression of pallial patterning genes. Molecular and Cellular Neuroscience. 2013;57:42-53.

137. Matsui T, Takano M, Yoshida K, Ono S, Fujisaki C, Matsuzaki Y, et al. Neural stem cells directly differentiated from partially reprogrammed fibroblasts rapidly acquire gliogenic competency. Stem Cells. 2012;30(6):1109-19.

138. Vierbuchen T, Ostermeier A, Pang ZP, Kokubu Y, Sudhof TC, Wernig M. Direct conversion of fibroblasts to functional neurons by defined factors. Nature. 2010;463(7284):1035-41.

139. Pang ZP, Yang N, Vierbuchen T, Ostermeier A, Fuentes DR, Yang TQ, et al. Induction of human neuronal cells by defined transcription factors. Nature. 2011;476(7359):220-3.

140. Chanda S, Ang CE, Davila J, Pak C, Mall M, Lee QY, et al. Generation of induced neuronal cells by the single reprogramming factor ASCL1. Stem cell reports. 2014;3(2):282-96.

141. Mall M, Kareta MS, Chanda S, Ahlenius H, Perotti N, Zhou B, et al. Myt1/ safeguards neuronal identity by actively repressing many non-neuronal fates. Nature. 2017;544(7649):245-9.

142. Heinrich C, Blum R, Gascon S, Masserdotti G, Tripathi P, Sanchez R, et al. Directing astroglia from the cerebral cortex into subtype specific functional neurons. PLoS biology. 2010;8(5):e1000373.

143. Thoma EC, Wischmeyer E, Offen N, Maurus K, Siren AL, Schartl M, et al. Ectopic expression of neurogenin 2 alone is sufficient to induce differentiation of embryonic stem cells into mature neurons. PLoS One. 2012;7(6):e38651.

144. Colasante G, Lignani G, Rubio A, Medrihan L, Yekhlef L, Sessa A, et al. Rapid conversion of fibroblasts into functional forebrain GABAergic interneurons by direct genetic reprogramming. Cell stem cell. 2015;17(6):719-34.

145. Mong J, Panman L, Alekseenko Z, Kee N, Stanton LW, Ericson J, et al. Transcription Factor-Induced Lineage Programming of Noradrenaline and Motor Neurons from Embryonic Stem Cells. Stem Cells. 2014;32(3):609-22.

146. Pfisterer U, Kirkeby A, Torper O, Wood J, Nelander J, Dufour A, et al. Direct conversion of human fibroblasts to dopaminergic neurons. Proceedings of the National Academy of Sciences. 2011;108(25):10343-8.

147. Son EY, Ichida JK, Wainger BJ, Toma JS, Rafuse VF, Woolf CJ, et al. Conversion of mouse and human fibroblasts into functional spinal motor neurons. Cell stem cell. 2011;9(3):205-18.

148. Xu Z, Jiang H, Zhong P, Yan Z, Chen S, Feng J. Direct conversion of human fibroblasts to induced serotonergic neurons. Mol Psychiatry. 2015.

149. Hester ME, Murtha MJ, Song S, Rao M, Miranda CJ, Meyer K, et al. Rapid and efficient generation of 
functional motor neurons from human pluripotent stem cells using gene delivered transcription factor codes. Molecular therapy. 2011;19(10):1905-12.

150. Kim J, Su SC, Wang H, Cheng AW, Cassady JP, Lodato MA, et al. Functional integration of dopaminergic neurons directly converted from mouse fibroblasts. Cell stem cell. 2011;9(5):413-9.

151. Gascon S, Masserdotti G, Russo GL, Gotz M. Direct Neuronal Reprogramming: Achievements, Hurdles, and New Roads to Success. Cell Stem Cell. 2017;21(1):18-34.

152. Xie X, Fu Y, Liu J. Chemical reprogramming and transdifferentiation. Curr Opin Genet Dev. 2017;46:104-13.

153. Caiazzo M, Dell'Anno MT, Dvoretskova E, Lazarevic D, Taverna S, Leo D, et al. Direct generation of functional dopaminergic neurons from mouse and human fibroblasts. Nature. 2011;476(7359):224-7.

154. Addis RC, Hsu F-C, Wright RL, Dichter MA, Coulter DA, Gearhart JD. Efficient conversion of astrocytes to functional midbrain dopaminergic neurons using a single polycistronic vector. PloS one. 2011;6(12):e28719.

155. Theka I, Caiazzo M, Dvoretskova E, Leo D, Ungaro F, Curreli S, et al. Rapid generation of functional dopaminergic neurons from human induced pluripotent stem cells through a single-step procedure using cell lineage transcription factors. Stem cells translational medicine. 2013;2(6):473-9.

156. Wasko NJ. Direct Reprogramming of Mouse Embryonic Fibroblasts Into GABAergic Neurons. 2013.

157. Mazzoni EO, Mahony S, Closser M, Morrison CA, Nedelec S, Williams DJ, et al. Synergistic binding of transcription factors to cell-specific enhancers programs motor neuron identity. Nature neuroscience. 2013;16(9):1219-27.

158. Barrero MJ, Boué S, Belmonte JCl. Epigenetic mechanisms that regulate cell identity. Cell stem cell. 2010;7(5):565-70.

159. Delgado-Morales R, Esteller M. Opening up the DNA methylome of dementia. Molecular psychiatry. 2017;22(4):485-96.

160. Goldberg AD, Allis CD, Bernstein E. Epigenetics: a landscape takes shape. Cell. 2007;128(4):635-8.

161. Lardenoije R, latrou A, Kenis G, Kompotis K, Steinbusch HW, Mastroeni D, et al. The epigenetics of aging and neurodegeneration. Prog Neurobiol. 2015;131:21-64.

162. Avgustinova A, Benitah SA. Epigenetic control of adult stem cell function. Nat Rev Mol Cell Bio. 2016;17(10):643.

163. Coskun V, Tsoa R, Sun YE. Epigenetic regulation of stem cells differentiating along the neural lineage. Curr Opin Neurobiol. 2012;22(5):762-7.

164. Lilja T, Heldring N, Hermanson O. Like a rolling histone: epigenetic regulation of neural stem cells and brain development by factors controlling histone acetylation and methylation. Biochimica et Biophysica Acta (BBA)-General Subjects. 2013;1830(2):2354-60.

165. Olynik BM, Rastegar M. The genetic and epigenetic journey of embryonic stem cells into mature neural cells. Front Genet. 2012;3:81.

166. Liu J, Githinji J, McLaughlin B, Wilczek K, Nolta J. Role of miRNAs in neuronal differentiation from human embryonic stem cell-derived neural stem cells. Stem Cell Rev. 2012;8(4):1129-37.

167. Lukovic D, Moreno-Manzano V, Klabusay M, Stojkovic M, Bhattacharya SS, Erceg S. Non-coding RNAs in pluripotency and neural differentiation of human pluripotent stem cells. Frontiers in genetics. 2014;5.

168. Esteller M. Non-coding RNAs in human disease. Nature reviews genetics. 2011;12(12):861-74.

169. Guil S, Esteller M. Cis-acting noncoding RNAs: friends and foes. Nature structural \& molecular biology. 2012;19(11):1068-75.

170. Smith ZD, Sindhu C, Meissner A. Molecular features of cellular reprogramming and development. Nat Rev Mol Cell Bio. 2016;17(3):139-54.

171. Hirabayashi $Y$, Gotoh Y. Epigenetic control of neural precursor cell fate during development. Nature reviews Neuroscience. 2010;11(6):377-88. 
172. Huang C, Wu JC. Epigenetic modulations of induced pluripotent stem cells: novel therapies and disease models. Drug Discovery Today: Disease Models. 2012;9(4):e153-e60.

173. van den Hurk M, Kenis G, Bardy C, van den Hove DL, Gage FH, Steinbusch HW, et al. Transcriptional and epigenetic mechanisms of cellular reprogramming to induced pluripotency. Epigenomics. 2016;8(8):1131-49.

174. Watanabe A, Yamada Y, Yamanaka S. Epigenetic regulation in pluripotent stem cells: a key to breaking the epigenetic barrier. Philosophical Transactions of the Royal Society B: Biological Sciences. 2013;368(1609):20120292.

175. Firas J, Liu X, Lim SM, Polo JM. Transcription factor-mediated reprogramming: epigenetics and therapeutic potential. Immunology and cell biology. 2015;93(3):284-9.

176. Chen T, Dent SY. Chromatin modifiers and remodellers: regulators of cellular differentiation. Nature Reviews Genetics. 2014;15(2):93-106.

177. Cheng L, Gao L, Guan W, Mao J, Hu W, Qu B, et al. Direct conversion of astrocytes into neuronal cells by drug cocktail. Cell research. 2015;25(11):1269-72.

178. Cheng L, Hu W, Quu B, Zhao J, Yu Y, Guan W, et al. Erratum: Generation of neural progenitor cells by chemical cocktails and hypoxia. Cell research. 2015;25(5):645-6.

179. Black JB, Adler AF, Wang HG, D'Ippolito AM, Hutchinson HA, Reddy TE, et al. Targeted Epigenetic Remodeling of Endogenous Loci by CRISPR/Cas9-Based Transcriptional Activators Directly Converts Fibroblasts to Neuronal Cells. Cell Stem Cell. 2016;19(3):406-14.

180. Chavez A, Scheiman J, Vora S, Pruitt BW, Tuttle M, E PRI, et al. Highly efficient Cas9-mediated transcriptional programming. Nat Methods. 2015;12(4):326-8.

181. Kearns NA, Genga RM, Enuameh MS, Garber M, Wolfe SA, Maehr R. Cas 9 effector-mediated regulation of transcription and differentiation in human pluripotent stem cells. Development. 2014;141(1):219-23.

182. Victor MB, Richner M, Hermanstyne TO, Ransdell JL, Sobieski C, Deng PY, et al. Generation of human striatal neurons by microRNA-dependent direct conversion of fibroblasts. Neuron. 2014;84(2):311-23.

183. Laufer BI, Singh SM. Strategies for precision modulation of gene expression by epigenome editing: an overview. Epigenetics \& chromatin. 2015;8(1):1.

184. Chakraborty S, Ji H, Kabadi AM, Gersbach CA, Christoforou N, Leong KW. A CRISPR/Cas9-based system for reprogramming cell lineage specification. Stem cell reports. 2014;3(6):940-7.

185. Wei S, Zou Q, Lai S, Zhang Q, Li L, Yan Q, et al. Conversion of embryonic stem cells into extraembryonic lineages by CRISPR-mediated activators. Sci Rep. 2016;6:19648.

186. Gao X, Yang J, Tsang JC, Ooi J, Wu D, Liu P. Reprogramming to pluripotency using designer TALE transcription factors targeting enhancers. Stem cell reports. 2013;1(2):183-97.

187. Balboa D, Weltner J, Eurola S, Trokovic R, Wartiovaara K, Otonkoski T. Conditionally Stabilized dCas9 Activator for Controlling Gene Expression in Human Cell Reprogramming and Differentiation. Stem cell reports. 2015;5(3):448-59.

188. Cano-Rodriguez D, Rots MG. Epigenetic editing: on the verge of reprogramming gene expression at will. Current genetic medicine reports. 2016;4(4):170-9.

189. Fu L, Zhu X, Yi F, Liu G-H, Belmonte JCl. Regenerative medicine: transdifferentiation in vivo. Cell Research. 2014;24(2):141-2.

190. Gascon S, Murenu E, Masserdotti G, Ortega F, Russo GL, Petrik D, et al. Identification and Successful Negotiation of a Metabolic Checkpoint in Direct Neuronal Reprogramming. Cell Stem Cell. 2016;18(3):396-409.

191. Guo Z, Zhang L, Wu Z, Chen Y, Wang F, Chen G. In vivo direct reprogramming of reactive glial cells into functional neurons after brain injury and in an Alzheimer's disease model. Cell Stem Cell. 2014;14(2):188-202. 
192. Torper O, Pfisterer U, Wolf DA, Pereira M, Lau S, Jakobsson J, et al. Generation of induced neurons via direct conversion in vivo. Proceedings of the National Academy of Sciences of the United States of America. 2013;110(17):7038-43.

193. Niu W, Zang T, Zou Y, Fang S, Smith DK, Bachoo R, et al. In vivo reprogramming of astrocytes to neuroblasts in the adult brain. Nature cell biology. 2013;15(10).

194. Ohori Y, Yamamoto S, Nagao M, Sugimori M, Yamamoto N, Nakamura K, et al. Growth factor treatment and genetic manipulation stimulate neurogenesis and oligodendrogenesis by endogenous neural progenitors in the injured adult spinal cord. J Neurosci. 2006;26(46):11948-60.

195. Li H, Chen G. In Vivo Reprogramming for CNS Repair: Regenerating Neurons from Endogenous Glial Cells. Neuron. 2016;91(4):728-38.

196. Srivastava D, DeWitt N. In Vivo Cellular Reprogramming: The Next Generation. Cell. 2016;166(6):1386-96.

197. Ong SG, Lee WH, Kodo K, Wu JC. MicroRNA-mediated regulation of differentiation and transdifferentiation in stem cells. Adv Drug Deliv Rev. 2015;88:3-15.

198. Stappert L, Roese-Koerner B, Brustle O. The role of microRNAs in human neural stem cells, neuronal differentiation and subtype specification. Cell Tissue Res. 2015;359(1):47-64.

199. Lopez-Ramirez MA, Nicoli S. Role of miRNAs and epigenetics in neural stem cell fate determination. Epigenetics. 2014;9(1):90-100.

200. He M, Liu Y, Wang X, Zhang MQ, Hannon GJ, Huang ZJ. Cell-type-based analysis of microRNA profiles in the mouse brain. Neuron. 2012;73(1):35-48.

201. Davis S, Lollo B, Freier S, Esau C. Improved targeting of miRNA with antisense oligonucleotides. Nucleic acids research. 2006;34(8):2294-304.

202. Yang N. An overview of viral and nonviral delivery systems for microRNA. International journal of pharmaceutical investigation. 2015;5(4):179.

203. Kim J, Inoue K, Ishii J, Vanti WB, Voronov SV, Murchison E, et al. A MicroRNA feedback circuit in midbrain dopamine neurons. Science. 2007;317(5842):1220-4.

204. Yang D, Li T, Wang Y, Tang Y, Cui H, Tang Y, et al. miR-132 regulates the differentiation of dopamine neurons by directly targeting Nurr1 expression. J Cell Sci. 2012;125(Pt 7):1673-82.

205. Abernathy DG, Kim WK, McCoy MJ, Lake AM, Ouwenga R, Lee SW, et al. MicroRNAs Induce a Permissive Chromatin Environment that Enables Neuronal Subtype-Specific Reprogramming of Adult Human Fibroblasts. Cell Stem Cell. 2017;21(3):332-48 e9.

206. Ambasudhan R, Talantova M, Coleman R, Yuan X, Zhu S, Lipton SA, et al. Direct reprogramming of adult human fibroblasts to functional neurons under defined conditions. Cell stem cell. 2011;9(2):1138.

207. Yoo AS, Sun AX, Li L, Shcheglovitov A, Portmann T, Li YL, et al. MicroRNA-mediated conversion of human fibroblasts to neurons. Nature. 2011;476(7359):228-U123.

208. Åkerblom M, Jakobsson J. MicroRNAs as neuronal fate determinants. The Neuroscientist. 2014;20(3):235-42.

209. Coolen M, Katz S, Bally-Cuif L. miR-9: a versatile regulator of neurogenesis. Frontiers in cellular neuroscience. 2013;7:220.

210. Meza-Sosa KF, Pedraza-Alva G, Pérez-Martínez L. microRNAs: key triggers of neuronal cell fate. Frontiers in cellular neuroscience. 2014;8:175.

211. Mateo F, Arenas E, Aguilar H, Serra-Musach J, De Garibay GR, Boni J, et al. Stem cell-like transcriptional reprogramming mediates metastatic resistance to mTOR inhibition. Oncogene. 2017;36(19):2737-49.

212. Medvedev S, Shevchenko A, Zakian S. Induced pluripotent stem cells: problems and advantages when applying them in regenerative medicine. Acta Naturae (англоязычная версия). 2010;2(2 (5)). 
213. Nishizawa M, Chonabayashi K, Nomura M, Tanaka A, Nakamura M, Inagaki A, et al. Epigenetic variation between human induced pluripotent stem cell lines is an indicator of differentiation capacity. Cell stem cell. 2016;19(3):341-54.

214. Kim K, Doi A, Wen B, Ng K, Zhao R, Cahan P, et al. Epigenetic memory in induced pluripotent stem cells. Nature. 2010;467(7313):285-90.

215. Kim H, Lee G, Ganat Y, Papapetrou EP, Lipchina I, Socci ND, et al. miR-371-3 expression predicts neural differentiation propensity in human pluripotent stem cells. Cell Stem Cell. 2011;8(6):695-706.

216. Masserdotti G, Gascón S, Götz M. Direct neuronal reprogramming: learning from and for development. Development. 2016;143(14):2494-510.

217. Rouaux C, Arlotta P. Direct lineage reprogramming of post-mitotic callosal neurons into corticofugal neurons in vivo. Nature cell biology. 2013;15(2):214-21.

218. Kee N, Volakakis N, Kirkeby A, Dahl L, Storvall H, Nolbrant S, et al. Single-Cell Analysis Reveals a Close Relationship between Differentiating Dopamine and Subthalamic Nucleus Neuronal Lineages. Cell Stem Cell. 2017;20(1):29-40.

219. Kirkeby A, Nolbrant S, Tiklova K, Heuer A, Kee N, Cardoso T, et al. Predictive Markers Guide Differentiation to Improve Graft Outcome in Clinical Translation of hESC-Based Therapy for Parkinson's Disease. Cell Stem Cell. 2017;20(1):135-48.

220. Fatehullah A, Tan SH, Barker N. Organoids as an in vitro model of human development and disease. Nature cell biology. 2016;18(3):246-54.

221. Lancaster MA, Knoblich JA. Organogenesis in a dish: modeling development and disease using organoid technologies. Science. 2014;345(6194).

222. Murphy SV, Atala A. 3D bioprinting of tissues and organs. Nature biotechnology. 2014;32(8):773-85.

223. Dezonne RS, Sartore RC, Nascimento JM, Saia-Cereda VM, Romão LF, Alves-Leon SV, et al. Derivation of functional human astrocytes from cerebral organoids. Scientific reports. 2017;7:45091.

224. Lancaster MA, Renner M, Martin CA, Wenzel D, Bicknell LS, Hurles ME, et al. Cerebral organoids model human brain development and microcephaly. Nature. 2013;501(7467):373-9.

225. Li Y, Muffat J, Omer A, Bosch I, Lancaster MA, Sur M, et al. Induction of expansion and folding in human cerebral organoids. Cell stem cell. 2017;20(3):385-96. e3.

226. Nascimento JM, Martins-de-Souza D. The proteome of schizophrenia. npj Schizophrenia. 2015;1(1):1-11

227. Fu R-H, Wang Y-C, Liu S-P, Shih T-R, Lin H-L, Chen Y-M, et al. Decellularization and recellularization technologies in tissue engineering. Cell transplantation. 2014;23(4-5):621-30. 



\section{Supplementary material}

Supplementary Table 1. Overview of the assays that have been used for the phenotypic characterization of the chemically defined differentiation systems for neuronal induction and differentiation.

\begin{tabular}{lllll}
\hline References Phenotypes & $\begin{array}{c}\text { Spe- } \\
\text { cies }\end{array}$ & $\begin{array}{l}\text { Starting cell } \\
\text { types }\end{array}$ & Cell sorting & \begin{tabular}{l} 
Phenotypic characterization \\
\cline { 3 - 4 }
\end{tabular}
\end{tabular}

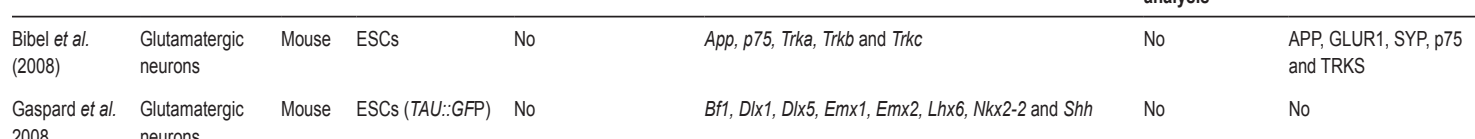

2008 -

$\begin{array}{lllll}\text { Eiraku et al. } & \text { Glutamatergic } & \text { Mouse } & \text { ESCs (Sox1::GFP } & \text { FACS of Bf1::Venus+ } \\ (2008) & \text { neurons } & \begin{array}{l}\text { and } \\ \text { human }\end{array} & \text { and Bf1::Venus) } & \text { cells }\end{array}$

Li et al. (2009) Glutamatergic Human ESCs No neurons

Espuny-Camacho et al. $(2013)$

Kim et al.

(2011c)

Shi et al.

(2012a;

$2012 b)$

Barberi et al. (2003)

$\begin{array}{llll}\begin{array}{l}\text { Maroof et al. } \\ \text { (2010) }\end{array} & \text { GABAergic } & \text { Mouse } & \text { ESCs (Lhx6::GFP) } \\ \text { neurons } & & \\ \text { Watanabe et } & \text { GABAergic } & \text { Mouse } & \text { ESCs (Sox1::G- } \\ \text { al. (2005) } & \text { neurons } & & \text { FP) }\end{array}$

\begin{tabular}{|c|c|c|c|}
\hline Li et al. (2009) & $\begin{array}{l}\text { GABAergic } \\
\text { neurons }\end{array}$ & Human & ESCs \\
\hline $\begin{array}{l}\text { Liu et al. } \\
\text { (2013) }\end{array}$ & $\begin{array}{l}\text { GABAergic } \\
\text { neurons }\end{array}$ & Human & ESCs and iPSCs \\
\hline $\begin{array}{l}\text { Carri et al. } \\
(2013)\end{array}$ & $\begin{array}{l}\text { GABAergic } \\
\text { neurons }\end{array}$ & Human & ESCs and iPSCs \\
\hline
\end{tabular}

Nicholas et al. GABAergic Human ESCs

(2013) neurons
GABAergic

Mouse ESCs

No

Glutamatergic Human ESCs and iPSCs No

Human ESCs and iPSCs No neurons neurons
BF1, DLX2, EMX1, EN1, GAPDH, GL13, HOXB4, NKX21, PAX6 and WNT3A

BF1, BLBP, TDGF1, MYC, CTIP2, CUX1, CUX2, DLX2, DNMT3B, EMX1, EMX2, ETV1, FOXP1, GAPDH, GBX2, GSX2, IRX3, KLF4, NANOG, NKX2-2, OCT3/4, TOX1, PAX6, RELN, REX1, SATB2, SOX1, SOX2, TBR2 TBR1, TERT, VGLUT1, VGLUT2 and YWHAZ

AFP, ASCL1, BF1, BMP4, DACH1, EMX2, GAPDH, GFAP, GLI3, HOXA4, HOXB4, MAP2, NANOG, NCAM1, NKX2-1, OCT3/4, PAX6, PSD95, SHH, SIX3, SOX1, SOX2, SYT1 and T/BRACHYURY

BF1, DLX1, EMX1, HOXB4, ISL1, NKX2-1, OTX1, PAX6, SOX1, SOX2 and TBR2

Microarray (Affymetrix Human

ACTB, GLI3, TBR1, GENOME U133 Plus 2.0 Array) GAD65/67 Microarray (Affy- No metrix Human GENOME U133 Plus 2.0 Array) No GBX2, ISL1, LIX1, LMO3, MSX1, NKX2-1, OTX1, OTX2,

Microarray No Chip)
FACS of Lhx6-GFP+ $\quad \mathrm{No}$

cells

ACS of Sox1-GFP+ $\mathrm{CDH} 1+$ and $\mathrm{OCT} 3 / 4+$ cells

No

FACS of NKX2-1$\mathrm{GFP}+$ cells
Bf1, Bmpr1b, Chat, Chrd, Dat, Dbx1, Dlx-1, Eaac1,

No n1, Fgf8, Fgfr3, Fst, Gad67, Gapdh, Gfap, Gli1, Gli3, Hb9, Irx3, IsI1, Lmx1b, Mag, Map2, Mbp, Msi1, Nes, Ngn2, Nkx2-2, Nkx6-1, Nurr1, Oct3/4, Olig2, Osp, Otx2, Pax2, Pax5, Pax6, Pax7, Pitx3, Shh, Sox10, SOX2 Tuj1 and Th

No

No No

Dlx5, Fgf5, Foxp1, Nodal, Oct3/4, Sox1, T/Brachyury, Tdgf1, Wnt1, Wnt10b, Wnt2b, Wnt3, Wnt3a, Rex1 and Zfp503

BF1, DLX2, EMX1, EN1, GAPDH, GLI3, HOXB4, NKX2- No 1. PAX6 and WNT3A

No

No

ARPP-21, ASCL1, DACH1, DARPP-32, DLX5, DLX6, No DRD1, EAR, EMX2, FOXG1, FOXP1, FOXP2, GAPDH, $P A X 6$ and $S I X 3$

ASCL1, BF1, CALB2, DLX2, GAD67, GAPDH, LHX6, NKX2-1, NPY, OLIG2, PV, POU5F1, SST and TUJ1

(Illumina Human HT-12 Bead-

No

SMAD1, SMAD5 SMAD, Phosphorylated-SMAD1, Phosphorylated-SMAD5 and Phosphorylated-SMAD8

ACTB, DARPP32, GAD65/67, GLI3 and TBR1

No

No and $\mathrm{PSCS}$ 


Immunohistochemistry

GABA, ISL1, NES, PAX6, RC2, TH, TUJ1, VGLUT1 and VGAT

ASCL1, BrdU, CHAT, COUP-TFI, COUP-TFII, CTIP2, CUX1, CALB2, CASP3, Cytokeratin, EN1, ETV1, FOXP2,GABRA6, GFAP, GFP, GSX2, HNF4, MAP2, MATH1, MKI67, Myosin, NKX2-1, NKX2-2, NES, OCT3/4, OTX1, OTX2, P73, PAX6, PECAM1, RET, RELN, SATB2, SOX5, TBR1, TBR2, TH, TLE4, TUJ1, VGAT, VGLUT1 and VGLUT2

BF1, BLBP, BrdU, CALB2, CAMKIIA, CDH2, COUP-TFI, CTIP2, CUX1,EMX1, TUBG1, GAD67, GFP, GRIA1, MAP2, OTX2, P73, Phosphorylated-H3, PROM1, PRKCA, RELN, SATB2, SOX1, SYP, TAU, TBR1, tBR2, TTR, TUJ1, VGLUT1 and ZO1

BF1, CTIP2, DARPP32, GABA, GAD65/67, HOXB4, NKX2-1, PAX6, TBR1, TUJ1 and VGLUT1

a-SMA, AFP, BF1, BLBP, BrdU, BRN2, CALR, CHAT, COUP-TFI, CTIP2, CUX1, FOXP2, GAD67, GFAP,GFP, Homer1, HuNu clone 235-1, MAP2, NANOG, NES, NKX2-1, OCT3/4, OTX1/2, PAX6, SAFB2, SOX2, SSEA3, SSEA4, SYP, TBR1, TBR2, TRA-1-60, TRA-1-81, TH, TUJ1, VGAT and VGLUT1

Alkaline phosphatase, CALR, FLAG M2, FORSE-1, GFAP, GFP, NANOG, NES, OCT3/4, SYN1, TRA-1-81 and TUJ1

Acetylated Tubulin, Atypical PKC, BF1, BrdU, BRN2, CD133, CDH2, CTIP2, CUX1, DCX, GAD67, GFAP, GFP, Gamma Tubulin, Homer1, MAP2, MKI67, MUNC13-1, NCAM, SOX2, NES, OTX1/2, PAX6, PSD95, Phosphorylated-H3, Phosphorylated-Ser55, Phosphorylated-Vimentin, S100, SATB2, SYP, TBR1, TBR2, Transferrin Receptor, TUJ1, VGLUT1 and Z01

5-HT, CNP, EN1, GABA, GFAP, HB9, MBP, MSI1, NCAM, NES, NG2, 01, 04, TH and TUJ1

Electrop-
hysiological
assessment
Whole cell
patch clamp
Whole cell
patch clamp

No

No

(1)

Whole cell

patch clamp

$\begin{array}{lll}\begin{array}{l}\text { Whole cell } \\ \text { patch clamp } \\ \text { (in vitro and ex }\end{array} & \text { Calcium Imaging } & \begin{array}{l}\text { Transplantation into } \\ \text { neonatal mice }\end{array} \\ \begin{array}{l}\text { vivo) } \\ \text { Whole cell }\end{array} & \text { No } & \text { No } \\ \text { patch clamp } & & \end{array}$

Whole cell patch clamp

No

BF1, CALB2, DLX2, GABA, GFP, KV3.1, LHX6, MKI67, NKX2-1, NPY, PV, SST and TBR1

5-HT, ASCL1, BrdU, CDH1, GABA, GAD65, GAD67, GFP, HOXB4, ISL1/2, NES, NKX2-1, NKX2-2, OCT3/4, OTX1, PAX2, PAX6, RC2, TH, TUJ1 and Phosphorylated-histone H3

BF1, CTIP2, DARPP32, GABA, GAD65/67, HOXB4, NKX2-1, PAX6, TBR1, TUJ1 and VGLUT1

BF1, CALB1, GABA, NNOS, NKX2-1, PV, SST and TUJ1

5-HT, A2A, BF1, CALB1, CALR, CHAT, CTIP2, DARPP-32, DRD2, FOXP1, FOXP2, GABA, GAD65/67, GATA4, GFAP, GSX2, MAP2ab, NES, NPY, OCT4, OTX2, PAX6, PV, SOX1, SST, SYP, TH, TUJ1, VGLUT1, p75 and a-SMA

ASCL1, BF1, CALB1, CDH2, CR, CHAT, CTIP2, CXCR4, DARPP32, DCX, DLX2, ETV1, GABA, GFAP, GFP, HuNu, ISL1, LHX6, MKI67, NEUN, NKX2-1, OLIG2, PAX6, PV, RAX, RFP, SST, TBR1, TH, TUJ1 and VGAT

$\begin{array}{lll}\begin{array}{l}\text { Biochemical } \\ \text { assessment }\end{array} & \begin{array}{l}\text { Transplantations/ } \\ \text { Co-culture assay }\end{array} & \begin{array}{l}\text { Other bioassays and } \\ \text { assessments }\end{array}\end{array}$

Calcium Imaging

No

Transplantation into prenatal mice (in utero), transplantation into neonatal mice and postnatal mouse brain slice co-culture assay

\section{Transplantation into} neonatal mice and embryonic mouse forebrain tissue co-culture assay No No

Axonal tracing Artificial synapse formation assay and electron microscopy

Embryonic mouse cortical brain slice co-culture assay

No

No
No

BrdU proliferation assay (Birth-date analysis)
BrdU proliferation assay (Birth-date analysis) and clonal analyses

BrdU proliferation assay (Birth-date analysis) 
Supplementary Table 1. (Continued).

\begin{tabular}{|c|c|c|c|c|c|c|c|}
\hline \multirow[t]{2}{*}{ References } & \multirow[t]{2}{*}{ Phenotypes } & \multirow{2}{*}{$\begin{array}{l}\text { Spe- } \\
\text { cies }\end{array}$} & \multirow{2}{*}{$\begin{array}{l}\text { Starting cell } \\
\text { types }\end{array}$} & \multirow[t]{2}{*}{ Cell sorting } & \multicolumn{3}{|l|}{ Phenotypic characterization } \\
\hline & & & & & (Quantitative) Polymerase chain reaction & $\begin{array}{l}\text { Genome-wide } \\
\text { expression } \\
\text { analysis }\end{array}$ & Western-Blot \\
\hline $\begin{array}{l}\text { Maroof et al. } \\
(2013)\end{array}$ & $\begin{array}{l}\text { GABAergic } \\
\text { neurons }\end{array}$ & Human & $\begin{array}{l}\text { ESCs } \\
(N K X 2-1:: G F P) \\
\text { and iPSCs }\end{array}$ & $\begin{array}{l}\text { FACS of NKX2-1- } \\
\text { GFP+ cells }\end{array}$ & No & $\begin{array}{l}\text { Microarray } \\
\text { (Illumina Human } \\
\text { HT-12 Bead- } \\
\text { Chip) }\end{array}$ & $\begin{array}{l}\text { GAPDH, LHX6 and } \\
\text { RAX }\end{array}$ \\
\hline $\begin{array}{l}\text { Lin et al. } \\
(2015)\end{array}$ & $\begin{array}{l}\text { GABAergic } \\
\text { neurons }\end{array}$ & Human & $\begin{array}{l}\text { Neural precursor } \\
\text { (ReNcell VM) }\end{array}$ & No & $\begin{array}{l}\text { ADORA2A, ARPP-21, ASCL1, BAX, BCL2, CALB1, } \\
\text { CDC20, CTIP2, DARPP32, DRD1, DRD2, EMX2, } \\
\text { FOXP1, FOXP2, GBX2, GFAP, GRIA1, GRIK5, GRIN2B, } \\
\text { HTT, MAP2, MBP, MEIS2, MYC, NES, NKX2-1, PAX6, } \\
\text { REST, RPL19, SOX2 and TUJ1 }\end{array}$ & No & No \\
\hline $\begin{array}{l}\text { Barberi et al. } \\
\text { (2003) }\end{array}$ & $\begin{array}{l}\text { Dopaminergic } \\
\text { neurons }\end{array}$ & Mouse & ESCs & No & $\begin{array}{l}\text { Bf1, Bmpr1b, Chat, Chrd, Dat, Dbx1, Dlx-1, Eaac1, } \\
\text { En1, Fgf8, Fgfr3, Fst, Gad67, Gapdh, Gfap, Gli1, Gli3, } \\
\text { Hb9, Irx3, Isl1, Lmx1b, Mag, Map2, Mbp, Msi1, Nes, } \\
\text { Ngn2, Nkx2-2, Nkx6-1, Nurr1, Oct3/4, Olig2, Osp, Otx2, } \\
\text { Pax2, Pax5, Pax6, Pax7, Pitx3, Shh, Sox10, SOX2, } \\
\text { Tuj1 and Th }\end{array}$ & No & No \\
\hline $\begin{array}{l}\text { Kawasaki et } \\
\text { al. }(2000)\end{array}$ & $\begin{array}{l}\text { Dopaminergic } \\
\text { neurons }\end{array}$ & Mouse & ESCs & No & Gapdh, Nurr1 and Pitx3 & No & No \\
\hline $\begin{array}{l}\text { Lee et al. } \\
(2000)\end{array}$ & $\begin{array}{l}\text { Dopaminergic } \\
\text { neurons }\end{array}$ & Mouse & ESCs & No & $\begin{array}{l}\text { Actb, En1, Fgfr3, Fgf8, Gli1, Nes, Nurr1, Otx1, Otx2, } \\
\text { Pax2, Pax5, Ret, Shh, Smo and Wnt1 }\end{array}$ & No & No \\
\hline $\begin{array}{l}\text { Lim et al. } \\
(2015)\end{array}$ & $\begin{array}{l}\text { Dopaminergic } \\
\text { neurons }\end{array}$ & Human & ESCs & No & $\begin{array}{l}\text { EN1, GAPDH, GIRK2, LMX1B, NURR1, OCT3/4, PITX3, } \\
\text { SMAD1, SMAD5, SMAD8, TH and TUJ1 }\end{array}$ & No & $\begin{array}{l}\text { ACTB, LMNB1, Phos- } \\
\text { phorylated-SMAD1/5/8 } \\
\text { and SMAD1 }\end{array}$ \\
\hline $\begin{array}{l}\text { Yan et al. } \\
(2005)\end{array}$ & $\begin{array}{l}\text { Dopaminergic } \\
\text { neurons }\end{array}$ & Human & ESCs & No & $\begin{array}{l}\text { BF1, EN1, GAPDH, GBX2, LMX1B, NKX6-1, NURR1, } \\
\text { PAX2, PITX3, SHH and WNT1 }\end{array}$ & No & No \\
\hline $\begin{array}{l}\text { Swistowski et } \\
\text { al. }(2010)\end{array}$ & $\begin{array}{l}\text { Dopaminergic } \\
\text { neurons }\end{array}$ & Human & iPSCs & No & $\begin{array}{l}\text { AADC, DAT, EN1, GIRK2, LMX1B, MSX1, NURR1, } \\
\text { OTX2, TH and VMAT }\end{array}$ & $\begin{array}{l}\text { Microarray } \\
\text { (Illumina Human } \\
\text { HT-12 Bead- } \\
\text { Chip) }\end{array}$ & $\begin{array}{l}\text { ACTB, CTNNB1, } \\
\text { OCT3/4, MYC and } \\
\text { NR5A2 }\end{array}$ \\
\hline $\begin{array}{l}\text { Cai et al. } \\
(2010)\end{array}$ & $\begin{array}{l}\text { Dopaminergic } \\
\text { neurons }\end{array}$ & Human & iPSCs & No & $\begin{array}{l}\text { ALDH1A1, CHAT, D BH, FOXA2, GAD67, GAPDH, } \\
\text { GLAST, LMX1A, MSX1, NURR1, PITX3, TH and TPH1 }\end{array}$ & No & No \\
\hline $\begin{array}{l}\text { Doi et al. } \\
\text { (2014) }\end{array}$ & $\begin{array}{l}\text { Dopaminergic } \\
\text { neurons }\end{array}$ & Human & iPSCs & FACS of CORIN+ cells & $\begin{array}{l}\text { EN1, FOXA2, GAPDH, GBX2, GSC, LMX1A, MAP2, } \\
\text { NANOG, NURR1, OCT3/4, OTX2, SIX3, SOX1, SOX17, } \\
\text { T/BRACHYURY and TH }\end{array}$ & $\begin{array}{l}\text { Microarray (Affy- } \\
\text { metrix GeneChip } \\
\text { Whole-Tran- } \\
\text { script Expres- } \\
\text { sion Arrays) }\end{array}$ & No \\
\hline $\begin{array}{l}\text { Kriks et al. } \\
\text { (2011) }\end{array}$ & $\begin{array}{l}\text { Dopaminergic } \\
\text { neurons }\end{array}$ & Human & ESCs and iPSCs & No & ASCL1, FOXA2, LMX1A, NR4A2 and PITX3 & $\begin{array}{l}\text { Microarray } \\
\text { (Illumina Human } \\
\text { HT-12 Bead- } \\
\text { Chip) }\end{array}$ & No \\
\hline Xi et al. (2012) & $\begin{array}{l}\text { Dopaminergic } \\
\text { neurons }\end{array}$ & $\begin{array}{l}\text { Human } \\
\text { and } \\
\text { pri- } \\
\text { mate }\end{array}$ & ESCs and iPSCs & No & No & No & No \\
\hline $\begin{array}{l}\text { Barberi et al. } \\
(2003)\end{array}$ & $\begin{array}{l}\text { Serotonergic } \\
\text { neurons }\end{array}$ & Mouse & ESCs & No & $\begin{array}{l}\text { Bf1, Bmpr1b, Chat, Chrd, Dat, Dbx1, Dlx-1, Eaac1, } \\
\text { En1, Fgf8, Fgfr3, Fst, Gad67, Gapdh, Gfap, Gli1, Gli3, } \\
\text { Hb9, Irx3, Isl1, Lmx1b, Mag, Map2, Mbp, Msi1, Nes, } \\
\text { Ngn2, Nkx2-2, Nkx6-1, Nurr1, Oct3/4, Olig2, Osp, Otx2, } \\
\text { Pax2, Pax5, Pax6, Pax7, Pitx3, Shh, Sox10, SOX2, } \\
\text { Tuj1 and Th }\end{array}$ & No & No \\
\hline
\end{tabular}




Immunohistochemistry

ASCL1, BF1, CALB1, CALB2, CHAT, DLX2, DCX, FOXA2, GABA, GAPDH, Gephyrin, GFAP, GFP, LHX6, MAP2, MKI67, NES, NKX2-1, NNOS, OLIG2, PV, PAX6, PSD95, RAX, SATB2, SC121, SST, TBR1, TH, TUJ1, VGAT, VGLUT1 and VIP

5-HT, ASCL1, BF1, CALB1, CALB2, CHAT, CTIP2, DARPP32, DRD2, GABA, GFAP, MAP2, No MEIS2, NES, NKX2-1, NPY, 01, PAX6, PV, SOX2, SST, TH, TUJ1 and VGLUT1

5-HT, CNP, EN1, GABA, GFAP, HB9, MBP, MSI1, NCAM, NES, NG2, 01, 04, TH and TUJ1 Whole cell patch clamp

5-HT, CDH1, DBH, FLK1, GAD, GFAP, KRT14, MF20, NCAM, NES, PDGFRA, RC2, SYP, $\quad$ No TH, TUJ1 and VACHT

5-HT, DBH, GABA, TH and TUJ1

ACTB, MKI67, NES, SMAD1/5/8, SMAD2/3, TH and TUJ1

5-HT, AADC, CTNNB1, CORIN, EN1, FOXA2, GABA, GIRK2, NCAM, HuNu, LMX1A, MAP2, Whole cell NES, NURR1, PCNA and TH patch clamp

Activity-dependent dopamine release (FAST)

AADC, BF1, CALB1, CCK8, DBH, EN1, GABA, NES, OCT3/4, OTX2, PAX6, PNMT, RET, SSEA4, SOX1, SYP, TH, TUJ1 and VMAT2

GALC, GFAP, GIRK2, HuNu, MSI1, NES, OCT3/4, SOX1, TH, TH clone TH-16 and TUJ1

ALDH1A1, LMX1A, MKI67, NES, HuNu, SOX2, TH and TRKB

5-HT, AADC, CORIN, FOXA2, GIRK2, HuNu, LMX1A, MKI67, NES, NEUN, NURR1, OTX2, PITX3, TH and TUJ1

5-HT, BF1, CALB1, Collagen, CTNNB1, Cytokeratin, DAT, DCX, ED1, FOXA2, GABA, GFAP, GFP, GIRK2, NCAM, HuNu, Human cell adhesion molecule, Human specific cytoplasm (SC-121), IBA1, LMX1A, MKI67, NES, NURR1, OCT3/4, OTX2, PAX6, PITX3, SYN1, TH, TUJ1 and VMAT2

5-HT, ALDH1A1, ASCL1, BF1, CORIN, EN1, FOXA2, FOXP1, GABA, GIRK2, HOXA2, HOXB1, LMX1A, LMX1B, NES, NGN2, NKX6-1, NURR1, PAX6, TH, TUJ1 and OTX2
Whole cell patch clamp

No

No

Whole cell patch clamp
Activity-dependent dopamine release (HPLC)

No
Activity-dependent dopamine release (HPLC)

Activity-dependent (HPLC)

No (HPLC) (HPLC)
Whole cell patch clamp Whole cell patch clamp
Calcium Imaging and GABA release analysis (ELISA)

Activity-dependent dopamine release (HPLC)

Transplantation into 6-OHDA lesioned mice
Transplantation into neonatal mice, mouse brain slice co-culture assay, slice migration analysis and human cortical projection neuron co-culture assay

\section{No}

No

Operetta - automated high content image analysis

ther bioassays and

assessments

No

Cytosolic and nuclear fractionation

\section{Electron microscopy}

Transplantation into 6-OHDA lesioned mice

No

No

\section{No}

No

Transplantation into 6-OHDA lesioned rats

No

No

No

Transplantation into 6-OHDA lesioned rats

No

Activity-dependent Transplantation into dopamine release 6-OHDA lesioned rats

Activity-dependent Transplantation into dopamine release 6-OHDA lesioned rats

\section{Activity-dependent 6-OHDA lesioned dopamine release mice and rats, and (HPLC) MPTP lesioned rhesus monkeys}

No No No

No No No 
Supplementary Table 1. (Continued).

\begin{tabular}{|c|c|c|c|c|c|c|c|}
\hline \multirow[t]{2}{*}{ References } & \multirow[t]{2}{*}{ Phenotypes } & \multirow{2}{*}{$\begin{array}{l}\text { Spe- } \\
\text { cies }\end{array}$} & \multirow{2}{*}{$\begin{array}{l}\text { Starting cell } \\
\text { types }\end{array}$} & \multirow[t]{2}{*}{ Cell sorting } & \multicolumn{3}{|l|}{ Phenotypic characterization } \\
\hline & & & & & (Quantitative) Polymerase chain reaction & $\begin{array}{l}\text { Genome-wide } \\
\text { expression } \\
\text { analysis }\end{array}$ & Western-Blot \\
\hline $\begin{array}{l}\text { Lee et al. } \\
(2000)\end{array}$ & $\begin{array}{l}\text { Serotonergic } \\
\text { neurons }\end{array}$ & Mouse & ESCs & No & $\begin{array}{l}\text { Actb, En1, Fgfr3, Fgf8, Gli1, Nes, Nurr1, Otx1, Otx2, } \\
\text { Pax2, Pax5, Ret, Shh, Smo and Wnt1 }\end{array}$ & No & No \\
\hline $\begin{array}{l}\text { Shimada et al. } \\
\text { (2012) }\end{array}$ & $\begin{array}{l}\text { Serotonergic } \\
\text { neurons }\end{array}$ & Mouse & $\begin{array}{l}\text { ESCs (ePet::EG- } \\
F P) \text { and iPSCs }\end{array}$ & $\begin{array}{l}\text { FACS of ePet-EGFP+ } \\
\text { cells. }\end{array}$ & $\begin{array}{l}\text { Actb, Ascl1, Chat, Gfap, Gad67, Map2, Mbp, Nes, Nkx2- } \\
\text { 2, Pet-1, Phox2b, Sert, Shh, Th and Tph2 }\end{array}$ & No & No \\
\hline $\begin{array}{l}\text { Kumar et al. } \\
(2009)\end{array}$ & $\begin{array}{l}\text { Serotonergic } \\
\text { neurons }\end{array}$ & Human & ESCs & No & $\begin{array}{l}\text { ASCL1, FEV, GAPDH, GATA2, LMX1B, NKX2-2 and } \\
\text { SERT }\end{array}$ & No & No \\
\hline $\begin{array}{l}\text { Vadodaria et } \\
\text { al. (2015) }\end{array}$ & $\begin{array}{l}\text { Serotonergic } \\
\text { neurons }\end{array}$ & Human & $\begin{array}{l}\text { ESCs (TPH2::G- } \\
F P, T P H 2:: T d T \\
\text { and SYN- } \\
\text { 1::dsRed) }\end{array}$ & $\begin{array}{l}\text { FACS of TPH2-GFP+ } \\
\text { and SYN1-dsRed+ } \\
\text { cells }\end{array}$ & FEV, GATA2, LHX6, LMX1B, NKX2-2 and TPH2 & No & No \\
\hline $\begin{array}{l}\text { Lu et al. } \\
\text { (2016) }\end{array}$ & $\begin{array}{l}\text { Serotonergic } \\
\text { neurons }\end{array}$ & Human & ESCs and iPSCs & $\begin{array}{l}\text { FACS OF NKX2-2+ } \\
\text { cells. }\end{array}$ & $\begin{array}{l}\text { BF1, CDH2, CDX2, DBX1, EGR2, EMX1, EN1, FEV, } \\
\text { GAPDH, GBX2, HOXA1, HOXA2, HOXA3, HOXA4, } \\
\text { HOXB1, HOXB2, HOXB3, HOXB4, HOXB6, HOXB8, } \\
\text { HOXC5, HOXC6, IRX3, LHX1, LMX1A, LMX1B, } \\
\text { NANOG, NKX2-1, NKX2-2, NKX6-1, OCT3/4, OLIG2, } \\
\text { OTX2, PAX6, PAX7, SERT, SIM1, SIX3, SOX1, SOX17, } \\
\text { T/BRACHYURY, TBR2 and TPH2 }\end{array}$ & No & $\begin{array}{l}\text { FEV, GAPDH and } \\
\text { SERT }\end{array}$ \\
\hline $\begin{array}{l}\text { Barberi et al. } \\
\text { (2003) }\end{array}$ & $\begin{array}{l}\text { Cholinergic/Mo- } \\
\text { tor neurons }\end{array}$ & Mouse & ESCs & No & $\begin{array}{l}\text { Bf1, Bmpr1b, Chat, Chrd, Dat, Dbx1, Dlx-1, Eaac1, En1, } \\
\text { Fgf8, Fgfr3, Fst, Gad67, Gapdh, Gfap, Gli1, Gli3, Hb9, } \\
\text { Irx3, Isl1, Lmx1b, Mag, Map2, Mbp, Msi1, Nes, Ngn2, } \\
\text { Nkx2-2, Nkx6-1, Nurr1, Oct3/4, Olig2, Osp, Otx2, Pax2, } \\
\text { Pax5, Pax6, Pax7, Pitx3, Shh, Sox10, SOX2, Tuj1 and Th }\end{array}$ & No & No \\
\hline $\begin{array}{l}\text { Wichterle et } \\
\text { al. (2002) }\end{array}$ & $\begin{array}{l}\text { Cholinergic/Mo- } \\
\text { tor neurons }\end{array}$ & Mouse & ESCs (Hb9:GFP) & $\begin{array}{l}\text { FACS of Hb9-GFP+ } \\
\text { cells }\end{array}$ & No & No & No \\
\hline Li et al. (2005) & $\begin{array}{l}\text { Cholinergic/Mo- } \\
\text { tor neurons }\end{array}$ & Human & ESCs & No & $\begin{array}{l}\text { GAPDH, HOXB1, HOXB6, HOXC5, HOXC8, IRX3, } \\
\text { NKX2-2, NKX6-1, OLIG2, OTX2, PAX6, SHH and SOX1 }\end{array}$ & No & No \\
\hline $\begin{array}{l}\text { Singh Roy et } \\
\text { al. (2005) }\end{array}$ & $\begin{array}{l}\text { Cholinergic/Mo- } \\
\text { tor neurons }\end{array}$ & Human & $\begin{array}{l}\text { ESCs }(H b 9: E G- \\
F P)\end{array}$ & $\begin{array}{l}\text { FACS of Hb9-EGFP+ } \\
\text { cells }\end{array}$ & EN1, ISL1, OCT3/4 and PAX7 & No & No \\
\hline $\begin{array}{l}\text { Dimos et al. } \\
\text { (2008) }\end{array}$ & $\begin{array}{l}\text { Cholinergic/Mo- } \\
\text { tor neurons }\end{array}$ & Human & ESCs and iPSCs & No & $\begin{array}{l}\text { MYC, FOXD3, GAPDH, KLF4, NANOG, OCT3/4, REX1, } \\
\text { SOD1, SOX3, TDGF1 and TERT }\end{array}$ & No & No \\
\hline $\begin{array}{l}\text { Qu et al. } \\
\text { (2014) }\end{array}$ & $\begin{array}{l}\text { Cholinergic/Mo- } \\
\text { tor neurons }\end{array}$ & Human & ESCs and iPSCs & No & $\begin{array}{l}\text { ARX, BCL2, BRN2, CACNA1A, CEP290, CHAT, CHX10, } \\
\text { CNTNAP2, COUP-TFI, COUP-TFII, CREBBP, CTNNA2, } \\
\text { EGFL7, EGR2, EMX2, EP3O0, EVI1, FEZFF1, FEZF2, } \\
\text { FOXA2, GAS1, GBX2, GNPAT, HB9, HES3, HESX1, } \\
\text { HESX1, HOXC4, HSPA5, KAT2B, LEF1, LHX2, MAFB, } \\
\text { MTPN, NCOA1, NCOA2, NCOA3, NEUROD1, NKX6-1, } \\
\text { OTX1, POU3F1, RARA, RARB, RARG, RAX, RORA, } \\
\text { RXRA, RXRB, RXRG, SDF4, SIX3, SMO, SOX3, ULK1, } \\
\text { WNT7B and ZEB2 }\end{array}$ & No & $\begin{array}{l}\text { GAPDH, NANOG, } \\
\text { OCT3/4, PAX6, SOX1 } \\
\text { and ZIC1 }\end{array}$ \\
\hline $\begin{array}{l}\text { Karumbay- } \\
\text { aram et al. } \\
\text { (2009) }\end{array}$ & $\begin{array}{l}\text { Cholinergic/Mo- } \\
\text { tor neurons }\end{array}$ & Human & $\begin{array}{l}\text { ESCs and iPSCs } \\
(H b 9: E G F P)\end{array}$ & No & No & No & No \\
\hline $\begin{array}{l}\text { Amoroso et } \\
\text { al. (2013) }\end{array}$ & $\begin{array}{l}\text { Cholinergic/Mo- } \\
\text { tor neurons }\end{array}$ & Human & $\begin{array}{l}\text { ESCs and iPSCs } \\
(H b 9: G F P)\end{array}$ & $\begin{array}{l}\text { FACS of Hb9-GFP+ } \\
\text { cells }\end{array}$ & $\begin{array}{l}\text { CHT1, FOXP1, HOXA5, HOXC6, HOXC8, HOXD9, } \\
\text { LHX3 and RALDH2 }\end{array}$ & $\begin{array}{l}\text { Whole tran- } \\
\text { scriptome RNA } \\
\text { sequencing } \\
\text { (Illumina HiSeq) }\end{array}$ & No \\
\hline $\begin{array}{l}\text { Shimojo et al. } \\
\text { (2015) }\end{array}$ & $\begin{array}{l}\text { Cholinergic/Mo- } \\
\text { tor neurons }\end{array}$ & Human & $\begin{array}{l}\text { ESCs and iPSCs } \\
\text { (HB9::Venus) }\end{array}$ & $\begin{array}{l}\text { FACS of HBg-Venus }+ \\
\text { cells. }\end{array}$ & $\begin{array}{l}\text { ACTB, CHAT, EBNA-1, HB9, ISL1, KLF4, MYCL, LIN28, } \\
\text { NANOG, NGN2, NKX2-2, OCT3/4, OLIG2, PAX6, SOX1 } \\
\text { and SOX2 }\end{array}$ & No & $\begin{array}{l}\text { ACTB, CHAT, HB9 } \\
\text { and ISL1 }\end{array}$ \\
\hline $\begin{array}{l}\text { Lee et al. } \\
(2007)\end{array}$ & $\begin{array}{l}\text { Cholinergic/Mo- } \\
\text { tor neurons }\end{array}$ & $\begin{array}{l}\text { Human } \\
\text { and } \\
\text { pri- } \\
\text { mate }\end{array}$ & $\begin{array}{l}\text { ESCS } \\
\text { (RUES1-EGFP) }\end{array}$ & No & $\begin{array}{l}\text { BF1, CHAT, GAPDH, HOXB4, ISL1, NGN2, NKX2-2, } \\
\text { NKX6-1, OLIG2, OLIG3, PAX6, PAX7, SOX1 and VACHT }\end{array}$ & $\begin{array}{l}\text { Microarray (Affy- } \\
\text { metrix Human } \\
\text { GENOME U133 } \\
\text { Plus } 2.0 \text { Array) }\end{array}$ & No \\
\hline
\end{tabular}

Neuronal phenotypes, species and starting cell types are summarized for each protocol according to their reference. Cell sorting techniques and the phenotypic characterization, consisting of assays such as (quantitative) polymerase chain reaction, genome-wide expression analysis, western-blot, immunohistochemistry, electrophysiological assessment, biochemical assessment, transplantation in to animal models, co-culture assays or other bioassays, are broadly outlined if applicable. 


\begin{tabular}{|c|c|c|c|c|}
\hline Immunohistochemistry & $\begin{array}{l}\text { Electrop- } \\
\text { hysiological } \\
\text { assessment }\end{array}$ & $\begin{array}{l}\text { Biochemical } \\
\text { assessment }\end{array}$ & $\begin{array}{l}\text { Transplantations/ } \\
\text { Co-culture assay }\end{array}$ & $\begin{array}{l}\text { Other bioassays and } \\
\text { assessments }\end{array}$ \\
\hline 5-HT, DBH, GABA, TH and TUJ1 & No & No & No & No \\
\hline 5-HT, GABA, MAP2, NES, TH and TUJ1 & $\begin{array}{l}\text { Whole cell } \\
\text { patch clamp }\end{array}$ & $\begin{array}{l}\text { Activity-dependent } \\
5 \text {-HT release } \\
\text { (HPLC) }\end{array}$ & No & No \\
\hline 5-HT, BLBP, GFAP, MAP2, NES, TPH, TUJ1 and Vimentin & No & $\begin{array}{l}\text { Activity-dependent } \\
\text { 5-HT release }\end{array}$ & No & $\begin{array}{l}5 \text {-HT imaging (Three-photon } \\
\text { microscopy) and analysis of the } \\
\text { relative } 5 \text {-HT content }\end{array}$ \\
\hline 5-HT, GABA, MAP2ab, TH, TPH and TPH2 & No & No & No & No \\
\hline $\begin{array}{l}\text { 5-HT, AADC, BF1, EN1, FEV, FOXA2, GABA, GAPDH, GATA2, GATA3, GFAP, HOXA2, } \\
\text { HOXA3, HOXB1, HOXB4, HTR1A, MKI67, NES, NKX2-2, NKX6-1, OCT3/4, OLIG2, OTX2, } \\
\text { PAX3/7, PAX6, PHOX2B, SERT, SOX1, SOX2, TH, TPH2, TUJ1 and VMAT2 }\end{array}$ & $\begin{array}{l}\text { Whole cell } \\
\text { patch clamp }\end{array}$ & $\begin{array}{l}\text { Activity-dependent } \\
\text { 5-HT release (UP- } \\
\text { LC-ESI-MS/MS) }\end{array}$ & No & No \\
\hline 5-HT, CNP, EN1, GABA, GFAP, HB9, MBP, MSI1, NCAM, NES, NG2, 01, O4, TH and TUJ1 & No & No & No & No \\
\hline $\begin{array}{l}\text { CHAT, CHX10, DBX1, EN1, GFP, HB9, HOXC5, HOXC6, IRX3, ISL1, LHX3, LIM1, LIM2, } \\
\text { NCAM, NEUN, Neurofilament, NKX2-2, NKX6-1, OLIG2, OTX2, PAX6, PAX7, SC1, SOX1, } \\
\text { SYB, SYN, TUJ1 and VACHT }\end{array}$ & No & No & $\begin{array}{l}\text { Chick spinal cords } \\
\text { (in ovo) }\end{array}$ & $\begin{array}{l}\text { BrdU proliferation assay (Birth-date } \\
\text { analysis) }\end{array}$ \\
\hline CHAT, HB9, ISL1/2, LHX3, NES, OLIG2, OTX2, PAX6, PAX7, SOX1, TUJ1 and VACHT & $\begin{array}{l}\text { Whole cell } \\
\text { patch clamp }\end{array}$ & No & $\begin{array}{l}\text { Mouse myocyte } \\
\text { co-culture }\end{array}$ & No \\
\hline CHAT, Desmin, GFP, HB9, ISL1, SSEA4, SV2, TRA 1-81 and TUJ1 & $\begin{array}{l}\text { Whole cell } \\
\text { patch clamp }\end{array}$ & No & $\begin{array}{l}\text { Rat muscle co-culture } \\
\text { assay }\end{array}$ & No \\
\hline $\begin{array}{l}\text { a-SMA, AFP, CHAT, Desmin, GFAP, HB9, ISL1/2, NANOG, SSEA-3, SSEA4, TE-7, TRA-1- } \\
\text { 60, TRA-1-81 and TUJ1 }\end{array}$ & No & No & No & No \\
\hline CHAT, CHX10, HB9, ISL1, MAP2, NANOG, OCT3/4, SMI-32, SV2, SYN1 and TUJ1 & $\begin{array}{l}\text { Whole cell } \\
\text { patch clamp }\end{array}$ & No & $\begin{array}{l}\text { Mouse myocyte } \\
\text { co-culture }\end{array}$ & $\begin{array}{l}\text { Synaptic structure and neuromus- } \\
\text { cular junction analysis (Multiphoton } \\
\text { microscopy) }\end{array}$ \\
\hline $\begin{array}{l}\text { BRN2, CHAT, HOXA3, HOXA5, HOXC6, HOXC8, ISL1, LHX3, NKX6-1, OLIG2, PAX6, PAX7, } \\
\text { NES, SOX1, SOX2, SOX3 and TUJ1 }\end{array}$ & $\begin{array}{l}\text { Whole cell } \\
\text { patch clamp }\end{array}$ & No & No & No \\
\hline BRN3A, FOXP1, GFP, HB9, ISL1, LHX3, NF-H, RALDH2 and TUJ1 & $\begin{array}{l}\text { Whole cell } \\
\text { patch clamp }\end{array}$ & Calcium Imaging & $\begin{array}{l}\text { Chick spinal cords } \\
\text { (in ovo) }\end{array}$ & No \\
\hline CHAT, GFP, HB9, ISL1, MYHC and TUJ1 & No & No & $\begin{array}{l}\text { Human myocyte } \\
\text { co-culture }\end{array}$ & No \\
\hline $\begin{array}{l}\text { BF1, CHAT, HB9, HOXB4, HOXC6, HOXC8, HuNu, ISL1, LHX3, NCAM, NKX2-2, OLIG2, } \\
\text { OTX2, PAX6, PAX7, SOX1 and TUJ1 }\end{array}$ & $\begin{array}{l}\text { Whole cell } \\
\text { patch clamp }\end{array}$ & $\begin{array}{l}\text { Activity-dependent } \\
\text { acetylcholine } \\
\text { release (HPLC) }\end{array}$ & $\begin{array}{l}\text { Chick eggs (in ovo) } \\
\text { and rat spinal cords } \\
\text { (in vivo) }\end{array}$ & No \\
\hline
\end{tabular}


Supplementary Table 2. Overview of the assays that have been used for the phenotypic characterization of the transcription factor-mediated neuronal reprogramming protocols.

\begin{tabular}{|c|c|c|c|c|c|c|c|}
\hline \multirow[t]{2}{*}{ References } & \multirow[t]{2}{*}{ Phenotypes } & \multirow{2}{*}{$\begin{array}{l}\text { Spe- } \\
\text { cies }\end{array}$} & \multirow{2}{*}{$\begin{array}{l}\text { Starting cell } \\
\text { types }\end{array}$} & \multirow[t]{2}{*}{ Cell sorting } & \multicolumn{3}{|l|}{ Phenotypic characterization } \\
\hline & & & & & (Quantitative) Polymerase chain reaction & $\begin{array}{l}\text { Genome-wide } \\
\text { expression } \\
\text { analysis }\end{array}$ & Western-Blot \\
\hline $\begin{array}{l}\text { Vierbuchen et } \\
\text { al. (2010) }\end{array}$ & $\begin{array}{l}\text { Glutamatergic } \\
\text { neurons }\end{array}$ & Mouse & $\begin{array}{l}\text { Fibroblasts } \\
\text { (Tau::EGFP) }\end{array}$ & $\begin{array}{l}\text { FACS of Tau-EGFP+ } \\
\text { cells }\end{array}$ & Actb, Sox1 and Sox10 & No & No \\
\hline
\end{tabular}

\begin{tabular}{|c|c|c|c|c|c|}
\hline $\begin{array}{l}\text { Heinrich et al. } \\
\text { (2010) }\end{array}$ & $\begin{array}{l}\text { Glutamatergic } \\
\text { neurons }\end{array}$ & Mouse & Astocytes & No & $\begin{array}{l}\text { Aldh1l1, Emx1, Emx2, Gapdh, Gfap, Glt1, Glu1, Ngn2, } \\
\text { S100ß, Sox2 and Tuj1 }\end{array}$ \\
\hline $\begin{array}{l}\text { Thoma et al. } \\
\text { (2012) }\end{array}$ & $\begin{array}{l}\text { Glutamatergic } \\
\text { neurons }\end{array}$ & Mouse & ESCs & No & $\begin{array}{l}\text { Afp, Dcx, Ef1a1, Gad1, Insulin, Math3, Myod1, Nanog, } \\
\text { Neun, Ngn2, Ngn2 (Transient), Ngn2 (Inducible), Oct3/4, } \\
\text { Olig2, Pax6, Pu1, Sox1, Th, Vglu2 and Vglut1 }\end{array}$ \\
\hline $\begin{array}{l}\text { Chanda et al. } \\
\text { (2014) }\end{array}$ & $\begin{array}{l}\text { Glutamatergic } \\
\text { neurons }\end{array}$ & $\begin{array}{l}\text { Mouse } \\
\text { and } \\
\text { human }\end{array}$ & $\begin{array}{l}\text { Fibroblasts } \\
\text { (Tau::EGFP) and } \\
\text { ESCs }\end{array}$ & No & Ascl1, Ngn2, Myt11, Brn2 and Gapdh \\
\hline $\begin{array}{l}\text { Pang et al. } \\
\text { (2011) }\end{array}$ & $\begin{array}{l}\text { Glutamatergic } \\
\text { neurons }\end{array}$ & Human & $\begin{array}{l}\text { Fibroblasts } \\
(\text { Tau::EGFP), } \\
\text { ESCs and iPSCs }\end{array}$ & No & $\begin{array}{l}\text { ACTB, ASCL1, AsCl1, BRN1, BRN2, Brn2, CAMKIIB, } \\
\text { DCX, GAD65, GAD67, GAPDH, GFAP, MAP2, MYT1L, } \\
\text { Myt1I, NCAM, NES, NEUROD1, Neurod1, OLIG2, P75, } \\
\text { PAX6, PV, SOX10, SOX9, SYN1, TH, TUJ1, VGAT, } \\
\text { VGLUT1, VGLUT2 and VGLUT3 }\end{array}$ \\
\hline Zhang et al. & Glutamatergic & Human & ESCs and iPSCs & No & No \\
\hline
\end{tabular}

(2013) neurons

\begin{tabular}{|c|c|c|c|c|c|c|c|}
\hline $\begin{array}{l}\text { Addis et al. } \\
\text { (2011) }\end{array}$ & $\begin{array}{l}\text { Dopaminergic } \\
\text { neurons }\end{array}$ & Mouse & $\begin{array}{l}\text { Astrocytes } \\
\text { (MAP2::CD4 and } \\
\text { MAP2::GCaMP3) } \\
\text { and fibroblasts }\end{array}$ & $\begin{array}{l}\text { MACS of MAP2-CD4+ } \\
\text { cells }\end{array}$ & $\begin{array}{l}\text { Aldh1a7, Brn2, Cacna1g, Calb1, Dat, Ddc, En1, Foxa2, } \\
\text { Gabra1, Gad65, Grin1, Kcnn3, Lmx1a, Msx1, Ngn2, } \\
\text { Pax2, Pax5, Pitx3, Scn3a, Syn2, Tau, Th, Vglut1 and } \\
\text { Vmat2 }\end{array}$ & No & No \\
\hline $\begin{array}{l}\text { Kim et al. } \\
\text { (2011b) }\end{array}$ & $\begin{array}{l}\text { Dopaminergic } \\
\text { neurons }\end{array}$ & Mouse & $\begin{array}{l}\text { Fibroblasts } \\
\text { (Pitx3::EGFP) }\end{array}$ & $\begin{array}{l}\text { FACS of Pitx3-EGFP+ } \\
\text { cells }\end{array}$ & Aadc, Dat, Th and Vmat2 & No & No \\
\hline $\begin{array}{l}\text { Caiazzo et al. } \\
\text { (2011) }\end{array}$ & $\begin{array}{l}\text { Dopaminergic } \\
\text { neurons }\end{array}$ & $\begin{array}{l}\text { Mouse } \\
\text { and } \\
\text { human }\end{array}$ & $\begin{array}{l}\text { Mouse fibroblasts } \\
\text { (TH::GFP) and } \\
\text { human fibroblasts }\end{array}$ & $\begin{array}{l}\text { FACS of TH-GFP+ } \\
\text { cells }\end{array}$ & $\begin{array}{l}\text { ALDH1A1, Aldh1a1, Ascl1 (Viral), DAT, Dat, Drd2, En1, } \\
\text { GAPDH, Gapdh, Lmx1a, Lmx1a (Viral), Lmx1b, Ngn2, } \\
\text { Nurr1, Nurr1 (Viral), Otx2, Pitx3, Sox2, TH, Th, VMAT2 } \\
\text { and Vmat2 }\end{array}$ & $\begin{array}{l}\text { Microarray (Affy- } \\
\text { metrix Gene- } \\
\text { Chip Mouse } \\
\text { Gene ST1.0) }\end{array}$ & No \\
\hline $\begin{array}{l}\text { Pfisterer et al. } \\
\text { (2011) }\end{array}$ & $\begin{array}{l}\text { Dopaminergic } \\
\text { neurons }\end{array}$ & Human & Fibroblasts & No & $\begin{array}{l}\text { ACTB, Ascl1 (Viral), BLBP, Brn2 (Viral), En1 (Viral), } \\
\text { Foxa2 (Viral), GAPDH, Gli1 (Viral), Lmx1a (Viral), Lmx1b } \\
\text { (Viral), MAP2, Msx1 (Viral), Myt1I (Viral), NGN2, Nurr1 } \\
\text { (Viral), Otx2 (Viral), Pax2 (Viral), Pax5 (Viral), PAX6, } \\
\text { PAX7, SOX1, SOX2 and SOX10 }\end{array}$ & No & No \\
\hline $\begin{array}{l}\text { Theka et al. } \\
\text { (2013) }\end{array}$ & $\begin{array}{l}\text { Dopaminergic } \\
\text { neurons }\end{array}$ & Human & iPSCs & No & $\begin{array}{l}\text { ALDH1A1, ASCL1, Ascl1 (Viral), CALB1, CORIN, DAT, } \\
\text { DDC, DRD2, EN1, FOXA2, GAPDH, GIRK2, LMX1B, } \\
\text { LMX1A, LmX1a (Viral), NURR1, Nurr1 (Viral), OTX2, } \\
\text { PITX3, TH and VMAT2 }\end{array}$ & No & No \\
\hline Wasko (2013) & $\begin{array}{l}\text { GABAergic } \\
\text { neurons }\end{array}$ & Mouse & Fibroblasts & No & No & No & No \\
\hline $\begin{array}{l}\text { Heinrich et al. } \\
(2010)\end{array}$ & $\begin{array}{l}\text { GABAergic } \\
\text { neurons }\end{array}$ & Mouse & Astocytes & No & $\begin{array}{l}\text { Aldh1l1, Emx1, Emx2, Gapdh, Gfap, Glt1, Glu1, Ngn2, } \\
\text { S100B, Sox2 and Tuj1 }\end{array}$ & No & No \\
\hline $\begin{array}{l}\text { Colasante et } \\
\text { al. (2015) }\end{array}$ & $\begin{array}{l}\text { GABAergic } \\
\text { neurons }\end{array}$ & $\begin{array}{l}\text { Mouse } \\
\text { and } \\
\text { human }\end{array}$ & $\begin{array}{l}\text { Mouse fibroblasts } \\
\text { (GAD67::GFP), } \\
\text { human firbroblasts } \\
\text { and human iPSCs }\end{array}$ & $\begin{array}{l}\text { FACS of GAD67- } \\
\text { GFP+ cells }\end{array}$ & $\begin{array}{l}\text { 18s, ACTB, Arx, Ascl1, Bf1, Calb1, Calb2, Chat, } \\
\text { Col24a1, Col9a2, Coup-tfl, Coup-tfll, Darpp32, DLX1, } \\
\text { DLX2,Dlx2, Dlx5, Dlx6, Erbb4, Etv1, GAD67, GAD65, } \\
\text { Gbx2, Gfap, Helt, Isl1, Kv3.1, Lhx6, Lhx8, Mafb, Mef2c, } \\
\text { Nkx2-1, Olig2, PV, Ptf1a, Reln, SST, Satb1, Sox2, Sox6, } \\
\text { Sp8, Tbx1, Tbx2, VGLUT1, Vglut1 and VIP }\end{array}$ & $\begin{array}{l}\text { Whole tran- } \\
\text { scriptome RNA } \\
\text { sequencing } \\
\text { (NEBNext Ultra } \\
\text { Directional RNA } \\
\text { Library Prep } \\
\text { Kit and Illumina } \\
\text { HiSeq 2500) }\end{array}$ & ASCL1, Sox2 and V5 \\
\hline
\end{tabular}




Immunohistochemistry

5-HT, BrdU, CHAT, CALB2, GABA, GAD65, GFAP, MAP2, NEUN, NKX2-2, OLIG1, PAX3, PAX6, PAX7, PRPH, SOX2, SYN1, TH, TUJ1 and VGLUT1

CALR, CAMKIla, GAD1, GFAP, GFP, MAP2, RFP, SYN1, TBR1, TBR2, TUJ1, VGAT and VGLUT1

MAP2, MAP2ab, MYC, NANOG, NR1, STAT3, SYN1, TAU, TH, TUJ1 and VGLUT1

GFP, MAP2, NEUN, SYN1, TUJ1, VGAT and VGLUT1

ASCL1, BrdU, BRN2, GAD65, GFAP, LU5, MAP2, NEUN, Neurofilament, PRPH, SOX10, SYN1, SYT1, TBR1, TH, TUJ1 and VGLUT1

GAD6, GFAP, MAP2, NANOG, NEUN, OCT3/4, SOX2, SYN1, TUJ1 and VGLUT1

GIRK2, OTX2, SYP, TH, TUJ1 and V5

AADC, DAT, EGFP, MAP2, PITX3, TH and TUJ1

AADC, ALDH1A1, BrdU, CALB1, DAT, DRD2, GFP, MAP2, OTX2, SYN1, SYT1, TH, TUJ1 and VMAT2

5-HT, AADC, BLBP, Collagen I, Collagen II, GABA, Glutamate, MAP2, NES, NGN2, NKX2-2, NURR1, P75, PAX6, PAX7, PRPH, SOX1, SOX2, SYP, TE-7, TH and TUJ1

ALDH1A1, CALB1, DDC, FOXA2, GFP, GIRK2, MAP2, NANOG, OCT3/4, SOX2, SYT1, TH, TRA-1-60, TUJ1 and VMAT2

GABA, MAP2, NEUN, TUJ1 and VGLUT1

CALR, CAMKIla, GAD1, GFAP, GFP, MAP2, RFP, SYN1, TBR1, TBR2, TUJ1, VGAT and VGLUT1

ARX, CALB1, CALB2, GABA, GAD65/67, GFP, HOMER1, KV3.1B, MAP2, NPY, NAV, PV, RFP, SST, SYT1, TUJ1 and VGAT

$\begin{array}{ll}\begin{array}{l}\text { Electro- } \\ \text { physiological }\end{array} & \text { Biochemical } \\ \text { assessment } & \end{array}$

Whole cell No
patch clamp

\begin{tabular}{|c|c|}
\hline Whole cell & Calcium imaging \\
\hline Whole cell & No \\
\hline
\end{tabular}

patch clamp

Whole cell

patch clamp

No

No

Whole cell

patch clamp

No

Mouse cortical neu
co-culture assay

Whole cell

patch clamp

Calcium imaging

Can

\section{Transplantations/ Other bioassays and \\ Co-culture assay \\ assessments}

Neonatal mouse neu- BrdU proliferation assay (Birth-

ron co-culture assay date analysis)

and postnatal mouse

astrocyte co-culture

assay

No

Mouse hippocampa

neuron co-culture

assay

Mouse hippocampal

neuron co-culture

assay

Mouse cortical neuron No

No

No

No

Glia-neuron co-culture assay and transplantation into mice striatum

Optogenetic based presynaptic assesment

Whole cell
patch clamp
Whole cell
patch clamp
Whole cell
patch clamp
(in vitro and in
vivo)

Whole cell

patch clamp

Whole cell patch clamp

No

Whole cell patch clamp

Whole cell patch clamp
Activity dependent

dopamine release

(HPLC) and calcium Imaging

Activity dependent dopamine release (HPLC)

Activity dependent dopamine release (HPLC) and FM464 assay

No

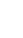

\section{Activity dependent Transplantation into No dopamine release postnatal mice (HPLC) \\ Calcium imaging No \\ GABA concentrati- Transplantation into}

on measurements adult mice hippocam(ELISA) pus (in vivo) and rat hippocampal co-culture assay

No

Southern blot

Evoked catecholamine exocytosis (Amperometric recording), bisulfite promotor sequencing (Th and Vmat2) and electron microscopy

No

No

No

Functional GABAergic neurotransmission (optogenetics), pre-embedding immunogold localization experiment (electron microscopy) and chromatin immunoprecipitation (for ASCL1, BF1 and SOX2) 
Supplementary Table 2. (Continued)

\begin{tabular}{|c|c|c|c|c|c|c|c|}
\hline \multirow[t]{2}{*}{ References } & \multirow[t]{2}{*}{ Phenotypes } & \multirow{2}{*}{$\begin{array}{l}\text { Spe- } \\
\text { cies }\end{array}$} & \multirow{2}{*}{$\begin{array}{l}\text { Starting cell } \\
\text { types }\end{array}$} & \multirow[t]{2}{*}{ Cell sorting } & \multicolumn{3}{|l|}{ Phenotypic characterization } \\
\hline & & & & & (Quantitative) Polymerase chain reaction & $\begin{array}{l}\text { Genome-wide } \\
\text { expression } \\
\text { analysis }\end{array}$ & Western-Blot \\
\hline $\begin{array}{l}\text { Xu et al. } \\
\text { (2015) }\end{array}$ & $\begin{array}{l}\text { Serotonergic } \\
\text { neurons }\end{array}$ & Human & Fibroblasts & No & $\begin{array}{l}\text { AADC, ALDH1A1, ASCL1, ASCL1 (Viral), CACNA1C, } \\
\text { FEV, FEV (Viral), FOXA2, FOXA2 (Viral), GAPDH, } \\
\text { LMX1B, LMX1B (Viral), MAP2, hp53ShRNA (Viral), } \\
\text { PCLO, SCN1A, SERT, SYN1, TPH1, TPH2, TUJ1 and } \\
\text { VMAT2 }\end{array}$ & No & No \\
\hline $\begin{array}{l}\text { Vadodaria et } \\
\text { al. (2015) }\end{array}$ & $\begin{array}{l}\text { Serotonergic } \\
\text { neurons }\end{array}$ & Human & $\begin{array}{l}\text { Fibroblasts } \\
\text { (TPH2::GFP and } \\
\text { SYN1::dsRed) }\end{array}$ & $\begin{array}{l}\text { FACS of TPH2-GFP+ } \\
\text { and SYN1-dsRed+ } \\
\text { cells }\end{array}$ & FEV, GATA2, LHX6, LMX1B, NKX2-2 and TPH2 & $\begin{array}{l}\text { Whole tran- } \\
\text { scriptome RNA } \\
\text { sequencing } \\
\text { (Illumina HiSeq } \\
\text { 2500) }\end{array}$ & $\begin{array}{l}\text { FEV, GAPDH and } \\
\text { NKX2-2 }\end{array}$ \\
\hline $\begin{array}{l}\text { Mazzoni et al. } \\
\text { (2013) }\end{array}$ & $\begin{array}{l}\text { Cholinergic/Mo- } \\
\text { tor neurons }\end{array}$ & Mouse & $\operatorname{ESCs}(H b 9:: G F P)$ & $\begin{array}{l}\text { FACS of Hb9-GFP+ } \\
\text { cells }\end{array}$ & Actb, Chat, Hb9, IsI1, Lhx3, Ngn2, Phox2a and Vacht & $\begin{array}{l}\text { Microarray (Affy- } \\
\text { metrix Mouse } \\
\text { Gene } 1.0 \text { ST) }\end{array}$ & V5 \\
\hline $\begin{array}{l}\text { Mong et al. } \\
\text { (2014) }\end{array}$ & $\begin{array}{l}\text { Cholinergic/Mo- } \\
\text { tor neurons }\end{array}$ & $\begin{array}{l}\text { Mouse } \\
\text { and } \\
\text { human }\end{array}$ & ESCs & $\begin{array}{l}\text { MACS of PSA- } \\
\text { NCAM+ cells }\end{array}$ & $\begin{array}{l}\text { CHAT, DBH, L19, ISL1, III1, PHOX2A, Phox2a, } \\
\text { PHOX2B, Phox2b, PRPH, NET, Net, Tbx20, Th, TIx3 } \\
\text { and Tuj1 }\end{array}$ & $\begin{array}{l}\text { Whole transcrip- } \\
\text { tome sequencing } \\
\text { (Affymetrix } \\
\text { Gene-Chip } \\
\text { Mouse Exon } \\
\text { ST1.1) }\end{array}$ & No \\
\hline $\begin{array}{l}\text { Son et al. } \\
\text { (2011) }\end{array}$ & $\begin{array}{l}\text { Cholinergic/Mo- } \\
\text { tor neurons }\end{array}$ & $\begin{array}{l}\text { Mouse } \\
\text { and } \\
\text { human }\end{array}$ & $\begin{array}{l}\text { Mouse fibroblasts } \\
(H b 9:: G F P) \text { and } \\
\text { human fibroblasts }\end{array}$ & $\begin{array}{l}\text { FACS of Hb9-GFP+ } \\
\text { cells }\end{array}$ & Ascl1, Brn2, Hb9, Isl1, LTR (Viral), Lhx3, Myt1l and Ngn2 & No & No \\
\hline $\begin{array}{l}\text { Hester et al. } \\
\text { (2011) }\end{array}$ & $\begin{array}{l}\text { Cholinergic/Mo- } \\
\text { tor neurons }\end{array}$ & Human & $\begin{array}{l}\text { ESCs (Hb9::GFP) } \\
\text { and iPSCs }\end{array}$ & No & $\begin{array}{l}\text { ACTB, CHAT, HB9, ISL1, LHX3, NANOG, NEUROD1, } \\
\text { OCT3/4, OLIG2, PAX6, REX1 and SOX2 }\end{array}$ & No & No \\
\hline $\begin{array}{l}\text { Mong et al. } \\
\text { (2014) }\end{array}$ & $\begin{array}{l}\text { Noradrenergic } \\
\text { neurons }\end{array}$ & $\begin{array}{l}\text { Mouse } \\
\text { and } \\
\text { human }\end{array}$ & ESCs & $\begin{array}{l}\text { MACS of PSA- } \\
\text { NCAM+ cells }\end{array}$ & $\begin{array}{l}\text { CHAT, DBH, L19, ISL1, III1, PHOX2A, Phox2a, } \\
\text { PHOX2B, Phox2b, PRPH, NET, Net, Tbx20, Th, TIX3 } \\
\text { and Tuj1 }\end{array}$ & $\begin{array}{l}\text { Microarray (Affy- } \\
\text { metrix Gene- } \\
\text { Chip Mouse } \\
\text { Exon ST1.1) }\end{array}$ & No \\
\hline
\end{tabular}

Neuronal phenotypes, species and starting cell types are summarized for each protocol according to their reference. Cell sorting techniques and the phenotypic characterization, consisting of assays such as (quantitative) polymerase chain reaction, genome-wide expression analysis, western-blot, immunohistochemistry, electrophysiological assessment, biochemical assessment, transplantation in to animal models, co-culture assays or other bioassays, are broadly outlined if applicable. 
Immunohistochemistry

\section{Immunohistochemistry}

5-HT, AADC, ALDH1A1, MAP2, NEUN, SERT, STX1A, TPH2, TUJ1 and VMAT2

5-HT, FEV, GABA, GATA2, GFAP, GFP, LMX1B, MAP2ab, NEUN, NKX2-2, SERT, TAU, TH, TPH, TPH2, TUJ1 and Vimentin

CHAT, HB9, ISL1, MKI67, NCAM, NEUN, NGN2, OLIG2, PHOX2B, SV2A, TUJ1, V5 and VACHT

NET, PHOX2A, PHOX2B, PSA-NCAM, PRPH, TH and TUJ1

ACHR, CHX10, HB9, ISL1, SYN1, TH and VACHT

ACTA1, CHAT, HB9, HOXC6, ISL1, LHX3, NANOG, NGN2, OCT3/4, OLIG2, OTX2, PAX6, RHD-BTX, TUJ1, SMI31, SOX2, SSEA4 and SV2A

NET, PHOX2A, PHOX2B, PSA-NCAM, PRPH, TH and TUJ1
Electro-

physiologica

assessment

Whole cell
patch clamp

patch clamp

Whole cell patch clamp

Whole cell patch clamp

No

No

Activity dependent

5-HT release and

5-HT uptake analy-

sis (both HPLC)

Calcium imaging

and 5-HT concen-

tration measure-

ments (ELISA)

No

No

No
Other bioassays and

assessments

No
Rat astrocyte co-culture Drug assay (SSRIs)

Ras

No

No
Chromatin immunoprecipitation (for Isl1 and V5), co-immunoprecipitation and DNA motif analysis High-throughput imaging drug assay

BrdU proliferation assay (Birthdate analysis)

$\begin{array}{llll}\begin{array}{l}\text { Whole cell } \\ \text { patch clamp }\end{array} & \text { No } & \begin{array}{l}\text { Mouse } \mathrm{C} 2 \mathrm{C} 12 \\ \text { myoblast co-culture } \\ \text { assay, chick myotube } \\ \text { co-culture assay, chick } \\ \text { eggs (in ovo), glia-neu- } \\ \text { ron co-culture assay }\end{array} & \begin{array}{l}\text { BrdU proliferation assay (Birth- } \\ \text { date analysis) }\end{array} \\ \begin{array}{l}\text { Whole cell } \\ \text { patch clamp }\end{array} & \text { No } & \begin{array}{l}\text { Mouse C2C12 myotube } \\ \text { co-culture assay }\end{array} & \text { No } \\ \text { No } & \text { No } & \text { No } & \text { High-throughtut imaging drug } \\ & & & \text { assay }\end{array}$


Supplementary Table 3. Overview of the assays that have been used for the phenotypic characterization of the epigenetic-based neuronal conversion approaches.

\begin{tabular}{|c|c|c|c|c|c|c|c|}
\hline \multirow[t]{2}{*}{ References } & \multirow[t]{2}{*}{ Phenotypes } & \multirow{2}{*}{$\begin{array}{l}\text { Spe- } \\
\text { cies }\end{array}$} & \multirow[t]{2}{*}{ Starting cell types } & \multirow[t]{2}{*}{ Cell sorting } & \multicolumn{3}{|l|}{ Phenotypic characterization } \\
\hline & & & & & (Quantitative) Polymerase chain reaction & $\begin{array}{l}\text { Genome-wide expressi- } \\
\text { on analysis }\end{array}$ & Western-Blot \\
\hline $\begin{array}{l}\text { Raciti et al. } \\
(2013)\end{array}$ & NPCs & Mouse & $\begin{array}{l}\text { Fibroblasts } \\
\text { (Trp53+/+, } \\
\text { Trp53-/-, Sox1+/+, } \\
\text { Sox1EGFP/+, } \\
\text { Tau+/+ and } \\
\text { TauEGFP/+) }\end{array}$ & $\begin{array}{l}\text { FACS of Sox1-EGFP+ } \\
\text { and Tau-EGFP+ cells }\end{array}$ & Bf1, Emx1, Emx2, Gapdh, Hes5, Ngn2 and Pax6 & No & No \\
\hline $\begin{array}{l}\text { Cheng et al. } \\
(2015 b)\end{array}$ & NPCs & $\begin{array}{l}\text { Mouse } \\
\text { and } \\
\text { human }\end{array}$ & $\begin{array}{l}\text { Mouse fibroblasts } \\
\text { and human urinary } \\
\text { cells }\end{array}$ & No & $\begin{array}{l}\text { Ascl1, Blbp, Brn2, NANOG, Nanog, NES, OCT3/4, } \\
\text { Oct3/4, PAX6, Pax6, SOX1, SOX2, Sox2, HPRT and Hprt }\end{array}$ & $\begin{array}{l}\text { Microarray }(8 \times 60 \text { K array } \\
\text { from Agilent Technologies) }\end{array}$ & No \\
\hline $\begin{array}{l}\text { Cheng et al. } \\
(2015 \mathrm{a})\end{array}$ & $\begin{array}{l}\text { Dopaminergic, } \\
\text { GABAergic, } \\
\text { glutamatergic } \\
\text { and cholinergic } \\
\text { motor neurons }\end{array}$ & Mouse & $\begin{array}{l}\text { Astrocytes } \\
\text { (GFAP::GFP and } \\
\text { Neurod1::GFP) }\end{array}$ & No & $\begin{array}{l}\text { Asl1, DII1, DII3, DIl4, DIx2, Hes1, Hes5, Hprt1, Jag1, } \\
\text { Jag2, Neurod1, Ngn2, Notch1, Notch2, Notch3, Notch4, } \\
\text { Pax6 and Sox2 }\end{array}$ & No & No \\
\hline $\begin{array}{l}\text { Black et al. } \\
(2016)\end{array}$ & $\begin{array}{l}\text { Neurons } \\
\text { (Unspecified) }\end{array}$ & Mouse & $\begin{array}{l}\text { Fibroblasts } \\
(S Y N 1:: R F P)\end{array}$ & No & $\begin{array}{l}\text { ASCL1, Ascl1 (Endogenous), Ascl1 (Total), Brn2 (Endog- } \\
\text { enous), Brn2 (Total), Myt1l (Endogenous), Myt1l (Total), } \\
\text { Tuj1, gRNA, GAPDH and Gapdh }\end{array}$ & No & $\begin{array}{l}\text { ASCL1, } \\
\text { BRN2, FLAG, } \\
\text { GAPDH and } \\
\text { MYT1L }\end{array}$ \\
\hline $\begin{array}{l}\text { Chavez et al. } \\
(2015)\end{array}$ & $\begin{array}{l}\text { Neurons } \\
\text { (Unspecified) }\end{array}$ & Human & iPSCs & No & $\begin{array}{l}\text { ACTB, ACTC1, ASCL1, MIAT, NEUROD1, NGN2, } \\
\text { RHOXF2, TTN and VEGF }\end{array}$ & No & No \\
\hline $\begin{array}{l}\text { Low et al. } \\
(2012)\end{array}$ & $\begin{array}{l}\text { Neurons } \\
\text { (Unspecified) }\end{array}$ & Mouse & NPCs & No & Actb, Gfap, Map2, Nes, Rest, Rip and Tuj1 & No & $\begin{array}{l}\text { ACTB and } \\
\text { REST }\end{array}$ \\
\hline $\begin{array}{l}\text { Yang et al. } \\
\text { (2012) }\end{array}$ & $\begin{array}{l}\text { Dopaminergic } \\
\text { neurons }\end{array}$ & Mouse & $\operatorname{ESCs}(T H:: G F P)$ & $\begin{array}{l}\text { FACS of TH-GFP+ } \\
\text { cells }\end{array}$ & Actb, Bdnf, Gdnf, Nurr1, Pitx3, Pre-miR-132 and Th & $\begin{array}{l}\text { RNA expression analysis } \\
\text { (TaqMan Low Density } \\
\text { Assay v2.0) }\end{array}$ & $\begin{array}{l}\text { ACTB and } \\
\text { NURR1 }\end{array}$ \\
\hline $\begin{array}{l}\text { Kimet al. } \\
(2007)\end{array}$ & $\begin{array}{l}\text { Dopaminergic } \\
\text { neurons }\end{array}$ & Mouse & ESCs & FACS of PITX3+ cells & ACTB, Actb, miR133b (Human) and miR133b (Mouse) & No & No \\
\hline $\begin{array}{l}\text { Stappert et al. } \\
\text { (2013) }\end{array}$ & $\begin{array}{l}\text { Dopaminergic } \\
\text { neurons }\end{array}$ & Human & NPCs & No & 18S, DAT, GAD67, NURR1 and TH & $\begin{array}{l}\text { MiRNA expression analysis } \\
\text { (ABI microRNA multiplex } \\
\text { TaqMan assay) }\end{array}$ & No \\
\hline $\begin{array}{l}\text { Victor et al. } \\
\text { (2014) }\end{array}$ & $\begin{array}{l}\text { GABAergic } \\
\text { neurons }\end{array}$ & Human & $\begin{array}{l}\text { Fibroblasts } \\
(\text { SYN1::EGFP) }\end{array}$ & FACS & $\begin{array}{l}\text { ANK2, ASCL1, BCl-XL, BDNF, CALB2, CHAT, CHRM4, } \\
\text { CTIP2, DRH, DCX, DDC, DLX1, DLX1 3'UTR, DLX2, } \\
\text { DLX2 3'UTR, DRD1, DRD2, GAD65, GAD67, GAPDH, } \\
\text { GPR6, GRP, HPRT1, HTR2C, HTR3A, LHX6, MAP2, } \\
\text { MYT1L, NCAM1, NES, NEUROD1, NKX2-1, OPRM1, } \\
\text { OTX1, OTX2, P75, PAX6, PCP2, PDYN, PENK, POU3F1, } \\
\text { PPP1R1B, Pri-miR-9 Loci 3, Pri-miR-124 Loci 1, PRPH, } \\
\text { PSD95, PVALB, RARB, RPS18, SCN2A, SCN3A, } \\
\text { SHANK3, SLC17A6, SLC17A8, SLC6A3, SLC6A4, } \\
\text { SST, TAC1, TAU, TBR1, TBR2, TH, TPH2, Transgenic } \\
\text { Pri-miR9124 and TUJ1 }\end{array}$ & $\begin{array}{l}\text { Single-cell gene expres- } \\
\text { sion analysis (Fluidigm } \\
\text { dynamic array) }\end{array}$ & No \\
\hline $\begin{array}{l}\text { Yoo et al. } \\
\text { (2011) }\end{array}$ & $\begin{array}{l}\text { Glutamatergic } \\
\text { and GABAergic } \\
\text { neurons }\end{array}$ & Human & Fibroblasts & $\begin{array}{l}\text { FACS of MAP2+1 } \\
\text { TUJ1+ cells }\end{array}$ & $\begin{array}{l}\text { 18S, BDNF, BSN, CACNA1C, CRIM1, CTIP2, CUX1, } \\
\text { DCX, DDC, DKK3, DLX1, DLX5, ETV1, FOXP1, FOXP2, } \\
\text { GAD67, GAPDH, GRP, HTR2C, LXN, MEF2C, NCAM, } \\
\text { NR4A3, NSE, OMA1, PCLO, PCP2, PERIPHERIN, PLX- } \\
\text { ND1, PPP1R1B-DARPP32F, S100A10, SATB2, SCN1A, } \\
\text { SCN2A, SCN3A, SCN8A, SEMA3E, SHANK3, SLC1A2, } \\
\text { SOX5, SYN1, SYT9, TAU, TBR1, TH, TIS21-BTG2, } \\
\text { TLE1, TLE4, TPM2, TUJ1, GRM5, UNC5D and VLGUT1 }\end{array}$ & $\begin{array}{l}\text { Single-cell gene expres- } \\
\text { sion analysis (Fluidigm } \\
\text { dynamic array) }\end{array}$ & $\begin{array}{l}\text { BAF53Aand } \\
\text { GAPDH }\end{array}$ \\
\hline $\begin{array}{l}\text { Ambasudhan } \\
\text { et al. (2011) }\end{array}$ & $\begin{array}{l}\text { Glutamatergic } \\
\text { and GABAergic } \\
\text { neurons }\end{array}$ & Human & Fibroblasts & No & $\begin{array}{l}\text { BAF53B, BRN2, BRN2 (Viral), KRT1, miR-124, MYT1L, } \\
\text { MYT1L(Viral), P4HA1, P75, PAX6, SOX1 and SOX10 }\end{array}$ & No & No \\
\hline $\begin{array}{l}\text { Abernathy et } \\
\text { al. (2017) }\end{array}$ & $\begin{array}{l}\text { Cholinergic } \\
\text { motor neurons }\end{array}$ & Human & Fibroblasts & No & $\begin{array}{l}\text { CHAT, COL13A1, HB9, HOXA7, HOXA9, HOXB4, } \\
\text { HOXB7, HOXC10, HOXC11, HOXC6, HOXC8, HOXC9, } \\
\text { HOXD11, HOXD8, HOXD9, HPRT, hsa-miR-218 S100A4, } \\
\text { RNU-44, SLC18A3 and VIM }\end{array}$ & $\begin{array}{l}\text { Microarray (llumina Total- } \\
\text { Prep kits for Agilent Human } \\
\text { 4x44Kv1), RNA-seq and } \\
\text { TRAP RNA-seq }\end{array}$ & No \\
\hline
\end{tabular}

Neuronal phenotypes, species and starting cell types are summarized for each protocol according to their reference. Cell sorting techniques and the phenotypic characterization, consisting of assays such as (quantitative) polymerase chain reaction, genome-wide expression analysis, western-blot, immunohistochemistry, electrophysiological assessment, biochemical assessment, transplantation in to animal models, co-culture assays or other bioassays, are broadly outlined if applicable. 


\begin{tabular}{|c|c|c|c|c|}
\hline Immunohistochemistry & $\begin{array}{l}\text { Electrop- } \\
\text { hysiological } \\
\text { assessment }\end{array}$ & $\begin{array}{l}\text { Biochemical } \\
\text { assessment }\end{array}$ & $\begin{array}{l}\text { Transplantations/ } \\
\text { Co-culture assay }\end{array}$ & Other bioassays and assessments \\
\hline BF1, BrdU, GFAP, MAP2, NANOG, NEUN, PAX6, SOX2 and TUJ1 & $\begin{array}{l}\text { Whole cell } \\
\text { patch clamp }\end{array}$ & No & $\begin{array}{l}\text { Transplantation into } \\
\text { neonatal mice }\end{array}$ & $\begin{array}{l}\text { BrdU proliferation assay (Birth-date } \\
\text { analysis) and NPC-differentiation } \\
\text { into neurons }\end{array}$ \\
\hline
\end{tabular}

GAD67, GFAP, Glutamate, MAP2, MBP, MKI67, NES, NEUN, OLIG2, PAX6, SOX2, SYN1 and TUJ1

CHAT, DCX, GAD67, GFP, MAP2, NEUN, SOX2, TH, TUJ1, VLGUT1 and VMAT2

ASCL1, BRN2, MAP2 and TUJ1

Neurofilament 200 and TUJ1

GFAP, MAP2, NES, 04 and TUJ1

MAP2, NES and TH

BrdU, GABA, miR-133, TH and TUJ1

BrdU, GAD65/67, TH and TUJ1

Ankyrin G, CTIP2, DARPP32, DLX5, FOXP1, GABA, GAD67, MAP2, NEUN, SCN1A, SYN1, TH, TUJ1 and VGLUT1

BAF45B, BAF45C, BAF53B, GABA, GRIN1, MAP2, Neurofilament 200, SCN1A, SV2, SYN1, TUJ1 and VGLUT1
Whole cell patch clamp

\section{Alkaline phosp} hatase analysis

Whole cell patch clamp

Whole cell patch clamp

No

No

No

No

No

Whole cell

patch clamp

(in vitro and $e x$

vivo) (HPLC)
Transplantation into embryonic mice

No

No

No

No

No

No dopamine release
No

No

No

Calcium imaging No and FM1-43 imaging
NPC-differentiation into neurons and glia

No
ChiP-seq and ChIP-qPCR (Brn2, Ascl 1 and $M y t 11-H 3 K 27 a c$ and H3K4me3)

No

Examination of neurite length and outgrowth

No

Apoptosis analysis (TUNEL) and Pitx3 luciferase assay

BrdU proliferation assay (Birth-date analysis) and non-radioactive northern blotting

Transplantation into neonatal NSG mice
No

EdU proliferation assay (Birth-date analysis)
GABA, GFAP, KRT1, MAP2, NEUN, NKX2-2, P75, PAX6, PERIPHERIN, PHD4, SOX2, SYN1, Whole cell No

TH, TUJ1, VGAT and VGLUT1

FSP1, MAP2, KI67, TUJ1, NEUN, NCAM, MNX1, SCN1A, ANKG, SV2, CHAT, SMI-32 patch clamp

Whole cell

patch clamp
No

\section{No}

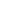

No

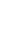

Human myotue co-cul-

ture assay 

Chapter 8

Establishment and characterization of a human neuronal in vitro model system for Alzheimer's disease using induced pluripotent stem cells: An exploratory approach

Renzo J.M. Riemens ${ }^{1,2}$, Katherine Basil ${ }^{1}$, Cengiz Akbulut ${ }^{3}$, Leon J. Schurgers ${ }^{3}$, Daniël L.A. van den Hove ${ }^{1,4}$, Jos C. Kleinjans ${ }^{5}$, Twan van den Beucken ${ }^{5 \dagger}$, Gunter Kenis ${ }^{1 \dagger}$

${ }^{1}$ Department of Psychiatry and Neuropsychology, School for Mental Health and Neuroscience (MHeNs), Maastricht University, Maastricht, the Netherlands.

${ }^{2}$ Institute of Human Genetics, Julius Maximilians University, Wuerzburg, Germany.

${ }^{3}$ Department of Biochemistry, Cardiovascular Research Institute Maastricht, Maastricht University, Maastricht, the Netherlands.

${ }^{4}$ Laboratory of Translational Neuroscience, Department of Psychiatry, Psychosomatics and Psychotherapy, University of Wuerzburg, Wuerzburg, Germany.

${ }^{5}$ Department of Toxicogenomics, GROW School for Oncology and Developmental Biology, Maastricht University, the Netherlands.

† Authors contributed equally to this work 



\begin{abstract}
In recent years, the establishment of induced pluripotent stem cells (iPSCs) has offered an alternative in vitro model system for Alzheimer's disease (AD). While still in their infancy, these developing models hold considerable promise to push forward the efforts to disentangle AD's pathophysiology. However, before the potential of these models can be fully realized, it is imperative to set up a robust neuronal differentiation protocol relevant for $A D$, as this represents a milestone that needs to be achieved in order to subsequently develop an adequate in vitro model. Here we present preliminary data on the characterization of a cortical forebrain differentiation protocol from iPSCs. Based on the step-wise application of neuralizing factors, iPSCs were differentiated towards neural progenitor cells (NPCs) and subsequently cortical neurons and glia, which represents cellular populations known to be affected in the pathophysiology of AD. Based on assessing the expression of key-lineage transcription factors at each stage of the differentiation process we confirmed that the cells adopted towards a cortical forebrain fate. Furthermore, we assessed the expression of Tenascin XB (TNXB) and the oxytocin receptor (OXTR), as we have previously identified TNXB and oxytocin $(O X T)$-signaling to be associated with $A D$ in genome-wide methylation screenings. Both genes were detectable after 14 days and their expression maintained up until 56 days of NPC differentiation. Taken together, we successfully established a differentiation protocol for cortical forebrain cells that will enable us to perform mechanistic investigation of candidate genes for AD.
\end{abstract}

\title{
Keywords
}

Induced pluripotent stem cells (iPSCs), Alzheimer's disease, cortical neurons, directed differentiation. 


\section{Introduction}

Research into $A D$ has changed rapidly over the last years as iPSCs derived from patients are now being widely explored as an alternative model system to study the disease in a more humanized in vitro setting. One could claim that the establishment of this technology represents as yet one of the most innovative biomedical advances of this century, mainly because these patient-specific cells contain the molecular information from the donors, and therefore might be able to provide new insights into the disease that could contribute to the development of (personalized) therapeutics. Another major innovative character of this approach is that, at least in theory, all major brain cell types can be obtained by directed differentiation of the iPSCs, which allows for cell-specific studies in vitro and the establishment of increasingly complex human co-culture systems relevant for $A D$ [1]. By applying this technology, pioneering studies have furthermore already demonstrated that cellular $A D$ hallmarks can be found in patient-derived neural cells differentiated from iPSCs when compared to those derived from healthy controls [2-7]. Thus, while still in their relative infancy, iPSC-based neural models are thought to hold considerable promise to push $A D$ research forward.

Although significant advances have been made in generating various neural cells from iPSCs, in vitro neuronal differentiation is actually not a process that is fully disciplined yet, since protocols for specific neural subtypes vary substantially in time scale and efficiency, both within and between different laboratories [1]. On that account, setting up a robust neuronal differentiation protocol relevant for $A D$ represents a milestone that needs to be achieved a priori in order to subsequently develop adequate AD models. In the present study, we therefore present preliminary data on the characterization of a cortical forebrain differentiation protocol from iPSCs. Based on the step-wise application of neural patterning factors, we differentiated iPSCs towards neural progenitor cells (NPCs) and subsequently cortical neurons and glia, which represent cellular populations known to be affected in the pathophysiology of AD [8]. We postulate that the application of this differentiation protocol on both patient- and healthy control-derived iPSCs might provide an appealing model to study $A D$. We furthermore assessed the expression of Tenascin XB (TNXB) and the oxytocin receptor (OXTR) in the differentiated neural cells. TNXB and oxytocin (OXT)-signaling have previously been associated with $A D$ pathophysiology, and iPSC-based studies could help in determining their specific molecular mechanisms in relation to the disease [9-11]. All in all, the pilot data presented here represents an exploratory approach characterizing a cortical forebrain differentiation protocol in iPSC with the eventual aim to apply this for the establishment of an AD-related disease model. 


\section{Materials and methods}

\section{Sample and ethics statement}

Peripheral blood samples were obtained from a healthy Southern European male that was in his late twenties at time of donation. Mononuclear blood cells isolated from the peripheral blood samples were reprogrammed towards iPSCs using the Epi5 $^{\mathrm{TM}}$ Episomal iPSC Reprogramming Kit (Invitrogen ${ }^{\mathrm{TM}}$, Thermo Fisher Scientific, Waltham, MA, USA) in combination with a Nucleofector ${ }^{\mathrm{TM}}$ Device (Lonza, Bazel, Switzerland). All laboratory procedures for iPSC reprogramming were performed at the Cardiovascular Research Institute Maastricht (CARIM; Maastricht University, Maastricht, the Netherlands). Clones of growing iPSC colonies were picked, expanded and characterized according to general quality control guidelines, including tests such as the evaluation of pluripotency marker expression, assessment of transgene silencing, karyotyping by G-banding, embryoid body (EB) induction and directed differentiation into the three embryonic germ layers [12]. A single clone, i.e. CARIMi001-A, which surpassed the quality control criteria, was selected and used in the present study. For further details on the used iPSC line, please refer to https://hpscreg.eu/cell-line/CARIMi001-A.

\section{Induced pluripotent stem cell maintenance}

iPSC colonies were maintained in Essential 8 flex medium (Gibco ${ }^{T M}$, Thermofisher Scientific) on plates coated with human embryonic stem cell-qualified Geltrex ${ }^{\mathrm{TM}}$ (Gibco ${ }^{\mathrm{TM}}$, Thermofisher Scientific) and regularly passaged upon confluence using UltraPure $^{\mathrm{TM}}$ EDTA (Invitrogen ${ }^{\mathrm{TM}}$, Thermo Fisher Scientific). Passaged iPSCs were incubated overnight with medium containing $10 \mu \mathrm{M}$ ROCK Inhibitor Y-27632 (Stemcell Technologies, Vancouver, Canada) in order to enhance cell survival and medium was refreshed every other day thereafter. Cells were regularly checked and verified to be free of mycoplasma contamination.

\section{Neural progenitor cell induction}

NPCs were differentiated based on a previously described cortical forebrain differentiation protocol, with small adaptations [13]. In brief, clumps of iPSC colonies were sparsely plated and grown up until profound single rounded colonies were present in the culture. The colonies were then enzymatically dissociated using collagenase IV $\left(\mathrm{Gibco}^{\mathrm{TM}}\right.$, Thermofisher Scientific) and transferred to ultra-lowattachment plates (Costar ${ }^{\circledR}$, Corning, New York, NY, USA) in order to generate freefloating EBs. The generating EBs were maintained in neuronal induction medium (NIM) on an orbital shaker for the first 24 hours and then as static culture for a total time of two weeks. The NIM consisted of DMEM/F-12 GlutaMAX ${ }^{\mathrm{TM}}$ (Gibco ${ }^{\mathrm{TM}}$, Thermofisher Scientific) with N-2 ${ }^{\mathrm{TM}}\left(1 \mathrm{X} ;\right.$ Gibco $^{\mathrm{TM}}$, Thermofisher Scientific) and B-27 $7^{\mathrm{TM}}$ (1X; Gibco ${ }^{\mathrm{TM}}$, Thermofisher Scientific), supplemented with $100 \mathrm{ng} / \mathrm{ml}$ recombinant human Noggin (Peprotech, Cranbury, NJ, USA) 100 nM LDN193189 (StemMacs ${ }^{\text {TM }}$, 
Miltenyi Biotec, Bergisch Gladbach, Germany) and 10 nM SB431542 (StemMacs ${ }^{\text {TM }}$, Miltenyi Biotec), i.e. dual SMAD inhibition [14]. After two weeks of incubation, EBs were plated onto poly-L-ornithine- (PLO; Sigma Aldrich, Saint Louis, MO, USA) and mouse laminin- (Gibco ${ }^{\mathrm{TM}}$, Thermofisher Scientific) coated plates in NIM containing 20 $\mathrm{ng} / \mathrm{ml}$ human fibroblast growth factors 2 (FGF2; Miltenyi Biotec) and $1 \mu \mathrm{g} / \mathrm{ml}$ laminin . After 48 hours, adhered EBs displaying outgrowth of neural projections were picked, dissociated using Accutase ${ }^{\circledast}$ (Corning) and plated onto PLO/laminin-coated plates in order to generate neuronal progenitor cells (NPCs). The NPCs were maintained at high density as monolayers in NIM containing $20 \mathrm{ng} / \mathrm{ml}$ human FGF2 and regularly passaged using Accutase ${ }^{\circledR}$. Passaged NPCs were incubated overnight with $10 \mu \mathrm{M}$ ROCK Inhibitor Y-27632 in order to enhance cell survival and medium was refreshed every other day thereafter.

\section{Cortical forebrain differentiation}

For terminal differentiation towards cortical neural cells, NPC were plated at lower densities $\left(25,000\right.$ to 50,000 cells $\left./ \mathrm{cm}^{2}\right)$ on PLO/laminin-coated plates, while maintained in neuronal differentiation medium (NDM) for up to 2, 4 or 8 weeks. NDM consisted of $\mathrm{N}-2$ and B-27 supplemented DMEM/F12:Neurobasal ${ }^{\mathrm{TM}}$ media $\left(1: 1 ;\right.$ Gibco $^{\mathrm{TM}}$, Thermofisher Scientific) with $20 \mathrm{ng} / \mathrm{ml}$ recombinant human brain-derived neurotrophic factor (BDNF; Peprotech), $20 \mathrm{ng} / \mathrm{ml}$ human recombinant glial cell-derived neurotrophic factor (GDNF; Peprotech), 1mM dibutyrl cyclic-AMP (dcAMP; Sigma-Aldrich), 200 nM ascorbic acid (AA; Stemcell Technologies), $1 \mu \mathrm{g} / \mathrm{ml}$ laminin, non-essential amino acids (NEAA (1X); Gibco ${ }^{\mathrm{TM}}$, Thermofisher Scientific) and antibiotic-antimycotic solution (1X; Gibco $^{\mathrm{TM}}$, Thermofisher Scientific). Plated NPCs were incubated overnight with $10 \mu \mathrm{M}$ ROCK Inhibitor Y-27632 in order to enhance cell survival and medium was refreshed every other day thereafter.

\section{Immunocytochemisty and cell quantification}

Cells were washed three times with $1 \mathrm{X}$ phosphate buffered saline (PBS) and subsequently fixated for 15 minutes at room temperature using $4 \%$ paraformaldehyde in PBS. The cells were then washed three times for 5 minutes each with 1X PBS and permeabilized using $0.1 \%$ Triton-X100 in PBS for 5 minutes. Following three subsequent washing steps with PBS lasting 5 minutes each, the cell were blocked for one hour at room temperature using $10 \%$ of bovine serum albumin (BSA) diluted in PBS. After blocking, cells were incubated at $4^{\circ} \mathrm{C}$ overnight with primary antibodies diluted in $10 \%$ BSA blocking solution. The next day, the cells were washed three times for 10 minutes using 1 X PBS. The cells were then incubated with the corresponding Alexa Fluor ${ }^{\circledR}$ conjugated secondary antibodies diluted in 10\% BSA for one hour at room temperature. Following incubation, the cells were washed three times for 10 minutes using 1X PBS and counterstained with Hoechst (1:500) diluted in PBS. Please refer to Supplementary Table $\mathrm{S} 1$ for an overview of used antibodies in this 
study. Fluorescent images were taken using an iX81 microscope (Olympus Life Science, Olympus Corporation, Waltham, MA, USA) with the Stereo Investigator software (MBF, Bioscience, Williston, VT, USA). For NPC cell counting, 15,000 cells were plated per well of a 24-well plate and fixated after 48 hours in culture. Images of the cells in five visual fields were randomly taken and loaded into the FIJI ImageJ software (https://imagej.nih.gov/ij/index.html) [15]. On average, $318 \pm 39$ cells per staining were manually counted using the ImageJ point tool function.

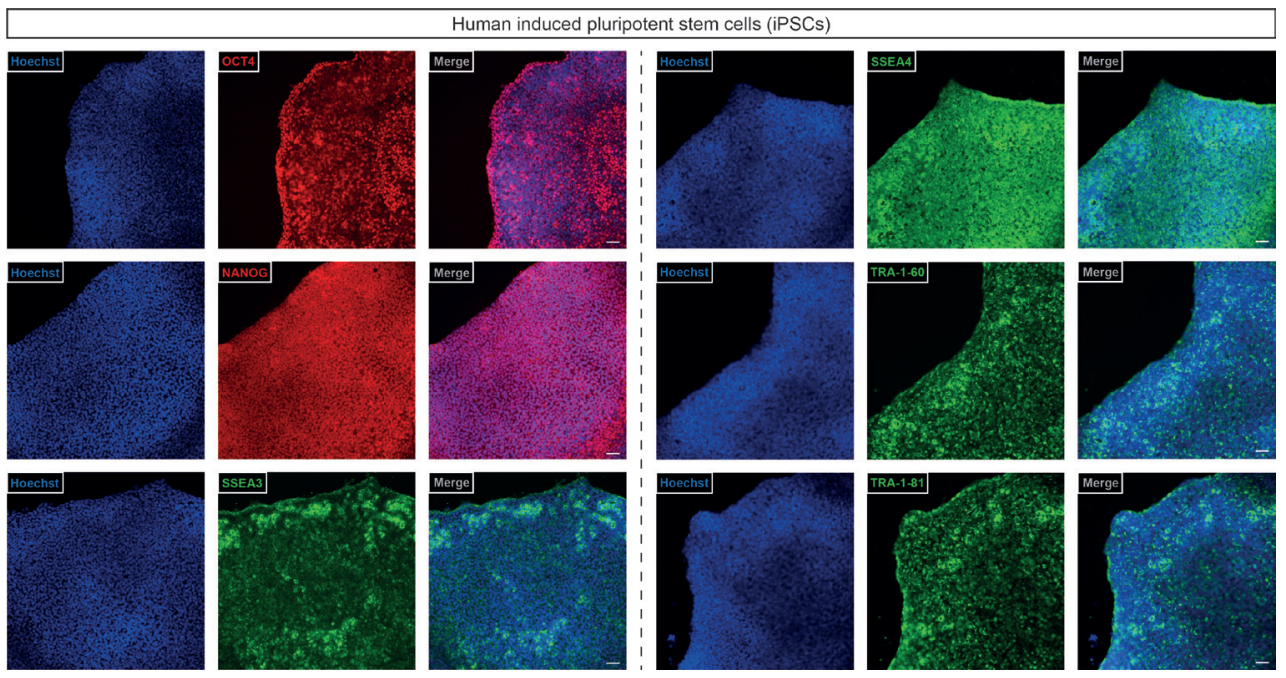

Figure 1. Characterization of stemness markers in human induced pluripotent stem cells (iPSCs). The human iPSC line, i.e. CARIMi001-A, was stained for the stemness markers OCT4, NANOG, SSEA3, SSEA4, TRA-1-60 and TRA-1-81. Images are displayed for the nuclear counterstaining with Hoechst, the respective stemness marker and the overlay of both images (Merge). Scale bars represent $100 \mu \mathrm{M}$.

\section{Results}

Characterization of human iPSCs and derived cortical forebrain neurons and glia First, the iPSC line used in the present study, i.e. CARIM-i001A, was stained for several commonly used iPSC markers. As shown in Figure 1, growing iPSC colonies exhibited a typical round morphology with well-defined sharp edges and tightly packed cells that stained positive for OCT4, NANOG, SSEA3, SSEA4, TRA-1-60 and TRA-1-81. Next, iPSCs were differentiated to NPCs via a combination of dual SMAD inhibition and EB formation (see Material and methods, as well as Figure 2 for details). After 4 weeks of NPC differentiation, cells were characterized for expression of NPC-specific markers. As shown in Figure 3, the NPCs stained positive for NESTIN (85.89 $\pm 7.14 \%)$, PAX6 $(93.10 \pm 5.85 \%)$ and SOX2 (91.28 $\pm 6.39 \%)$. Double labeling with each of these markers revealed that $82.13 \pm 5.81 \%$ of the Hoechst-positive cells stained for both NESTIN and PAX6, 85.69 $\pm 8.62 \%$ for NESTIN and SOX2, and $88.44 \pm 8.06 \%$ for SOX2 and PAX6, indicating that most of the iPSCs differentiated into NPCs. 


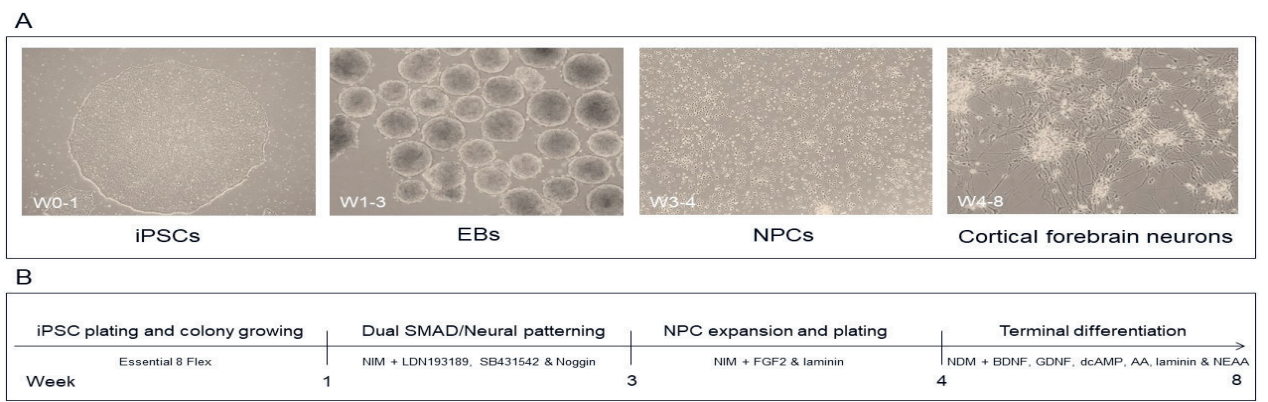

Figure 2. Overview of the cortical forebrain differentiation protocol in induced pluripotent stem cells (iPSCs). A. Brightfield images represent examples of the cells at different stages in the process of neuronal differentiation, from iPSCs to embryoid bodies (EBs), to neural progenitor cells (NPCs), to cortical forebrain neurons. B. The timeline displays the overview of the method for generating human cortical forebrain neurons by inhibition of dual SMAD signaling combined with EB formation, the use of neuronal induction medium (NIM) supplemented with fibroblast growth factor-2 (FGF2) for NPC maintenance and subsequently terminal differentiation towards neurons by using neuronal differentiation medium (NDM) supplemented with, brain-derived neurotrophic factor (BDNF), glial cellderived neurotrophic factor (GDNF), dibutyrl cyclic-AMP (dcAMP), ascorbic acid (AA), $1 \mu \mathrm{g} / \mathrm{ml}$ laminin, non-essential amino acids (NEAA). The differentiation process starting from the iPSCs towards the cortical forebrain neurons takes approximately 8 weeks.

NPCs were subsequently differentiated towards cortical forebrain cells (see Material and methods, as well as Figure 2 for details) for a total of 4 weeks. While after 2 weeks of differentiation the cells had lost the expression of the pluripotency gene OCT4, expression of the neuronal marker TUJ could be detected (Figure $4 A)$. A more elaborate characterization after 4 weeks of differentiation furthermore confirmed the expression of the neuronal markers MAP2AB, DCX and TUJ1, as well as the glial marker GFAP (Figure 4B). In addition, after 4 weeks of differentiation the neural cells expressed cortical forebrain markers, including CTIP2, a cortical pyramidal neuronal marker found in layer $\mathrm{VI}-\mathrm{V}$, and the telencephalic marker FOXG1 (Figure 4C) [16]. Overall, these data show that we successfully generated neurons, as well as glia cells, from these iPSCs with a cortical forebrain identity that was confirmed based on the expression of the aforementioned key lineagespecific markers. 

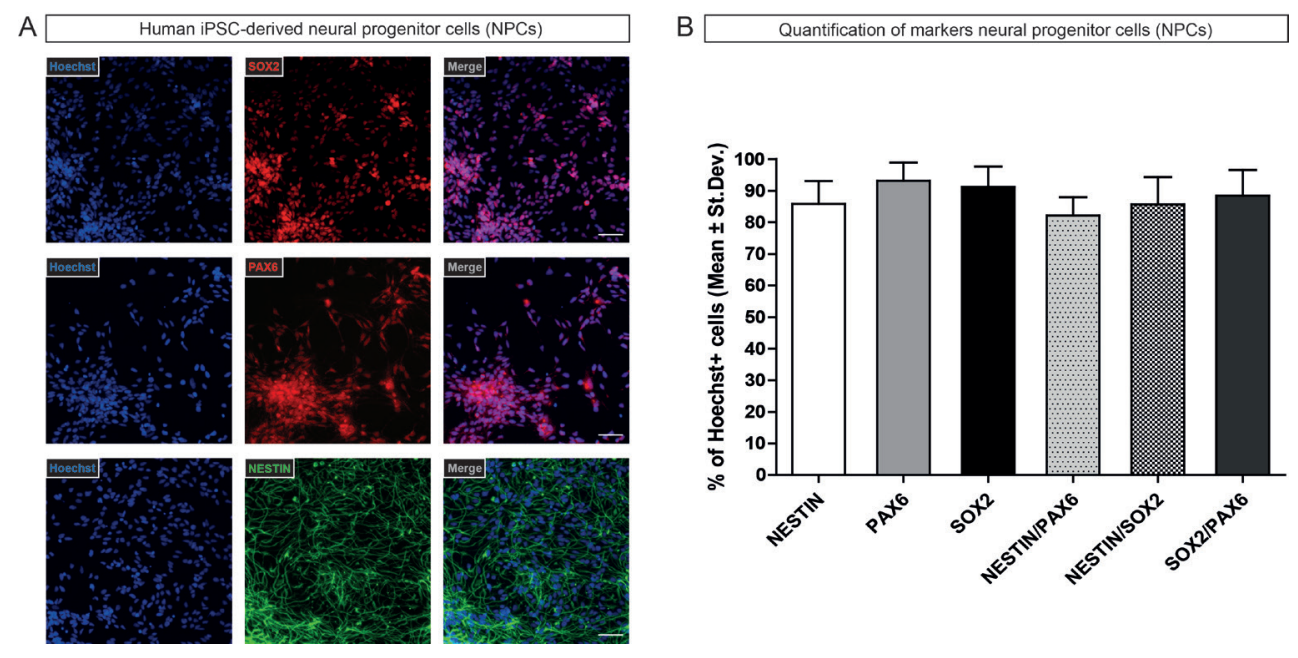

Figure 3. Characterization of the neural progenitor cells (NPCs) derived from the human induced pluripotent stem cells (iPSCs). A. Representative fluorescent images of the NPCs staining positive for markers such as NESTIN, SOX2 and PAX6. Images are displayed for the nuclear counterstaining with Hoechst, the respective NPC marker and the overlay of both images (Merge). Scale bars represent $100 \mu \mathrm{M}$. B. Quantification of these markers demonstrated that most of the iPSC-derived NPCs stained double positive for NESTIN, PAX6 and SOX2.

\section{Cortical forebrain neural cells express TNXB and OXTR}

We furthermore assessed the expression of TNXB and OXTR in the differentiated neural cells. Deregulation in the TNXB and OXT, as well as OXT-signaling have previously been associated with $A D$ and, specifically, OXT administration has therefore been proposed as a potential therapeutic approach for dementia $[9,10$, 17-20]. In order to use the iPSC-derived cortical forebrain cells for mechanistic studies targeting these candidates, it is crucial to confirm that the cells described here express the related proteins. Expression of both TNXB and OXTR was assessed at three time-intervals, i.e. after 14 days, 28 days and 56 days of NPCdifferentiation, in order to determine potential timely differences in their expression during the differentiation and maturation of the neural cells. As shown in Figure 5 , expression of both TNXB and OXTR could be identified in the differentiated cortical forebrain neurons, as early as 14 days and maintained up to 56 days of NPC-differentiation. Immunoreactivity of TNXB could be identified extracellularly, whereas OXTR expression was detected at the soma of both TUJ1-postivite and TUJ1-negative cells. These data therefore suggest that the cortical forebrain protocol described here might represent an appealing model system to study processes related to TNXB and OXT signaling in the context of AD. 


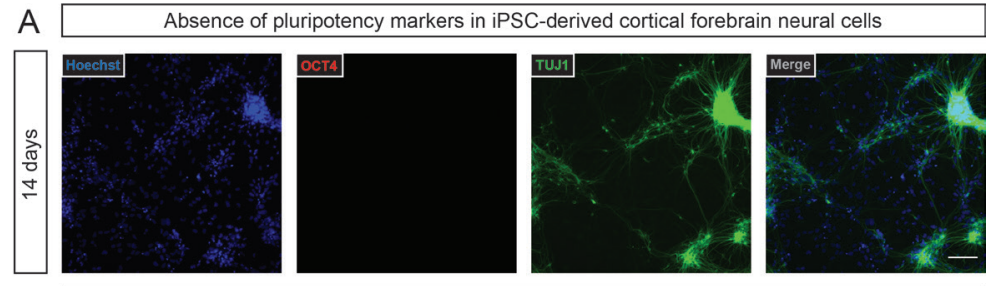

$\mathrm{B}$

Expression of neuron and glia markers in iPSC-derived cortical forebrain neural cells

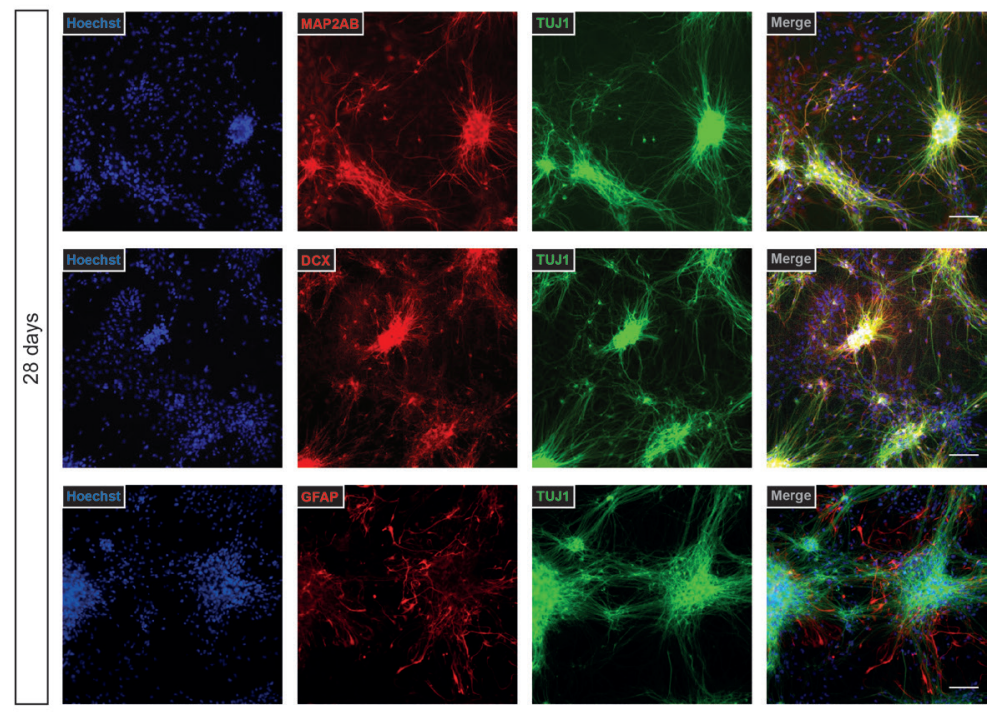

C

Expression of cortical forebrain markers in iPSC-derived neural cells
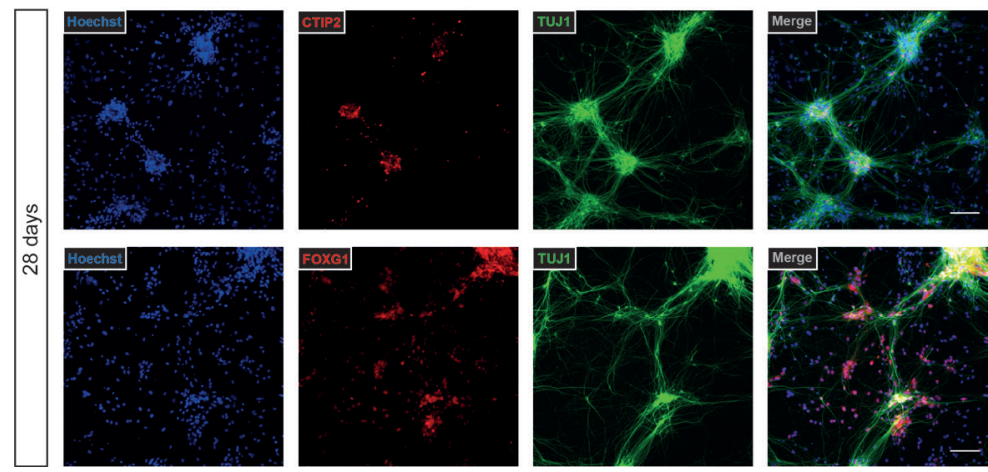

Figure 4. Characterization of the cortical forebrain neurons and glia derived from the human induced pluripotent stem cells (iPSCs). A. Expression of pluripotency markers such as OCT4 was not detected in the differentiated cortical forebrain cells 14 days after the start of neural progenitor cell (NPC) differentiation. B. Representative fluorescent images of the cells staining positive for the neuronal markers MAP2AB, TUJ1, DCX, as well as the glial marker GFAP 28 days after start of NPC differentiation. Images are displayed for the nuclear counterstaining with Hoechst, the respective marker and the overlay of both images (Merge). C. Cells furthermore expressed cortical forebrain markers, including CTIP2, which is cortical pyramidal neuronal marker found in layer VI-V, as well as the telencephalic marker FOXG1. Scale bars represent $100 \mu \mathrm{M}$. 


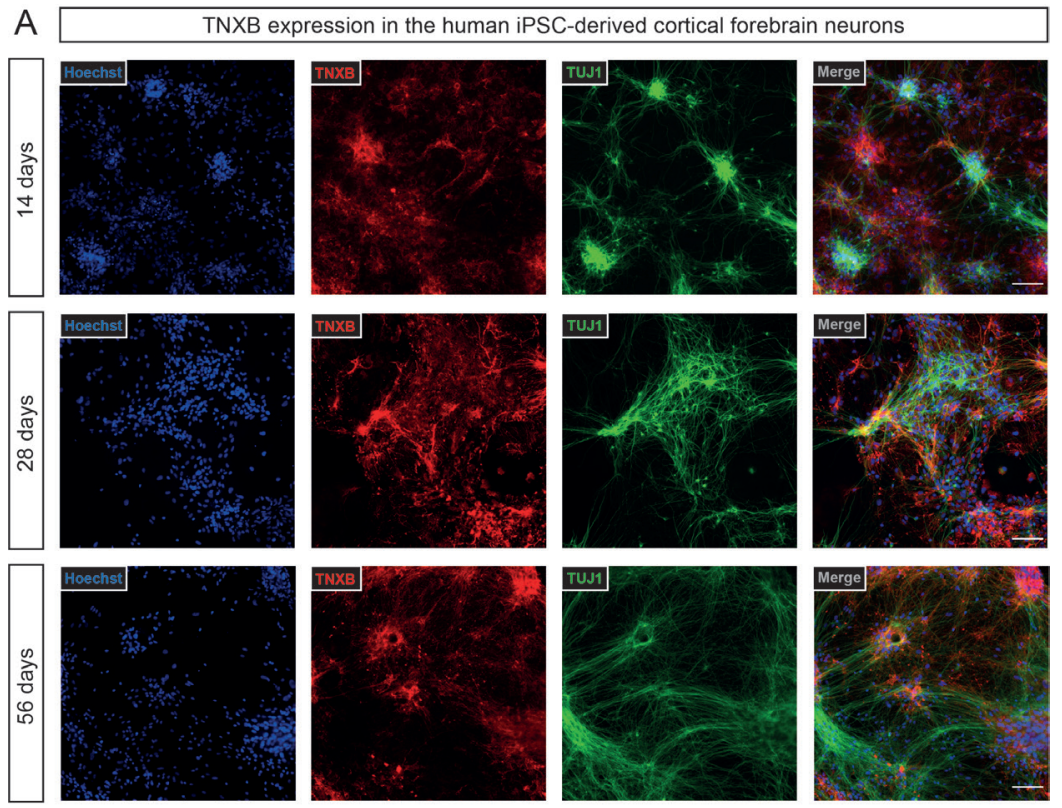

B OXTR expression in the human iPSC-derived cortical forebrain neurons
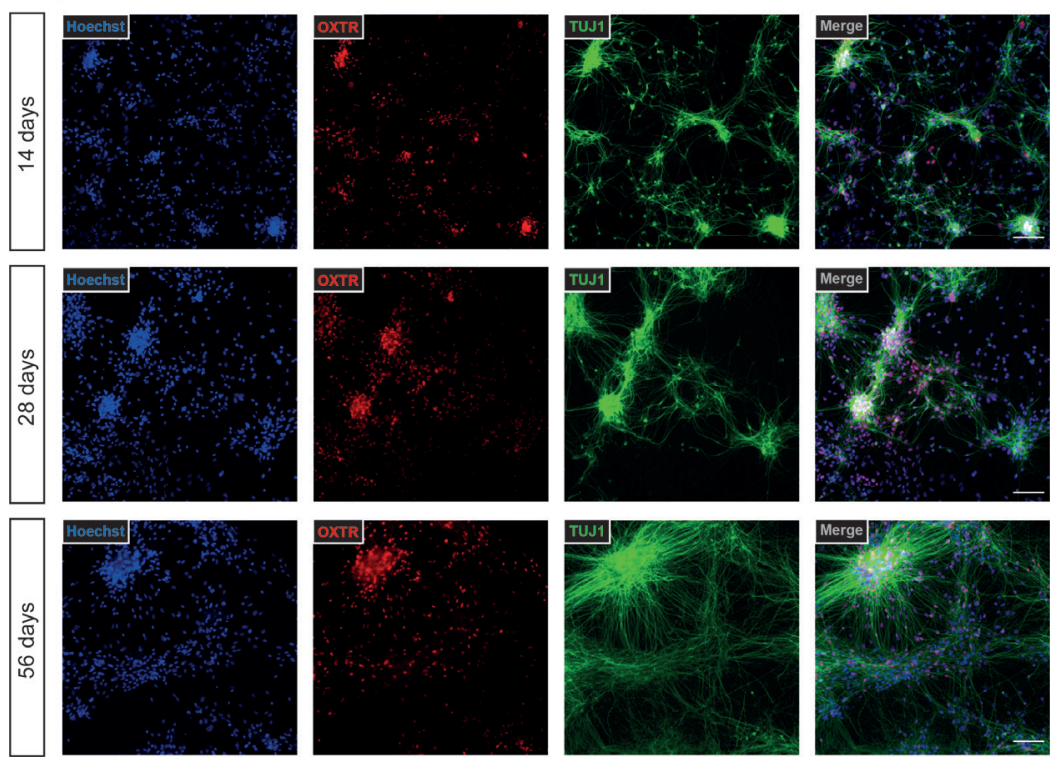

Figure 5. Expression of Tenascin XB (TNXB) and the oxytocin receptor (OXTR) in the cortical forebrain neurons and glia. Images show the expression of both TNXB (A) and OXTR (B), which were assessed at three time-intervals, i.e. after 14 days, 28 days and 56 days of neural progenitor cell (NPC)-differentiation. Images are displayed for the nuclear counterstaining with Hoechst, TNXB or OXTR and the overlay of both images (Merge). Expression of both TNXB and OXTR could be identified in the differentiated cortical forebrain neurons after 14 days and maintained up until 56 days of differentiation. Scale bars represent $100 \mu \mathrm{M}$. 


\section{Discussion}

In the present study, we described the first steps in establishing and characterizing a cortical forebrain differentiation protocol in iPSCs. The findings described here represent preliminary data of a currently ongoing project aimed at developing in vitro model systems for the study of AD. For this purpose, the stemness of the iPSC-line, which was used for neuronal differentiation, was first confirmed based on assessing the expression of pluripotency markers such as OCT4, NANOG, SSEA3, SSEA4, TRA-1-60 and TRA-1-81. Next, cells were differentiated based on a chemical stimulation with neuralizing factors known to be involved in guiding development towards a cortical forebrain fate [13]. At each step of the differentiation process, the cells were monitored for their expression of key-lineage-specific markers. As such, we demonstrated that the cells successfully adopted to a forebrain fate and differentiated into NPCs, and subsequently both neurons and glia. Successful derivation of NPCs was confirmed by the presence of markers such as NESTIN, SOX2 and PAX6 (Figure 3). Quantification of these markers furthermore revealed that most of the iPSCs differentiated towards NPCs ( $>82 \%)$ with a comparable efficiency as described previously [13]. Immunofluorescent examination of the differentiated NPCs verified the presence of the neuronal markers such as MAP2AB, DCX and TUJ1, and the astrocytic marker GFAP. Additionally, the presence of cortical forebrain markers, including CTIP2 and FOXG1, were identified. Overall, these preliminary findings confirmed the successful differentiation of the iPSCs in a heterogeneous mixed population of cortical forebrain neurons and glia.

As a next step in the characterization process of the iPSC-derived neuronal cells, it would be imperative to identify the neurotransmitter subtypes that are present in the differentiated cultures. In general, iPSCs that are differentiated in vitro towards a cortical forebrain fate produce neurons that predominantly express glutamatergic and gamma-aminobutyric acid (GABA)-ergic markers [1]. For instance, in a study using a similar differentiation protocol it was found that the majority of the iPSCderived neurons $(\sim 80 \%)$ were glutamatergic and a smaller subset $(\sim 20 \%)$ was GABAergic [13]. The eventual derivation of these subtypes is, however, dependent on a variety of technological and biological factors, and variation between, as well as within laboratories, is commonly observed while using the exact same protocol [1]. Furthermore, previous studies have demonstrated that the differentiation propensity for specific neurotransmitter subtypes also depends on the iPSC line used, as processes such as epigenetic memory and complete reprogramming of the cells might affect differentiation efficiency [1, 21]. It would, therefore, be interesting to explore the ratios between these subtypes, as well as other neuronal cells, in the cultures. In addition, next to the expression of both neuronal and glial markers, it would be important to confirm that the neuronal cells derived from the iPSC also display the functional characteristics of their in vivo equivalents. As 
such, another important milestone to be achieved, would be to confirm that the derived neurons are electrophysiologically active, assessed either by means of calcium imaging or by patch-clamp recordings. Overall, such measures, as well as the ability to replicate these finding in different batches and other iPSC lines, are crucial to address the robustness of the differentiation protocol.

After further confirmation of the aforementioned characteristics, the protocol described here could potentially be used for the establishment of a cortical in vitro model, allowing mechanistic studies related to AD. In an attempt to address this potential already at an early stage, we then quantified the expression of TNXB and OXTR in the iPSC-derived cortical cultures. As previously demonstrated in Chapter 4 of this thesis, epigenetic and genetic deregulation of TNXB have been associated with $A D$ pathophysiology in multiple regions of the $A D$ brain, including the cortex [9, 17-19, 22]. In addition, epigenetic deregulation of the OXT gene and alterations in OXT signaling have previously been implicated in AD or associated disease phenotypes [10, 20, 23, 24]. Taken together, these studies demonstrated that both genes and related signaling pathways might be functionally involved in $A D$; hence, the availability of iPSC-derived cortical neurons from both AD patients and healthy controls might represent an appealing approach to study their role in the disease at the molecular level. Here, as a crucial first step, we confirm the presence of TNXB and OXTR protein in the iPSC-derived cortical cells at different time intervals during the differentiation; both proteins could be detected in the differentiated neural cells already at 14 days and maintained up until 56 days of NPC-differentiation. TNXB is a member of the tenascin family of extracellular matrix glycoproteins [25], and, in line with that, we found TNXB immunoreactivity extracellularly in our differentiated cultures. OXTR expression on the other hand, has mainly been observed in neural cell somas with little expression in the neurites [26]. Although a further detailed characterization remains necessary, we also detected OXTR immunoreactivity in the somas of the iPSC-derived neural cells. Thus, the data presented here demonstrate that the cortical forebrain cells might represent an appealing model system to study mechanistic processes related to TNXB and OXT signaling in the context of AD.

As a next step in validating these cultures for the use of an AD disease model it would be highly informative to explore the toxicological responses of the neural cell towards disease-related environmental insults, including amyloid beta proteins. In combination with an MTT assay one would be able to assess cell viability and to determine a dose-toxicity curve towards these peptides. A subsequent analysis using both patient- and healthy control-derived cortical neural cells differentiated from iPSCs might then potentially demonstrate whether toxicological responses could differ in these cultures. Within this context, it would furthermore 
be interesting to interrogate whether genes such as TNXB and OXT or related signaling pathways could mediate resilience or susceptibility towards such insults. Although for TNXB little is known yet about its mechanistic role in the disease, administration of OXT has e.g. recently been shown to reverse amyloid betainduced synaptic impairments in mouse hippocampal slices [27]. As such, it would be interesting to address whether similar effects can be identified in human iPSCderived neural cultures and to apply this platform for further interrogation of the underlying molecular mechanisms mediating these effects.

Aside from the possibilities addressed above, the iPSC-derived cortical neurons obtained by the protocol described in the present manuscript, offer a plethora of other future research opportunities for $A D$. For instance, it is increasingly recognized that environmental risk factors with complex gene-environment interactions, play a crucial role in reinforcing $A D$ pathogenesis. However, the exact molecular nature of these interactions, as well as their temporal relationship with the development of $A D$, remain largely unknown. Furthermore, studies have shown that exposure to risk factors can bring about epigenomic, transcriptomic, proteomic and metabolomic changes related to $A D$, which can bring about sustained alterations in molecular processes leading into the manifestation of the full-blown disease. These observations that advocate for the role of environmental risk factors in combination with genetic and epigenetic factors, could nowadays be modeled and, hence, studied more thoroughly in iPSC-derived neuronal cells. Within this framework, the recent availability of editing systems based on CRISPR-Cas9, as well as the use of isogenic lines even adds another dimension to these possibilities. All in all, it is therefore anticipated that future efforts specifically focusing on the associations between environmental factors and the aforementioned multi-omics modalities will aid in developing a better understanding about their involvement in AD. Furthermore, studies aimed at comparing both multi-omics profiles between iPSCderived and ex vivo neuronal sub-populations, obtained from both AD patients and healthy controls, would represent another fascinating example that might not only provide us with new knowledge about molecular disease mechanisms, but also could aid in further mapping the strengths and weaknesses of iPSC-derived models.

Finally, next to the cortical neurons, it would also be interesting to explore whether other neural subtypes, e.g. serotonergic neurons, can be obtained from the iPSCs using adapted protocols based on alternative patterning factors. Hence, this would allow one to explore whether the aforementioned environmental factors and molecular alterations relevant to AD behave similarly or differently when studying them in other neural cells derived from iPSCs. Moreover, the availability of different iPSC-derived neural populations also allows for future co-cultures or complex 3D 
models using scaffolds-based and scaffold-free systems, e.g. gels and spheroids, respectively [28]. These latter complex cultures are emerging as an innovative and advanced alternative driven by their resemblance to in vivo environmental and architectural characteristics. In fact, these model systems allow for complex intercellular communication and the establishment of intricate spatial organizations that provide physical and chemical cues, which might even be essential for the study of human brain diseases at both cellular and molecular levels. Furthermore, it has even been shown that 3D environments can promote better neuronal differentiation and neural network formation [28-32]. Alternatively, a combination of both in vitro differentiation and subsequent transplantation into animal models might represent another opportunity for AD modelling [33]. Such approaches might even capture pathological phenotypes that could not be detected when cells are only cultured in vitro. All in all, the availability of iPSC-derived neurons represents an exciting new area to explore for future AD studies.

\section{Acknowledgements}

The authors would like to thank Dr. Theodora Saridaki and Daria Kaloujskaia for their assistance during the project. This research was made possible by BReIN (Brightlands e-infrastructure for Neurohealth), an initiative which is co-funded by the Province of Limburg, Maastricht University and Maastricht University Medical Centre + in the Netherlands.

\section{References}

1. Riemens RJ, van den Hove DL, Esteller M, Delgado-Morales R. Directing neuronal cell fate in vitro: achievements and challenges. Progress in neurobiology. 2018;168:42-68.

2. Israel MA, Yuan SH, Bardy C, Reyna SM, Mu Y, Herrera C, et al. Probing sporadic and familial Alzheimer's disease using induced pluripotent stem cells. Nature. 2012;482(7384):216-20.

3. Yagi T, Ito D, Okada Y, Akamatsu W, Nihei Y, Yoshizaki T, et al. Modeling familial Alzheimer's disease with induced pluripotent stem cells. Hum Mol Genet. 2011;20(23):4530-9.

4. Kondo T, Asai M, Tsukita K, Kutoku Y, Ohsawa Y, Sunada Y, et al. Modeling Alzheimer's disease with iPSCs reveals stress phenotypes associated with intracellular Abeta and differential drug responsiveness. Cell Stem Cell. 2013;12(4):487-96.

5. Muratore CR, Rice HC, Srikanth P, Callahan DG, Shin T, Benjamin LN, et al. The familial Alzheimer's disease APPV717I mutation alters APP processing and Tau expression in iPSC-derived neurons. Hum Mol Genet. 2014;23(13):3523-36.

6. Young JE, Boulanger-Weill J, Williams DA, Woodruff G, Buen F, Revilla AC, et al. Elucidating molecular phenotypes caused by the SORL1 Alzheimer's disease genetic risk factor using human induced pluripotent stem cells. Cell Stem Cell. 2015;16(4):373-85.

7. Sproul AA, Jacob S, Pre D, Kim SH, Nestor MW, Navarro-Sobrino M, et al. Characterization and molecular profiling of PSEN1 familial Alzheimer's disease iPSC-derived neural progenitors. PLoS One. 2014;9(1):e84547.

8. Gasparoni G, Bultmann S, Lutsik P, Kraus TF, Sordon S, VIcek J, et al. DNA methylation analysis on purified neurons and glia dissects age and Alzheimer's disease-specific changes in the human cortex. Epigenetics \& chromatin. 2018;11(1):41. 
9. Lunnon K, Smith R, Hannon E, De Jager PL, Srivastava G, Volta M, et al. Methylomic profiling implicates cortical deregulation of ANK1 in Alzheimer's disease. Nat Neurosci. 2014;17(9):1164-70.

10. Lardenoije R, Roubroeks JA, Pishva E, Leber M, Wagner H, latrou A, et al. Alzheimer's diseaseassociated (hydroxy) methylomic changes in the brain and blood. Clinical epigenetics. 2019;11(1):164.

11. Riemens RJM, Pishva E, latrou A, Roubroeks J, Nolz J, Lardenoije R, et al. Brain-region- and cell type-specific epigenetic profiling strongly implicates a role for dysregulation of TNXB and other loci in the brainstem in Alzheimer's disease. 2021.

12. Sullivan S, Stacey GN, Akazawa C, Aoyama N, Baptista R, Bedford P, et al. Quality control guidelines for clinical-grade human induced pluripotent stem cell lines. Regenerative medicine. 2018;13(7):859-66.

13. Vadodaria KC, Ji Y, Skime M, PaquolaA, Nelson T, Hall-Flavin D, et al. Serotonin-induced hyperactivity in SSRI-resistant major depressive disorder patient-derived neurons. Molecular psychiatry. 2019;24(6):795-807.

14. Chambers SM, Fasano CA, Papapetrou EP, Tomishima M, Sadelain M, Studer L. Highly efficient neural conversion of human ES and iPS cells by dual inhibition of SMAD signaling. Nat Biotechnol. 2009;27(3):275-80.

15. Rasband WS. National Institutes of Health, Bethesda, Maryland, USA. http://imagej nih gov/ij/. 2011.

16. Espuny-Camacho I, Michelsen KA, Gall D, Linaro D, Hasche A, Bonnefont J, et al. Pyramidal neurons derived from human pluripotent stem cells integrate efficiently into mouse brain circuits in vivo. Neuron. 2013;77(3):440-56.

17. Chang T, editor Tau Network Genes in a Genome Wide Association Study of Progressive Supranuclear Palsy. MOVEMENT DISORDERS; 2017: WILEY 111 RIVER ST, HOBOKEN 07030-5774, NJ USA.

18. Chapuis J, Hot D, Hansmannel F, Kerdraon O, Ferreira S, Hubans C, et al. Transcriptomic and genetic studies identify IL-33 as a candidate gene for Alzheimer's disease. Molecular psychiatry. 2009;14(11):1004-16.

19. Nazarian A, Yashin Al, Kulminski AM. Genome-wide analysis of genetic predisposition to Alzheimer's disease and related sex disparities. Alzheimer's research \& therapy. 2019;11(1):5.

20. Haas BW, Filkowski MM, Cochran RN, Denison L, Ishak A, Nishitani S, et al. Epigenetic modification of OXT and human sociability. Proceedings of the National Academy of Sciences. 2016;113(27):E3816-E23.

21. Kim JE, O‘Sullivan ML, Sanchez CA, Hwang M, Israel MA, Brennand K, et al. Investigating synapse formation and function using human pluripotent stem cell-derived neurons. Proceedings of the National Academy of Sciences of the United States of America. 2011;108(7):3005-10.

22. Sherva R, Baldwin CT, Inzelberg R, Vardarajan B, Cupples LA, Lunetta K, et al. Identification of novel candidate genes for Alzheimer's disease by autozygosity mapping using genome wide SNP data. Journal of Alzheimer's Disease. 2011;23(2):349-59.

23. Cong L, Jia J, Qin W, Ren Y, Sun Y. Genome-wide analysis of DNA methylation in an APP/PS1 mouse model of Alzheimer's disease. Acta neurologica Belgica. 2014;114(3):195-206.

24. Tampi RR, Maksimowski M, Ahmed M, Tampi DJ. Oxytocin for frontotemporal dementia: a systematic review. Therapeutic advances in psychopharmacology. 2017;7(1):48-53.

25. Aktar R, Peiris M, Fikree A, Cibert-Goton V, Walmsley M, Tough IR, et al. The extracellular matrix glycoprotein tenascin-X regulates peripheral sensory and motor neurones. The Journal of physiology. 2018;596(17):4237-51.

26. Tan Y, Singhal SM, Harden SW, Cahill KM, Nguyen D-TM, Colon-Perez LM, et al. Oxytocin receptors are expressed by glutamatergic prefrontal cortical neurons that selectively modulate social recognition. Journal of Neuroscience. 2019;39(17):3249-63.

27. Takahashi J, Yamada D, Ueta Y, Iwai T, Koga E, Tanabe M, et al. Oxytocin reverses A impairment of hippocampal synaptic plasticity in mice. Biochemical and Biophysical Research Communications. 2020;528(1):174-8. 
28. Hernández-Sapiéns MA, Reza-Zaldívar EE, Cevallos RR, Márquez-Aguirre AL, Gazarian K, CanalesAguirre AA. A three-dimensional Alzheimer's disease cell culture model using iPSC-Derived neurons carrying A246E mutation in PSEN1. Frontiers in Cellular Neuroscience. 2020;14.

29. Choi SH, Kim YH, Hebisch M, Sliwinski C, Lee S, D'Avanzo C, et al. A three-dimensional human neural cell culture model of Alzheimer's disease. Nature. 2014;515(7526):274-8.

30. Ravi M, Paramesh V, Kaviya S, Anuradha E, Solomon FP. 3D cell culture systems: advantages and applications. Journal of cellular physiology. 2015;230(1):16-26.

31. Zhang D, Pekkanen-Mattila M, Shahsavani M, Falk A, Teixeira Al, Herland A. A 3D Alzheimer's disease culture model and the induction of P21-activated kinase mediated sensing in iPSC derived neurons. Biomaterials. 2014;35(5):1420-8.

32. Fang Y, Eglen RM. Three-dimensional cell cultures in drug discovery and development. Slas discovery: Advancing Life Sciences R\&D. 2017;22(5):456-72.

33. McQuade A, Kang YJ, Hasselmann J, Jairaman A, Sotelo A, Coburn M, et al. Gene expression and functional deficits underlie TREM2-knockout microglia responses in human models of Alzheimer's disease. Nature communications. 2020;11(1):1-17. 


\section{Supplementary material}

Supplementary table 1. Overview antibodies

\begin{tabular}{|c|c|c|c|c|}
\hline \multicolumn{5}{|l|}{ Primary antibodies } \\
\hline Antibody & Host & Catalogue Number & Company & Dilution \\
\hline ОСТ4 & Goat & ab27985 & Abcam & $1: 1000$ \\
\hline NANOG & Rabbit & ab5731 & Sigma Aldrich & $1: 200$ \\
\hline SSEA3 & Rat & 330302 & BioLegend & $1: 200$ \\
\hline SSEA4 & Mouse & 330402 & BioLegend & $1: 200$ \\
\hline TRA-1-60 & Mouse & 330602 & BioLegend & $1: 1000$ \\
\hline TRA-1-81 & Mouse & 330702 & BioLegend & $1: 500$ \\
\hline PAX6 & Rabbit & ab5790 & Abcam & $1: 200$ \\
\hline NESTIN & Mouse & MAB5326 & Merck Millipore & $1: 200$ \\
\hline sox2 & Goat & EB07378 & Everest Biotech & $1: 200$ \\
\hline MAP2AB & Chicken & $\mathrm{CH} 22103$ & Neuromics & $1: 200$ \\
\hline TUJ1 & Mouse & MAB1637 & Sigma Aldrich & $1: 500$ \\
\hline DCX & Goat & ab113435 & Abcam & $1: 200$ \\
\hline CTIP2 & Rat & ab18465 & Abcam & $1: 200$ \\
\hline FOXG1 & Rabbit & ab18259 & Abcam & $1: 200$ \\
\hline GFAP & Rabbit & $a b 7260$ & Abcam & $1: 200$ \\
\hline OXTR & Rabbit & ab217212 & Abcam & $1: 200$ \\
\hline TNXB & Rabbit & AB19011 & Merck Millipore & $1: 200$ \\
\hline \multicolumn{5}{|l|}{ Secundary antibodies } \\
\hline Antibody & Host & Catalogue Number & Company & Dilution \\
\hline Anti-Goat Alexa 647 & Donkey & A-21447 & $\begin{array}{l}\text { ThermoFisher Scientific, } \\
\text { Invitrogen }\end{array}$ & $1: 100$ \\
\hline Anti-Rabbit Alexa 488 & Donkey & A-21206 & $\begin{array}{l}\text { ThermoFisher Scientific, } \\
\text { Invitrogen }\end{array}$ & $1: 100$ \\
\hline Anti-Rabbit Alexa 594 & Donkey & A-21207 & $\begin{array}{l}\text { ThermoFisher Scientific, } \\
\text { Invitrogen }\end{array}$ & $1: 100$ \\
\hline Anti-Mouse Alexa 488 & Donkey & A-21202 & $\begin{array}{l}\text { ThermoFisher Scientific, } \\
\text { Invitrogen }\end{array}$ & $1: 100$ \\
\hline Anti-Rat Alexa 488 & Donkey & A-21208 & $\begin{array}{l}\text { ThermoFisher Scientific, } \\
\text { Invitrogen }\end{array}$ & $1: 100$ \\
\hline Anti-Rat Alexa 594 & Donkey & A-21209 & $\begin{array}{l}\text { ThermoFisher Scientific, } \\
\text { Invitrogen }\end{array}$ & $1: 100$ \\
\hline Anti-Chicken Alexa 647 & Donkey & $703-605-155$ & $\begin{array}{l}\text { Jackson ImmunoResearch } \\
\text { Europe Ltd }\end{array}$ & $1: 100$ \\
\hline
\end{tabular}

Overview of the used antibodies in the present study. Displayed are the target of the antibody, the host species, the catalogue number, the company and dilution that was used for each respective antibody. 
Chapter 9

General discussion 

In this section, the major aims, key findings, strengths, limitations and future perspectives of the research collected in this thesis will be discussed.

\section{Discussion of major aims and key findings}

In this thesis, the importance of epigenetic mechanisms, with a major focus on DNA methylation and hydroxymethylation, in the development and course of sporadic Alzheimer's disease (SAD) were addressed. As emphasized throughout the dissertation, an individual's epigenotype at the level of both aforementioned DNA modifications can be altered as a response towards (e.g. adverse) environmental stimuli, leading to changes in gene expression patterns that can affect molecular and cellular processes [1]. These epigenetic changes can occur throughout life and may contribute directly to the development of SAD pathology or interact with the embedded effects of other contributing factors, such as genetic risk factors, to both induce and influence SAD development and progression [2]. In recent years, an increasing number of epigenome-wide association studies (EWAS) have therefore been performed in SAD (reviewed in Chapter 2), where state-of-the-art Illumina microarray technology was applied in combination with more targeted approaches using pyrosequencing on various patient-derived (typically cortical) brain tissues and blood samples to identify disease-associated changes at the level of DNA (hydroxy)methylation [3-12]. As such, these studies have identified distinct and overlapping loci that are dysregulated in SAD patients' brain and/or blood, emphasizing their crucial involvement in the disease. Even though further validation and functional interrogation of these epigenetic signatures remains necessary, the identification of these loci is highly valuable for SAD research, as they provide novel molecular leads that deepen our knowledge on underlying disease mechanisms that subsequently aid in the development of future therapeutic interventions and potential disease biomarker assays.

In spite of the importance of these findings for SAD research, it is well-known that neuroepigenomic studies still come along with numerous challenges. These include the limited genomic coverage of the commonly applied Illumina arrays, the relative low statistical power of EWAS, the (a)specificity of current methodologies, issues related to cell-type specificity of epigenetic marks, variations in target tissue compositions, a lack of understanding in terms of causality and a current scarcity in multi-omics approaches (reviewed in Chapter 2), some of which will also be addressed in more detail in the section 'Strengths and limitations' that can be found below. Altogether, these issues challenge the correct interpretation of current epigenetic findings in relation to sAD, thereby limiting our understanding of their exact functional implications and causal relationships in the etiopathogenesis of $A D$. It has therefore been debated that future endeavors aimed at incorporating these considerations into their study designs can substantially increase the reliability and significance of the derived epigenetic data. 
Hence, the first part of this thesis (Chapters 3 and 4 ) builds upon this notion and aims at overcoming reoccurring methodological challenges in neuroepigenomic studies, thereby allowing for the production of more conclusive data. Issues that were tackled included the (a)specificity of current (bisulfite) methodologies, as well as the tissue and cell-type specificity of epigenetic marks, which will be discussed in the more detail in the following sections.

It is well-known that classical bisulfite treatment does not allow for the discrimination between DNA methylation and hydroxymethylation, i.e. between 5-methylcytosine (5-mC) and 5-hydroxymethylcytosine (5-hmC), respectively [13, 14]. In fact, Illumina bead arrays and other sequencing-based approaches, such as pyrosequencing, which have relied on this technique, have previously acquired cumulative measures on the levels of both modifications rather than each of them individually. For this reason, earlier studies in SAD utilizing this classical bisulfite approach might have overestimated, underestimated, or even overlooked relevant effects related to these epigenetic marks. Thus, in an attempt to overcome this issue, Chapter 3 presented an alternative approach for pyrosequencing based on a highly selective chemical oxidation using $\mathrm{KRuO} 4$, which converts 5 -hmC into 5 -formylcytosine (5-fC) bases [13]. While after classical bisulfite treatment and subsequent polymerase chain reaction (PCR)-based amplification, both $5-\mathrm{mC}$ and $5-\mathrm{hmC}$ are read as a cytosine, 5 -fC bases are read as a thymine at the pyrosequencing step, hence allowing one to distinguish between $5-\mathrm{mC}$ and 5 -hmC in the context of single cytosine-guaninephosphate $(\mathrm{CpG})$ sites [13]. Oxidative bisulfite pyrosequencing - the technology described in this chapter - therefore provides a more accurate readout of the levels of $5-\mathrm{mC}$ as compared to classical bisulfite pyrosequencing, while at the same time allowing the user to estimate levels of 5-hmC after quantitative subtraction of the bisulfite and oxidative bisulfite data obtained for the same DNA sample.

In order to demonstrate the efficacy of this modified approach, three spike-in pyrosequencing controls were developed. These can be added to any given DNA sample prior to (oxidative) bisulfite treatment. By utilizing these standards, it was demonstrated that the oxidative bisulfite pyrosequencing approach can be successfully applied for the detection of 5-mC and 5-hmC, as well as unmodified cytosine (5$\mathrm{uC}$ ) bases, the latter of which can be ascertained by subtracting the bisulfite signal from $100 \%$ [12]. In order to further address the sensitivity of the method, the $5-\mathrm{hmC}$ conversion efficiency was estimated by using the spike-in pyrosequencing controls, revealing that a $5-\mathrm{hmC}$ conversion efficiency $>88 \%$ per $\mathrm{CpG}$ site could be obtained, which is similar to previous studies using the oxidative bisulfite technology with other sequencing approaches, e.g. Sanger sequencing [14]. In addition, factors such as an insufficient bisulfite incubation time were demonstrated to negatively influence the 5-hmC conversion efficiency. Next, it was shown that levels of all three aforementioned 
modifications could be detected following this approach targeting OXT and DNAJB13 in both post-mortem brain tissue and cultured induced pluripotent stem cells (iPSCs), which are tissues known to be enriched in all three modifications [15-18]. Finally, it was confirmed that the 5-hmC spike-in control could be used as internal pyrosequencing control that does not interfere with the analysis of the accompanying sample, and vice versa. Thus, the oxidative bisulfite pyrosequencing protocol presented in this thesis offers a standardized approach for the targeted detection of 5-mC, 5-hmC and 5-uC that could greatly benefit future neuroepigenomic studies, even outside the field of SAD research.

In addition to the (a)specificity of current (bisulfite) methodologies, the second issue that was tackled in the first part of the thesis was the reoccurring challenge of tissue and cell-type specificity of epigenetic marks, obtained from heterogeneous bulk tissues used for epigenetic profiling (see Chapter 4). Aside from inducing noise in epigenetic profiles, the use of bulk tissues, as commonly applied in SAD studies, could mask cell-type specific epigenetic modifications related to the disease, as changes in one cell type could dilute or oppose changes in another, thereby obscuring important cell-specific alterations when analyzed in bulk tissue [19]. By utilizing a combination of laser capture microdissection (LCM) and limiting dilution bisulfite pyrosequencing (LDBSP) [20], Chapter 4 described a novel approach for targeted methylation analysis of single alleles derived from small pools of individually isolated neurons. As such, this approach offers a workable solution for the aforementioned issue, as it allows for targeted methylation profiling in a limited number of isolated brain cells.

For this purpose, dorsal raphe nucleus (DRN) tissue sections were stained for a neuronal subtype-specific marker (i.e. serotonin (5-HT)), after which immuno-positive neurons were individually isolated using LCM and subsequently processed in pools of 50 cells for LDBSP analysis, targeting DNAJB13, PGLYRP1, OXT, RHBDF2, C3 and $L M X 1 B$ simultaneously. In contrast with previous studies [20-25], LDBSP on pools of 50 neurons rendered a considerable amount ( $8.77 \%$ on average per gene) of the downstream PCR reactions with more than one target allele. In order to correct the LDBSP data for these multi-allele reactions, a novel data analysis approach was then developed. This novel approach, which compromises a CpG-site calling procedure combined with an integrated in-depth analysis of the raw $\mathrm{CpG}$ methylation values, aims at avoiding unintentionally induced bias due to the blunt exclusion of reactions that seem to contain more than one target allele. When comparing this novel approach to the traditional method for the downstream data analysis, which is based on excluding multi-allele reactions, significant differences in the combined CpG methylation rates for $L M X 1 B$, as well as for individual $C p G$ sites in $L M X 1 B$, $R H B D F 2$ and $O X T$, were identified. These findings emphasized that the derived LDBSP methylation data can differ significantly depending on the method that is 
applied for the downstream analysis. This implies that it is crucial to correct for multiallele reactions instead of excluding them when encountered utilizing LDBSP, as this could potentially influence experimental data negatively, either by inducing bias or by reducing or reinforcing effect sizes, for example. Overall, the approach proposed here provides the user with a more accurate estimation of the DNA methylation status of each target gene in the analyzed cell pools, thereby adding further validity to the data. In conclusion, LDBSP in combination with LCM offers a novel and alternative strategy to single cell(type) bisulfite sequencing techniques that can be applied for the study of DNA methylation marks in the human brain. It is anticipated that similar strategies using a limited number of isolated cells in combination with LDBSP will be increasingly valuable for future neuroepigenomic studies, also outside the scope of SAD.

Another major focus in the first part of this thesis (see Chapter 5) was centered on the importance and (unmet) need for profiling epigenetic signatures in early affected regions of the SAD brain, including regions of the brainstem such as the DRN and locus coeruleus (LC), which has not been performed previously. When looking at the bulk of EWAS that has been conducted in SAD to date (see Chapter 2), one would realize that these studies have primarily assessed epigenetic profiles in either cortical regions or the hippocampus. Even though one cannot deny (later) involvement of limbic and cortical areas of the brain in relation to the cognitive symptoms, recent evidence suggest that early dysregulation in both the aforementioned brainstem nuclei accounts for the earliest, largely non-cognitive symptomatology [26-28]. Furthermore, studies have shown that tauopathy may begin earlier than previously thought and possibly in the brainstem rather than in transentorhinal regions [26-29], suggesting a potential causal role in the pathogenesis of SAD. In line with this view, it is currently thought that from these brainstem nuclei pathology could spread to subcortical areas, including the hippocampus, and, subsequently, to areas of the neocortex, marking the clinical stages of the disease. Other evidence for a central role of the brainstem in SAD comes from magnetic resonance imaging studies, demonstrating significant volume reductions and structural deformations in these brainstem regions of SAD patients [ 30 , 31]. Thus, interrogating the epigenetic landscape in early affected regions such as the DRN and LC could potentially improve our understanding on the role of epigenetic mechanisms involved in the early stages, as well as during the course of the disease.

Chapter 5 therefore presented the first large-scale epigenetic study in the DRN and LC, consisting of two EWAS combined with a similar oxidative bisulfite approach as described in Chapter 3. Furthermore, these discovery EWAS were complemented with a targeted pyrosequencing validation study in both brainstem regions using the same patient cohort, as well as a cell-subtype specific validation analysis in the DRN using an independent cohort (relying on the combinatory LCM-LDBSP approach described in Chapter 4). During the EWAS, Braak stage-associated alterations at the 
level of 5-mC, 5-hmC and 5-uC were quantified in bulk tissues derived from both the DRN and the LC by using the Illumina Infinium MethylationEPIC BeadChip array. A strong overlapping dysregulation in the Tenascin XB (TNXB) gene was identified in both brainstem regions assessed, next to previously identified and novel epigenetic loci, which might play a pivotal role in the early development and course of SAD. In the subsequent validation study using bisulfite pyrosequencing in the same patient cohort, it was confirmed that demethylation of TNXB was associated with increasing Braak stage in both the DRN and LC. These findings were then validated in an independent patient cohort targeting TNXB in single serotonergic and non-serotonergic cells isolated from the DRN by using the LCM-LDBSP approach and an adapted bisulfite pyrosequencing protocol for each of the cell types, respectively. When comparing the bisulfite methylation levels of TNXB between SAD patients and controls in serotonergic and non-serotonergic cells, a significant interaction between cell-type and experimental condition was identified. Strikingly, SAD-associated methylation profiles in TNXB were opposite in serotonergic neurons and non-serotonergic cells, the latter of which resembled the initially acquired EWAS and pyrosequencing data.

Taken together, these results strongly advocate for an important role of epigenetic dysregulation in $T N X B$ in the (etio)pathophysiology of the disease. Interestingly, epigenetic dysregulation in TNXB has previously also been annotated in another SAD EWAS [5], next to genetic variation in this locus that has been associated with a risk of developing the disease [32-34], as well as with another tau-related disorder [35]. TNXB expresses a glycoprotein with anti-adhesive properties, but its exact physiological role in the brain is not completely understood [36]. As such, more research on the exact function of $T N X B$, the interplay between genetic and epigenetic variation in this locus, as well as further functional interrogation of this SAD neuropathology-associated epigenetic signature, remains necessary in order to develop a better understanding of its exact role in SAD. Another major outcome of this study was that the associated dysregulation in TNXB in the DRN was both dependent on the disease phenotype and the cell type analyzed, which supports the notion that epigenetic data derived from heterogeneous post-mortem bulk tissue should be interpreted with caution, as changes in one cell type could negate or mask changes in another. Overall, this finding has crucial implications for future planned epigenetic studies in AD, as it warrants the need for single cell (-type) neuroepigenetic analyses, opposite to the more common bulk tissue analyses that have been performed to date. Future efforts aimed at dissecting intercellular differences of epigenetic marks are therefore anticipated to aid in developing a more complete understanding of their role in the disease and will be increasingly important for neuroepigenomic studies into SAD.

While still dedicated to the pathophysiology of SAD, the second part of the thesis (Chapters 6, 7 and 8) deviates from profiling epigenetic signatures in patient-derived 
brain tissues or cells and addresses the potential of iPSC-based models for studying the disease. The capacity to differentiate iPSCs into disease-relevant neural subtypes has empowered them as an exciting tool for the generation of patient-specific 'brain in a dish' models that could fill the gap between pre-clinical and clinical SAD research and may, eventually, largely replace experimental animal studies [37-39]. The second part of the thesis therefore commences with a review paper presented in Chapter 6 , summarizing the pioneering studies that have explored the use of these stem cells for SAD modelling. Recent findings have demonstrated that cells harboring patientspecific backgrounds do manifest pathological phenotypes in vitro, such as increased levels of amyloid beta peptides and hyperphosphorylation of tau protein, amongst others [40-44]. For this reason, patient-derived iPSC models are thought to be extremely valuable for fundamental research into $S A D$, as they allow one to interrogate functional effects of genetic, epigenetic and transcriptional variants linked to risk, as well as to protective, environmental factors. Within the field of neuroepigenomics, iPSC-based models in combination with epigenetic editing systems might even offer appealing avenues to dissect causality of epigenetic signatures in view of disease pathology, and vice versa [19]. Such endeavors might significantly contribute to elaborate our understanding on the mechanisms underlying the disease and could aid in the development of therapeutic interventions.

In spite of their potential, these aforementioned studies have also proven that a high degree of variability in terms of detectable disease hallmarks is common when comparing differentiated neural cells from different patient-derived iPSC lines. While some of this might be explained by the heterogeneous nature of $S A D$, it is likely that other sources of variability related to the models play a role in this as well. Given that iPSC models in SAD represent a relative nascent area of research, it is therefore crucial to dissect each of these sources of variability and to assess how these could impact the interpretation of the findings in relation to SAD. Examples of such sources (that were also reviewed in Chapter 6) include genetic alterations induced in response to iPSC reprogramming, cell type origin-dependent neural differentiation bias, loss of potential aging traits in IPSC lines due to reprogramming processes of the epigenome and a lack of consensus in neural differentiation protocols between different studies. In addition, much work is still needed in order to define and establish adequate reference panels for control cell lines, as well as to assess the impact of somatic cell mosaicism that can affect disease modelling, and clonal selectivity of donor cells for iPSC reprogramming.

Expanding on the potential of patient-specific in vitro disease models, Chapter 7 follows with a thorough review on directed and direct neural differentiation protocols using iPSCs and other somatic cells. The progress that has been made to date in generating human and mouse anatomically-specified, i.e. attributable 
to specific regions of the brain, neural precursor cells (NPCs) and differentiated neuronal subtypes are being summarized, including glutamatergic-, GABAergic, dopaminergic, serotonergic- and cholinergic- neurons. Overall, over the last years, increasing knowledge on developmental signaling pathways and gene regulatory networks has guided the design of current in vitro neuronal differentiation and cellular reprogramming strategies. Moreover, recent studies have demonstrated that within these networks of patterning molecules and transcription factors that orchestrate neuronal induction and differentiation, the epigenetic machinery is essential for finetuning genetic programs that coordinate distinct developmental processes, as well as shaping neuronal identities at a phenotypic resolution $[45,46]$. For this reason, different kinds of methods relying on chemically defined systems using neuralizing patterning factors, transcription factor-mediated reprogramming strategies and epigenetic-based approaches for the derivation of the aforementioned subtypes are currently being developed. Overall, patient-derived neurons obtained via directed differentiation of iPSCs or direct reprogramming of somatic cells promise to fill an important niche between studies in humans and animal models in deciphering disease mechanisms and identifying therapeutic avenues for neurodegenerative- and other psychiatric diseases.

Although recent scientific efforts begin to tap into this potential, pioneering studies have also highlighted challenges in directing neuronal differentiation that must be overcome for it to be fully realized. In fact, in vitro neuronal differentiation is actually not a process that is fully disciplined yet, given the great variability in protocol efficiencies and the production of unwanted cell types in the obtained neural cultures that are observed both within and between laboratories. Furthermore, differentiation protocols for multiple neuronal subtypes remain unestablished, especially when considering very specific anatomical subtypes, or they remain unstable and do not allow one to obtain the terminally differentiated neuron with its entire functional mature characteristics. As such, currently, emphasis is payed toward efforts to increase the efficiency of current protocols, including combinatory approaches and the use of cell sorting techniques, among others (reviewed in Chapter 7). Taken together, the establishment of robust protocols to obtain functional neurons in vitro is essential for studying human brain cell functions, as well as disease modelling, drug discovery and regenerative medicine, both within and outside the scope of SAD research. Future efforts aimed at overcoming these aforementioned limitations are therefore anticipated to aid in realizing the full potential of this platform.

Finally, in Chapter 8, a pilot study is presented that combines efforts described in previous sections and aims at characterizing a cortical forebrain differentiation protocol from iPSCs, with the ultimate goal to apply this approach for the establishment of a SAD-related in vitro model. For this purpose, the iPSC-line used for neuronal 
differentiation was first characterized for the expression of pluripotency markers such as OCT4, NANOG, SSEA3, SSEA4, TRA-1-60 and TRA-1-81, in order to confirm their stemness. Next, the cells were differentiated towards NPCs based on the formation of free-floating embryoid bodies (EBs) combined with the inhibition of dual SMAD signaling [47], which favors development into the neural lineage. These obtained NPCs were then phenotypically characterized by means of immunofluorescence markers for e.g. NESTIN, PAX6 and SOX2. Double labeling with each of these markers revealed that most of the iPSCs differentiated into NPCs (>82\%). These cells were then further differentiated towards a cortical forebrain fate by maintaining them in medium supplemented with both neural patterning- and growth factors. Following this 8-week differentiation protocol, the expression of neuronal markers, including MAP2AB, DCX and TUJ1, and the glial marker GFAP, was confirmed in the cultures. Moreover, the cells lost the expression of pluripotency markers such as OCT4 and typically expressed cortical forebrain markers such as CTIP2 and FOXG1. Furthermore, as TNXB and oxytocin (OXT)-signaling have previously been associated with SAD based on EWAS screenings, expression of both TNXB and the oxytocin receptor (OXTR) was confirmed in the differentiated neural cells by a immunofluorescence staining, suggesting that the cultures might represent an appealing model system to study processes related to both genes in the context of sAD. Overall, these data showed that the applied protocol successfully generated neurons, as well as glial cells, from these iPSCs, with a cortical forebrain identity that will enable future mechanistic investigation of candidate genes for SAD.

As a next step in the characterization, it would be interesting to explore the (ratios of) neurotransmitter subtypes that are present in the differentiated cultures, such as glutamatergic and GABAergic neurons, and to assess whether these cells also display the functional characteristics of their in vivo equivalents, such as their electrophysiological properties. These measures and the ability to replicate the presented findings in different batches and other iPSC lines will be crucial to address the robustness of the differentiation protocol. In addition, to further address the potential for these cultures for the use as a disease model, it would be highly informative to explore the responses of the neural cell towards SAD-related environmental stimuli, including amyloid beta, and assess whether potential toxicological responses might differ in these cultures when comparing healthy control and patient-derived neural cells differentiated from iPSCs. Within this context, it would furthermore be interesting to interrogate whether genes such as TNXB and OXT or related signaling pathways could mediate susceptibility or resilience towards such exposures. In addition, by using alternative differentiation protocols, or by adapting the one proposed in this chapter by including additional patterning factors, allowing for the derivation of serotonergicor noradrenergic neurons that are found in the DRN and LC, respectively, it would be highly fascinating to assess whether similar or different cellular and molecular 
responses can be identified in different cell types. In conclusion, the differentiation method proposed in this chapter provides an important stepping-stone for future functional studies using iPSC-derived brain cells for SAD research.

\section{Strengths and limitations}

The research collected in the first part of this thesis aims at interrogating epigenetic signatures in the (etio)pathophysiology of SAD, with a major focus on identifying dysregulation in early affected brainstem regions that might reflect changes associated with the most incipient stages of the disease. Aside from gaining new insights into underlying disease mechanisms of SAD, a major strength of the projects presented here is the establishment and application of state-of-the-art methodologies. To our knowledge, the method presented in Chapter 3 represents the first fully optimized approach combining pyrosequencing with oxidative bisulfite technology, allowing one to distinguish between 'true' DNA methylation and DNA hydroxymethylation, i.e. 5-mC and 5-hmC, respectively, in a locus-specific manner. Moreover, the novel developed DNA spike-in controls that can be applied in conjunction with the described protocol enables to confirm the successful conversion of the oxidative bisulfite-converted DNA, hence ensuring methodological consistency of the described procedure. This approach in combination with these novel spike-in controls therefore provides future opportunities for more standardized, thorough and affordable interrogation of both DNA modifications using pyrosequencing in a wide variety of pathological conditions, including SAD. Before the introduction of this oxidative bisulfite conversion step, only the combined signal of methylation and hydroxymethylation could be obtained. As repeatedly addressed in this thesis, this has been a major issue in neuroepigenomic studies to date, especially when applied on brain tissue, where DNA hydroxymethylation is known to be more abundant as compared to other bodily tissues. It should therefore be emphasized that previous (brain-related) studies relying on the classical bisulfite approach have been unable to discriminate between the two and, hence, should be interpreted with caution.

In relation to the aforementioned strength, Chapter 5 describes the first study to date that targets DRN and LC bulk tissues derived from the brainstem of SAD patients using the Illumina Infinium MethylationEPIC BeadChip array in combination with an oxidative bisulfite treatment of the isolated target DNA from these samples. Aside from representing one of the pioneering studies applying the oxidative bisulfite approach for genome-wide profiling in SAD, the use of the novel EPIC array technology furthermore provides a higher genomic coverage when compared to previous studies that have commonly relied on its predecessor, i.e. the Illumina BeadChip 450K array. In fact, the EPIC array technology has nearly doubled the number of target $\mathrm{CpG}$ sites across the genome (i.e. from 485.512 to 866.836 probes [48]), now also including previously untargeted enhancer regions, hence allowing for the interrogation of additional sites 
in relation to the disease. In addition to the value of dealing with one of the most devastating disorders world-wide, the effort to interrogate epigenetic signatures in previously overlooked brainstem structures, i.e. the DRN and LC, makes the presented study stand out from similar endeavors targeting other brain regions that are affected in more advanced stages of SAD, which is a major asset of this thesis. To this end, overlapping and unique epigenomic profiles associated with disease progression described by Braak stage were identified, which have set the pillars for future replication studies and meta-analyses, as well as for mechanistic and functional research into their role in the disease.

Another significant strength from the first part of this thesis is the approach described in Chapter 4 and applied in Chapter 5, which relies on a combination of LCM and LDBSP and allows for targeted (bisulfite) methylation profiling of single alleles derived from a limited number of isolated neurons. Initially, the input requirements for targeted approaches such as pyrosequencing, as well as for genome-wide methods such as arrays, required hundreds of thousands or even millions of cells. Owing to technological developments as shown in Chapter 4, this is changing with numerous epigenetic features now assayable at the single-cell level. Understanding of DNA methylation signatures in the past was therefore mostly based on studies that utilized these techniques for quantifying the average epigenetic states of bulk cell populations and tissues, similar to what was performed in Chapter 5 where the EPIC array was applied on heterogeneous brainstem tissue homogenates. Even though these studies using bulk tissues are still highly informative and have revealed meaningful diseaseassociated signatures, such studies generally come along with a small signal-tonoise ratio, while the accompanying measurements that represent average tissue profiles cannot always be extrapolated to single cell populations or certain cell type niches within or between different brain regions, e.g. cells that display disease-related pathology compared to those that do not. Different cell types are known to differ in their epigenetic profiles, independent of disease-related changes, and manifestation of the latter could easily occur in a cell-dependent manner, as demonstrated in Chapter 5 , or only under very specific circumstances, such as within specific time intervals or only upon exposure to certain environmental cues. Evidently, for many biological questions, interrogation of epigenetic marks is most informative when studied at a single-cell level, where intercellular differences can be dissected leading to a more refined understanding of their contribution to the disease. All in all, such cellspecific approaches are therefore of immense added value and are anticipated to be increasingly considered in future neuroepigenomic studies, both within and outside the scope of SAD.

The second part of the thesis is focused on addressing the potential of iPSC-based models in SAD. A clear strength of this platform, which is also addressed in Chapters 
6, 7 and 8 , lies in the fact that patient-derived cells contain a pathogenic background and, hence, it is anticipated that these cells manifest pathological processes in vitro, thereby offering a promising avenue for SAD modeling. In fact, the use of disease-relevant neural cells by differentiating iPSCs along the neural lineage, as demonstrated in Chapters 7 and 8 , offers an alternative approach to study the underlying neuropathological mechanisms in a humanized, personalized, and cell subtype-specific manner. As such, iPSC-derived neuronal populations generated from SAD patients with known pathogenic or susceptible backgrounds can be studied, (epi)genetically probed, exposed to environmental factors and treated with drug libraries to investigate their molecular and cellular effects. For these reasons, there has been a growing body of research over the past years to adopt rapidly improving iPSC-derived model systems of SAD for fundamental research applications, as well as for the assessment of drugs prior to the initiation of clinical trials [49-53]. Another clear advantage of these model systems is their potential to reduce the need for animal experimentation and hopefully increase the translatability of preclinical studies towards clinical interventions. Although still a relative nascent area of investigation, it is anticipated that future efforts in this field will broaden our knowledge on the underlying pathophysiology of many brain disorders, including SAD.

In spite of the aforementioned strengths, the work described in this thesis is also subject to both general and more specific limitations. While the oxidative bisulfite technology used enables the detection of 'true' DNA methylation and hydroxymethylation, it should be recognized that this approach does still not take into consideration other (less prevalent, but functionally relevant) $\mathrm{CpG}$ modifications such as 5-fC and 5-carboxylcytosine (5-caC). In reality, the signals of both 5-fC and 5 -caC are entwined with the signal ascertained for $5-\mathrm{uC}$, which is currently considered inversely proportional to the bisulfite read-out and can be obtained by quantitative subtraction of the bisulfite signal from $100 \%$, as demonstrated in Chapter 3 . In fact, a bisulfite treatment of 5-uC, 5-fC and 5-caC bases results in a conversion to uracil and, subsequently, to thymine after the PCR amplification step, which makes it impossible to distinguish between the three modifications. As the abundancy of 5-fC and 5-caC in the brain is known to be relatively low [18], even when compared to 5 -hmC, it is not expected that they will have a profound impact on the obtained data. This could therefore be regarded as a minor limitation, even though still important to consider given that it affects both the pyrosequencing approach described in Chapter 3 and the array data in Chapter 5.

While both of the previous chapters allow for the detection of 5-mC and 5-hmC, the method described in Chapter 4 relies on the combination of LCM and LDBSP and hence still provides the unsegregated signals, as oxidative bisulfite treatment had not been implemented yet. In fact, it is important to acknowledge that for LDBSP, which 
relies on the analysis of single alleles, it is impossible to obtain a 5-hmC measurement based on this technology. Even when implementing the oxidative module, one can not to run both the bisulfite and oxidative bisulfite approach on a single DNA molecule in parallel, which would be necessary to derive a readout for 5 -hmC. It would therefore require other methodological advances and alternative strategies (such as for example proposed in [54]) in order to obtain a direct 5-hmC read-out from a single DNA molecule, opposite to the indirect method that is used for the oxidative bisulfite technology. Nonetheless, future implementation of the oxidative module for LDBSP could still allow for the derivation of the 5-mC signal only, thereby providing a more detailed profile of 'true' DNA methylation patterns on a single allele-level.

Another limitation stemming from the indirect method to determine 5-hmC and 5-uC levels using the oxidative bisulfite technology is related to the 'naïve' subtraction of the oxidative- and the bisulfite signals that is described in Chapter 3 . Due to technological variation and the need for paired measurements derived from the same DNA target sample, the oxidative bisulfite method is subject to noise that can affect the correct readout for (relative small levels of) 5 -hmC. This issue is further exemplified by two kinds of overshooting values when estimating the 5-hmC and 5-uC levels following quantitative subtraction. As demonstrated in Chapter 3, subtraction of the oxidative bisulfite signal from the combined bisulfite signal for estimating 5 -hm $\mathrm{C}$ can result in the derivation of negative values, which are biologically meaningless and represent false differences in scores between the paired bisulfite-only and the oxidative bisulfite data sets, due to background noise. On the other hand, when combining the levels of 5-uC, $5-\mathrm{mC}$ and 5 -hmC after quantitative subtraction, levels can also exceed $100 \%$ due to similar experimental noise. These overshooting sites may even constitute a substantial proportion of total $\mathrm{CpG}$ sites, depending on the technical variation and the degree of 5 -hmC content in the analyzed samples. In order to exclude negative values, strict detection thresholds have commonly been applied, leading into a substantial dropout of CpG sites from related (Illumina array) data sets. Alternatively, novel methods such as a maximum likelihood estimation (MLE) can nowadays be used to determine the levels of these modifications when dealing with oxidative bisulfite-based 5-mC, 5 -hmC and 5-uC data [55,56], as also demonstrated in Chapter 5. This approach is consistent in that $5-\mathrm{hmC}$ levels are non-negative and all three modifications together never sum over $100 \%$, consequently leading to less CpG dropouts from the data set. Thus, this alternative therefore provides a more suitable approach for estimating the levels of these modifications, even though still subject to experimental variation and noise that would require further methodological developments to be overcome.

Another limitation associated with data analysis of Illumina arrays is related to the identification of differentially modified regions, in which DNA methylation and hydroxymethylation are treated equally. However, next to their distinct biological 
roles, these two modifications are not equally abundant in the brain. In fact, DNA hydroxymethylation is not as enriched as DNA methylation, and, as such, by using identical data analysis strategies for the identification of differentially hydroxymethylated regions (DHRs) and differentially methylated regions (DMRs), current approaches tend to contempt the former. This is demonstrated from the analysis performed in Chapter 5, where neither for the DRN nor for the LC a DHR associated with Braak stage was identified. These findings therefore suggest that the spatial correlation analysis for DHRs might need to be optimized, e.g. by adjusting the sliding window, if one wishes to identify meaningful altered regions at the level of 5 -hmC. Similarly, next to treating the different modifications equally, current methods also asses each of these modifications independently, while alterations at a locus at the level of multiple modifications can also have functional consequences. Future methods should therefore also be aimed at integrating changes within specific sliding windows at the level of multiple modifications, rather than looking at each of these modifications individually.

Two more specific minor limitations faced in Chapter 4 are related to the (novel) data analysis method that is used for LDBSP on pools of neurons isolated with LCM. While the novel approach presented here reduces data bias by omitting the need for excluding multi-allele reactions (as conducted in the traditional analysis pipeline), it is limited in detecting only a maximum of three DNA molecules present in a single reaction. In fact, it extrapolates the number of target alleles present based on arbitrary thresholds that were set for the raw $\mathrm{CpG}$ methylation values obtained after pyrosequencing. Within the boundaries of these thresholds, i.e. (1) $\leq 8.33 \%$ and $\geq 91.33 \%$, (2) $50 \pm 8.33 \%$, and (3) $33.33 \pm 8.33 \%$ and $66.66 \pm 8.33 \%$, one can make a relative safe estimation on whether a reaction contains one, two or three alleles, respectively. However, with increasing numbers, the estimation based on $\mathrm{CpG}$ methylation values becomes rather ambiguous, as one also needs to consider potential technical variation that might affect these methylation values. The method proposed in this chapter will most likely classify these reactions as representing three alleles. Despite the fact that the methylation data obtained from these reactions would now be partially corrected by this novel data analysis pipeline (when compared to the traditional pipeline), this correction is still imperfect in such a scenario. Hence, overall, it is strongly advised to dilute the DNA sample properly in the first place to ensure that the proportion of reactions that contain more than three alleles is either negligible or, preferable, zero. In addition to the aforementioned notion and independent of the performed downstream analysis, LDBSP does also not allow one to identify multi-allele reactions that show an identical pattern of $\mathrm{CpG}$ methylation. In this specific scenario, and irrespective of whether a reaction contains one, two, or three or more alleles, all CpG methylation values will approach $0 \%$ or $100 \%$, for unmethylated and methylated sites, respectively. These reactions therefore display a typical binary methylation 
pattern that is expected for a single DNA molecule, while in reality more alleles were present. In general, this becomes a bigger challenge when, due to biological factors, a target locus is completely methylated or unmethylated. It is therefore advisable to always target a substantial number of $\mathrm{CpG}$ sites per target gene ( 5 or more) in order to increase the chance of obtaining, and hence detecting, at least one site with a 'nonbinary' methylation status on one of the target alleles.

Several other limitations that should be addressed regarding the first part of this thesis are related to the brainstem EWAS described in Chapter 5, of which the most common ones are also summarized in Chapter 2. A recurring limitation of these studies is the small to moderate sample sizes that are being used, which limits the statistical power to detect significant differences. This poses a problem particularly when aiming to identify differentially modified positions at the level of 5-uC, 5-mC and 5-hmC, which generally show very small effect sizes. In addition, the significance thresholds for assigning these positions after multiple testing correction is also extremely conservative, as a $p<9.0 \mathrm{E}-08$ has been established for achieving genome-wide significance when utilizing the Illumina Infinium MethylationEPIC BeadChip array [57]. This is exemplified in the EWAS performed in Chapter 5, where all the top-ranked differentially modified positions for both brainstem regions were nominally significant at $p<0.001$, but none of them reached the genome-wide significance level after multiple testing correction. As the collection of bigger sample sizes remains an ongoing challenge, it would therefore require further development of alternative bioinformatics approaches to strengthen these results. In addition, it will be imperative to conduct replication studies in independent patient cohorts and perform meta-analyses to confirm the obtained findings. Recently, the first cross-cortex meta-analysis in SAD using data from 1,408 donors from six independent studies identified $220 \mathrm{CpG}$ sites associated with neuropathology, annotated to 121 genes, of which 96 genes had not been previously reported at experiment-wide significance, which further emphasizes the importance of these type of analyses [58].

While the Illumina Infinium MethylationEPIC BeadChip provides a higher coverage compared to its predecessor, the probes present on the chip still only cover about $3 \%$ of the total number of $\mathrm{CpG}$ sites that are found within the human genome. Moreover, non$\mathrm{CpG}$ sites, including $\mathrm{CpA}, \mathrm{CpT}$ and $\mathrm{CpC}$, as well as non-cytosine modified positions are not covered by this technology. Future epigenetic studies in SAD should therefore also be complemented with whole-genome-sequencing based approaches, which could provide a broader coverage, and thus would allow for a deeper understanding of disease-related signatures. Nevertheless, these might oppose a restriction given their high costs, i.e. per individual and in view of the concomitant demand for larger study populations (due to the accompanying decrease in statistical power), making currently used array-based approaches still a more preferred strategy in most cases. 
As also addressed in Chapters 4 and 5 , as well as strongly emphasized throughout the thesis, another major issue of EWAS is related to the cellular heterogeneity of bulk cell populations that can produce noise and bias in experimental data when used for epigenetic profiling. While single cell analyses evidently provides a more robust alternative, another way of dealing with this issue is the application of cell deconvolution approaches for downstream bioinformatics analyses [59]. However, to date, these algorithms for the human brain are only available for the frontal cortex $[12,60]$, while in the EWAS of this thesis brainstem nuclei were targeted. These therefore render limited advantage for such an analysis, as cortical brain regions differ significantly in their cellular proportions when compared to the DRN and the LC. As such, during the analysis performed in Chapter 5 , surrogate variables that are known to correct for unmeasured confounding factors, including cell type composition, were included in the statistical model. While currently offering a valid approach, it would require further development of bioinformatic tools and alternative algorithms that would allow one to correct for the cell type composition in different structures of the human brain, especially for more specialized nuclei such as the DRN and the LC.

While the major focus of the epigenetic studies presented in this thesis is on identifying disease-related changes at the level of DNA modifications, it is also important to consider their biological relevance and coherent limitations. In fact, transcriptional regulation is not only mediated by DNA modifications, but also by histone modifications and non-coding RNAs, as well as by underlying genetic factors [61]. Hence, significant changes at a single $\mathrm{CpG}$ site or even in a bigger region of a gene might not directly result in (negative) functional alterations. In addition, the tendency to reduce complex disorders such as SAD to a single genetic locus opposes another major issue, as it shifts the attention towards more specific and single molecular or cellular systems, hence one might lose sight on the importance of 'the bigger picture'. Of course, by reducing a complex disorder like SAD into smaller elements, one hopes that when such elements become elucidated, it will be easier to tie them together and understand the disease in a more holistic fashion as well. However, we should not deny that for complex disorders such as SAD, an immense interacting network of numerous distinct (epi)genetic risk, disease-modifying- and protective loci, and associated molecular pathways and cellular mechanisms, are likely to underlie the development and course of the disease. As such, one should be cautious and careful in claiming that single genes as those identified by EWAS are explaining the disease and/or related endophenotype(s). In fact, in future endeavors, it would be more meaningful to integrate current available epigenomic data sets into more multi-omics- and even multi system-levels-orientated approaches, using both genomics, epigenomics, transcriptomics and proteomics as well as other (e.g. imaging, clinical phenotyping) data obtained from the same patient cohorts. Overall, this might provide clarity on the contribution of each of these omics layers and might aid in disentangling the complex pathophysiology of SAD. 
In contrast to data obtained in GWAS, there is the issue of causality when dealing with results obtained through EWAS. In EWAS, it is simply impossible to conclude whether epigenetic changes associated with $S A D$ are causally involved, whether they are a consequence of its pathogenesis or whether they represent an epiphenomenon [19]. In view of SAD, it is likely a combination of both, where some epigenetic signatures identified in EWAS could represent early causal changes and others changes that are the result of pathological processes involving e.g. amyloid beta or neurofibrillary tangle formation. In order to elucidate their exact contribution, it requires future longitudinal studies and approaches combining epigenetic editing systems in both in vivo animal models and in vitro culture systems, using iPSC-derived neural cells, for example.

Aside from the challenges faced in neuroepigenomic studies on SAD, the second part of the thesis, which addresses to potential of iPSC-based model systems for SAD, is also subject to several limitations. While iPSC-derived neural cells are portrayed as breakthrough models for SAD and many other brain disorders, one should not overlook the fact that this area of investigation is still very nascent and, hence, highly experimental in nature. At the moment, it therefore comes with potentially even more limitations than direct translational features, which all need to be addressed in future studies if one wishes to develop robust model systems that could directly benefit preclinical and clinical research and, eventually, healthcare. The field currently has the tendency to go ahead of itself and directly starts looking for opportunities to use these patients' iPSC-derived neural cultures for interrogating pathological mechanisms related to the disease of interest, whilst the associated models themselves still require further validation in order to do this efficiently and correctly. In fact, given their juvenile and artificial character, it will be paramount to dissect and subsequently optimize each of the contributing factors that could affect establishment of a valid in vitro disease model for SAD. In this context, one could think of the molecular and cellular consequences of reprogramming processes, effects of varying differentiation protocols, the use of different donor cell types, the role of different culture conditions, e.g. 2D or 3D models, and other potential environmental influences that have been addressed above. All of these factors might affect the derivation of clinically relevant mature/aged brains cells, as well as the manifestation of disease hallmarks in vitro, hence influencing potential experimental outcomes when not studied in a controlled setting. Many of these general limitations or those specifically related to the establishment of SAD models are also reviewed in Chapters 6 and 7 , and will require attention in future studies to strengthen the potential of this platform.

Finally, one of the most important considerations related to iPSC-models is that the acceptable quality control criteria attributed to iPSCs and their differentiated neural progeny should be defined in more detail for both fundamental and clinical 
applications, as this aspect has not been extensively clarified to date. Evidently, this is also difficult to address, especially for SAD patient-derived cells, because of our limited knowledge on the molecular and cellular mechanisms underlying this complex and heterogeneous disease. After all, the aim is to produce human brain cells that mimic their in vivo counterparts, both in healthy and diseased conditions. However, the similarity and differences between these cells that are produced under laboratory conditions and those obtained from a human brain have not been extensively characterized yet. Thus, efforts aimed at the development of a consistent and reliable translational iPSC model with well-defined cellular and molecular characteristics is therefore a vital pre-requisite to realize and understand their full potential. Welldefined standardized reprogramming, direct differentiation and directed differentiation protocols and strategies to gauge the magnitude, as well as to improve the maturation status of the cells will be highly valuable in this respect. As such, the study presented in Chapter 8 is also subject to these aforementioned limitations. While the successful differentiation into a specific neural lineage was determined based on the expression of lineage-specific transcription factors, further functional tests and a more detailed molecular characterization remains necessary. All in all, such more detailed analyses are needed if one wishes to conclude that these derived cultures are similar to cells found in the human brain, and hence could form the basis for the establishment of a reliable SAD model system.

\section{Future perspectives}

The key findings, strengths and limitations that were addressed above provide exciting opportunities for future SAD-related studies. On the one hand, the identified dysregulated epigenetic loci and associated molecular pathways in the SAD brainstem, the presented state-of-the-art methodologies for the quantification of (specific) epigenetic marks in bulk tissues or single cells, as well as the iPSC models, provide a solid foundation for future mechanistic studies on the etiopathophysiology of the disease. On the other hand, the limitations that have been described might by themselves lead to new research ideas and the development of improved methodologies by inspiring other researchers to tackle these in future studies. Overall, the knowledge that is gained from the work collected in this thesis could provide guidance for the development of new scientific breakthroughs and inventions within the field of neuroepigenomics in SAD. While moving this field forward, it remains crucial to put both the research findings and their corresponding technological-, bioinformatics- and logistical limitations into perspective in order to assign the right credibility to the data that are being obtained from these studies. Future efforts should therefore not only be focused on acquiring more elaborated epigenetic data sets in $S A D$, but should also be aimed at overcoming the remaining challenges in order to strengthen and further elucidate the exact contribution, as well as significance, of the obtained epigenetic signatures associated with this disease. 
The continuous development of (genome-wide) approaches for interrogating the epigenome together with an increasing number of initiatives aimed at establishing high-quality patient tissue banks could greatly benefit future endeavors. In fact, the availability of brain tissue linked to extensive records on patient demographics would allow for larger epigenetic screenings with increasing sample sizes. Furthermore, they could provide a great source for independent replication studies. Such efforts, as well as the use of meta-analyses, will be highly beneficial for the field to confirm previous observations. Moreover, as the use of bulk tissues might dilute disease-associated effects due to tissue heterogeneity, it will be crucial to implement more single-cell(type) based approaches in future studies, allowing one to dissect cell-specific contributions of epigenetic aberrations in the SAD brain. Furthermore, the field would highly benefit from more sequencing-based techniques that provide a higher genomic coverage, which could be combined with methods for the segregation of various epigenetic marks, such as in the case of DNA methylation and hydroxymethylation by means of oxidative bisulfite sequencing. Aside from targeting brain tissues or cells, it would furthermore be highly informative to include more accessible tissues into the study designs, such as blood samples from the same patients, as this would allow for addressing potential overlap and differences in disease-associated signatures that could allow for the establishment of biomarkers and diagnostic tools. In this respect, and considering the dynamic nature of epigenetic alterations, it will be highly informative to perform wellestablished longitudinal studies that aid in developing a better understanding on the temporal association of these epigenetic marks with the disease.

Importantly, it shall not be overlooked that methodological advances should be accompanied by the development of robust bioinformatics tools to handle epigenetic data sets. In fact, advances in statistical approaches and mathematical modelling serve as the backbone for taking full advantage of such data. Furthermore, emphasis should be put on future efforts to integrate epigenomic data into bigger multiomics and multi-level (systems biology/medicine) data sets, including genomics, transcriptomics, proteomics, and demographic and phenotypical data, among others, in order to dissect the functional consequences of changes in epigenetic marks that would be necessary to address the significance of these alterations in the disease causation and progression. In fact, it is anticipated that more advanced bioinformatics tools such as machine learning, computational modeling and systems biology approaches will be highly valuable in the attempt to differentiate the molecular cascades that induce or drive SAD from those that merely represent a consequence of it or just an epiphenomenon, e.g. epigenetic changes that occur during normal aging. As such, the establishment of future (inter)national collaborations in which assets from these different scientific disciplines are merged, e.g. involving clinicians, molecular neurobiologists and data scientists, are indispensable for the field to thrive.

In light of future iPSC models, all of the aforementioned efforts will also be highly 
instrumental. In fact, the availability of extensive and well-established tissue banks having both brain and other bodily tissues available from the same patients would allow for more reliable and detailed comparisons of ex vivo neural cells with iPSCdifferentiated- or direct reprogrammed neural cultures. Such efforts could not only contribute to elaborating knowledge on molecular mechanisms underlying the disease, but also aid in further validating these novel humanized in vitro models. By studying these cells at the multi-omics level, it should be possible to obtain fingerprints of both iPSCs and their differentiated progeny, which allows one to assess how reprogramming affects biological processes, e.g. aging marks and epigenetic memory of the donor cells, thus solving many of the current challenges accompanying the use of these cells. Furthermore, the availability of such matched tissues could allow for the establishment of well-characterized cell panels of both healthy and diseased individuals, which would greatly benefiting future research into SAD. The availability of such well-defined iPSC and differentiated neural cultures might furthermore provide robust studies on the causality of epigenetic marks by using e.g. epigenetic editing systems in combination with exposures to environmental insults, and vice versa.

To conclude, the studies compiled in this thesis offer novel insights into both the role of epigenetic mechanisms in SAD, as well as into current state-of-the-art methodologies that are being used to study this disease. Hence, the research and accompanying strengths and limitations presented here, set the pillars for future endeavors to clarify the functional consequences of the identified epigenetic signatures in the etiology and progression of SAD. Altogether, the work presented in this thesis contributes to the global apprehension of contributing and modifying factors in relation to SAD that are crucially important for other scientists in the field aiming to further advance our current understanding, as well as to combat this devastating neurodegenerative disorder.

\section{References}

1. Liu X, Jiao B, Shen L. The epigenetics of Alzheimer's disease: Factors and therapeutic implications. Frontiers in genetics. 2018;9:579.

2. Lahiri DK, Maloney B, Zawia NH. The LEARn model: an epigenetic explanation for idiopathic neurobiological diseases. Molecular psychiatry. 2009;14(11):992-1003.

3. Bakulski KM, Dolinoy DC, Sartor MA, Paulson HL, Konen JR, Lieberman AP, et al. Genome-wide DNA methylation differences between late-onset Alzheimer's disease and cognitively normal controls in human frontal cortex. Journal of Alzheimer's Disease. 2012;29(3):571-88.

4. Sanchez-Mut JV, Aso E, Heyn H, Matsuda T, Bock C, Ferrer I, et al. Promoter hypermethylation of the phosphatase DUSP22 mediates PKA-dependent TAU phosphorylation and CREB activation in Alzheimer's disease. Hippocampus. 2014;24(4):363-8.

5. Lunnon K, Smith R, Hannon E, De Jager PL, Srivastava G, Volta M, et al. Methylomic profiling implicates cortical deregulation of ANK1 in Alzheimer's disease. Nat Neurosci. 2014;17(9):1164-70.

6. De Jager PL, Srivastava G, Lunnon K, Burgess J, Schalkwyk LC, Yu L, et al. Alzheimer's disease: early alterations in brain DNA methylation at ANK1, BIN1, RHBDF2 and other loci. Nat Neurosci. 
2014;17(9):1156-63.

7. Watson CT, Roussos P, Garg P, Ho DJ, Azam N, Katsel PL, et al. Genome-wide DNA methylation profiling in the superior temporal gyrus reveals epigenetic signatures associated with Alzheimer's disease. Genome medicine. 2016;8(1):1-14.

8. Gasparoni G, Bultmann S, Lutsik P, Kraus TF, Sordon S, Vlcek J, et al. DNA methylation analysis on purified neurons and glia dissects age and Alzheimer's disease-specific changes in the human cortex. Epigenetics \& chromatin. 2018;11(1):41.

9. Smith RG, Hannon E, De Jager PL, Chibnik L, Lott SJ, Condliffe D, et al. Elevated DNA methylation across a 48-kb region spanning the HOXA gene cluster is associated with Alzheimer's disease neuropathology. Alzheimer's \& Dementia. 2018;14(12):1580-8.

10. Semick SA, Bharadwaj RA, Collado-Torres L, Tao R, Shin JH, Deep-Soboslay A, et al. Integrated DNA methylation and gene expression profiling across multiple brain regions implicate novel genes in Alzheimer's disease. Acta neuropathologica. 2019;137(4):557-69.

11. Lardenoije R, Roubroeks JA, Pishva E, Leber M, Wagner H, latrou A, et al. Alzheimer's diseaseassociated (hydroxy) methylomic changes in the brain and blood. Clinical epigenetics. 2019;11(1):164.

12. Smith AR, Smith RG, Pishva E, Hannon E, Roubroeks JAY, Burrage J, et al. Parallel profiling of DNA methylation and hydroxymethylation highlights neuropathology-associated epigenetic variation in Alzheimer's disease. Clin Epigenetics. 2019;11(1):52.

13. Booth MJ, Ost TW, Beraldi D, Bell NM, Branco MR, Reik W, et al. Oxidative bisulfite sequencing of 5-methylcytosine and 5-hydroxymethylcytosine. Nat Protoc. 2013;8(10):1841-51.

14. Booth MJ, Branco MR, Ficz G, Oxley D, Krueger F, Reik W, et al. Quantitative sequencing of 5-methylcytosine and 5-hydroxymethylcytosine at single-base resolution. Science. 2012;336(6083):934-7.

15. Kriaucionis $\mathrm{S}$, Heintz $\mathrm{N}$. The nuclear DNA base 5-hydroxymethylcytosine is present in Purkinje neurons and the brain. Science. 2009;324(5929):929-30.

16. SzwagierczakA, Bultmann S, Schmidt CS, Spada F, Leonhardt H. Sensitive enzymatic quantification of 5-hydroxymethylcytosine in genomic DNA. Nucleic acids research. 2010;38(19):e181-e.

17. Cheng $\mathrm{Y}, \mathrm{Xie} \mathrm{N}$, Jin P, Wang T. DNA methylation and hydroxymethylation in stem cells. Cell biochemistry and function. 2015;33(4):161-73.

18. Song C-X, He C. Potential functional roles of DNA demethylation intermediates. Trends in biochemical sciences. 2013;38(10):480-4.

19. van den Hove DL, Riemens RJ, Koulousakis P, Pishva E. Epigenome-wide association studies in Alzheimer's disease; Achievements and challenges. Brain Pathology. 2020.

20. Hajj NE, Kuhtz J, Haaf T. Limiting Dilution Bisulfite Pyrosequencing(R): A Method for Methylation Analysis of Individual DNA Molecules in a Single or a Few Cells. Methods Mol Biol. 2015;1315:22139.

21. El Hajj N, Trapphoff T, Linke M, May A, Hansmann T, Kuhtz J, et al. Limiting dilution bisulfite (pyro) sequencing reveals parent-specific methylation patterns in single early mouse embryos and bovine oocytes. Epigenetics. 2011;6(10):1176-88.

22. Mattern F, Heinzmann J, Herrmann D, Lucas-Hahn A, Haaf T, Niemann H. Gene-specific profiling of DNA methylation and mRNA expression in bovine oocytes derived from follicles of different size categories. Reprod Fertil Dev. 2017;29(10):2040-51.

23. Trapphoff T, El Hajj N, Zechner U, Haaf T, Eichenlaub-Ritter U. DNA integrity, growth pattern, spindle formation, chromosomal constitution and imprinting patterns of mouse oocytes from vitrified preantral follicles. Human reproduction. 2010;25(12):3025-42.

24. Heinzmann J, Hansmann T, Herrmann D, Wrenzycki C, Zechner U, Haaf T, et al. Epigenetic profile of developmentally important genes in bovine oocytes. Molecular reproduction and development. 
2011;78(3):188-201.

25. Diederich M, Hansmann T, Heinzmann J, Barg-Kues B, Herrmann D, Aldag P, et al. DNA methylation and mRNA expression profiles in bovine oocytes derived from prepubertal and adult donors. Reproduction. 2012;144(3):319.

26. Simic G, Stanic G, Mladinov M, Jovanov-Milosevic N, Kostovic I, Hof PR. Does Alzheimer's disease begin in the brainstem? Neuropathology and applied neurobiology. 2009;35(6):532-54.

27. latrou A, Kenis G, Rutten BP, Lunnon K, van den Hove DL. Epigenetic dysregulation of brainstem nuclei in the pathogenesis of Alzheimer's disease: looking in the correct place at the right time? Cell Mol Life Sci. 2017;74(3):509-23.

28. Braak H, Thal DR, Ghebremedhin E, Del Tredici K. Stages of the pathologic process in Alzheimer disease: age categories from 1 to 100 years. Journal of Neuropathology \& Experimental Neurology. 2011;70(11):960-9.

29. Grinberg L, Rüb U, Ferretti R, Nitrini R, Farfel J, Polichiso L, et al. The dorsal raphe nucleus shows phospho-tau neurofibrillary changes before the transentorhinal region in Alzheimer's disease. A precocious onset? Neuropathology and applied neurobiology. 2009;35(4):406-16.

30. Lee JH, Ryan J, Andreescu C, Aizenstein H, Lim HK. Brainstem morphological changes in Alzheimer's disease. Neuroreport. 2015;26(7):411.

31. Ji X, Wang H, Zhu M, He Y, Zhang H, Chen X, et al. Brainstem atrophy in the early stage of Alzheimer's disease: a voxel-based morphometry study. Brain Imaging and Behavior. 2021;15(1):49-59.

32. Chapuis J, Hot D, Hansmannel F, Kerdraon O, Ferreira S, Hubans C, et al. Transcriptomic and genetic studies identify IL-33 as a candidate gene for Alzheimer's disease. Molecular psychiatry. 2009;14(11):1004-16.

33. Sherva R, Baldwin CT, Inzelberg R, Vardarajan B, Cupples LA, Lunetta K, et al. Identification of novel candidate genes for Alzheimer's disease by autozygosity mapping using genome wide SNP data. Journal of Alzheimer's Disease. 2011;23(2):349-59.

34. Nazarian A, Yashin Al, Kulminski AM. Genome-wide analysis of genetic predisposition to Alzheimer's disease and related sex disparities. Alzheimer's research \& therapy. 2019;11(1):5.

35. Chang T, editor Tau Network Genes in a Genome Wide Association Study of Progressive Supranuclear Palsy. MOVEMENT DISORDERS; 2017: WILEY 111 RIVER ST, HOBOKEN 07030-5774, NJ USA.

36. Valcourt U, Alcaraz LB, Exposito J-Y, Lethias C, Bartholin L. Tenascin-X: beyond the architectural function. Cell adhesion \& migration. 2015;9(1-2):154-65.

37. Sullivan SE, Young-Pearse TL. Induced pluripotent stem cells as a discovery tool for Alzheimers disease. Brain Res. 2015.

38. Yang J, Li S, He XB, Cheng C, Le W. Induced pluripotent stem cells in Alzheimer's disease: applications for disease modeling and cell-replacement therapy. Mol Neurodegener. 2016;11(1):39.

39. Mungenast AE, Siegert S, Tsai LH. Modeling Alzheimer's disease with human induced pluripotent stem (iPS) cells. Mol Cell Neurosci. 2016;73:13-31.

40. Israel MA, Yuan SH, Bardy C, Reyna SM, Mu Y, Herrera C, et al. Probing sporadic and familial Alzheimer's disease using induced pluripotent stem cells. Nature. 2012;482(7384):216-20.

41. Kondo T, Asai M, Tsukita K, Kutoku Y, Ohsawa Y, Sunada Y, et al. Modeling Alzheimer's disease with iPSCs reveals stress phenotypes associated with intracellular Abeta and differential drug responsiveness. Cell Stem Cell. 2013;12(4):487-96.

42. Foveau B, Correia AS, Hébert SS, Rainone S, Potvin O, Kergoat M-J, et al. Stem cell-derived neurons as cellular models of sporadic Alzheimer's disease. Journal of Alzheimer's Disease. 2019;67(3):893910.

43. Duan L, Bhattacharyya BJ, Belmadani A, Pan L, Miller RJ, Kessler JA. Stem cell derived basal forebrain cholinergic neurons from Alzheimer's disease patients are more susceptible to cell death. 
Mol Neurodegener. 2014;9:3.

44. Ochalek A, Mihalik B, Avci HX, Chandrasekaran A, Téglási A, Bock I, et al. Neurons derived from sporadic Alzheimer's disease iPSCs reveal elevated TAU hyperphosphorylation, increased amyloid levels, and GSK3B activation. Alzheimer's research \& therapy. 2017;9(1):1-19.

45. Feng JA, Fouse S, Fan GP. Epigenetic regulation of neural gene expression and neuronal function. Pediatr Res. 2007;61(5):58r-63r.

46. Fitzsimons CP, van Bodegraven E, Schouten M, Lardenoije R, Kompotis K, Kenis G, et al. Epigenetic regulation of adult neural stem cells: implications for Alzheimer's disease. Mol Neurodegener. 2014;9:25.

47. Chambers SM, Fasano CA, Papapetrou EP, Tomishima M, Sadelain M, Studer L. Highly efficient neural conversion of human ES and iPS cells by dual inhibition of SMAD signaling. Nat Biotechnol. 2009;27(3):275-80.

48. Fernandez-Jimenez N, Allard C, Bouchard L, Perron P, Bustamante M, Bilbao JR, et al. Comparison of Illumina 450K and EPIC arrays in placental DNA methylation. Epigenetics. 2019;14(12):1177-82.

49. Wojda U, Kuznicki J. Alzheimer's disease modeling: ups, downs, and perspectives for human induced pluripotent stem cells. J Alzheimers Dis. 2013;34(3):563-88.

50. Han F, Liu C, Huang J, Chen J, Wei C, Geng X, et al. The application of patient-derived induced pluripotent stem cells for modeling and treatment of Alzheimer's disease. Brain Science Advances. 2019;5(1):21-40.

51. Haston KM, Finkbeiner S. Clinical Trials in a Dish: The Potential of Pluripotent Stem Cells to Develop Therapies for Neurodegenerative Diseases. Annu Rev Pharmacol Toxicol. 2015.

52. Riemens R, Kenis G, van den Beucken T. Human-induced pluripotent stem cells as a model for studying sporadic Alzheimer's disease. Neurobiology of learning and memory. 2020:107318.

53. Ooi L, Sidhu K, PoljakA, Sutherland G, O'Connor MD, Sachdev P, et al. Induced pluripotent stem cells as tools for disease modelling and drug discovery in Alzheimer's disease. J Neural Transm (Vienna). 2013;120(1):103-11.

54. Mooijman D, Dey SS, Boisset J-C, Crosetto N, Van Oudenaarden A. Single-cell $5 \mathrm{hmC}$ sequencing reveals chromosome-wide cell-to-cell variability and enables lineage reconstruction. Nature biotechnology. 2016;34(8):852-6.

55. Qu J, Zhou M, Song Q, Hong EE, Smith AD. MLML: consistent simultaneous estimates of DNA methylation and hydroxymethylation. Bioinformatics. 2013;29(20):2645-6.

56. Kiihl SF, Martinez-Garrido MJ, Domingo-Relloso A, Bermudez J, Tellez-Plaza M. MLML2R: an R package for maximum likelihood estimation of DNA methylation and hydroxymethylation proportions. Statistical applications in genetics and molecular biology. 2019;18(1).

57. Mansell G, Gorrie-Stone TJ, Bao Y, Kumari M, Schalkwyk LS, Mill J, et al. Guidance for DNA methylation studies: statistical insights from the Illumina EPIC array. BMC genomics. 2019;20(1):115.

58. Smith R, Pishva E, Shireby G, Smith AR, Roubroeks JA, Hannon E, et al. Meta-analysis of epigenomewide association studies in Alzheime's disease highlights 220 differentially methylated loci across cortex. BioRxiv. 2020.

59. Titus AJ, Gallimore RM, Salas LA, Christensen BC. Cell-type deconvolution from DNA methylation: a review of recent applications. Human molecular genetics. 2017;26(R2):R216-R24.

60. Guintivano J, Aryee MJ, Kaminsky ZA. A cell epigenotype specific model for the correction of brain cellular heterogeneity bias and its application to age, brain region and major depression. Epigenetics. 2013;8(3):290-302.

61. Lardenoije R, latrou A, Kenis G, Kompotis K, Steinbusch HW, Mastroeni D, et al. The epigenetics of aging and neurodegeneration. Prog Neurobiol. 2015;131:21-64. 
Chapter 10

\section{Summary}



Chapter 1 introduced the reader to the field of neuroepigenomics in sporadic Alzheimer's disease (sAD), as well as to the use of induced pluripotent stem cell (iPSC)-based models for studying this neurodegenerative disorder. After this brief introduction, an overview of the general structure of the thesis was presented, which could be divide into two main parts focused on each of the aforementioned research lines was presented. In more detail, the studies presented in the first part of this thesis were centered on the role of epigenetic dysregulation in the etiopathophysiology of SAD. This first part, which includes Chapters 2-5, consisted of one perspective paper, two methodological research papers and one original research article. The work in the second part of this thesis, focused on the application and establishment of iPSC-based models for SAD, e.g. in view of mechanistic studies into epigenetic dysregulation. The second part, consisting of Chapters 6-8, presented two in-depth review articles and one exploratory pilot study.

In Chapter 2, a perspective paper on epigenome-wide association studies (EWAS), or more specifically methylome-wide association studies (MWAS), in SAD, was presented. Epigenetic mechanisms, which mediate the interaction between the genome and the environment, are thought to provide a mechanistic explanation in view of the etiopathogenesis of SAD. In this paper, all studies that have been performed to date targeting various (cortical) regions of the brain, as well as peripheral blood samples, derived from patients and matched non-demented controls, were briefly reviewed. Furthermore, relevant caveats in relation to these studies that challenge the interpretation of the experimental outcomes, including genomic coverage, statistical power, specificity of the epigenetic marks assessed, cell-type specificity and composition, causal inference and multi-omics, were discussed. Finally, an outlook on possible solutions that might overcome these challenges, including methodological developments and advances in related data science disciplines, was provided. Overall, what was concluded from this chapter is that although MWAS are highly relevant in the context of SAD research, it remains vital to address the aforementioned caveats in future studies in order to produce more meaningful data, which could significantly increase our understanding about the processes underlying the disease.

In view of these challenges, Chapter 3 described a standardized protocol for oxidative bisulfite pyrosequencing that allows for an accurate discrimination between DNA methylation and hydroxymethylation, i.e. between 5 -methylcytosine $(5-\mathrm{mC})$ and 5-hydroxymethylcytosine $(5-\mathrm{hmC})$ bases, in the context of cytosinephosphate-guanine $(\mathrm{CpG})$ sites. The pyrosequencing protocol described in this chapter provides a workable solution for the issue of modification-specificity as faced in neuroepigenomic studies of SAD. The approach relies on a highly selective 
chemical oxidation using $\mathrm{KRuO}_{4}$ that is applied prior to the bisulfite treatment, polymerase chain reaction (PCR) and pyrosequencing steps. Moreover, a novel spike-in DNA standard set that allows the user to accurately determine the oxidative bisulfite conversion efficiency was developed for specifically this approach. As a proof-of-principle, the protocol was conducted on both post-mortem brain tissue and cultured iPSCs, demonstrating that $5-\mathrm{mC}$ and $5-\mathrm{hmC}$, as well as unmodified cytosine (5-uC) bases, could be detected in OXT and DNAJB13. Furthermore, it was confirmed that the novel $(5-\mathrm{hmC})$ spike-in control could be used as an internal pyrosequencing control that does not interfere with the analysis of the accompanying sample, and vice versa. Such innovative approaches as presented in this chapter are crucial for moving the field of neuroepigenomics in SAD forward, as they aid in increasing the validity of the data.

Chapter 4 introduced a novel approach for DNA methylation profiling in small pools of 50 neurons, which relied on a combination of limiting dilution bisulfite pyrosequencing (LDBSP) and laser capture microdissection (LCM). This method allows one to determine $\mathrm{CpG}$ site methylation rates on single alleles in a multitargeted and cell type-specific manner, hence providing a solution to the issue of cellular heterogeneity that is encountered in neuroepigenomic studies of SAD. The general working procedure of LDBSP on cells isolated from post-mortem brain tissue using LCM was described in this chapter. Furthermore, as proof-of-principle, a targeted methylation analysis of DNAJB13, PGLYRP1, RHBDF2, C3, LMX1B and OXT was performed. Interestingly, LDBSP on these pools of neurons often rendered downstream reactions with more than one DNA molecule, a scenario that rarely occurs when conducting LDBSP on a one-cell-sample or just a few cells. As such, an adapted data analysis pipeline for LDBSP was developed, allowing one to include and correct $\mathrm{CpG}$ methylation rates derived from multi-allele reactions. Overall, the method described in this chapter provides the user with a more accurate estimation of the DNA methylation status of each target gene in the analyzed cell pools, thereby adding further validity to the data. In addition, it was demonstrated that the efficiency of LDBSP on neurons isolated with LCM is similar to the efficiency achieved in previously published studies using this technique on other isolated cell types. It is anticipated that single cell(type) approaches as described in this chapter will be increasingly valuable for future neuroepigenomic studies in SAD.

Chapter 5 represented the first large-scale epigenetic analysis in the brainstem of SAD to date, targeting the dorsal raphe nuclei (DRN) and locus coeruleus (LC), thereby complementing the body of EWAS reviewed in Chapter 2. Differentially modified positions and regions in bulk tissues obtained from both brainstem nuclei were identified at the level of DNA methylation, hydroxymethylation and 
unmodified cytosines by using the Illumina Infinium MethylationEPIC BeadChip array in combination with an oxidative bisulfite treatment of the isolated target DNA (similar to what was described in Chapter 3). Aside from a strong overlapping dysregulation in the Tenascin XB gene (TNXB) in both the DRN and LC, common and novel epigenetic signatures compared to previous studies targeting the SAD brain were discovered, all of which may play a pivotal role in the etiopathogenesis of SAD. A subsequent bisulfite pyrosequencing analysis in the same patient cohort confirmed the observed dysregulation of TNXB in both of the brainstem regions assessed. As a follow up on these discovery findings, a targeted analysis of TNXB was performed in an independent patient cohort, assessing methylomic signatures in single serotonergic neurons and non-serotonergic cells isolated from the DRN by means of LCM. This study therefore represented the first cell subtype-specific validation analysis performed in the brainstem to date, relying on the LCM-LDBSP approach that was described in Chapter 4. Strikingly, when comparing the bisulfite methylation levels of $T N X B$ between SAD patients and controls in serotonergic and non-serotonergic neurons, a significant interaction between cell-type and experimental condition was identified. The SAD-associated methylation profiles were opposite in the serotonergic neurons and non-serotonergic cells, the latter of which resembled the EWAS data. As such, this study demonstrated for the first time that epigenetic signatures within the DRN can strongly dependent on both the disease phenotype and the cell type analyzed. These findings therefore emphasize the importance of future cell type-specific neuroepigenomic studies in SAD, which was already briefly addressed in Chapters $\mathbf{2}$ and $\mathbf{4}$.

Chapter 6 presented a review paper on the potential value of iPSCs for SAD research. Since their discovery, these cells have been offering a promising avenue to fill the translational gap between pre-clinical and clinical SAD research, by allowing the establishment of patient-specific in vitro disease models that can be applied for fundamental research and drug discovery. It is anticipated that efforts in this field will deepen our knowledge on various underlying disease mechanisms and aid in the establishment of therapeutic interventions. All pioneering studies utilizing iPSCs from SAD patients were reviewed in this chapter, demonstrating that the iPSC-derived neural cells recapitulate neuropathological processes of the disease, although with quite a high degree of variability in terms of their presence and severity. Therefore, sources of variability related to the model in addition to those that might be explained by the heterogeneous nature of SAD were critically assessed. What followed from this chapter is that developing iPSC models for SAD remains a challenging endeavor due to the multifactorial nature of the disease, the nearly life-long disease progression and the high degree of inter-individual heterogeneity that might be reflected in cells obtained from patients. Aside from the variability associated with the disease, several methodological factors, such 
as those related to iPSC generation and (neural) differentiation, further impact on this degree of variation. Thus, future efforts aimed at extensively characterizing the iPSC-derived models and sources of variability in fine detail, are necessary if one wishes to establish robust disease models for the study of SAD. Nonetheless, whilst taking into account these considerations, the developing iPSC models clearly provide an exciting avenue in SAD research.

Within the framework of developing iPSC-based models, Chapter 7 presented an in-depth review article on directed- and direct neural differentiation protocols starting from stem cells and somatic cells, respectively. As addressed in this review, insights from basic research and developmental biology have guided the design of current protocols and numerous approaches for the derivation of regional-specific glutamatergic, dopaminergic, GABAergic, serotonergic, and cholinergic/motor neurons have become available. Approaches for the derivation of each of these disease-relevant neural subtypes were summarized, relying on either chemically defined systems using patterning factors, transcription factormediated reprogramming and epigenetic-based strategies. This review furthermore highlighted that although distinct approaches have shown to be successful in directing neuronal cell fate in vitro, their refinement and optimization, as well as the search for alternative strategies, remains necessary to help realize the full potential of the eventually derived neuronal populations. Furthermore, existing protocols are still limited in the number of neuronal subtypes whose induction is fully established, and different cultivation protocols for each subtype exist. As such, future detailed characterization of the cellular and molecular characteristics involved in guiding stem cell differentiation and somatic cell reprogramming along the neural lineage is expected to contribute to the development of highly robust protocols. Evidently, these efforts could aid in the development of highly meaningful in vitro disease models for disorders such as SAD.

Finally, Chapter 8 comprised an exploratory pilot study, in which the knowledge of Chapters 6 and $\mathbf{7}$ was combined in an effort to establish and characterize a cortical forebrain differentiation protocol from iPSCs. The overarching aim of this chapter was to use this differentiation protocol for the establishment of an in vitro model relevant for SAD. First, the stemness of the iPSC-line, which was used for neural differentiation, was confirmed based on the expression of pluripotency markers such as OCT4, NANOG, SSEA3, SSEA4, TRA-1-60 and TRA-1-81. Next, by the sequential application of neural patterning factors, iPSCs were differentiated to neural progenitor cells (NPCs) and subsequently to cortical neurons and glia. At each stage of the differentiation process, the expression of key-lineage transcription factors was assessed, which confirmed that the cells progressed towards a cortical forebrain fate. Successful derivation of NPCs was confirmed by the expression of 
NESTIN, SOX2 and PAX6. Quantification of these markers furthermore revealed that the iPSCs differentiated towards relative homogeneous population of NPCs $(>82 \%)$. Immunofluorescent examination following differentiation of the NPCs verified the presence of the neuronal markers such as MAP2AB, DCX and TUJ1, and the astrocytic marker GFAP. Additionally, the presence of cortical forebrain markers, including CTIP2 and FOXG1, were identified in the differentiated cultures. Overall, these preliminary findings confirmed that the protocol described in this chapter was successful in differentiating the iPSCs into a mixed population of cortical forebrain neurons and glia. Although further functional characterization of these neural cells remains necessary, it was postulated that the protocol applied in this chapter can be used for the establishment of a cortical in vitro disease model system for $S A D$. In order to further explore this potential, expression of TNXB and the oxytocin receptor (OXTR) in the iPSC-derived cortical cultures was assessed. As previously demonstrated in Chapter $\mathbf{4}$ of this thesis, epigenetic and genetic dysregulation of $T N X B$ have been associated with AD pathophysiology in multiple regions of the $S A D$ brain. In addition, epigenetic deregulation of the oxytocin gene (OXT) and alterations in OXT signaling have previously also been implicated in SAD or associated disease phenotypes. Interestingly, expression of both TNXB and OXTR could be detected in the differentiated neural cells already at 14 days and maintained up until 56 days of NPC-differentiation, suggesting that these cortical forebrain cells might represent an appealing model system to study mechanistic processes related to TNXB and OXT signaling in the context of SAD. All in all, efforts as attained in this chapter are imperative if one wishes to establish disease-relevant iPSC-based models. 

Chapter 11

Samenvatting 

Hoofdstuk 1 introduceerde de lezer op het gebied van neuroepigenetica in relatie tot de sporadische ziekte van Alzheimer (SAD), evenals op het gebruik van geïnduceerde pluripotente stamcel (iPSC)-gebaseerde modellen voor het bestuderen van deze neurodegeneratieve aandoening. $\mathrm{Na}$ deze korte inleiding werd een overzicht gepresenteerd van de algemene structuur van het proefschrift, dat zou kunnen worden onderverdeeld in twee hoofddelen gericht op elk van de bovengenoemde onderzoekslijnen. In meer detail, de studies gepresenteerd in het eerste deel van dit proefschrift zijn gericht op de rol van epigenetische ontregelingen in de etiopathofysiologie van SAD. Dit eerste deel, dat de Hoofdstukken 2-5 omvat, bestond uit een perspectief artikel, twee methodologische onderzoekartikelen en een origineel onderzoekartikel. Het werk in het tweede deel van dit proefschrift was gericht op de toepassing en totstandkoming van iPSC-gebaseerde modellen voor SAD, bijvoorbeeld met het oog op mechanistische studies naar epigenetische ontregeling. Het tweede deel, bestaande uit de Hoofdstukken 6-8, bevatte twee diepgaande overzichtsartikelen en een verkennende pilotstudie.

In Hoofdstuk 2, wordteen perspectiefartikel overepigenoom-bredeassociatiestudies (EWAS), of meer specifiek methylome-brede associatiestudies (MWAS), in SAD gepresenteerd. Epigenetische mechanismen, die de interactie tussen het genoom en de omgeving mediëren, worden verondersteld een mechanistische verklaring te bieden met het oog op de etiopathogenese van SAD. In dit artikel werden alle onderzoeken die tot nu toe zijn uitgevoerd, gericht op verschillende (corticale) hersengebieden, evenals perifere bloedmonsters, afkomstig van patiënten en overeenkomende niet-demente controlepersonen, kort besproken. Verder werden relevante kanttekeningen met betrekking tot deze onderzoeken die de interpretatie van de onderzoeksresultaten beïnvloeden besproken, waaronder genomische dekking, statistische bewijskracht, specificiteit van de geëvalueerde epigenetische kenmerken, celtypespecificiteit en samenstelling, causale inferentie en multiomica. Ten slotte werd een vooruitblik gegeven op mogelijke oplossingen om deze uitdagingen het hoofd te bieden, bestaande uit methodologische ontwikkelingen en vorderingen in gerelateerde datawetenschap-disciplines. In het algemeen werd uit dit hoofdstuk geconcludeerd dat, hoewel MWAS zeer relevant zijn in de context van SAD-onderzoek, het essentieel blijft om de bovengenoemde kanttekeningen in toekomstige studies aan te pakken om meer zinvolle gegevens te produceren, die het begrip van de processen die ten grondslag liggen aan de ziekte aanzienlijk zouden kunnen vergroten.

In relatie tot bovengenoemde uitdagingen, beschreef Hoofdstuk 3 een gestandaardiseerd protocol voor oxidatieve bisulfiet pyrosequentie bepaling waarmee een nauwkeurig onderscheid kan worden gemaakt tussen DNAmethylatie en hydroxymethylatie, dat wil zeggen tussen 5-methylcytosine (5- 
$\mathrm{mC}$ ) en 5-hydroxymethylcytosine (5-hmC) basen, in de context van cytosinefosfaat-guanine $(\mathrm{CpG})$ posities. Het pyrosequentie bepaling protocol dat in dit hoofdstuk wordt beschreven, biedt een werkbare oplossing voor het probleem van modificatiespecificiteit waarmee neuroepigenetische onderzoeken naar SAD worden geconfronteerd. De aanpak is gebaseerd op een zeer selectieve chemische oxidatie met behulp van $\mathrm{KRuO} 4$ die wordt toegepast voorafgaand aan de bisulfiet behandeling, polymerasekettingreactie (PCR) en pyrosequentie bepaling stappen. Bovendien werd specifiek voor deze methode een nieuwe spike-in DNA-standaard set ontwikkeld waarmee de gebruiker nauwkeurig de oxidatieve bisulfiet conversieefficiëntie kan bepalen. Om het principe aan te tonen werd het protocol uitgevoerd op zowel post-mortem hersenweefsel als iPSCs, waarmee werd aangetoond dat 5-mC en 5-hmC, evenals niet gemodificeerde cytosine (5-uC) basen, kunnen worden gedetecteerd in OXT en DNAJB13. Bovendien werd bevestigd dat de nieuwe (5-hmC) spike-in controle zou kunnen worden gebruikt als een interne pyrosequentie bepaling-controle die de analyse van het doelmonster niet verstoort, en andersom. Dergelijke innovatieve benaderingen zoals gepresenteerd in dit hoofdstuk zijn cruciaal om het veld van neuroepigenetica in SAD vooruit te helpen, omdat deze de validiteit van de onderzoeksresultaten vergroten.

Hoofdstuk 4 introduceerde een nieuwe methode voor DNA-methylatie profilering in kleine clusters van 50 neuronen, die gebaseerd was op een combinatie van limiting dilution bisulfite pyrosequencing (LDBSP) en laser capture microdissectie (LCM). Met deze methode kan men de methylatie graad van CpG posities op afzonderlijke allelen en op een meervoudig-gerichte en celtype-specifieke manier bepalen, waardoor er een oplossing wordt geboden voor het probleem van cellulaire heterogeniteit dat wordt aangetroffen in neuroepigenetisch onderzoek naar SAD. De algemene werkwijze van LDBSP op cellen geïsoleerd uit postmortem hersenweefsel met behulp van LCM werd in dit hoofdstuk beschreven. Bovendien werd om het principe aan te tonen een gerichte methylatie analyse van DNAJB13, PGLYRP1, RHBDF2, C3, LMX1B en OXT uitgevoerd. Interessant is dat LDBSP op deze clusters van neuronen vaak afgeleide reacties vertoonde met meer dan één DNA-molecuul, een scenario dat zelden voorkomt bij het uitvoeren van LDBSP op een eencellig monster of slechts een paar cellen. Als zodanig werd een aangepaste methode voor de analyse van de onderzoeksresultaten voor LDBSP ontwikkeld, die het mogelijk maakt om de methylatie graad van CpG posities die zijn verkregen van multi-allelreacties op te nemen en te corrigeren. Over het algemeen biedt de methode die in dit hoofdstuk wordt beschreven de gebruiker een nauwkeurigere schatting van de DNA-methylatie status van elk doel gen in de geanalyseerde cel clusters, waardoor de validiteit van de onderzoeksresultaten toenemen. Bovendien werd aangetoond dat de efficiëntie van LDBSP op neuronen geïsoleerd met LCM vergelijkbaar is met de efficiëntie 
die werd bereikt in eerder gepubliceerde onderzoeken met deze techniek op andere geïsoleerde celtypen. Verwacht wordt dat analyses van een individuele cel(type), zoals beschreven in dit hoofdstuk, steeds waardevoller zullen worden voor toekomstige neuroepigenetische studies naar SAD.

Hoofdstuk 5 vertegenwoordigde de eerste grootschalige epigenetische analyse in de hersenstam van SAD tot nu toe, gericht op de dorsale raphe nuclei (DRN) en locus coeruleus (LC), en vormt daarmee een aanvulling op het geheel van EWAS besproken in Hoofdstuk 2. Differentiaal gemodificeerde posities en regio's in bulkweefsels verkregen uit beide hersenstamkernen werden geïdentificeerd op het niveau van DNA-methylatie, hydroxymethylatie en niet gemodificeerde cytosines door gebruik te maken van de Illumina Infinium MethylationEPIC BeadChiparray in combinatie met een oxidatieve bisulfiet behandeling van het geïsoleerde doel-DNA (vergelijkbaar met wat werd beschreven in Hoofdstuk 3). Naast een sterke overlappende ontregeling in het Tenascin XB gen (TNXB) in zowel de DRN als de LC, werden gemeenschappelijke en nieuwe epigenetische patronen ontdekt in vergelijking met eerdere onderzoeken gericht op de sAD-hersenen, die allemaal een cruciale rol kunnen spelen in de etiopathogenese van SAD. Een daaropvolgende analyse gebruikmakend van een bisulfiet pyrosequentie bepaling in hetzelfde patiënten cohort bevestigde de waargenomen ontregeling van TNXB in beide onderzochte hersenstamgebieden. Als vervolg op deze bevindingen werd een gerichte analyse van TNXB uitgevoerd in een onafhankelijk patiënten cohort, waarbij methylatie patronen werden onderzocht in enkele serotonerge neuronen en niet-serotonerge cellen geïsoleerd uit de DRN door middel van LCM. Deze studie vertegenwoordigde daarom de eerste cel subtype-specifieke validatie analyse die tot nu toe in de hersenstam is uitgevoerd, gebaseerd op de LCMLDBSP-methode die werd beschreven in Hoofdstuk 4. Opvallend is dat bij het vergelijken van de bisulfiet methylatie niveaus in TNXB tussen SAD-patiënten en controles in serotonerge en niet-serotonerge neuronen, een significante interactie tussen celtype en experimentele conditie werd geïdentificeerd. De SAD-geassocieerde methylatieprofielen waren tegengesteld in de serotonerge neuronen en niet-serotonerge cellen, waarvan de laatste vergelijkbaar waren met de EWAS-onderzoeksresultaten. Als zodanig toonde deze studie voor het eerst aan dat epigenetische patronen binnen de DRN sterk kunnen afhangen van zowel het ziektebeeld als het geanalyseerde celtype. Deze bevindingen benadrukken daarom het belang van toekomstige celtype-specifieke neuroepigenetische studies naar SAD, wat al kort aan de orde kwam in de Hoofdstukken 2 en 4.

Hoofdstuk 6 presenteerde een overzichtsartikel over de potentiële waarde van iPSCs voor SAD-onderzoek. Sinds hun ontdekking bieden deze cellen een veelbelovende manier om de translationele kloof tussen preklinisch en klinisch 
sAD-onderzoek te dichten, door het opzetten van patiënt-specifieke in vitro ziektemodellen die kunnen worden toegepast voor fundamenteel onderzoek en ontdekking van geneesmiddelen. Verwacht wordt dat inspanningen op dit gebied onze kennis over verschillende onderliggende ziektemechanismen zullen verdiepen en zullen helpen bij het opzetten van therapeutische interventies. Alle baanbrekende onderzoeken met iPSCs van SAD-patiënten werden in dit hoofdstuk besproken, welke hebben aangetoond dat de iPSC-afgeleide neurale cellen de neuropathologische processen van de ziekte recapituleren, hoewel met een vrij hoge mate van variabiliteit in termen van hun aanwezigheid en ernst. Derhalve werden bronnen van variabiliteit gerelateerd aan het model, naast degene die zouden kunnen worden verklaard door de heterogene aard van SAD, kritisch belicht. Voortvloeiend uit dit hoofdstuk, is dat het ontwikkelen van iPSC-modellen voor SAD een uitdagende inspanning blijft vanwege de multifactoriële aard van de ziekte, de bijna levenslange ziekteprogressie en de hoge mate van interindividuele heterogeniteit die kan worden weerspiegeld in cellen die zijn verkregen van patiënten. Afgezien van de variabiliteit die met de ziekte gepaard gaat, hebben verschillende methodologische factoren, zoals die gerelateerd aan iPSC-generatie en (neurale) differentiatie, een verdere invloed op deze mate van variatie. Toekomstige inspanningen gericht op het uitgebreid karakteriseren van de iPSCafgeleide modellen en bronnen van variabiliteit in fijn detail, zijn dus noodzakelijk als men robuuste ziektemodellen wil opzetten voor de studie van SAD. Ondanks deze overwegingen bieden de zich ontwikkelende iPSC-modellen duidelijk een opwindende weg in SAD onderzoek.

In het kader van het ontwikkelen van iPSC-gebaseerde modellen, presenteerde Hoofdstuk 7 een diepgaand overzichtsartikel over aangestuurde en directe neurale differentiatieprotocollen, uitgaande van respectievelijk stamcellen en somatische cellen. Zoals in dit overzicht wordt besproken, hebben inzichten uit fundamenteel onderzoek en ontwikkelingsbiologie het ontwerp van huidige protocollen geleid en zijn er talloze methoden beschikbaar voor de afleiding van regionaal-specifieke glutamaterge, dopaminerge, GABAergische, serotonerge en cholinerge/motor neuronen. Methoden voor de afleiding van elk van deze ziekte gerelateerde neurale subtypes werden samengevat, gebaseerd op ofwel chemisch gedefinieerde systemen met behulp van patroonfactoren, op transcriptiefactorengemedieerde herprogrammering en op epigenetische-gebaseerde strategieën. Dit review benadrukte verder dat hoewel verschillende methoden succesvol zijn gebleken bij het aansturen van de lotsbestemming van neuronale cellen in vitro, hun verfijning en optimalisatie, evenals het zoeken naar alternatieve strategieën, noodzakelijk blijven om het volledige potentieel van de uiteindelijk afgeleide neuronale populaties te helpen realiseren. Bovendien zijn de bestaande protocollen nog steeds beperkt in het aantal neuronale subtypen waarvan de inductie volledig 
is vastgesteld, en er bestaan verschillende kweekprotocollen voor elk subtype. Als zodanig wordt verwacht dat toekomstige gedetailleerde karakterisering van de cellulaire en moleculaire kenmerken die betrokken zijn bij het begeleiden van stamceldifferentiatie en somatische cel herprogrammering langs de neurale lijn, zal bijdragen aan de ontwikkeling van zeer robuuste protocollen. Het is evident dat deze inspanningen kunnen helpen bij de ontwikkeling van zeer zinvolle in vitro ziektemodellen voor aandoeningen zoals SAD.

Ten slotte omvatte Hoofdstuk 8 een verkennende pilotstudie, waarin de kennis van Hoofdstuk 6 en 7 werd gecombineerd in een poging om een corticale voorhersendifferentiatieprotocol vanuit iPSCs op te zetten en te karakteriseren. Het overkoepelende doel van dit hoofdstuk was om dit differentiatieprotocol te gebruiken voor het opzetten van een in vitro model dat relevant is voor SAD. Ten eerste werd de stamcel karakteristieken van de iPSC-lijn, die werd gebruikt voor neurale differentiatie, bevestigd op basis van de expressie van pluripotente markers zoals OCT4, NANOG, SSEA3, SSEA4, TRA-1-60 en TRA-1-81. Vervolgens werden iPSCs door de opeenvolgende toepassing van neurale patroonfactoren gedifferentieerd naar neurale voorlopercellen (NPCs) en vervolgens naar corticale neuronen en glia. In elke fase van het differentiatieproces werd de expressie van belangrijke afstamming transcriptiefactoren beoordeeld, wat bevestigde dat de cellen vorderde naar een corticale voorhersenen lotsbestemming. Succesvolle afleiding van NPCs werd bevestigd door de expressie van NESTIN, SOX2 en PAX6. Kwantificering van deze markers onthulde verder dat de iPSCs differentieerde naar een relatief homogene populatie van NPCs (> 82\%). Immunofluorescentie bepaling na differentiatie van de NPCs bevestigde de aanwezigheid van de neuronale markers zoals MAP2AB, DCX en TUJ1, en de astrocytische marker GFAP. Bovendien werd de aanwezigheid van markers van de corticale voorhersenen, waaronder CTIP2 en FOXG1, geïdentificeerd in de gedifferentieerde culturen. Over het algemeen bevestigden deze voorlopige bevindingen dat het protocol dat in dit hoofdstuk wordt beschreven, succesvol was in het differentiëren van de iPSCs in een gemengde populatie van corticale frontale neuronen en glia. Hoewel verdere functionele karakterisering van deze neurale cellen noodzakelijk blijft, werd gepostuleerd dat het protocol dat in dit hoofdstuk wordt toegepast gebruikt kan worden voor het opzetten van een corticaal in vitro ziektemodelsysteem voor sAD. Om dit potentieel verder te onderzoeken, werd de expressie van TNXB en de oxytocinereceptor (OXTR) in de iPSC-afgeleide corticale culturen vastgesteld. Zoals eerder aangetoond in Hoofdstuk 4 van dit proefschrift, zijn epigenetische en genetische ontregeling van TNXB geassocieerd met $A D$ pathofysiologie in meerdere regio's van de SAD hersenen. Bovendien was epigenetische dysregulatie van het oxytocine-gen (OXT) en veranderingen in OXT-signalering eerder ook betrokken bij SAD of geassocieerde ziektefenotypes. Interessant is 
dat expressie van zowel TNXB als OXTR al na 14 dagen in de gedifferentieerde neurale cellen kon worden gedetecteerd en werd gehandhaafd tot 56 dagen na NPC-differentiatie, wat suggereert dat deze corticale voorhersenencellen een aantrekkelijk modelsysteem zouden kunnen zijn om mechanistische processen gerelateerd aan TNXB en OXT-signalering te bestuderen in de context van SAD. Al met al zijn de inspanningen die in dit hoofdstuk werden verricht noodzakelijk als men ziektegerelateerde iPSC-gebaseerde modellen wil ontwikkelen. 
Chapter 12

Zusammenfassung 

Kapitel 1 führte den Leser in das Gebiet der Neuroepigenomik bei sporadischer Alzheimer-Krankheit (SAD) sowie in die Verwendung von auf induzierten pluripotenten Stammzellen (iPSC) basierenden Modellen zur Untersuchung dieser neurodegenerativen Störung ein. Nach dieser kurzen Einführung wurde ein Überblick über die allgemeine Struktur der Arbeit gegeben, der in zwei Hauptteile unterteilt werden konnte, die sich auf jede der oben genannten Forschungslinien konzentrierten. Ausführlicher, konzentrierten sich die im ersten Teil dieser Arbeit vorgestellten Studien auf die Rolle der epigenetischen Dysregulation in der Ätiopathophysiologie von SAD. Dieser erste Teil, der die Kapitel 2 bis $\mathbf{5}$ enthält, bestand aus einem Perspektivpapier, zwei methodischen Forschungspapieren und einem Original-Forschungsartikel. Die Arbeit im zweiten Teil dieser Arbeit konzentrierte sich auf die Anwendung und Etablierung von iPSC-basierten Modellen für $S A D$, zum Beispiel im Hinblick auf mechanistische Studien zur epigenetischen Dysregulation. Der zweite Teil, bestehend aus den Kapiteln 6-8, enthielt zwei ausführliche Übersichtsartikel und eine explorative Pilotstudie.

In Kapitel 2, wurde ein Perspektivpapier zu epigenomweiten Assoziationsstudien (EWAS) oder genauer zu methylomweiten Assoziationsstudien (MWAS) in SAD vorgestellt. Es wird angenommen, dass epigenetische Mechanismen, die die Wechselwirkung zwischen Genom und Umwelt vermitteln, eine mechanistische Erklärung im Hinblick auf die Ätiopathogenese von SAD liefern. In diesem Artikel wurden alle bisher durchgeführten Studien, die auf verschiedene (kortikale) Regionen des Gehirns abzielten, sowie periphere Blutproben, die von Patienten stammen und mit nicht dementen Kontrollen übereinstimmen, kurz besprochen. Darüber hinaus wurden relevante Vorbehalte in Bezug auf diese Studien diskutiert, die die Interpretation der experimentellen Ergebnisse in Frage stellen, einschließlich der genomischen Abdeckung, der statistischen Aussagekraft, der Spezifität der bewerteten epigenetischen Markierungen, der Spezifität und Zusammensetzung des Zelltyps, der kausalen Inferenz und der Multi-Omics. Schließlich wurde ein Ausblick auf mögliche Lösungen gegeben, die diese Herausforderungen bewältigen könnten, einschließlich methodischer Entwicklungen und Fortschritte in verwandten datenwissenschaftlichen Disziplinen. Insgesamt wurde aus diesem Kapitel der Schluss gezogen, dass MWAS zwar im Kontext der SAD-Forschung von hoher Relevanz sind, es jedoch weiterhin wichtig ist, die oben genannten Vorbehalte in zukünftigen Studien zu berücksichtigen, um aussagekräftigere Daten zu erhalten, die unser Verständnis der Prozesse die der Krankheit zugrunde liegen erheblich verbessern könnten.

In Anbetracht dieser Herausforderungen wurde in Kapitel $\mathbf{3}$ ein standardisiertes Protokoll für die oxidative Bisulfit Pyrosequenzierung beschrieben, das eine genaue Unterscheidung zwischen DNA-Methylierung und -Hydroxymethylierung ermöglicht, 
das heißt zwischen 5-Methylcytosin (5-mC) und 5-Hydroxymethylcytosin (5-hmC)Basen in der Kontext von Cytosin-Phosphat-Guanin (CpG)-Stellen. Das in diesem Kapitel beschriebene Pyrosequenzierungsprotokoll bietet eine praktikable Lösung für das Problem der Modifikationsspezifität, mit dem neuroepigenomische Studien von SAD konfrontiert sind. Der Ansatz beruht auf einer hochselektiven chemischen Oxidation unter Verwendung von $\mathrm{KRuO} 4$, die vor der Bisulfitbehandlung, der Polymerasekettenreaktion (PCR) und den Pyrosequenzierungs schritten angewendet wird. Darüber hinaus wurde für diese Methode ein neuartiger Spike-In-DNA-Standardsatz entwickelt, mit dem der Benutzer die Effizienz der oxidativen Bisulfitumwandlung genau bestimmen kann. Als Beweis des Prinzips wurde das Protokoll sowohl an post mortem Hirngewebe als auch an iPSCs durchgeführt, was zeigte, dass $5-\mathrm{mC}$ und $5-\mathrm{hmC}$ sowie unmodifizierte Cytosin (5-uC)-Basen in OXT und DNAJB13 nachgewiesen werden konnte. Darüber hinaus wurde bestätigt, dass die neuartige $(5-\mathrm{hmC})$ Spike-In-Kontrolle als interne Pyrosequenzierungskontrolle verwendet werden kann, die die Analyse der Ziel Probe nicht beeinträchtigt, und umgekehrt. Solche innovativen Ansätze, wie sie in diesem Kapitel vorgestellt werden, sind entscheidend, um das Gebiet der Neuroepigenomik in SAD voranzubringen, da sie dazu beitragen die Gültigkeit der Daten zu erhöhen.

In Kapitel 4 wurde eine neuartigere Methode für die Erstellung von DNAMethylierungsprofilen in kleinen Kolonien von 50 Neuronen vorgestellt, der auf einer Kombination aus Grenzverdünnungs-Bisulfit-Pyrosequenzierung (LDBSP) und Laser-Capture-Mikrodissektion (LCM) beruhte. Diese Methode ermöglicht es, die Methylierungsraten der CpG-Stelle auf einzelnen Allelen auf eine vielfach zielgerichtete und zelltypspezifische Weise zu bestimmen, wodurch eine Lösung für das Problem der zellulären Heterogenität bereitgestellt wird das in neuroepigenomischen Studien von SAD auftritt. In diesem Kapitel wurde das allgemeine Arbeitsverfahren von LDBSP an Zellen beschrieben, die mit LCM aus post mortem Hirngewebe isoliert wurden. Darüber hinaus wurde als Beweis des Prinzips eine gezielte Methylierungsanalyse der DNAJB13, $P G L Y R P 1, R H B D F 2, C 3, L M X 1 B$ und $O X T$ durchgeführt. Interessanterweise führte LDBSP in diesen Neuronekolonien häufig zu nachgeschalteten Reaktionen mit mehr als einem DNA-Molekül, ein Szenario das selten auftritt wenn LDBSP an einer Einzellprobe oder nur wenigen Zellen durchgeführt wird. Als solches wurde eine angepasste Datenanalyse-Pipeline für LDBSP entwickelt, die es ermöglicht CpG-Methylierungsraten die aus Multi-Allel-Reaktionen abgeleitet wurden einzuschließen und zu korrigieren. Insgesamt bietet die in diesem Kapitel beschriebene Methode dem Benutzer eine genauere Schätzung des DNAMethylierungsstatus jedes Zielgens in den analysierten Zellpools, wodurch die Daten weiter validiert werden. Darüber hinaus wurde gezeigt, dass die Effizienz 
von LDBSP bei mit LCM isolierten Neuronen ähnlich der Effizienz ist, die in zuvor veröffentlichten Studien unter Verwendung dieser Technik bei anderen isolierten Zelltypen erzielt wurde. Es wird erwartet, dass Einzelzell(typ)ansätze, wie sie in diesem Kapitel beschrieben werden, für zukünftige neuroepigenomische Studien in SAD zunehmend wertvoll sein werden.

Kapitel 5 stellte die erste groß angelegte epigenetische Analyse im Hirnstamm von SAD dar, die auf die dorsalen Raphekerne (DRN) und den Locus coeruleus (LC) abzielte und damit die in Kapitel 2 besprochenen EWAS-Literaturergänzte. Aus beiden Hirnstammkernen erhaltene Massengewebe wurden Differential modifizierte Positionen und Regionen identifiziert auf der Ebene der DNA-Methylierung, -Hydroxymethylierung und unmodifizierten Cytosine unter Verwendung des Illumina Infinium MethylationEPIC BeadChip-Arrays in Kombination mit einer oxidativen Bisulfit-Behandlung der isolierten Ziel-DNA (ähnlich wie in Kapitel 3 beschrieben). Abgesehen von einer stark überlappenden Dysregulation im Tenascin XB-Gen (TNXB), sowohl im DRN als auch im LC, wurden gemeinsame und neuartige epigenetische Signaturen im Vergleich zu früheren Studien zum sAD-Gehirn entdeckt, die alle eine entscheidende Rolle bei der Ätiopathogenese von SAD spielen können. Eine anschließende Bisulfit-Pyrosequenzierungsanalyse in derselben Patientenkohorte bestätigte die beobachtete Dysregulation von $T N X B$ in beiden untersuchten Hirnstammregionen. Im Anschluss an diese Entdeckungsergebnisse wurde eine gezielte Analyse von TNXB in einer unabhängigen Patientenkohorte durchgeführt, wobei die methylomischen Signaturen in einzelnen serotonergen Neuronen und nicht serotonergen Zellen bewertet wurden, die mittels LCM aus dem DRN isoliert wurden. Diese Studie stellte daher die erste zellsubtypspezifische Validierungsanalyse dar, die bisher im Hirnstamm durchgeführt wurde und sich auf den in Kapitel 4 beschriebenen LCM-LDBSP-Ansatz stützte. Bemerkenswerterweise beim Vergleich der Bisulfitmethylierungsniveaus von TNXB zwischen SAD-Patienten und Kontrollen bei Serotonergen und nicht serotonergen Neuronen wurde eine signifikante Wechselwirkung zwischen Zelltyp und experimentellem Zustand identifiziert. Die SAD-assoziierten Methylierungsprofile waren in den serotonergen Neuronen und nicht serotonergen Zellen entgegengesetzt, wobei letztere den EWAS-Daten ähnelten. Als solche zeigte diese Studie zum ersten Mal, dass epigenetische Signaturen innerhalb des DRN sowohl vom Krankheitsbild als auch vom analysierten Zelltyp stark abhängen können. Diese Ergebnisse unterstreichen daher die Bedeutung zukünftiger zelltypspezifischer neuroepigenomischer Studien bei sAD, auf die bereits in den Kapiteln 2 und $\mathbf{4}$ kurz eingegangen wurde.

In Kapitel 6 wurde ein Übersichtsartikel über den potenziellen Wert von iPSCs für die sAD-Forschung vorgestellt. Seit ihrer Entdeckung bieten diese Zellen einen vielversprechenden Weg, um die Translationslücke zwischen 
präklinischer und klinischer sAD-Forschung zu schließen, indem sie die Erstellung patientenspezifischer In-vitro-Krankheitsmodelle ermöglichen, die für die Grundlagenforschung und die Wirkstoffentdeckung angewendet werden können. Es wird erwartet, dass die Bemühungen auf diesem Gebiet unser Wissen über verschiedene zugrundeliegende Krankheitsmechanismen vertiefen und bei der Etablierung therapeutischer Interventionen helfen werden. Alle wegweisenden Studien unter Verwendung von iPSCs von SAD-Patienten wurden in diesem Kapitel überprüft, die zeigen, dass die von iPSC abgeleiteten Nervenzellen neuropathologische Prozesse der Krankheit rekapitulieren, wenn auch mit einem recht hohen Grad an Variabilität hinsichtlich ihres Vorhandenseins und ihrer Schwere. Daher wurden Variabilitätsquellen in Bezug auf das Modell zusätzlich zu denen, die durch die heterogene Natur von SAD erklärt werden könnten, kritisch diskutiert. Aus diesem Kapitel geht hervor, dass die Entwicklung von iPSC-Modellen für SAD ein herausforderndes Unterfangen bleibt aufgrund der multifaktoriellen Natur der Krankheit, des nahezu lebenslangen Fortschreitens der Krankheit und des hohen Grads an interindividueller Heterogenität, die sich in Zellen von Patienten widerspiegeln könnte. Abgesehen von der mit der Krankheit verbundenen Variabilität wirken sich verschiedene methodische Faktoren, wie die mit der iPSC-Erzeugung und der (neuronalen) Differenzierung verbundenen, weiter auf diesen Variationsgrad aus. Daher sind zukünftige Anstrengungen erforderlich, um die von iPSC abgeleiteten Modelle und Variabilitätsquellen detailliert zu charakterisieren, wenn robuste Krankheitsmodelle für die Untersuchung von SAD erstellt werden sollen. Unter Berücksichtigung dieser Überlegungen bieten die sich entwickelnden iPSC-Modelle jedoch eindeutig einen spannenden Ansatzin der sAD-Forschung.

Im Verhältnis zu der Entwicklung von iPSC-basierten Modellen wurde in Kapitel 7 ein ausführlicher Übersichtsartikel zu Protokollen zur gerichteten und direkten neuronalen Differenzierung vorgestellt ausgehend von beziehungsweise Stammzellen und somatischen Zellen. Wie in dieser Rezension diskutiers, haben Erkenntnisse aus der Grundlagenforschung und der Entwicklungsbiologie das Design aktueller Protokolle geleitet, und es sind zahlreiche Ansätze zur Ableitung regionalspezifischer glutamaterger, dopaminerger, GABAerger, serotonerger und cholinerger/motor Neuronen verfügbar geworden. Ansätze zur Ableitung jedes dieser krankheitsrelevanten neuronalen Subtypen wurden zusammengefasst, wobei entweder chemisch definierte Systeme unter Verwendung von Musterfaktoren, Transkriptionsfaktor-vermittelte Reprogrammierung und epigenetische Strategien zugrunde gelegt wurden. In dieser Übersicht wurde ferner hervorgehoben, dass sich zwar unterschiedliche Ansätze als erfolgreich erwiesen haben, um das Schicksal neuronaler Zellen in vitro zu steuern, ihre Verfeinerung und Optimierung sowie die Suche nach alternativen Strategien jedoch weiterhin erforderlich sind, 
um das volle Potenzial der letztendlich abgeleiteten neuronalen Populationen auszuschöpfen. Darüber hinaus sind bestehende Protokolle in der Anzahl der neuronalen Subtypen, deren Induktion vollständig etabliert ist, immer noch begrenzt, und es existieren unterschiedliche Kultivierungsprotokolle für jeden Subtyp. Daher wird erwartet, dass die zukünftige detaillierte Charakterisierung der zellulären und molekularen Eigenschaften, die bei der Steuerung der Stammzelldifferenzierung und der Reprogrammierung somatischer Zellen entlang der neuralen Linie eine Rolle spielen, zur Entwicklung hoch robuster Protokolle beiträgt. Offensichtlich könnten diese Bemühungen zur Entwicklung hoch aussagekräftiger In-vitroKrankheitsmodelle für Erkrankungen wie SAD beitragen.

Schließlich umfasste Kapitel 8 eine explorative Pilotstudie, in der die Kenntnisse der Kapitel 6 und 7 kombiniert wurden, um ein Differenzierungsprotokoll für das kortikale Vorderhirn von iPSCs zu etablieren und zu charakterisieren. Das übergeordnete Ziel dieses Kapitels war es, dieses Differenzierungsprotokoll zur Erstellung eines für SAD relevanten In-vitro-Modells zu verwenden. Zunächst wurde der Stamm der iPSC-Linie, die zur neuronalen Differenzierung verwendet wurde, anhand der Expression von Pluripotenzmarkern wie OCT4, NANOG, SSEA3, SSEA4, TRA-160 und TRA-1-81 bestätigt. Als nächstes wurden durch die sequentielle Anwendung neuronaler Strukturierungsfaktoren iPSCs zu neuralen Vorläuferzellen (NPCs) und anschließend zu kortikalen Neuronen und Glia differenziert. In jeder Phase des Differenzierungsprozesses wurde die Expression von Transkriptionsfaktoren der Schlüssellinie bewertet, was bestätigte, dass die Zellen in Richtung eines Schicksals des kortikalen Vorderhirns voranschritten. Die erfolgreiche Ableitung von NPCs wurde durch die Expression von NESTIN, SOX2 und PAX6 bestätigt. Die Quantifizierung dieser Marker ergab weiterhin, dass sich die iPSCs in Richtung einer relativ homogenen Population von NPCs (>82\%) differenzierten. Die Immunfluoreszenzuntersuchung nach Differenzierung der NPCs bestätigte das Vorhandensein der neuronalen Marker wie MAP2AB, DCX und TUJ1 sowie des Astrozytenmarkers GFAP. Zusätzlich wurde das Vorhandensein von kortikalen Vorderhirnmarkern, einschließlich CTIP2 und FOXG1, in den differenzierten Kulturen identifiziert. Insgesamt bestätigten diese vorläufigen Ergebnisse, dass das in diesem Kapitel beschriebene Protokoll die iPSCs erfolgreich in eine gemischte Population von kortikalen Vorderhirnneuronen und Glia differenzierte. Obwohl eine weitere funktionelle Charakterisierung dieser neuralen Zellen weiterhin erforderlich ist, wurde postuliert, dass das in diesem Kapitel angewandte Protokoll zur Einrichtung eines kortikalen In-vitro-Krankheitsmodellsystems für SAD verwendet werden kann. Um dieses Potenzial weiter zu untersuchen, wurde die Expression von TNXB und des Oxytocinrezeptors (OXTR) in den von iPSC abgeleiteten kortikalen Kulturen bewertet. Wie bereits in Kapitel 4 dieser Arbeit gezeigt, wurde die epigenetische und genetische Dysregulation von 
TNXB in mehreren Regionen des SAD-Gehirns mit der AD-Pathophysiologie in Verbindung gebracht. Darüber hinaus wurden die epigenetische Deregulierung des Oxytocin-Gens (OXT) und Veränderungen der OXT-Signalübertragung zuvor auch mit SAD- oder assoziierten Krankheitsphänotypen in Verbindung gebracht. Interessanterweise konnte die Expression von TNXB und OXTR bereits nach 14 Tagen in den differenzierten Nervenzellen nachgewiesen und bis zu 56 Tage nach NPC-Differenzierung aufrechterhalten werden, was darauf hindeutet, dass diese kortikalen Vorderhirnzellen ein ansprechendes Modellsystem zur Untersuchung mechanistischer Prozesse im Zusammenhang mit TNXB und OXT-Signalisierung im Kontext von SAD darstellen könnten. Alles in allem sind die in diesem Kapitel unternommenen Anstrengungen unerlässlich, wenn krankheitsrelevante iPSCbasierte Modelle erstellt werden sollen. 
Chapter 13

Impact paragraph 

In this section, the scientific and societal impact of the research described in this thesis will be discussed.

\section{Scientific impact}

In spite of the enormous intellectual and financial investments into sporadic Alzheimer's disease (SAD) research over the last century, there is still considerable debate regarding the underlying causes of the disease and the precise mechanisms behind disease progression. Curing SAD when the disease is already in a more advanced stage is unlikely, as the damage done to the patient's brain seems irreversible at this point. Moreover, current diagnostic tools fall short in detecting the development of SAD in a timely manner that could improve treatment outcome. While early detection and intervention appear to be key in treating $S A D$, at this moment, even when detected at an early stage, available treatment options solely provide symptomatic relief rather than disease-modifying benefits; hence, gradual development of the full-blown disease phenotype is still inevitable. Thus, there is an unmet scientific need to develop a better understanding of early sAD-associated molecular mechanisms, which in turn could aid in the establishment of therapeutic interventions and diagnostic alternatives.

In recent years, the role of epigenetic mechanisms in SAD has received increasing attention, as they are thought to provide a mechanistic link between environmental exposures and both the development and course of the disease. In fact, the identification of SAD-associated epigenetic profiles is anticipated to provide us with novel cues towards affected molecular mechanisms and interacting environmental factors that could explain the complex underpinnings of the disease. Within this context, efforts aimed at interrogating brain regions with early manifestation of disease processes are of particular importance, as it is thought that the observed molecular changes in these brain areas are more relevant for sAD's etiology and pathophysiology. The scientific interest in epigenetic mechanisms is furthermore instigated by their clinical potential, as they could serve as biomarkers (when targeting e.g. blood), allowing earlier detection of SAD and/or provide opportunities for more detailed disease stratification. Their reversible nature furthermore makes them a realistic target for future preventive treatment strategies and pharmacological interventions.

Despite the growing number of epigenetic studies in SAD (reviewed in Chapter 2), the field of neuroepigenomics remains a relatively nascent area of investigation. Recent advances in microarray and sequencing technologies show that both whole genome-scale and targeted studies on the epigenome across much larger sample collections are now conceivable. It is important to acknowledge, however, that a plain exhaustive search for contributing factors, as has been employed in 
genetic studies of SAD, is unlikely to be fruitful for neuroepigenomic research. In reality, studies aiming to identify epigenetic mechanisms in complex diseases such as SAD need to consider several important issues related to study design, methodological limitations, tissue/cell type-specificity of epigenetic marks, and inference on causality, among others (reviewed in Chapter 2). As such, a more systematic approach towards understanding the role of epigenetic mechanisms in SAD is essential. While pushing this field forward, it will be crucial to remain cautious and scrutinize each of these issues thoroughly; next to extensively profiling brain tissues and/or cells derived from independent patient cohorts - if one wishes to provide conclusive insights into the underlying mechanisms of the disease.

The research described in the first part of this thesis builds upon this notion and highlights the scientific impact of the presented studies. More specifically, Chapter 3 describes an approach for targeted epigenetic profiling allowing one to distinguish between different epigenetic marks, i.e. DNA methylation and hydroxymethylation. Many of the existing methods used to interrogate the epigenome in SAD have been unable to specifically discriminate between these two closely related, but functionally distinct, epigenetic marks. As such, previous data obtained from these studies could be confounded and epigenetic effects related to SAD could have been overlooked and/or over- or underestimated. Thus, the work presented in this chapter represents an elegant solution towards overcoming methodological limitations as currently encountered in neuroepigenetic studies. The incorporation of such standardized protocols is therefore crucial for future neuroepigenomic research, even outside the field of $S A D$, as they will attribute further meaning and validity to the experimental outcomes.

Chapter 4 describes how a second challenge in the field, i.e. that of cellular heterogeneity, is being tackled, hence contributing to directing future neuroepigenomic research into more fruitful avenues. It is well-known that epigenetic profiles vary substantially between different cell types, even in the healthy human brain. Hence, a potential problem arises when heterogeneous bulk tissue samples, such as those from the brain, are being used for epigenomic profiling. Epigenetic marks in one cell type may oppose or dilute those in another, potentially obscuring important cell type-specific changes when analyzed all together. This issue is intensified when heterogeneous samples from healthy individuals are compared to those derived from SAD patients at different stages of the disease, which is common practice. Aside from sampling-induced variation between the tissues, differences in cellular composition e.g. as a result from neurodegeneration and increased immune activation as observed in SAD can significantly affect the cellular proportions of different brain samples. Overall, such variation can tremendously 
affect epigenetic data, resulting in experimental differences that in reality are not attributable to the disease, or, alternatively, masking actual differences. A practical solution to this issue, however, would be to profile individual cells (or cell types) isolated from these heterogeneous tissue samples. For this reason, Chapter 4 describes a novel approach relying on a combination of laser capture microdissection (LCM) and limiting dilution bisulfite pyrosequencing (LDBSP) that allows for targeted methylation profiling in individually isolated cells. This novel approach or similar strategies using other isolation techniques in combination with LDBSP will be increasingly valuable for future neuroepigenomic studies, even outside the scope of SAD.

Chapter 5 represents the first large-scale epigenetic analysis performed in the brainstem of $S A D$ to date, targeting both the dorsal raphe nuclei (DRN) and locus coeruleus (LC). Here, the state-of-the-art techniques described in the previous chapters are being combined in an effort to obtain more in-depth knowledge on the exact contribution of epigenetic mechanisms in these brain regions affected early in $S A D$. The scientific impact of this work is therefore threefold. First, this study is among the first of its kind to examine different epigenetic marks in the SAD brain simultaneously, including both DNA methylation and hydroxymethylation. Second, in contrast with previous studies that targeted (primarily cortical) brain regions affected in more advanced stages of $S A D$, the work in this chapter aimed at identifying potential disease-specific epigenetic marks in the brainstem, which are indicative of the more incipient stages of SAD. Third, the bulk tissue analysis in the DRN described here was complemented by a targeted cell-specific validation study were individual cells isolated from this brainstem region were analyzed. While replication of the obtained findings in independent patient cohorts remains necessary, the identified genes presented here could serve as pillars for future mechanistic studies into SAD. Moreover, this is the first study to date demonstrating that epigenetic signatures within the DRN are strongly dependent upon the cell type analyzed. Overall, the conclusion we can draw from this work is that future studies will need to implement single cell(type) analyses next to targeting heterogeneous bulk tissue samples.

In the second part of the thesis, starting from Chapter 6, the focus moves away from epigenetic profiling and, instead, insights into the potential of human induced pluripotent stem cell (iPSC)-based models for SAD research are being offered. An overview of the studies to date, exploring the use of iPSC-based models for SAD research, is presented, which includes but is not limited to neuroepigenomic studies. Furthermore, opportunities, challenges and considerations related to their use are being addressed. Despite the fact that further detailed characterization and validation of iPSC-based models remains necessary, overall, they are projected to 
significantly advance our current understanding of many disease processes and to revolutionize approaches for the identification of therapeutics for SAD. In fact, the recent availability of iPSC-based models, cellular reprogramming techniques and directed neural differentiation protocols, which are reviewed in Chapter 7, are anticipated to overcome the persistent translational gap between preclinical and clinical studies into SAD, emphasizing their scientific impact. Furthermore, it is anticipated that the establishment of robust iPSC-based in vitro models will contribute to reducing the need for animal experimentation.

Chapter 8 describes an exploratory approach on the characterization of a protocol to differentiate iPSCs cortical forebrain cells, with the ultimate aim to establish an SAD-relevant in vitro model. Even though the findings should be regarded as preliminary and the iPSC-derived neural cells are in need of further characterization and validation, the availability of such disease-relevant cultures offers appealing opportunities for future SAD studies. In this regard, iPSC-derived neural cells can be applied for modelling complex gene-environment interactions, by e.g. exposing cells derived from healthy individuals and SAD patients with different genetic backgrounds to environmental insults. This would allow more detailed studies on the cellular and molecular responses to these insults, even at an epigenetic level, for example. Furthermore, within this framework, the use of epigenome editing tools in these cultures could broaden our understanding on their causal involvement into sAD. All in all, also the iPSC 'adds' to neuroepigenomic research into SAD.

\section{Anticipated societal impact}

At this stage, it is important to acknowledge that the research described in this thesis is very fundamental and exploratory in nature. Therefore, the studies presented here will likely not have a direct impact on society in the short run, but mainly offer novel insights into methodological advances and the disease itself that are important for other scientists in the field. In spite of that, and while replication of the epigenetic analysis performed in the brainstem (Chapter 5) is crucial, the identified genes could be further investigated for their mechanistic and functional roles in $\mathrm{SAD}$, and, as such, might turn out to be targets for treatment strategies in future studies. All in all, the work presented in this thesis, ranging from systematic studies on the contribution of epigenetic mechanisms in SAD (Chapters 2, 3, 4 and 5) to the development of clinically relevant human disease models (Chapters 6, 7 and 8), serve as pillars that could maneuver future studies towards novel directions, aiding in the generation of new knowledge, and eventually, new applications that may benefit the clinic and, as such, society. In essence, expanding our current understanding on factors contributing to disease causation and progression are crucial if one wish to develop a cure for SAD. As such, it will furthermore be crucial to embed the generated data from this thesis within larger efforts that 
aim to combine multiple molecular layers, including genomics, epigenomics, transcriptomics, proteomics, metabolomics and other data modalities, in order to deepen our understanding of the mechanisms underlying SAD. It is anticipated that such endeavors will allow for better knowledge-driven development of therapeutics that could directly impact on society.

Having that said, it is important to recognize that advances in the management of other common diseases and the improvement in general health, have resulted in an increasingly elderly population so that the prevalence of SAD is expected to increase significantly in the upcoming years. Given the lack of treatments and (early) diagnostics for SAD, the disease is currently acknowledged as one of the most costly to society, with an immense socioeconomic burden. In fact, patients require the support of multiple stakeholders across the healthcare system and social care sectors, as well as from their direct family members and friends, hence affecting not only the patient, but society as a whole. This also means that the cost of care is not only captured by the healthcare systems but also in the personal sector, impacting the quality of life of patients, caregivers and patients' relatives. I therefore strongly believe that even the smallest achievements in fundamental research contribute 'to the greater good' and thus, the work presented in this thesis might hopefully contribute, in the long-term, to alleviating this social and economic burden. Of course, this will not happen in a matter of days, but the pillars that are set by fundamental research, like described in the present thesis, might inspire other researchers and, as such, contribute to the development of novel ideas that could eventually lead to revolutionary scientific breakthroughs. 

Chapter 14

Curriculum vitae 

Renzo Jan Maria Riemens was born on May $13^{\text {th }} 1992$ in Terneuzen, the Netherlands. He grew up in Clinge and went to primary school at Basisschool Sint Bernardus. Renzo subsequently moved to Hulst where he attended high school at Reynaertcollege and obtained his Atheneum degree in 2011. In the same year, he started his Bachelor in Biomedical Sciences with a specialization in Molecular life sciences (Minors: (i) Neurosciences and Toxicology, and (ii) Diseased Cells) at Maastricht University in Maastricht (Figure 1).

During his Bachelor internship, Renzo studied the effect of precedent physical activity on hippocampal volumetric changes in a mice model of social defeat under supervision of Dr. Ehsan Pishva and Prof. Dr. Daniël van den Hove at the Department of Psychiatry and Neuropsychology. He successfully obtained his Bachelor's degree in 2014, after which he entered the prestige Research Master programme in Cognitive and clinical Neuroscience with a specialization in Fundamental Neuroscience at Maastricht University. For the compulsory 9-month internship of the aforementioned programme, under the distal supervision of Prof. Dr. Daniël van den Hove, Renzo joined the research team of Dr. Raul Delgado-Morales and Prof. Dr. Manel Esteller at Institut d'Investigació Biomédica de Bellvitge (IDIBELL), Barcelona, Spain. During his Research Master internship, Renzo was assigned to a project aimed at characterizing and optimizing a neuronal in vitro model system derived from human neural stem cells.

After successfully completing his Research Master studies in 2016, Renzo started a joint-PhD programme between Maastricht University and Julius Maximilians-University, Wuerzburg, Germany, under the primary supervision of Prof. Dr. Daniël van den Hove and Prof. Dr Thomas Haaf, respectively. During the first 6 months of his PhD studies, Renzo continued his work at IDIBELL, where he took the opportunity to further develop his skills in (stem) cell culture techniques. In 2017, Renzo physically joined the laboratory of Prof. Dr. Thomas Haaf at the Institute of Human Genetics (Julius Maximilians-University), where he, as part of the European EPI-AD project (http://www. epi-ad.eu/), coordinated and managed the research dedicated to work package 3 of the grant proposal.

Within the scope of this work package, he developed a targeted DNA methylationprofiling pipeline for single laser capture microdissected cells that he applied for the study of epigenetic dysregulation in single serotonergic neurons isolated from the dorsal raphe nucleus (DRN) of Alzheimer's disease (AD) patients and healthy controls. During his time at the Julius Maximilians-University, he furthermore visited the Laboratory of Translational Neuroscience of Prof. Dr. Klaus-Peter Lesch at the department of Psychiatry, Psychosomatics and Psychotherapy, where he expanded his skills in cultivating and differentiating human induced pluripotent stem cells (iPSCs) 
towards specific neural populations.

In addition, during his time in Germany, Renzo initiated and participated in several other collaborations focused on assessing the role of epigenetic mechanisms in human disease, including AD among others, together with members of the groups of Prof. Dr. Thomas Haaf, Dr. Rohit Kulkarni (Islet Cell and Regenerative Biology, Joslin Diabetes Center, Department of Medicine, Brigham and Women's Hospital, Harvard Stem Cell Institute, Harvard Medical School, Boston), Dr. André Mégarbané (Institut Jérôme Lejeune, Paris, France) and Dr. Alfredo Ramirez (Division of Neurogenetics and Molecular Psychiatry, Department of Psychiatry and Psychotherapy, University of Cologne, Germany).

In 2019, Renzo moved back to the Department of Psychiatry and Neuropsychology at Maastricht University, where he joined the Neuroepigenetics group of Prof. Dr. Daniël van den Hove for the second half of his PhD. In the same year, he was awarded with a European Graduate School of Neuroscience (EURON) mobility grant, which he used to visit the group of Dr. Tim Vanmierlo at the Biomedical Research Institute, Faculty of Medicine and Life Sciences, University of Hasselt, Hasselt, Belgium.

During his research stay in Hasselt, Renzo developed a pipeline for targeted DNA hydroxymethylation analysis using pyrosequencing and he conducted a study within the scope of the EPI-AD project, for which he validated epigenome wide association study (EWAS) data by means of pyrosequencing in the locus coeruleus (LC) and DRN derived from AD patients and healthy controls. During the summer of 2019, in order to complement his previous research findings, Renzo undertook another research stay at the laboratory of Prof. Dr. Thomas Haaf, where he conducted a cell-specific methylation analysis in non-serotonergic cells using pyrosequencing in the DRN of AD patients and healthy controls.

In the same year, as part of the Brightlands e-Infrastructure for Neurohealth (BReIN) project at Maastricht University, Renzo was assigned as coordinating and managing researcher on work package 2 of the grant proposal, where he, under the supervision of Dr Gunter Kenis, Dr. Twan van den Beucken and Prof. Dr. Jos Kleinjans, aimed at developing iPSC-based neural models in the context of sporadic AD research.

During his PhD, Renzo has furthermore been a practical supervisor and lecturer in several Bachelor and Master courses at Maastricht University. In addition, has supervised numerous Bachelor, Master and $\mathrm{PhD}$ students during their internships and/ or in their research projects. Next to teaching others, Renzo also has been expanding his own skills over the course of his $\mathrm{PhD}$, taking workshops, courses and/or lectures in stem cell technology, bioinformatics, human neuroanatomy and neuroepigenetics to evolve his own research. 
Furthermore, Renzo has been actively organizing sessions and presenting his research at (more than) 8 national and international conferences, including the Dutch Neuroscience Meeting, The Alzheimer's Association International Conference (AAIC) and the Epigenomics of Common Diseases conference, among others. In 2020, Renzo was awarded with a Kootstra Talent Fellowship for young talented scientific researchers by the Maastricht UMC+ Research Committee (WMUMC+) to fund his ow post-doc position. Currently, Renzo is a post-doc fellow in the group of Prof. Dr. Daniël van den Hove and Dr. Gunter Kenis, further expanding his research on neuroepigenomics in AD trough the implementation of both targeted and epigenomewide (single cell) approaches, as well as iPSC-based model systems.

Dr. Renzo Jan Maria Riemens

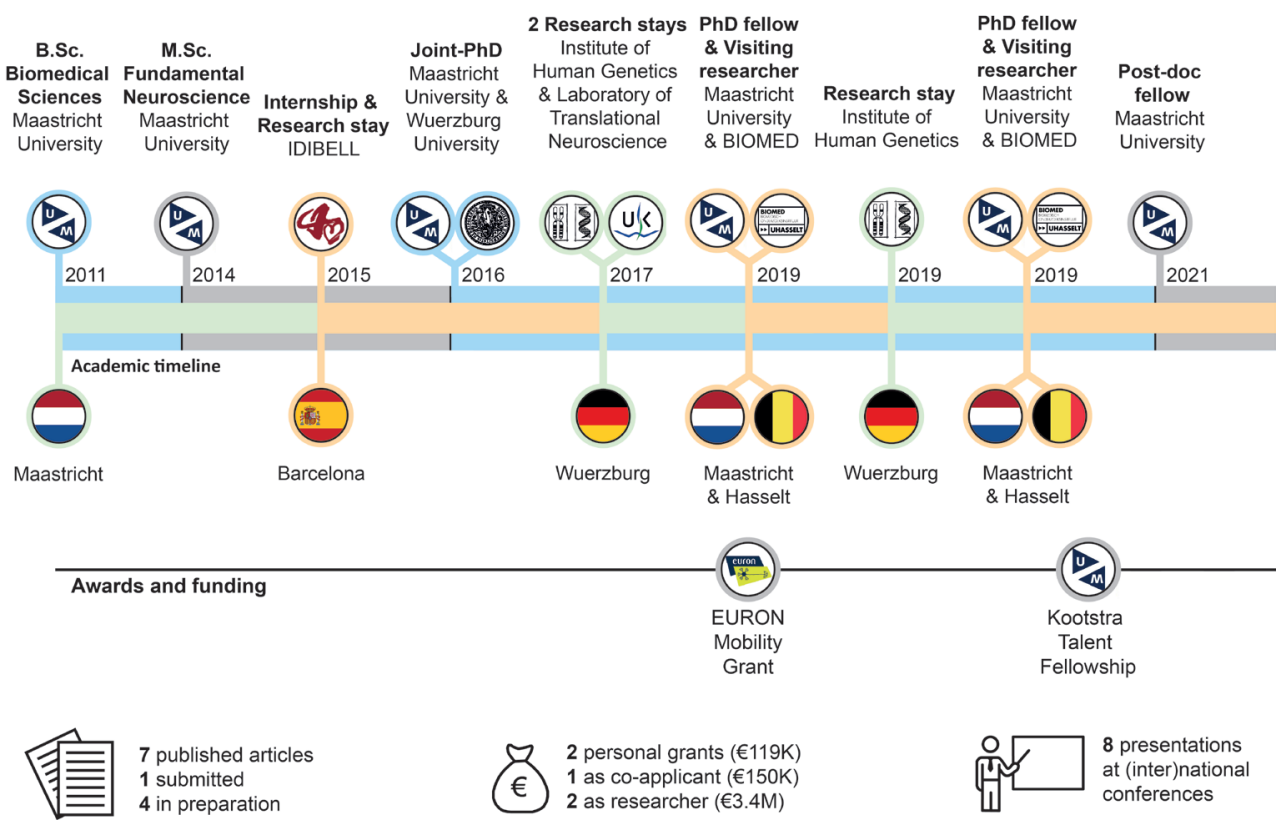

Figure 1. Overview of academic carreer (2011-2021) 

Chapter 15

List of publications 



\section{Published}

\section{Research articles}

- De Jesus DF, Orime K, Kaminska D, Kimura T, Basile G, Wang CH, Haertle L, Riemens RJM, Brown NK, Hu J, Männistö V, Silva AM, Dirice E, Tseng Y, Haaf T, Pihlajamaki J, Kulkarni RN. Parental metabolic syndrome epigenetically reprograms offspring hepatic lipid metabolism in mice. Journal of Clinical Investigation. 2020 Apr 6. pii: 127502.

- Haertle L, Müller T, Lardenoije R, Maierhofer A, Dittrich M, Riemens RJM, Stora S, Roche M, Leber M, Riedel-Heller S, Wagner M, Scherer M, Ravel A, Mircher C, Cieuta-Walti C, Durand S, van de Hove DLA, Hoffmann P, Ramirez A, Haaf T, El Hajj N, Mégarbané A. Methylomic profiling in trisomy 21 identifies cognitionand Alzheimer's disease-related dysregulation. Clinical Epigenetics. 2019 Dec 16;11(1):195.

- Lippert J, Appenzeller S, Liang R, Sbiera S, Kircher S, Altier B, Nanda I, Weigand I, Gehrig A, Steinhauer S, Riemens RJM, Muller CR, Kroiss M, Rost S, Rosenwald A, Fassnacht M, Ronchi CL. Targeted molecular analysis in adrenocortical carcinomas: a way towards improved personalized prognostication. Journal of Clinical Endocrinology \& Metabolism. 2018 Dec 1;103(12):4511-4523.

\section{Review articles}

- Riemens RJM, Kenis G, van den Beucken T. Human-induced pluripotent stem cells as a model for studying sporadic Alzheimer's disease. Neurobiology of Learning and Memory. 2020 Nov; 175:107318.

- Riemens RJM, van den Hove DLA, Esteller M, Delgado-Morales R. Directing neuronal cell fate in vitro: Achievements and challenges. Progress in Neurobiology. 2018 Apr 10. pii: S0301-0082(17)30182-X.

\section{Perspective articles}

- Riemens RJM*, van den Hove DLA*, Koulousakis P*, Pishva E. Epigenome-wide association studies in Alzheimer's Disease; Achievements and Challenges. Brain Pathology. 2020 Sep;30(5):978-983.

\section{Book chapters}

- Riemens RJM, Soares ES, Esteller M, Delgado-Morales R. Stem Cell Technology for (Epi)genetic Brain Disorders. Advances in Experimental Medicine and Biology. 2017;978:443-475. 


\section{Abstract publications}

- Riemens RJM, EPI-AD Consortium. Alzheimer's disease-associated (hydroxy)methylomic changes in the brain and blood: Genetics/omics and systems biology. Alzheimer's \& Dementia. Alzheimer's Association International Conference. 2020. 16, e042083.

\section{Submitted articles}

- Tiane A, Schepers M, Riemens RJM, Rombaut B, Vandormael P, Somers V, Prickaerts $\mathrm{J}$, Hellings $\mathrm{N}$, van den Hove DLA*, Vanmierlo $\mathrm{T}^{*}$. DNA methylation regulates the expression of the negative transcriptional regulators ID2 and ID4 during OPC differentiation. Cellular and Molecular Life Sciences. Submitted.

\section{Articles in preparation}

- Riemens RJM, Kenis G, Mastroeni D, Haaf T, van den Hove DLA. Targeted detection of unmodified cytosine, 5-methylcytosine and 5-hydroxymethylcytosine levels at single $C p G$ sites by oxidative bisulfite pyrosequencing. In preparation.

- Riemens RJM, Kenis G, Nolz J, Susano Chaves SC, Durous D, Pishva E, Mastroeni D, van Steen K, Haaf T, van den Hove DLA. Targeted methylation profiling of single laser-capture microdissected post-mortem brain cells by adapted limiting dilution bisulfite pyrosequencing (LDBSP). In preparation.

- Riemens RJM, Pishva E, latrou A, Roubroeks J, Nolz J, Lardenoije R, Ali M, Del Sol A, Delgado-Morales R, Esteller M, Kenis G, Rutten B, Lesch KP, Mill J, Mastroeni D, Ramirez A, Haaf T, Lunnon K, van den Hove DLA. Brain-region- and cell type-specific epigenetic profiling strongly implicates a role for dysregulation of TNXB and other loci in the brainstem in Alzheimer's disease. In preparation.

- Riemens RJM, Basil K, Akbulut C, Schurgers LJ, van den Hove DLA, Kleinjans $\mathrm{JC}$, van den Beucken $\mathrm{T}^{*}$, Kenis $\mathrm{G}^{*}$. Establishment and characterization of a human neuronal in vitro model system for Alzheimer's disease using induced pluripotent stem cells: An exploratory approach. In preparation. 
Chapter 16

\section{Statement of individual author contributions}





\section{Dissertation Based on Several Published Manuscripts}

Statement of individual author contributions and of legal second publication rights

Publication 1 (Chapter 2):

\section{Epigenome-wide association studies in Alzheimer's disease; Achievements and challenges}

Renzo J.M. Riemens ${ }^{1,3^{*}}$, Daniël L.A. van den Hove ${ }^{1,2 *}$, Philippos Koulousakis ${ }^{1 *}$, Ehsan Pishva ${ }^{1,4}$

1'Department of Psychiatry and Neuropsychology, School for Mental Health and Neuroscience (MHeNs), Maastricht University, Maastricht, the Netherlands.

${ }^{2}$ Division of Molecular Psychiatry, Laboratory of Translational Neuroscience, Center of Mental Health, Department of Psychiatry, University of Würzburg, Würzburg, Germany.

${ }^{3}$ Institute of Human Genetics, Julius Maximilians University, Würzburg, Germany.

${ }^{4}$ College of Medicine and Health, University of Exeter Medical School, Exeter University,

Exeter, UK.

${ }^{*}$ Authors contributed equally to this work.

Brain Pathology. 2020 Sep;30(5):978-983.

Doi: $10.1111 / \mathrm{bpa} .12880$.

\section{Participated in}

Study Design

Methods Development

Data Collection

Data Analysis and Interpretation

Manuscript Writing

Writing of Introduction

Writing of Materials \& Methods

Writing of Discussion

Writing of First Draft
Author Initials

Responsibility decreasing from left to right

N.A.

N.A.

N.A.

RJMR,

DvdH,

PK, EP 


\section{Publication 2 (Chapter 3):}

Targeted detection of unmodified cytosine, 5-methylcytosine and 5-hydroxymethylcytosine levels at single CpG sites by oxidative bisulfite pyrosequencing

Renzo J.M. Riemens ${ }^{1,2}$, Gunter Kenis ${ }^{1}$, Diego Mastroeni ${ }^{3}$, Thomas Haaf ${ }^{2}$, Daniël L.A. van den Hove ${ }^{1,4}$

${ }^{1}$ Department of Psychiatry and Neuropsychology, School for Mental Health and Neuroscience (MHeNs), Maastricht University, Maastricht, the Netherlands. ${ }^{2}$ Institute of Human Genetics, Julius Maximilians University, Wuerzburg, Germany.

${ }^{3}$ L.J. Roberts Alzheimer's Disease Center, Banner Sun Health Research Institute, Sun City, Arizona, United States of America.

${ }^{4}$ Laboratory of Translational Neuroscience, Department of Psychiatry, Psychosomatics and Psychotherapy, University of Wuerzburg, Wuerzburg, Germany.

To be submitted

\section{Participated in}

\section{Author Initials}

Responsibility decreasing from left to right

Study Design RJMR DvdH GK $\mathrm{TH}$ DM
Data Collection

Data Analysis and Interpretation

Manuscript Writing

Writing of Introduction

Writing of Materials \& Methods

Writing of Discussion

Writing of First Draft
RJMR

$\begin{array}{llll}\text { RJMR } & \text { DvdH } & \text { GK } & \text { TH } \\ \text { RJMR } & \text { DvdH } & \text { GK } & \text { TH }\end{array}$




\section{Publication 3 (Chapter 4):}

Targeted methylation profiling of single laser-capture microdissected postmortem brain cells by adapted limiting dilution bisulfite pyrosequencing (LDBSP)

Renzo J.M. Riemens ${ }^{1,2}$, Gunter Kenis ${ }^{1}$, Jennifer Nolz ${ }^{3}$, Sonia C. Susano Chaves ${ }^{1}$, Diane Duroux ${ }^{4}$, Ehsan Pishva ${ }^{1}$, Diego Mastroeni ${ }^{3}$, Kristel van Steen ${ }^{4,5}$, Thomas Haaf $^{2}$, Daniël L.A. van den Hove ${ }^{1,6}$

1Department of Psychiatry and Neuropsychology, School for Mental Health and Neuroscience (MHeNs), Maastricht University, Maastricht, the Netherlands.

${ }^{2}$ Institute of Human Genetics, Julius Maximilians University, Wuerzburg, Germany. ${ }^{3}$ Biodesign Institute, Neurodegenerative Disease Research Center, Arizona State University, Tempe, Arizona, United States of America.

${ }^{4}$ WELBIO, GIGA-R Medical Genomics - BIO3, University of Liège, Liège, Belgium; Department of Human Genetics - Systems Medicine, University of Leuven, Leuven, Belgium.

${ }^{5}$ Department of Human Genetics - Systems Medicine, University of Leuven, Leuven, Belgium.

${ }^{6}$ Laboratory of Translational Neuroscience, Department of Psychiatry, Psychosomatics and Psychotherapy, University of Wuerzburg, Wuerzburg, Germany.

To be submitted

Participated in

Study Design

Methods Development

Data Collection

Data Analysis and Interpretation RJMR

Manuscript Writing

Writing of Introduction

Writing of Materials \&

RJMR DvdH GK

Methods

Writing of Discussion

Writing of First Draft

Author Initials

RJMR

Responsibility decreasing from left to right

TH, RJMR GK JN, EP

DvdH DM

DvdH GK, KvS,

SCS DD

EP 


\section{Publication 4 (Chapter 5):}

Brain-region- and cell type-specific epigenetic profiling strongly implicates a role for dysregulation of TNXB and other loci in the brainstem in Alzheimer's disease

Renzo J.M. Riemens ${ }^{1,2 *}$, Ehsan Pishva ${ }^{1,3 *}$, Artemis latrou ${ }^{4}$, Janou Roubroeks ${ }^{3}$, Jennifer Nolz ${ }^{5}$, Roy Lardenoije ${ }^{1,6}$, Muhammad Ali ${ }^{1,7}$, Antonio Del Sol ${ }^{7}$, Raul DelgadoMorales $^{1,8}$, Manel Esteller Badosa ${ }^{9}$, Gunter Kenis ${ }^{1}$, Bart Rutten ${ }^{1}$, Klaus-Peter Lesch $^{10}$, Jonathan Mill ${ }^{3}$, Diego Mastroeni ${ }^{1,5,11}$, Alfredo Ramirez ${ }^{12}$, Thomas Haaf T2 Katie Lunnon ${ }^{3 \dagger}$, Daniël L.A. van den Hove ${ }^{1,10 \dagger}$

${ }^{1}$ Department of Psychiatry and Neuropsychology, School for Mental Health and Neuroscience (MHeNs), Maastricht University, Maastricht, the Netherlands.

${ }^{2}$ Institute of Human Genetics, Julius Maximilians University, Wuerzburg, Germany.

${ }^{3}$ University of Exeter Medical School, University of Exeter, Exeter, United Kingdom.

${ }^{4}$ Rush Alzheimer's Neurodisease Center, Rush University Medical Center, Chicago, Illinois, United States of America.

${ }^{5}$ Biodesign Institute, Neurodegenerative Disease Research Center, Arizona State University, Tempe, Arizona, United States of America.

${ }^{6}$ Department of Psychiatry and Psychotherapy, University Medical Center Göttingen, Göttingen, Germany.

${ }^{7}$ Luxembourg Centre for Systems Biomedicine (LCSB), University of Luxembourg, Esch-surAlzette, Luxembourg.

${ }^{8}$ Cancer Epigenetics and Biology Program (PEBC), Bellvitge Biomedical Research Institute IDIBELL, L'Hospitalet del Llobregat, Barcelona, Catalonia, Spain.

9 Josep Carreras Leukaemia Research Institute (IJC), Badalona, Barcelona, Spain; Centro de Investigacion Biomedica en Red Cancer (CIBERONC), Madrid, Spain; Institucio Catalana de Recerca I Estudis Avançats (ICREA), Barcelona, Catalonia, Spain; Physiological Sciences Department, School of Medicine and Health Sciences, University of Barcelona (UB), Barcelona, Catalonia, Spain.

${ }^{10}$ Laboratory of Translational Neuroscience, Department of Psychiatry, Psychosomatics and Psychotherapy, University of Wuerzburg, Wuerzburg, Germany.

${ }^{11}$ L.J. Roberts Center for Alzheimer's Research Banner Sun Health Research Institute, Sun City, Arizona, United States of America.

${ }^{12}$ Division for Neurogenetics and Molecular Psychiatry, Department of Psychiatry and Psychotherapy, Medical Faculty, University of Cologne, Cologne, Germany; Department for Neurodegenerative Diseases and Geriatric Psychiatry, University of Bonn, Bonn, Germany.

${ }^{*}$ Authors contributed equally to this work

${ }^{\dagger}$ Authors contributed equally to this work

To be submitted 
Participated in

Author Initials

Responsibility decreasing from left to right

Study Design

Methods Development

Data Collection

Data Analysis and Interpretation

Manuscript Writing

Writing of Introduction

Writing of Materials \& Methods

Writing of Discussion

Writing of First Draft

DvdH, KL TH RJMR, Remaining

EP authors

RJMR, EP

RJMR, EP DvdH KL Remaining authors

RJMR, EP DvdH KL Remaining authors 


\section{Publication 5 (Chapter 6):}

\section{Human-induced pluripotent stem cells as a model for studying sporadic}

Alzheimer's disease

\section{Renzo J.M. Riemens ${ }^{1,2}$, Gunter Kenis ${ }^{1}$, Twan van den Beucken ${ }^{3}$}

${ }^{1}$ Department of Psychiatry \& Neuropsychology, Graduate School MHeNS (School for Mental Health and Neuroscience), allocated with the Faculty Health Medicine and Life Sciences of Maastricht University, Maastricht, the Netherlands.

2Institute of Human Genetics, Julius Maximilian University, Wuerzburg, Germany.

${ }^{3}$ Department of Toxicogenomics, Graduate School GROW (Research School for Oncology and Developmental Biology), allocated with the Faculty Health Medicine and Life Sciences of Maastricht University, Maastricht, the Netherlands.

Neurobiology of Learning and Memory. 2020 Sep 22;107318.

Doi: 10.1016/j.nlm.2020.107318.

\section{Participated in}

Study Design

Methods Development

Data Collection

Data Analysis and Interpretation

Manuscript Writing

Writing of Introduction

Writing of Materials \& Methods

Writing of Discussion

Writing of First Draft

\section{Author Initials}

Responsibility decreasing from left to right

N.A.

N.A.

N.A.

RJMR, TvdB GK 


\section{Publication 6 (Chapter 7):}

\section{Directing neuronal cell fate in vitro: achievements and challenges}

Renzo J.M. Riemens ${ }^{1,2,3}$, Daniël L.A. van den Hove ${ }^{3,4}$ Manel Esteller ${ }^{1,5,6}$, Raul Delgado-Morales ${ }^{1,3}$

${ }^{1}$ Cancer Epigenetics and Biology Program (PEBC), Bellvitge Biomedical Research Institute - IDIBELL, L'Hospitalet del Llobregat, Barcelona, Catalonia, Spain.

${ }^{2}$ Institute of Human Genetics, Julius Maximilians University, Wuerzburg, Germany. ${ }^{3}$ Department of Psychiatry and Neuropsychology, School for Mental Health and Neuroscience (MHeNs), Maastricht University, Maastricht, the Netherlands. ${ }^{4}$ Laboratory of Translational Neuroscience, Department of Psychiatry, Psychosomatics and Psychotherapy, University of Wuerzburg, Wuerzburg, Germany.

${ }^{5}$ Department of Physiological Sciences II, School of Medicine, University of Barcelona, Barcelona, Catalonia, Spain.

${ }^{6}$ Institucio Catalana de Recerca i Estudis Avançats (ICREA), Barcelona, Catalonia, Spain.

Prog Neurobiol. 2018 Sep;168:42-68.

Doi: 10.1016/j.pneurobio.2018.04.003.

\section{Participated in}

\section{Author Initials}

Responsibility decreasing from left to right

Study Design

N.A.

Methods Development

Data Collection

N.A.

Data Analysis and Interpretation

N.A.

Manuscript Writing

Writing of Introduction

RJMR RDM DvdH ME

Writing of Materials \& Methods

Writing of Discussion

Writing of First Draft 


\section{Publication 7 (Chapter 8):}

Establishment and characterization of a human neuronal in vitro model system for Alzheimer's disease using induced pluripotent stem cells: An exploratory approach

Renzo J.M. Riemens ${ }^{1,2}$, Katherine Basil ${ }^{1}$, Cengiz Akbulut ${ }^{3}$, Leon J. Schurgers ${ }^{3}$, Daniël L.A. van den Hove ${ }^{1,4}$, Jos C. Kleinjans ${ }^{5}$, Twan van den Beucken ${ }^{5+}$, Gunter Kenis $^{1+}$

1Department of Psychiatry and Neuropsychology, School for Mental Health and Neuroscience (MHeNs), Maastricht University, Maastricht, the Netherlands. ${ }^{2}$ Institute of Human Genetics, Julius Maximilians University, Wuerzburg, Germany. ${ }^{3}$ Department of Biochemistry, Cardiovascular Research Institute Maastricht, Maastricht University, Maastricht, the Netherlands.

${ }^{4}$ Laboratory of Translational Neuroscience, Department of Psychiatry, Psychosomatics and Psychotherapy, University of Wuerzburg, Wuerzburg, Germany.

${ }^{5}$ Department of Toxicogenomics, GROW School for Oncology and Developmental Biology, Maastricht University, the Netherlands.

† Authors contributed equally to this work

To be submitted

\section{Participated in}

Study Design

Methods Development

Data Collection

Data Analysis and Interpretation

Manuscript Writing

Writing of Introduction

Writing of Materials \& Methods

Writing of Discussion

Writing of First Draft

\section{Author Initials}

Responsibility decreasing from left to right

RJMR, KB, GK TvdB Remaining

CA

authors

RJMR

RJMR GK TvdB

RJMR GK TvdB Remaining

authors 
The doctoral researcher confirms that she/he has obtained permission from both the publishers and the co-authors for legal second publication.

The doctoral researcher and the primary supervisor confirm the correctness of the above-mentioned assessment.

Doctoral Researcher's Name

Primary Supervisor's Name
Place, Date

Place, Date
Signature

Signature 


\section{Dissertation Based on Several Published Manuscripts}

Statement of individual author contributions to figures/tables/chapters included in the manuscripts

Publication 1 (Chapter 2):

\section{Epigenome-wide association studies in Alzheimer's disease; Achievements and challenges}

Renzo J.M. Riemens ${ }^{1,3 *}$, Daniël L.A. van den Hove ${ }^{1,2 *}$, Philippos Koulousakis ${ }^{1 *}$, Ehsan Pishva ${ }^{1,4}$

${ }^{1}$ Department of Psychiatry and Neuropsychology, School for Mental Health and Neuroscience (MHeNs), Maastricht University, Maastricht, the Netherlands.

${ }^{2}$ Division of Molecular Psychiatry, Laboratory of Translational Neuroscience, Center of Mental Health, Department of Psychiatry, University of Würzburg, Würzburg, Germany. ${ }^{3}$ Institute of Human Genetics, Julius Maximilians University, Würzburg, Germany.

${ }^{4}$ College of Medicine and Health, University of Exeter Medical School, Exeter University, Exeter, UK.

${ }^{*}$ Authors contributed equally to this work.

Brain Pathology. 2020 Sep;30(5):978-983.

Doi: 10.1111/bpa.12880.

$\begin{array}{ll}\text { Table(s) } & \begin{array}{l}\text { Author Initials } \\ \text { Responsibility decreasing from left to right }\end{array}\end{array}$

\begin{tabular}{llll}
\hline Table 1 & PK & RJMR & DvdH
\end{tabular}




\section{Publication 2 (Chapter 3):}

Targeted detection of unmodified cytosine, 5-methylcytosine and 5-hydroxymethylcytosine levels at single CpG sites by oxidative bisulfite pyrosequencing

Renzo J.M. Riemens ${ }^{1,2}$, Gunter Kenis ${ }^{1}$, Diego Mastroeni ${ }^{3}$, Thomas Haaf ${ }^{2}$, Daniël L.A. van den Hove ${ }^{1,4}$

1'Department of Psychiatry and Neuropsychology, School for Mental Health and Neuroscience (MHeNs), Maastricht University, Maastricht, the Netherlands.

${ }^{2}$ Institute of Human Genetics, Julius Maximilians University, Wuerzburg, Germany.

${ }^{3}$ L.J. Roberts Alzheimer's Disease Center, Banner Sun Health Research Institute, Sun City, Arizona, United States of America.

${ }^{4}$ Laboratory of Translational Neuroscience, Department of Psychiatry, Psychosomatics and Psychotherapy, University of Wuerzburg, Wuerzburg, Germany.

To be submitted

\section{Figure(s) Author Initials}

Responsibility decreasing from left to right

\begin{tabular}{llll}
\hline Figure 1 & RJMR & DvdH & GK \\
Figure 2 & RJMR & DvdH & GK \\
Figure 3 & RJMR & DvdH & GK \\
\hline Table(s) & \multicolumn{2}{l}{ Author Initials } & \\
& Responsibility decreasing from left to right \\
\hline Table 1 & RJMR & DvdH & GK \\
Table 2 & RJMR & DvdH & GK \\
Table 3 & RJMR & DvdH & GK \\
Table 4 & RJMR & DvdH & GK \\
Table 5 & RJMR & DvdH & GK \\
Supplementary Table 1 & RJMR & DvdH & GK \\
Supplementary Table 2 & RJMR & DvdH & GK \\
Supplementary Table 3 & RJMR & DvdH & GK \\
\hline
\end{tabular}




\section{Publication 3 (Chapter 4):}

Targeted methylation profiling of single laser-capture microdissected postmortem brain cells by adapted limiting dilution bisulfite pyrosequencing (LDBSP)

Renzo J.M. Riemens ${ }^{1,2}$, Gunter Kenis ${ }^{1}$, Jennifer Nolz ${ }^{3}$, Sonia C. Susano Chaves ${ }^{1}$, Diane Duroux ${ }^{4}$, Ehsan Pishva ${ }^{1}$, Diego Mastroeni ${ }^{3}$, Kristel van Steen ${ }^{4,5}$, Thomas Haaf', Daniël L.A. van den Hove ${ }^{1,6}$

${ }^{1}$ Department of Psychiatry and Neuropsychology, School for Mental Health and Neuroscience (MHeNs), Maastricht University, Maastricht, the Netherlands.

${ }^{2}$ Institute of Human Genetics, Julius Maximilians University, Wuerzburg, Germany.

${ }^{3}$ Biodesign Institute, Neurodegenerative Disease Research Center, Arizona State University, Tempe, Arizona, United States of America.

${ }^{4}$ WELBIO, GIGA-R Medical Genomics - BIO3, University of Liège, Liège, Belgium;

Department of Human Genetics - Systems Medicine, University of Leuven, Leuven,

Belgium.

${ }^{5}$ Department of Human Genetics - Systems Medicine, University of Leuven, Leuven,

Belgium.

${ }^{6}$ Laboratory of Translational Neuroscience, Department of Psychiatry, Psychosomatics and Psychotherapy, University of Wuerzburg, Wuerzburg, Germany.

To be submitted

Figure(s) Author Initials

Responsibility decreasing from left to right

\begin{tabular}{llll}
\hline Figure 1 & RJMR & DvdH & GK \\
Figure 2 & RJMR & DvdH & GK \\
\hline
\end{tabular}

\begin{tabular}{ll}
\hline Table(s) & Author Initials \\
& Responsibility dect
\end{tabular}

\begin{tabular}{llll}
\hline Table 1 & RJMR & DvdH & GK
\end{tabular}

$\begin{array}{lll}\text { Table } 2 \text { RJMR } & \text { DvdH }\end{array}$

Supplementary RJMR DvdH GK

Supplementary RJMR DvdH GK 


\section{Publication 4 (Chapter 5):}

\section{Brain-region- and cell type-specific epigenetic profiling strongly implicates a role for dysregulation of TNXB and other loci in the brainstem in Alzheimer's disease}

Renzo J.M. Riemens ${ }^{1,2 *}$, Ehsan Pishva ${ }^{1,3 *}$, Artemis latrou ${ }^{4}$, Janou Roubroeks ${ }^{3}$, Jennifer Nolz ${ }^{5}$, Roy Lardenoije ${ }^{1,6}$, Muhammad Alii, ${ }^{1,7}$, Antonio Del Sol ${ }^{7}$, Raul Delgado-Morales ${ }^{1,8}$, Manel Esteller Badosa ${ }^{9}$, Gunter Kenis ${ }^{1}$, Bart Rutten ${ }^{1}$, Klaus-Peter Lesch $^{10}$, Jonathan Mill ${ }^{3}$, Diego Mastroeni ${ }^{1,5,11}$, Alfredo Ramirez ${ }^{12}$, Thomas Haaf $\mathrm{T}^{2}$, Katie Lunnon ${ }^{3 \dagger}$, Daniël L.A. van den Hove ${ }^{1,10 \dagger}$

1Department of Psychiatry and Neuropsychology, School for Mental Health and Neuroscience (MHeNs), Maastricht University, Maastricht, the Netherlands.

${ }^{2}$ Institute of Human Genetics, Julius Maximilians University, Wuerzburg, Germany.

${ }^{3}$ University of Exeter Medical School, University of Exeter, Exeter, United Kingdom.

${ }^{4}$ Rush Alzheimer's Neurodisease Center, Rush University Medical Center, Chicago, Illinois, United States of America.

${ }^{5}$ Biodesign Institute, Neurodegenerative Disease Research Center, Arizona State University, Tempe, Arizona, United States of America.

${ }^{6}$ Department of Psychiatry and Psychotherapy, University Medical Center Göttingen, Göttingen, Germany.

${ }^{7}$ Luxembourg Centre for Systems Biomedicine (LCSB), University of Luxembourg, Eschsur-Alzette, Luxembourg.

${ }^{8}$ Cancer Epigenetics and Biology Program (PEBC), Bellvitge Biomedical Research Institute - IDIBELL, L'Hospitalet del Llobregat, Barcelona, Catalonia, Spain.

${ }^{9}$ Josep Carreras Leukaemia Research Institute (IJC), Badalona, Barcelona, Spain; Centro de Investigacion Biomedica en Red Cancer (CIBERONC), Madrid, Spain; Institucio Catalana de Recerca I Estudis Avançats (ICREA), Barcelona, Catalonia, Spain; Physiological Sciences Department, School of Medicine and Health Sciences, University of Barcelona (UB), Barcelona, Catalonia, Spain.

${ }^{10}$ Laboratory of Translational Neuroscience, Department of Psychiatry, Psychosomatics and Psychotherapy, University of Wuerzburg, Wuerzburg, Germany.

${ }^{11}$ L.J. Roberts Center for Alzheimer's Research Banner Sun Health Research Institute, Sun City, Arizona, United States of America.

${ }^{12}$ Division for Neurogenetics and Molecular Psychiatry, Department of Psychiatry and Psychotherapy, Medical Faculty, University of Cologne, Cologne, Germany; Department for Neurodegenerative Diseases and Geriatric Psychiatry, University of Bonn, Bonn, Germany.

* Authors contributed equally to this work

+ Authors contributed equally to this work

To be submitted 


\begin{tabular}{|c|c|c|}
\hline \multirow{2}{*}{$\begin{array}{l}\text { Figure(s) } \\
\text { Figure } 1\end{array}$} & \multicolumn{2}{|c|}{$\begin{array}{l}\text { Author Initials } \\
\text { Responsibility decreasing from left to right }\end{array}$} \\
\hline & RJMR, EP & $\mathrm{DvdH}$ \\
\hline Figure 2 & RJMR & DvdH \\
\hline Supplementary Figure 1 & RJMR, EP & DvdH \\
\hline Table(s) & \multicolumn{2}{|c|}{$\begin{array}{l}\text { Author Initials } \\
\text { Responsibility decreasing from left to right }\end{array}$} \\
\hline Table 1 & RJMR, EP & $\mathrm{DvdH}$ \\
\hline Table 2 & RJMR, EP & DvdH \\
\hline Table 3 & RJMR, EP & DvdH \\
\hline Table 4 & RJMR, EP & DvdH \\
\hline Table 5 & RJMR, EP & $\mathrm{DvdH}$ \\
\hline Supplementary Table 1 & RJMR, EP & DvdH \\
\hline Supplementary Table 2 & RJMR, EP & $\mathrm{DvdH}$ \\
\hline Supplementary Table 3 & RJMR, EP & DvdH \\
\hline Supplementary Table 4 & RJMR, EP & DvdH \\
\hline Supplementary Table 5 & RJMR, EP & DvdH \\
\hline Supplementary Table 6 & RJMR, EP & DvdH \\
\hline Supplementary Table 7 & RJMR, EP & DvdH \\
\hline Supplementary Table 8 & RJMR, EP & DvdH \\
\hline Supplementary Table 9 & RJMR, EP & DvdH \\
\hline Supplementary Table 10 & RJMR, EP & DvdH \\
\hline Supplementary Table 11 & RJMR, EP & DvdH \\
\hline Supplementary Table 12 & RJMR, EP & $\mathrm{DvdH}$ \\
\hline Supplementary Table 13 & RJMR & DvdH \\
\hline Supplementary Table 14 & RJMR & DvdH \\
\hline
\end{tabular}




\section{Publication 5 (Chapter 6):}

Human-induced pluripotent stem cells as a model for studying sporadic Alzheimer's disease

Renzo J.M. Riemens ${ }^{1,2}$, Gunter Kenis ${ }^{1}$, Twan van den Beucken ${ }^{3}$

1'Department of Psychiatry \& Neuropsychology, Graduate School MHeNS (School for Mental Health and Neuroscience), allocated with the Faculty Health Medicine and Life Sciences of Maastricht University, Maastricht, the Netherlands.

${ }^{2}$ Institute of Human Genetics, Julius Maximilian University, Wuerzburg, Germany. ${ }^{3}$ Department of Toxicogenomics, Graduate School GROW (Research School for Oncology and Developmental Biology), allocated with the Faculty Health Medicine and Life Sciences of Maastricht University, Maastricht, the Netherlands.

Neurobiology of Learning and Memory. 2020 Sep 22;107318.

Doi: 10.1016/j.nlm.2020.107318.

\begin{tabular}{ll}
\hline Figure(s) & $\begin{array}{l}\text { Author Initials } \\
\text { Responsibility decreasing from left to right }\end{array}$ \\
\hline Figure 1 & TvdB \\
\hline Table(s) & $\begin{array}{l}\text { Author Initials } \\
\text { Responsibility decreasing from left to right }\end{array}$ \\
\hline Table 1 & RJMR, TvdB \\
\hline
\end{tabular}




\section{Publication 6 (Chapter 7):}

\section{Directing neuronal cell fate in vitro: achievements and challenges}

Renzo J.M. Riemens ${ }^{1,2,3}$, Daniël L.A. van den Hove ${ }^{3,4}$ Manel Esteller ${ }^{1,5,6}$, Raul Delgado-Morales ${ }^{1,3}$

${ }^{1}$ Cancer Epigenetics and Biology Program (PEBC), Bellvitge Biomedical Research Institute - IDIBELL, L'Hospitalet del Llobregat, Barcelona, Catalonia, Spain.

${ }^{2}$ Institute of Human Genetics, Julius Maximilians University, Wuerzburg, Germany.

${ }^{3}$ Department of Psychiatry and Neuropsychology, School for Mental Health and

Neuroscience (MHeNs), Maastricht University, Maastricht, the Netherlands.

${ }^{4}$ Laboratory of Translational Neuroscience, Department of Psychiatry, Psychosomatics and Psychotherapy, University of Wuerzburg, Wuerzburg, Germany.

${ }^{5}$ Department of Physiological Sciences II, School of Medicine, University of Barcelona, Barcelona, Catalonia, Spain.

${ }^{6}$ Institucio Catalana de Recerca i Estudis Avançats (ICREA), Barcelona, Catalonia, Spain.

Prog Neurobiol. 2018 Sep;168:42-68.

Doi: 10.1016/j.pneurobio.2018.04.003.

\begin{tabular}{lll}
\hline Figure(s) & \multicolumn{2}{l}{ Author Initials } \\
& Responsibility decreasing from left to right & \\
\hline Figure 1 & RJMR & DM, DvdH \\
Figure 2 & RJMR & DM, DvdH \\
Figure 3 & RJMR & DM, DvdH \\
Figure 4 & RJMR & DM, DvdH \\
\hline Table(s) & Author Initials & \\
& Responsibility decreasing from left to right \\
\hline Table 1 & RJMR & DM, DvdH \\
Table 2 & RJMR & DM, DvdH \\
Table 3 & RJMR & DM, DvdH \\
Supplementary Table 1 & RJMR & DM, DvdH \\
Supplementary Table 2 & RJMR & DM, DvdH \\
Supplementary Table 3 & RJMR & DM, DvdH \\
\hline
\end{tabular}




\section{Publication 7 (Chapter 8):}

Establishment and characterization of a human neuronal in vitro model system for Alzheimer's disease using induced pluripotent stem cells: An exploratory approach

Renzo J.M. Riemens ${ }^{1,2}$, Katherine Basil ${ }^{1}$, Cengiz Akbulut ${ }^{3}$, Leon J. Schurgers ${ }^{3}$, Daniël L.A. van den Hove ${ }^{1,4}$, Jos C. Kleinjans ${ }^{5}$, Twan van den Beucken ${ }^{5+}$, Gunter Kenis ${ }^{1 \dagger}$

1'Department of Psychiatry and Neuropsychology, School for Mental Health and Neuroscience (MHeNs), Maastricht University, Maastricht, the Netherlands.

${ }^{2}$ Institute of Human Genetics, Julius Maximilians University, Wuerzburg, Germany.

${ }^{3}$ Department of Biochemistry, Cardiovascular Research Institute Maastricht, Maastricht University, Maastricht, the Netherlands.

${ }^{4}$ Laboratory of Translational Neuroscience, Department of Psychiatry, Psychosomatics and Psychotherapy, University of Wuerzburg, Wuerzburg, Germany.

${ }^{5}$ Department of Toxicogenomics, GROW School for Oncology and Developmental Biology, Maastricht University, the Netherlands.

† Authors contributed equally to this work

To be submitted

Figure(s) Author Initials

Responsibility decreasing from left to right

\begin{tabular}{lll}
\hline Figure 1 & RJMR
\end{tabular}

$\begin{array}{lll}\text { Figure } 2 \text { RJMR GK } & \text { G }\end{array}$

$\begin{array}{lll}\text { Figure } 3 & \text { RJMR }\end{array}$

$\begin{array}{lll}\text { Figure } 4 & \text { RJMR }\end{array}$

$\begin{array}{lll}\text { Figure } 5 & \text { RJMR } & \text { GK }\end{array}$

Table(s) Author Initials

Responsibility decreasing from left to right

\begin{tabular}{lll}
\hline Supplementary Table 1 & RJMR & GK \\
\hline
\end{tabular}

I also confirm my primary supervisor's acceptance.

Doctoral Researcher's Name

$$
\text { Place, Date }
$$

Signature 

Chapter 17

Affidavit 



\section{Affidavit}

I hereby confirm that my thesis entitled "Neuroepigenomics in Alzheimer's disease: The single cell ADds" is the result of my own work. I did not receive any help or support from commercial consultants. All sources and/or materials applied are listed and specified in the thesis.

Furthermore, I confirm that this thesis has not yet been submitted as part of another examination process neither in identical nor in similar form.

Place, Date

Signature

\section{Beëdigde verklaring}

Hierbij verklaar ik dat mijn proefschrift getiteld "Neuroepigenomics in Alzheimer's disease: The single cell $A D d s$ " het resultaat is van mijn eigen werk. Ik heb geen hulp of ondersteuning gekregen van commerciële consulenten. Alle toegepaste bronnen en/of materialen zijn beschreven en gespecificeerd in het proefschrift.

Verder bevestig ik dat dit proefschrift nog niet is ingediend als onderdeel van een ander examenproces, noch in identieke noch in vergelijkbare vorm.

Plaats, Datum

Handtekening

\section{Eidestattliche Erklärung}

Hiermit erkläre ich an Eides statt, die Dissertation "Neuroepigenomics in Alzheimer's disease: The single cell ADds" eigneständig, das heißt insbesondere selbständig und ohne Hilfe einse kommerziellen Promotionsberaters, angefertigt und keine anderen als die von mir angegebenen Quellen un Hilfsmittel verwended zu haben.

Ich erkläre außerdem, dass die Dissertation weder in gleicher noch in ähnlicher Form bereits in einem andere Prüfungsverfahren vorgelegen hat. 

Chapter 18

Acknowledgements 

I would not be where I am today without the help of many - both during my time as a PhD student and before that. Looking back, I have been very fortunate to always be surrounded by and to come across with amazing people, who all have been playing their parts in me becoming the researcher, and perhaps even more important, the person that I am today. Therefore, for anyone, who stood next to me and/or who contributed to my work in any form or by any means (even when not explicitly mentioned in this section), I want you to know that I am extremely grateful, and I want to thank you for your support, your encouragement and for the joyful moments or pain that we shared together during these years.

Having that said, I would like to take the opportunity to thank and to express my sincerest gratitude to a number of people in particular. First and foremost, I would like to thank my promotion team, who made it possible for me to embark on this joint-PhD adventure and who gave me the liberty to develop myself as an academic researcher. It has been a real pleasure and a truly enriching experience to conduct this joint-PhD programme between Maastricht University and the JuliusMaximilians-University.

Daniël, from the very first moment we met during my Bachelor up until the very end of my PhD you have always encouraged me to get the best out of myself and to "go that extra (s)mile"! Honestly, you have been a true inspiration and my role model all along. Your "enthusiasm for neuroscience is contagious" and a strong drive for me to try to "be a carrier". It cannot be stressed enough that you are the perfect example of a supervisor one could wish for, and that the way you treat the people under your supervision should become the golden standard for every other staff member within (and even outside of) academia. I would like to thank you for believing in me from the very beginning, for trusting in my abilities, for guiding me, for always being there when necessary, for providing a listening ear, for being honest, for being critical, for taking care, for being patient, for convincing me that "things well be all right" and for sharing your thoughts on any matter. I am more than grateful for all the time and effort you have invested in me. Doing a PhD under your wings has been truly EPIC and I am really looking forward to my time as a post-doc in your NeuroEPICgenetics group! After all these years, I think we can agree: "science is fun" indeed!

Bart, thank you for believing in me and supporting me from the start, even before I embarked on this joint-PhD adventure. My time abroad did not allow us to meet on a frequent basis during my time as $\mathrm{PhD}$ student, but when we did, you always had some valuable input for my research. I think it is safe to say that without your enthusiasm and faith in me I would not be where I am today, for which I want to express my sincerest gratitude. 
Gunter, you are really one of the most caring, knowledgeable and intelligent people that I know. I really look up to you and I have learned so much from you over the years! Thank you for supervising me, sharing your expertise with me, for supporting me and for having faith in me. Your contributions and insights into my research have been truly invaluable. If it had not been for you, Daniël and I would still be trying to figure out how to estimate alleles in a single reaction and my $\mathrm{PhD}$ would have never come to an end. Our weekly meetings, which were not only used to catch up on work, but also on life, have been strongly motivating me to keep on pushing my boundaries week after week.

Professor Haaf, my sincerest appreciation for guiding me and for providing me with such freedom in your laboratory. Your dedication to science and hard-working attitude are truly inspiring. I will always remember your visits in my office that started every time with the question "Und?" and ended with the phrase "Na ja, gut!", which reflected your generous interest in my developing projects and your cautious optimism for the next milestone(s) to be achieved. Aside from the brief updates from my side, our chats in-between these phrases were filled with in-depth discussions on both science and on how to developing a successful career, which really have been eye-opening - so thank you for that!

Professor Förster, I still vividly remember the day I came to your office to ask whether you would be willing to join my supervisor committee in Wuerzburg. After our inspiring chat on doing translational research and the role of epigenetics in human brain disorders, you answered this question with a yes without hesitation, for which I am truly grateful. I specifically want to thank you for the time that you have invested in me and for joining me in this journey as a PhD student.

Professor Lesch, It has been a great honor and pleasure to be able to work with you and your group. The experience in cultivating and working with human stem cells that I gained during the time in your laboratory has significantly contributed to my skillset, from which I still highly benefit today. It has been a truly enriching experience for which I want to express my sincerest gratitude.

A special mention and thank you also goes out to the (other) members of my assessment committee.

Jos, my whole journey as a PhD student would not have been so successful if it had not been for you and Daniël to accept me in the Fundamental Neuroscience (FN) programme. I strongly believe that being part of $\mathrm{FN}$ has significantly contributed to my professional and personal development, both during my time as a research master student, as well as during my $\mathrm{PhD}$. I would therefore like to thank you for encouraging 
me from the start and teaching me along the way. It is also a great pleasure to be able to end my PhD together with you and to have you as the chair of my assessment committee. I genuinely hope I can keep on surprising you in the future!

Tim, I have gotten to know you as an amazing scientist with a great sense of dry humor to match. Your elaborated knowledge on a wide variety of different laboratory techniques is truly inspiring, and your witty, unexpected jokes during our labmeetings are hilarious. You definitely know how to keep the perfect balance between a serious working attitude and having fun at work. Thank you for all your invaluable advice on my research and for assisting me with my Pyrosequencing experiments in Hasselt. To be able to work with you and your group in BIOMED has been very rewarding and a great pleasure!

I am equally thankful to Prof. Dr. Paul Lucassen, Prof. Dr. Geraldine ZimmerBensch and Dr. Angelika Schmitt-Böhrer for being on my assessment committee. Thank you for taking the time to read and to assess my thesis.

I would also like to express my special thanks to Prof. Dr. Manel Esteller. Thank you for granting me the opportunity to work in your laboratory. The expertise that I acquired during my time in IDIBELL has contributed significantly to develop myself as a researcher and allowed me to excel during my time as a PhD student, for which I am really grateful.

A warm and special thank you also goes to Raul. It was a great pleasure to be able to get to know you and to be able to work with you! You were not only a great supervisor during my time in IDIBELL, but also an amazing friend. Really, you are the best! I hope my questions on laboratory- and science-related matters are not haunting you in your dreams up until this day. Thank you for your patience, for guiding me, for teaching me how to become more confident with my work and for showing me how to be more independent in the laboratory. We will meet again! I am sure!

I would also like to express my sincerest gratitude to Jos, Twan, Marcel and everybody else within BReIN or at the Department of Toxicogenomics at Maastricht University that has (in)directly contributed to my work. Thank you for involving me and granting me the opportunity to work together with you on this project.

I am also extremely grateful to Gabriele and Nicole for organizing all the legal matters in relation to this joint-PhD degree, as well as to Rachelle for organizing all the administration of my PhD thesis in Maastricht. You did an amazing job!

Then I want to thank my paranymphs for all their support over the years and for their help with my thesis defense. 
Dean, we have been partners in crime (both literally and as a figure of speech), from the early days at the university up until today, where we both are about to obtain our $\mathrm{PhD}$ degrees soon. I would like to thank you for being there as a great friend and for sharing all these memorable moments together with me during these past years, both inside and outside of academia. From brushing our teeth with coffee early in the morning, just before rush-hour was about to start to the university, to our "hotbox sessions" until late at night, where we played loud electronic music in the university library (read worked our asses off). From changing lightbulbs with lemons during our evenings going to the "hippos", to drinking beers at the Ramblas, to performing as DJs in Wuerzburg, and so on... It has been absolutely amazing and the "schweinerei" definitely kept me going even during the toughest moments where there was little time to sleep and only time to work.

Philippos, aside from being a Greek, a German and a Dutch friend, a college and my office-mate, you have also fulfilled the role as my psychologist during the last (crucial) phase of my PhD. I would therefore like to thank you for simply being there for me, for the free therapy sessions, and for providing me with your (well-appreciated) constructive criticism. Yes, when reading this you can finally say: "Er hat schließlich seine Doktorarbeit eingereicht. Wer hätte das erwartet?". I am very happy we will remain colleagues for a while, but be aware that everything you say can be used against you and that you still have to defend your PhD thesis one day. Of note, your amazing dance moves, your passion for dressing up as a medieval soldier and your love for watching gaming streams (even during working hours) have not gone unnoticed. Overall, it has been a blast of Oxytocin getting to know you and irrespective of the results that you will require in the remaining years of your $\mathrm{PhD}, \mathrm{I}$ am convinced that our friendship has reduced my risk of developing Alzheimer's disease in the future.

A massive thank you also goes to the Neuroepigenetics group at the Department of Psychiatry and Neuropsychology at Maastricht University. Thank you for sharing all your expertise with me, for your support in the laboratory, for your (career) advice, for the pizza meetings (read journal clubs), for the coffee-moments, for all the enjoyable (online) gatherings outside of the laboratory and simply for the lovely chats in the hallway.

Ehsan, a special thank you to you! Thanks for guiding me and working together with from the start of my Bachelor internship up until the very end of my PhD, for your straight-to-the-point advice and for always being there in case I was in desperate need for bioinformatics expertise. You really can breakdown bioinformatics to a level where even I understand it. A wise man once told me (read Gunter): "You need to be an expert in order to be able to explain complicated things in a very simple way". This definitely also applies to you and it is something I truly admire. 
Chris, "lunch?". As you can see, I kept my promise to give you your own paragraph in my acknowledgements section. Thank you for being such a caring, funny and lovely office-mate, as well as for being a true friend and a "Pyro Ranger". Your personality, your sense of humor and the weird facts that you bring to the office every day, significantly $(p<0.001)$ contribute to the positive working atmosphere. Perhaps this is also one of the reasons why I do not want to leave our office yet - so please stop asking.

Katherine, aside from being a great researcher you have also proven to be an exceptional neuroethicist, something that is ever more important today and truly admirable. I want to thank you for sharing all your stem cell-expertise with me and for assisting me in setting up my iPSC experiments in Maastricht.

Clara, I am still not sure whether I should talk to you in Dutch or in English or maybe use a combination of both - if that ever makes sense. I want to thank you for sharing all the ins and outs of writing a $\mathrm{PhD}$ thesis with me. This has been extremely helpful. I wish you all the best of luck during your time as a post-doc in Boston, I am sure you will do an amazing job!

Roy, Janou, Artemis and Nicole, we met each other for the first time even before my $\mathrm{PhD}$ and our paths have crossed multiple times over the years; during research electives, during (online) meetings, at conferences, in the office, in the laboratory or during the EPI-AD meetings for example. I would like to thank you all for taking me by the hand and showing me around in any of these occasions!

Last, but not least: Lars, Rick, Ali, David, Manon, Martin, Alix, Ghazi and Laurence, you have been such amazing colleagues! I would like to thank you for all the useful comments and advice on my research, presentations, laboratory issues, talks and so on. The brainstorms we had during our labmeetings were always very fruitful and rewarding. I has been a pleasure to work with you.

A special mention and thank you also goes to the technician team at the Department of Psychiatry and Neuropsychology. Hellen, Denise, Sandra, Barbie, Wouter and Theodora, you were always readily available to share your expertise, to provide me with materials or equipment, and to assist me in my experiments whenever needed. Your efforts have really helped me to develop my skillset over the years, so many thanks for that!

I also would like to thank all my other colleagues, PhD students, post-docs and senior scientists (past and present) at the Department of Psychiatry and Neuropsychology for the nice working atmosphere and for making my time as a PhD student in 
Maastricht memorable: Ellis, Glenn, Christian, Roman, Jeroen, Shengua, Tanya, Faisal, Caterina, Jackson, An, Qian, Aryo, Mathijs, Sarah, Rose, Perla, Sylvana, Christian, Jana, Daan, Roel, Marina, Maarten, Ralph, Nikita, Nynke, Faris, Martijn, Thomas, Wenting, Margot, Lonne, Wouter, Stijn, Gowoon, David, Tom, Marie-Thérèse, Ankie, Damaris, Mario, Harry, Pilar, Ali, Govert and everybody else that I might have forgotten.

I also want to thank my colleagues from BIOMED, who made my research stay in Hasselt extremely enjoyable and a great success.

Assia, it was a heroic battle, but we managed to tame the beast and the first articles are either in submission or close to being published. I really think we can pat each other on the back: "Go go Pyro Rangers!" Yes, with the beast, I mean the Pyrosequencer Q48 Autoprep, the monster that appeared at the end of our quest from BIOMED to Hasselt University, and the only thing that stood in-between us and our data. I would like to thank you for fighting this battle with me, and more importantly, for winning it together! Your enthusiasm, positive- and hard-working attitude definitely play an important part in this victory. Like Tim said: We are true "Pyro-neers"!

Melissa and Ben, I have never felt so welcomed and directly integrated into a new laboratory as compared to when I arrived for the first time to BIOMED. Mainly thanks to you! Melissa, everything you touch in the laboratory seems to turn into gold. Know that the next time when I am in desperate need for earthshattering data, you will be one of the first people I will contact to collaborate with. Ben, if you are ever in doubt whether becoming a scientist is your true ambition; perhaps you might consider finding a way to make money out of "memes". For whoever reads this: All credits and copyrights of the "Pyro Rangers" go to Ben.

Of course, I also want to thank all my friends and colleagues from AG Haaf at the Institute of Human Genetics in Wuerzburg.

Diese Felix und Diese Alex! I think we can conclude that a whole lot of Greek food, half-liter beers and Ouzo are the perfect combination to achieve the highest level of productivity. I would like to thank you both for these memorable moments in-between our hard working days, for teaching me the ins and outs of limiting dilution bisulfite pyrosequencing (Felix) and for the help with the ICT-related issues I was facing in Wuerzburg (Alex) - AZERTY keyboards and the German version of Windows are simply not my favorite. Felix, if you might ever wonder what to buy me as a gift for obtaining my PhD degree: I am still in desperate need for a CD of "Die Flippers". We should really start planning our trip to Amsterdam, invite Alex and "not limit our dilutions" at another Greek or Italian restaurant. 
Nady, thank you for helping me out in the laboratory, for being approachable for any science-related question and for being my first go-to lunch mate during my time in Wuerzburg. The way you are developing your career is truly inspiring! No doubt, you will succeed! I like to believe that you are currently driving your Ferrari, Maserati, Rolls Royce or any other supercar to the laboratory in Qatar. Please do not forget that I am still waiting for the invitation to come over and drive these supercars ones together. Perhaps we can directly plan another lunch at a fancy restaurant and talk some more science.

Larissa, you were literally just one door away in case I was in need for advice, might it be anything work-related or even life in general. You are really one of the most open and approachable people that I met during my time in Wuerzburg with whom I could share anything, even the struggles of learning a new language like Spanish. Thank you for always being there whenever necessary!

Tabea, I do not know whether I should start with thanking you or with providing you with my apologies for involving you in all these complicated Germany-NetherlandsBelgium constructions and for making you ship materials from Wuerzburg to all over Europe - let us hope the concerned companies will never read this. I guess I owe you both! It cannot be emphasized enough that you really did an amazing job!

Ruth, a big thank you to you for always being available, for saving my day so many times and for making my life in Wuerzburg much easier! Your kindness, generosity, and willingness to help others stands as a shining example for every secretary. From helping me to find a place to stay in Wuerzburg, to making sure I also could keep my place, to assisting me with filling-out legal documents, to organizing grant money and bills for my projects, to rescuing my e-mail account and so on; Not a single job was too big, for which I want to express my sincerest gratitude!

Indrajit, Caroline, Michaela, Ramya, Anna, Julia, Julia, Julia, Paulina, Barbara and Laura, I really enjoyed spending time and working together with you. Thank you for being such amazing colleagues, for introducing me to the German Biergarten, for the Weinprobe (which really could not be considered as a "tasting") and for showing me the Weinfeste in Wuerzburg. It was a whole lot of fun!

A special mention also goes to Daniel, Reinhard, Eva, Marcus, Tobias, Tamara, Julianne and everybody else from the Institute of Human Genetics that have worked with me. Thank you for your assistance and invaluable advice during these years!

Then I would like to thank everybody from the Laboratory of Translational Neuroscience in Wuerzburg, and specifically: 
Johanna, our paths in Barcelona just missed each other and when you came back to Wuerzburg, I was just about to leave and move to Maastricht. In spite of that, it always felt like we have known each other for years. Thank you for being such an extremely joyful and kind person, for introducing me to Kiliani (after my time in Wuerzburg - I clearly did something wrong there), for helping me out in the laboratory and for providing me a place to sleep whenever necessary. Know that you are always welcome and that you always will have a place to stay here with us!

Charline, Maria, and Julia, thank you for showing me the ins and outs of working with stem cells during my time in Wuerzburg. I have learned a lot from you and I am very grateful for the time and effort that you have invested in teaching me.

I also would like to thank and to express my appreciation to everybody from PEBC1 and PEBC2 at IDIBELL during my time in Barcelona.

Edilene, my favorite Brazilian! A special mention and thank you also goes to you. The energy and enthusiasm that radiates from you is truly admirable and unique! You are such an amazing and kind person. Thank you for being so patient with me and for always being there for me during my time in Barcelona. And, of course, for taking care of my ReN(zo)cells VM, whenever I decided to travel back to the Netherlands - I bet people are still trying to figure out whether we came up with this name ourselves. It really was a blast getting to know you and I truly hope our paths will cross once again soon!

Davide, who would ever expect that it is possible to obtain a $\mathrm{PhD}$ degree by just putting water into water the entire day? If you are still in doubt, please find proof of that here. Thank you for being a great friend and for being the most stereotypical Italian person in existence - Let's face it: During our chats, your hands and fingers were always doing the talking. Our time together in Barcelona was really too short! We should meet up once again soon to catch-up on life while enjoying a game of "Exploding Kittens" and your mother's home-made Limoncello.

Sònia, David, Catia, Cristina, Humberto, Raquel, Fer, Pere, Miguel, Aida, Sebas, Carles, Maxime, Holger, Manu, Laia, Carmen, Anna, Marta, Rute and Olga. Thanks for making me feel home regardless of my very poor Spanish or Catalan back then, for guiding me, for sharing all your expertise and for the amazing gatherings outside of the laboratory. My time in Barcelona has really been one of the highlight during my time as a PhD- and as a research master student. Some of the Spanish habits that I acquired during my time with you, such as the time and size of meals for example, will be forever engrained in my daily routines. Thank you for the amazing memories! I look back with a lot of joy and a smile on my face! 
I am also grateful to my wonderful Bachelor and Master students: Dasha, Natalie, Julianne, Manas, Sonia and Ziva. Thank you not only for your contribution to my projects, but also for everything that I have learned from you. I hope that you all enjoyed your internships and that you learned as much from me as I learned from you! Please keep me up-to-date about all your future endeavors; I am sure you will all achieve great success!

Of course, I also would like to thank everybody outside of academia and my work, and specifically my closest friends and family.

Jonas, Koen, Tijs, Fieneke, Niels, Wouter, Chaira, Tom, Marlon, Joeri, Sam, Brian and Thomas, you really helped me to find the right balance between work and life, and were always there so I could take my mind off work and clear my head. I want to thank you for being part of my life, for all the joyful moments during parties, gatherings, festivals or visits, and for the post-cards or messages that I received over the years while being in the distance - This really kept me going.

This also applies to Michiel. We started our studies at the same time in Maastricht and soon we both will be doctors - Although you are the only one that can really treat patients, but that is just a minor detail. I think we can be proud! Who would have ever expected that?

Then fom the bottom of my heart, I am extremely grateful to my parents, Willem and Ingeborg, and to my brother, Silvano. I am so blessed to have you in my life. I want to thank you deeply for all what you have done for me, for your endless support and encouragement, for all the sacrifices that you made, for helping me move around all the time, for all the guidance that you gave me and for molding me into the person that I have become today. Without you I would not have made it this far! Really!

Anaís, you have been my solid rock, my other better half and the person that has always been there for me during these years. You are the one who knows me best, both during good and during bad times, the one that cheers me up and the one that picks me up to put me back on my feet whenever necessary. Thanks for helping me to put things in perspective, for believing in me, for reminding me of my own capabilities and for simply being the person who you are. I am really looking forward to the time and adventures that lay ahead of us!

Finally, I would like take a moment to acknowledge my grand- and godparents, Jan and Maria, who have always been a true inspiration and a strong drive behind my success. Your memory will be forever with me. 
